\title{
NEBULISERS FOR THE GENERATION OF LIPOSOMAL AEROSOLS
}

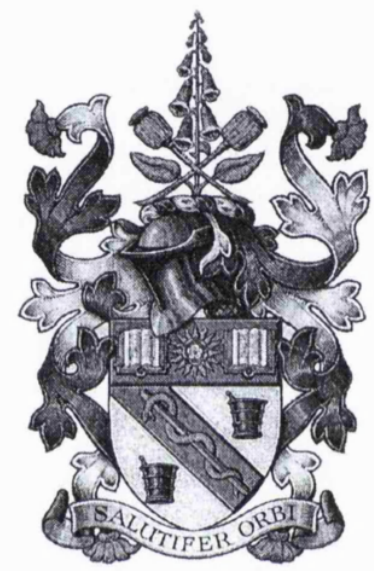

\section{Paul Anthony Bridges \\ BPharm MRPharmS}

\author{
School of Pharmacy \\ University of London \\ London
}

June 1997

Submitted in fulfilment of the requirements for the degree of Doctor of Philosophy 
ProQuest Number: 10104800

All rights reserved

INFORMATION TO ALL USERS

The quality of this reproduction is dependent upon the quality of the copy submitted.

In the unlikely event that the author did not send a complete manuscript and there are missing pages, these will be noted. Also, if material had to be removed, a note will indicate the deletion.

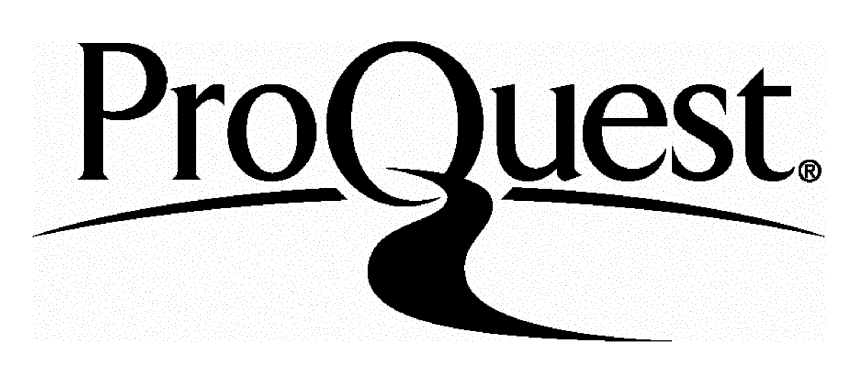

ProQuest 10104800

Published by ProQuest LLC(2016). Copyright of the Dissertation is held by the Author.

All rights reserved.

This work is protected against unauthorized copying under Title 17, United States Code. Microform Edition $\odot$ ProQuest LLC.

\author{
ProQuest LLC \\ 789 East Eisenhower Parkway \\ P.O. Box 1346 \\ Ann Arbor, MI 48106-1346
}




\begin{abstract}
The thesis details investigations into the use of various types of medical nebuliser for the generation of liposomal aerosols. Chapter 1 provides a comprehensive introduction to the theory and practice of drug delivery to the respiratory tract. It also reviews the potential applications of inhaled liposomes to drug delivery, and the devices used to generate liposomal aerosols.
\end{abstract}

A preliminary investigation into the physicochemical attributes of liposomes which may. influence aerosol generation is detailed in chapter 2 . These include studies of the stability of liposome bilayers to disruptive energy (ie. membrane extrusion and sonication), investigations of the surface tension and viscosity of liposomes, and also the release of a liposomally entrapped hydrophilic marker. Chapter 3 demonstrates how these physicochemical attributes may influence nebulised liposomal aerosols. Most notably, the aerosol droplet size and output rate are proportional to the concentration of the formulation. The residual liposome concentration is determined by the mean liposome size. The droplet size, nebulisation time, aerosol output, and residual liposome concentration are all significantly influenced by the particular jet nebuliser model selected for aerosol generation. The relative stability of different liposome formulations to nebulisation is also determined by a variety of factors, as revealed in chapter 4 of the thesis. The release of an entrapped marker, and the reduction in liposome size during nebulisation, is influenced by the nebuliser model, and also the liposome size and bilayer composition.

Chapter 5 investigates the freeze drying of liposomes. The chapter concludes that liposomes for nebulisation may be freeze dried in the presence of a cryoprotectant, without influencing the aerosol produced following subsequent rehydration. Chapter 6 reveals that the droplet size produced from ultrasonic nebulised liposomes is relatively large, and the output inefficient from concentrated formulations. In addition, ultrasonic nebulisation is associated with similar liposome stability concerns as jet nebulisation. 
Title page

Abstract.

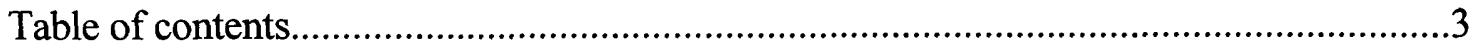

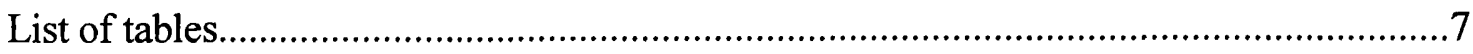

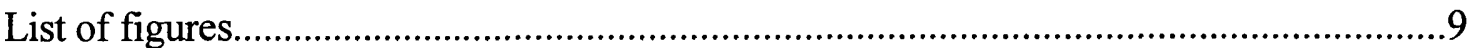

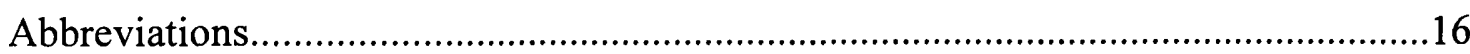

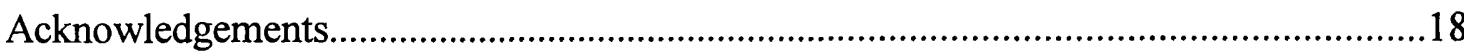

\section{INTRODUCTION}

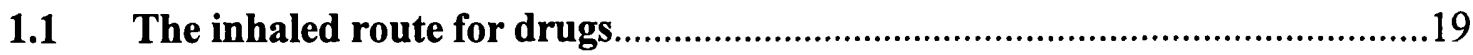

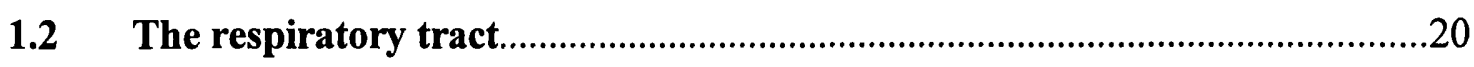

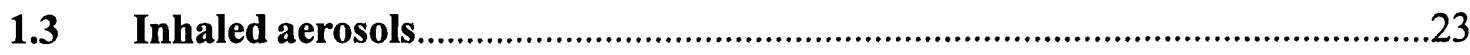

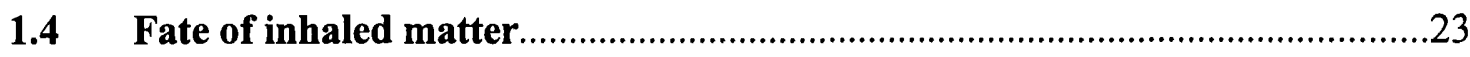

1.4.1 Deposition of particles.........................................................................23

1.4.2 Clearance of deposited particles.............................................................26

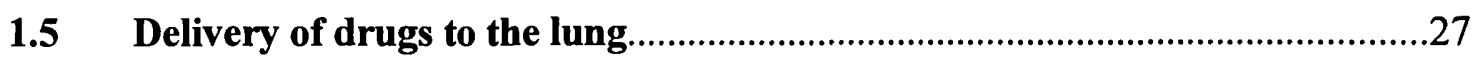

1.5.1 Inhaled drugs for local action...............................................................27

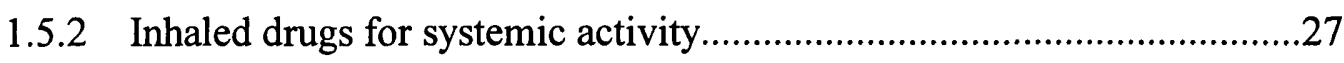

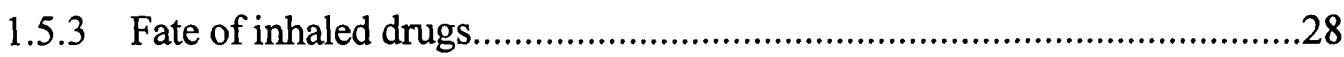

1.5.4 Characteristics of therapeutic aerosols..................................................30

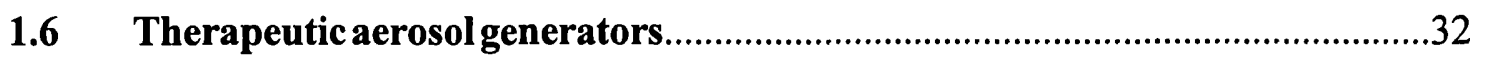

1.6.1 Metereddoseinhalers............................................................................32

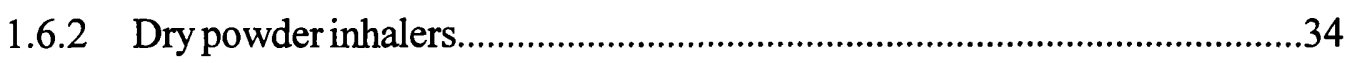

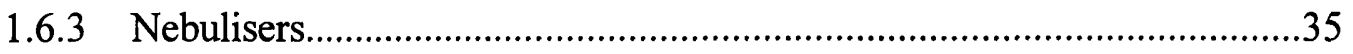

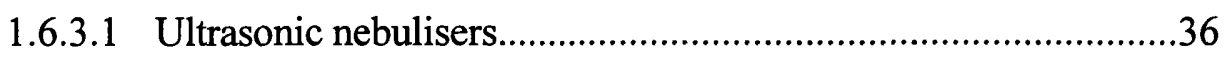

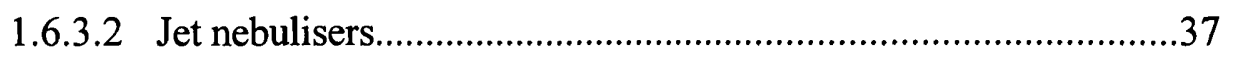

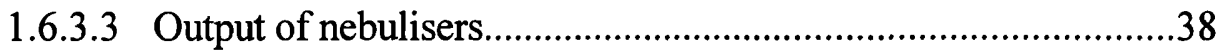

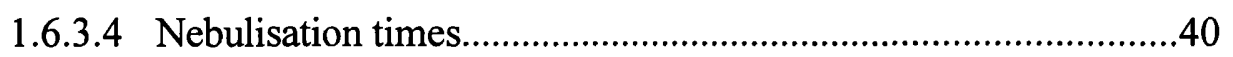

1.6.3.5 Temperature and concentration effects.......................................40

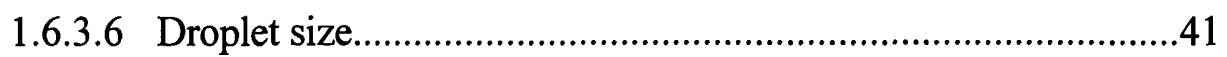

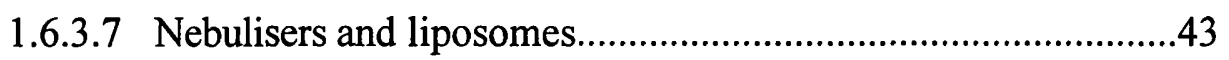

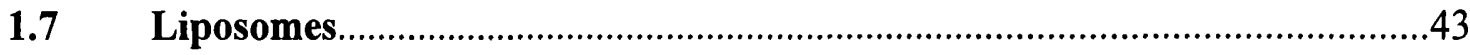

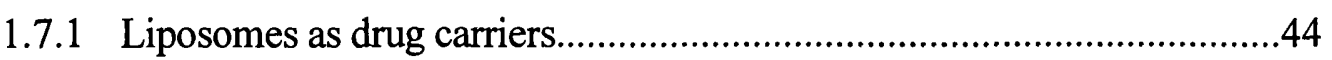

1.7.2 Medical applications of liposomes......................................................46

1.7.3 Disadvantages of liposomes as drug carriers...........................................48

1.8 The pulmonary delivery of liposomes..........................................................48

1.9 Liposomes as drug carriers to the respiratory tract.......................................52

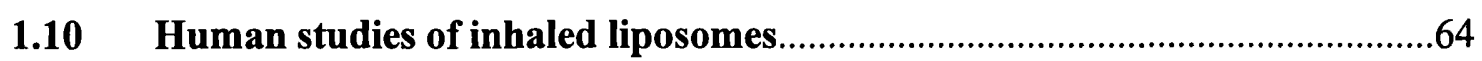


1.11 Devices used for the aerosolisation of liposomes .68

1.12 Aims of the study. .71

2 PHYSICOCHEMICAL PROPERTIES OF LIPOSOMES

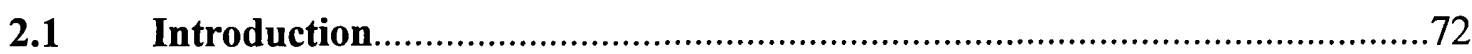

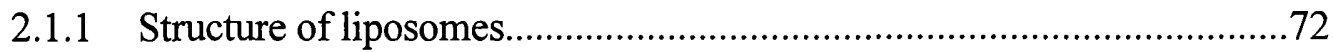

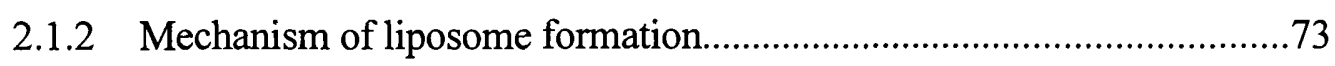

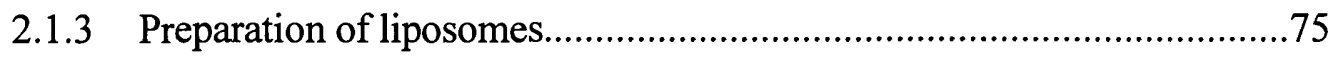

2.1.4 Release of entrapped agents from liposomes.......................................78

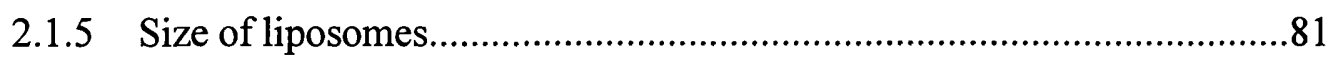

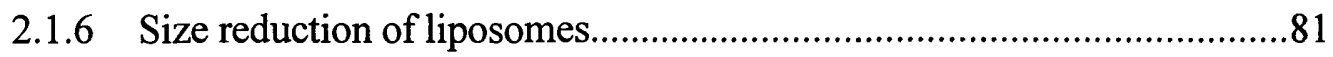

2.1.7 Entrapment and release of soluble solutes by liposomes...........................82

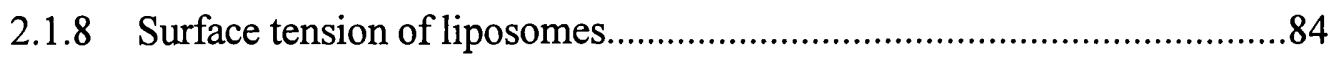

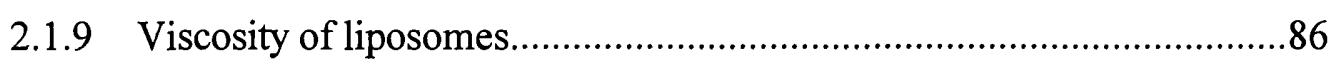

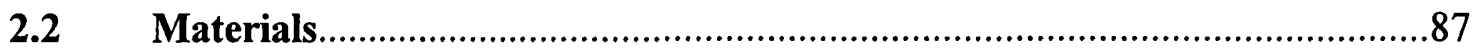

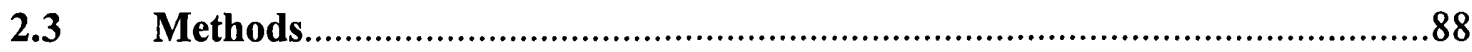

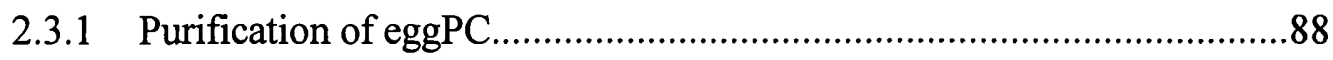

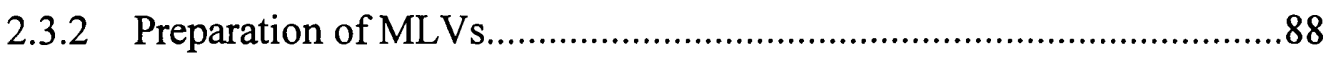

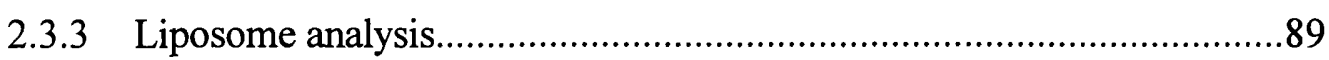

2.3.4 Size reduction of prepared liposomes.............................................89

2.3.4.1 Extrusion through polycarbonate membrane filters................89

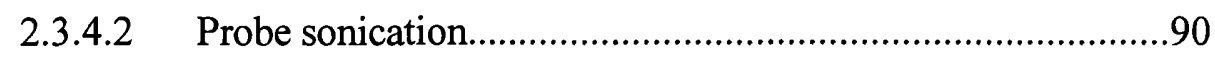

2.3.5 Liposomal entrapment and release of SCG.....................................90

2.3.5.1 SCG standard curve.......................................................90

2.3.5.2 Preparation of SCG-containing liposomes..............................91

2.3.5.3 Assessment of SCG entrapment in SCG-containing liposomes..........................................................................91

2.3.5.4 The release of SCG from SCG-containing liposomes...............91

2.3.6 The surface tension of liposomes...................................................92

2.3.7 The viscosity of liposomes............................................................92

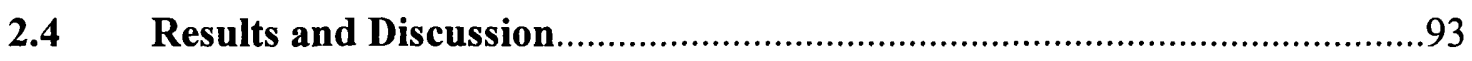

2.4.1 Purification of eggPC....................................................................93

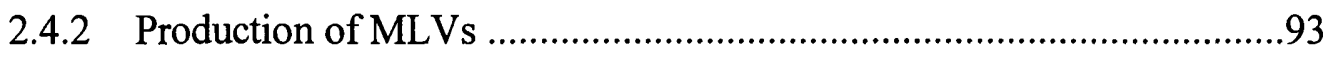

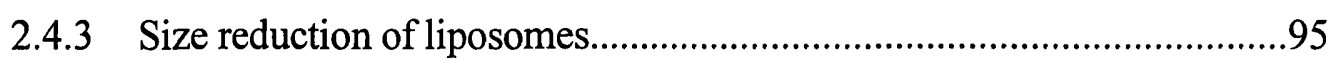

2.4.4 Entrapment of SCG by liposomes................................................97

2.4.5 Release of SCG from SCG-containing liposomes................................100

2.4.6 Surface tension of liposomes................................................................. 104

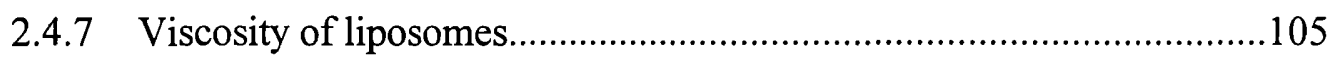

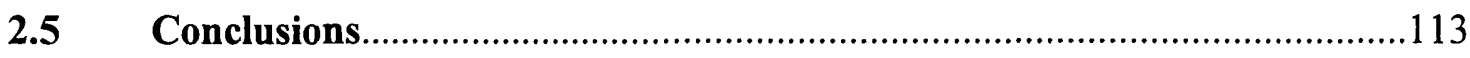



FROM JET NEBULISERS

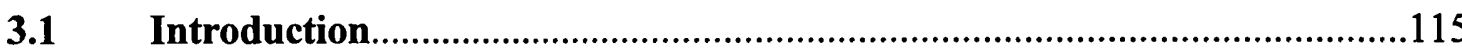

3.1.1 Sizing of nebulised droplets........................................................119

3.1.2 The output of liposomal aerosols from jet nebulisers.........................123

3.1.3 Concentration of liposomes during nebulisation.................................124

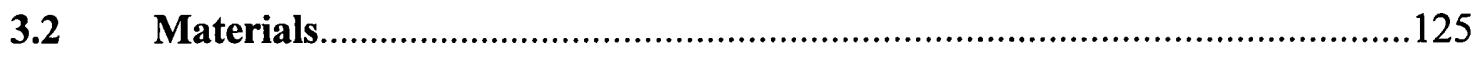

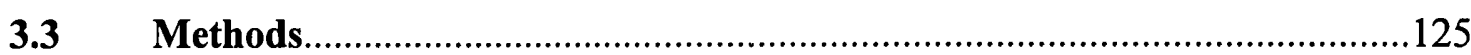

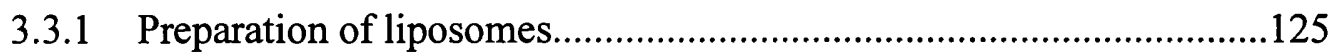

3.3.2 Assessment of liposomal aerosols.....................................................126

3.3.3 The effects of liposome concentration on emitted droplet size.............126

3.3.4 The influence of lipid concentration on the aerosol output.....................127

3.3.5 Concentration of liposomes during nebulisation................................127

3.3.6 Examination of nebuliser component orifices...................................128

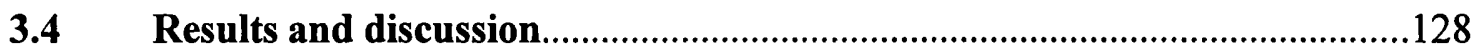

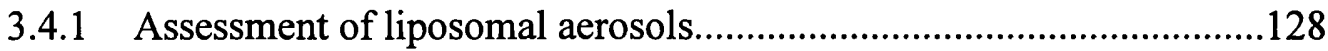

3.4.2 Effects of liposome concentration on droplet size..............................136

3.4.3 The output of liposomal aerosols from jet nebulisers...........................150

3.4.4 Concentration of liposomes during nebulisation................................159

3.4.5 Examination of nebuliser component orifices......................................173

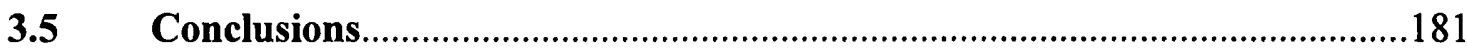

4 THE STABILITY OF LIPOSOMES TO NEBULISATION

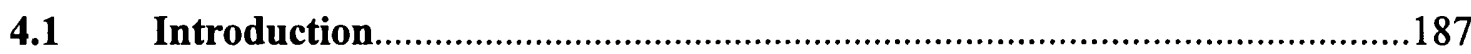

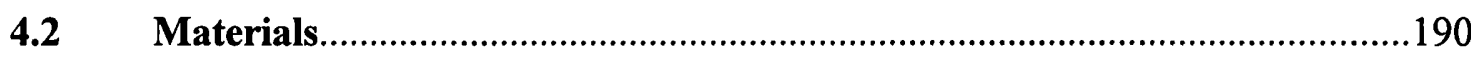

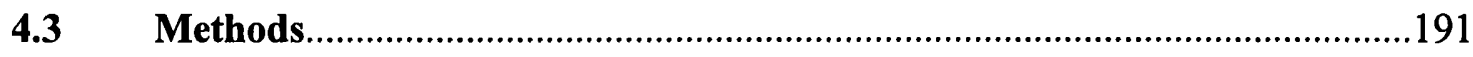

4.3.1 The effects of nebulisation on the size of residual liposomes..................191

4.3.2 The influence of liposome size and nebulisation time on the stability of liposomes to nebulisation...................................................191

4.3.3 The release of SCG from SCG-containing liposomes during

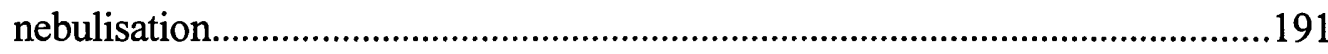

4.3.4 The sampling of aerosolised liposomes.............................................193

4.3.5 The intra-nebuliser variability in the generation of liposomal aerosols.. 193

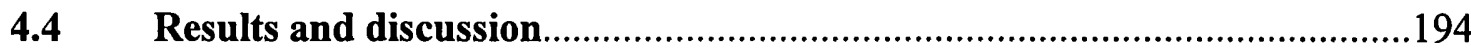

4.4.1 The effects of nebulisation on the size of residual liposomes.................194

4.4.2 The influence of liposome size and nebulisation time on residual liposomes...................................................................201

4.4.3 The release of SCG from liposomes during nebulisation..................211

4.4.4 The collection of aerosolised liposomes.........................................221

4.4.5 The intra-nebuliser variability in the generation of liposomal aerosols. 231

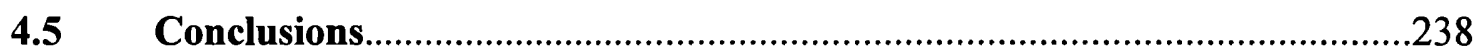


5 FREEZE DRYING OF LIPOSOMES FOR NEBULISATION

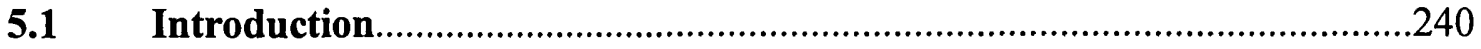

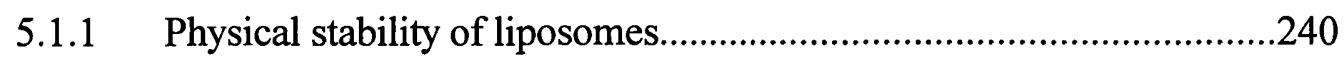

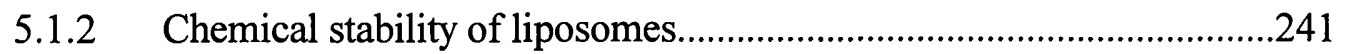

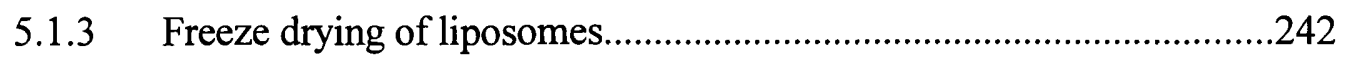

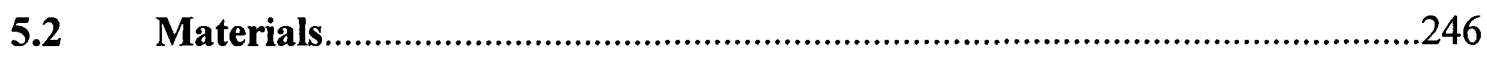

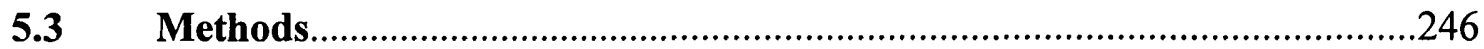

5.3.1 Freeze drying ofliposomes.............................................................246

5.3.2 Nebulisation of rehydrated freeze dried liposomes.........................247

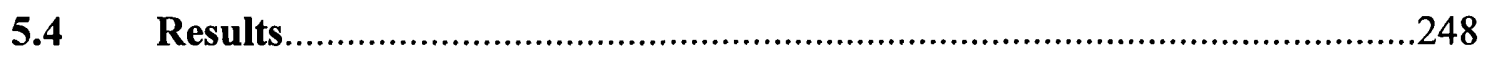

5.4.1 Freeze drying of liposomes............................................................248

5.4.2 Nebulisation of rehydrated freeze dried liposomes..............................260

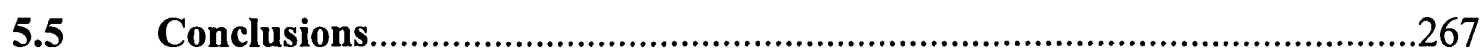

6 ULTRASONIC NEBULISATION OF LIPOSOMES

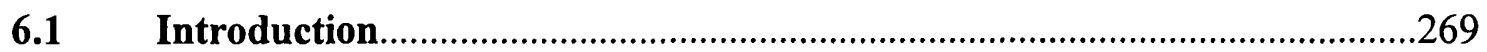

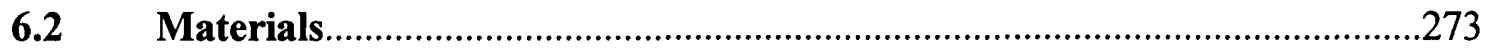

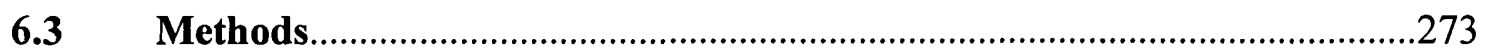

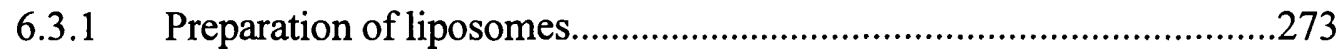

6.3.2 Characterization of liposomal aerosols produced by the

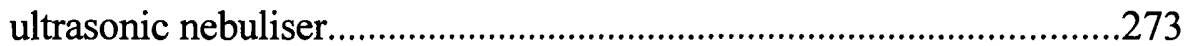

6.3.3 Characterization of liposomes delivered in aerosols produced by the ultrasonic nebuliser..............................................273

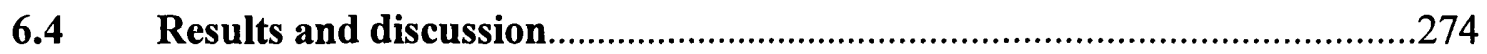

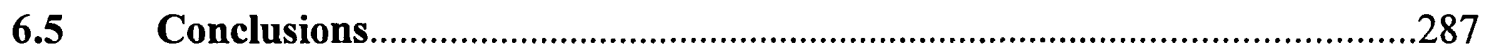

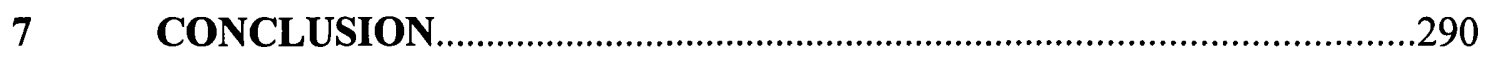

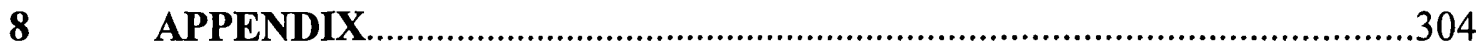

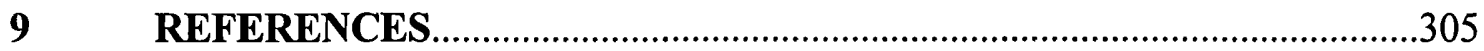




\section{LIST OF TABLES}

Table 2.1 Fatty acid composition of phosphatidylcholine extracted from eggs (eggPC) and soy bean (soyPC). .73

Table 2.2 Temperatures of the principle phase transition ( $\mathrm{Tm})$ of phospholipid commonly used in the preparation of liposomes. .79

Table 2.3 Settings for the Carri-Med CSL Controlled Stress Rheometer. 92

Table 2.4 Mean size (VMD) and span of liposomes prepared by the thin-film technique... 93

Table 2.5 Reduction in mean liposome size by sonication. .94

Table 2.6 Entrapment of SCG by different liposome formulations as determined by UV assay. .99

Table 2.7 Entrapment of SCG by SCG-containing liposomes. .98

Table 2.8 Half life of SCG release from SCG-containing liposomes. 103

Table 3.1 The effect of the driving gas flow rate on the size (VMD), and span of liposomal aerosols

Table 3.2 Influence of the lipid concentration of nebulised liposomes on the mean droplet VMD produced by various jet nebulisers. 140

Table 3.3 Output (\% of total mass added) of eggPC/chol (1:1) liposomes nebulised from two jet nebulisers.

Table 3.4 Increase in the residual sodium chloride $(\mathrm{NaCl})$ concentration following nebulisation with various jet nebulisers. 160

Table 3.5 Increase in the residual lipid concentration following nebulisation... 161

Table 4.1 The mean droplet VMD produced by various jet nebulisers during nebulisation of eggPC liposomes ( $5 \mu \mathrm{m}$ mean size, $20 \mathrm{mg} / \mathrm{ml}$ lipid concentration) at $81 / \mathrm{min}$ .194

Table 4.2 Reduction in the mean size of $5 \mu \mathrm{m}$ liposomes following nebulisation as a function of the bilayer composition. 196

Table 4.3 Reduction in the span of residual $5 \mu \mathrm{m}$ mean size eggPC and eggPC/chol (1:1) liposomes during nebulisation with various jet nebulisers. 200

Table 4.4 The mean droplet VMD and time taken for the aerosol output to become Intermittent for each jet nebuliser (calculated from the nebulisation of $20 \mathrm{mg} / \mathrm{ml} 5 \mu \mathrm{m}$ eggPC liposomes with a flow rate of $7 \mathrm{~L} /$ minute). 202

Table 4.5 Size of residual liposomes following nebulisation of liposome formulations with an initial mean size of 2.5 and $5 \mu \mathrm{m}$, to $50 \%$ and $100 \%$ of the nebulisation time...203 
Table 4.6 Decrease in the mean size of residual liposomes following the nebulisation of liposome formulations with an initial mean size of 2.5 and $5 \mu \mathrm{m}$ to $50 \%$ and $100 \%$ of the nebulisation time.

Table 4.7 Rate of residual liposome size reduction following nebulisation of 5 and 2.5 $\mu \mathrm{m}$ liposomes to $50 \%$ and $100 \%$ of the nebulisation time. .208

Table 4.8 Rate of residual liposome size reduction during nebulisation as a function of the initial liposome size, and bilayer composition (mean of all nebulisers)

Table 4.9 Entrapment of SCG by variously sized eggPC and eggPC/chol (1:1)liposomes (\%/100 mg lipid).

Table 4.10 Loss of SCG (mg/ml of residual liposomes) from eggPC and eggPC/chol (1:1) ( 2.5 and $5 \mu \mathrm{m}$ initial mean size) liposome formulations during nebulisation at gas flow rates of 4 and $7 \mathrm{~L} /$ minute .214

Table 4.11 The size of residual liposomes, and liposomes deposited within the upper and lower stages of the TI following nebulisation of $5 \mu \mathrm{m}$ liposomes with the Pari-LC and Sidestream nebulisers for $50 \%$ and $100 \%$ of the nebulisation time.

Table 4.12 The size of residual liposomes, and liposomes deposited within the upper and lower stages of the TI following nebulisation of $2.5 \mu \mathrm{m}$ liposomes with the Pari-LC and Sidestream nebulisers for $50 \%$ and $100 \%$ of the nebulisation time. .222

Table 4.13 The mean VMD and span of aerosols emitted from the Pari-LC and Sidestream nebulisers at different driving gas flows. .231

Table 5.1 The size characteristics of eggPC and eggPC/chol (1:1) liposome formulations, freeze-dried in the presence or absence of trehalose (tr). .249

Table 5.2 The VMD $(\mu \mathrm{m})$ and span of aerosol droplets produced during the nebulisation of freeze-dried and rehydrated liposomes (f-d-r), and non-freeze-dried liposomes with the Pari-LC nebuliser .260

Table 5.3 The rate of liposome size reduction during nebulisation of liposomes freezedried in the presence/absence of trehalose $(+\mathrm{Tr} /-\mathrm{Tr})$, and that of non-freeze-dried eggPC/chol (1:1) liposome formulations. .262

Table 6.1 Mean size and span of liposomes remaining in the nebuliser reservoir during nebulisation in a Medix Electronic nebuliser. .281

Table 6.2 The size of residual liposomes, and liposomes deposited within each stage of the TI at $50 \%$ and $100 \%$ (300 s and $600 \mathrm{~s}$ for the Medix) of the nebulisation time. .285

Table 6.3 The size of residual liposomes, and liposomes deposited within each stage of the TI, following nebulisation to $50 \%$ and $100 \%$ of the nebulisation time with jet nebulisers. .285 


\section{LIST OF FIGURES}

Figure 1.1 The human lung and the generations of the airways...................................20

Figure 1.2 The metered dose inhaler, with attached spacer device..............................32

Figure 1.3 The Spinhaler ${ }^{\circledR}$ dry powder inhaler device................................................ 34

Figure 1.4 The Pari IS-2, an example of a modern jet nebuliser....................................37

Figure 1.5 Structure of the liposome in its different forms.........................................44

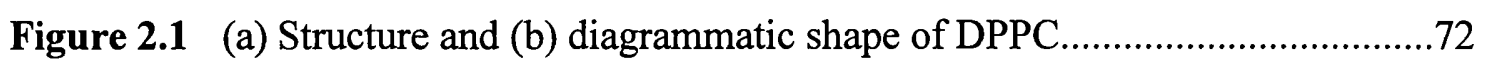

Figure 2.2 The packing arrangement (phase) of different phospholipids when added to an aqueous environment, and its dependence on molecular shape. .74

Figure 2.3 The reversible main phase transition of liposome bilayers from the ordered ( $\mathrm{gel}$ ) to the disordered (liquid crystalline) state that occurs as temperature is increased $(+\Delta \mathrm{T})$ above the transition temperature $\left(T_{m}\right)$ of the phospholipids................................78

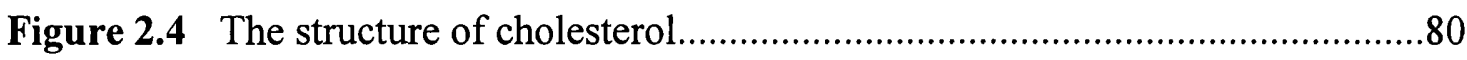

Figure 2.5 The reduction in the mean size (VMD) of eggPC, eggPC/chol (1:1), and $\mathrm{DPPC} / \mathrm{chol}(1: 1)$ liposomes, following extrusion through 5, 3 and $1 \mu \mathrm{m}$ membrane filters.

Figure 2.6 The reduction in the mean size (VMD) of eggPC, eggPC/chol (1:1), and $\mathrm{DPPC} / \mathrm{chol}(1: 1)$ liposomes, following probe sonication to approximately $1 \mu \mathrm{m} . \ldots \ldots \ldots . . .96$

Figure 2.7 Calibration curve for the UV absorbance of SCG solutions...........................97

Figure 2.8 Release of SCG from eggPC MLVs........................................................ 101

Figure 2.9 Release of SCG from eggPC/chol (1:1) MLVs.......................................101

Figure 2.10 Release of SCG from DPPC/chol (1:1) MLVs........................................... 102

Figure 2.11 The surface tension of eggPC and eggPC/chol (1:1) liposomes, and the influence of increasing the lipid concentration.

Figure 2.12 Rheological response (shear rate $v s$ shear stress) of eggPC liposomes of varying concentration

Figure 2.13 Rheological response (shear rate $v s$ shear stress) of eggPC/chol $(1: 1)$ liposomes of varying concentration. .106

Figure 2.14 The relationship between the lipid concentration and the viscosity of eggPC and eggPC/chol (1:1) liposomes. Shear stress $=0.75 \mathrm{Nm}^{-2}, 0.52 \mathrm{Nm}^{-2}$ .107

Figure 2.15 The viscosity response of eggPC liposomes of varying lipid concentration as the applied shear stress is increased. 108 
Figure 2.16 The viscosity response of eggPC/chol (1:1) liposomes of varying lipid concentration as the applied shear stress is increased.................................................108

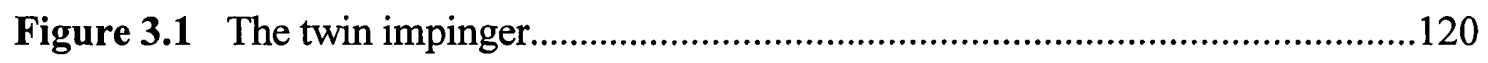

Figure 3.2 Arrangement of optical components in the Malvern 2600c laser diffraction sizer.

Figure 3.3 The Malvern 2600c laser diffraction sizer equipment, used to analyse a nebulised aerosol.

Figure 3.4 The mean volume median diameter (VMD) of aerosols delivered from the Cirrus (a) and Pari-LC (b) nebulisers during nebulisation of eggPC liposomes.. 129

Figure 3.5 The mean volume median diameter (VMD) of aerosols delivered from the Sidestream (a) and Ventstream (b) nebulisers during nebulisation of eggPC liposomes...130 Figure 3.6 Effect of driving gas flow rate on the volume median diameter (VMD) of droplets produced from the Cirrus, Pari-LC, Sidestream and Ventstream nebulisers. 133

Figure 3.7 Effect of driving gas flow rate on the span of droplets produced from the Cirrus, Pari-LC, Sidestream and Ventstream nebulisers. .134

Figure 3.8 Mean VMD of droplets emitted from the Cirrus nebuliser with time, during nebulisation of (a) eggPC, $1 \mu \mathrm{m}$; (b) eggPC/chol, $1 \mu \mathrm{m}$; (c) eggPC, $5 \mu \mathrm{m}$; and (d) eggPC/chol, $5 \mu \mathrm{m}$ liposomes at lipid concentrations of $2.5 \mathrm{mg} / \mathrm{ml}, 5 \mathrm{mg} / \mathrm{ml}, 10 \mathrm{mg} / \mathrm{ml}, 20$ $\mathrm{mg} / \mathrm{ml}, 40 \mathrm{mg} / \mathrm{ml}, 80 \mathrm{mg} / \mathrm{ml}$.

Figure 3.9 Mean VMD of droplets emitted from the Pari-LC nebuliser with time, during nebulisation of (a) eggPC, $1 \mu \mathrm{m}$; (b) eggPC/chol, $1 \mu \mathrm{m}$; (c) eggPC, $5 \mu \mathrm{m}$; and (d) eggPC/chol, $5 \mu \mathrm{m}$ liposomes with lipid concentrations of $2.5 \mathrm{mg} / \mathrm{ml}, 5 \mathrm{mg} / \mathrm{ml}, 10 \mathrm{mg} / \mathrm{ml}$, $20 \mathrm{mg} / \mathrm{ml}, 40 \mathrm{mg} / \mathrm{ml}$, and $80 \mathrm{mg} / \mathrm{ml}$. .138

Figure 3.10 Mean VMD of droplets emitted from the Sidestream nebuliser with time, during nebulisation of (a) eggPC, $1 \mu \mathrm{m}$; (b) eggPC/chol, $1 \mu \mathrm{m}$; (c) eggPC, $5 \mu \mathrm{m}$; and (d) eggPC/chol, $5 \mu \mathrm{m}$ liposomes with lipid concentrations of $2.5 \mathrm{mg} / \mathrm{ml}, 5 \mathrm{mg} / \mathrm{ml}, 10 \mathrm{mg} / \mathrm{ml}$, $20 \mathrm{mg} / \mathrm{ml}, 40 \mathrm{mg} / \mathrm{ml}$, and $80 \mathrm{mg} / \mathrm{ml}$.

Figure 3.11 Change in the mean VMD of aerosol droplets produced from the nebulisation of eggPC and eggPC/chol liposomes as the lipid concentration is increased.

Figure 3.12 Influence of lipid concentration on the polydispersity of aerosol droplets produced during the nebulisation of $1 \mu \mathrm{m}$ eggPC and $5 \mu \mathrm{m}$ eggPC; $1 \mu \mathrm{m}$ eggPC/chol (1:1) and, $5 \mu \mathrm{m}$ eggPC/chol (1:1) liposome using (a) Cirrus (b) Pari-LC and (c ) Sidestream nebulisers. .142

Figure 3.13 Schematic diagram of droplet formation from an nebuliser nozzle. 143 
Figure 3.14 Increase in the mean droplet size (VMD) between the initial 90s and final 90 seconds of nebulisation, for the Cirrus, Pari-LC and Sidestream nebulisers

Figure 3.15 The output of nebulised $1 \mu \mathrm{m}$ and $5 \mu \mathrm{m}$ mean size eggPC/chol (1:1) liposomes from the Pari-LC nebuliser as a function of the lipid concentration

Figure 3.16 The output of nebulised $1 \mu \mathrm{m}$ and $5 \mu \mathrm{m}$ mean size eggPC/chol $(1: 1)$ liposomes from the Sidestream nebuliser as a function of the lipid concentration.

Figure 3.17 Rate of fluid output from the Pari-LC nebuliser during nebulisation of $1 \mu \mathrm{m}$ mean size eggPC/chol $(1: 1)$ liposomes.

Figure 3.18 Rate of fluid output from the Pari-LC nebuliser during nebulisation of $5 \mu \mathrm{m}$ mean size eggPC/chol (1:1) liposomes.

Figure 3.19 Rate of fluid output from the Sidestream nebuliser during nebulisation of 1 $\mu \mathrm{m}$ mean size eggPC/chol (1:1) liposomes. 156

Figure 3.20 Rate of fluid output from the Sidestream nebuliser during nebulisation of $5 \mu \mathrm{m}$ mean size eggPC/chol (1:1) liposomes. 156

Figure 3.21 Increase in the residual sodium chloride concentration following nebulisation as a function of the nebulisation time of each nebuliser studied. .161

Figure 3.22 Increase in the residual lipid concentration of (a) approximately $0.6 \mu \mathrm{m}$ mean size liposomes, and (b) $1 \mu \mathrm{m}$ mean size liposomes, as a function of the nebulisation time of different nebulisers. 164

Figure 3.23 Increase in the residual lipid concentration of (a) $2.5 \mu \mathrm{m}$ mean size liposomes, and (b) $5 \mu \mathrm{m}$ mean size liposomes, as a function of the nebulisation time of different nebulisers. 165

Figure 3.24 Increase in the residual lipid concentration during nebulisation of variously sized liposomes. 166

Figure 3.25 Increase in the residual lipid concentration of liposomes following nebulisation as a function of the mean liposome size (mean of all nebulisers studied)....166 Figure 3.26 Increase in the residual lipid concentration of liposomes following nebulisation with the Cirrus (a) and Pari-LC (b) nebulisers. 168

Figure 3.27 Increase in the residual lipid concentration of liposomes following nebulisation with Respirgard II (a) and Sidestream nebulisers.

Figure 3.28 Increase in the residual lipid concentration of liposomes during nebulisation (mean of all liposome sizes) as a function of the volume median diameter (VMD) of droplets produced by various nebuliser models. 170 
Figure 3.29 Increase in the lipid concentration of residual liposomes during nebulisation of approximately $0.6 \mu \mathrm{m}$ (a), and $1 \mu \mathrm{m}$ mean size liposomes (b), as a function of the mean droplet size (VMD) produced by the Cirrus, Pari-LC, Respirgard II, and Sidestream nebulisers. .171

Figure 3.30 Increase in the lipid concentration of residual liposomes during nebulisation of approximately $2.5 \mu \mathrm{m}$ (a) and $5 \mu \mathrm{m}$ mean size liposomes (b), as a function of the mean droplet size (VMD) produced by the Cirrus, Pari-LC, Respirgard II, and Sidestream nebulisers. .172

Figure 3.31 Schematic diagrams of the various orifices within each jet-nebuliser with baffles and unit top/mouthpiece removed (not to scale). Letters/numerals in each diagram refer to the various scanning electron micrographs illustrated in figures 3.30 to $3.32 \ldots . . .175$ Figure 3.32 Scanning electron micrographs of the various orifices within the Pari-LC jetnebuliser.

Figure 3.33 Scanning electron micrographs of the various orifices within the Respirgard II jet-nebuliser.

Figure 3.34 Scanning electron micrographs of the various orifices within the Cirrus and Sidestream nebulisers. .178

Figure 3.35 Relationship between the diameter of the gas jet orifice of various jet nebulisers and the size of aerosol droplets produced. 180

Figure 4.1 The mean size of residual eggPC and DPPC/chol (1:1) liposomes during nebulisation with the (a) Cirrus (b) Pari-LC (c) Respirgard II and (d) Sidestream nebulisers. .195

Figure 4.2 The relationship between the droplet size produced by a jet nebuliser and the percentage reduction in the mean residual liposome size .198

Figure 4.3 The effect of nebulisation with various jet nebulisers on the polydispersity (span) of vesicle sizes within eggPC and DPPC/chol (1:1) residual liposomes. .200 Figure 4.4 The effect of nebulisation with the (a) Cirrus and (b) Pari-LC nebuliser on the residual liposome size of eggPC and eggPC/chol (1:1) liposomes with an initial mean size of $5 \mu \mathrm{m}$ and $2.5 \mu \mathrm{m}$. .205

Figure 4.5 The effect of nebulisation with the (a) Respirgard II and (b) Sidestream nebuliser on the residual liposome size of eggPC and eggPC/chol (1:1) liposomes with an initial mean size of $5 \mu \mathrm{m}$ and $2.5 \mu \mathrm{m}$. .206 
Figure 4.6 The relationship between the droplet size produced by a jet nebuliser and the reduction in the mean vesicle size caused to residual (a) eggPC and (b) eggPC/chol (1:1) $5 \mu \mathrm{m}$ and $2.5 \mu \mathrm{m}$ liposomes. .210

Figure 4.7 The proportion of liposomally entrapped SCG released from 5, 2.5 and $1 \mu \mathrm{m}$ mean size eggPC and eggPC/chol (1:1) liposomes during nebulisation with the Pari-LC nebuliser, operated at a driving gas flow rate of (a) $4 \mathrm{~L} /$ minute and (b) $7 \mathrm{~L} /$ minute......215 Figure 4.8 The proportion of liposomally entrapped SCG released from 5, 2.5 and $1 \mu \mathrm{m}$ mean size eggPC and eggPC/chol (1:1) liposomes during nebulisation with the Sidestream nebulisers, operated at a driving gas flow rate of (a) $4 \mathrm{~L} /$ minute and (b) $7 \mathrm{~L} /$ minute.....216 Figure 4.9 The relationship between the initial size of eggPC and eggPC/chol (1:1) liposomes and the fraction of entrapped SCG released during nebulisation (mean of all nebulisers studied

Figure 4.10 The mean size of residual liposomes and liposomes deposited in the upper and lower stages of the twin impinger during nebulisation of liposomes with an initial mean size of $2.5 \mu \mathrm{m}$.

Figure 4.11 The mean size of residual liposomes and liposomes deposited in the upper and lower stages of the twin impinger during nebulisation of liposomes with an initial mean size of $5 \mu \mathrm{m}$ .224

Figure 4.12 The distribution of vesicle sizes in the population of residual liposomes and liposomes deposited in the upper and lower stages of the twin impinger during nebulisation of liposomes with an initial mean size of $2.5 \mu \mathrm{m}$. .225

Figure 4.13 The distribution of vesicle sizes in the population of residual liposomes and liposomes deposited in the upper and lower stages of the twin impinger during nebulisation of liposomes with an initial mean size of $5 \mu \mathrm{m}$. .226

Figure 4.14 The mean volume median diameter (VMD) and span of aerosols produced from eggPC/chol (1:1) liposomes using five different jet nebulisers of the same Pari-LC (numbered 1 to 5) driven at driving gas flow rates of 4 and $7 \mathrm{~L} /$ minute

Figure 4.15 The mean volume median diameter (VMD) and span of aerosols produced eggPC/chol (1:1) liposomes using five different jet nebulisers of the Sidestream model (numbered 1 to 5) driven at driving gas flow rates of 4 and $7 \mathrm{~L} /$ minute. .233

Figure 4.16 The relationship between the mean aerosol droplet size produced by five different Pari-LC nebulisers (operated at driving gas flow rates of 4 and $7 \mathrm{~L} /$ minute) and the reduction in the residual eggPC and eggPC/chol (1:1) liposome size .235 
Figure 4.17 The relationship between the mean aerosol droplet size produced by five different Sidestream nebulisers (operated at driving gas flow rates of 4 and $7 \mathrm{~L} /$ minute) and the reduction in the residual eggPC and eggPC/chol (1:1) liposome size....................236

Figure 5.1 The phase diagram and eutectic point for water.....................................243

Figure 5.2 (a) The mean size and (b) polydispersity (as determined by the span) of reconstituted eggPC $(\square / /)$ and eggPC/chol (1:1) liposome formulations previously freezedried in the presence and absence of trehalose.

.250

Figure 5.3 The change in (a) the mean size and (b) the polydispersity (span) of reconstituted eggPC and eggPC/chol (1:1) liposome formulations following freeze-drying in the presence and absence of trehalose

Figure 5.4 Scanning electron micrographs of freeze-dried eggPC/chol liposomes (initial mean size $1 \mu \mathrm{m})$ .255

Figure 5.5 Scanning electron micrographs of freeze-dried eggPC/chol liposomes (initial mean size $2.5 \mu \mathrm{m})$. .256

Figure 5.6 Scanning electron micrographs of freeze-dried eggPC/chol (1:1) liposomes prepared with the cryoprotectant trehalose (initial mean size $1 \mu \mathrm{m}$ ). .257

Figure 5.7 Scanning electron micrographs freeze-dried of eggPC/chol (1:1) liposomes (initial mean size $2.5 \mu \mathrm{m}$ ) prepared with the cryoprotectant trehalose. .258

Figure 5.8 Proposed mechanism of the trehalose-mediated stabilisation of liposomes during freeze-drying and subsequent rehydration. .259

Figure 5.9 A comparison between the change in droplet size recorded by McCallion et al., (1996) as the size of suspended latex spheres is increased and that produced as the redispersed liposome size (following freeze-drying) is increased in the present study....261 Figure 5.10 The mean size of freeze-dried and reconstituted eggPC/chol (1:1) liposome formulations during nebulisation into a twin-impinger. .263

Figure 5.11 A comparison between the rate of residual liposome damage during the initial and latter stages of the Pari-LC nebulisation of conventional eggPC/chol (1:1) liposomes and eggPC/chol (1:1) liposomes previously freeze-dried and rehydrated .264

Figure 5.12 The polydispersity (span) of freeze-dried and reconstituted eggPC/chol (1:1) liposomes during nebulisation into a twin-impinger. .266

Figure 6.1 Schematic diagram of a typical ultrasonic nebulizer .270

Figure 6.2 The mean droplet size (VMD) of liposome aerosols produced from the Medix Electronic nebuliser during the nebulisation of (a) eggPC and (b) eggPC/chol (1:1) liposomes of varying lipid concentration. 
Figure 6.3 The polydispersity of liposome aerosols produced from the Medix Electronic nebuliser during the nebulisation of (a) eggPC and (b) eggPC/chol (1:1) liposomes of varying lipid concentration.. .276

Figure 6.4 The influence of lipid concentration on the volume median diameter (VMD) of droplets formed upon the nebulisation of eggPC and eggPC/chol (1:1) liposomes with the Medix Electronic ultrasonic nebuliser. .279

Figure 6.5 The mean size of residual liposomes and liposomes deposited in the upper stage and lower stage of the twin impinger following nebulisation with the Medix

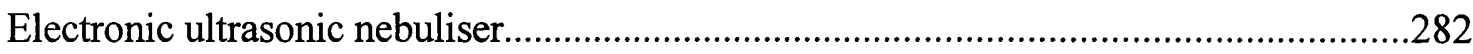

Figure 8.1 Proposed mechanism of liposome aerosolisation from a jet nebuliser......295 


\begin{tabular}{|c|c|c|}
\hline${ }^{99 \mathrm{~m}} \mathrm{Tc}$ & - & technetium \\
\hline$\alpha-\mathrm{T}$ & - & $\alpha$-tocopherol \\
\hline AIDS & - & acquired immuno-deficiency syndrome \\
\hline ampB & - & amphotericin-B \\
\hline $\mathrm{ARBC}$ & - & artificial red blood cell \\
\hline ARDS & - & adult respiratory distress syndrome \\
\hline AUC & - & area under the curve \\
\hline $\mathrm{Bec}$ & - & beclomethasone dipropionate \\
\hline CAT & - & chloramphenicol acetyltransferase \\
\hline $\mathrm{CBC}$ & - & critical bilayer concentration \\
\hline $\mathrm{CF}$ & - & carboxyfluoroscein \\
\hline $\mathrm{CFC}$ & - & chlorofluorocarbon \\
\hline CFTR & - & cystic fibrosis transmembrane conductance receptor \\
\hline chol & - & cholesterol \\
\hline $\mathrm{CI}$ & - & cascade impactor \\
\hline $\mathrm{CMC}$ & - & critical micelle concentration \\
\hline CMD & - & count median diameter \\
\hline CsA & - & cyclosporin A \\
\hline DCP & - & dicetyl phosphate \\
\hline DLPC & - & dilauroyl phosphatidylcholine \\
\hline DMPC & - & dimyristoyl phosphatidylcholine \\
\hline DMPG & - & dimyristoyl phosphatidylglycerol \\
\hline DNA & - & deoxyribonucleic acid \\
\hline DPI & - & dry powder inhalers \\
\hline DPPC & - & dipalmitoyl phosphatidylcholine \\
\hline DPPG & - & dipalmitoyl phosphatidylglycerol \\
\hline DSPC & - & distearoyl phosphatidylcholine \\
\hline DSPG & - & distearoyl phosphatidylcholine \\
\hline DTPA & - & diethylene triamine pentaacetic acid \\
\hline EDTA & - & ethylenediaminetetraacetic acid \\
\hline eggPC & - & egg phosphatidylcholine \\
\hline $\mathrm{fd}$ & - & freeze dried \\
\hline FLEF & - & free and liposome-encapsulated fentanyl \\
\hline FRLE & - & foetal rat lung epithelial \\
\hline GIT & - & gastro-intestinal tract \\
\hline GSD & - & geometric standard deviation \\
\hline GTN & - & glyceryltrinitrate \\
\hline $\mathrm{h}$ & - & hours \\
\hline HFC & - & hydrofluorocarbon \\
\hline HFC-134a & - & tetrafluorethane \\
\hline
\end{tabular}




\begin{tabular}{|c|c|c|}
\hline IL-2 & - & interleukin-2 \\
\hline IT & - & intratracheal \\
\hline IV & - & intravenous \\
\hline 1 & - & litres \\
\hline LDH & - & lactate dehydrogenase \\
\hline lip-ampB & - & liposomal amphotericin B \\
\hline LUV & - & large unilamellar vesicle \\
\hline lysoPC & - & lysophosphatidylcholine \\
\hline $\mathrm{Mab}$ & - & monoclonal antibody \\
\hline MDI & - & metered dose inhalers \\
\hline $\min$ & - & minute \\
\hline MLI & - & multi-stage liquid impinger \\
\hline MLV & - & multilamellar vesicle \\
\hline MMAD & - & mass median aerodynamic diameter \\
\hline MMD & - & mass median diameter \\
\hline MPS & - & mononuclear phagocyte system \\
\hline MRT & - & mean residence time \\
\hline NHS & - & national health service \\
\hline NRDS & - & neonatal respiratory distress syndrome \\
\hline $\mathrm{PC}$ & - & phosphatidyl choline \\
\hline PE & - & phosphatidylethanolamine \\
\hline PG & - & phosphatidyl glycerol \\
\hline PEG & - & polyethylene glycol \\
\hline PI & - & phosphatidylinositol \\
\hline PP & - & packing parameter \\
\hline PS & - & phosphatidylserine \\
\hline REV & - & reverse phase evaporation vesicle \\
\hline rhDNase & - & recombinant human deoxyribonuclease 1 \\
\hline SA & - & stearylamine \\
\hline SCG & - & sodium cromoglycate \\
\hline SEM & - & scanning electron microscope \\
\hline SOD & - & superoxide dismutase \\
\hline soyPC & - & soy phosphatidylcholine \\
\hline SP & - & surfactant protein \\
\hline SUV & - & small unilamellar vesicle \\
\hline TI & - & twin impinger \\
\hline $\mathrm{T}_{\mathrm{m}}$ & - & main phase transition temperature \\
\hline $\mathrm{t}$ & - & time \\
\hline $\operatorname{tr}$ & - & trehalose \\
\hline UV & - & ultraviọlet \\
\hline VMD & - & volume median diameter \\
\hline
\end{tabular}




\section{ACKNOWLEDGMENTS}

I gratefully acknowledge the financial support which the Royal Pharmaceutical Society of Great Britain, and RP Scherer provided for this $\mathrm{PhD}$ project. Also, the Edward's Kenilworth Schools Charity for their generous bursary.

A sincere thankyou to my supervisor, Dr. Kevin Taylor, for his enthusiasm and guidance throughout this project. Thanks also to Dr. Orla McCallion, Miss Kammy Leung, Mr. Dave McCarthy and the other staff and students of the School of Pharmacy, University of London who assisted in the project.

My thanks and love to all my family, to whom the preface "when the PhD's finished..." has become all too familiar. In particular, I am indebted to my Mother and Father for their encouragement and invaluable support throughout my education. Also to my Grandfather, the "number one pharmacist".

Finally, all my love and heartfelt thanks to Michelle for her patience and understanding throughout my studies. Without her, this thesis would never have been completed. 


\section{INTRODUCTION}

\subsection{The inhaled route for drugs}

The pharmacological consequences of the inhalation of certain airborne materials was recognised by many ancient civilisations. Records show that steam inhalations were used by followers of Hippocrates (460 to $370 \mathrm{BC}$ ), and Galen (131 to $201 \mathrm{AD}$ ), for the treatment of laryngeal and pulmonary disorders. Furthermore, the leaves of the Atropa belladonna plant were smoked in India as a treatment for cough at least 4000 years ago (Grossman, 1994). The first recorded use of an inhaled medicament in Western medicine was in England in 1802, when Datura ferox was used to treat asthma (Hilman, 1991). In addition, the asthma cigarette, composed of Datura stramonium and Atropa belladonna, was commonly used up until the late nineteenth century for its bronchodilatory ingredients (Grossman, 1994). It is now recognised that the pulmonary route for drug delivery requires a sophisticated delivery device to achieve efficient drug administration, as access to sites within the respiratory tract is difficult. Early, primitive devices generated aerosols that were inherently unstable, producing large particles which failed to efficiently penetrate the airways. However, at the turn of this century inhalation therapy was improved by the use of delivery systems such as atomisers, that were capable of the more efficient deposition of drugs within the lungs. The management of pulmonary diseases, such as asthma, bronchitis, and pneumonia, has since been revolutionised by the development of compact devices, such as the metered dose and dry powder inhalers, which are capable of targeting drugs to the lung. As a result of such developments, a wide variety of drugs are now commonly inhaled for action within the lung. More recently, significant research has been undertaken into the investigation of the lung as a route for the systemic delivery of novel protein and peptide drugs (eg. Patton and Platz, 1992). Such work has included the delivery of insulin (eg. Elliott et al., 1987), hormones such as leuprolide (Adjei and Garren, 1990), and human growth hormone (Patton et al., 1990). Delivery of genes, by aerosol, for incorporation into lung cells has also received considerable interest (eg. Brigham et al., 1989). Pulmonary delivery avoids various problems, such as acid and enzyme mediated denaturation in the stomach, and the invasive nature of injection, that are commonly associated with more traditional routes of peptide administration. A number of sophisticated delivery systems are being developed, with the aim of delivering such therapeutic agents to the lung, and also to achieve sustained release of conventional lung 
targeted drugs. Such studies have included the use of cyclodextrins (eg. Cabral Marques et al., 1991), microspheres (Lim et al., 1989) and liposomes (eg. Taylor et al., 1989). Of these, the delivery of liposomes to the lung has received the greatest interest. Indeed, an account of the successful treatment of a diseased patient with nebulised liposomal amphotericin B (ampB) aerosol, has recently been published (Purcell and Corris, 1995). The research into liposomal aerosols has almost exclusively concerned nebulised systems, principally due to their ease of formulation, and it is the nebulisation of liposomes that is the subject of the present thesis.

\subsection{The respiratory tract}

The lungs are delicate structures protected by the bony rib cage, and are the site of gaseous exchange in mammals, providing oxygenation of blood, and the removal of carbon dioxide. They also have a protective, and cleansing role (Forrest, 1993). Only about $10 \%$ of the total lung volume is solid tissue, the remainder being filled with air and blood.
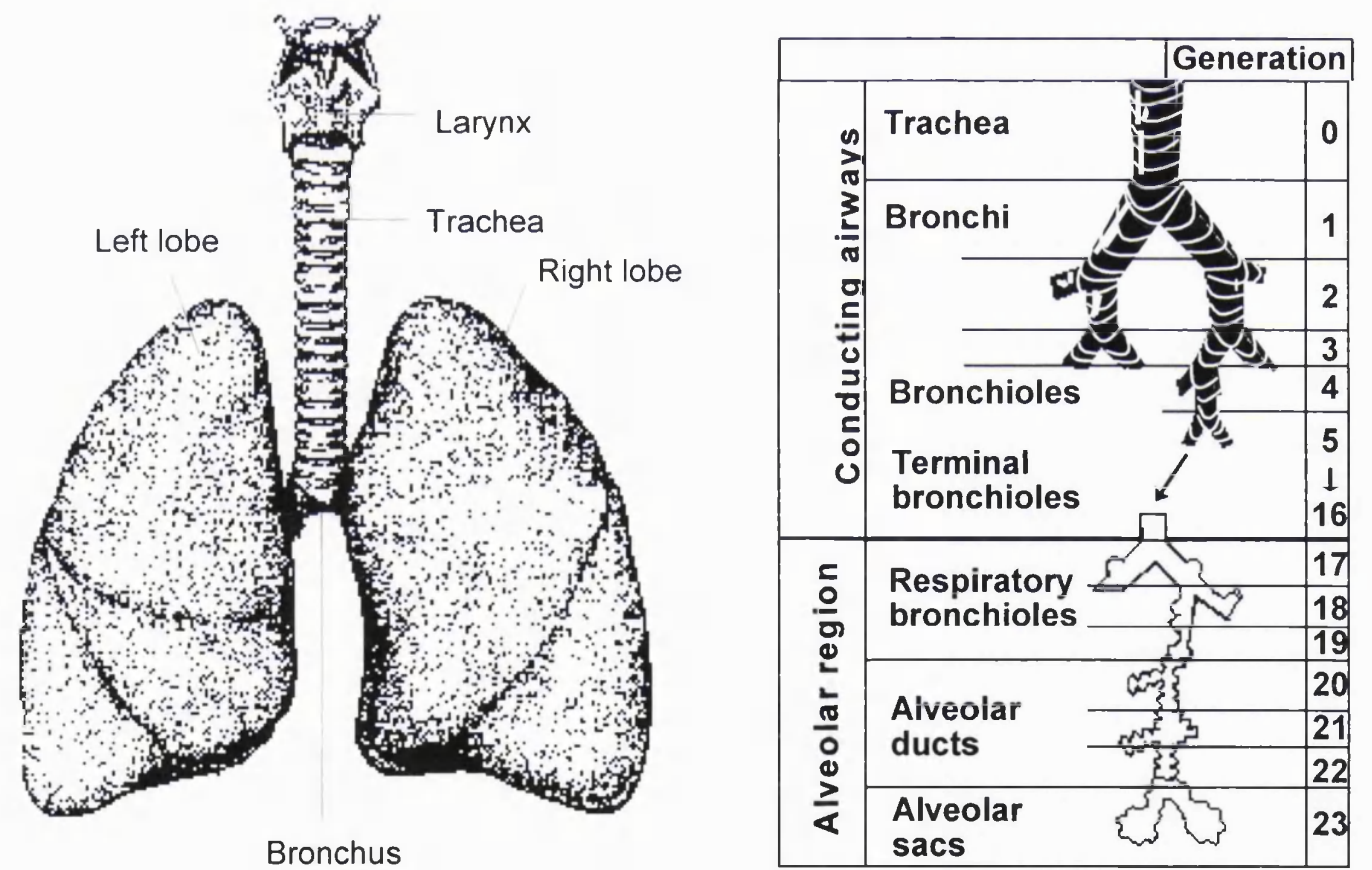

Figure 1.1 The human lung and the generations of the airways.

The respiratory system is frequently classified into the upper respiratory tract, consisting of the nose, mouth, nasal and paranasal passages, eustachian tubes, pharynx, oesophagus, 
and larynx; and the lower respiratory tract, consisting of the air passages (bronchioles) and alveoli. The trachea and bronchi are often included as part of the upper respiratory tract.

Upon inspiration, an airstream is drawn into the respiratory tract via the mouth or nose. The nasal passages contain hairs that serve to filter out the majority of the coarse airborne particles. The air then passes over the posterior conchae, which provide a large, moist surface area, with an extensive blood supply, serving to warm and humidify the incoming air. It then passes the oropharynx, larynx, and through the trachea. In this oropharyngeal region the epithelium is comprised of ciliated cells, covered by a mucous blanket. The mucus layer traps particles, and they are moved to the pharynx from where they are swallowed (part of the mucociliary clearance mechanism of the lung, see section 1.4.2). Subsequent to the trachea the respiratory tract bifurcates into two bronchi, conducting the airstream into the right and left lobes of the lung. From this point the lungs follow a pattern of regular dichotomy (fig. 1.1). The airstream follows the lung divisions into two equivalent branches, each successive one being termed a generation. The tracheobronchial region comprises the conducting airways, extending from generation 0 (the trachea), to generation 16 (the terminal bronchioles). The pulmonary, or alveolar, region comprises the alveolar airways extending from generation 17 (the respiratory bronchioles) to generation 23 (the alveolar sacs)(Weibel, 1963).

The thick muscular walls of the conducting airways limit the extent of gaseous exchange in the region. Instead, their principle function is to transport air to the alveolar region, and to filter out airborne particles. The epithelium is ciliated, and covered by mucus, produced by numerous goblet cells. The mucous layer helps to moisten the inspired air, and prevents drying of the airway walls. Vigorous beating of the cilia drives the mucus blanket towards the larynx, serving to clear any deposited insoluble particles from the lung by mucociliary clearance. Lymphatic vessels surround the arteries and bronchi, extending down to the bronchioles, which protect the lungs from allergens and pathogens. These drain into lymph nodes present along the second and third generation bronchi.

As successive bifurcations occur, supportive cartilaginous rings gradually diminish in number, and the bronchi become bronchioles. The muscular walls thin, and the cross sectional area of each airway decreases. However, there is a dramatic increase in the total 
cross-sectional area of the airways, due to the bifurcations that occur (Weibel, 1963). A progressive reduction in airflow results, until in the alveolar region the movement of air is by diffusion alone. Moving from the conducting to the alveolar region also sees a significant change in the histology of the airways. The goblet cells reduce in number, and are replaced by Clara cells, which secrete a less viscous fluid. The bronchioles branch into alveolar ducts lined with alveoli, which total approximately 300 million in number (Douglas, 1987). The alveolar region provides a gaseous exchange membrane covering a surface area of approximately $143 \mathrm{M}^{2}$, depending on the body mass, and an air-blood barrier thickness of approximately 0.2 to $0.5 \mu \mathrm{m}$ (Weibel et al., 1976; Gehr et al., 1978). The red blood cells contained in the respiratory capillaries are therefore in intimate contact with the respiratory space. Gaseous exchange of carbon dioxide and oxygen is thus facilitated by a number of structural features - the large surface area, the thin absorption barrier, and also the fact that the whole cardiac output perfuses the lungs in very fine capillaries.

\section{Histology of the alveolus}

The alveolus is comprised of five main cell types. The attenuated type I pneumocytes provide the diffusion medium for the respiratory gases. Approximately 0.1 to $0.3 \mu \mathrm{m}$ in thickness, they account for about $5 \%$ of the epithelial cells, yet cover approximately $95 \%$ of the alveolus (Weibel et al., 1976). The greater part of their surface area is comprised of thin squamous extensions which, lacking cell organelles, present a minimal barrier to gas diffusion. However, they consequently have a poor ability for self repair. The cuboidal type II pneumocytes are 10 to $15 \mu \mathrm{m}$ in thickness and act as progenitors for Type I cells, if required following cell damage. However, their principal function is to secrete lung surfactant, which is a complex mixture of lipoproteins responsible for maintaining the integrity of the airways(Taeusch et al., 1983)(see section 1.8). Type III pneumocytes also cover $40 \%$ of the alveolar surface in rat lung, but they remain to be confirmed in humans (Forrest, 1993). Alveolar macrophages roam the alveolar surface (Crapo et al., 1982). Sparse in number, they migrate from the blood to the respiratory surface, serving to ingest and clear any deposited particulate matter which may have evaded the tracheobronchial filtration mechanisms. Capillary endothelial cells form an extensive network of blood capillaries which surround the alveoli, and fibroblasts located in thicker areas of the alveolar wall provide supportive bulk. 


\subsection{Inhaled Aerosols}

An aerosol may be defined as a dispersion of solid or liquid particles, typically less than 50 $\mu \mathrm{m}$ in size, dispersed in a gas (Gonda, 1988). The human lung processes 10000 to 20000 litres of air a day, which is estimated to contain between 100 and 1000000 particles per $\mathrm{cm}^{3}$ (Brain and Valberg, 1979). The particles in the atmosphere may be as large as $5000 \mu \mathrm{m}$ rain droplets. However, particles in inspired "aged" air are generally between 0.01 and 1 $\mu \mathrm{m}$ in diameter, ranging from bacteria and viruses, to dusts and pollens (Brain and Valberg, 1979). In addition to these environmental particles, may be particles deliberately inhaled for therapy, diagnosis, or research. Penetration of such particles into the lung presents a significant toxic challenge, as the lung is a highly sensitive organ, reliant as it is on the intimate contact between the respiratory membrane and the blood for gaseous exchange. A variety of mechanisms therefore protect it from the build-up of inhaled matter.

\subsection{Fate of inhaled matter}

\subsubsection{Deposition of particles}

Particles that enter the respiratory tract may be deposited by a combination of mechanisms.

\section{Inertial impaction}

Inertial impaction is the principal mechanism for the deposition of particles within the respiratory tract that are larger than about $5 \mu \mathrm{m}$ (Padfield, 1987; Hilman, 1991). The inertia of a large airborne particle causes it to follow its original path when the airstream is deflected, due to viscous drag force. If the momentum is large enough, the particle will follow the straight line motion and deposit. The greater majority of the coarse particles in inspired air are deposited by inertial impaction on hairs, and air deflecting channels within the nose, or in the throat and bronchi bifurcations (Task Group on Lung Dynamics, 1966). These are then translocated by mucociliary clearance towards the pharynx, from where they are swallowed. The probability of inertial deposition follows equation 1.1 (Stuart, 1973):

$$
I \propto \frac{U_{t} U \sin \theta}{g R}
$$

where:

$I=$ inertial deposition, $U=$ velocity of the airstream, $U_{t}=$ terminal settling velocity of the particle, $g=$ gravitational constant, $\theta=$ Angle of the bend, $R=$ radius of the airway. 
Larger particles therefore have increased terminal settling velocities, and thus a greater probability of deposition. The small total cross-sectional area of the conducting airways means that the velocity of airflow in this region is very high, and encourages particle impaction. In addition, the obstructed entrance to the trachea via the larynx creates turbulent flow in the trachea and bronchi, further encouraging particle impaction.

\section{Gravitational sedimentation}

Smaller particles (up to about to $5 \mu \mathrm{m}$ ) generally evade inertial impaction, and are able to move with the airstream to penetrate the lower airways (Stahlhofen et al, 1980). In these areas the airflow velocity is reduced, and becomes laminar in nature. As a result, inertial impaction is minimised, and deposition by gravitational sedimentation predominates. This is the chief mechanism of deposition beyond the 10 th airways generation (Hilman, 1991). The rate of sedimentation is dictated by the particle density as shown in equation 1.2 (Stuart, 1973).

$$
U_{t}=\frac{(\sigma-\rho) g d^{2}}{18 \gamma}
$$

where:

[equation 1.2]

$U_{t}=$ terminal settling velocity, $\sigma=$ particle density, $\rho=$ density of air, $\gamma=$ viscosity of the air, $d=$ particle diameter.

\section{Diffusion}

Particles with a mean size of up to about $2 \mu \mathrm{m}$ generally evade impaction and sedimentation in the upper airways, and therefore penetrate deeper into the alveolar region to deposit by sedimentation (Stahlhofen et al., 1980). Particles smaller than about $0.5 \mu \mathrm{m}$ are able to penetrate the alveoli, where the airflow velocity approaches zero (Morrow, 1964). Under these circumstances the particles may be displaced from the airstream by diffusion due to random collisions with gas molecules (Stuart, 1973).

\section{Electrostatic attraction and interception}

Two additional, but less important processes, also contribute to deposition in the lung (Gonda, 1992). Electrostatic attraction causes deposition of charged particles. Such particles induce an "image charge" in the respiratory surface, due to repulsion of like 
charges. As a result, a net force of attraction between the airway walls and the inspired particle is established, causing the particle to become deposited (Brain and Valberg, 1979). Alternatively, electrostatic attraction may occur due to Van der Waals' forces (Gonda, 1992). Interception occurs when the centre of gravity of a particle is in the middle of a flowing airstream, yet the particle shape causes the extremities to contact with the respiratory surface (Brain and Valberg, 1979). Interception occurs predominantly for smaller, elongated particles, such as mineral fibres (Harris and Fraser, 1976). It is postulated that reducing the particle size to less than about $0.6 \mu \mathrm{m}$ in size encourages the number that are inhaled and exhaled with air without deposition (Morrow, 1974). Despite this, particles smaller than about $0.6 \mu \mathrm{m}$ are efficient at penetrating the alveolar region.

Particle deposition within the lung generally reaches a maximum at a particle size of approximately $5 \mu \mathrm{m}$, and more specifically at about 2 to $3 \mu \mathrm{m}$ for alveolar deposition (Stahlhofen et al., 1980). However, the precise in vivo pattern of deposition of an inhaled solid or liquid particle has varied significantly between researchers. This is in part attributable to the influence that breathing patterns have on particle deposition. The average respiratory rate is approximately 15 breaths per minute. This produces a tidal volume of about $500 \mathrm{ml}$, and a residence time of approximately 3 seconds. A slowing of the breathing rate prolongs an inspired aerosols' residence time within the lung, and as a result increases sedimentation. Furthermore, a fast inspiratory flow rate and a shallow inspiratory volume will cause a shift in particle deposition to the proximal lung. Conversely slow, deep breathing, followed by breath-holding, increases deposition within the alveoli (Gonda, 1990). Therefore, inter-subject variations in breathing patterns produce significant differences in the deposition and fate of inhaled particles. Ilowite et al. (1987) demonstrated that the pattern of deposition of particles in the lung, from a pressurised inhaler, was subject to greater than $60 \%$ variation between patients, when no effort to control the breathing pattern was made, falling to $16 \%$ with controlled breathing. A significant change in aerosolised particle deposition also occurs with a change from mouth to nasal inhalation (Everard et al., 1993). The nasal passages have a smaller cross-sectional area, more acute bends, and larger impaction surfaces, which encourages the deposition of particles. The deposition pattern of aerosols may be further altered by inflammation, oedema, and airway obstruction, associated with pulmonary diseases such as asthma, chronic obstructive airways disease, and cystic fibrosis (Brain and Blanchard, 1993). 


\subsubsection{Clearance of deposited particles}

The lung has evolved to minimise the penetration of airborne materials into the airways. Despite this, vast numbers of particles settle on the respiratory membrane, and present a significant toxic challenge to the lungs. It is therefore imperative that particles are cleared rapidly from the airways. A build up of particles would impair gaseous exchange, and may cause inflammatory responses, infection, and absorption into the systemic circulation. To avoid this, the respiratory tract is protected by a number of clearance mechanisms.

\section{Clearance of particles from the conducting airways}

The principal clearance mechanism of the conducting airways is termed the mucociliary escalator (Clarke and Pavia, 1981), which extends from the larynx to the terminal bronchioles (Camner et al., 1971). A highly viscid mucous blanket covers the tracheobronchial membrane, and is propelled proximally by the ciliary activity of the columnar epithelial cells. Poorly soluble particles, which deposit within the conducting airways, are carried with the mucous flow towards the larynx to be expirated, or swallowed. The mucociliary clearance rate becomes significantly reduced towards the non-ciliated periphery of the lung (Serafini et al., 1976), and this forms the basis of the strategy of targeting drug particles and microcarriers to the alveolar region, in order to achieve extended drug residence times (Niven, 1992 ).

\section{Clearance from the alveolar region}

The clearance of insoluble materials deposited uniformly throughout the lung has been shown to follow a biphasic profile. Whereas insoluble particles depositing in the ciliated airways are cleared within a matter of several hours by mucociliary clearance, those in the non-ciliated alveolar region may remain for a matter of days or weeks (Brain and Blanchard, 1993). The principle defence mechanism within the alveolar region is provided by the phagocytic activity of the alveolar macrophages. Pinocytosis into Type I cells may also provide a limited clearance of particles into the lymphatics and interstitium, if macrophage clearance become saturated (Adamson and Bowden, 1981). The alveolar macrophages form part of the mononuclear phagocyte system (MPS), and are cleared from the lung, with their ingested payload, via the mucociliary escalator, or through the lymphatics via the interstitium. Clearance by mucociliary and macrophage action is in competition with drug dissolution, and subsequent absorption through the membrane. 


\subsection{Delivery of drugs to the lung}

The inhalation and subsequent deposition of particulates is exploited medically for the investigation, prophylaxis, and treatment of a number of diseases. Inhaled drugs for lung activity include antimicrobials (eg. pentamidine), anticholinergic bronchodilators (eg. ipratropium bromide), $\beta_{2}$-adrenergic bronchodilators (eg. salbutamol), corticosteroids (eg. beclomethasone dipropionate), enzymes (eg. dornase alfa), mucolytics (eg. acetylcysteine), and asthma prophylactics (eg. sodium cromoglycate). Those inhaled for systemic activity include ergotamine for migraine (British National Formulary, 1996).

\subsubsection{Inhaled drugs for local action}

The inhalation of an aerosolised drug allows the rapid attainment of therapeutic levels directly at the site of action. In so doing, a prompt pharmacological response produced by a minimal dose may be given. For instance, the $\beta_{2}$-adrenergic bronchodilators, such as salbutamol and terbutaline, are widely used in aerosol form as a treatment for airways obstruction in asthma (British National Formulary, 1996). The inhalation of drug particles allows direct action of the agonist on the target $\beta_{2}$-adrenoreceptors of the airway smooth muscle. This causes prompt relaxation of the smooth muscle, and a rapid easing of breathing. Salbutamol is administered at a dose of $200 \mu \mathrm{g}$ by inhalation, compared with 2 to $8 \mathrm{mg}$ via the oral route. Therefore, pulmonary delivery allows a 10 to 40 -fold reduction in the dose necessary to achieve a similar response to that given by the oral route. As a result, the aerosol treatment is associated with significantly reduced adverse systemic effects, compared with the orally dosed formulation (British National Formulary, 1996).

\subsubsection{Inhaled drugs for systemic activity}

The lung may also offer significant advantages over the oral or intravenously injected (IV) routes for the delivery of systemically acting agents. Administration of drugs by injection is disadvantaged by the fact that it is inconvenient. It is also more hazardous than other routes, as it by-passes the body's defence mechanisms. Similarly, the oral route may be associated with significant drug degradation, due to the elevated acid and enzyme levels of the gastro-intestinal tract (GIT). The relatively mild environment of the lung ( $\mathrm{pH} \sim 5.4$ to 7.4, Less and Shanker, 1977) makes the pulmonary route more suitable for the delivery of acid labile drugs than the GIT, which has a $\mathrm{pH}$ as low as 2 . In addition, delivery via the lung avoids "first-pass" metabolism of the drug within the liver. The vast surface area, and 
highly permeable alveolar membrane may facilitate the rapid absorption of deposited drugs into the systemic circulation. Drug absorption may be further promoted by absorption through aqueous pores, and/or phagocytosis of insoluble particles by alveolar macrophages. Furthermore, the lung provides a favoured route for certain drugs which are poorly absorbed from the GIT. An example is sodium cromoglycate (SCG) which exhibits poor oral bioavailability due to its hydrophilic nature. When delivered via the lung significant blood levels may be achieved, due to a carrier-mediated transport system which promotes absorption (Gonda, 1990). The lungs may therefore provide a convenient, and relatively safe means of delivering drugs efficiently to the systemic circulation by a non-invasive route. However, the pulmonary delivery of drugs for systemic or local activity is not without its problems. Firstly, the generation of particles suitable for pulmonary administration requires a sophisticated delivery system. Aerosol administration devices are therefore relatively expensive to develop and manufacture, and often demand a high degree of skill for efficient operation. Secondly, hydrophobic drugs prove difficult to formulate as aerosols, and local irritation may result from deposition of insoluble particulates within the lung. Furthermore, the efficient clearance mechanisms operating within the lung conspire to remove deposited drugs from their sites of action. This means multiple dosing is often necessary, which may challenge compliance, increase therapy cost and also the likelihood of adverse effects. However, the introduction of drug carriers, such as liposomes, may address these difficulties (Niven, 1992b).

\subsubsection{Fate of inhaled drugs}

Deposition of drug droplets, or solid particles, within the lung follows the same mechanisms discussed for particles in general (section 1.4.1). It is therefore particle size dependant, and may occur throughout the respiratory tract. Drugs administered to the lung may be deposited as a solution, or as solid particles, and will have one of a number of possible fates. Firstly, drug may be transported to the mucous blanket of the tracheobronchial mucosa, and cleared by mucociliary action to the pharynx. From here the drug may be swallowed (which may result in subsequent oral absorption), or coughed up. Alternatively, the drug may become sequestered within the lung by cellular pinocytosis, or phagocytosis by alveolar macrophages. The particle may then be cleared from the lung by mucociliary clearance, or alternatively by transport via the lymphatics to the bloodstream (Lauweryns and Baert, 1977). Finally, if the solid particle is small enough to dissolve 
rapidly, or if the drug is in solution, drug may diffuse through the respiratory epithelium and avoid these clearance mechanisms entirely. However, absorption of drug molecules through the epithelium of the conducting airways is impaired by the thick mucus layer. Furthermore, once dissolved within the mucus, the epithelial absorption of the drug will be in competition with the clearance of mucus from the lung by ciliary action, or alternatively with enzymatic degradation within the mucus (Thompson, 1992). The epithelial cells are connected by tight junctions, and so the drug entity must diffuse directly through the cell bilayers. As a result, the absorption is proportional to the drugs' lipid solubility, size, and charge (Thompson, 1992). Finally, having diffused through the epithelium and basement membrane, the drug may bind with receptors on tissues, glands or other cells.

Absorption of substances is maximal in the alveolar region where the interstitium and surfactant layer are at their thinnest (Schanker, 1978). The lipid membranes present an effective barrier to large molecular hydrophilic substances and charged substances, relative to their apparent oil/water partition coefficient (Burton and Schanker, 1974; Effros, 1993). Tight junctions link the epithelial cells in both the alveolar region and the conducting airways. This makes the absorption of hydrophilic molecules inversely related to the molecular weight, as the only possible means of membrane penetration is through the tight junctions (Enna and Schanker, 1972a, 1972b). However, the pulmonary absorption of hydrophilic materials was found to be faster than that predicted from similar absorption mechanisms in the GIT, which was taken to be suggestive of absorption via aqueous pores (Schanker, 1978). Carrier mediated adsorption may also aid the passage of certain hydrophilic substances, including SCG (Gonda, 1990). The absorption of drugs may also be influenced by certain pathological states. For example, smoking causes damage to the epithelial lining, which may lead to an increased absorption of agents (Minty et al., 1981).

Once adsorbed into pulmonary tissue, the drug may be deactivated by a wide variety of enzymes. However, studies demonstrate that few of the traditional compounds delivered to the lung are metabolised significantly during passage through the respiratory tissue (Damani, 1987). Despite this, deposited drug particles have a finite time in which to dissolve and diffuse to the target receptor sites, before being cleared from the lung. Removal of drugs from the site of action by any of the above mechanisms imposes a limit on the duration of action of inhaled drugs. This is significant, as it has been shown that 
asthma symptoms follow a diurnal pattern, with significantly impaired respiratory function between midnight and 8 am (Smolensky et al., 1987). Therefore, an inhaled anti-asthma drug should ideally give protection from symptoms during the 6 to 8 hours of sleep. This is usually not possible using traditional bronchodilators, due to their rapid clearance from the lung. Although a notable exception is salmeterol, which has a longer duration of activity. Attempts have been made to produce sustained release of drugs in the lung using insoluble salts or precipitates (Niven and Byron, 1988). Targeting of such particles to the non-ciliated airways may produce a reduced rate of drug clearance, and hence an increased duration of action, relative to the rate of drug dissolution. However, whilst pulmonary drug delivery for systemic drug activity may be best achieved using lipophilic agents, which are rapidly absorbed from sites of deposition, there is general consensus that sustained drug action in the lung is likely to necessitate a drug carrier (Niven, 1992). This may be achieved with microspheric encapsulation (eg. Lim et al., 1989), however most promising results have been produced with liposomal carriers (section 1.9).

An alternative strategy for the targeting of the lung for diagnosis, prophylaxis, or therapy, involves delivery via the vasculature following IV injection. For instance, IV administered liposomes, with diameters greater than about 5 to $8 \mu \mathrm{m}$ collect within the small bore capillaries which serve the lung. This has been exploited to deliver certain anti-cancer agents to lung tissue (eg. Fidler et al., 1980; Abra et al., 1984).

\subsubsection{Characteristics of therapeutic aerosols}

It is important that aerosol delivery systems are characterised during development. The physical property of aerosols most commonly quoted is the aerodynamic diameter. This is the diameter of a spherical particle, with a density of $1 \mathrm{~g} / \mathrm{cm}^{3}$, that settles with the same rate as the particle being described. Particles in therapeutic aerosols are usually approximately spherical, with diameters greater than $0.5 \mu \mathrm{m}$. For such particles, the aerodynamic diameter is described by equation 2.3 (Swift, 1980).

$$
d_{A}=d \sqrt{\frac{\rho}{\rho_{o}}}
$$

where:

[equation 1.3]

$d_{A}=$ aerodynamic diameter, $d=$ diameter, $\rho=$ particle density, $\rho_{o}=$ 'unit density' $\left(1 \mathrm{gm} / \mathrm{cm}^{3}\right)$ 
Therapeutic aerosols are usually manipulated to produce an aerodynamic diameter of between 1 and $5 \mu \mathrm{m}$, in order to facilitate pulmonary deposition. As previously stated, a particle diameter of below about $5 \mu \mathrm{m}$ to $6 \mu \mathrm{m}$ will promote particle deposition within the lung. However, the highly saturated nature of the airways (relative humidity is in excess of $99 \%$ at $37^{\circ} \mathrm{C}$, Gonda and Byron, 1978; Morrow, 1986) causes most hydrophilic agents delivered as solid particles, or hypertonic aqueous droplets to absorb moisture as they enter the lung, causing hygroscopic growth (Morrow, 1986). Conversely, isotonic aqueous droplets tend to evaporate as they leave the generator. The aerosol size may thus change significantly following generation, and subsequent inhalation (Brain and Blanchard, 1993).

Therapeutic aerosols are characteristically heterodisperse in nature (Brain and Valberg, 1979). A logarithmic normal ("log-normal") population of particle sizes is generally the most accurate description of the distribution (O'Callaghan and Barry, 1997). The size characteristics of the aerosol distribution may be determined from the count median diameter (CMD), which denotes the size at which half of the aerosol particles are contained in larger sizes, and half in smaller sizes. This may be quoted in conjunction with the geometric standard deviation (GSD), which is a measure of the polydispersity of the aerosol, representing the ratio of diameters corresponding to 84.3 and 50 per cent of the particles. However, it is often more useful to define an aerosol by the mass of particles, rather than the number, as the largest particles hold a disproportionately large fraction of the aerosol mass. This is because the mass of a particle is proportional to the cube of its diameter. As such, the mass median diameter is commonly used (equation 1.4).

$$
\ln M M D=\ln C M D+3(\ln G S D)^{2}
$$

\section{[equation 1.4]}

The mass median diameter (MMD) is the size such that $50 \%$ of the aerosol mass is contained in particles larger, and $50 \%$ smaller, than this size. A knowledge of the MMD allows calculation of the mass median aerodynamic diameter (MMAD) thus (Gonda, 1988):

$$
M M A D=M M D \sqrt{\frac{\rho}{\rho_{o}}}
$$

where:

$$
\rho=\text { density of the particle, } \rho_{\mathrm{o}}=\text { 'unit density' }\left(1 \mathrm{gm} / \mathrm{cm}^{3}\right)
$$


If the GSD is large, as is often the case in therapeutic aerosols, then as few as $1 \%$ of the total number of particles have a mass larger than the MMD. The methods employed to determine these values are discussed in chapter 3 .

\subsection{Therapeutic aerosol generators}

The formation of a stable aerosol requires the input of energy, in order to increase the total surface area of the system, and to transport the aerosolised particles away from the device. There are three types of device used clinically to provide such energy to therapeutic systems, namely metered dose inhalers, dry powder inhalers, and nebulisers.

\subsubsection{Metered Dose Inhalers (MDI)}

The MDI has become the most popular inhalation device since its conception over 40 years ago (Smith and Benstein, 1996). Introduced as the 'Medihaler' in 1956, the MDI is a selfcontained unit, containing a micronised suspension, or solution of drug, in a solvent composed of a mixture of propellants, traditionally chlorofluorocarbons (CFCs). This mixture is contained in a pressurised bottle or canister, equipped with a metering valve, set to release a precise volume (25 to $100 \mu \mathrm{l}$, Hallworth, 1987)(figure 1.2). The unit is held in a plastic sleeve, fitted with a mouthpiece. Upon actuation, the valve releases the dose at high velocity through the mouthpiece. The highly volatile CFC liquid evaporates rapidly, leaving a cloud of drug containing droplets, or solid particles, to be inhaled.

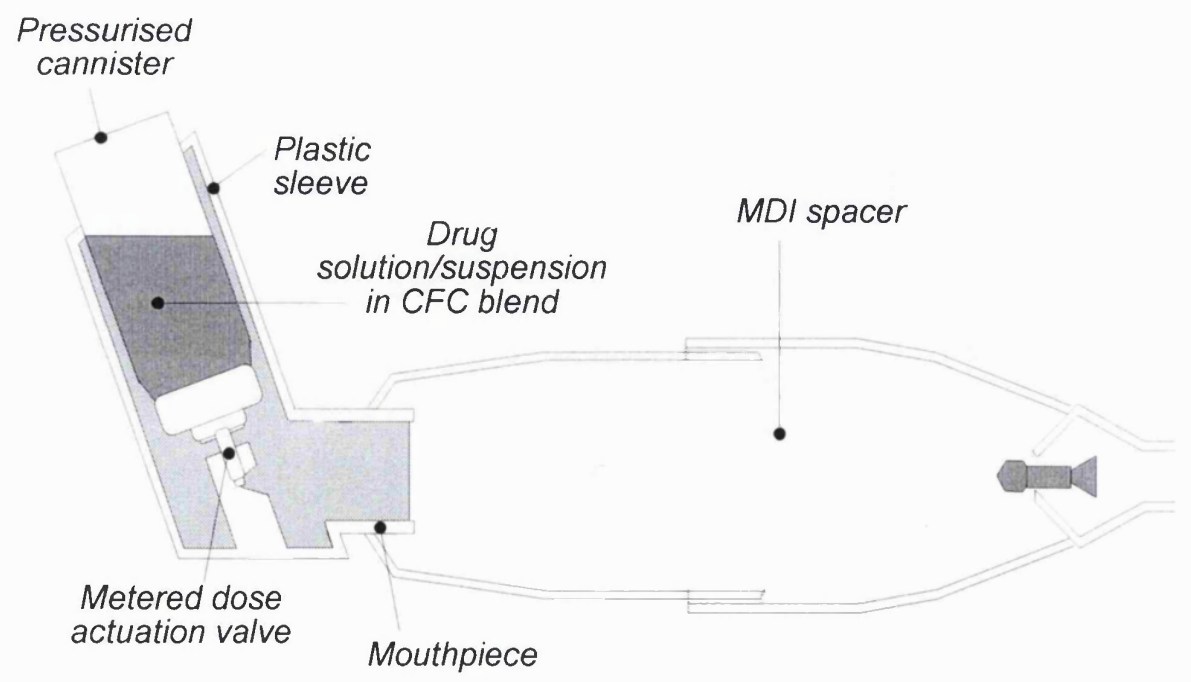

Figure 1.2 The metered dose inhaler, with attached spacer device. 
The MDI provides a chemically inert, tamper-proof storage unit for drugs, which is portable and readily accepted by patients. However, there are a number of significant disadvantages that hinder their use. Droplets leaving the inhaler are initially large, (MMAD $>30 \mu \mathrm{m})$, and are travelling at high speed $\left(>30 \mid \mathrm{ms}^{-1}\right)(\mathrm{Newman}, 1991)$, causing them to have a large inertia, such that the greater proportion sediment in the mouth and throat. As a result, only about $10 \%$ of the dose reaches the lungs (Newman et al., 1981; Newman, 1991). Lung deposition may be improved by the adoption of a slow, deep breathing technique, followed by breath holding. This reduces the velocity of the inspired aerosol, and therefore encourages sedimentation in the lung. However, many patients have insufficient dexterity to co-ordinate breathing with actuation, particularly during an asthmatic episode. As a result, the actual dose delivered to the site of action may be significantly less than $10 \%$ (Newman et al., 1981; Canny and Levison, 1988; Newman, 1991). This limits drug activity, and so increases dosing frequencies, and the risk of extra-pulmonary adverse effects, such as oral candidiasis with inhaled steroids (Salzman and Pyszczynski, 1988).

Extension chambers, or spacers, added to the MDI mouthpiece may improve the performance of MDIs. Spacers range from large volume chambers (eg. Volumatic ${ }^{\circledR}$, Allen and Hanbury's), to small extensions to the MDI mouthpiece (eg. Easibreathe ${ }^{\circledR}$, Baker Norton). In each case, the spacer causes a delay in the inhalation of the aerosol cloud, reducing the aerosol velocity, and allowing longer for propellant evaporation to occur. The momentum of the aerosol prior to inhalation is thus reduced, causing a reduction in oropharyngeal deposition, and an enhancement of lung deposition, particularly in poor coordinators (Newman et al., 1981; Toogood et al., 1982). Despite this, spacers have failed to achieve widespread use, due primarily to the reluctance of patients to use such cumbersome devices, and also the development of more efficient, breath activated MDIs, such as the Autohaler ${ }^{\circledR}$ (3M Pharmaceuticals). The Autohaler is primed before use by raising a lever on the device that is connected to an internal spring. The flow of inspired air through the device draws a vane forward, releasing the spring and thus actuating the aerosol. The Autohaler avoids the need for co-ordinated breathing, and may be operated at reduced inspiratory flow rates. However, the continued development of MDIs is threatened by their reliance on CFC propellants, which have been implicated in the depletion of atmospheric ozone. As a result, a worldwide restriction on the development of CFCs has now been implemented in accordance with the 1987 Montreal Protocol, and the subsequent 
London revision in 1989, sponsored by the United Nations Environment Programme, and signed by more than 150 countries. Further to this, the European Communities have adopted a regulation banning their continued production after June 1997 (Morén, 1993; Whitham and Eagle, 1994). Although a limited exemption has been granted for medical inhalers, this is only until a suitable alternative propellant is developed. European regulatory approval for the medical use of the first non-ozone depleting propellant, tetrafluoroethane 134a (a hydrofluorocarbon, HFC-134a), was granted in 1994. However, since this time only $3 \mathrm{M}$ Pharmaceuticals have introduced a CFC-free inhaler (Airomir ${ }^{(8)}$ ). This is largely because there are major differences in the properties of HFC-134a, compared with conventional CFC propellants, thus necessitating major reformulation of existing MDI systems (Dalby et al., 1990). Most notably, HFC-134a has a reduced stability, and a greater polarity than CFCs, and is thus a poor solvent for conventional MDI excipients, such as surfactants (Morén, 1993). In addition, the outlawing of HFCs, and other similar "greenhouse gas" propellants, has already been predicted (Tansey, 1995). With these problems in mind, there has been a drive to develop non-propellant based methods of aerosol generation, notably dry powder inhalers and nebulisers.

\subsubsection{Dry powder inhalers}

Dry powder inhalers (DPIs) were introduced over 20 years ago in attempt to overcome the co-ordination difficulties that patients experienced with MDIs. The first DPI was the Spinhaler $^{\circledR}$ (Fisons Pharmaceuticals)(fig. 1.3).

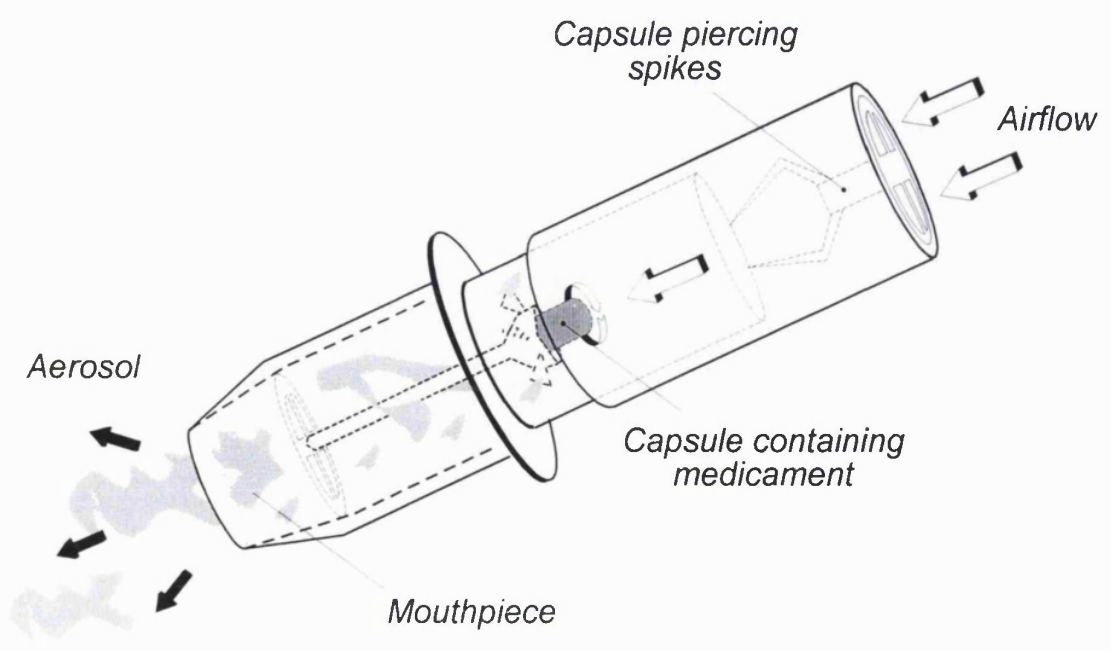

Figure 1.3 The Spinhaler ${ }^{\circledR}$ (Fisons Pharmaceuticals) dry powder inhaler device. 
The Spinhaler ${ }^{\circledR}$ is loaded with a gelatin capsule containing SCG powder, which is pierced by spikes within the device upon priming. An internal rotor spins in response to the patients inspired airflow, violently rotating the capsule, and thus liberating the particles into the inspired airstream. The Spinhaler ${ }^{\circledR}$ and also the Rotahaler (Glaxo Pharmaceuticals), dominated the DPI market for a long period. However, each was disadvantaged by the fact that they were single-dose devices. More convenient multi-dose devices have recently been introduced, including the Turbohaler ${ }^{\circledR}$ (Astra Pharmaceuticals), and Accuhaler ${ }^{\circledR}$ (Glaxo Pharmaceuticals). DPIs do not require the level of inhalation or actuation co-ordination that is required to operate MDIs, as they release aerosol during inspiration. In addition, they do not contain CFCs. However, they remain significantly less popular than MDIs. Their limited acceptance is attributable to a number of factors. Firstly, the penetration of drug into the lungs is generally less efficient than that produced by MDIs or nebulisers (Zainudin et al., 1990), and techniques such as breath holding will not improve the deposited fraction (Hansen and Pederson, 1989). Large agglomerates of drug and carrier are drawn into the airstream, meaning that few of the aerosolised particles lie within the respirable range (Davies, 1975). This, and the hygroscopic nature of the particles, means that a significant proportion undergo impaction in the mouth, often leading to irritation and cough (Smith and Bernstein, 1996). The second disadvantage of the DPI is that they often require the manipulation of small drug loaded capsules, or foil disks. This may prove difficult for patients experiencing an asthmatic episode, and those with diseases such as arthritis. Lastly, they require high inspiratory flow rates in order to disperse the powder into small particles. This may not be possible for certain patients, including children and the infirm, and means that drug delivery is subject to wide inter-patient variation (Virchow et al., 1994). The Turbohaler ${ }^{\circledR}$ presently represents the cutting edge of DPI technology, as a breath-operated, multidose device. However, the use of DPIs remains relatively uncommon. Despite this, the desire for a propellant-free, portable multidose inhaler, means that they are likely to undergo continued research and development.

\subsubsection{Nebulisers}

Nebulisers have been used in one form or another for centuries, serving to convert medical solutions, or suspensions, into respirable droplets. The precursor of modern nebulisers was the hand operated bulb nebuliser introduced in 1938 (McPherson, 1985). Squeezing a rubber bulb released a spray of bronchodilator solution from a glass bottle, which the 
patient inhaled. The device was inconvenient, as the drug had to be reconstituted prior to use, gave unpredictable doses, and significant oxidation of the medicament within the solution occurred. However, the advent of plastics, and portable, oil-free compressors lead to dramatic improvements in nebuliser design and performance, and there are now a wide variety of nebulisers based on ultrasonic, or gas jet designs (Grossman, 1994). Modern nebulisers have numerous advantages over MDIs, and DPIs, for the delivery of agents to the respiratory tract. Firstly, they are simple to use, providing a continuous aerosol supply, and permit the inhalation of droplets during normal tidal breathing. This is particularly beneficial to patients who find it difficult to achieve the breathe co-ordination necessary to use an MDI efficiently (such as infants, patients on mechanical ventilators, and the infirm). Secondly, large volumes of drug solution/suspension can be delivered to the patient, permitting the delivery of large therapeutic doses. Nebulisers may also be used to formulate hydrophilic drugs that prove impossible to formulate in a MDI or DPI (eg. dornase alfa, Pulmozyme ${ }^{\circledR}$, Roche), and they may be used to simultaneously administer mixtures of drugs. Despite these advantages, the use of nebulisers has been limited due to their reliance on compressed air drivers, gas cylinders, and power supplies. Nebulisers also achieve a poor level of lung deposition. For instance, Zainudin et al. (1990) demonstrated that only $9.9 \%$ of the available drug was deposited in the lung when delivered by nebuliser. Nebulisers also remain relatively expensive, and as such are not available on NHS prescription. However, the disadvantages of nebulisers are rapidly being addressed as nebuliser technology advances, driven by the need for CFC-free devices. Significantly, the nebuliser remains the most popular aerosol generating device used during the development of novel inhalation systems, such as peptides and liposomes, due to their relative ease of formulation.

\subsubsection{Ultrasonic nebulisers}

\section{Principles of operation}

The first of the two available types of nebuliser, the ultrasonic device, is considered in detail in chapter 6 . Briefly, ultrasonic nebulisers rely on the production of high frequency soundwaves (1-2 MHz) induced by the vibration of a piezo-electric crystal. These waves are transmitted through a coupling fluid to a chamber containing the nebuliser fluid. The vibrations create a high concentration fountain of droplets at the liquid surface, which contain a polydisperse distribution of droplet sizes. The smaller droplets are entrained into 
the inspired airstream, with or without the aid of an electric fan. Larger droplets are trapped on strategically placed obstacles, the "baffles", to fall back to the fluid reservoir. This ensures that the emitted aerosol is of a more "respirable" size. Many different models of ultrasonic nebuliser are available commercially, ranging from small domiciliary nebulisers, with a $10 \mathrm{ml}$ capacity, to large volume $(>100 \mathrm{ml})$ nebulisers, designed for continuous aerosol generation.

\subsubsection{Jet nebulisers}

The second type of nebuliser is the gas jet operated device, reliant upon high velocity gas for operation (fig. 1.4). These "jet nebulisers" have become established as the most popular type of nebuliser in the UK, and are commonly used for the delivery of aerosols in hospitals, and to an increasing extent in patients' homes (Leong, 1992). The modern jet nebulisers are made of lightweight injection moulded plastics, and most have a maximum fluid capacity of approximately $10 \mathrm{ml}$ (Kendrick et al., 1997).

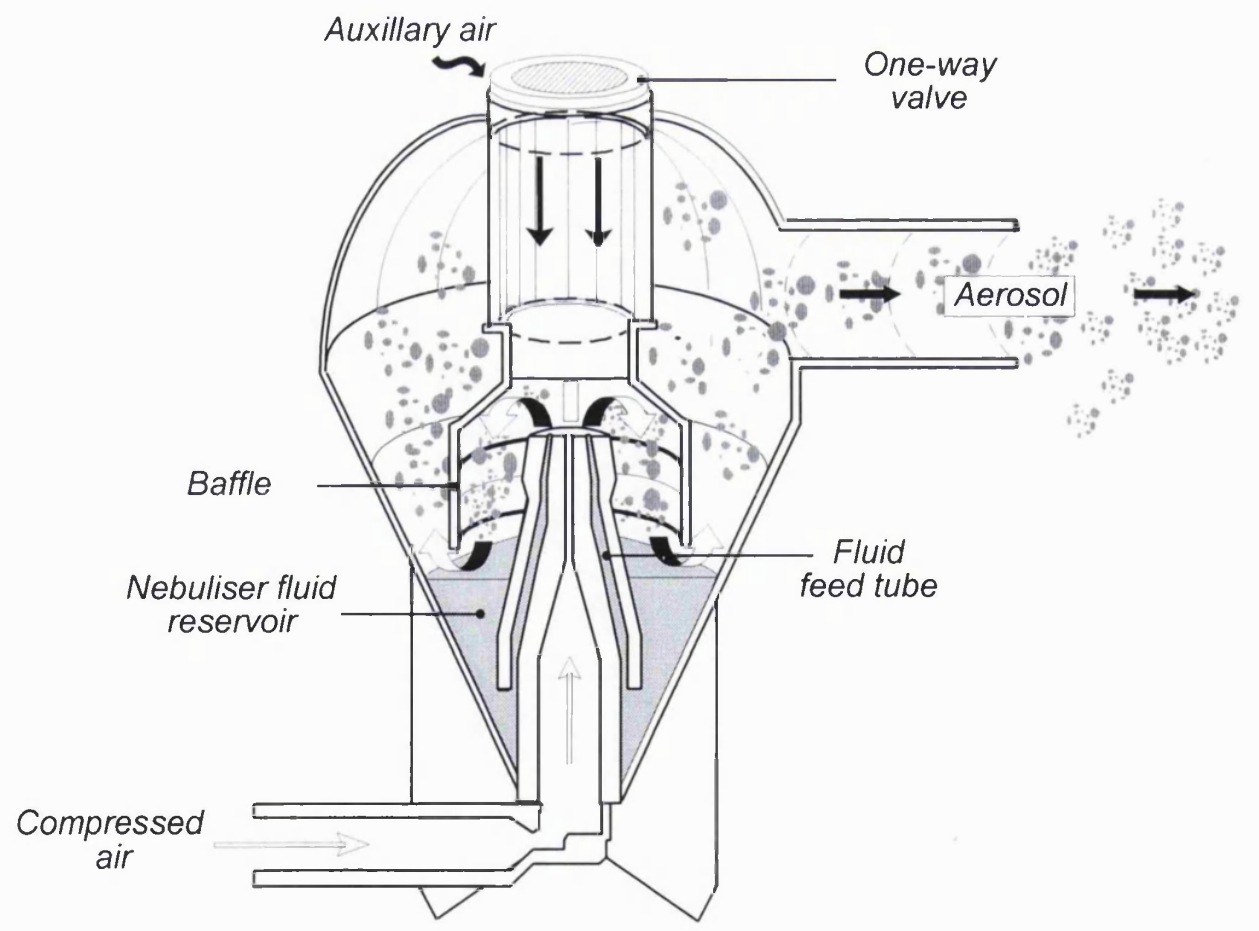

Figure 1.4 The Pari IS- $2^{\otimes}$ (Pari-Werk), an example of a modern jet nebuliser. It incorporates baffles, a breath enhanced, open vent, one-way valve system, and an inverted cone fluid reservoir. These features serve to maximise the respirable output. 
Air, or oxygen, from compressors, cylinders, or hospital gas-lines, is forced through the nebuliser chamber containing the drug solution/suspension. The jet of gas passes out of a narrow hole, the Venturi nozzle, either tangentially (concentric nebulisers, e.g. Respirgard $\mathrm{II}^{\circledR}$, Marquest), or co-axially (eg. Inspiron Mini-neb ${ }^{\circledR}$, Bard) creating an area of negative pressure adjacent to a fluid feed capillary (the Bernoulli effect). The end of the fluid feed capillary is immersed in the nebuliser fluid, which is drawn up the capillary in response to the pressure gradient, ultimately emerging into the gas stream. As it does, fine filaments are formed, which collapse under the influence of surface tension to yield primary aerosol droplets (see chapter 3). Large, non-respirable droplets represent greater than $99 \%$ of the primary aerosol mass (May, 1973). These droplets have a large inertia, and fail to negotiate strategically placed baffles, included to optimise the emitted respirable fraction. The impacted droplets fall back to the reservoir to be recycled, leaving smaller, more respirable droplets to evade the baffles in the secondary aerosol, which is delivered to the patient via a face-mask or mouthpiece.

\subsubsection{Output of nebulisers}

Nebulisers are operated continuously for extended periods. However, in so doing, aerosol is released from the nebuliser during the two-thirds of the breathing cycle that constitutes the non-inspiratory phase. As a result, a significant proportion of the aerosol is unavailable for inhalation. Devices such as holding chambers may minimise this problem. The aerosol is released into the chamber, where it is held during exhalation due to the closing of a one way valve (Thomas et al., 1988). The stored aerosol is then made available during the next phase of inhalation. Alternatively, a finger operated interrupter may be used by the patient to halt gas flow at the time of expiration (eg. Pari- $L^{\circledR}{ }^{\circledR}$, Pari-Werk). Although useful, such remedies have largely been made obsolete due to the development of breath-enhanced nebulisers. Such nebulisers (eg. Pari-LC ${ }^{\circledR}$, Pari-Werk) contain a vent in the nebuliser chamber wall. Air is drawn through the vent in response to the reduced pressure created upon inspiration, and supplements the gas flow carrying aerosol to the patient. This boosts the nebuliser performance, by supplementing the air supplied to it with the patient's own breath. Useful aerosol production is therefore increased to the point where it matches the patient's tidal volume, and in so doing greatly enhances the nebuliser performance (Dennis, 1995). A valve may also be included in the vent, which closes upon expiration, with the result that aerosol is only produced due to the compressed gas supply alone. Aerosol is thus 
saved for the next period of inspiration, and hence drug losses are minimised. In so doing, the breath-enhanced nebulisers may produce approximately twice the useful aerosol output achieved by conventional nebulisers (Knoch and Wunderlich, 1994). Despite such advances, a significant volume of the fluid added to a nebuliser, usually about $1 \mathrm{ml}$, is inevitably retained within the device (Clay et al., 1983b). This volume of fluid is distributed on internal structures, such as the walls and baffles, as a residual or "dead" volume. This lost dose may be reduced by tapping the nebuliser, which dislodges some of the retained droplets, and makes them available for reatomization (Everard et al., 1994). Alternatively, the residual fraction may be reduced by the use of larger fill volumes, as the internal surface area, and hence the dead volume, is fixed (Clay et al., 1983b). This may be achieved by the use of diluents. In addition, the more advanced nebulisers (eg. Pari-LC ${ }^{\circledR}$, Pari-Werk) have a chamber shaped as an inverted cone, which maximises the volume of fluid available to the fluid feed capillaries towards the end of nebulisation.

A nebuliser must be designed to retain as many large droplets as possible without reducing the aerosol output rate to levels that become a threat to patient tolerance. The output rate from nebulisers may vary markedly depending on a number of factors, including the physicochemical properties of the formulation (see chapter 3). For instance, as the surface tension of a fluid is reduced impacted droplets adhere less to the internal structures. A greater number of droplets are therefore made available for re-nebulisation, and hence the aerosol output is increased (Davis et al., 1978; O'Callaghan and Barry, 1997). Conversely, solutions with increased viscosities resist droplet formation from the fluid jets, and adhere to the baffles and walls of the chamber. As a result, they yield reduced output rates (McCallion et al., 1995). The output of aerosol from jet nebulisers may be independent of gas flow rate (Clay et al., 1983b; Kradjan and Lakshminarayan, 1985). However, Hess et al. (1989) demonstrated that output was increased by $50 \%$ with an increase in flow rate from 6 to $8 \mathrm{~L} / \mathrm{min}$.

It is more informative to talk in terms of the respirable aerosol output from nebulisers. This may be defined as the proportion of an emitted aerosol that is contained in droplets with a "respirable diameter", that is less than about 5 to $6 \mu \mathrm{m}$ (Newman and Clarke, 1983). Unlike the total aerosol output, the respirable output is dependent on the driving gas flow rate used, with higher gas flow rates producing an increased respirable output (see chapter 3 ). 


\subsubsection{Nebulisation times}

The point that nebulisation is halted in clinical use remains largely arbitrary. It is now advised that nebulisation should last no longer than 10 minutes, in order to optimise compliance (Nebuliser Project Group, 1997). Nebulisation is either stopped after a specific period of time, or alternatively at 'dryness'. Nebulisation for a fixed time aids patient compliance, and is simpler for nursing staff to follow. However, output rates vary significantly, depending on the nebuliser, formulation, fill volume and driving gas flow rate (eg. Kradjan and Lakshminarayan, 1985; Kendrick et al., 1997). Consequently, nebulising for a fixed time may produce a significant variation in the amount of drug delivered to the patient if the nebuliser, formulation, or conditions are altered. For this reason, nebulising to a specific point of aerosol production is a common practice. The sputtering time is the point at which the aerosol output becomes visibly intermittent, in response to a fall in the residual volume to below a critical level. Air is drawn up the fluid feed capillaries, and hence the aerosol output becomes erratic. The clinical time is the point at which aerosol delivery is ceased once sputtering has been observed, and the total time is the point at which no further aerosol is produced from the device (Kradjan and Lakshminarayan, 1985). Newman et al. (1986) further defined the nebulisation time as the point representing 30 seconds after the last visible aerosol release. Different nebulisers give different nebulisation times, due to differing baffling efficiencies, presence or absence of open vent systems, and variations in the design and construction of other internal structures. The physicochemical properties of the fluid will also influence the nebulisation time, with lower viscosities and surface tensions yielding more rapid aerosol output rates, and hence reduced nebulisation times (McCallion et al., 1995; chapter 3). Operation of jet nebulisers at higher gas flow rates is also associated with reduced nebulisation times. Ultrasonic devices are less flexible in this regard, as they are limited to one or two power settings.

\subsubsection{Temperature and concentration effects}

The temperature of fluid within a jet nebuliser may fall by as much as $10-15^{\circ} \mathrm{C}$ during nebulisation, as solvent evaporation saturates the relatively dry gas jet (Mercer, 1973; Ferron et al., 1976; Cockroft et al., 1989). This may cause the precipitation of drugs that are poorly soluble, such as mucolytics and antimicrobials (Taylor et al., 1992). Additionally, patients with sensitised airways, such as asthmatics, have been shown to experience bronchoconstriction when cold solutions have been inhaled (Lewis, 1983). The evaporation 
of solvent also causes a rise in the concentration of dissolved or dispersed materials with time (Niven et al., 1991b; Stapleton and Finlay, 1995; see chapter 3). Detrimental effects on droplet size, and output rate may result as the viscosity rises in response to the falling temperature and increasing concentration (Davis, 1978). The absence of gas flow, and the highly saturated internal environment means that minimal evaporation occurs during ultrasonic nebulisation. Typically the temperature of ultrasonically nebulised fluids increases during operation. This is because most of the energy supplied by the piezoelectric crystal becomes expended as heat, rather than in droplet formation (Mercer et al., 1968). Due to this, ultrasonic nebulisers may prove useful for delivering low solubility drugs, such as pentamidine isethionate, that are susceptible to precipitation as jet nebulised solutions cool (Taylor and Hoare, 1993). Conversely, they may be less suitable for certain heat labile formulations, including proteins (Cipolla et al., 1994).

\subsubsection{Droplet size}

The droplet size produced by a nebuliser is critical in determining the in vivo efficacy of a nebulised formulation (O'Callaghan and Barry, 1997). The droplet sizes produced by jet and ultrasonic nebulisers are discussed at length in chapter 3 and 6 respectively. For efficient delivery of drugs to the lung, a nebuliser must be capable of producing droplets below about 5 to $6 \mu \mathrm{m}$ in size (Stahlhofen et al., 1980; Newman et al., 1986). Although this figure is rather arbitrary, droplets that are larger than this will tend to deposit in the mouth and throat. For certain therapies, such as the antibiotic treatment of Pneumocystis carinii pneumonia, penetration into the non-ciliated regions of the lower periphery is required (Thomas et al., 1991a). This necessitates a droplet size of less than about $2 \mu \mathrm{m}$ (Stahlhofen et al., 1980; Newman and Clarke, 1983). In vitro measurement of droplet size is the most commonly used indicator of a nebuliser's potential clinical performance. A variety of methods have been employed to measure droplet size, but those based on cascade impaction, and laser diffraction, discussed in chapter 3, are most popular.

There are many different jet and ultrasonic nebulisers available to the clinician, and each may vary markedly in design. The design and geometry of internal nebuliser structures will influence the formation and retention of aerosol droplets. As a result, the aerosol size characteristics may vary significantly between devices. For instance, variation in the diameter of the Venturi jet between jet nebuliser models causes the pressure differential 
between the capillary end and the fluid reservoir to vary. The fluid therefore emerges from the capillary at different rates in different nebulisers, and consequently the resulting droplet size varies. Similarly, ultrasonic nebulisers vary in the formation and transmission of soundwaves between models. In addition, high efficiency baffles may be characteristic of nebulisers that produce smaller secondary droplet sizes. Furthermore, the droplet size may vary between individual nebulisers of the same model, due to variations in production, or progressive wear of structures within the device upon repeated use (Alvine et al., 1992; see chapter 4).

\section{Factors influencing the droplet size produced from jet nebulisers}

The factors influencing the droplet size produced by a jet nebuliser are discussed in detail in chapter 3. The mean aerosol size is principally determined by the gas flow rate. An increase in gas flow leads to increases in the shear forces to which droplet forming filaments are exposed. In addition, the droplets attain a higher velocity, and as such have a greater momentum, resulting in enhanced deposition on baffles. Atomization theory indicates that the physicochemical properties of the nebulised fluid will influence the droplet size produced (Glukohv, 1969; Rizkalla and Lefebvre, 1974). However, this remains to be conclusively proven, as the droplet size is a complex function of the nebuliser design, and the fluid properties (Davis, 1978). In addition, changes in the primary aerosol characteristics may not be reflected in the emitted aerosol, due to baffling.

\section{Factors influencing the droplet size produced from ultrasonic nebulisers}

The production of droplets from ultrasonic nebulisers is discussed in chapter 6. Briefly, aerosolised droplets are generated by ultrasonic nebulisers as a result of the formation and collapse of cavities within the liquid. This results in the development of standing capillary waves, the crests of which break up to form droplets. The primary droplet size distribution is dependent on the alternating frequency, and also on properties of the nebulised fluid, namely surface tension and viscosity. The influence of the nebuliser fluid properties is discussed more fully in chapter 3. As with jet nebulisers, the secondary droplet size is determined by the primary droplet size, and the design of structures such as baffles.

Despite the fact that the production of a small droplet size is used as an indicator of an efficient nebuliser, there are relatively few studies that demonstrate clear clinical benefits 
of producing smaller droplet size. Certain studies fail to show any correlation between droplet size and clinical response (Douglas et al., 1986; Hadfield et al., 1986). However, this may be due to inter-patient variability in breathing pattern (Pavia et al., 1977), or to the polydispersity of the aerosols. Alternatively, it may be because many aerosolised drugs, such as bronchodilators, are used at super-therapeutic doses. However, studies involving terbutaline (Clay et al., 1986), and isoprenaline (Patel et al., 1990), have indeed demonstrated a correlation between droplet size and the clinical response (Clarke, 1995). Furthermore, an increased bronchodilator response was produced when nebulised salbutamol droplets were reduced in size by $40 \%$ (Johnson et al., 1989). Despite such studies demonstrating that improved aerosol quality correlates with improved therapy, nebuliser manufacturers rarely include directions concerning variables such as fill volume and driving gas flow rate. In addition, the aerosol size is seldom determined by clinicians (Mallol, 1993). In fact, in a survey of hospital doctors, only $3 \%$ specified the driving gas flow rate when prescribing for nebulised medications (O'Driscoll and Cochrane, 1987). The present study investigated the importance of such variables during liposome nebulisation.

\subsubsection{Nebulisers and liposomes}

The subject of this thesis is the nebulisation of liposomes. Liposomes are microscopic lipid vesicles that encapsulate a portion of aqueous phase within their structure. In so doing, they may act as targeted carriers of hydrophilic, or hydrophobic drugs to the lung. They may also control drug release within the lung, thus increasing local tissue concentrations and minimising release to extrapulmonary sites (Niven, 1996). Although DPI and MDI devices have been investigated, nebulisers are the simplest means of aerosolising liposomes, and have been used in each of the human studies conducted thus far.

\subsection{Liposomes}

Liposomes were first described in 1965 (Bangham et al., 1965). The constituents and mechanisms of liposome manufacture are discussed in chapter 2. Briefly, they are formed when phospholipids, or certain other amphiphiles are swollen in excess aqueous phase (Lasic, 1988). The molecules arrange into ordered structures, commonly producing the characteristic "onion-layer" appearance of multilamellar liposomes (fig 1.5). The hydrophilic groupings of the phospholipids surround channels of aqueous phase, and the hydrophobic groupings aggregate in lipid rich channels. 


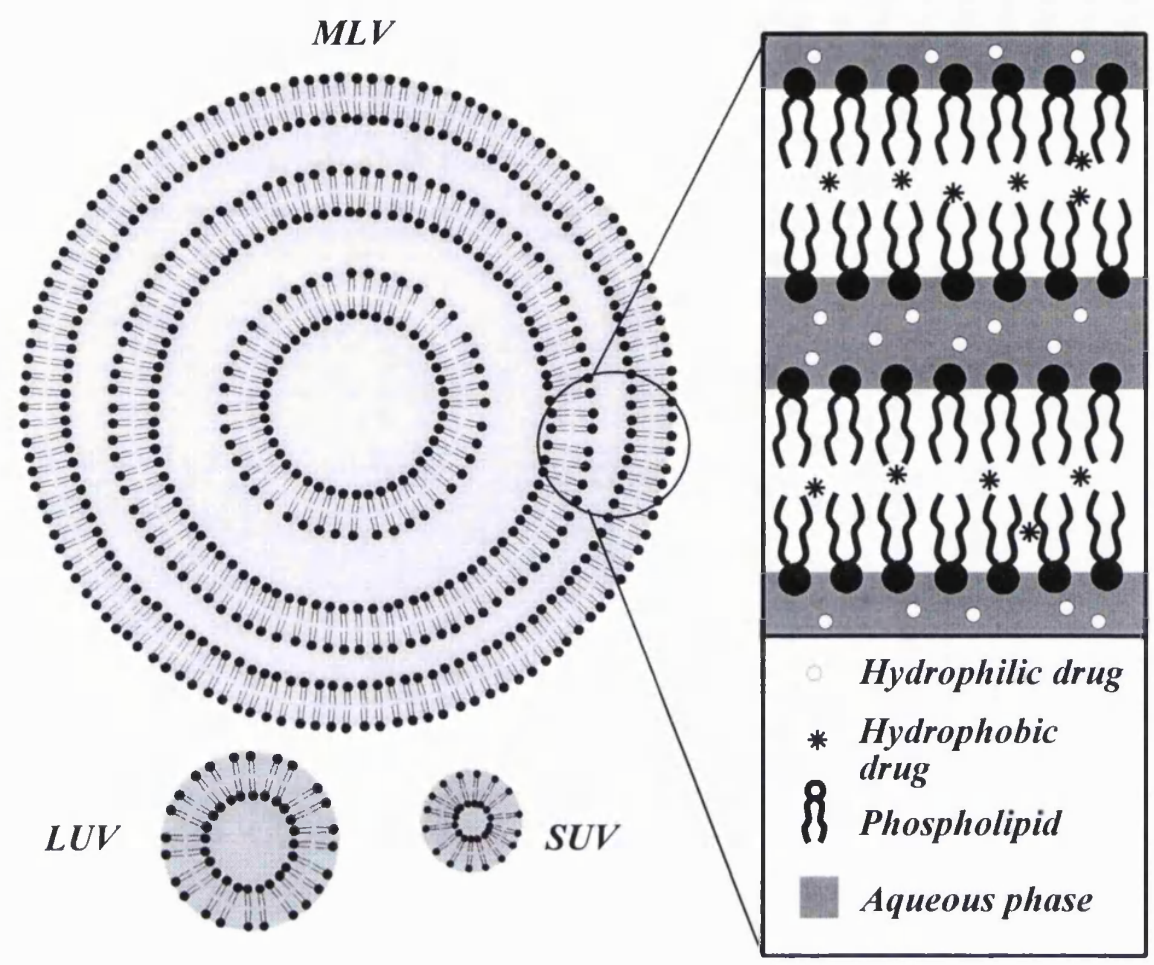

Figure 1.5 Structure of the liposome in its different forms. Multilamellar vesicle $(M L V)$; large unilamellar vesicle ( $L U V)$; and small unilamellar vesicle $(S U V)$. The amphiphilic nature of the liposome allows drugs of varying physicochemical nature to be encapsulated in different regions (see caption).

\subsubsection{Liposomes as drug carriers}

\section{Liposomes can encapsulate a wide variety drugs}

Liposomes can encapsulate a wide variety of drugs, making them useful for a range of diagnostic and therapeutic applications. The liposome entraps a portion of aqueous phase within a closed bilayer structure following vesicle formation. In so doing, accompanying dissolved aqueous material is encapsulated. All hydrophilic drugs have the potential for entrapment within liposomes, with the encapsulation efficiency being principally dependent on the volume of aqueous phase that is incorporated. This in turn will depend on the mean liposome size, number of bilayers, and the lipid concentration (New, 1990). Hydrophobic agents may be added to the lipid phase during liposome preparation, resulting in partitioning into the repeating lipid bilayers upon vesicularisation. The encapsulation efficiency in this instance largely depends on the lipid concentration, the transition state of 
the bilayers and the oil/water partition coefficient of the drug (see chapter 2). Amphiphilic drugs interact with both the hydrophilic channels, and the hydrophobic bilayer regions. Agents may also be covalently bound to the liposome. An example is the "stealth" liposome, which provides extended residence times within circulation due to bonding of the outer liposomal phospholipids to hydrophilic polymers such as polyethylene glycols (PEGs) (Allen and Chonn, 1987; Woodle and Lasic, 1992). The bilayer surface may also be modified in such a way that it will interact specifically with certain structures in the biological environment (Crommelin and Schreier, 1994). For instance, the phospholipids may be coupled to monoclonal antibodies to produce "immunoliposomes", capable of targeting specific cell surface antigens within the body. Upon administration the immunoliposomes concentrate at targeted cell surfaces, such as lung endothelial cells (Hughes et al., 1989). Electrostatic interaction may also bind therapeutic materials, such as interleukin-2 (IL-2), to liposomal phospholipids (Bergers et al., 1993). In such a case the concentration of charge holding constituents, and the ionic strength of the aqueous environment influence the efficiency of encapsulation.

\section{Liposomes are relatively non-toxic}

A prerequisite of a drug carrier system is that it should produce no toxic effects following administration. In addition, following delivery of drug to the site of action the carrier system must be cleared from the system rapidly, and without toxic metabolites. One of the principal advantages of liposomes is that it they are prepared from materials found in naturally high levels within the body. The similarity of liposomes to the constituents of living membranes allows them to be administered without significant adverse effects. In this way, the liposome may be considered to be both biocompatible and biodegradable.

\section{A wide variety of liposome types and sizes may be produced}

A variety of liposome types may be prepared relatively easily, in a range of sizes. A number of preparatory methods may be used. Each involves a lipid hydration step, but beyond this the combination of constituents and conditions employed is overwhelming (see chapter 2).

\section{Liposomes may control the release of entrapped materials}

With careful engineering the liposome may provide a means of controlling the release of entrapped agents. The use of cholesterol (chol) in varying amounts, and the selection of 
different phospholipids changes the bilayer packing properties, and as a result the bilayers' apparent "rigidity" (Senior, 1987) (see chapter 2). Phospholipid bilayers may be in either the liquid-crystalline ("fluid") or gel state ("solid"), dependent on temperature. An increase in temperature excites the phospholipid molecules to the point, called the main phase transition temperature $\left(\mathrm{T}_{\mathrm{m}}\right)$, where the bilayer is transformed from the gel to the liquidcrystalline state. The $T_{m}$ for a specific phospholipid may vary between $-15{ }^{\circ} \mathrm{C}$ for phosphatidylcholine derived from eggs (eggPC), to more than $50{ }^{\circ} \mathrm{C}$ for a phospholipid such as distearoyl phosphatidylcholine (DSPC) (Crommelin and Schreier, 1994). At temperatures greater than the $T_{m}$, the bilayer is relatively fluid, and thus relatively permeable to the release of entrapped materials. Furthermore, the inclusion of cholesterol in the bilayers increases their stability to the extent that the $T_{m}$ may become abolished entirely (Senior, 1987). As each phospholipid produces bilayers with a characteristic $T_{m}$, the selection of the appropriate bilayer constituents provides the potential for controlling the release of the entrapped materials. In so doing, a variety of release rates, appropriate to different therapeutic requirements, may be produced.

\subsubsection{Medical applications of liposomes}

Liposomes have proved to be highly versatile drug carriers, and since their discovery have been investigated for a variety of applications. It is beyond the scope of this introduction to explore thesejin any detail.Instead, an indication of the diverse areas of therapeutics to which liposomes have been applied will be presented.

The simplest pharmaceutical application of liposomes is as a non-toxic solubilizing vehicle for poorly soluble drugs (Lidgate et al., 1988). AmBisome ${ }^{\circledR}$ (Vestar Ltd.), the first liposomal product to be granted a product licence in the UK, uses liposomes for such an application. It is a lyophilised powder consisting of hydrogenated soy phosphatidylcholine (soyPC), DSPC, cholesterol, and $\alpha$-tocopherol ( $\alpha-\mathrm{T})$. Upon reconstitution the lipids form liposomes, encapsulating as they do the lipophilic antifungal agent, amphotericin-B $(\mathrm{ampB})$. The incorporation of ampB within liposomes avoids the need for potentially toxic co-solvents, and results in a reduced toxicity due to reduced delivery of free ampB to human cells (Lopez-Berestein, 1988). However, the majority of research into the therapeutic applications of liposomes has concentrated on their ability to target drugs to specific cell types, and tissues, following intravenous administration. Liposomes tend to be 
rapidly removed from circulation by hepatic Kupffer cells, and macrophages of the spleen and bone marrow, which comprise the MPS (Senior, 1987; Crommelin and Schreier, 1994). This restricts the delivery of encapsulated drugs to these sites, and limits the liposome's ability to prolong drug action. However, this clearance mechanism may be exploited when tissues of the MPS are therapeutic targets (eg. Gabizon et al., 1986). For instance leishmania infected Kupffer cells accumulate antimony-containing liposomes following injection, leading to a reduction in exposure of the drug to non-infected cells, and to an improved efficacy (New et al., 1978). The MPS may also be exploited as a sustained release reservoir, taking up drug loaded liposomes, and slowly releasing drug back into circulation, upon intracellular degradation (Ellens et al., 1982). More recently, strategies have been developed to avoid the uptake of liposomes by the MPS, thus allowing targeting of other cellular sites, and also prolonging drug release. This has been achieved with the use of smaller liposomes $(<100 \mathrm{~nm})$ with bilayers in the gel state at $37^{\circ} \mathrm{C}(\mathrm{eg} . \mathrm{DSPC})(\mathrm{Hwang}$ et al., 1987); the inclusion of bilayer cholesterol (Kirby et al., 1980); and coating with hydrophilic materials such as gangliosides and PEGs (Allen and Chonn, 1987; Woodle and Lasic, 1992). However, even if MPS uptake is minimised liposomes are generally unable to penetrate healthy endothelium, and as such tend to be retained in blood vessels following injection. Enhanced extravasation of the liposomes may be achieved in areas of inflammation, or tumours, where the endothelium is often more permeable (Crommelin and Schreier, 1994), but generally extravasation remains an elusive goal.

Recent advances in antibody technology have enabled targeting of specific cells within the body with drug loaded liposomes. Monoclonal antibodies (Mab) which recognise specific cell surface antigens may be incorporated into the liposomes, causing them to concentrate at the target cell surface following injection into the circulation. In so doing, the liposome may either release its drug payload at the cell surface, or become internalised into the target cell prior to the release of drug (Hughes et al., 1989). Other applications of injected liposomes include the carriage of immunostimulants for tumorcidal macrophage activation (Gregoriadis, 1990), and also the delivery of DNA into cells (Nicalau and Cudd, 1989).

One of the/principal advantages of liposomal drug delivery is the potential of controlling drug release. Drug delivery to the eye provides a useful example. The residence time of free drug within the eye, following topical administration, is often very short, due to the fast 
turnover of the tear film. Meisner et al. (1989b) showed that positively charged liposomes interact with the conjunctiva, prolonging contact with the eye, and hence reducing the ocular clearance of entrapped atropine. This allows a reduction of dosing frequencies, and also enhances the penetration of the ocular tissues. Liposomes have also provided a useful system for enhancing the delivery of dermally/transdermally directed drugs (Egbaria and Weiner, 1990). However, one of the most researched applications of liposome technology has been their use as inhaled drug carriers. The lung is readily accessible to inhaled liposomes, permitting local drug targeting for topical effects. This provides the potential for prompt drug action, with reduced extra pulmonary adverse effects. In so doing the liposomes may improve the therapeutic index of the inhaled drug. The lung residence times of inhaled agents may be increased significantly by encapsulation in liposomes (eg. Farr et al., 1994), and they provide a means of solubilising hydrophobic drugs into aqueous based inhalation systems (Vidgren et al., 1995). Liposomally entrapped agents may also be inhaled for systemic action, producing sustained plasma levels, compared with the equivalent non-liposomal dose (eg. Taylor et al., 1989). Liposomal formulations for inhalation, being the subject of this thesis, are discussed at length in sections 1.8 to 1.11 .

\subsubsection{Disadvantages of liposomes as drug carriers}

Despite intense research, and significant advancements in liposome technology, there remains very few marketed liposomal products. The development of liposomal products has been hindered by a variety of factors, including their cost, poor drug encapsulation efficiencies, relative instability, and difficulties in production scale-up (see chapter 2). Despite this, significant progresses have been made towards the development of liposomal products for delivery to the lung.

\subsection{The pulmonary delivery of liposomes}

\section{Liposomes and lung surfactant}

A distinct advantage of liposomal drug carriers is that they are composed of materials which are found in naturally high quantities in the body. They are therefore biocompatible and biodegradable. The lung contains high levels of phospholipid and cholesterol (10 to 20 $\%$ of the lung's dry weight, King and Clements, 1985), principally contained in lung surfactant, which are the most common components of liposomes. As such, liposomes settling on the respiratory surfaces would be expected to present minimal physiological 
disturbance. Lung surfactant is a complex mixture responsible for the maintenance of airway integrity. Without it, the smaller alveoli of the lung would remain in a deflated state following exhalation, due to the elevated surface tension at the air interface (Notter and Morrow, 1975). The surfactant reduces this surface tension by the action of the phospholipid fraction, comprising primarily saturated and unsaturated phosphatidylcholines, in particular dipalmitoyl phosphatidylcholine (DPPC). It also contains small amounts of phosphatidylserine (PS), phosphatidylinositol (PI), phosphatidylethanolamine (PE), and lysophosphatidylcholine (lysoPC). Efficient surfactant activity is also dependent on a smaller protein fraction, comprised of three glycoproteins, surfactant proteins A, B and C (SP-A/B/C)(Schäfer, 1992). The surfactant phospholipids are adsorbed as a monomolecular film at the air interface of the alveoli. As the film is compressed during exhalation, phospholipid molecules leave the film, and become sequestered in the underlying aqueous phase. As the breathing cycle enters the inspiratory phase, the film becomes expanded, causing the sequestered phospholipids to rejoin the interface, thus maintaining the reduced surface tension. This movement of surfactant to the interface is accelerated by the presence of the hydrophobic surfactant proteins, ensuring that there is no loss of film coverage during inspiration (Schäfer, 1992). The importance of lung surfactant is illustrated by the effects of the often fatal Neonatal, and Adult, Respiratory Distress Syndromes (A/NRDS). NRDS occurs in neonates as a result of under developed surfactant, and surfactant loss in ARDS may result from sepsis. The mortality from ARDS and NRDS may be reduced by the administration of exogenous phospholipids in nebulised preparations such as Exosurf ${ }^{\circledR}$ (Glaxo-Wellcome), which contains dispersed DPPC as its major component (Weg et al., 1994).

The development of liposomes as drug carriers to the lung has been aided by the many investigations of lung surfactant, as the fate of deposited liposomal phospholipid is intrinsically linked to that of surfactant. Ivey et al. (1977) were the first to report the delivery of phospholipid vesicles to human lungs, as a surfactant replacement therapy for NRDS, and the fate of exogenous phospholipid in the lung has since been well documented (Taeusch et al., 1983). The rate of synthesis and turnover of surfactant is influenced by a number of factors, including alveolar ventilation, cholinergic/adrenergic mediation, and parenchymal pH (King and Clements, 1985). Alveolar type II cells synthesise the phospholipid fraction, which is then combined with surfactant proteins in lamellar bodies, 
and extruded into the alveolar space (Williams, 1977). In healthy lung, the clearance of surfactant balances surfactant secretion to form a continuous film. Protein regulated surfactant uptake by Type II cells, and to a lesser extent by macrophages, has also been demonstrated with exogenous liposomal phospholipid (Claypool et al., 1984).

Clearance of liposomes from deposition sites in the lung limits the duration of drug release. Surfactant clearance, and by inference that of liposomal phospholipid, may also occur via mucociliary clearance, the lymphatics, macrophage ingestion, enzymatic degradation at the respiratory surface, or by reuptake into alveolar cells (Reifenrath, 1983; Miles et al., 1985; Stern et al., 1986; Schäfer, 1992). It has been demonstrated that exogenous phospholipids become associated with the endogenous surfactant pool, following administration to rabbit lung (Hallman et al., 1981). However, an earlier study demonstrated that nebulised DPPC liposomes had a shorter residence time within the lung than endogenous DPPC, following deposition. This was attributed to rapid clearance via type I cells (Geiger et al., 1975). Radiolabelled SUVs variously composed of $l$-DPPC and $d$-DPPC, with and without DPPG, were administered intratracheally to rabbits. The $l$ - and $d$-isomers were cleared at identical rates from the lung, indicating that enzymatic clearance of the administered phospholipid may not represent a major clearance route. This is significant, as it indicates that potentially irritating degradation products are unlikely to be produced as a consequence of clearance. DPPG-containing liposomes became cleared at twice the rate of the pure DPPC vesicles. This was interpreted as being indicative of a predominance of physical as opposed to chemical mediated clearance, as the presence of liposomal DPPG increases fusion with mammalian cells (Papahadjopoulos et al., 1973a). Petenazzo et al. (1989) argued that DPPC/DPPG/chol liposomes were cleared more slowly from rabbit lung than similarly administered surfactant, due to the existence of separate pathways for exogenous and endogenous phospholipid. However, it may instead have been due to variations in their distribution within the lung, or to differences in liposome size and dose (Taylor and Farr, 1993). That said, McCalden (1990) reported that liposomal phospholipid took longer to enter the systemic circulation than non-liposomal lipid, due to delayed breakdown of the liposomes on the respiratory surface. Furthermore, in vitro analysis by Fisher et al. (1987), indicated that cellular uptake of surfactant was influenced more by metabolic inhibitors than the similar uptake of liposomal lipid. This, the authors concluded, was further evidence that exogenous and endogenous lipids are cleared from the lung in different ways. 
Despite this, research indicates that liposome-containing droplets are handled by the lung in a different manner to conventional inhaled drug particles, in that processing, uptake, and recycling by Type II pneumocytes seems to occur as for natural surfactant. In so doing, liposomal phospholipid produces minimal disturbance of normal metabolic processes (Mihalko et al., 1988).

\section{Liposomal aerosol safety studies}

The relative safety of inhaled liposomes has been confirmed in a wide range of studies. Gonzalez-Rothi et al. (1991) examined the impact of liposomes on the phagocytic functioning of alveolar macrophages. No untoward effects of liposome treatment were detected, with regard to either functional or morphological parameters. Additionally, intratracheal (IT) instillation of up to $500 \mathrm{mg}$ of phospholipid mixtures to rabbits failed to elicit any untoward physiological or histological signs. The results suggested that the uptake of liposomes by alveolar macrophages was, to a certain extent, dependent upon the liposome constituents, as eggPC/DPPC (9:1) liposomes were more avidly taken up by macrophages than other types. Wyde et al. (1988) noted no histopathological changes in mouse lung following 120 minute exposure to empty and enviroxime-containing liposomal aerosols. Additional studies demonstrated the safety of liposomes when delivered to sheep (Schreier et al., 1992), and mice (Myers et al., 1993; Waldrep, 1995). Perhaps more significantly, the administration of exogenous lipid to neonates has been performed for the last 20 years without reported problems. Indeed, marketing approval for phospholipid aerosols used for the treatment of NRDS, would have required complete demonstration of their safety. Furthermore, Weg et al. (1994) demonstrated that prolonged administration of Exosurf ${ }^{\circledR}$ was well tolerated by patients with ARDS, and no toxicological responses have been reported in the various studies of inhaled liposomes performed with volunteers (Farr et al., 1985; Taylor et al., 1989; Barker et al., 1994; Hung et al., 1995; Vidgren et al., 1995; Waldrep et al., 1997). Thomas et al. (1991b) made the first objective study of liposome safety in man. No change of lung function was demonstrable up to $6 \mathrm{~h}$ post-inhalation of a soyPC liposome aerosol, and no irritation, or other side-effects were reported. This has recently been supported by studies of inhaled liposomes by healthy volunteers, and also diseased patients (Purcell and Corris, 1995; Waldrep et al., 1997). Current data therefore indicates that inhaled liposome formulations are safe, as was inferred from their similarity to natural lung surfactant. However, as the development of liposomes for inhalation 
progresses further, more stringent safety testing will be required. Many of the pulmonary applications to which liposomes appear to be most appropriate as drug carriers (eg. prophylactic antibiotics, gene therapy) will involve their chronic administration, for which safety data is lacking. Different liposome formulations may also give differing toxicological profiles, and hence the safety of a variety of phospholipids, and other constituents, must be tested. For instance, high cholesterol content liposomes have been shown to sensitize arterial smooth muscle to agonists, attributable to the action of cholesterol on the cell membrane (Broderick and Tulenko, 1984). It remains to be determined whether similar effects occur in bronchiolar smooth muscle. It is also possible that certain diseases may render the lung more sensitive to inhaled liposomes, and as such, safety studies are required in relevant diseases.

\subsection{Liposomes as drug carriers to the respiratory tract}

Liposomes have been investigated as drug carriers to the lung via indirect (that is, via the vasculature) and direct administration (that is, via inhalation). The capillaries of the lung are among the finest in the body, and are thus sensitive to blockage by larger particles (Abra et al., 1984; Hunt et al., 1979). This makes it possible to target the lung with appropriately sized, injected encapsulated drugs. Indeed, an early strategy for lung surfactant replacement therapy by such a mechanism was suggested by Zachman and Tsao (1980). This was based on the observation that intravenously injected liposomal PC was taken up by lung cell organelles. A number of researchers have since noted that liposomes bearing a negative charge (Fidler et al., 1980), or incorporating certain sugar derivatives (Takada et al., 1984), are accumulated within the lung. Such targeting of the lung has been suggested for the treatment of lung tumours (Fidler et al., 1980; Mayhew and Rustum, 1983), but remains largely unexplored for other applications. The dilution effect of systemic distribution means that high doses are usually required to produce a therapeutic response in target organs, such as the lung, following IV administration. Consequently, adverse effects often limit such therapy (Szoka, 1990; Gregoriadis and Florence, 1993). The majority of research concerning the pulmonary delivery of liposomal drugs has therefore involved topical administration to the lung via inhalation. Inhaled formulations provide a convenient means of achieving a rapid onset of drug action at the target site, often without significant extra pulmonary effects. However, the activity of conventional aerosol formulations is typically short lived due to rapid drug clearance from deposition sites. Due to this, multiple dosing 
is required, which increases the likelihood of adverse effects. It has long been accepted that achieving a sustained drug action in the lung would be of benefit to a wide range of inhaled drugs, and it is for this reason that extensive research has concentrated on the application of liposomes to pulmonary drug delivery (Niven, 1992). Liposomes have been shown to prolong the release of entrapped agents in a variety of situations, and have been investigated as a means of increasing organ specificity, reduce systemic absorption, and thus improve the therapeutic index of a variety of drugs. As such, they have great potential for improving therapies directed to the lung.

\section{The potential of inhaled liposomes as drug carriers}

Juliano et al. (1979) demonstrated that radiolabelled liposomes were retained within the small airways and alveoli of rabbit lungs for extended periods following topical administration, with little of the radiolabel becoming lost from the lung to the systemic circulation. In another study eggPC/chol/stearylamine (25:7:1) multilamellar liposomes were used to encapsulate the hydrophilic drug cytosine arabinoside. The formulation was administered as an IT bolus to anaesthetised rats, and the drug became distributed throughout the lung (McCullough and Juliano, 1979). This, and a subsequent study (Juliano and McCullough, 1980), demonstrated prolonged retention of the encapsulated drug within the lung, with a clearance half-life of 6 hours, compared with 20 minutes for the free drug solution. The rapid and extensive systemic absorption of free Ara-C was associated with widespread extra-pulmonary tissue damage, whilst the liposomal drug produced only "modest effects".

The systemic absorption of agents entrapped within inhaled liposomal formulations was further investigated by Woolfrey et al. (1988), using the model hydrophilic agent CF. Neutral dimyristoyl phosphatidylcholine (DMPC)/chol (1:1) and negatively charged DMPC/chol/dicetyl phosphate (DCP) (1:1:0.2) liposomes were prepared, and administered to anaesthetised rats by IT installation. The absorption of CF was found to be lipid dose dependent, with an increase in lipid dose being associated with a faster absorption rate. It was postulated that increased lipid levels initiated biological clearance processes, such as mucociliary clearance, phagocytosis, and/or lymphatic removal, thus speeding drug clearance. The absorption of CF from the charged liposomes was found to occur at least twice as rapidly, and to a greater extent, than that from the neutral liposomes. The authors 
concluded that systemic absorption was not a consequence of the absorption of intact liposomes. The possibilities of using inhaled liposomes as carriers for systemically acting drugs has also been explored using the model hydrophobic drug atropine. Liposomal atropine was shown to provide increased levels in rat lung for prolonged periods, compared with free atropine (Shek et al., 1988). Subsequently, Meisner et al. (1989a) delivered atropine by IT administration to anaesthetised rabbits. The results indicated a similar distribution and retention of the lipophilic drug within the lung as had been demonstrated with hydrophilic molecules. Free atropine was rapidly absorbed into the systemic circulation, making it extensively available to extra pulmonary sites. At 48 hours postinhalation, $20.9 \%$ of the liposomal drug remained associated with lung tissue, compared with only $4.1 \%$ of the free atropine solution. Urine analysis demonstrated that liposome encapsulation altered the distribution of drug to the systemic circulation, causing a less rapid elimination, compared with the free drug. A relatively constant, but reduced, concentration of drug was redistributed to the spleen and kidneys from the liposomal formulation. Tissue analysis demonstrated that an initial peak of atropine appeared in the liver one hour after dosing of the liposomal formulation. This was attributed to selective uptake of intact liposomes, or of liposome-drug fragments by the Kupffer cells, a theory supported by the fact that IT-instilled DPPC liposomes were preferentially accumulated in the liver. However, the authors cast doubt on the possibility that intact MLVs were being absorbed from the lung.

The use of liposomes as solubilising systems for pulmonary directed drugs was further investigated by Waldrep et al. (1993), using the immunosuppressant cyclosporin A (CsA). CsA is commonly administered orally (dissolved in arachis oil), or intravenously (dissolved in polyoxyethylated castor oil), to treat pulmonary allograft rejection. However, the clinical success of the formulation is limited by adverse effects, related to the drug and vehicle, and also to poor penetration of the drug into pulmonary tissue. Topical delivery of the agent to the lung has the potential benefits of producing therapeutic levels at the target site, thus reducing systemic adverse effects. However, formulation of CsA as an aerosol had previously been difficult to achieve, due to the molecule's hydrophobicity. Consequently, Waldrep et al. (1993) investigated several liposomal carriers, in order to formulate the drug for delivery by jet nebuliser. The different phospholipid formulations were assessed according to the aerosol size, and the efficiency of drug delivery. An estimate of respiratory 
tract delivery was made using a multi-stage impinger, and a computer model. Liposomal CsA aerosol was calculated as giving deposition within the peripheral airways, with minimal deposition in the mouth or larynx. The results indicated that pulmonary deposition of CsA-liposomes would produce significantly higher lung levels than the systemically dosed drug.

The delivery of drugs of varying physicochemical natures was investigated by Mihalko et al. (1988), following IT instillation to rat lungs. Using extruded eggPC/PG/chol (5:1:4) multilamellar liposomes, the pulmonary absorption of three model compounds, $\left[{ }^{14} \mathrm{C}\right]$ benzylpenicillin, $\left[{ }^{125} \mathrm{I}\right]$ oxytocin, and $\left[{ }^{14} \mathrm{C}\right]$ glyceryltrinitrate $(\mathrm{GTN})$ was studied. Liposomal formulations of the hydrophilic agents, benzylpenicillin and oxytocin, produced absorption rate constants that were equivalent to the free drug solution. However, the area under the plasma concentration curve (AUC) for each was significantly smaller for the encapsulated drug. As such, the amount of drug retained within the lung was significantly greater for the liposomal formulations. Based on the results, it was determined that the absorption of the hydrophiles from the lung into the bloodstream was the rate determining step of absorption, as opposed to the release of drug from the deposited vesicles. However, the amount of drug that entered the circulation depended on the amount of free drug in the lung, which was itself dependent on drug release from the liposomes. By contrast, liposomal GTN produced similar plasma levels whether delivered IV, or IT. This indicated that diffusion of the drug through the bilayers, and the lung membrane, was unimpaired due to its hydrophobic nature, and consequently similar absorption kinetics were evident via each route. The authors concluded that prolonged local activity, and sustained systemic delivery from the lung, was feasible for hydrophilic drugs that could be efficiently encapsulated in liposomes.

\section{Anti-asthma agents}

Orciprenaline is a potent bronchodilator, but has limited clinical value in asthma due to cardiovascular effects, related to systemic $\beta$-agonist activity. For this reason, $\mathrm{McCalden}$ et al. (1989b) investigated the liposomal delivery of the drug, using MLVs administered by IT bolus to anaesthetised guinea pigs. Drug was retained within the lung for up to 14 times longer for cholesterol-containing liposomal formulations, compared with the free drug solution. The liposomal formulations were also shown to produce a prompt bronchodialator effect in cat lungs, due to the presence of free drug, whilst leakage of drug from liposomes 
caused an overall prolonging of activity (McCalden et al., 1989a). Additional experiments demonstrated that liposomal orciprenaline protected against histamine induced bronchoconstriction for considerably longer than the equivalent free drug (Kamarei et al., 1989). Initially, the protective effects of liposomal orciprenaline were equivalent to those afforded by free drug, due to the presence of unencapsulated drug. However, long term protection afforded by the liposomal drug was significantly greater than that produced by the free agent. In addition, the liposomal formulations gave markedly reduced systemic effects, as evidenced by reduced hypotension and tachycardia (McCalden et al., 1989b). Subsequent studies showed that liposomal salbutamol produced a similarly prolonged activity to that shown with orciprenaline (McCalden, 1990). The author remarked that merely encapsulating a $\beta$-agonist was not sufficient to ensure a sustained pharmacological effect. The drug must be of sufficient potency to ensure that the small free drug fraction, supplemented by leakage from the liposomes, provides tissue concentrations of drug sufficient to produce a bronchodilatory effect. The release rate of drug from the liposomes was another important factor determining the efficacy of inhaled preparations. Encapsulated drugs with half-lives in excess of 17 hours failed to protect against histamine induced bronchoconstriction, because insufficient free drug was released to the receptor sites.

Fielding and Abra (1992) demonstrated that the lung residence of terbutaline was extended almost 14-fold by liposome encapsulation. The drug residence time in guinea pig lung following IT instillation was dependent on the liposome size, and the bilayer constituents. Lung clearance of DPPC/DPPG (95:5) liposomal terbutaline formulations was as rapid as that of the free agent, with a half-life in the lung of 1.3 hours. However, the addition of cholesterol increased the half-life to 17.9 hours. Changes in the lipid concentration had little effect, beyond altering the dose of terbutaline delivered to the lung. The prolonged drug release afforded by large, unfiltered liposomes, was in part attributed to the extra barrier to drug diffusion presented by the multilamellar bilayers.

\section{Pulmonary antioxidants}

The ability of liposomes to modulate the pharmacokinetics of entrapped agents has been exploited with great success for pulmonary antioxidant therapy. The lung is protected from damaging reactive oxygen species by antioxidant defence mechanisms, consisting of enzymes such as superoxide dismutase (SOD), catalase, glutathione peroxidase and 
glutathione reductase. However, instances of oxidative stress may cause a saturation of these defences, leading to extensive oxidative damage to cellular components. Traditionally, therapies have attempted to increase the antioxidant capacity of the lung cells by systemic delivery of therapeutic enzymes. However, this approach has achieved limited success since the enzymes are greatly diluted by systemic distribution, and undergo significant enzymatic degradation (Jurima-Romet et al., 1990). Delivery via inhalation has given variable success. This is largely due to the unfavourable physicochemical properties of the various antioxidants. Catalase, SOD and glutathione have difficulty penetrating cellular membranes due to their hydrophilic nature, and are thus cleared rapidly from the lung (Jurima-Romet and Shek, 1991). By contrast, the antioxidant $\alpha-\mathrm{T}$ is highly lipophilic, and hence difficult to formulate as an aerosol (Suntres et al., 1993). Padmanabhan et al. (1985) used negatively charged eggPC/PS (1:1) MLVs to deliver SOD, and catalase, by IT instillation to anaesthetised rats. The rats were then exposed to potentially damaging pure oxygen. The results demonstrated clearly the benefits of the liposomal carrier, with significant enhancement of enzyme activity compared with the free enzymes. The protection afforded by the liposomal enzymes was attributed to retention of the vesicles within the lung, producing elevated levels up to 72 hours following administration.

Lung administered liposomal $\alpha$-T has also proved a promising antioxidant formulation. In addition, it produces significant anti-inflammatory properties when administered to the lung in liposomes (Suntres and Shek, 1995). $\alpha$-T evidently reduces the formation of lipid peroxides from cell bilayers following oxidative stress, and also inhibits the influx of neutrophils from the circulation (Suntres and Shek, 1995). In liposomal form, $\alpha$-T is readily formulated as an aerosol, and has proved successful in the treatment of oxidant induced pulmonary damage (Suntres et al., 1992; Suntres et al., 1993; Suntres and Shek, 1994). Suntres and Shek (1994) determined that DPPC/ $\alpha-T$ (7:3) liposomes, containing the water soluble antioxidant glutathione, were retained for extended periods within rat lung, providing sustained release of glutathione up to 48 hours post instillation. Liposomes containing both $\alpha$-T and glutathione were more effective at protecting against induced membrane lipid peroxidation, than those containing either $\alpha$-T or glutathione alone. The authors concluded that the incorporation of $\alpha$-T in liposomes may serve as a valuable antioxidant drug delivery system, particularly when formulated as this "dual antioxidant formulation" (Suntres and Shek, 1995). Briscoe et al. (1995) recently demonstrated that 
SOD was delivered more efficiently to cultured foetal rat lung epithelial (FRLE) cells following entrapment in liposomes. The liposomes were taken up by the FRLE cells as if part of normal lung surfactant, exhibiting no evident toxicity. However, the cellular uptake of SOD was doubled when SP-A was included in the liposomes. This was due to the expression of SP-A receptors by FRLE cells, which when bound to the liposomal SP-A, initiate an increase in cellular phospholipid uptake. A significantly elevated intracellular uptake of liposomally associated SOD resulted. This supported the work of Buckley et al. (1987), who demonstrated that the antioxidant effects of liposome formulations were largely attributable to liposome-mediated augmentation of cellular penetration. It also concurred with Freeman et al. (1983), who studied liposomal SOD. Buckley et al (1987), concluded that such augmentation was likely to be achieved more readily by topical delivery to the lung, as alternative routes required high concentrations due to their nonspecific targeting. Jurima-Romet et al. (1990) further postulated that encapsulation within liposomes also protected the enzymes from degradation at the site of administration.

The liposomal encapsulation of antioxidants has facilitated their penetration into pulmonary mitochondria and endoplasmic reticulum, the primary sources of reactive species (Shek et al., 1994). Evidently, liposomes bind to the plasma membrane causing the phospholipids to become internalised through endocytosis. Once within the cell, the liposomes become degraded by lysosomal enzymes, causing antioxidant release. Alternatively, the liposomal phospholipid bilayers may fuse with the plasma, or lysosomal membranes of the cell (Freeman et al., 1983). Interestingly, survival figures for rats indicated a protective effect of liposomes alone. This supported the finding that liposomal DPPC protected against oxidative injury to endothelial cells (Freeman et al., 1983). This is perhaps not surprising, as phospholipids and cholesterol are vital components of lung surfactant, and of cell membranes. They may therefore be expected to afford protection against tissue insult.

\section{Respiratory antimicrobials}

The use of liposomes as carriers of antibiotics to the lung has received particular interest, due in part to the currently high morbidity rate in AIDS patients, due to opportunistic respiratory tract infections (Agostini et al., 1993). This is often attributable to drug solubility and toxicity problems, and the failure of conventional anti-infective therapies in targeting the lung (Gilbert, 1996). Debs et al. (1987) compared the delivery of free and 
liposomal pentamidine, administered to mice via the IV, and pulmonary routes. Pentamidine is a drug used to treat Pneumocystis carinii pneumonia. However, its use is restricted by severe toxicity responses, due to uptake by organs such as the liver. Liposomal delivery of the drug via the pulmonary route was proposed as a means of reducing such toxicity. The hydrophilic molecule was encapsulated in PC/PS/chol MLVs, and aerosol delivery achieved by use of a jet nebuliser. Whilst IV pentamidine resulted in significant uptake by the liver, and achieved poor drug levels in the lung, both free and liposomal pentamidine produced high, sustained lung levels following nebulisation. Sequestration of pentamidine within the lung was responsible for an absence of clearance up to 48 hours, and the fact that there were negligible extra-pulmonary effects. Phagocytosis of the liposomes by alveolar macrophages was revealed by electron microscopy, suggesting that the liposomes followed a similar intracellular clearance pathway to endogenous surfactant. The authors concluded that liposomal pentamidine aerosol may provide site specific delivery to infected pulmonary cells, with therapeutic efficacy only being limited by the access to the alveolar space. The uptake of liposomes by alveolar macrophages was further demonstrated by Forsgren et al. (1990), Gonzalez-Rothi et al. (1991), and Myers et al. (1993). Gonzalez-Rothi et al. (1991) noted that liposomes lacking cholesterol lost a significant proportion of entrapped CF over 24 hours, whilst those containing cholesterol were relatively stable. The in vitro exposure to liposomes produced a mean increase in macrophage cell surface area of $27 \%$, over 4 days. However, no detrimental effects on other morphological or functional features were noted. Such macrophage-mediated clearance has been exploited to treat intracellular infection using amikacin-loaded liposomes (Wichert et al. 1992). Amikacin is an antibiotic agent that is highly active against intracellular alveolar macrophage infections, caused by Mycobacterium. Delivery within small liposomes provided a means of specifically targeting the infected cells, delivering high concentrations of drug. Significant antibiotic activity was observed, and rapid phagocytosis was suggested. The authors predicted that intracellular release of amikacin may be achieved in vivo by targeting of the macrophages with liposomes. Schreier et al. (1992) continued this work in the sheep lung. Liposomally entrapped amikacin was administered to healthy sheep, using small MLVs of soyPC/PG (7:3), and soyPC/PG/chol (4:3:1). A large fraction of the amikacin was retained within the lungs of the animals, with approximately $40 \%$ systemically absorbed. The mean residence time of amikacin was increased from 4 hours for free drug, to 17 hours for drug entrapped within soyPC/PG/chol 
liposomes. Cholesterol-containing liposomes were thus retained for extended periods within the lung, making a large fraction of the amikacin dose available for macrophage uptake. The study confirmed that liposomes could be used to deliver antibiotics to target sites within the lung, and also to provide a sustained release reservoir of the drug.

The use of liposomes as carriers of antimicrobials to the lung was further investigated using the antiviral agent enviroxime (Wyde et al., 1988). Enviroxime is a highly active inhibitor of rhinovirus replication in vitro. However the in vivo efficacy of the agent when administered as a spray has proved variable, largely due to its poor aqueous solubility. Formulation as an aerosol may be achieved by the use of alcohol. However, this causes local irritation. Incorporation of enviroxime within liposomes, and delivery by aerosol, permitted a significantly greater concentration of enviroxime to be delivered to the respiratory tract following nebulisation, with demonstrable levels evident in the lungs of mice. When tested against tissue cell cultures the free and liposomal enviroxime produced equivalent efficacies, yet the liposomal drug was significantly less toxic (Six et al., 1989). From the same laboratory, Gilbert et al. (1988) reported the delivery of liposomal enviroxime aerosols to the nasal mucosa of five healthy volunteers. The eggPC liposomes delivered large amounts of enviroxime to the nasal mucosa of each subject, without apparent adverse effects. The authors concluded that liposomes were an especially useful method for the delivery of hydrophobic agents, in order to treat respiratory infections. This was further illustrated with lip-ampB. The failure to target the lungs effectively means that high doses of intravenous ampB are frequently required for AIDS patients with opportunistic lung mycoses. This increases the likelihood of adverse effects, and therapy frequently fails (Purcell and Corris, 1995). Aerosolised lip-ampB has been shown to produce elevated pulmonary concentrations of the anti-fungal agent in murine models, whilst exhibiting a low systemic toxicity. The inhaled formulations were more effective at prolonging survival than the equivalent IV doses, and also proved to be efficacious against systemic infections (Gilbert et al., 1992, 1994). Further to this, nebulised lip-ampB was found to be effective, when used both prophylactically and therapeutically, at reducing the number of Cryptococcus organisms within the lung (Gilbert, 1996). It has also been shown to provide effective prophylaxis protection against Aspergillus and Cryptococcus challenge (Allen et al., 1994a, 1994b; Gilbert and Proffitt, 1996). Experiments such as these, clearly demonstrate that aerosolised liposomal antibiotics may be effective at the treatment and 
prophylaxis of a variety of local and systemic infections. In directing encapsulated drugs to the lung, therapeutic and prophylactic levels may be achieved, both locally and systemically, whilst minimising extra pulmonary effects. However, care must be taken during formulation, For instance liposomal tobramycin failed to control Pseudomonas aeruginosa infection in mice, due primarily to an overly prolonged drug release (Omri et al., 1994).

\section{Protein and peptide drugs}

The lung represents an attractive route for the delivery of protein and peptide drugs, due to the fact that it may be targeted by a non-invasive means. For example, insulin produces a significant hypoglycaemic effect following IT instillation, or aerosolisation, into rat lungs (Colthorpe et al., 1992). Liu et al. (1993) administered insulin-containing, $1.9 \mu \mathrm{m}$ mean size DPPC/chol (7:2) REVs, by IT bolus to anaesthetised rats. Two liposomal formulations were tested. The first presented the insulin encapsulated in liposomes, with free insulin separated by ultrafiltration. The second was a control experiment, with free insulin mixed with blank liposomes. The liposomal formulations produced a considerable increase in the pulmonary uptake of insulin, compared with free drug solution. This was the case whether insulin was encapsulated, or simply mixed with the liposomes. In addition, the duration of the resultant hypoglycaemia was significantly enhanced from a $t_{\max }$ of 100 minutes for free insulin, to greater than 240 minutes for insulin plus blank liposomes. The bioavailability of insulin was also significantly increased by the liposomes. Free insulin yielded a mean bioavailability of $14.7 \%$, increasing to $26.2 \%$ for insulin plus blank liposomes, and to 30.3 $\%$ for the liposomally entrapped drug. The increased bioavailability of liposomal insulin was reflected by a marked enhancement of the hypoglycaemic response. Interestingly, the difference in bioavailability between each of the liposome formulations was not statistically significant. This was attributed to the fact that absorption enhancement was brought about by increased levels of DPPC at the respiratory membrane. Speeding of the turnover of surfactant within alveolar cells resulted, bringing about an increased interaction of insulin with the alveolar membrane, and thus an increased absorption. Alternatively, binding of free insulin molecules to the liposomes may have occurred. In either case, it indicated that the entrapment procedure may have been omitted.

Pulmonary delivery of peptides using liposomal carriers was further explored by Bennett 
et al. (1994), as a means of controlling the systemic absorption of the decapeptide detirelix, following pulmonary administration to dogs. Detirelix, a luteinising hormone releasing hormone, would benefit from a non-invasive route of delivery, such as via the lung. However, reproducible pulmonary absorption of simple solutions proved difficult to achieve, and so liposomes were investigated as a means of improving absorption. Three detirelix formulations were prepared - as free drug solution, encapsulated in DSPC/distearoyl phosphatidylglycerol (DSPG)/chol (52/8.4/39.6) liposomes, and also partially encapsulated. Each of the liposomal formulations produced a sustained release of detirelix to the systemic circulation. The half-lives of drug appearance in plasma were increased from 8.2 hours for the free peptide, to $19,16.3$, and 21.6 hours for partially entrapped (IT), partially entrapped (aerosol), and fully entrapped (IT). The mean residence time (MRT) of detirelix within the lung was markedly increased by encapsulation. Free drug gave a MRT of 14 hours. However, the partially entrapped formulations gave similarly increased MRTs for both IT and aerosol administration, with values of 31.4 and 28.2 hours respectively. The fully entrapped formulation yielded the most prolonged MRT, at 57.4 hours. However, loss of liposomal detirelix due to mucociliary clearance, or phagocytic degradation was indicated by a reduced bioavailability. The results differ from those for liposomal insulin (Liu et al., 1993), as the rate of detirelix absorption was limited by the amount of free drug in the lung. This in turn was controlled by the release rate of detirelix from the deposited liposomes. The authors speculated that the strong affinity of detirelix for phospholipids encouraged binding to both liposomal, and surfactant molecules. As a result the free, partially encapsulated, and fully encapsulated detirelix produced slower absorption than that observed for other similar peptides. Manipulation of peptide structure to include groupings which bind to phospholipids may exploit this phenomenon, providing the potential for enhanced liposome entrapment, and extended pulmonary residence. Similarly promising results have been demonstrated for liposomal IL-2, which yielded a significantly improved efficacy compared with free drug dosing, following nebulisation to dogs (Khanna et al., 1996).

\section{Gene therapy}

Liposomes have increasingly been used as carriers of genetic material. A number of human diseases are attributable to dysfunction, or absence of fully functioning genetic sequences. The first study to target functioning DNA to live animal lung cells was that reported by 
Bingham et al. (1989), where cationic liposomes were used to deliver a functioning prokaryotic gene, which encoded for the intracellular enzyme, chloramphenicol acetyltransferase (CAT). IT delivery of liposomal CAT to lungs of mice gave an increased, and longer lasting expression of the gene in the lung to that achieved IV. This confirmed the ability of liposomes to localise genetic material within the lungs, and to maximise exposure of target sites. A subsequent study by Hazinski et al. (1991) further demonstrated the delivery of liposomal genes connected to CAT to rat lung. CAT activity was detectable 24 hours after IT injection, and this remained for 48 hours, with negligible extra pulmonary activity. The authors concluded that liposome:DNA complexes were useful for the initial evaluation of therapeutic genes, avoiding the need to use retroviral vectors, and allowing the targeting of lung cells in gene therapy. Further studies demonstrated that type II cells of the epithelium expressed CAT genes following liposomal IT administration to rats (Hazinski, 1992). Furthermore, more than $70 \%$ of the cells within the lungs of mice expressed the gene following a single dose of cationic liposome-CAT aerosol (Debs et al. 1994). Such expression was long lasting, and exhibited no toxic effects. The efficient transfection of genes to lung cells has also been demonstrated using nebulised DNAliposomes, with increasing quantities of gene expression detectable up to 7 days postinhalation (Canonico et al., 1992).

Yoshimura et al. (1992) demonstrated that the human cystic fibrosis transmembrane conductance receptor (CFTR) gene could be effectively expressed within the lungs of mice, following IT administration, using a cationic liposomal vector. The expression was detectable in the murine model for at least 4 weeks following administration. Cystic fibrosis is the most common fatal hereditary disorder of Caucasians, affecting 1 in 2000 newborns (Coutelle and Williamson, 1996). It is a chronic obstructive disease of childhood, characterised by the secretion of an abnormal mucus from the pulmonary exocrine glands. Recent advances in genetic technology have identified the genetic mutation responsible, as being located in the long arm of chromosome 7 (band q31). This leads to the deletion of a single residue from a 1480 -amino acid protein, coded for by the gene. This protein, the CFTR, serves as the chloride channel in cell membranes of the lung and other organs, and is responsible for regulation of the intra-, and extra-cellular concentration of chloride and sodium ions. The absence, or defect of the CFTR, as in cystic fibrosis, leads to chloride imbalance, and ultimately to excessive sodium resorption. Pulmonary manifestations of this 
imbalance include the production of an overly viscous mucus, causing obstruction within the lung, and associated secondary diseases. As a consequence, $90 \%$ of cystic fibrosis mortality is due to respiratory failure (Fick and Stillwell, 1989). The fact that the epithelium of the airways is most implicated, makes aerosol delivered treatment the most appropriate for therapy (Coutelle and Williamson, 1996). Due to their proven ability to carry drugs into human cells, liposomes were investigated as a carrier to achieve the intracellular delivery of the CFTR gene. Unlike viral vectors, liposomes are non-immunogenic, being degraded via natural clearance mechanisms. Alton et al. (1993) used a nebuliser to deliver CFTR to cystic fibrosis mutant mice. The process of gene transfer was first evaluated using a reporter gene ( $\beta$-galactoside). The nebulisation of cationic liposome-gene complexes delivered genes to the entire airways of the mice, with an absence of untoward effects. The authors concluded that the delivery of genes could be performed easily, and with minimal discomfort utilising nebulised formulations. Correction of cystic fibrosis was then demonstrated following nebulisation of liposomal CFTR. However, complete correction was only demonstrable in certain animals. This may be improved in human studies, as inhalation will be more efficient. However, the mouse lungs lacked the viscous secretions which are characteristic of cystic fibrosis, and as such the authors predicted delivery would be less efficient in such human subjects. Due to the rather inefficient action of liposomal CFTR, large quantities of DNA-liposome complexes need to be delivered to the lung (Alton et al, 1993). This raises practical concerns, as concentrated DNA-liposome formulations are highly viscous, and as such may prove difficult to aerosolise efficiently (Yoshimura et al., 1992). Thus far, Phase I clinical trials have delivered cationic liposomeDNA complexes via the nasal route, showing a transient correction of cystic fibrosis, with an apparent lack of adverse effects (Caplen, et al, 1995). This is remarkable progress towards an effective treatment for cystic fibrosis, if one considers that the CFTR gene was only discovered at the beginning of the decade.

\subsection{Human studies of liposomal aerosols}

The first clinical use of phospholipid aerosols was reported in a study of NRDS therapy (Ivey et al., 1977). Since this time, human studies have remained scarce. However, the results of the few that have been performed have been highly encouraging. The first study of the deposition and clearance of liposomes in human lung was made in 1985 (Farr et al., 1985). A Hudson ${ }^{\circledR}$ jet nebuliser was used to administer ${ }^{99 \mathrm{~m}}$ Tc-DPPC MLVs (mean size 5.7 
$\mu \mathrm{m})$, and SUVs (70 $\mathrm{nm})$, to the lungs of 4 healthy volunteers. Each formulation produced a similar deposition of radioactivity throughout the lung. The study demonstrated that the liposome deposition and clearance was dependent on the aerosol characteristics, and the patient breathing pattern, and independent of the liposome characteristics. The liposomally associated technetium was retained within the lung for extended periods, yielding mean retention levels of between 76.8 and $87.5 \%$ at $6 \mathrm{~h}$ post inhalation. This compared with less than $25 \%$ activity remaining at 1 hour for the free technetium. The clearance of deposited vesicles from the lung was dependant on their site of deposition, with short term loss attributed to deposition, and subsequent mucociliary clearance, in the tracheobronchial region. By contrast, activity recorded at 20 hours was taken to be representative of vesicles deposited in the non-ciliated, alveolar region. The loss of activity from the alveolar region indicated that the alveolar deposited liposomes were cleared at a more rapid rate than may be predicted from consideration of particulate disposition theories. This suggested that the liposomes were cleared from the lung by faster clearance mechanisms than those described for insoluble particles. However, disassociation and resultant rapid clearance of the bound radiolabel may equally have been responsible.

${ }^{99 \mathrm{~m}}$ Tc was also employed as a marker by Barker et al. (1994), in a subsequent study of inhaled liposomes. The pulmonary delivery of a liposomally entrapped aqueous marker, ${ }^{99 \mathrm{~m}}$ Tc-DTPA, was studied using $0.9 \mu \mathrm{m}$ mean size, DPPC/chol (1:1) MLVs, delivered by the Respirgard $\mathrm{II}^{\circledR}$ nebuliser. Widespread distribution of activity throughout the lung was recorded following nebulisation, and the liposomes were shown to effectively prolong the residence time of the agent within the lung. There was an initial rapid clearance of radiolabel, up to 4 hours, attributed to the presence of free ${ }^{99 \mathrm{~m}} \mathrm{Tc}-\mathrm{DTPA}$, and to liposomes deposited and cleared from the upper airways. There then followed a period of slow decline in activity. At 5 hours post-inhalation $58.5 \%$ of the initial liposomal radioactivity was evident in the lung, compared to $16.8 \%$ for the equivalent free ${ }^{99 \mathrm{~m}} \mathrm{Tc}-\mathrm{DTPA}$ solution. At 24 hours the liposomal formulation was still yielding local activity levels of $44.7 \%$. This was attributed to the dose deposited in the alveolar region, and was further evidence of the ability of liposomes to sustain the release of hydrophilic agents deposited within the lungs.

The potential ability of liposomes to localise drug activity within the lung, and to improve therapeutic activity lead Vidgren et al. (1995) to investigate the in vivo effects of an inhaled 
liposomal glucocorticoid. ${ }^{99 \mathrm{~m}} \mathrm{Tc}$-labelled beclomethasone dipripionate $(\mathrm{Bec})$ was included in dilauroyl phosphatidylcholine liposomes (Bec-DLPC). Two experiments were performed, one a preliminary animal study, and the second a volunteer study. In each case the Bec-DLPC liposomes were administered unchanged with regard to size, with sizes ranging from less than $1 \mu \mathrm{m}$ to about $50 \mu \mathrm{m}$. The first experiment involved the in vivo assessment of the labelling technique, with Bec-DLPC liposomes administered intranasally to mice, and the distribution of radioactivity in different tissues detected using a gamma camera. Approximately $75 \%$ of the administered dose was distributed to the lungs. Free technetium was cleared from the lungs within minutes, whereas the retention of the liposomal label was prolonged, with $50 \%$ detectable at 24 hours. The second experiment involved a study of the deposition and clearance of ${ }^{99 \mathrm{~m}} \mathrm{Tc}$ Bec-DLPC liposomes within the lungs of six normal volunteers, following nebulisation with the Aerotech $\mathrm{II}^{\circledR}$ and Spira ${ }^{\circledR}$ jet nebulisers. Deposition modelling, based on the droplet size produced by each nebuliser, revealed that deposition occurred throughout the lung. Pulmonary deposition was calculated to be $17 \%$ for the Aerotech II, and $20 \%$ for the Spira nebuliser. Gamma camera analysis showed there to be a slow clearance of the deposited radioactivity over a 3 hour period, with $93 \%$ of the deposited activity from the Aerotech II, and $82 \%$ from the Spira, retained at 3 hours. This confirmed that liposomal entrapment of Bec achieved retention of the drug for extended periods within the lung. However, disassociation of the radiolabel within the lung could not be ruled out. Gamma camera analysis revealed interesting differences between the liposome deposition and clearance patterns achieved with each of the nebulisers. Oropharyngeal deposition was greater (38\% versus $26 \%$ ) for the Aerotech II, and lung deposition reduced (14\% versus $17 \%$ ) for the Spira nebuliser. This was attributed to differences in the droplet size produced by each nebuliser. Mean aerosol sizes produced were $1.2 \mu \mathrm{m}$ and $3.2 \mu \mathrm{m}$, for the Aerotech II and the Spira respectively. The larger droplet size produced by the Spira increased the deposition of liposomes in the mouth and throat, and thus reduced pulmonary deposition. The liposomes were also cleared at a faster rate, due to the larger proportion of the dose deposited in regions where rapid mucociliary clearance operates. The authors concluded that prolonged anti-inflammatory effects may be produced by the use of nebulised Bec-DLPC liposome formulations. In addition the liposomal aerosols were well tolerated, and lacked adverse effects, when nebulised to asthmatics (Waldrep et al., 1997). 
Taylor et al. (1989), were the first to demonstrate the ability of inhaled liposomes to control the release of drugs to the systemic circulation of man. SCG-containing DPPC/chol (1:1) REVs were extruded to $1.2 \mu \mathrm{m}$, and delivered to five healthy volunteers using a Hudson ${ }^{\circledR}$ jet nebuliser. The results demonstrated a prolonged release of SCG to the systemic circulation, producing significant plasma levels at 25 hours post inhalation. By contrast, free drug was rapidly absorbed, and absent from 24 hour samples. The in vivo release of liposomal drug was more rapid than that predicted from in vitro models. This may have been due to alveolar macrophage activity, vesicle degradation by phospholipases, phospholipid exchange with surfactant, and/or fusion with epithelial cells. Having predicted steady state levels following repeated administration, the authors concluded that therapeutic levels may be achieved through once daily dosing of the liposomal system.

The sustained delivery of drugs to the systemic circulation was further demonstrated using fentanyl (Hung et al. 1995). Nebulisation of fentanyl solution for post-operative analgesia is of limited value due to rapid lung clearance, and hence short activity. Liposomes were investigated as a carrier system, due to their potential to control the absorption of drugs from the lung. A mixture of free and liposome-encapsulated fentanyl (FLEF) was delivered, using a Powermist ${ }^{\circledR}$ jet nebuliser, to the lungs of 10 healthy volunteers. The aerosol formulation was well tolerated, with no evidence of pulmonary irritation. The inhaled FLEF produced a more delayed onset than an equivalent IV dose, and at 8 hours post-inhalation the mean drug level remained close to the therapeutic threshold. By contrast, the levels following IV dosing had fallen to sub-therapeutic levels. At 8, and 24 hours post administration, the inhaled FLEF retained $22 \%$, and $11 \%$ of the deposited dose, compared to $3 \%$, and $1 \%$ for the IV dose. The study demonstrated a number of advantages for inhaled FLEF for providing post-operative pain relief. Firstly, it provided a simple, and non-invasive route of administration, avoiding oral complications in patients with GIT dysfunction. Secondly, the pulmonary route by-passed the extensive first-pass metabolism associated with oral absorption of fentanyl. Free fentanyl included in the FLEF ensured that rapid attainment of therapeutic drug levels was achieved, and the liposomal fraction provided a prolonged drug action. It was concluded that inhaled FLEF was preferable to transmucosal fentanyl which provides a rapid onset, but short duration of drug of action.

The promising results of inhaled liposomes in volunteers were followed recently by the first 
documented use of a nebulised liposomal drug for the treatment of a diseased patient (Purcell and Corris, 1995). An asthmatic patient had presented with pulmonary Aspergillus fumigatus, infection for which IV and intrapleural ampB therapy had failed. Liposomal encapsulation of the drug (lip-ampB) successfully protected against bronchospasm produced in response to nebulised free drug, and following three days of twice daily inhaled lip-ampB, the patient was rendered apyrexial. Within four weeks the sputum and pleural fluid were negative for Aspergillus, and there was no relapse after one year. The true success of the nebulised lip-ampB was difficult to assess, due to the fact that it was coadministered with oral itraconazole. However, the study demonstrated that nebulised lipampB was well tolerated by patients with pulmonary disease. Renal function, which had been left seriously impaired following intravenous ampB administration, had returned to normal following transferral to nebulised lip-ampB. The only acknowledged drawback, was the extreme cost of the therapy. The six week course of inhaled lip-ampB cost $£ 10,000$. This was compared with a cost of $£ 300$ for the conventional ampB therapy .

\subsection{Devices used for the aerosolisation of liposomes}

In each of the human studies so far performed, a jet nebuliser has been used to administer the liposomes. Jet nebulisers have a number of advantages that make them more appropriate to the aerosolisation of liposomes than alternative devices. The MDI is largely limited to those preparations that may be formulated with lipophilic propellants. Farr et al. (1987) assessed the feasibility of delivering liposomes from MDIs, by including phospholipids in CFC blends. The authors suggested in situ liposome formation once the phospholipid was in the saturated lung environment. However, increasing the phospholipid concentration in the formulation reduced the vapour pressure of the propellant, to the extent that the respirable fraction was markedly reduced. Another study used a novel device to prepare liposomes in situ, following MDI actuation (McGurk et al., 1986). The method involved the mixing of organic and aqueous phases upon aerosol actuation, and proved highly effective with regard to drug entrapment. However, no aerosol size analysis was performed. In view of the fact that the system contained a large proportion of non-volatile components, it was likely to give a poor aerosol quality. In a subsequent study, Farr et al. (1989) concluded that the MDI approach to liposome aerosolisation was limited by the small quantities of phospholipid that are delivered, due to the need to achieve respirable droplet sizes. However, a novel approach to maximising hydrophilic drug delivery from MDI 
devices is detailed in a US patent (Radhakrish, et al., 1990). Preformed liposomes are dehydrated, and packed into a CFC blend in which the phospholipids are poorly soluble. Upon inhalation the solvent system evaporates, allowing liposomes to hydrate within the lung. This allows high entrapment efficiencies to be achieved prior to inclusion in the device. However, drug and phospholipid stability would need to be ensured.

Schreier et al. (1994) investigated the use of a DPI to aerosolise liposomes. Liposomes were prepared in the presence of lactose, with entrapped CF. The liposomes were then freeze dried, and aerosolised using the Spinhaler ${ }^{\circledR}$. However, the results were poor, demonstrating that the respirable fraction of the aerosol was low. Ho (1995) further investigated the DPI system using freeze dried DPPC liposomes. Samples of the liposome powder were milled at varying speeds, and for increasing periods, in order to reduce the freeze dried liposome particle size. Liposome samples milled for short periods, and at low speeds, yielded highly inefficient aerosols. However, the aerosol characteristics were improved by increasing the milling speed and time. The author concluded that the DPI-liposome delivery system may be formulated to give satisfactory aerosol characteristics, by due regard to the sample preparation. However, the issue of liposome stability required further investigation, as milling at high speeds, and for extended periods, significantly heated the sample. Indeed, instability was indicated by the fact that the size of the rehydrated liposomes increased by up to 42-fold following milling. An additional concern is that the respirable fraction was defined as those particles with a MMD less than $10 \mu \mathrm{m}$.

There is therefore a lack of data demonstrating whether DPI or MDIs are appropriate to the delivery of liposomes to the lower respiratory tract. Whilst this remains the case, the development of liposomes will concentrate on nebulisation. Nebulisers have the distinct advantage of being capable of atomising aqueous based formulations. As such, drug containing liposomes may be prepared using conventional techniques, and loaded directly into the device without the need for further processing. Additionally, nebulisers are capable of delivering large dose volumes, over extended periods of time. A potential disadvantage of the use of nebulisers is the question of liposome stability. High shear forces operate within jet nebulisers, and liposome damage has been demonstrated during nebulisation. This is exhibited as a reduction in liposome size, and an associated loss of entrapped hydrophilic solute (Taylor et al., 1990b). However, damage may be minimised by judicious 
liposome design (see chapter 4). Heating of the nebulised fluid during ultrasonic nebulisation may also raise concerns regarding liposome stability (Barber and Shek, 1989).

Various researchers have demonstrated that jet nebulisers may be used to produce liposomal aerosols with characteristics that may be predicted to achieve efficient deposition within the lung (eg. Barker et al., 1994). However, there have been remarkably few attempts made to characterise the process of liposome aerosolisation in such devices. A number of researchers have noted that liposomes are relatively unstable during nebulisation (Debs et al., 1987; Gilbert et al., 1988; Niven and Schreier, 1990; Taylor et al., 1990b; Niven et al., 1991a; Niven et al., 1992a; Vidgren et al. 1995). Despite this, only Niven and Schreier (1990), Niven et al. (1991a, 1992a), Taylor et al. (1990b), Waldrep et al. (1994) and Vidgren et al (1995), have undertaken systematic investigations of the generation of liposomal aerosols from nebulisers.

Niven and Schreier (1990) and Taylor et al. (1990b), demonstrated that the droplet size produced by jet nebulisers was independent of the liposome size characteristics. Furthermore, the leakage of entrapped CF was independent of the lipid concentration used in the formulations (Niven and Schreier, 1990). However, liposomes that incorporated stearylamine (SA) were more stable to nebulisation, due to the rigid bilayers that the molecule produced (Niven and Schreier, 1990). The influence of bilayer state on the retention of solute was further demonstrated by results showing that entrapped $\mathrm{CF}$ release was maximal at temperatures above the $T_{m}$ of the bilayers (Niven and Schreier, 1990). Later studies showed that for maximum stability the mean size of the liposomes should be reduced to below that of the nebulised droplets (Niven et al., 1991a). This supported earlier work, showing that retention of an aqueous phase marker was improved for liposomes with sizes reduced by extrusion (Taylor et al. 1990b). To ensure improved retention of entrapped solute, the driving gas flow rate, and nebulisation time should also be minimised (Niven et al., 1991a). As the authors themselves acknowledge, these studies provide useful, but limited information regarding the nebulisation of liposomes, as a wider variety of nebulisers, drugs, and also liposome types, sizes, and concentrations have been used in nebulised formulations (Niven and Schreier, 1990). The present study was designed to provide further information, in order to improve the current understanding of the processes involved in the nebulisation of liposomes. 


\subsection{Aims of the study}

In a review of inhaled drug delivery systems, Atkins et al. (1992) commented that "although widely used, nebulisers are a highly variable, in general poorly understood means of achieving respiratory drug delivery, despite successful therapeutic use for many years". Despite this, over the last 10 years a wide variety of liposome formulations have been developed for delivery by nebuliser to the human lung. There has, however, been little attempt to standardise the formulation variables of such delivery systems. In particular, there has been minimal research directed towards the nebuliser component. This is despite the fact that there are a vast array of nebulisers available with which the liposomes may be aerosolised (Kendrick et al., 1997). This problem is further compounded by the fact that the nebulisers may be operated under wide ranging conditions, and that this is usually disregarded by both clinicians, and nebuliser manufacturers (O'Driscoll and Cochrane, 1987). Furthermore, in a study of hospital prescriptions for nebulised formulations, as few as $7 \%$ were found to be written correctly (Craig et al., 1989).

The aim of the present study was to characterise a representative sample of modern jet nebulisers, with regard to their ability to generate liposomal aerosols. Such devices have been used in each of the liposome aerosol human studies to date. Each nebuliser operates under the same basic principle, yet different models often differ significantly in their design. The present study aimed to correlate the design of the nebuliser with its ability to aerosolise liposomes, and thus to explore the processes by which liposomes become aerosolised. In addition, the processing of various liposome formulations by different jet nebulisers was investigated. From this, the characteristics of the liposome and nebuliser components of the nebulised liposome system that achieved optimal liposomal aerosol characteristics were hoped to be established.

The study, it was envisaged, would yield information that would be applicable not only to the nebulisation of liposomes, but also to suspensions and other sensitive materials, such as proteins and peptide drugs, which are increasingly being developed for delivery to the airways by jet nebulisation. Conscious of the potential future developments in inhaled liposomal aerosol formulations, the stability of liposomes to ultrasonic nebulisation, and the potential application of freeze-drying technology were also investigated. 


\section{PHYSICOCHEMICAL PROPERTIES OF LIPOSOMES}

\subsection{Introduction}

Liposomes are complex formulations, and changes in their physicochemical nature may be of significance to their performance as a nebulised system. For instance, the primary droplet size produced by nebulisers is dependant on the physicochemical properties of the nebuliser fluid (Lang, 1962; Mercer 1973).

\subsubsection{Structure of liposomes}

The principal constituents of liposomes are phospholipids, and it is their amphiphilic nature that is crucial to liposome formation. Phospholipids have a backbone of either glycerol (glycerophospholipids), or sphingosine (sphingophospholipids). Phosphatidylcholines (PCs) containing glycerophospholipids are the most abundant animal and plant derived phospholipid, and are also found to a lesser extent in bacteria (Harwood, 1994). The dipalmitoyl phosphatidylcholine (DPPC) (fig. 2.1a) molecule has a backbone of glycerol, with the hydroxyl groups of positions 1 and 2 esterified to long chain fatty acids. In PCs the phosphate and attached alcohol moiety provides the polar head group of the molecule, and is connected to the hydrophobic acyl chains by the glycerol "bridge" (fig. 2.1b). The fatty acids confer the lipid character of the phospholipid molecule, and may differ in the number of carbon atoms, and the degree of unsaturation. This provides the basis of phospholipid classification.

( a )

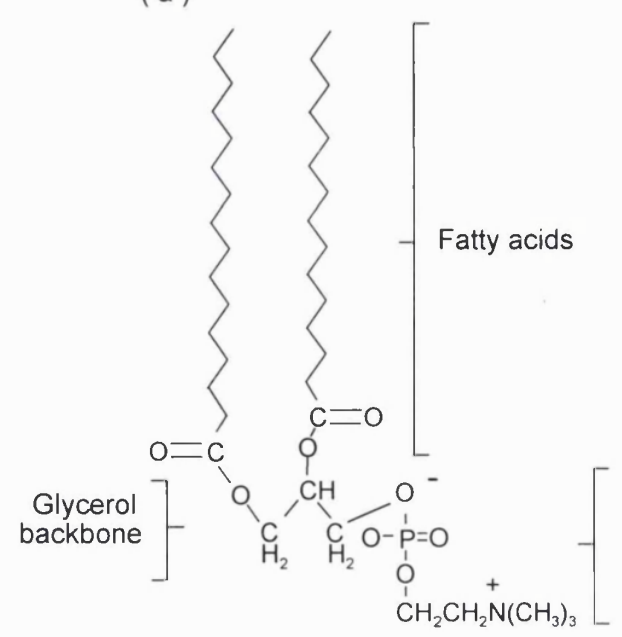

(b)

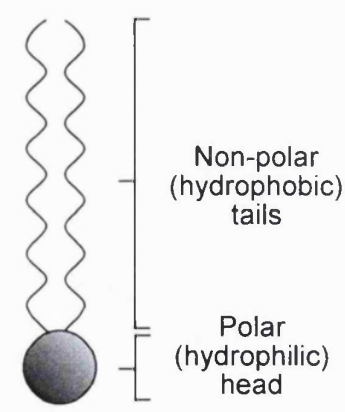

Phosphorylated alcohol (Phosphatidylcholine)

Figure 2.1 (a) Structure and (b) diagrammatic shape of DPPC. 
Two of the most common fatty acids in PC from natural sources are oleic acid (18:1), with an eighteen carbon length acyl chain bearing a single double bond, and palmitic acid (16:0), with a fully saturated sixteen carbon acyl chain (Gunstone, 1994). However, a wide variety of fatty acids may be present, and differences can alter the behaviour of the molecule. The fatty acid composition of hen's egg and soy-bean derived PC is shown in table 2.1 .

\begin{tabular}{|c|c|c|}
\hline \multicolumn{2}{|c|}{$\begin{array}{r}\text { Table 2.1 Fatty acid composition of phosphatidylcholine extracted from } \\
\text { eggs (eggPC) and soy bean (soyPC)(Vemuri and Rhodes, 1995) }\end{array}$} \\
\hline Fatty acid composition & \multicolumn{2}{|c|}{ Phosphatidylcholine (\%) } \\
\cline { 2 - 3 } & eggPC & soyPC \\
\hline Palmitic (16:0) & 32 & 12 \\
\hline Palmitoleic (16:1) & 1.5 & $<0.2$ \\
\hline Stearic (18:0) & 16 & 2.3 \\
\hline Oleic (18:1) & 26 & 10 \\
\hline Linoleic (18:2) & 13 & 68 \\
\hline Linolenic (18:3) & $<0.3$ & 5 \\
\hline Arachiodonic (20:4) & 4.8 & $<0.1$ \\
\hline Docosapentaenoic (22:5) & 4.0 & $<0.1$ \\
\hline
\end{tabular}

Phosphoglycerides, such as PC and phosphatidylethanolamine (PE), are widespread in nature, providing the structural support for biological membranes. Hen's egg yolk, and soy bean provide a relatively cheap source of phospholipids, but yield a mixture of phosphatides. They therefore require purification prior to use (Bangham, 1974)(see section 2.3.1). DPPC and DMPC may be produced synthetically, ensuring a higher purity source of liposomal phospholipid. However, each of these are very expensive.

\subsubsection{Mechanism of liposome formation}

A liposome forms when a phospholipid, and certain other amphiphiles, are swollen in an excess of aqueous phase. Most amphiphiles self aggregate to form micelles at a certain critical concentration, depending on the amphiphile and the conditions. In phospholipids, the hydrophobic fatty acid chains, and the hydrophilic polar head regions attempt to segregate into separate hydrophilic and hydrophobic environments. However, the long fatty 
acid tail chains inhibit micelle formation, and instead, the molecules produce the repeating bilayer structure which is characteristic of liposomes. The fatty acid chains of the molecules turn in to exclude water, whilst the polar head regions associate with the aqueous phase. This causes the molecules to vesiculate, enclosing an aqueous core, and repeating aqueous channels (Lasic, 1988)(see fig. 1.12). Israelachvili et al. (1977, 1980) classified phospholipids, glycolipids, glycerides, and steroids, in terms of their behaviour during hydration. From this, a "packing parameter" (PP) was established (equation 2.1).

$$
P P=\frac{V}{A L_{c}}
$$

where:

[equation 2.1]

$P P=$ packing parameter,$V=$ hydrophobic volume, $A=$ average surface area occupied by the polar region at air/water interface, $L_{C}=$ length of hydrophobic region.

Amphiphiles with a PP of approximately 1.0 are cylinder-like in structure, and readily form liposomes. However, as the PP deviates from 1.0 the molecules tend towards the formation of micelles, or hexagonally packed structures (fig. 2.2). Changes in $\mathrm{pH}$, or ionic strength, may lead to changes in the aggregation state (polymorphism), and hence produce liposome instability (Israelachvili et al., 1980).

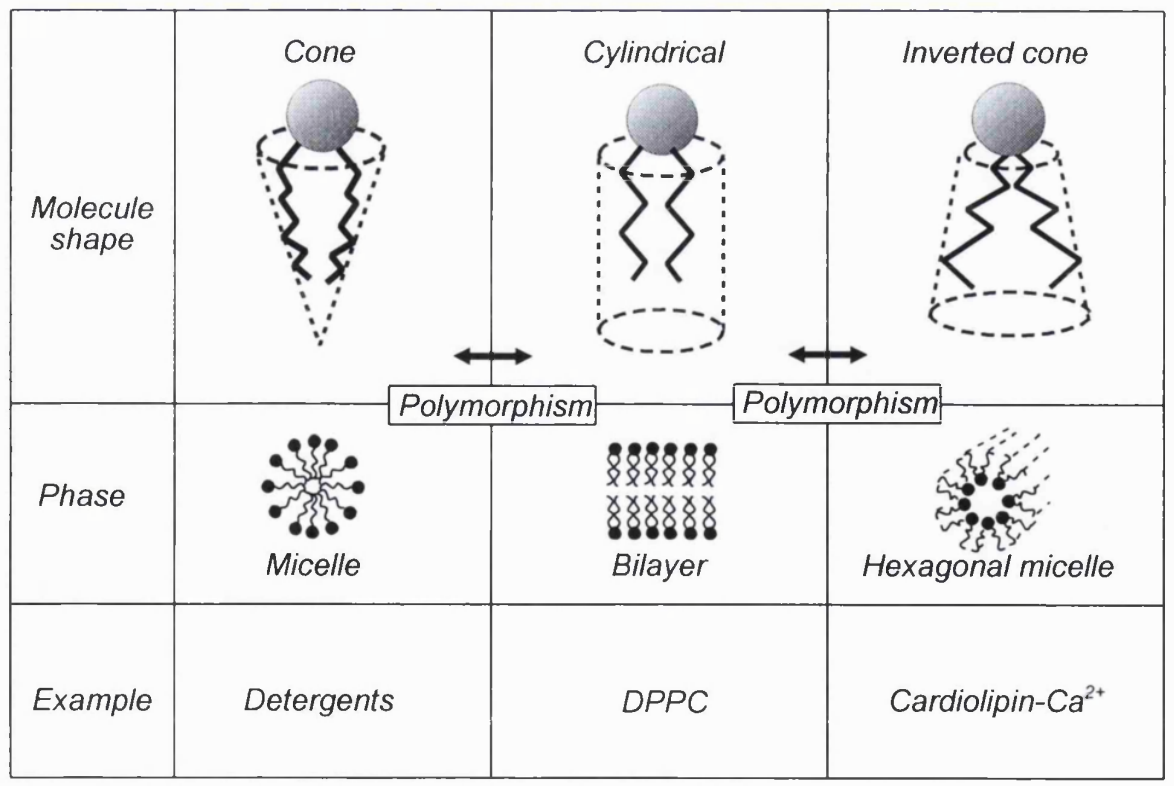

Figure 2.2 The packing arrangement (phase) of different phospholipids when added to an aqueous environment, and its dependence on molecular shape (adapted from Israelachvili et al., 1977; 1980). 
When dispersed in an aqueous environment, at a temperature above their $T_{m}$ (see 1.7.1), most phospholipids bearing two fatty acid chains will form liposomes. The size, lamellarity, structure, and other properties will depend, to an extent, on the method of preparation, the lipid type (Szoka and Papahadjopoulos, 1980), and the presence of additional materials, such as cholesterol and drugs (McIntosh, 1978; Bally et al., 1990).

\subsubsection{Preparation of liposomes}

Liposomes of widely varying morphological character may be prepared relatively simply by a variety of methods. Commonly used techniques include mechanical methods, such as Bangham's "thin-film" (Bangham et al., 1965), and the French-press techniques (Barenholz et al., 1979); detergent removal techniques (Rhoden and Goldin, 1979); reverse-phase evaporation (Szoka and Papahadjopoulos, 1978); solvent infusion (Deamer and Bangham, 1976); and freeze-thaw techniques (Mayer et al., 1986). Each involves a lipid hydration step, but beyond this the combination of constituents and conditions that may be used is overwhelming. Contrary to certain reports, the formation of liposomes is not strictly a spontaneous process (Lasic, 1990). This is evidenced by the fact that all liposomes will eventually break down into a simple two phase system of lipid and water. When water is added to a dry phospholipid film, the film will hydrate as it absorbs the water molecules. However, the liposomes fail to detach from the phospholipid mass unless a small amount of energy is provided to the system (for example by mechanical shaking) (Lasic, 1990).

The classification of liposomes has yet to be standardised, and as such remains confusing. Traditionally, liposomes have been classified according to the method of preparation, as different techniques yield characteristic liposome types. For example $R E V s$ are formed when a water-in-oil emulsion of phospholipid(s) and buffer is prepared, usually by sonication, in excess organic phase (Szoka and Papahadjopoulos, 1978). The organic phase is then removed under reduced pressure, causing the lipid coated aqueous droplets to form a gel, and then a smooth paste consisting of liposomes. Similarly, ether infusion vesicles are formed when lipids dissolved in diethyl ether are slowly injected into warm water (Deamer and Bangham, 1976). The ether is then removed from the mixture by application of a vacuum. French press vesicles are formed following the extrusion of lipid dispersions through a French Press at $4{ }^{\circ} \mathrm{C}$. A single extrusion yields a heterogeneous population of vesicles. Repeated extrusion yields progressively smaller vesicle sizes, and reduces their 
heterodispersity (Barenholz et al., 1979). Such classification, based on the method of preparation, is of limited use, as small changes in the constituents, or preparative conditions, may significantly alter the liposome morphology, and thus their potential applications. It is thus more common to classify liposomes according to their structure, most commonly their size and lamellarity.

Multilamellar vesicles (MLVs) may be manufactured with common laboratory equipment using the "thin-film" method described by Bangham et al. (1965). A thin film of phospholipid is formed on the walls of a round bottomed flask, following evaporation from an organic solvent. The film is then hydrated with aqueous phase (containing any water soluble components), at a temperature above the $T_{m}$ of the constituent phospholipid(s). Subsequent agitation then produces liposomes. The thin-film method is widely used in research, and produces a population of MLVs that range in size from less than $0.2 \mu \mathrm{m}$ up to about $10 \mu \mathrm{m}$ (Cullis et al., 1989). Each liposome possesses a variable number of concentric bilayers, producing the characteristic "onion-layer" appearance (fig. 1.12). MLVs may vary significantly with regard to size, bilayer number, and encapsulation efficiency, depending on the hydration time, the thickness of the phospholipid film, and also the lipid, and aqueous phase constituents (Lasic, 1993). For most pharmaceutical applications a more defined size distribution than is produced using the thin-film technique is required. This may be achieved by extrusion of the MLVs through membrane filters (Olson et al., 1979), exposing them to sonication (Papahadjopoulos and Watkins, 1967), or to freeze-thaw techniques (Gruner et al., 1985) (section 2.1.6). These relatively straightforward techniques yield vesicles that have a more defined, and uniform size distribution. By the selection of appropriate processing conditions, small unilamellar vesicles (SUVs) may be prepared. For instance, the passage of liposomes through a French press strips bilayers from the MLVs, to form a vesicle bearing a single bilayer. These SUVs range in size from about 20 to $100 \mathrm{~nm}$ (Lasic, 1988), are homogeneous, and simple to separate from contaminating MLVs. SUVs may also be prepared by ether infusion (Deamer and Bangham, 1976), or ethanol injection (Batzri and Korn, 1973). This involves the introduction of phospholipids dissolved in an organic solvent into stirred aqueous solution, at a temperature above the $T_{m}$ of the phospholipid(s). However SUVs are more commonly produced by the sonication of phospholipid mixtures (Papahadjopoulos and Watkins, 1967; Huang, 1969)(section 2.1.6). SUVs have the disadvantage of providing a poor encapsulation efficiency, usually in the 
range of 0.1 to $1.0 \%$, depending on the lipid concentration (Szoka and Papahadjopoulos, 1980). This limits their applicability to drug delivery. Of more use for the entrapment of hydrophilic materials are the higher capacity large unilamellar vesicles (LUVs), which posses a single bilayer, and range in size from 0.1 to about $1 \mu \mathrm{m}$ (Lasic, 1988). LUVs are capable of entrapping significantly greater volumes of aqueous phase than SUVs or MLVs, with entrapment efficiencies of 60-65 \% being reported (Szoka and Papahadjopoulos, 1978). They may be prepared by reverse phase evaporation, (Szoka and Papahadjopoulos, 1978), and ether injection (Deamer and Bangham, 1976), as described above. Alternatively, a calcium induced fusion technique has been detailed (Papahadjopoulos, 1978). This involves the treatment of SUVs with calcium ions, which induces fusion of the vesicles into cylindrical multilamellar structures, termed cochleate cylinders. Upon addition of ethylenediaminetetraacetic acid (EDTA), the calcium ions are chelated, transforming the cylinders into LUVs. A potential disadvantage of LUVs is that their preparation exposes encapsulated drugs to high concentrations of organic solvents. This may cause drug instability, particularly in biologically active molecules, such as proteins. Adoption of the dehydration/rehydration technique detailed by Shew and Deamer (1985) avoids this problem. This involves the dehydration of SUVs, which fuse, and upon reconstitution with aqueous phase (containing agents to be entrapped), yield LUVs.

The preparation of MLVs is relatively simple, and for this reason they are the most commonly used liposomes in research. The MLV has a low free energy state, and as such will form almost spontaneously when dry phospholipid is swollen in excess aqueous phase (Lasic, 1988). However, the input of energy is required to prevent the hydration of only the outer layers of the phospholipid particles (Lasic, 1993). The arrangement of phospholipids into bilayers is energetically favourable, as it removes hydrophobic regions of the molecule from the water. SUVs and LUVs are less thermodynamically favourable, and their preparation thus requires increased energy expenditure. Consequently, their formation is less straightforward, requiring more severe conditions. Under normal conditions SUVs are stable for at best several weeks, after which time the vesicles tend to aggregate and fuse, yielding LUVs. LUVs undergo a similar transformation, yielding MLVs in time (Lasic, 1990). MLVs may therefore be prepared with greater confidence in their stability. However, they are also ultimately transformed into a two phase system, consisting of discrete regions of separate water, and phospholipid "stacked bilayers" (Lasic, 1988). 


\subsubsection{Release of entrapped agents from liposomes}

A driving force behind liposome research has been their ability to control the release of entrapped materials. Liposomes may be engineered to provide specific drug release characteristics, depending on the pharmacological requirements of the formulation. Materials entrapped within the aqueous compartment(s) of the liposome are separated from the external environment by 1 or more phospholipid bilayers. These present permeability barriers to the entrapped drug, thus retarding drug efflux. The composition of the phospholipid bilayer is critical in determining the rate of efflux of entrapped materials, and may be adjusted accordingly to influence the rate. An important factor controlling the release of entrapped agents is the transition state of the bilayer components (fig. 2.3)(Szoka and Papahadjopoulos, 1980).

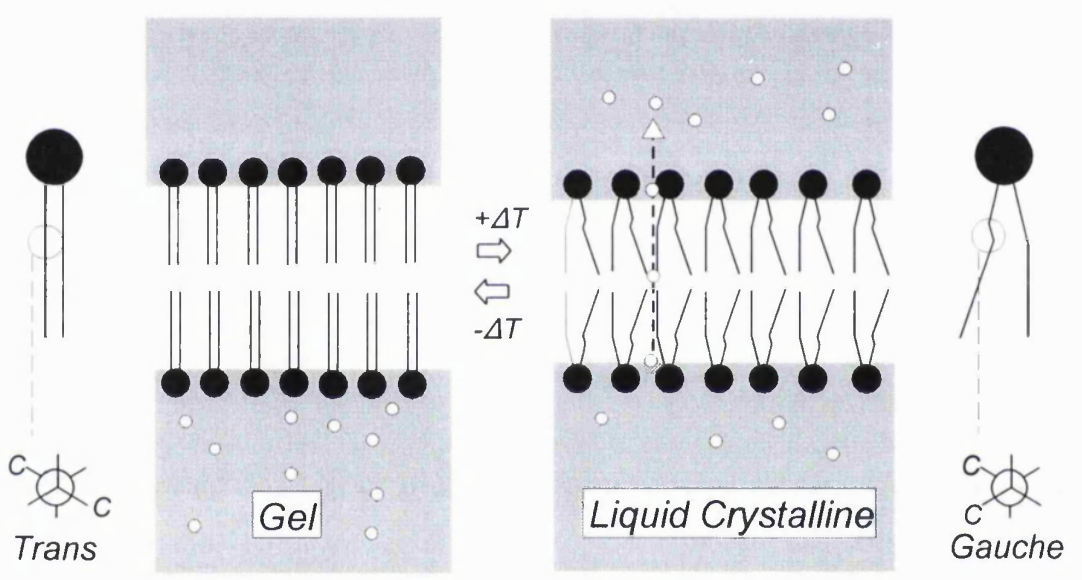

Figure 2.3 The reversible main phase transition of liposome bilayers from the ordered (gel), to the disordered (liquid crystalline) state, that occurs as temperature is increased $[+\Delta \mathrm{T}]$ above the principal transition temperature $\left(T_{m}\right)$ of the phospholipids. Hydrophilic drug [0] release [ $[\nabla]$ is facilitated by such a transition.

Phospholipid bilayers exist in a gel or liquid-crystalline state, depending on the bilayer constituents, and the ambient temperature. Low temperatures, long acyl chains, and an absence of double bonds, favour the gel state (Cevc, 1992). In the gel state, the fatty acid chains of the phospholipids assume an ordered, well defined structure, which presents a considerable barrier to hydrophilic drug release. As the temperature is increased the rotational movement of the hydrocarbon chains increases, until a critical level of excitation is attained. The fatty acid chains then tend towards adoption of the gauche conformation, rather than the predominantly trans straight chain configuration adopted at lower 
temperatures (fig. 2.3). The trans to gauche change requires an activation energy of approximately $3 \mathrm{kcal} \mathrm{mol}^{-1}$, and has an enthalpy in the order of $0.4 \mathrm{kcal} \mathrm{mol}^{-1}(\mathrm{New}, 1990)$. As the temperature rises an increased number of carbon atoms attain the gauche configuration, and for increased periods of time, with the result that increased bilayer packing defects are evident. At the $T_{m}$ of the membrane, the gel and liquid-crystalline states co-exist in different parts of the same membrane, and at areas where the two states interface the bilayer defects are at their greatest. Due to this, the permeability of the bilayer to solutes reaches a maximum at around the $T_{m}$ (Papahadjopoulos et al., 1973b). The bilayer becomes expanded, and its overall thickness becomes reduced (New, 1990). This point represents the acyl chain "melting point", and the bilayer becomes transformed from the ordered, gel state to the disordered, liquid-crystalline state. In the relatively fluid liquid-crystalline state the passive diffusion of entrapped agents through the bilayers is facilitated, and hence drug efflux is promoted. Each phospholipid has a unique $T_{m}$ (table 2.2). Liposome bilayers composed of mixtures of phospholipids will give a principle transition point intermediate to that of the component phospholipids (New, 1990).

\begin{tabular}{|c|c|}
\hline \multicolumn{2}{|c|}{$\begin{array}{c}\text { Table 2.2 Temperatures of the principal phase transition }\left(\mathrm{T}_{\mathrm{m}}\right) \\
\text { of } \\
\text { phospholipids commonly used in the preparation of liposomes }\end{array}$} \\
\hline Phospholipid & $\begin{array}{c}\text { Transition } \\
\text { Temperature }\left({ }^{\circ} \mathbf{C}\right)\end{array}$ \\
\hline Dilauroyl phosphatidylcholine & -1.8 \\
\hline Dimyristoyl phosphatidylcholine & 23.9 \\
\hline Dipalmitoyl phosphatidylcholine & 41.4 \\
\hline Distearoyl phosphatidylcholine & 54.9 \\
\hline \multicolumn{2}{|c|}{ (Mabrey and Sturtevant, 1978) } \\
\hline
\end{tabular}

The packing of phospholipid molecules within liposome bilayers significantly restricts the motion of the constituent molecules, even in the liquid-crystalline state. The incorporation of cholesterol in liposome bilayers has been shown to further stabilise liquid-crystalline state bilayers, by reducing the phospholipid chain mobility (Keough, 1973; Clejan, et al., 1979; Senior, 1987). Cholesterol alone does not form bilayers, but is readily incorporated into phospholipid bilayers, forming stable liposomes at a 1:1 mole ratio of $\mathrm{PC} / \mathrm{chol}$ 
(Lundberg, 1977). The molecule, a sterol, is an abundant component of natural membranes, and is dominated by a planar steroid nucleus, and an aliphatic side chain (fig. 2.4). The $3 \beta$ hydroxyl group of the molecule is thought to hydrogen bond to carbonyl groups of PC molecules within the liposome bilayers (Brockerhoff, 1974), and in so doing orientates itself at the hydrophilic-hydrophobic interface within the PC molecule, in the very centre of the bilayer (Szoka and Papahadjopoulos, 1980). The steroid nucleus is then positioned adjacent to the first 10 carbon atoms of the fatty acid chains, and its presence inhibits their motion. The ability of these atoms to undergo trans-gauche transformation is therefore impeded. The consequence of this, is an elevation of the $T_{m}$ of the bilayer with increasing cholesterol concentration (Senior, 1987). Once a concentration of $50 \mathrm{~mol} \%$ is reached the enthalpy of phase change is reduced to zero, with the result that the phase transition is abolished (Senior, 1987; New, 1990). The inclusion of cholesterol in liquid-crystalline state liposome bilayers therefore causes a retardation of drug release (De Gier et al., 1968; Papahadjopoulos et al., 1973b), and also reduces drug leakage that occurs in vivo due to interaction with plasma proteins. However, the molecule may, to an extent, fluidise gel state bilayers due to a disturbance of the ordered packing (Ladbroke et al., 1968).

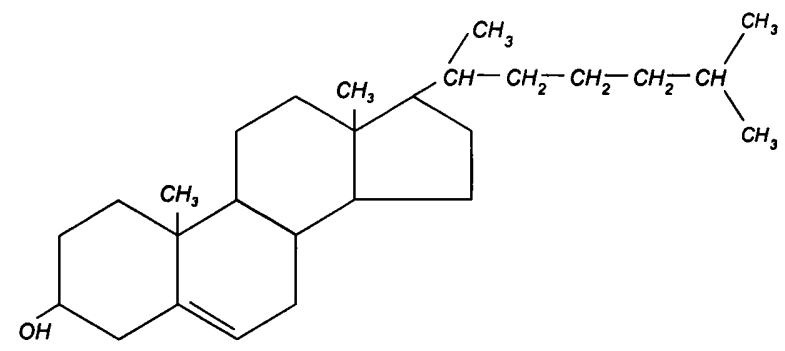

Figure 2.4 The structure of cholesterol.

The use of charged phospholipids has also been shown to influence the release rate of different ionic species (Bangham et al., 1965). Stearylamine (SA) is commonly used to confer a positive charge, and PS a negative charge to the liposome bilayers. Positively charged bilayers are largely impermeable to cations, with bilayers of all charges being generally more permeable to anions than cations (Bangham et al., 1965). It is thus clear that with careful engineering liposomes may provide a means of controlling the rate of release of a variety of drugs, as necessitated by different therapeutic applications. 


\subsubsection{Size of liposomes}

For medical applications the mean size of the liposomes is an important consideration. Liposomes may be prepared as SUVs, with a minimum possible diameter in the region of $20 \mathrm{~nm}$, LUVs with diameters of approximately $1 \mu \mathrm{m}$, and MLVs with diameters up to 10 $\mu \mathrm{m}$ or more. Large MLVs may be favoured if a gradual, sustained release of solute is desired, as they contain a greater number of lipid bilayers, which retard solute efflux (Szoka and Papahadjopoulos, 1980). However, there is an increased clearance of larger liposomes from the circulation, by cells of the MPS (Cullis et al., 1987). Despite this, larger liposomes may be favoured, as smaller liposomes tend to entrap reduced quantities of hydrophilic materials, due to their reduced internal aqueous volume. The liposome size is less critical for the carriage of lipid soluble compounds, as the entrapment is largely determined by the mass of liposomal lipid. With regard to the nebulisation of liposomes, a variety of sizes have been employed. Liposomes which are smaller than the aerosol droplets have the significant advantage of being more stable to jet nebulisation (Taylor et al., 1990b; Niven et al., 1991a). Small liposomes may thus be favoured when the retention of hydrophilic solute during aerosolisation is important. However, in many of the liposomal aerosol studies involving the delivery of hydrophobic materials, large MLVs have been used (eg. Vidgren et al., 1995). This has the advantage of simplifying the preparation process, and may prolong the in vivo release of the drug. For instance, Fielding and Abra (1992) demonstrated that $3.9 \mu \mathrm{m}$ liposomes yielded a threefold increase in an entrapped drug's half-life in the lung, compared to that produced by the equivalent $270 \mathrm{~nm}$ liposomes. This may be attributed to an increased number of bilayers exposed to phospholipases and serum proteins, to reduced clearance by macrophages, and/or to a retarding of drug diffusion.

\subsubsection{Size reduction of liposomes}

The experiments in the present study required liposomes of defined sizes, and there are a variety of procedures available to achieve this. Severe physical treatment of liposomal bilayers causes damage, yielding a population of vesicles with a reduced mean size (Lasic, 1993). This may be accomplished using high shear homogenizers/micro-fluidizers (Mayhew et al., 1984; Talsma et al., 1990a), French-press cells (Barenholz et al., 1979), sonicators (Papahadjopoulos and Watkins, 1967; Huang, 1969), or membrane extrusion filters (Olson et al., 1979). The latter two methods are most commonly used in research (New, 1990). 


\section{Polycarbonate membrane extrusion}

Extrusion of liposomes through polycarbonate membrane filters with defined pore sizes produces a well controlled reduction in the mean liposome size. The method was developed by Olson et al. (1979), and is convenient and highly reproducible. Polycarbonate filters with diameters from $30 \mathrm{~nm}$ to several $\mu \mathrm{m}$ are available. As large liposomes are forced through the filter pores, the phospholipid bilayers deform and stretch, until the applied pressure reaches a critical level, and the bilayers rupture, facilitating the free passage of the liposome through the pore. The liposome then reseals on the reverse of the filter with a reduced diameter.

\section{Sonication}

Sonication of liposomes subjects the phospholipid bilayers to ultrasonic energy (Papahadjopoulos and Watkins, 1967; Huang, 1969). Indirect sonication is achieved by placing the liposomes in a container within a sonication bath, and direct by surrounding the tip of a sonicator with the liposomes. In each case, the energy imparted by ultrasound waves disrupts the phospholipid bilayers which, if held at a temperature above the $T_{m}$ of the bilayer phospholipids, reanneal to form smaller liposomes. The indirect method has the advantage of avoiding contamination of the sample by the ultrasound source. However, the energy imparted is relatively low, and hence size reduction takes longer to complete (New, 1990). Therefore, direct, or "probe", sonication is more commonly employed (Lasic, 1993).

\subsubsection{Entrapment and release of soluble solutes by liposomes}

The ability of liposomes to entrap hydrophilic materials is determined by the efficiency of the entrapment of aqueous phase. Following removal of the unincorporated fraction, liposome formulations may provide an effective sustained release reservoir for entrapped materials. The entrapped material is released from the vesicles as a function of its physicochemical properties, and also the bilayer integrity. Therefore, hydrophilic markers provide a useful indicator of the integrity of the liposome bilayers. Any disruption of the bilayer packing, such as that occurring during nebulisation, is accompanied by an increase in the concentration of unentrapped marker within the continuous phase (eg. Niven and Schreier, 1990). The use of readily detectable hydrophilic solutes, such as CF and SCG, has increased the information regarding liposome morphology, and their behaviour in pharmaceutical applications (Weinstein et al., 1977; Taylor et al., 1990a). Knowledge of 
the in vitro release of such solutes from liposome systems also allows predictions to be made of the potential in vivo behaviour of the formulation (eg. Taylor et al., 1989). The present thesis aimed to determine the behaviour of liposomes during nebulisation, and among the experiments employed was a study of the release of the hydrophilic molecule, SCG, during aerosolisation (chapter 4). SCG is a molecule that is widely used in the prophylaxis of asthma. It is water soluble, and readily incorporated into the aqueous phase of liposomes, without partitioning into the bilayers (Taylor et al., 1990a). Quantitative analysis of the molecule in liposomal formulations may be accurately achieved by spectroscopy, due to its strong ultraviolet (UV) absorption maxima at $326 \mathrm{~nm}$. Conversion of absorbance values to concentration figures is simply achieved, as the absorption follows the Beer-Lambert law. Taylor et al. (1990a) successfully studied solute entrapment, and release, from a variety of MLV and REV formulations, using this technique.

\section{Separation of unentrapped hydrophilic solutes}

The encapsulation of hydrophilic solutes such as SCG within MLVs is an inefficient process. An encapsulation efficiency of 5 to $10 \%$ is commonly produced with MLVs, although this is critically dependent on the lipid concentration (New, 1990). Consequently, a large proportion of the hydrophilic solute remains unentrapped within the externalised aqueous phase of liposomes following preparation. For studies of solute release, and for most proposed pharmaceutical applications, removal of this fraction is necessary. Due to the large size difference between liposomes and entrapped solutes, this separation is readily achieved according to size, using column chromatography, dialysis, or centrifugation.

Polydextran beads are often used as a separation material for liposomes. The beads are porous, possessing tortuous paths into which dissolved solute enters, but which exclude liposomes by virtue of their size. The liposomes are therefore eluted rapidly, leaving the solute retained on the column for extended periods. Potential disadvantages of the method include the fact that significant retention of liposomes on the column may occur (New, 1990). Unentrapped solute removal from liposomes may also be achieved by a variety of dialysis techniques (eg. Abra et al., 1984). The liposome sample is separated from a buffer using a semi-permeable membrane, causing the unentrapped solute to attempt to achieve an equilibrium state with the more dilute external buffer. By circulating the buffer, a concentration gradient is maintained, equilibrium on each side of the membrane is never 
established, and hence free solute is removed from the liposomes. Disadvantages of the technique include the fact that it may be extremely slow. Liposomes may also be separated from unentrapped solute by virtue of their increased density. Large MLVs may be separated from unentrapped solute by spinning at $100000 \mathrm{~g}$, increasing to 10 to 20 hours at 200000 $\mathrm{g}$ for SUVs (New, 1990). Such a centrifugation technique for unentrapped solute removal was developed for use in the present study.

\subsubsection{Surface tension of liposomes}

Variations in the surface tension properties of different fluids have been shown to influence the atomisation of liquids (Rizkalla and Lefebvre, 1974). The phenomenon of surface tension exists because molecules at the surface of a liquid are subjected to forces of attraction from similar molecules in the liquid bulk. The net inward attraction means that the surface of the liquid is in a state of lateral tension, hence the term "surface tension". As a consequence of this, nebulised drops of liquid take the form of spheres in an attempt to minimise their surface area to volume ratio. Work must be done in order to increase the surface area of fluids during nebulisation (Fell, 1988; Florence and Attwood, 1988), and therefore surface tension controls the formation, stabilization, and destruction of nebulised droplets (Mercer, 1973). It is thus surprising to note a relative lack of published data regarding the effects of surface tension on nebulised fluids, and indeed none concerning nebulised liposomes. The aim of the present experiment was to determine the changes in surface tension that occur as the concentration of liposomes is increased. Of particular interest, was the change in surface tension occurring over the range of concentrations likely to be encountered in nebulised liposome formulations. This was investigated with the commonly used Whilhelmy plate technique of surface tension determination. The apparatus consists of a glass plate immersed in the liquid under study. The liquid container is lowered, and the detachment force as the glass plate breaks free of the liquid surface is recorded, allowing the surface tension to be calculated (equation 2.2, Fell, 1988).

$$
\gamma=\frac{W_{L}-W_{T}}{2(L+T)}
$$

where:

$\gamma=$ Surface tension, $W_{L}=$ Weight of plate prior to detachment, $W_{T}=$ Weight of plate following detachment, $T=$ Plate thickness, $L=$ Length of plate. 
The constituents of liposomes are surfactants, a class of molecule that concentrate at the surface of aqueous liquids, in order to remove hydrophobic groupings from the aqueous environment, and thus achieve a minimum free energy state. In so doing, the amphiphiles replace a proportion of the water molecules at the air-liquid interface, reducing the net intermolecular attraction between adjacent groupings at the surface, thus reducing the surface tension. The formation of liposomes by amphiphiles such as PC is attributable to their behaviour when hydrated in water, which in turn is determined by a phenomenon termed the hydrophobic effect (Tanford, 1980; Cevc and Marsh, 1987).

Phospholipids such as PC are insoluble in water, and as a result have a barely detectable critical micelle concentration $(\mathrm{CMC})\left(\mathrm{eg} . \mathrm{DPPC}=4.6 \times 10^{-10} \mathrm{M}\right.$, Smith and Tanford, 1972; $\mathrm{DMPC} \sim 5.0 \times 10^{-8} \mathrm{M}, \mathrm{Cevc}$ and Marsh, 1987). However, the polar regions of the molecule enable water to be soluble in the phospholipid, swelling the lipid to form ordered, liquid crystalline structures. Upon agitation with excess water, the swollen lipid structures bud-off the phospholipid mass to form myelin figures, and ultimately energetically favoured liposomes. A combination of the low solubility of the acyl chains, and the strong hydrogen bonds of the water molecules, dictates that PC forms such liquid-crystalline structures in aqueous environments.

Water is a highly structured liquid, with an ice-like tetrahedral hydrogen-bonded organization (Cevc and Marsh, 1987). Any solute added to the water will disrupt these bonds, and therefore stress the water structure. The disruption of the highly favoured water structure by the hydrophobic acyl chains of PC is thus an energetically unfavourable event. The free energy disturbance of the phospholipid-water system is reduced by hydrogen bonding of the water to the polar regions of the phospholipid (eg. the phosphate group of PC), and the self association of the fatty acid chains. Those water hydrogen bonds that remain disrupted minimise free energy by reassembling into a "cage" of water surrounding hydrophobic contact areas (Cevc and Marsh, 1987). As a result, phospholipids with long acyl chains assume lamellar structures. Such behaviour suggests that the surface tension of liposome forming phospholipids may differ significantly from that of other amphiphiles, due to their predisposition for vesicle formation. This was investigated in the experiments detailed in chapter 3. 


\subsubsection{Viscosity of liposomes}

In addition to surface tension, the viscosity of a fluid has been shown to influence droplet formation (Rizkalla and Lefebvre, 1974). Fluid emerging from an aerosol nozzle does so as a sheet, with its diameter being determined by liquid properties such as viscosity, surface tension, and density (Byron, 1987). A higher viscosity liquid will tend to create thicker sheets, and hence yield larger droplet sizes. Therefore, in the study of the generation of liposomal aerosols, it is was desirable to determine the viscosity of liposomes, and also the relationship between the viscosity and liposome concentration.

Newton (c.1700) was the first to define the resistance of a fluid to deformation, or flow. He described the rate of flow as being directly proportional to the applied stress, with a constant of proportionality being viscosity. Fluids that obey the Newton formula are termed Newtonian, those that deviate are non-Newtonian. Therefore, in simple terms, the viscosity of a liquid is its resistance to movement. A highly viscous fluid, such as syrup, will be less willing to flow than a low viscosity fluid, such as water. There are a variety of instruments used to determine the viscosity of Newtonian fluids. Those based on stress-driven, that is gravity, flow include capillary viscometers (U-tube, suspended level) and falling sphere viscometers. However, many pharmaceutical fluids are non-Newtonian in nature (Marriott, 1988). The viscosity of such fluids cannot be accurately determined from these simple viscometers, as they determine the viscosity at a single value of shear. Several more advanced machines have therefore been developed, that permit controlled shear measurements to be made.

Rotational viscometers operate on the principle that an object rotating in a fluid will experience a drag that is proportional to the viscosity of the surrounding fluid. Such machines allow a wide range of shear rates to be recorded, and are applicable to both Newtonian and non-Newtonian fluids. The Carri-Med CSL rheometer, used in the present study, is a cone-plate measuring device, consisting of a flat circular plate below a wide angle cone. The sample to be analysed is place between the tip of the cone and the plate. The cone is lowered until only a small gap remains between the cone and the plate. The cone is then rotated, and torque registered by the instrument through the cone body. Temperature controlled measurements of viscosity may then be accurately made, over a wide range of shear stresses. The viscosity is determined by application of the following 
equation (Marriott, 1988):

$$
\eta=\frac{3 \omega T}{2 \pi r^{3} \alpha}
$$

where:

[equation 2.3]

$\eta=$ viscosity,$T=$ torque, $\omega=$ angular velocity of the plate, $\alpha=$ angle between cone and plate, $r=$ radius of the cone.

\subsection{Materials}

Aluminium oxide

(active, neutral, Brockmann grade I)

$\mathrm{BDH}, \mathrm{UK}$

Butanone (ethyl methylketone),

BDH, UK

Anala-R, (99.5\%)

Chloroform, Anala-R (99-99.4\%)

- $\quad$ BDH, UK

Cholesterol, Anala-R (99-99.4\%)

- $\quad \mathrm{BDH}, \mathrm{UK}$

Deionised water

- Whatman WR50

RO/Deioniser, Whatman UK

Dipalmitoylphosphatidyl choline $(99 \%+)$

- $\quad$ Sigma Chemicals, USA

Iodine

Lecithin from eggs (90\%)

- $\quad$ BDH, UK

Methanol, Anala-R (99.8\%)

- $\quad$ Sigma Chemicals, USA

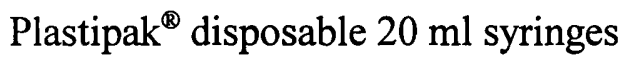

- $\quad$ BDH, UK

Polycarbonate membrane filters,

$2.5 \mathrm{~mm}$ diameter

(1 and $5 \mu \mathrm{m}$ pore size)

- $\quad$ Nucleopore, UK

Sodium cromoglycate

- $\quad$ Fisons, UK

Tris-(hydroxymethyl)-aminomethane,

Anala-R (99-99.4\%)

BDH, UK

Triton X-100

(iso-octylphenoxypolyethoxyethanol) $(98+\%)$

BDH, UK 


\subsection{Methods}

\subsubsection{Purification of eggPC}

EggPC was purified using the column chromatography technique developed by Bangham et al. (1974). A glass chromatography column (600 $\mathrm{mm} \times 30 \mathrm{~mm})$ was filled with chloroform, and the tap end plugged with glass wool. Aluminium oxide was slowly added to the column, until $5 \mathrm{~cm}$ short of the top. $10 \mathrm{~g}$ of unpurified eggPC was added to $20 \mathrm{ml}$ of chloroform, and allowed to dissolve overnight, at between 2 and $5{ }^{\circ} \mathrm{C}$. The resulting eggPC solution was added to the column, taking care not to disturb the aluminium oxide. The tap was opened, and the eggPC allowed to drain into the aluminium oxide, until $1 \mathrm{~mm}$ proud of the solid packing. A primary eluent consisting of $90 \mathrm{ml}$ chloroform/methanol (8:1) was then drained in to the column until $1 \mathrm{~mm}$ proud of the solid packing, followed by a secondary eluent consisting of $240 \mathrm{ml}$ of chloroform/methanol (3:1). $30 \mathrm{ml}$ fractions were collected as the secondary eluent eluted from the column, and a sample of each fraction spotted on to a silica gel thin layer chromatography plate (Polgram sil-N-HR, Camlab, UK). This was then eluted in a glass chromatography tank, with a mobile phase consisting of chloroform/methanol/water (14:6:1). The plates were air dried, before being visualised by placing in a jar of iodine vapour for approximately 10 seconds. The fractions containing phosphatidylcholine were visualised as black spots' $(\mathrm{Rf}=$ approximately 0.5$)$. These were then recombined, added to a round bottomed flask, and the solvent driven off under reduced pressure using a rotary evaporator (Buchi, Switzerland), depositing the PC as a thin film on the inner walls of the flask. The flask was flushed with nitrogen, and $5 \mathrm{ml}$ of butanone added to dissolve the eggPC. This solution was then poured into $1000 \mathrm{ml}$ of acetone, precipitating the purified eggPC. The purified eggPC was then stored for 24 hours under acetone, in a screw topped amber glass jar, at $-25^{\circ} \mathrm{c}$. This removed the final traces of soluble pigment from the purified eggPC. The acetone, containing dissolved pigment, was then replaced with fresh acetone, before storage at $-25{ }^{\circ} \mathrm{C}$.

\subsubsection{Preparation of MLVs}

Liposomes were prepared following the principles of the thin-film technique described by Bangham et al. (1965). The required amount of lipid constituents, comprising eggPC or DPPC, with or without equimolar cholesterol (chol), were weighed in the appropriate proportions into a round-bottomed flask. A $100 \mathrm{ml}$ flask was used for final lipid concentrations to $40 \mathrm{mg} / \mathrm{ml}$. For lipid concentrations of $40 \mathrm{mg} / \mathrm{ml}$, and above, a $1 \mathrm{~L}$ flask 
was used to provide an increased surface area, and ensure the production of a thin, uniform film. The minimum volume of chloroform necessary to dissolve the lipid constituents was added to the flask, and the organic solvent carefully removed at $40^{\circ} \mathrm{C}$ under vacuum using the rotary evaporator. The rotary speed was carefully controlled to ensure a thin film of lipid was deposited uniformly around the inner walls of the flask. The flask was then flushed with nitrogen for 5 minutes. The required volume of deionised water, containing any required aqueous phase constituents (ie. SCG, or trehalose), was then added to the flask, with approximately thirty $3 \mathrm{~mm}$ glass beads. The solution was carefully swirled around the walls, and the sealed flask maintained at a hydration temperature of $40{ }^{\circ} \mathrm{C}$ (for eggPC); or $56^{\circ} \mathrm{C}$ (for eggPC/chol (1:1) and DPPC/chol (1:1) preparations), for $1 \mathrm{~h}$ using a water bath. The flask was then shaken for 10 minutes on a mechanical shaker (G8070014, Galenkamp, Germany) set to the maximum shaking amplitude. The resulting vesicles were then annealed at the appropriate hydration temperature for 30 minutes, before transferral into a screw topped glass vial, and storage under nitrogen gas, at a temperature of 2 to $5^{\circ} \mathrm{C}$.

\subsubsection{Liposome analysis}

The Malvern 2600c laser diffraction sizer (Malvern Instruments, Malvern, UK) was used to analyse the size distribution of the prepared liposomes. A $63 \mathrm{~mm}$ Fourier lens was assembled, and the sizer programmed to make "Particles in Liquid" (PIL) measurements. The sample cell was filled with double distilled, deionised water, and liposomes were added dropwise until the ideal obscuration value was attained (as determined by the Malvern software). The computed mean volume median diameter (VMD), and span value [(90\% undersize $-10 \%$ undersize/50\% undersize)], of the liposome sample were then recorded.

\subsubsection{Size reduction of prepared liposomes}

\subsubsection{Extrusion through polycarbonate membrane filters}

The prepared liposomes were reduced in size to approximately 5,3 or $1 \mu \mathrm{m}$, by extrusion through polycarbonate membrane filters. A $20 \mathrm{ml}$ disposable plastic syringe was used to repeatedly inject $40 \mathrm{ml}$ of liposomes through a single $5 \mu \mathrm{m}$ pore size $25 \mathrm{~mm}$ diameter polycarbonate membrane filter, held in a $25 \mathrm{~mm}$ Luer-lok ${ }^{\circledR}$ filter holder. The liposomes were maintained at a temperature that was above their respective hydration temperature (see section 2.3.2) during filtration. Following each extrusion the liposome samples were 
analysed, using the Malvern 2600c laser diffraction sizer. Once a mean liposome size of approximately 4 to $5 \mu \mathrm{m}$ was recorded by laser diffraction, the filter was changed to a $3 \mu \mathrm{m}$ pore size, and extrusion continued. A $1 \mu \mathrm{m}$ pore size was used once the liposome size was approximately 1.5 to $2 \mu \mathrm{m}$. The extrusion was halted once the mean VMD of the liposomes had reached the required size of 5,3 , or $1 \mu \mathrm{m}$, and the precise size determined by laser diffraction (as detailed in section 2.3.3).

\subsubsection{Probe sonication}

Probe sonication was studied as an alternative method for liposome size reduction. $50 \mathrm{ml}$ of liposomes were added to a large volume, round bottomed, glass sonication vessel. 15 second periods of sonication ( $5 \mu \mathrm{m}$ amplitude, peak to peak) were delivered to the liposomes, using a probe sonicator (150 W, MSE, UK). The $10 \mathrm{~mm}$ diameter probe was sunk to a depth of $4 \mathrm{~mm}$, and a stream of nitrogen gas directed at the fluid surface of the liposomes. The sonication was interspersed by 30 second periods of cooling (monitored by thermometer), using a jacket of ice if necessary, to maintain the liposomes at $40{ }^{\circ} \mathrm{C}$ (eggPC) or $56{ }^{\circ} \mathrm{C}$ (eggPC/chol (1:1) and DPPC/chol (1:1) liposomes). The liposome size was monitored by laser diffraction at intervals during sonication, until the desired liposome size was obtained. Contaminating titanium was removed from the dispersions by centrifugation at $10000 \mathrm{~g}$ for 30 minutes in a bench a centrifuge (Minor $\mathrm{S}, \mathrm{MSE}, \mathrm{UK}$ ), followed by redispersal of the lipid pellet by gentle vortex mixing (Rotamixer Deluxe, Hook and Taylor, UK), for a sufficient period of time such that the liposomes were deaggregated, and the desired mean size was produced (approximately 2 minutes). The liposome size was then rechecked using laser diffraction analysis in order to determine their precise final mean size.

\subsubsection{Liposomal entrapment and release of SCG}

\subsubsection{SCG standard curve}

A stock solution of SCG $(30 \mathrm{mg} / \mathrm{ml})$ in deionised water was prepared, and diluted to produce a range of SCG concentrations. Samples of each were added to a spectroscopy cuvette (1 cm path length) and examined, using a UV spectrometer (PE 554 UV-VIS, Perkin Elmer Ltd, UK), at a wavelength of $326 \mathrm{~nm}$. The results were plotted to produce an equation describing the relationship between concentration and absorption. 


\subsubsection{Preparation of SCG-containing liposomes}

DPPC/chol (1:1), eggPC/chol (1:1), and eggPC liposomes were prepared by the film method. The liposomes were hydrated with $30 \mathrm{mg} / \mathrm{ml}$ SCG solution, to a total lipid concentration of $10 \mathrm{mg} / \mathrm{ml}$. Following preparation, the liposomes were reduced to a mean size of approximately 5 , or $1 \mu \mathrm{m}$, by extrusion through membrane filters (see section 2.3.4.1). The final liposome size was then checked using laser diffraction sizing.

\subsubsection{Assessment of SCG entrapment in SCG-containing liposomes}

The ultracentrifuge used was a MSE Superspeed 75 (MSE, UK) pre-equilibrated to $10{ }^{\circ} \mathrm{C}$, and the centrifugation times quoted were holding times. $500 \mu \mathrm{l}$ of SCG liposomes were added to 8 centrifuge tubes, and the total volume made up to $10 \mathrm{ml}$ with deionised water. The tubes were spun at $200000 \mathrm{~g}$ for 30 minutes at $10{ }^{\circ} \mathrm{C}$. The eluate was then decanted from the tubes. A further $10 \mathrm{ml}$ of water was then added to the tubes, and the tubes respun at $200000 \mathrm{~g}$ for an additional 20 minutes in order to "wash" the pellets. The eluate was then decanted. Ten $\mathrm{ml}$ of Triton X-100 in deionised water $(10 \% \mathrm{v} / \mathrm{v})$ was added to each tube. The liposome pellet was redispersed using a Rotamixer (Rotamixer Deluxe, Hook and Taylor, UK) for 3 minutes, and the liposomes incubated in a sealed tube at $56^{\circ} \mathrm{C}$ for 6 hours. The surfactant, Triton $\mathrm{X}-100$, did not interfere with the UV assay, at the concentration used. The tubes were then cooled, and respun to deposit the dispersed lipid at $200000 \mathrm{~g}$ for 30 minutes in the ultracentrifuge. $5 \mathrm{ml}$ of the eluate was then added to a spectrophotometer cuvette (path length $1 \mathrm{~cm}$ ), and the sample analysed at $326 \mathrm{~nm}$ in an ultraviolet spectrophotometer (Perkin Elmer 554 UV-VIS, Perkin Elmer, UK). The absorbance readings were adjusted for the dilutions made, and the concentration of the entrapped SCG determined by reference to the standard curve.

\subsubsection{The release of SCG from SCG-containing liposomes}

SCG-containing liposomes were prepared as detailed in section 2.3.5.2. Unentrapped SCG was removed by the ultracentrifugation method (section 2.3.5.3). Nine $\mathrm{ml}$ of liposomes was then added to a $10 \mathrm{~cm}$ length of sealed dialysis sac $(\mathrm{BDH}, \mathrm{UK})$ held in a beaker containing $200 \mathrm{ml}$ of deionised water, maintained at $37^{\circ} \mathrm{C}$. The water was stirred continuously using a $2 \mathrm{~cm}$ magnetic stirrer. Five $\mathrm{ml}$ samples were drawn out of the beaker at intervals, added to a glass spectroscopy cuvette $(1 \mathrm{~cm}$ path length), and analysed at a wavelength of $326 \mathrm{~nm}$ using the UV spectrophotometer. The sample was returned to the beaker following analysis. 


\subsubsection{The surface tension of liposomes}

EggPC and eggPC/chol (1:1) liposomes were prepared by the thin-film method (section 2.3.2), to a lipid concentration of $80 \mathrm{mg} / \mathrm{ml}$. The mean vesicle size was reduced to $2.5 \mu \mathrm{m}$ by probe sonication. The size of the liposomes was monitored using laser diffraction analysis, and contaminating titanium removed by bench centrifugation at $10000 \mathrm{~g}$ for 30 minutes. The liposomes were redispersed using a Vortex mixer, and analysed using the Dynamic Contact Angle analyser (DCA-312; Cahn Instruments, USA). $25 \mathrm{ml}$ of liposomes were mixed thoroughly using a stirring rod, and maintained at $27^{\circ} \mathrm{C}$ during measurements. Surface tension values were computed from the receding curve of the distance/force plot. The liposomes were diluted by withdrawing $50 \%$ of the dispersion and replacing with an equal volume of deionised water $\left(\right.$ at $27^{\circ} \mathrm{C}$ ). The liposomes were then allowed to equilibrate for 5 minutes before repeated analysis.

\subsubsection{The viscosity of liposomes}

EggPC and eggPC/chol (1:1) liposomes were reduced in size to $1 \mu \mathrm{m}$ by extrusion. Total lipid concentrations of 2.5 to $80 \mathrm{mg} / \mathrm{ml}$ were prepared, by dilution with deionised water. The viscosity of each liposome sample was then analysed, using the Carri-Med CSL Controlled Stress Rheometer (Carri-Med Ltd, Dorking, England). The rheometer was programmed as detailed in table 2.3 .

\begin{tabular}{|c|c|c|c|}
\hline \multicolumn{4}{|c|}{ Table 2.3 Settings for the Carri-Med CSL } \\
Controlled Stress Rheometer \\
\hline Variable & Setting & Variable & Setting \\
\hline System & Cone and plate & mode & shear stress \\
\hline Cone diameter & $6 \mathrm{~cm}$ & temperature & $25^{\circ} \mathrm{C}$ \\
\hline Cone angle & $2{ }^{\circ}$ & Start/end stress & $0 / 0.75 \mathrm{Nm}^{-2}$ \\
\hline System gap & $56 \mu \mathrm{m}$ & Stress mode & Linear $^{-4}$ \\
\hline System inertia & $4.7 \mu \mathrm{Nms}^{-2}$ & Ascent & 1 minute \\
\hline Preshear stress & $0 \mathrm{Nm}^{2}$ & Descent & 0 \\
\hline
\end{tabular}




\subsection{Results and Discussion}

\subsubsection{Purification of eggPC}

Under the conditions of the separation, free fatty acids and pigments contained in the eggPC mixture were eluted with the primary eluent at about 1 column volume. PC is eluted at 2 to 3 column volumes, with the more polyunsaturated species appearing first (Bangham et al., 1974). Storage of the purified eggPC under acetone removed the residual acetonesoluble contaminants, such as triglyceride fats, steroids, and pigments. This was evidenced by the fact that the solvent became a vivid orange/yellow colour with time.

\subsubsection{Production of MLVs}

The eggPC, eggPC/chol (1:1) and DPPC/chol (1:1) liposomes prepared by the thin-film technique were opaque white fluids. Size analysis revealed that each liposome formulation contained a heterogeneous spread of vesicle sizes, with a mean VMD of about $6 \mu \mathrm{m}$ (table 2.4). Liposomes formed by the thin-film method are typically large in size, usually in the range of 0.6 to $5 \mu \mathrm{m}$ (Bangham et al., 1965), although some authors report sizes up to 10 $\mu \mathrm{m}$ (Vemuri and Rhodes, 1995).

\begin{tabular}{|c|c|c|}
\hline \multicolumn{3}{|c|}{$\begin{array}{l}\text { Table 2.4 Mean size (VMD) and span of liposomes prepared by } \\
\text { the thin-film technique. }[\mathrm{n}=3 ; \pm \text { sd }]\end{array}$} \\
\hline Liposomes & Mean VMD $(\mu \mathrm{m})$ & Span \\
\hline eggPC & $5.98( \pm 0.07)$ & $1.68( \pm 0.04)$ \\
\hline $\operatorname{eggPC/chol~(1:1)~}$ & $6.30( \pm 0.38)$ & $1.77( \pm 0.07)$ \\
\hline DPPC/chol (1:1) & $6.40( \pm 0.17)$ & $1.60( \pm 0.02)$ \\
\hline
\end{tabular}

The method of liposome preparation can markedly alter the properties of the prepared liposomes (Szoka and Papahadjopoulos, 1980). A thin film of lipid is desirable in order to obtain efficient hydration of the liposome bilayers, as this provides an increased surface area to volume ratio. Thick films hydrate inefficiently, and yield undispersed lipid particles. Olson et al. (1979) reported that the hydration time is an important determinant of the mean liposome size. A MLV preparation hydrated for 30 minutes will yield vesicles with reduced mean diameters compared with those formed after 2 hours hydration. 
The liposomes prepared with cholesterol had an increased mean size compared with the eggPC liposomes, although this proved insignificant when tested $(p<0.05)$. However, there was a significant difference between the size of the eggPC and DPPC/chol (1:1) liposomes $(p<0.05)$. Such a trend towards an increase in the size of liposomes, caused by cholesterol, has been demonstrated previously by a number of authors, including McIntosh (1978), who noted that the inclusion of equimolar cholesterol yielded an increase in the bilayer width of DPPC liposomes. Similar results have been demonstrated for SUVs, with liposomes prepared from eggPC/chol (1:0.5) having a $30 \%$ greater size than eggPC alone (Johnson, 1973). Newman and Huang, (1975) noted that the radius of eggPC liposomes was increased monotonically as cholesterol was incorporated at increasing concentrations. Evidently, at higher concentrations cholesterol molecules intercalate between adjacent phospholipid molecules, resulting in the phospholipid rearranging in order to fully hydrate the polar head region (McIntosh, 1978; Rhoden and Goldin, 1979). There is also an increase in the polar head-head spacing (Newman and Huang, 1975). The DPPC/chol (1:1) liposomes contained a significantly reduced spread of liposome sizes compared with the eggPC containing formulations $(\mathrm{p}<0.05)$, as evidenced by the reduced span value. However, the span of sizes observed for eggPC liposomes, like the mean size, was not significantly influenced by the presence of cholesterol $(\mathrm{p}<0.05)$.

The thin-film technique provided a simple method for the preparation of liposomes for nebulisation. The size of the liposomes so prepared was relatively large (approximately 6 $\mu \mathrm{m})$, which has important consequences for their nebulisation. It has been shown that liposomes with a mean size greater than that of the nebulised droplet size are relatively unstable to the nebulisation process (Taylor et al., 1990b). However, the liposome size has been deemed to be of less significance to hydrophobic solutes, which remain associated with the liposome bilayers during disruption. As such, large multilamellar liposomes are commonly used for the aerosol delivery of lipophilic entities (eg. Vidgren et al., 1995). However, the mean liposome size may be critical to the retention of hydrophilic solutes, which become released from liposomes during bilayer disruption (Taylor et al., 1990b). As a result, many researchers reduce the size of the liposomes for nebulisation, if retention of a hydrophilic solute is a requirement (eg. Barker et al., 1994). The liposomes produced by the thin-film method were larger than $5 \mu \mathrm{m}$, which was significant as most nebulisers strive to emit aerosol droplets that are of this size, or below. Attempting to nebulise liposomes 
that are of a size greater than the emitted droplets is likely to be accompanied by a significant degree of liposome instability, and so size reduction techniques were employed. Despite this, the ability to prepare liposomes of a size greater than the emitted droplets was deemed advantageous, in that their nebulisation may provide more information regarding the mechanism of nebulisation.

\subsubsection{Size reduction of liposomes}

Extrusion of liposomes through polycarbonate membrane filters was facilitated by maintaining the liposomes at a temperature above the $T_{m}$ of the liposome bilayers. At temperatures below the $T_{m}$ clogging of the filter pores occurred, preventing continued passage of the dispersion through the filter, to the extent that fracture of the membrane sometimes resulted, due to the increased applied pressures. Increasing the temperature of the liposomes to above the $T_{m}$ of the bilayer lipids fluidises the bilayers, facilitating the deformation and rupture of vesicles during extrusion as the applied pressure is increased. Even so, the bilayer composition of the liposome was found to influence the number of passages through the filters required to reduce the size of the liposomes (fig. 2.5).

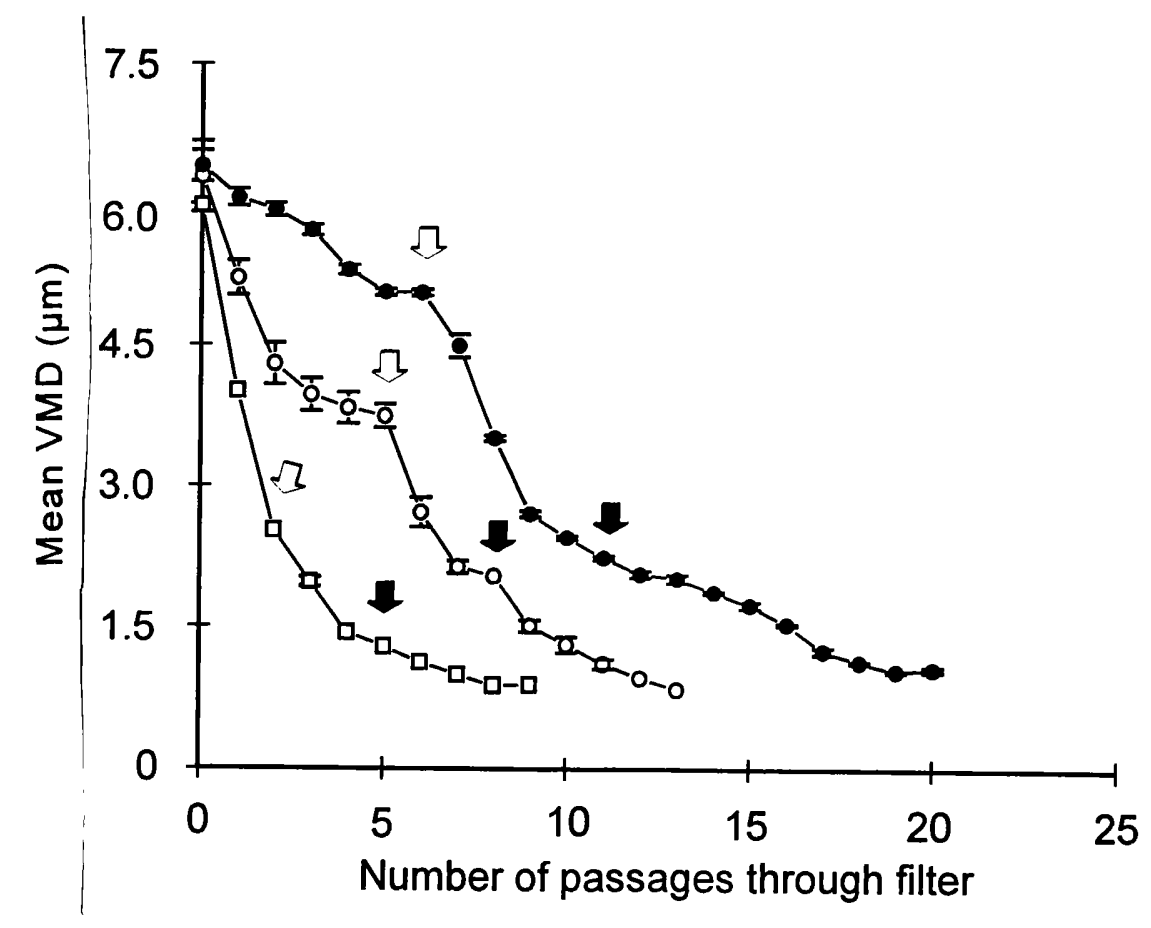

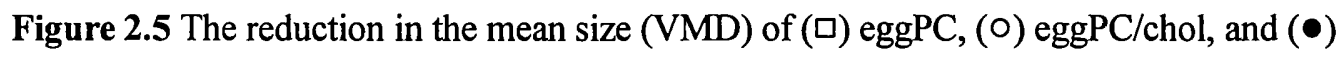
DPPC/chol (1:1) liposomes, following extrusion through 5, $3(\triangleright)$ and $1 \mu \mathrm{m}(\Leftrightarrow)$ membrane filters. $[\mathrm{n}=3 ; \pm \mathrm{sd}]$. 
Probe sonication was also effective at reducing the mean liposome size (fig. 2.6). As determined for membrane extrusion, the eggPC liposomes, having relatively fluid bilayers, required a reduced duration of sonication in order to reduce their mean size to $1 \mu \mathrm{m}$, compared with the cholesterol-containing gel state liposomes. The eggPC/chol (1:1) and DPPC/chol (1:1) liposomes, with relatively stable bilayers, required an extended duration of sonication (fig. 2.6). In contrast to the results obtained for the membrane extrusion technique, the cholesterol containing liposomes each required a similar sonication exposure time to be reduced to $1 \mu \mathrm{m}$.

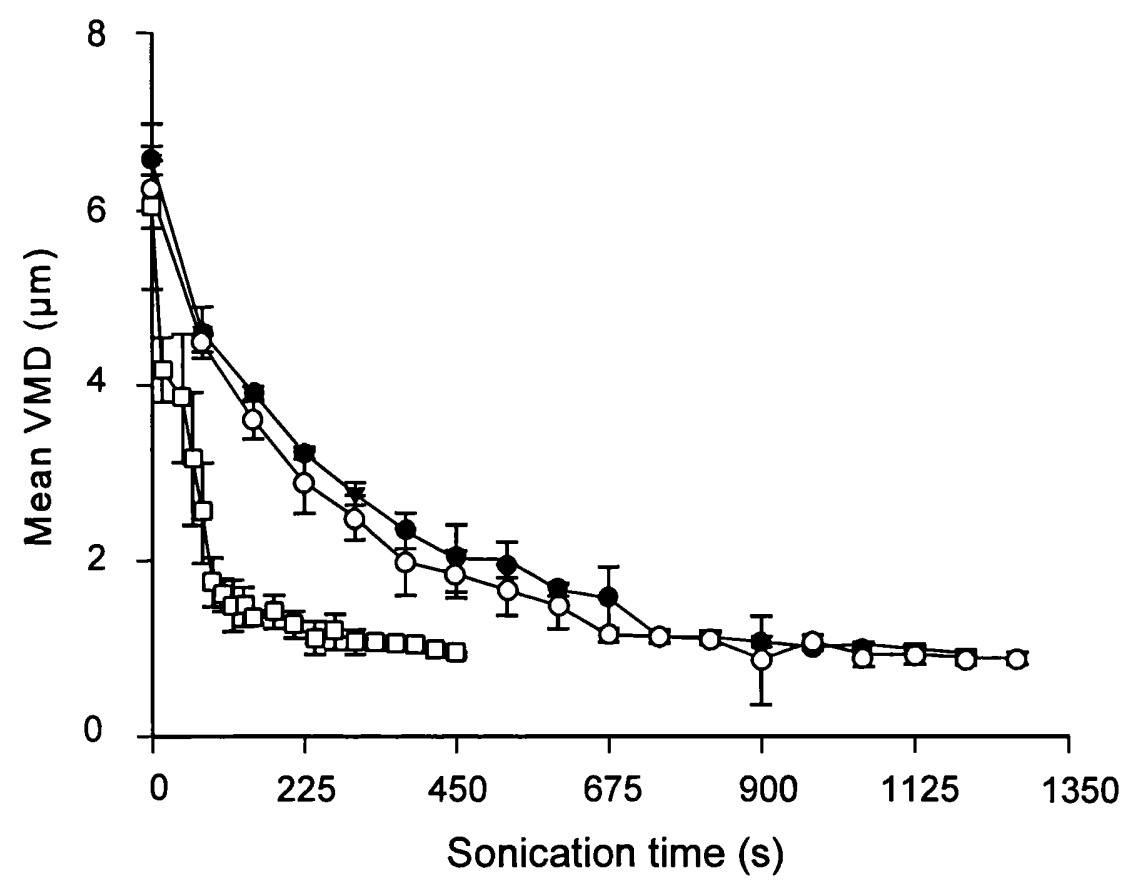

Figure 2.6 The reduction in the mean size (VMD) of ( $\square$ ) eggPC, (0) eggPC/chol (1:1), and $(\bullet) \mathrm{DPPC} / \mathrm{chol}(1: 1)$ liposomes, following probe sonication to approximately $1 \mu \mathrm{m}$. $[\mathrm{n}=3 ; \pm \mathrm{sd}]$.

The polydispersity of the liposomes was reduced by both probe sonication, and extrusion. Extrusion reduced the mean span $( \pm \mathrm{sd})$ of the liposomes from $1.62(0.05), 1.74(0.06)$ and $1.65(0.04)$, to $1.15(0.07), 1.13(0.03)$ and $1.16(0.06)$, for eggPC, eggPC/chol (1:1) and DPPC/chol (1:1) liposomes respectively. Similarly, probe sonication caused a fall to 1.10 $(0.05), 1.11(0.11)$ and $1.16(0.17)$. However, the probe sonication method proved to be less reproducible than the membrane extrusion technique. Prediction of the precise end point of sonication was difficult, which meant regular checks were necessary in order to 
determine the precise size of the liposomes. This may be attributed to the difficulty in assembling the apparatus. The precise position and depth of the probe within the liposome sample significantly influences the ultrasonic energy imparted to the liposomes (New, 1990). As a result, the amount of bilayer disruption that occurs varies considerably. It also proved difficult to control the temperature of the liposomes, and the cooling that was necessary greatly extended the duration of the procedure. The sonication technique thus proved rather labourious, as the liposome size had to be checked at regular intervals during size reduction. Szoka and Paphadjopoulos (1980) drew similar conclusions in a review of liposome preparative techniques, describing the probe sonication method of liposome size reduction as "...in general difficult to standardise". Contamination of the liposomes with metal ions derived from the probe was an additional concern, and a bench centrifugation step was necessary in order to remove contaminating titanium. The stability of the phospholipids to sonication may also be a concern, as lipid peroxidation may be induced by sonication (Crommelin and Schreier, 1994). Nevertheless, the probe sonication technique proved useful when membrane filters were unavailable, due to supply difficulties.

\subsubsection{Entrapment of SCG by liposomes}

The entrapment of SCG was determined by reference to the standard curve obtained for SCG solutions (fig. 2.7).

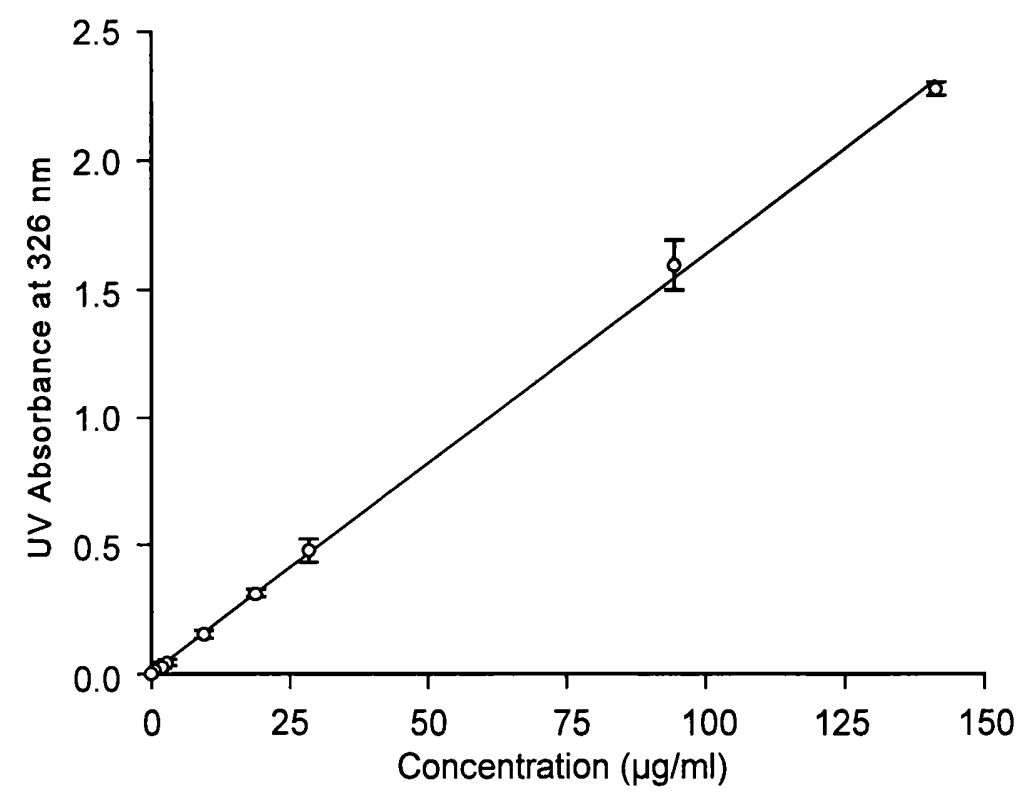

Figure 2.7 Calibration curve for the UV absorbance of SCG solutions. [Equation of line: Absorbance $=0.016308 \times$ Concentration $+0.004391 ; \mathrm{r}=$ $0.999][\mathrm{n}=3 ; \pm \mathrm{sd}]$ 
The efficiency of SCG entrapment was determined according to equation 2.4, yielding the values presented in table 2.5 .

$$
\text { Entrapment }=\frac{U V_{a}}{U V_{b}} \times 100
$$

where:

[equation 2.4]

$U v_{a}=\mathrm{UV}$ absorption of aqueous phase liberated from liposomes. $U v_{b}=\mathrm{UV}$ absorption of total aqueous phase.

Table 2.5 Entrapment of SCG by liposomes as determined by UV assay.

\begin{tabular}{|c|c|c|c|c|}
\hline & & {$[\mathrm{n}=3 ; \pm \mathrm{sd}]$} & & \\
\hline \multirow[b]{2}{*}{ Liposome } & \multicolumn{2}{|c|}{$1 \mu \mathrm{m}$ liposomes } & \multicolumn{2}{|c|}{$5 \mu \mathrm{m}$ liposomes } \\
\hline & $\begin{array}{c}\text { Mass } \\
\text { entrapped } \\
(\mu \mathrm{g} / \mathrm{ml})\end{array}$ & $\begin{array}{c}\text { Entrapment } \\
\text { (per } 100 \mathrm{mg} \\
\text { lipid) \% }\end{array}$ & $\begin{array}{c}\text { Mass } \\
\text { entrapped } \\
(\mu \mathrm{g} / \mathrm{ml})\end{array}$ & $\begin{array}{c}\text { Entrapment } \\
\text { (per } 100 \mathrm{mg} \\
\text { lipid) \% }\end{array}$ \\
\hline eggPC & $\begin{array}{c}348 \\
( \pm 52)\end{array}$ & $\begin{array}{c}3.9 \\
( \pm 0.6)\end{array}$ & $\begin{array}{c}369 \\
( \pm 40)\end{array}$ & $\begin{array}{c}4.1 \\
( \pm 0.5)\end{array}$ \\
\hline $\begin{array}{c}\operatorname{eggPC} / \text { chol } \\
(1: 1)\end{array}$ & $\begin{array}{c}408 \\
( \pm 41)\end{array}$ & $\begin{array}{c}4.2 \\
( \pm 0.4)\end{array}$ & $\begin{array}{c}388 \\
( \pm 41)\end{array}$ & $\begin{array}{c}4.4 \\
( \pm 0.4)\end{array}$ \\
\hline $\begin{array}{c}\text { DPPC/chol } \\
(1: 1)\end{array}$ & $\begin{array}{c}527 \\
( \pm 117)\end{array}$ & $\begin{array}{c}5.6 \\
( \pm 1)\end{array}$ & $\begin{array}{c}646 \\
( \pm 121)\end{array}$ & $\begin{array}{c}6.9 \\
( \pm 1.9)\end{array}$ \\
\hline
\end{tabular}

The efficiency of SCG entrapment was in close agreement with that previously reported for $1 \mu \mathrm{m}$ liposomes (Taylor et al., 1990a) (table 2.6). However, Taylor et al. (1990a) determined that eggPC/chol (1:1) liposomes encapsulated a greater proportion of SCG than the equivalent DPPC/chol $(1: 1)$ liposomes. This is in contrast to the results of the present study, which found no significant difference in entrapment between each of the liposome formulations studied $(\mathrm{p}<0.05)$. This may be attributed to differences in methodology. Firstly, the study by Taylor et al. (1990a) investigated the entrapment provided by unprocessed liposomes, whilst the liposomes included in the present study were extruded 
to ensure a reduced, and defined size. Secondly, the present study employed two 30 minute periods of centrifugation for solute removal, compared with only a single 30 minute period utilised by Taylor et al. (1990a). It may be postulated that a prolonged centrifugation time may cause a greater stress to the eggPC/chol (1:1) liposomes, than to the DPPC/chol (1:1) liposomes, as indicated by the fact that DPPC/chol (1:1) liposomes exhibit an increased stability to extrusion (section 2.4.3). This effect may have manifested itself as an increased release of entrapped solute from eggPC/chol (1:1) liposomes during the extended centrifugation period employed in the present study. This may also be responsible for the reduced efficiency of SCG entrapment exhibited by eggPC liposomes presently. In addition, phospholipids from natural sources vary significantly between batches (Singleton et al., 1965), which may result in variations in the entrapment observed by different researchers.

\begin{tabular}{|c|c|c|}
\hline \multicolumn{3}{|c|}{$\begin{array}{l}\text { Table 2.6 Entrapment of SCG by different liposome } \\
\text { formulations. }[ \pm \mathrm{sd} ; \mathrm{n}=3]\end{array}$} \\
\hline Liposome & $\begin{array}{c}\text { Bridges } \\
\text { (this study) }\end{array}$ & $\begin{array}{c}\text { Taylor et al. } \\
\text { (1990a) }\end{array}$ \\
\hline eggPC & $\begin{array}{l}3.9( \pm 0.6)^{\mathrm{a}} \\
4.1( \pm 0.5)^{\mathrm{b}}\end{array}$ & $3.3( \pm 0.2)^{c}$ \\
\hline $\begin{array}{c}\operatorname{eggPC} / \mathrm{chol} \\
(1: 1)\end{array}$ & $\begin{array}{l}4.2( \pm 0.4)^{\mathrm{a}} \\
4.4( \pm 0.4)^{\mathrm{b}}\end{array}$ & $6.3( \pm 0.4)^{d}$ \\
\hline $\begin{array}{c}\text { DPPC/chol } \\
(1: 1)\end{array}$ & $\begin{array}{l}5.6( \pm 0.4)^{\mathrm{a}} \\
6.9( \pm 1.9)^{\mathrm{b}}\end{array}$ & $5.35( \pm 0.4)^{\mathrm{e}}$ \\
\hline
\end{tabular}

The $5 \mu \mathrm{m}$ mean size liposomes yielded a 5,5 , and $19 \%$ greater entrapment of SCG compared with the $1 \mu \mathrm{m}$ liposomes, for eggPC, eggPC/chol (1:1), and DPPC/chol $(1: 1)$ liposomes respectively (table 2.6). However, when tested statistically these do not represent significant differences $(p<0.05)$. It is difficult to assess the relationship between the vesicle size and the entrapped volume of hydrophilic solutes. There is likely to be a similar number of lipid bilayers in the liposomes of each size, with the reduction in trapped aqueous phase 
per vesicle in the smaller liposomes being compensated for by the greater number of smaller vesicles. Therefore, one may indeed expect there to be no demonstrable difference in the entrapment of SCG provided by liposomes of each size. However, this takes no account of the size of the inner core of aqueous phase, which may contribute a disproportionately large fraction of the entrapped aqueous volume (Szoka and Papahadjopoulos, 1980). Reducing the size of a spherical bilayer encapsulating an inner core of aqueous phase will reduce the captured volume, per mol of lipid (Szoka and Papahadjopoulos, 1980). This has been confirmed in a number of studies, with larger MLVs yielding significantly increased entrapment volumes (New, 1990). The entrapment efficiency is determined by the relative amounts of water and lipid present in the preparation, whereas the volume of aqueous phase encapsulated per mole of lipid is determined by the liposome size, and number of lamellae (Szoka and Papahadjopoulos, 1980). It may therefore be likely that the differences in the liposome size were not large enough to demonstrate the expected increase in entrapment with larger liposomes. Despite this, there was a trend towards an increased entrapment with large liposomes.

The inclusion of cholesterol in the liposome bilayers yielded a trend towards an improvement in the entrapment of SCG. Similarly, Taylor et al. (1990a) demonstrated a significantly increased SCG entrapment within cholesterol-containing liposomes. An increased SCG entrapment may be expected for cholesterol containing liposomes, as the incorporation of cholesterol causes an increase in the width of the phospholipid bilayers (McIntosh, 1978). This results in a greater volume of aqueous phase becoming entrapped, and therefore yields an improved entrapment efficiency. Despite this, the increase in SCG entrapment produced by the inclusion of cholesterol $\mid$ was insufficient to demonstrate a significant increase in the experiments $(\mathrm{p}<0.05)$. However, a significant increase in SCG entrapment may have been demonstrated had the experiment been repeated more than 3 times, as the increase was found to be significant at the $90 \%$ confidence level.

\subsubsection{Release of SCG from SCG-containing liposomes}

Of the liposome formulations studied, those composed of pure eggPC released entrapped SCG most rapidly upon incubation at $37^{\circ} \mathrm{C}$, producing an efflux half-life of 3.8 hours (fig. 2.8). The incorporation of equimolar cholesterol stabilised the bilayers, and prolonged the release of SCG, such that the efflux half-life was increased significantly (fig. 2.9)(table 2.7). 


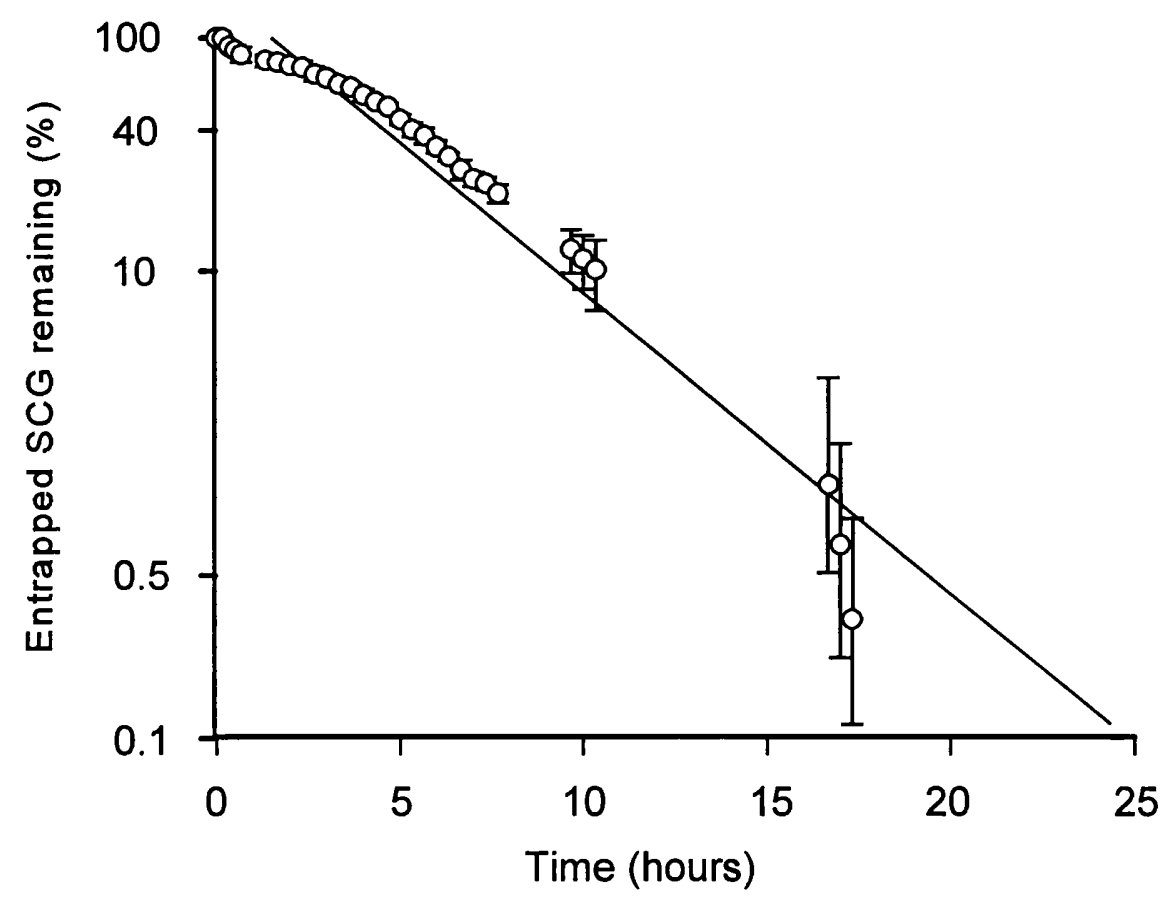

Figure 2.8 Release of SCG from eggPC MLVs.

Equation of line: $\log y=-0.129 x+2.192 ; r=0.964$. [n=3; \pm sd]

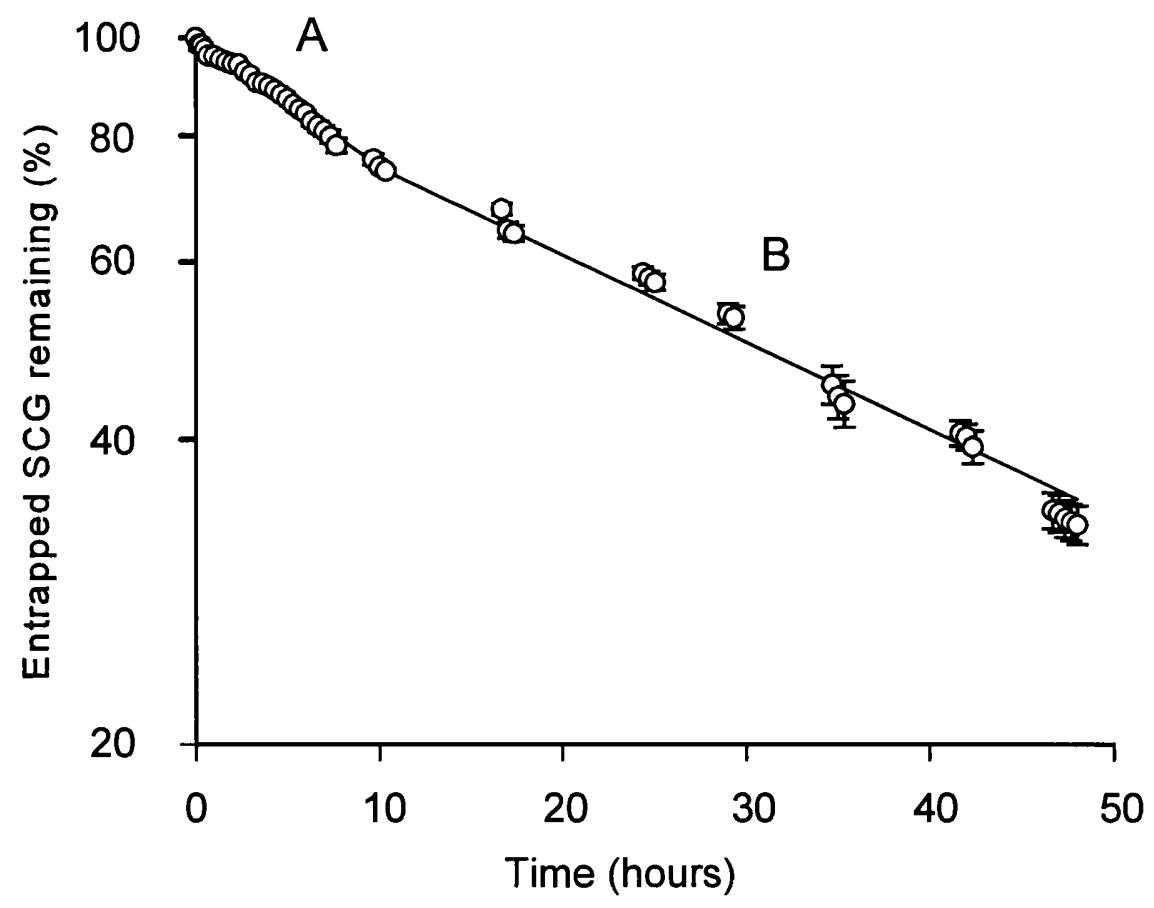

Figure 2.9 Release of SCG from eggPC/chol (1:1) MLVs.

[A] Initial phase, and [B] terminal phase of biphasic release profile. [ $n=3 ; \pm s d]$ 


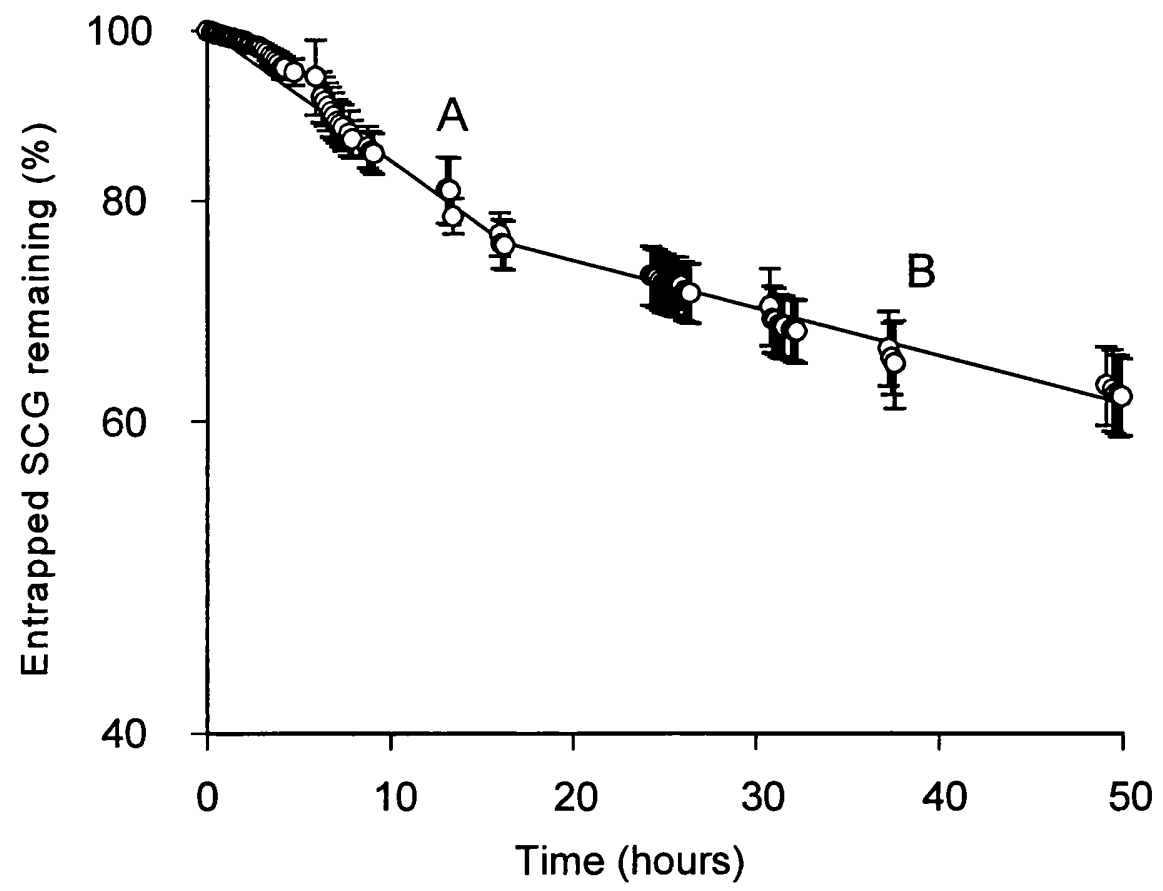

Figure 2.10 Release of SCG from DPPC/chol (1:1) MLVs.

[A] Initial phase, and [B] terminal phase of biphasic release profile. [ $n=3 ; \pm s d]$

The release of SCG from DPPC/chol (1:1) liposomes was found to be more prolonged (fig. 2.10). This result contrasts with the normally accepted effects of cholesterol on gel-state phospholipid bilayers. Cholesterol is often associated with a fluidisation of bilayers existing at temperatures below their main Tm (Ladbroke et al., 1968), such that they are made more permeable to solute efflux. However, the failure of the present results to demonstrate this is in agreement with those previously published by Taylor et al. (1990a). This was attributed to hydrogen bonding of SCG to the phospholipid polar head regions. Taylor et al. (1990a) postulated that such binding prevents the fluidising effects of cholesterol. Such an interaction has previously been demonstrated for the thiocyanate anion ( $\left.\mathrm{SCN}^{-}\right)$, at the polar group-water interface of PC bilayers (Chapman et al., 1977).

A feature of the release of SCG from the cholesterol-containing liposomes was the indication of a biphasic pattern of release (fig. 2.9 and 2.10). This was attributable to the initial rapid release of surface associated molecules. However, the biphasic profile was absent from the eggPC release graph. This may be due to the minimal barrier to SCG efflux 
presented by the relatively fluid SCG bilayers. The period of initial rapid SCG release observed from the cholesterol containing liposomes was very much reduced compared to that shown by Taylor et al. (1990a)(table 2.7). This may have been due to the fact that the two studies differ in the protocol used to separate unentrapped SCG from the liposomes. The present study employed an additional 'washing' step of centrifugation in order to remove traces of surface adsorbed SCG. This contrasted with the method of Taylor et al. (1990a) which omitted such a step. It would therefore be expected that the SCG-containing liposomes prepared in the present study would have less adsorbed SCG associated with the purified liposomes, and thus would produce a less marked biphasic release of SCG.

\begin{tabular}{|c|c|c|}
\hline \multicolumn{3}{|c|}{ Table 2.7 Half life of SCG release from SCG-containing liposomes. } \\
\hline & Bridges (this study) & Taylor et al. (1990a) \\
\hline eggPC & $3.8 \mathrm{~h}$ & $\sim 1 \mathrm{~h}$. \\
\hline eggPC/chol (1:1) & $24.13 \mathrm{~h}^{\mathrm{A}} ; 31.03 \mathrm{~h}^{\mathrm{B}}$ & $84.5 \mathrm{~h}^{\mathrm{B}}$ \\
\hline DPPC/chol (1:1) & $37.9 \mathrm{~h}^{\mathrm{A}} ; 116.87 \mathrm{~h}^{\mathrm{B}}$ & $138.6 \mathrm{~h}^{\mathrm{B}}$ \\
\hline \multicolumn{3}{|c|}{${ }^{\mathrm{A}}$ initial, and ${ }^{\mathrm{B}}$ terminal phase of SCG release profile. $^{2}$} \\
\hline
\end{tabular}

The half-life of SCG release from the cholesterol-containing liposomes demonstrated that the rate was lower than that determined by Taylor et al. (1990a) for similar MLV preparations (table 2.7). One possible explanation is that the liposomes used in presently were reduced in size to $1 \mu \mathrm{m}$, whilst those studied by Taylor et al. (1990a) were larger, unprocessed vesicles. Larger MLVs have more bilayers through which entrapped SCG must traverse before being released to the continuous phase. As a result, the release of solute is more prolonged (Gruner et al., 1985). The smaller liposomes of the present study each had fewer bilayers, and an increased surface area to volume ratio for diffusion, and hence SCG release was more rapid. Such observations have been noted for hydrophilic solutes in vivo, although this is likely to have been also due to altered interactions with biological processes (Fielding and Abra, 1992). The more rapid SCG release observed in the present study may also have been due to differences in the batch, and purity of the eggPC. This is supported by the fact that the half-lives for the terminal phase of SCG release (" $B$ ") from the liposomes containing the synthetic phospholipid, DPPC, are in closer agreement between researchers. 


\subsubsection{Surface tension of liposomes}

Figure 2.11 shows the surface tension response of liposomes as the total bilayer lipid concentration of the formulation was increased. The liposomes exhibited a concentration dependant surface tension response, which is characteristic of surface active molecules (surfactants). A sharp fall in surface tension was evident as the liposome concentration was increased between zero and $1.25 \mathrm{mg} / \mathrm{ml}$. However, apart from readings made at the two most dilute concentrations, there was no apparent change in surface tension with an increase in lipid concentration. Furthermore, the inclusion of cholesterol had no significant effect on the surface tension of the formulations $(p<0.05)$.

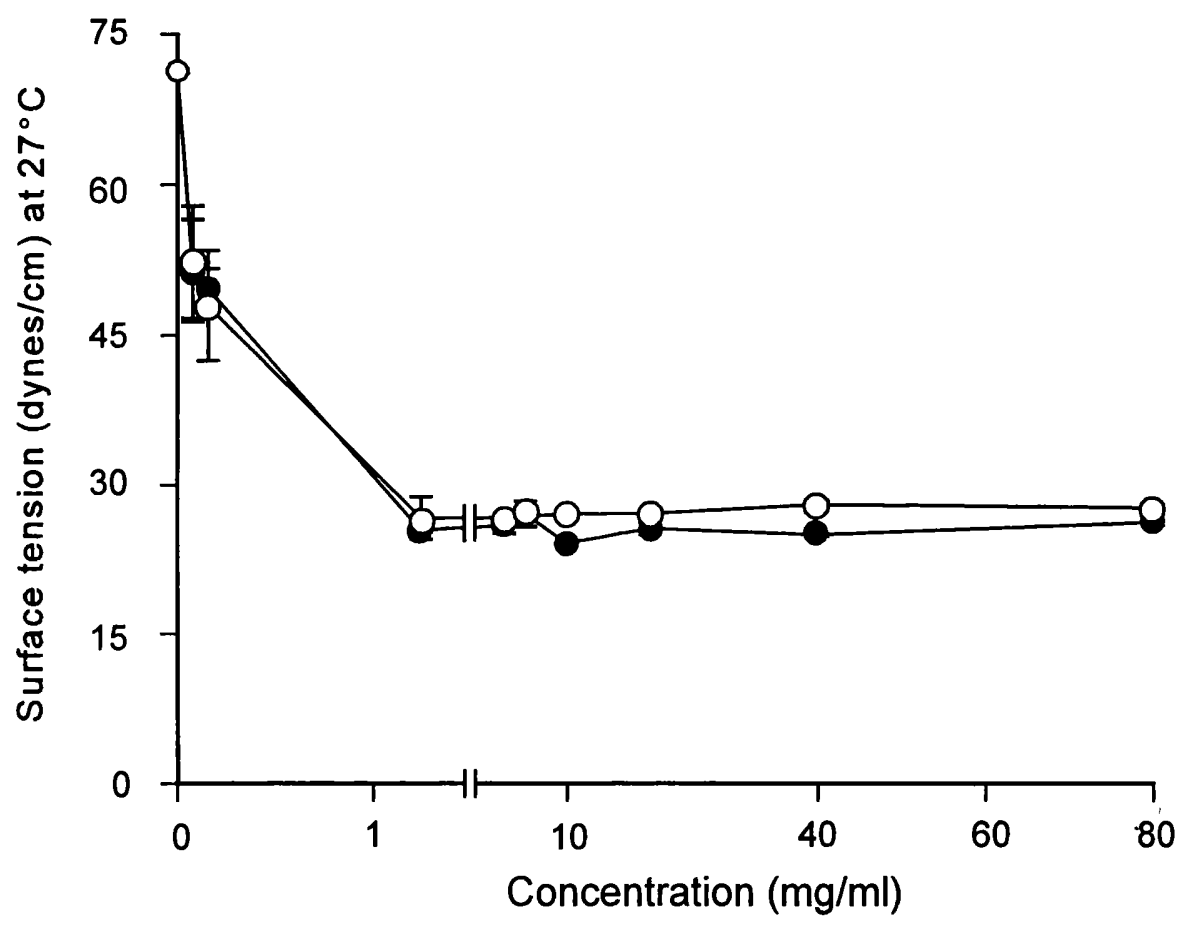

Figure 2.11 The surface tension of eggPC $(0)$ and eggPC/chol $(1: 1)(\bullet)$ liposomes, and the influence of increasing the lipid concentration. $[n=3 ; \pm s d]$

The amphiphilic nature of phospholipid molecules makes it energetically favourable for them to adsorb at the air-liquid interface. The molecules orientate themselves in order to remove the hydrophobic groupings from the aqueous environment, and hence minimise the free energy state. This reduces the attractive forces between adjacent water molecules, and in so doing surface contraction is reduced. As a result, the surface tension became lowered from 72 dynes $/ \mathrm{cm}$ for pure water, to approximately 30 dynes $/ \mathrm{cm}$. 
No further fall in surface tension was evident with further increases in lipid concentration above $1.25 \mathrm{mg} / \mathrm{ml}$. For amphiphiles, such a minima is commonly known as the critical micelle concentration (CMC), and represents the point at which free molecules become sufficiently concentrated to allow them to associate into micelle structures. Additional molecules fail to produce any further effects at the liquid/air interface as they become incorporated into micelles. The lack of data points below a lipid concentration of 1.25 $\mathrm{mg} / \mathrm{ml}$ makes the determination of a precise CMC value for the liposomes difficult. Indeed CMC values are very small for liposome forming phospholipids, to the extent that they are practically impossible to measure (Tanford, 1980). CMC values have been reported for a number of phospholipids (eg. DPPC: $3.45 \times 10^{-7} \mathrm{mg} / \mathrm{ml}$, Smith and Tanford, 1972; DMPC: $\sim 3.39 \times 10^{-5} \mathrm{mg} / \mathrm{ml}$, Cevc and Marsh, 1987). However, no CMC value has been defined for eggPC. Robinson (1960), determined that aqueous dispersions of eggPC scattered light down to a concentration less than $5 \times 10^{-4} \mathrm{mg} / \mathrm{ml}$, and as such, no assignment of CMC was justified. Furthermore, Bangham (1979) commented that the CMC for liposomes was "vanishingly small", and that as such there would be few molecules to recruit into the fluid interface. Very few free phospholipid molecules are present in solution, and as a result there is little experimental evidence of a spontaneous equilibrium between free molecules and aggregates (Johnson and Davenport, 1971). It is also perhaps more correct to define a critical bilayer concentration (CBC) for liposomal phospholipids. In the present experiment a less marked reduction in surface tension was noted at a lipid concentration below 1.25 $\mathrm{mg} / \mathrm{ml}$. However, this was unlikely to represent a concentration below the CBC of eggPC or eggPC/chol (1:1), as the documented CMC values for phospholipids are well below this concentration. Indeed, Smith and Tanford (1972) reported that it was not possible to determine the CMC of DPPC by surface tension measurements. Instead, the increased surface tension of the liposomes evident for the most dilute samples may have represented inefficient mixing of the water of dilution with the liposomes.

\subsubsection{Viscosity of liposomes}

The shear rate versus shear stress plots for eggPC and eggPC/chol (1:1) liposomes are presented in fig. 2.12 and 2.13. From these plots the liposome dispersions were calculated to yield viscosities ranging from $2.4( \pm 0.02)$ to $4.6( \pm 0.04) \mathrm{mPa} . \mathrm{s}$ (shear stresses of 0.521 to $0.75 \mathrm{Nm}^{-2}$ ) at a temperature of $25^{\circ} \mathrm{C}$ (fig. 2.14). 


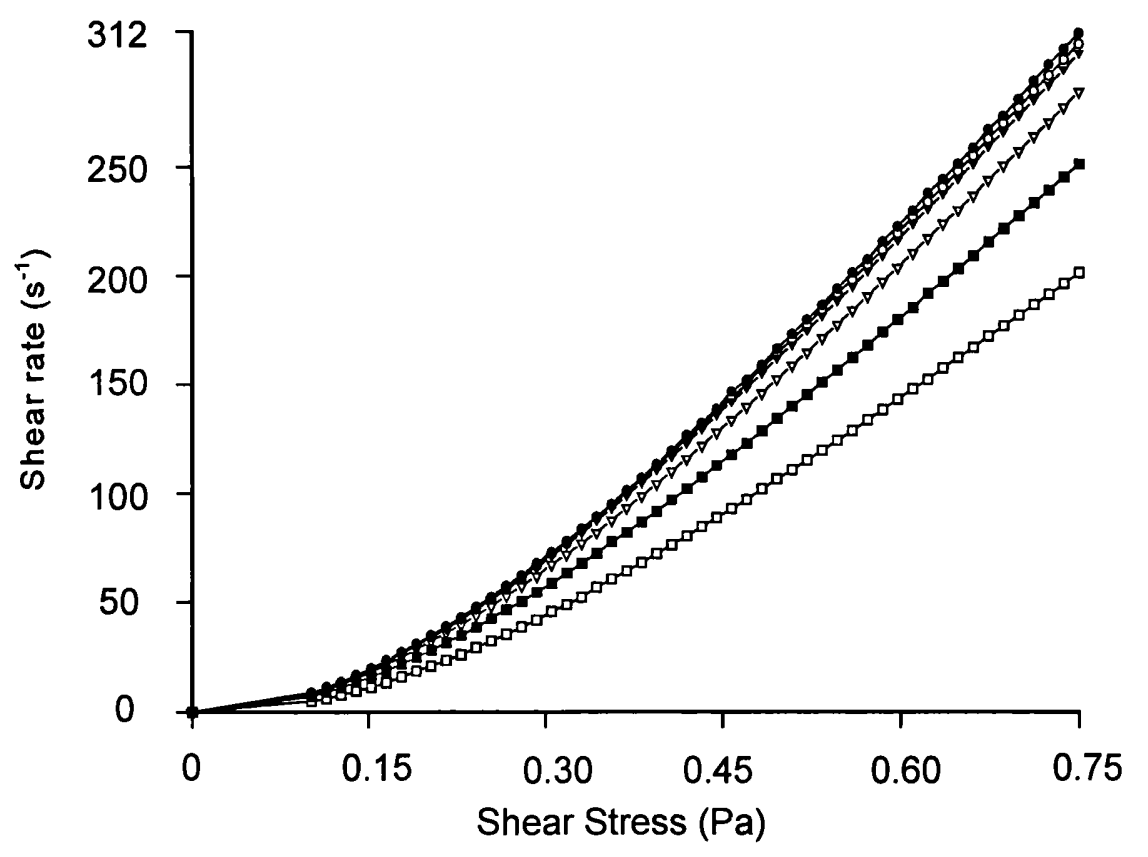

Figure 2.12 Rheological response (shear rate $v s$ shear stress) of eggPC liposomes of varying lipid concentration. (ㅁ) $80 \mathrm{mg} / \mathrm{ml} ;(-) 40 \mathrm{mg} / \mathrm{ml} ;(\nabla) 20 \mathrm{mg} / \mathrm{ml} ;(\nabla)$ $10 \mathrm{mg} / \mathrm{ml}$; (०) $5 \mathrm{mg} / \mathrm{ml}(\bullet) 2.5 \mathrm{mg} / \mathrm{ml}$. [n=3]

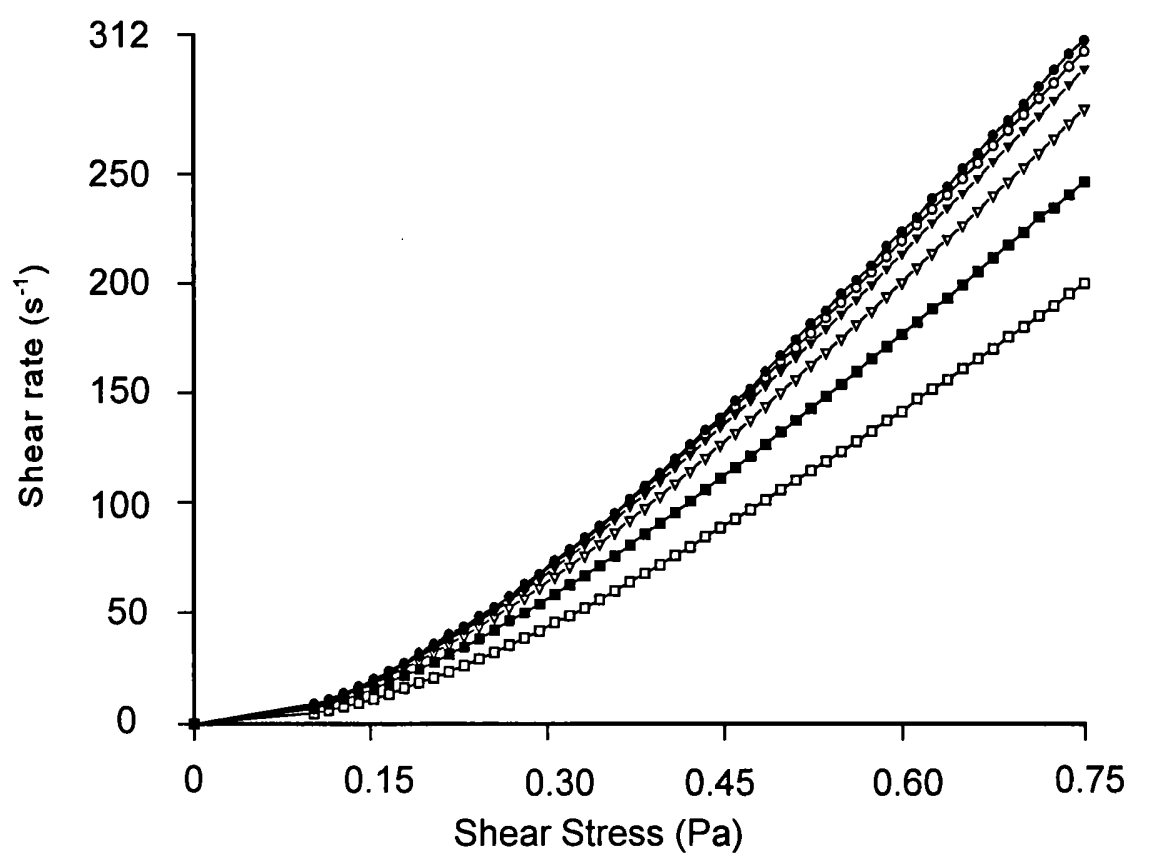

Figure 2.13 Rheological response (shear rate $v s$ shear stress) of eggPC/chol (1:1)

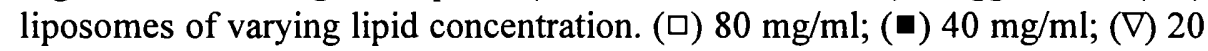
$\mathrm{mg} / \mathrm{ml} ;(\boldsymbol{\nabla}) 10 \mathrm{mg} / \mathrm{ml} ;(0) 5 \mathrm{mg} / \mathrm{ml}(\bullet) 2.5 \mathrm{mg} / \mathrm{ml}$. [n=3] 
The liposome dispersions yielded viscosities intermediate to the range produced by most common pharmaceutical fluids. For instance, water and ethanol have a viscosity of 1.002 and $1.2 \mathrm{mPa}$.s respectively. High viscosity fluids include olive and castor oil, with viscosities of 84 and $986 \mathrm{mPa}$.s respectively (values from Marriott, 1988). However, it is significant to note that the viscosity response of the liposomes was found to vary with the applied shear stress (fig. 2.15 and 2.16). As such, the dispersions exhibited a degree of nonNewtonian behaviour. Consequently, apparent viscosity values must be quoted at defined shear stress values. Each liposome formulation gave a linear increase in viscosity with increasing lipid concentration (fig. 2.14). An increase in lipid concentration from 2.5 to 80 $\mathrm{mg} / \mathrm{ml}$ produced a mean rise in viscosity of $56.3 \%$ for eggPC liposomes, and $58.5 \%$ for the eggPC/chol (1:1) liposomes.

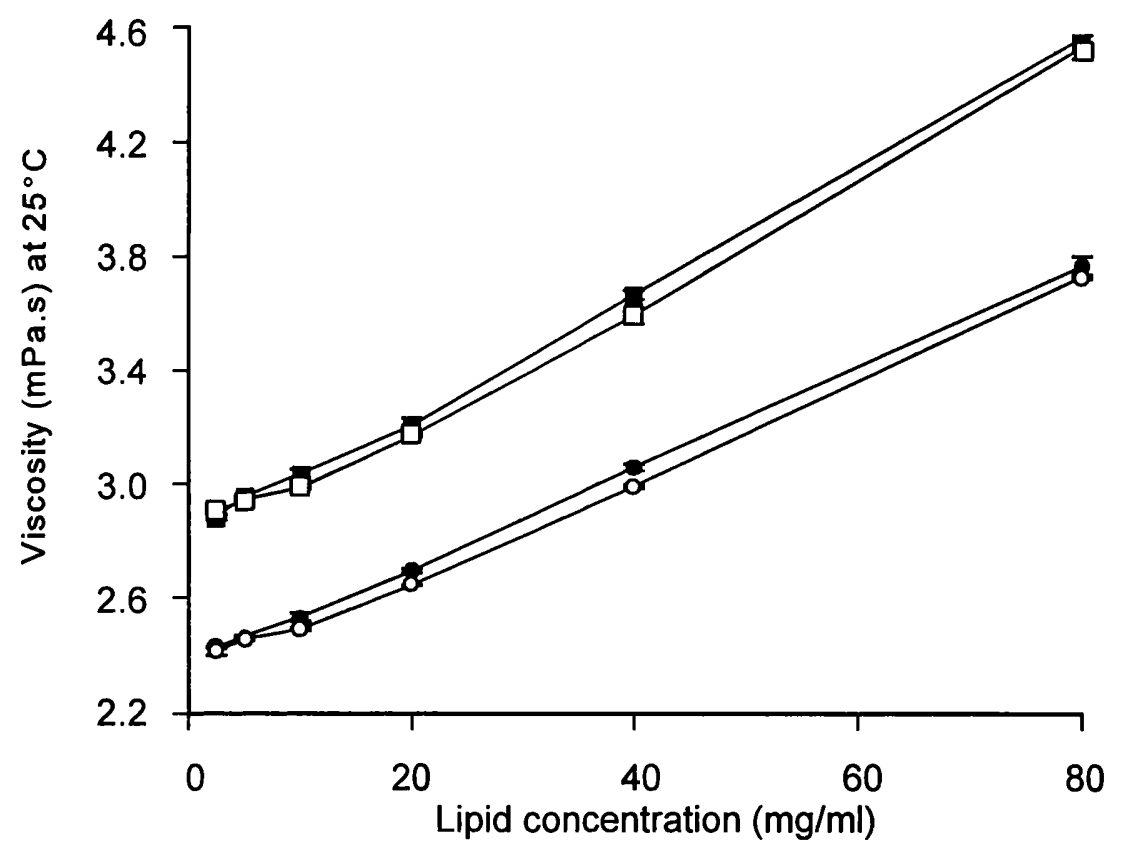

Figure 2.14 The relationship between the lipid concentration and the viscosity of eggPC (OD) and eggPC/chol (1:1) liposomes (OD). [Shear stress $=0.75 \mathrm{Nm}^{-2}$ (O), $\left.\left.0.52 \mathrm{Nm}^{-2}(\square \square)\right][\mathrm{n}=3 ; \pm \mathrm{sd})\right]$

More complex systems, such as those commonly used in pharmaceuticals, frequently deviate from Newton's law. Such formulations are typified by colloidal systems (Marriott, 1988). The complex nature of these systems causes a time dependent response to the applied shear, producing a non-linear viscosity plot (Florence and Attwood, 1988). 


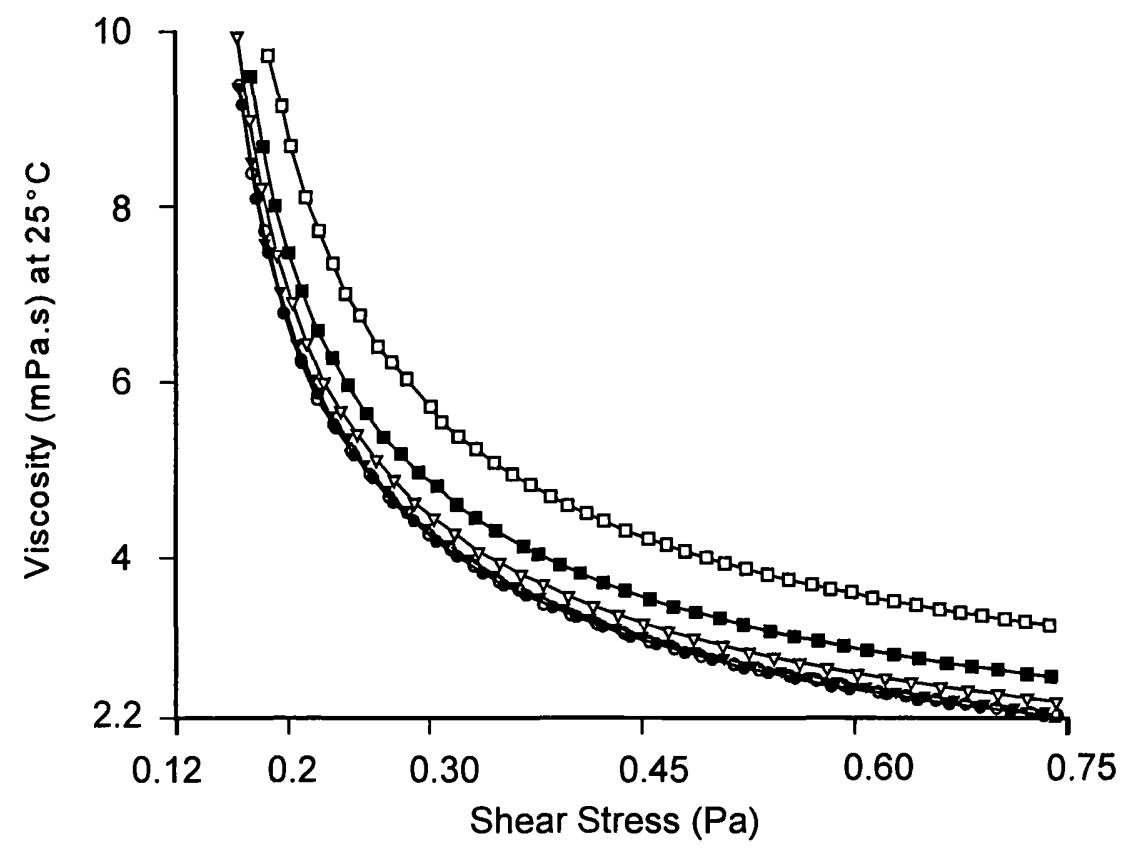

Figure 2.15 The viscosity response of eggPC liposomes of varying lipid concentration as the applied shear stress is increased. Lipid concentration: (ㅁ) 80

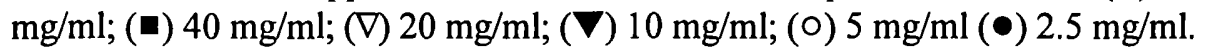
$[\mathrm{n}=3$ ]

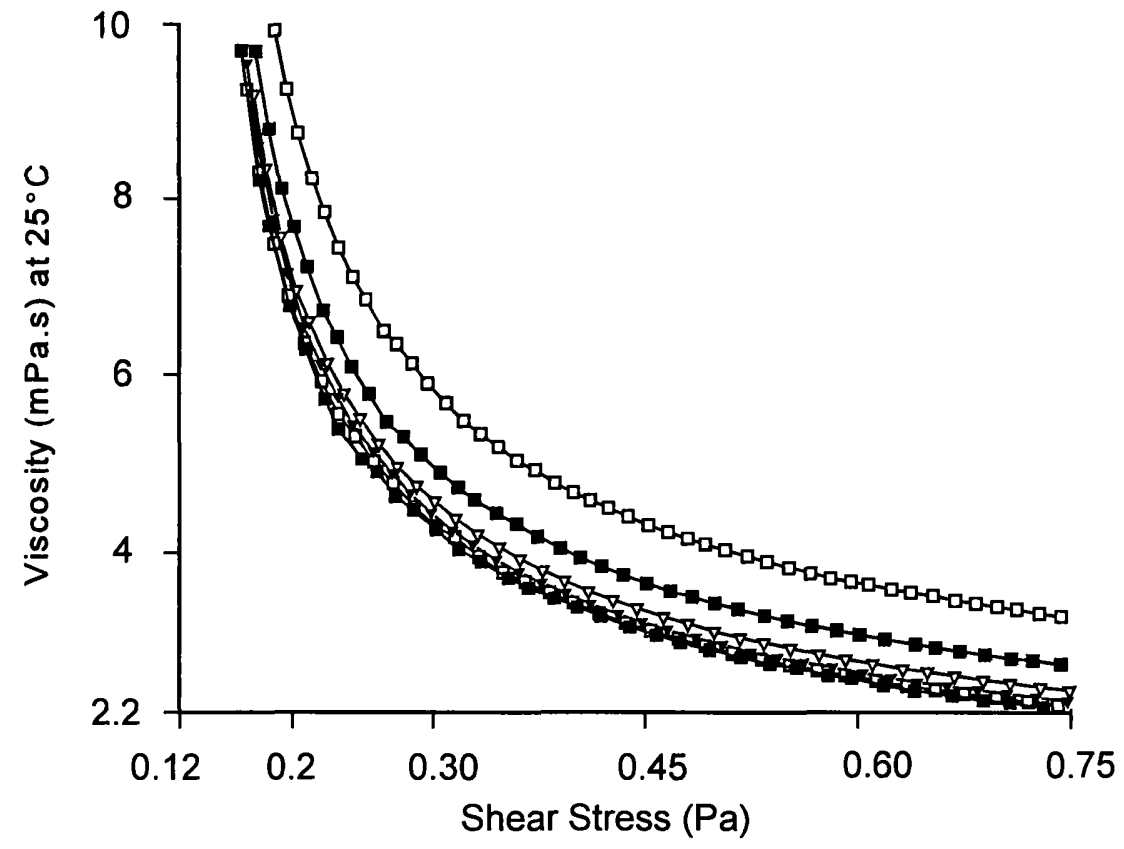

Figure 2.16 The viscosity response of eggPC/chol (1:1) liposomes of varying lipid concentration as the applied shear stress is increased. Lipid concentration:

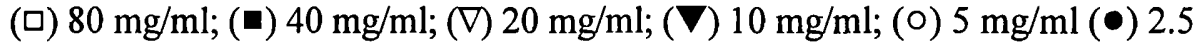
$\mathrm{mg} / \mathrm{ml}$. [n=3] 
The plot of shear stress vs shear rate for the liposomes produced a slope exhibiting a varying gradient, and hence changing viscosity, indicating non-Newtonian flow characteristics. As the shear stress was increased, the shear rate became increasingly large, indicating an increasing flow and hence a reduced viscosity (fig. 2.12 and 2.13). The slope intersects at the origin and as such the flow was best described as being pseudoplastic (Florence and Attwood, 1988). Pseudoplastic materials are commonly aqueous dispersions of high molecular weight molecules, which tend to aggregate and hence resist flow at reduced shear (Marriott, 1988).

Brinkman (1952), provided one of the first documented studies of the rheology of colloidal systems. He developed a mathematical formula, describing the relationship between the concentration of a suspension and its flow properties, which determined that the viscosity of a colloidal suspension was directly related to its concentration, as shown in the present experiment. Brinkman (1952) concluded that additional particles present in the continuous phase of a suspension cause hydrodynamic obstacles to the flow of adjacent particles, causing an increase in the fluid viscosity. In a subsequent study, Arakawa et al. (1975) demonstrated that suspended $10 \mu \mathrm{m}$ diameter poly (phthaloyl $l$-lysine) microcapsules exhibited a non-Newtonian, pseudoplastic flow. Similar pseudoplastic behaviour was also reported for gelatin microcapsules containing castor oil (Navari, et al., 1969). The Casson equation may be used to describe the flow of such suspensions (Arakawa et al., 1975). The equation was first introduced in an attempt to describe the flow behaviour of printing inks, which form aggregates at rest, yielding elevated viscosities. These aggregates dissociate at elevated shear, and hence the viscosity becomes reduced. The shear stress is found to have the following relationship with the shear rate:

$$
\mathrm{F}=\mathrm{k}_{\mathrm{o}}+\mathrm{k}_{\mathrm{l}} \mathrm{D}
$$

where:

\section{[equation 2.5]}

$$
F=\text { shear stress, } k_{o} \text { and } k_{l}=\text { constants, } D=\text { shear rate }
$$

The Casson equation is likely to be applicable to liposomes, as suggested by the present results. However, conventional suspension systems may provide only a limited approximation to the present liposomal system as, unlike liposomal phospholipid bilayers, 
they are generally non-deformable. There is little published data concerning the rheology of liposomes. Huang (1969) noted an increase in viscosity when the concentration of eggPC liposomes was increased from 40 to $140 \mathrm{mg} / \mathrm{ml}$. However, analysis at different shear rates revealed negligible variations in viscosity. From this, the authors concluded that the vesicles did not deform when made to flow, and thus behaved as Newtonian fluids. This contrasts with the results of the present study, which may be explained by the more sensitive method for viscosity determination used presently.

The majority of the rheological studies to have involved liposomes have been performed on artificial red blood cells (ARBCs), during the development of blood substitute systems (Tsuchida et al., 1988). Liposomes satisfy many of the criteria outlined for an ideal blood substitute system, as established by the National Research Council in 1963 (Vidal-Naquet et al., 1989). They provide a haemoglobin carrier that is non-allergenic, has colloidal osmotic properties, a circulation half-life approaching 12 hours, and a satisfactory shelf-life. They also produce no toxic adverse effects, or pyrogenicity. One of the principal considerations in the design of a ARBC system is that it has rheological properties which are similar to those of natural erythrocytes. Liposomes, being deformable and of a suitable size, will be a satisfactory ARBC system, provided their viscosity response to flow is similar to that of erythrocytes in blood vessels. The work on the rheology of erythrocytes, and $\mathrm{ARBCs}$, therefore provides relevant data by which to compare that produced by the present rheological study. Erythrocytes are deformable colloidal vesicles with an outer membrane consisting of a double phospholipid bilayer. As is the case with liposomes, this bilayer may contain additional non-phospholipid structures, such as proteins. Research in the 1960s, determined that whole blood, like other biological fluids containing suspended materials, exhibited a shear-rate dependent (pseudoplastic) viscosity response, similar to that observed in the present experiment (Haynes and Burton, 1959; Wells et al., 1961). The viscosity of blood was dependent on the shear rate, and the volume concentration of the erythrocytes (Haynes, 1961; Wells et al., 1962). Such a response was attributed to a reversible interaction between adjacent blood cells under low shear (Wells et al., 1962). It was suggested that the non-Newtonian characteristics of blood were attributable to the "coherence of suspended particles with one another". This coherence was attributed to the production of an "aggregation and association network" of erythrocytes under low shear. With an increase in shear this network becomes disrupted, yielding an increased flow. Upon 
removal of shear the network reforms producing an associated rise in the resistance to flow, and hence an increase in viscosity (Wells et al., 1962). A similar mechanism is likely to operate in liposomal systems, with the interactions between adjacent liposomes becoming increased at elevated concentrations.

The pseudoplastic response may also be attributable to the deformable nature of liposomes. Navari et al. (1969), stated that the pseudoplastic behaviour of erythrocytes was due to the deformation of the cell membranes, in addition to their aggregation. The non-Newtonian response of liposomes may therefore be attributable to their tendency to self-aggregate, and to their relatively elastic nature. Liposome aggregates at rest resist the tendency to flow as shear is applied to the system. Once this shear reaches a critical level the aggregates dissociate under the stress, the flow becomes enhanced, and the viscosity falls. As further shear is applied the phospholipid bilayers become distorted and the liposomes deform. Gruner (1987) states that lipid bilayers are “...sheets of materials endowed with elastic properties such as tensile strength, compressibility and bending moduli". Such properties would be expected to confer non-Newtonian properties. As the applied shear is reduced the liposomes recover, aggregates reform, and as a result the viscosity increases.

Arakawa et al. (1975) described the flow properties of erythrocyte suspensions as approximating to that of "...deformable liquid drops..." when subjected to flow. However, in contrast to previous research, Arakawa et al. (1975) failed to detect aggregates in erythrocyte or microcapsule samples. Instead, the authors attributed the non-Newtonian flow to the vesicle deformation which occurred at elevated shear stresses. The authors further noted that erythrocyte surface membranes play an important role in determining the flow properties, as membrane bound carboxymethylchitin increased the viscosity of erythrocyte suspensions, relative to liposomal blood substitutes. This is significant, in so much as it may be assumed that the same is true for liposomes. That is, the viscosity response of a phospholipid vesicle dispersion is significantly dictated by the phospholipid bilayer properties. The small rise in viscosity (approximately $1 \%$ for each lipid concentration) elicited by the inclusion of cholesterol in the present experiment supports this (fig. 2.14). This proved significant at each lipid concentration tested, except for the most dilute $(2.5 \mathrm{mg} / \mathrm{ml})$, and the most concentrated $(80 \mathrm{mg} / \mathrm{ml})$, for each formulation $(p<0.05)$. The rise in viscosity can be attributed to the stabilisation of the liposome bilayers 
that cholesterol causes. Intercalation of cholesterol between the disordered eggPC molecules restricts their motility (Keough et al., 1973; Senior, 1987). As a result, the liposome bilayers are made more rigid under the experimental conditions, and hence resist deformation as the shear is increased. As such, more shear must be applied to the eggPC/chol (1:1) liposomes to produce an equivalent flow to that of the eggPC liposomes.

With regard to rheological studies of liposomes, Tsuchida et al. (1988) determined that haemoglobin-containing unilamellar vesicles had a reduced viscosity compared with whole human blood. This was supported by Kato et al. (1983), who went on to demonstrate that eggPC liposomes were non-Newtonian in nature. The liposomes exhibited a similar pseudoplastic flow to that recorded in the present study, and the viscosity increased linearly with the concentration of vesicles in the suspension. Djordjevich et al. (1987) measured the viscosity of $3 \mu \mathrm{m}$ diameter eggPC/chol liposomes, with and without $\mathrm{DCP}$, using a temperature controlled cone and plate rheometer. Shear rate, temperature and concentration were found to be the major determinants of the viscosity of the samples, with a pronounced rise in viscosity recorded as the concentration of the liposomes was increased. Again, the liposomes were found to be non-Newtonian fluids, with a marked decrease in viscosity evident as the shear rate was increased from 1.15 to $450 \mathrm{~s}^{-1}$. As presently determined, the viscosity was influenced by the inclusion of bilayer additives. The negative charge imparted to the liposome surface by bilayer DCP reduced the observed viscosity, through a reduction in the number of aggregates. However, Vidal-Naquet et al. (1989) determined that soyPC/chol/DMPG $(5: 4: 1)$ liposomes were strongly non-Newtonian, despite the incorporation of the charged DMPG, included to inhibit vesicle aggregation. The liposome preparation was found to be a "shear-thinning fluid", with viscosity values that decreased markedly as the shear rate increased between 2 and $1000 \mathrm{~s}^{-1}$. This provides further evidence for the role of the liposome bilayers in determining the rheological properties.

Jopski et al. (1990) investigated the viscosity of liposomes with a similar bilayer composition to those studied presently. EggPC and eggPC/chol (1:0.25) liposomes were studied, with and without haemoglobin, using a variable rate viscometer. The liposomes were found to produce a similar non-Newtonian response to that documented in this chapter, producing viscosity values ranging from approximately 3.2 to $6.4 \mathrm{mPa}$.s (eggPC and eggPC/chol $17 \mathrm{mg} / \mathrm{ml}$ respectively). However, a greater dependence of viscosity on the 
liposome concentration and bilayer composition was demonstrated. An $80 \%$ increase in viscosity was observed with a change in concentration from 17 to $23 \mathrm{mg} / \mathrm{ml}$. A similar rise in liposome concentration would be predicted to give only a $4 \%$ rise in viscosity (at a shear stress of $0.305 \mathrm{~Pa}$ ) based on the results of the present study. The inclusion of cholesterol by Jopski et al. (1990) also had a greater impact, yielding an increase in viscosity of approximately $100 \%$. This compared with an increase of only $1 \%$ observed in the present study. These discrepancies may be explained by differences in methodology. Jopski et al. (1990) varied the shear rate applied by a cylinder rotational viscometer at $37^{\circ} \mathrm{C}$, whereas the present study used a variable stress Carrimed-CSL viscometer at $25^{\circ} \mathrm{C}$. Perhaps more importantly, the liposome size was not defined by Jopski et al. (1990). Human erythrocytes have a diameter of approximately 7 to $8 \mu \mathrm{m}$. This makes large, unfiltered liposomes most appropriate to their role as an ARBC. Such liposomes may produce more demonstrable effects as the concentration, or bilayer composition, is varied, compared with the small 1 $\mu \mathrm{m}$ liposomes used presently.

\subsection{Conclusions}

Large multilamellar liposomes may be prepared relatively simply from eggPC, eggPC/chol(1:1) and DPPC/chol (1:1) mixtures, utilising a thin-film hydration technique based on that described by Bangham et al. (1974). The liposomes so prepared are polydispersed, with a relatively large mean size of approximately $6 \mu \mathrm{m}$. The mean liposome size, and polydispersity, may be reduced by extrusion through membrane filters, or by sonication. Membrane extrusion was selected as the technique of choice due to the labourious nature of sonication. That said, sonication proved a useful substitute when the membrane filters could not be obtained, due to supply difficulties. The size reduction by either technique was hindered by the incorporation of cholesterol in the eggPC liposome bilayers. The eggPC bilayers are in the liquid-crystalline state at room temperature, and as such are relatively simple to reduce in size. However, the addition of equimolar cholesterol to the eggPC bilayers yields liposomes with a bilayer stability representative of gel-state liposomes. Therefore, the relatively inexpensive phospholipid eggPC may be used to model the physical attributes of widely differing liposomal formulations by the addition of cholesterol.

Incorporation of the hydrophilic solute SCG within the entrapped aqueous phase of 
liposomes produced by the thin-film technique was demonstrated. Increased diameters, and the presence of cholesterol, were associated with a trend towards an increase in entrapment. However, the present study determined that these effects were not significant. The entrapment of SCG was relatively inefficient, and thus necessitated the removal of the unentrapped solute fraction prior to the solute release studies. This was reliably achieved using an ultracentrifugational technique. Release of SCG occurs rapidly from the relatively fluid bilayers of eggPC liposomes. However, the inclusion of equimolar cholesterol stabilises bilayers to the extent that drug release from eggPC/chol (1:1) is greatly extended. $\mathrm{EggPC} / \mathrm{chol}(1: 1)$ liposomes may therefore be used in in vitro studies to approximate to DPPC containing liposomes. The results of solute release from eggPC/chol (1:1) liposomes during nebulisation may therefore be extrapolated to reflect the likely release from DPPC liposomes, which are more commonly employed in nebulised liposome studies, yet are very much more expensive to prepare.

The surface tension of eggPC, and eggPC/chol (1:1) liposomes, was found to be approximately half that of pure water, due to the amphiphilic nature of the phospholipids. This may have consequences for the aerosol output, and droplet size produced from nebulised liposomal formulations. The surface tension of liposome dispersions is relatively constant over a wide range of lipid concentrations. It is therefore unlikely that the nebulisation of liposomes with differing bilayer lipid concentrations will be influenced by surface tension effects. Significantly, the surface tension of nebulised fluids has been shown to have less of an influence on the emitted aerosol droplet size compared with viscosity (McCallion et al. (1995).

The viscosity of eggPC and eggPC/chol (1:1) liposomes was shown to increase linearly as the total lipid concentration of the formulation was increased. The liposomes examined in the present study responded to applied shear in a non-Newtonian, pseudoplastic manner, exhibiting a reduced viscosity at elevated shear stresses. This "shear thinning" behaviour may be attributable to the presence of vesicle aggregates at low shear, and to the deformable nature of the multilamellar bilayers. The latter theory is supported by the fact that gel state, cholesterol-containing liposomes, which are more rigid in nature, give elevated viscosities. 


\section{FACTORS INFLUENCING THE LIPOSOMAL AEROSOL OUTPUT FROM JET NEBULISERS}

\subsection{Introduction}

Nebulisers are simple to use, and capable of delivering a large volume of therapeutic aerosol to sites deep within the lung. For these reasons, nebulisers have been extensively researched for their potential for the pulmonary administration of liposomes. Unlike MDI or DPI devices, the liposomes may be filled directly into the device following preparation, without requiring additional processing. However, concerns have been expressed regarding the stability of liposomes to ultrasonic nebulisation, due to the significant rise in temperature that occurs during operation (Barber and Shek, 1993). It is for these reasons that jet nebulisers have been used in each of the clinical studies involving liposomal aerosols (Farr et al., 1985; Taylor et al., 1989; Barker et al., 1994; Purcell and Cullis, 1995; Vidgren et al., 1995; Waldrep et al., 1997). Consequently, the experiments detailed in the present thesis principally concerned the aerosolisation of liposomes using jet nebulisers. However, due consideration was given to the ultrasonic nebulisation of liposomes in the experiments detailed in chapter 6 .

There are a wide range of jet nebulisers available, each capable of delivering a heterodisperse population of aerosol droplets to the lung. However, careful consideration must be made of the aerosol characteristics that each nebuliser model yields, when used in conjunction with a particular nebulised formulation, as different models vary significantly in design and construction. The optimum mean size of particles for maximal deposition within the lower respiratory tract is taken to be below about 5 to $6 \mu \mathrm{m}$ (Task Group on Lung Dynamics, 1966; Stahlofen et al., 1980; Newman and Clarke, 1983). However, this is rather arbitrary, as a number of patient and delivery device variables may influence the site of droplet deposition. Despite this, the consensus is that nebulised droplets with a mean size less than approximately $5 \mu \mathrm{m}$ are "respirable" (Nebuliser Project Group, 1997). Targeting of specific sites within the lung may require a more precise control of droplet size. For instance, the alveolar region may be targeted in order to minimise mucociliary clearance of material which occurs within the conducting airways. In so doing, a more prolonged residence of the nebulised agent within the lung may be achieved. This is likely to be a goal for nebulised liposome formulations which are designed to produce sustained 
release of entrapped materials (Barker et al., 1994). Such a deposition target demands that the nebuliser produces droplets with a mean size of approximately $2 \mu \mathrm{m}$ (Stahlhofen et al., 1980; Newman and Clarke, 1983; Clay et al., 1986). Ilowite et al. (1991) determined that antibiotic therapy may be enhanced by reference to the particle size produced by the nebuliser. In particular, the efficacy of nebulised pentamidine for the treatment of pneumocystis infection of the alveoli, has been markedly improved by the development of nebulisers capable of producing appropriately small droplets (Smalldone et al., 1988; Simonds et al., 1989a; Simonds et al., 1989b). For example, pentamidine delivered from the System 22 Mizer nebuliser yielded double the incidence of adverse effects in patients, compared with that from the Respirgard nebuliser (Thomas et al., 1991a). This was attributed to the fact that the Respirgard nebuliser, producing a mean droplet size of $2.1 \mu \mathrm{m}$, achieved a greater deposition of pentamidine in the alveolar region of the lung, compared with the System 22 Mizer device which yielded a mean droplet size of $5.1 \mu \mathrm{m}$. It is therefore important that the droplet size produced by a nebulised system is determined prior to use, in order to ensure it is capable of delivering the agent to the appropriate region of the lung (Dickenson and Smalldone, 1990). The experiments of chapter 3 evaluated a number of jet nebulisers, driven by a variety of driving gas flow rates, for aerosolisation of liposomes. In addition, the formulation variables specific to the liposome component of the systems were investigated, as the physicochemical properties of fluids may influence their ability to form droplets (eg. Mercer, 1973; Davis, 1978; McCallion et al., 1995). The principal differences between liposome dispersions of low and high concentrations are the viscosity of the fluid (chapter 2), and the total number of vesicle bilayers present. It may be predicted that a change in these properties, as a result of varying liposome concentration, will alter the droplet size characteristics produced by a nebuliser. This is based on a number of published mathematical expressions, which describe the factors that influence droplet size. Glukhov (1969) related the diameter of primary droplets to the surface tension of the fluid thus:-

$$
D_{m} \propto D_{L}\left(2 \gamma / \rho_{a} \cdot V^{2} \cdot D_{L}\right)^{0.45}
$$

Where:

$D_{m}=$ diameter of droplet of average volume, $D_{L}=$ diameter of liquid orifice, $V=$ relative velocity between air and liquid, $\rho_{a}=$ density of air, $\gamma=$ liquid surface tension. 
However, there is no term in equation 3.1 that describes the influence of fluid viscosity on droplet size. An alternative model, proposed by Rizkalla et al. (1974), in a study of air-blast atomisers, stressed the importance that both surface tension and viscosity have upon the droplet size (equation 3.2).

$$
D_{m}=521 V_{a}^{-1} \cdot \gamma^{0.5} \cdot \rho_{l}^{0.75}\left(1+\frac{W_{l}}{W_{a}}\right)+0.037 \eta^{0.85}(\gamma \rho)^{1.2}\left(1+\frac{W_{l}}{W_{a}}\right)^{2}
$$

where:

[equation 3.2]

$D_{m}=$ diameter of droplet of average volume, $\gamma=$ liquid surface tension, $V=$ Velocity of air $\rho_{l}=$ liquid density, $W_{a}=$ mass flow rate (air), $W_{l}=$ mass flow rate (liquid), $\eta=$ viscosity.

Research by Lorenzetto and Lefebvre (1977), confirmed that an increase in the liquid viscosity or surface tension produced a rise in the primary aerosol droplet size. This was attributed to the stabilisation of the liquid-air interface to disruptive forces that each affords. Similar work with simple spray nozzles further supports the proposition that the droplet size is proportional to the surface tension, and viscosity of the fluid (Dorman, 1952; Hasson and Mizrhai, 1961). However, Dorman (1952) concluded that the increase in droplet size brought about by an increase in the viscosity of insecticides, was small enough to be ignored during mathematical modelling. Furthermore, a number of studies have failed to provide evidence that surface tension influences the size of sprayed droplets (Walkenhurst and Dautrebande, 1964; Kochetov et al., 1965). More recently, Sterk et al. (1983) demonstrated that increasing the concentration of solute within a nebuliser fluid resulted in an increase in the emitted droplet size. The authors attributed this to the lowering in surface tension brought about by the increased nebuliser fluid concentration. However, the authors later conceded that the droplet size effects of surface tension were impossible to distinguish from those resulting from a rise in the fluid viscosity (Sterk et al., 1984). The passage of high velocity gas through the jet nebuliser causes significant evaporative losses of water from the nebuliser fluid. This results in a marked fall in the fluid temperature (Mercer, 1973; Ferron et al., 1976), and a rise in the solute concentration (Sterk et al., 1983). Each will result in changes in the fluid surface tension and viscosity, as nebulisation proceeds. Clay et al. (1982) noted that there was a significant reduction in the aerosol production rate in response to temperature-dependent changes in surface tension and viscosity. However, little effect on droplet size may be observed (Clay et al., 1983b). The 
droplet size produced by the Collison nebuliser (May, 1973), and the Laskin aerosol generator (Hinds et al. 1983), were similarly unaffected by the use of fluids with widely differing viscosities. Subsequent studies with antibiotic solutions also failed to demonstrate changes in the respirable fraction, with varying fluid surface tensions (Newman et al., 1987: Hurley et al., 1994), and viscosities (Newman et al., 1987). Contrary to atomization theory, Searls and Snyder (1936), recorded a decrease in the emitted droplet size, as the viscosity of atomised fluids was increased. This was supported by McCallion and Patel (1996), who determined that the droplet sizes produced from the Cirrus, Pari-LC, and Sidestream jet nebulisers were inversely proportional to viscosity. This was attributed to the fact that less liquid entered the fluid feed capillaries of the nebuliser as the viscosity was raised. Energy imparted by the gas flow was therefore expended on a smaller volume of fluid. Hence the energy for atomization was increased, and the droplet size reduced.

It is thus apparent that much of the published research concerning nebulised aerosols either fails to support atomization theory, or produces completely contradictory results. A likely explanation for this, is the presence of baffles within the modern jet nebuliser. An increase in the mean size of primary droplets, accompanying a rise in surface tension or viscosity, may be compensated for by an increase in the deposition of droplets on the baffles. Larger droplets are therefore retained within the nebuliser, and as a result the effects of viscosity and surface tension are masked (Newman et al., 1987). This is supported by the fact that a decrease in aerosol output rate is a well documented consequence of nebulising fluids with increased surface tensions (Davis et al., 1978; Clay et al., 1983b: Smye et al., 1990), and also viscosities (McCallion and Patel, 1996).

Such poor correlation of the nebulised droplet size with the physical properties of the nebulised fluid, means that it is important that the aerosol produced from a nebulised formulation is characterised prior to use, when delivered by a particular nebuliser (Newman et al., 1987). This may be especially important for nebulised liposome preparations, which are more complex systems than the conventional nebulised solutions. Changing the lipid concentration of liposome formulations has been shown to influence the viscosity of the fluid (chapter 2). This was therefore investigated in the experiments of chapter 3 , as it may have significant effects on the emitted aerosol droplet size. Further to this, the effect on the rate of aerosol output was investigated. 


\subsubsection{Sizing of nebulised droplets}

A variety of non-fluid factors determine the droplet size produced by jet nebulisers. Principal among these are the nebuliser design, and the driving gas flow rate. Despite operating under the same basic principles, different jet nebuliser models often differ markedly in their design and construction. As a result, they produce aerosols with significantly differing characteristics. For instance, the mean aerosol size produced from liposome suspensions, by 18 different jet nebulisers, was found to vary between $0.9 \mu \mathrm{m}$ and $7.2 \mu \mathrm{m}$ (Waldrep et al., 1993). Such variability is compounded by the use of different operating conditions. Current aerosol research recognises the need to size aerosols as a means of objectively comparing nebulisers. In vitro measurement of the aerosol size distribution is the most commonly used indicator of a formulation's potential in vivo efficacy following nebulisation, and such measurements correlate well with in vivo results (Ho et al., 1988; Clarke, 1995). The most appropriate sizing methods are those based on inertial impaction and laser diffraction, which sample the aerosol cloud generated from a nebuliser during normal, continuous operation. However, each method is potentially flawed in that neither can reproduce the effects of changes in the relative humidity of the surrounding air as the aerosol cloud enters the lung in vivo. The emitted droplets encounter wide extremes of temperature and humidity as they travel from the nebuliser to the site of deposition in the lung. The consequent coagulation, evaporation or condensation of droplets occurring within the emitted aerosol cloud may all have a significant impact on the aerosol droplet size during inhalation (Porstendörfer et al., 1977; Phipps and Gonda, 1990). The measured droplet size, and hence the extrapolated in vivo efficacy, may therefore depend on the selected method of sampling (Clark , 1995).

\section{Methods based on inertial impaction}

The cascade impactor (CI), and multi-stage liquid impinger (MLI), are aerodynamic sizing techniques that are commonly used for therapeutic aerosol analysis. Each device contains a series of stacked, and connected horizontal trays which provide dry (CI), or wet (MLI) impaction surfaces. These are representative of the different generations of airway in the lung. The nozzles connecting each stage are of increasingly narrow diameter and accelerate the aerosol that is drawn through it, causing it to become fractionated according to the MMAD of the particles (Hallworth, and Andrews, 1976). A particle deposits on an impaction slide when its inertia is sufficient to overcome the drag force that acts to retain 
the droplet within the airstream. Therefore, the smaller the particle, the further down the impactor it is able to penetrate before impaction. The larger particles of the aerosol are thus deposited on the stage(s) representative of the larger airways, as their increased momentum prevents them from flowing around the corners of the impactor nozzles. Withdrawal and analysis of the contents of each stage by chemical, radioactive, or other means, allows a size distribution for the droplets to be produced. However, the use of cascade impaction analysis has a number of significant disadvantages. The high velocity airstream used with the technique, typically 28.3 to $60 \mathrm{l} / \mathrm{min}$, causes significant evaporative loss from the droplets, thus changing the recorded aerosol size distribution. In addition, droplet bounce-off, blowoff, re-entrainment, and wall losses may significantly distort the size distribution (Ho et al., 1987; Hallworth, 1993). Further to these problems, is the fact that the technique is highly laborious. In this respect, the two-stage, or twin impinger (TI) may be more appropriate (fig. 3.1).

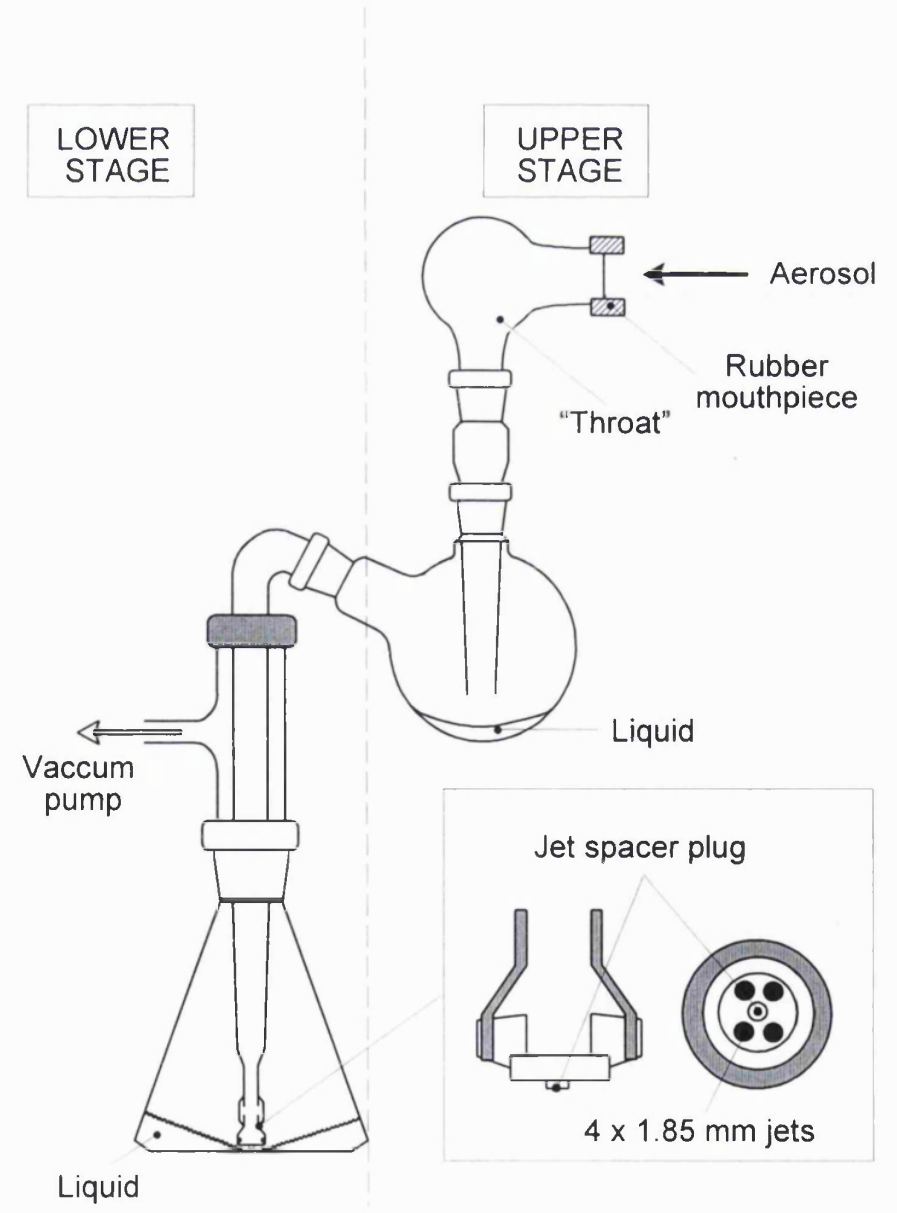

Figure 3.1 The twin impinger. 
The TI provides a more simple device for aerosol analysis than is provided by either the CI or the MLI. The Type I TI was developed as a glass separator, with an upper stage representative of stages 1 and 2 of a CI, and the lower stages of 3, 4 and the filter. A revised Type II version was then introduced, with Quickfit joints, and employing liquid impingement (Hallworth and Westmoreland, 1987). Air is drawn through the device at a flow rate of $60 \mathrm{l} / \mathrm{min}$ by means of a vacuum pump, entraining the aerosol introduced at the inlet into a glass bulb which simulates the oropharynx. Large particles deposit in the $90^{\circ}$ angle of the bulb, (the "throat"). Smaller particles pass into the upper impingement stage, where the fraction with a MMAD larger than $6.4 \mu \mathrm{m}$ deposits. Those smaller than $6.4 \mu \mathrm{m}$ avoid deposition, and deposit in the lower stage. The device is therefore useful for coarse analysis of an aerosol, separating it into a fraction likely to give oropharyngeal (or nonrespirable) deposition, and that with a potential for pulmonary (or respirable) deposition. Another similar twin impinger device, Apparatus B, is also included in the BP. The TI was designed specifically for the analysis of MDIs, and is included in the British 1993, and US XXII (Single Stage Impactor no. 3) Pharmacopoeias as a standard method for the identification of "good" and "poor" aerosols. With small modifications to the aerosol inlet the apparatus is equally applicable to aerosols generated from nebulisers. The apparatus provides an accurate method for aerosol analysis, that has proved to be capable of differentiating between subtly differing aerosol formulations (Holzner and Müller, 1995). It also provides a useful means of collecting aerosols, readily allowing sampling of the particles deposited within the liquid in each stage. The principle drawback of the TI, is the fact that it provides little information regarding the character of the respirable aerosol fraction. No indication regarding the aerosol size distribution can be calculated, beyond the percentage of the aerosol having potential for deposition within the lung (that is, a respirable fraction).

\section{Fraunhofer laser diffraction analysis}

Currently, the most popular technique for the analysis of nebulised aerosols is that based on the principle of laser light diffraction. The most widely employed instrument for aerosol analysis by this technique is the Malvern Instruments 2600c laser particle sizer (Malvern Instruments Ltd., Malvern, UK)(fig. 3.2/3.3). The analyser uses the Fraunhofer principle of diffraction to measure the size of dispersed particles. A low energy, $5 \mathrm{~mW}$, spatially filtered helium laser, with a diameter of $1 \mathrm{~cm}$, is continuously fired through the aerosol 
sample. The laser light is focussed on concentric photo detector rings by a Fourier transform lens, and a signal computed that is proportional to the energy received. The method measures particles related to their physical nature. When a particle is significantly larger than the wavelength of light $(400-700 \mathrm{~nm})$, the scatter of light that it produces in a beam is principally in a forward direction, producing minimal changes in the scatter angle. This is the Fraunhofer principle - large particles in the beam will diffract light at small angles, whereas small particles diffract light at high angles.

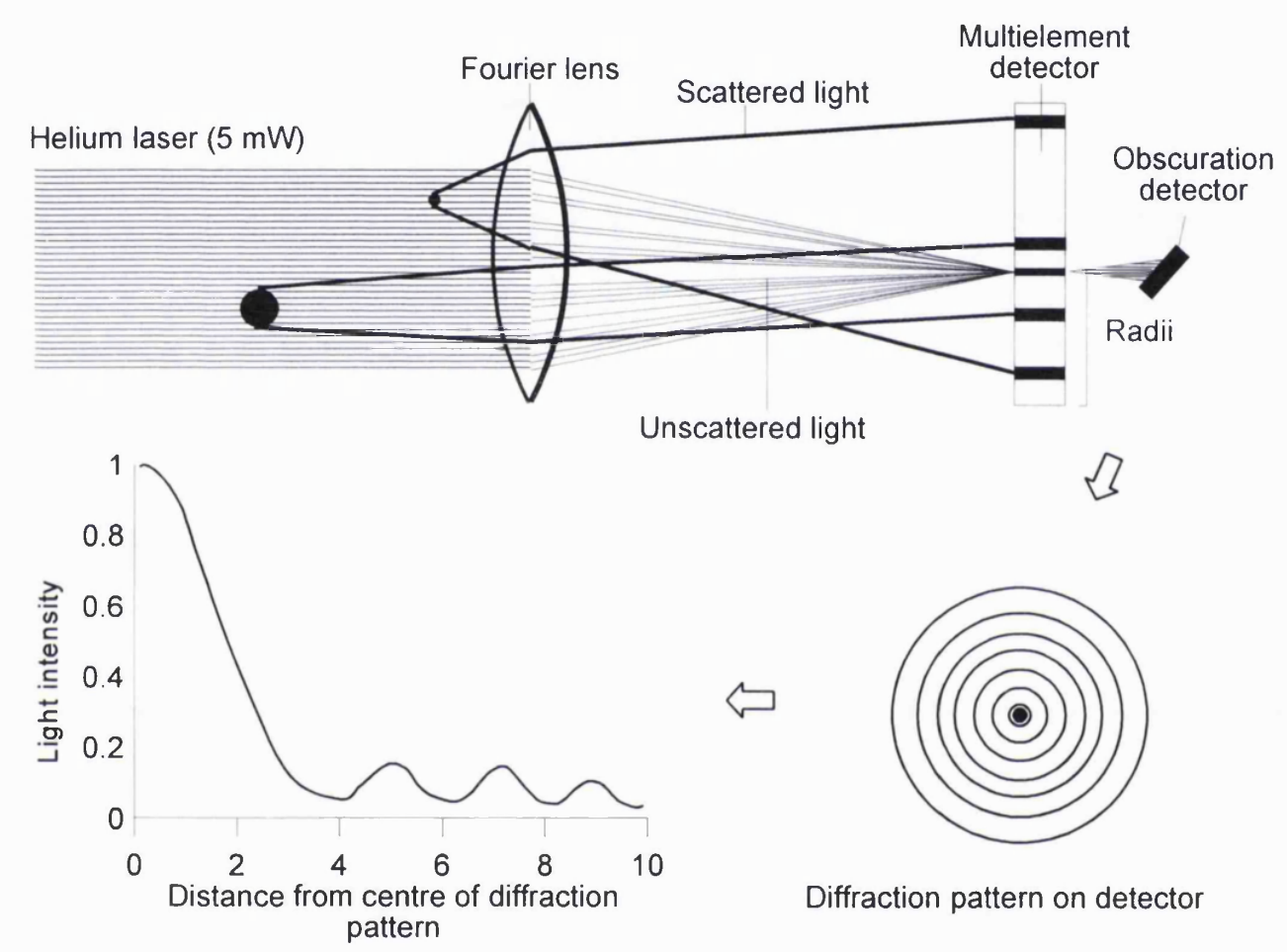

Figure 3.2 Arrangement of the optical components of the Malvern $2600 c^{\circ}$ laser diffraction sizer (Malvern Instruments, UK).

The angle of diffraction produced as laser light strikes a particle is thus inversely proportional to the radius of the particle, and the laser light is received on the Fourier lens as a regular pattern related to the particle's volume distribution (fig. 3.2). A model independent computer program is used to determine the size distribution corresponding to the light scattering data, allowing an equivalent sphere diameter to be computed. The percentage of the aerosol volume contained in each of 16 size bands on a log-diameter scale 
is then plotted, allowing the VMD to be determined. The laser diffraction sizing method is a convenient, and highly reproducible means of determining aerosol size, and is applicable to any liquid or solid particle, providing the refractive index is sufficiently different to that of the suspending medium. An aerosol may be sampled during continuous operation, at a distance from the mouthpiece equivalent to that of the throat when in normal clinical use (fig. 3.3). The aerosol droplets equilibrate to ambient conditions prior to sampling, and the measurements more accurately reflect the size distribution that enters the respiratory tract, than those produced by inertial impaction methods (Clark , 1995). The technique provides an accurate, and convenient means for the determination of the droplet size of nebulised aerosols (Ho et al., 1988). Clark (1995) demonstrated that it provides realistic information regarding the droplet size, that correlates well with the dose delivered to the lungs in vivo. Due to these significant advantages, the aerosols of the present study were analysed by laser diffraction.

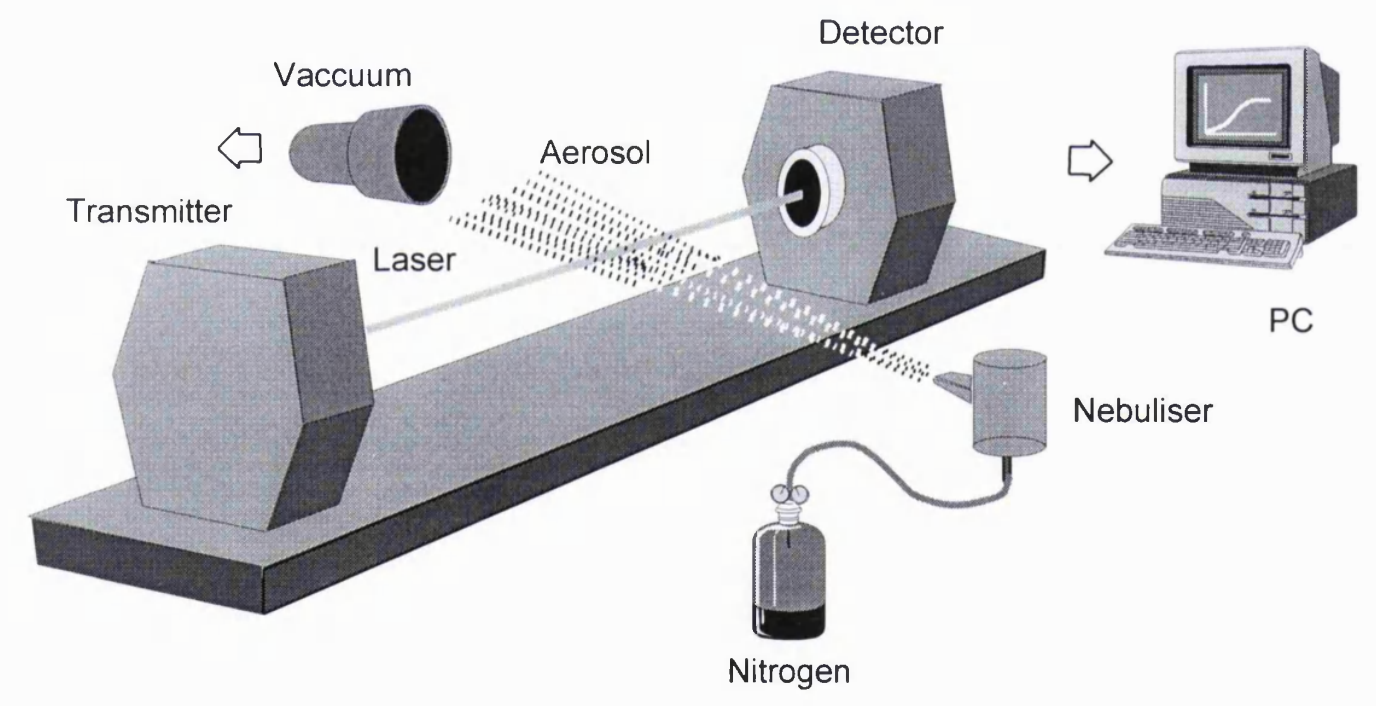

Figure 3.3 The Malvern 2600c laser diffraction sizer equipment, used to analyse a nebulised aerosol.

\subsubsection{The output of liposomal aerosols from jet nebulisers}

The output of liposomal aerosols, generated from different jet nebuliser models was determined in chapter 3 . Therapeutic fluids are commonly nebulised for a specific period of time in clinical situations. This is usually until the output becomes intermittent 
(sputtering), or until aerosol output ceases (Kradjan and Lakshminarayan, 1985). It is therefore desirable to have knowledge of the uniformity of liposome-aerosol delivery during these nebulisation periods. A fall in the nebuliser fluid temperature is a commonly observed phenomenon during jet nebulisation (Clay et al., 1983b; Taylor et al., 1992). This may have important implications for the aerosol output, due to changes in the surface tension and viscosity of the nebulised fluid (Davis, 1978). The rate of liposomal aerosol output during the course of nebulisation was therefore monitored, and related to changes in the physicochemical properties of the liposome dispersion.

\subsubsection{Concentration of liposomes during nebulisation}

A wide variety of liposome sizes have been used in the clinical studies of nebulised liposomal aerosols. These have ranged from SUVs (Farr et al., 1985), to liposomes extruded to a mean size of approximately $1 \mu \mathrm{m}$ (Barker et al., 1994), to large, unprocessed liposomes (eg. Vidgren et al., 1995). However, the size of liposomes used in nebulised formulations may be critical. A marked reduction in mean liposome size has been observed during jet nebulisation, indicating a degree of liposome instability (Taylor et al., 1990b; Niven et al., 1991a). Liposome stability may be improved by reducing their size prior to nebulisation (Taylor et al., 1990b; Niven et al., 1991a). From this, it is postulated that nebulisers exhibit a degree of size selective behaviour towards liposomes, failing to release dispersed liposomes above a certain critical size, until they become processed to a smaller size. This would lead to liposomes with a diameter greater than a certain size being predominantly retained within the nebuliser, with the result that a significant proportion of the aerosol output is comprised of water, smaller vesicles, and the products of large liposome rupture.

To test the hypothesis proposed above, the concentration of two fluids, the first a simple solution, the second liposomes, which remain within the residual fluid of nebulisers during operation, were investigated. The aim was to determine the extent of the concentration of variously sized liposomes during nebulisation. If a size selective process was indeed occurring during nebulisation, then an increased concentrating effect would be observed with larger mean size liposome formulations, over and above that caused by evaporative loss of solvent alone. 


\subsection{Materials}

\section{Reagents}

Cholesterol, Anala-R (99-99.4\%)

BDH, UK

Deionised water

WR50, RO/Deioniser, Whatman, UK

Lecithin from eggs (90\%), purified

Sigma Chemicals, USA

as detailed in section 2.3.1.1.

Methanol, Anala-R (99.8\%)

- $\quad$ BDH, UK

Polycarbonate membrane filters,

$2.5 \mathrm{~mm}$ diameter

( 1 and $5 \mu \mathrm{m}$ pore size)

- $\quad$ Nucleopore, UK

Sodium chloride, Anala-R (99.8\%)

BDH, UK

\section{Nebulisers}

Cirrus nebuliser

- $\quad$ DHD Intersurgical, UK

Pari-LC nebuliser

- $\quad$ Pari-Werk, Germany

Respirgard II nebuliser

- $\quad$ Marquest, USA

Sidestream nebuliser

Ventstream nebuliser

- $\quad$ Medic Aid, UK

- $\quad$ Medic Aid, UK

\subsection{Methods}

\subsubsection{Preparation of liposomes}

Previously purified eggPC was used to prepare eggPC and eggPC/chol (1:1) liposomes by the "thin-film" method (section 2.3.2). Total lipid concentrations of $80 \mathrm{mg} / \mathrm{ml}$ were prepared with deionised water as the aqueous phase, and diluted where necessary to concentrations between 2.5 and $80 \mathrm{mg} / \mathrm{ml}$. The mean liposome size was reduced to that required, by a combination of probe sonication, and/or repeated sequential extrusion through 5, 3 and $1 \mu \mathrm{m}$ polycarbonate membrane filters (section 2.3.4). Samples exposed to probe sonication were spun at $10000 \mathrm{~g}$ for 30 minutes in a bench centrifuge (Minor $\mathrm{S}$, MSE, UK), in order to remove contaminating titanium liberated from the sonicator probe. The liposomes were redispersed using a vortex mixer, until the mean liposome size was approximately equal to that obtained prior to centrifugation. The resulting mean vesicle size was then determined using the laser diffraction sizer (Malvern 2600c, Malvern Instruments, $\mathrm{UK}$ ) (section 2.3.3). 


\subsubsection{Assessment of liposomal aerosols}

EggPC liposomes $(20 \mathrm{mg} / \mathrm{ml}$ total lipid concentration, $1 \mu \mathrm{m}$ mean size) were filled into the Cirrus, Pari-LC, Sidestream, or Ventstream nebuliser to a volume of $5 \mathrm{ml}$. The mouthpiece of the nebuliser was clamped a distance of $0.5 \mathrm{~cm}$ from the edge of the Malvern laser diffraction sizer detection lens ( $63 \mathrm{~mm}$ focal length), right angles to, and $2.5 \mathrm{~cm}$ from, the laser beam (see fig. 3.3). The vertical position was adjusted such that the diffraction sizer laser beam passed through the centre of the emitted aerosol cloud. Each nebuliser was then operated in turn to dryness, using nitrogen gas delivered from a cylinder, at a flow rate of $5,6,7$, and $8 \mathrm{l} / \mathrm{min}$. The aerosol cloud was drawn through the laser by means of a vacuum nozzle clamped $10 \mathrm{~cm}$ from the laser beam, parallel to and facing the nebuliser. Background readings were made prior to nebulisation. The nebuliser was operated until aerosol output ceased, and the Malvern 2600c programmed to take "Liquid Droplet Spray" (LDS) measurements, every 30 seconds. Ten seconds was allowed prior to the first measurement, so that the nitrogen gas flow rate through the nebuliser became stabilised. The Malvern detector lens was covered with cardboard between each reading, to minimize the deposition of aerosolised liposomes on the lens during the experiment. The cardboard was removed 5 seconds prior to each reading to allow the aerosol cloud to equilibrate. The VMD of the aerosol sample at each time period was calculated, and plots of VMD vs time produced. The point at which the emitted aerosol cloud became intermittent was recorded. The earliest time recorded for each nebuliser was then used as the end point of nebulisation, and the mean droplet VMD and span for each experiment calculated over the time period prior to this point.

\subsubsection{The effects of liposome concentration on droplet size}

A Cirrus, Pari-LC, and Sidestream jet nebuliser were used to aerosolise eggPC, and eggPC/chol (1:1) liposomes ( $1 \mu \mathrm{m}$ mean size), prepared by the thin-film method with deionised water to concentrations ranging from 2.5 to $80 \mathrm{mg} / \mathrm{ml}$. Each nebuliser was driven at a driving gas flow rate that had been shown in the previous experiment to produce a mean droplet VMD of approximately $2 \mu \mathrm{m}$. The nitrogen gas was therefore delivered at a flow rate of $81 / \mathrm{min}$ for the Cirrus and Pari-LC nebulisers, and at $71 / \mathrm{min}$ for the Sidestream nebuliser. The aerosol was drawn through the laser of the Malvern 2600c diffraction sizer, and the aerosol analysed as detailed in section 3.3.2. Measurements were made in triplicate, and the VMD of the aerosol prior to the sputtering phase calculated. 


\subsubsection{The influence of lipid concentration on the aerosol output}

EggPC and eggPC/chol (1:1) liposomes, mean size $5 \mu \mathrm{m}$ and $1 \mu \mathrm{m}$, were produced in a range of lipid concentrations. The liposomes were aerosolised using the Pari-LC, and Sidestream nebulisers, at a nitrogen gas flow rate of $51 / \mathrm{min}$. The time taken for each nebuliser to reach the sputtering phase, and to cease producing aerosol entirely, was recorded for $10 \mathrm{mg} / \mathrm{ml}$ eggPC/chol (1:1) liposomes. The aerosol produced by each nebuliser was drawn into a glass twin impinger (British Pharmacopoeia Appendix XVII C, Apparatus A), with a $60 \mathrm{l} / \mathrm{min}$ flow rate. The impinger was merely used as a means of drawing the aerosol cloud away from the nebuliser, not as a sampling device. The output of aerosol from each nebuliser was determined at $50 \%$ and $100 \%$ of the time to sputtering, and also following 2 minutes of sputtering, by mass measurements.

\subsubsection{Concentration of liposomes during nebulisation}

\section{Evaporative losses of solvent occurring during nebulisation of a simple solution}

A $30 \mathrm{mg} / \mathrm{ml}$ solution of sodium chloride was prepared, and $5 \mathrm{ml}$ nebulised with the PariLC, Cirrus, Sidestream, and Respirgard nebulisers, operated at $71 / \mathrm{min}$. The time taken to reach the sputtering phase was recorded for each device. A further $5 \mathrm{ml}$ of saline solution was then nebulised by each nebuliser. A $1 \mathrm{ml}$ sample of the residual sodium chloride solution was then withdrawn from each of the nebuliser chambers at the sputtering time, and added to a preweighed test tube. In addition, a $1 \mathrm{ml}$ sample of the original $30 \mathrm{mg} / \mathrm{ml}$ sodium chloride solution was added to a separate test tube as a control. The tubes were then incubated at $60^{\circ} \mathrm{C}$ in an oven for 12 hours, in order to evaporate off the solvent. The tubes were cooled to room temperature, and then reweighed. The mass of sodium chloride within each tube was determined, allowing the concentration of sodium chloride within the residual fluid of each nebuliser at the sputtering time to be calculated.

\section{Evaporative losses of solvent occurring during nebulisation of liposomes}

EggPC/chol (1:1) liposomes were prepared by the thin film method, to a concentration of $10 \mathrm{mg} / \mathrm{ml}$. The mean liposome size was reduced to $5,2.5,1$, or approximately $0.6 \mu \mathrm{m}$ by initial extrusion through $5 \mu \mathrm{m}$ filters, and then probe sonication to approximately $2.5 \mu \mathrm{m}$. The $1 \mu \mathrm{m}$ liposomes were then prepared by extrusion of $2.5 \mu \mathrm{m}$ liposomes through $1 \mu \mathrm{m}$ membrane filters. A sample of $1 \mu \mathrm{m}$ liposomes was then exposed to continued sonication, until shown by laser diffraction to be approximately $0.6 \mu \mathrm{m}$ in size (a total of approximately 
21 minutes sonication, calculated from extrapolation of figure 2.6). Contaminating titanium was removed from sonicated samples by centrifugation for 10 minutes at $10000 \mathrm{~g}$ (Minor $\mathrm{S}, \mathrm{MSE}, \mathrm{UK})$. The liposomes were redispersed by vortex mixing, and an indication of the mean vesicle size given by laser diffraction analysis prior to use. $1 \mathrm{ml}$ of each liposome sample was then added to preweighed test tubes, with $9 \mathrm{ml}$ of deionised water. The tubes were spun at $200000 \mathrm{~g}$ in an ultracentrifuge (Superspeed 75, MSE, UK) for 30 minutes at $10^{\circ} \mathrm{C}$. The eluate was decanted carefully from the lipid pellet. The tubes were incubated at $60^{\circ} \mathrm{C}$ for 12 hours in an oven. The samples were removed, equilibrated to room temperature, and reweighed, to establish the mean control mass of lipid which remained following dehydration of the liposome samples.

Each liposome preparation was then nebulised, using a fill volume of $5 \mathrm{ml}$, following the protocol detailed for the sodium chloride solution. The mass of lipid that remained following nebulisation to sputtering was determined by withdrawl of a $1 \mathrm{ml}$ sample, and dehydration as detailed previously for the controls. The change in lipid concentration, due to evaporative losses during nebulisation, was then determined by reference to the control masses of dehydrated lipid.

\subsubsection{Examination of nebuliser component orifices}

Various internal components from each nebuliser were removed, mounted on microscope stubs, and vacuum coated with a $30 \mathrm{~nm}$ thick layer of gold. Scanning electron micrographs were then taken at an accelerating voltage of 10 to $15 \mathrm{kV}$ (Phillips XL20, Phillips, UK).

\subsection{Results and discussion}

\subsubsection{Assessment of liposomal aerosols}

The Cirrus, Pari-LC, Sidestream, and Ventstream jet nebulisers studied are among those commonly used in clinical practice, and typify the diverse nature of commercially available designs (Nebuliser Project Group, 1997). The mean droplet size produced by each, during nebulisation of $1 \mu \mathrm{m}$ mean size eggPC liposomes, is illustrated in figure 3.4 and 3.5. The mean droplet size was relatively stable throughout the time to sputtering. However, once the volume of residual fluid had fallen to below a critical level, the aerosol production became intermittent, and the nebulisers were seen to spit and "sputter". The droplet size for each profile was therefore determined prior to this point. 
(a)
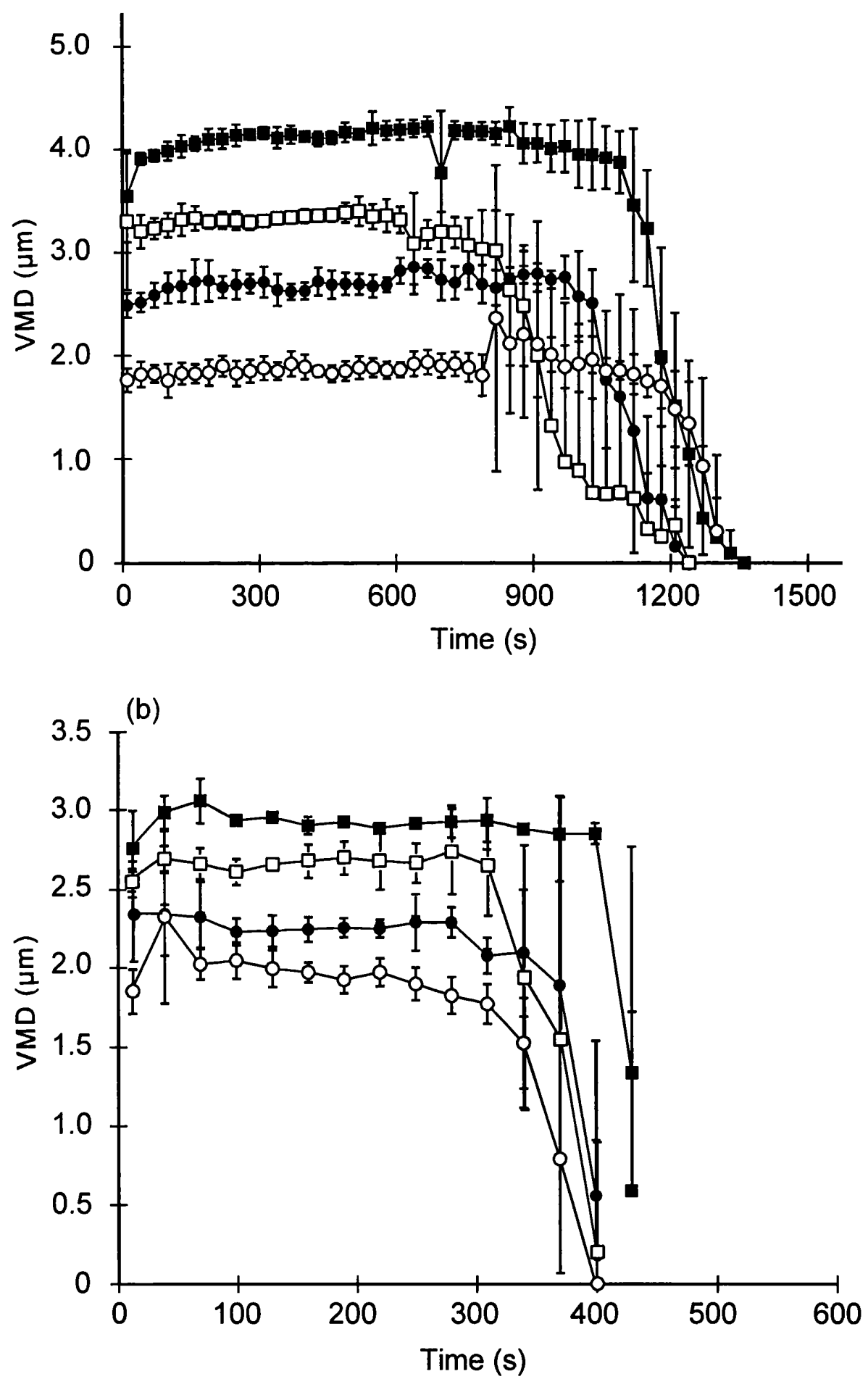

Figure 3.4 The mean volume median diameter (VMD) of aerosols delivered from the Cirrus (a) and Pari-LC (b) nebulisers during nebulisation of eggPC liposomes. Each nebuliser was operated at driving gas flow rates of $(\square) 5,(\square) 6,(\bullet) 7$, and $(O) 8 \mathrm{l} / \mathrm{min}$. $[ \pm \mathrm{sd} ; \mathrm{n}=6]$ 
(a)

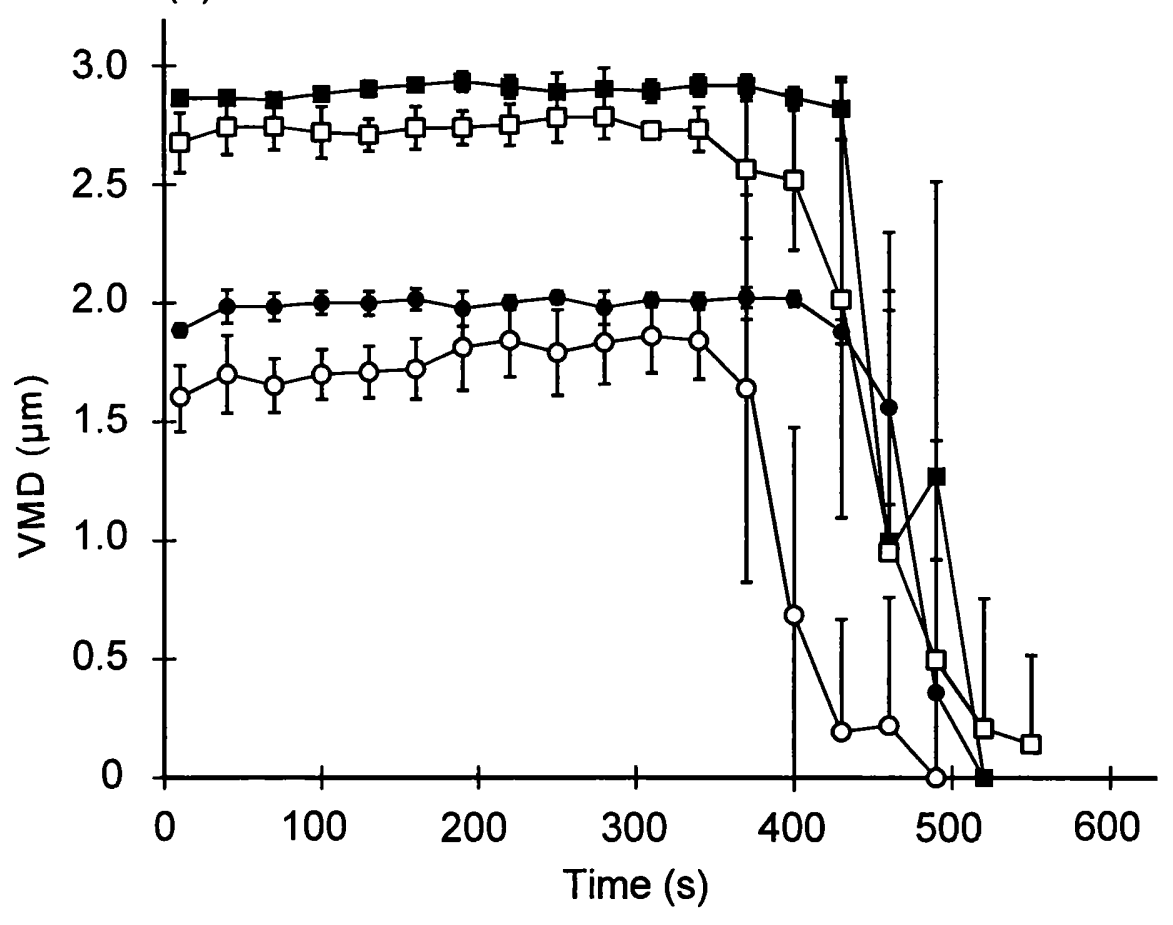

(b)

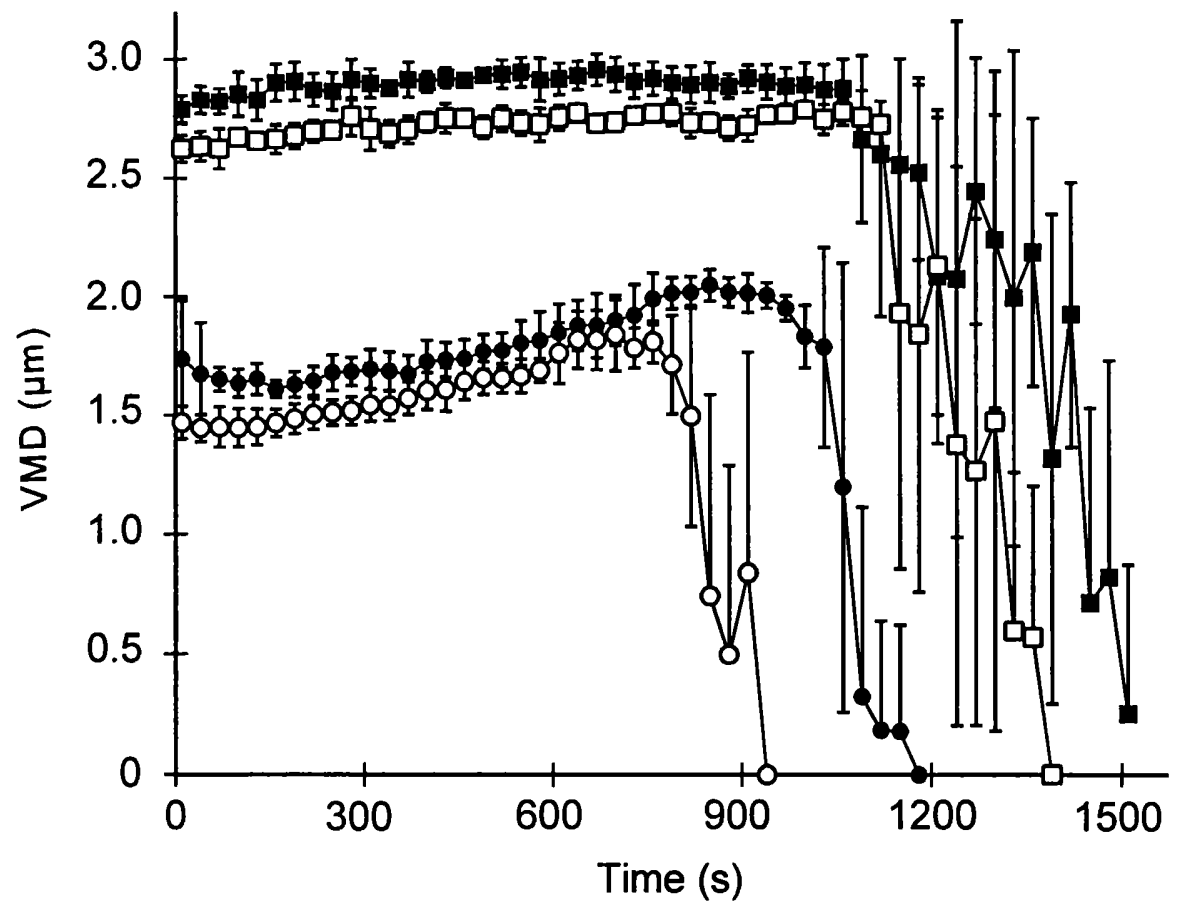

Figure 3.5 The mean volume median diameter (VMD) of aerosols delivered from the Sidestream (a) and Ventstream (b) nebulisers during nebulisation of eggPC liposomes. Each nebuliser operated at driving gas flow rates of $(\square) 5,(\square) 6,(\bullet) 7$, and $(O) 81 / \mathrm{min}$. $[ \pm$ sd; $n=6]$ 
Each of the nebulisers studied produced droplets that may be considered to be "respirable", having mean VMDs less than $5 \mu \mathrm{m}$ (Nebuliser Project Group, 1997). However, there was found to be a significant variation in the droplet size produced by certain of the nebuliser models at a given flow rate $(\mathrm{p}<0.05)$. The Cirrus produced aerosols with a mean VMD between 1.9 to $4.1 \mu \mathrm{m}$ (fig. 3.4a); the Pari-LC 2.0 to $2.9 \mu \mathrm{m}$ (fig. 3.4b); the Sidestream 1.7 to $2.9 \mu \mathrm{m}$ (fig. 3.5a); and the Ventstream 1.6 to $2.9 \mu \mathrm{m}$ (fig. 3.5b), for gas flow rates of 5 to $8 \mathrm{l} / \mathrm{min}$ (table 3.1). Each nebulised aerosol was heterodisperse in nature, as reflected by the span values (table 3.1). The Pari-LC nebuliser produced aerosols with the greatest spread of droplet sizes (span values of 2.30 to 2.56 ), and the Sidestream the least (1.74 to 2.27).

Table 3.1 The effect of the driving gas flow rate on the size (VMD), and span of

\begin{tabular}{|c|c|c|c|c|c|}
\hline \multirow{2}{*}{ Nebuliser } & & \multicolumn{4}{|c|}{ Driving gas flow rate (1/min) } \\
\cline { 3 - 6 } & & 5 & $\mathbf{6}$ & 7 & $\mathbf{8}$ \\
\hline \multirow{2}{*}{ Cirrus } & VMD $(\boldsymbol{\mu m})$ & $4.06(0.01)$ & $3.27(0.11)$ & $2.70(0.08)$ & $1.86(0.05)$ \\
\cline { 2 - 6 } & Span & $1.92(0.06)$ & $2.03(0.04)$ & $2.31(0.09)$ & $2.56(0.11)$ \\
\hline \multirow{2}{*}{ Pari-LC } & VMD $(\boldsymbol{\mu m})$ & $2.92(0.08)$ & $2.66(0.04)$ & $2.45(0.05)$ & $1.97(0.06)$ \\
\cline { 2 - 6 } & Span & $2.30(0.09)$ & $2.46(0.08)$ & $2.69(0.08)$ & $2.84(0.07)$ \\
\hline \multirow{2}{*}{ Sidestream } & VMD $(\boldsymbol{\mu m})$ & $2.89(0.03)$ & $2.72(0.06)$ & $1.99(0.03)$ & $1.74(0.09)$ \\
\cline { 2 - 6 } & Span & $1.74(0.07)$ & $1.84(0.06)$ & $2.09(0.07)$ & $2.27(0.08)$ \\
\hline \multirow{2}{*}{ Ventstream } & VMD $(\mu \mathrm{mm})$ & $2.90(0.03)$ & $2.68(0.19)$ & $1.80(0.14)$ & $1.61(0.13)$ \\
\cline { 2 - 6 } & Span & $1.86(0.04)$ & $2.02(0.07)$ & $2.17(0.03)$ & $2.42(0.07)$ \\
\hline
\end{tabular}

The design and geometry of the constituent parts of each nebuliser model determines the relative gas jet speeds, and the efficiency of the impaction, and re-entrainment of aerosol droplets (Dennis and Hendrick, 1992). Droplet formation is influenced by differences in the diameter and orientation of the capillaries between each nebuliser, whilst the emission of droplets is controlled by the size, geometry and design of the baffles, and other internal structures (O'Callaghan and Barry, 1997). These differences explain the variations observed in the mean droplet size and size distribution, produced by different nebuliser models, when operated at equivalent flow rates. The Sidestream and Ventstream nebulisers only differ in 
the design of an open vent, included to increase the aerosol output efficiency during the patients inspiratory phase of breathing. The Ventstream nebuliser incorporates a one-way valve, which opens and closes the vent in synchronicity with the patients breathing, thus producing maximal useful aerosol output. This is termed a breath -assisted, open vent design. By contrast, the Sidestream lacks such a valve, and is thus an open vent nebuliser. As there was negligible inspiratory flow provided by the vacuum during the experiment, the vent did not influence the nebuliser performance. Consequently, the two nebulisers yielded very similar aerosols. Indeed, only at the 7 and $81 / \mathrm{min}$ flow rates were the aerosols produced by the Sidestream and Ventstream nebulisers statistically different $(p<0.05)$. Of the nebulisers tested, the Cirrus, operated at a reduced flow rate, was likely to be most unsuitable for achieving alveolar deposition of liposomes, as it produced a mean droplet size that was significantly larger than the $2 \mu \mathrm{m}$ size generally accepted as being necessary for efficient penetration of droplets to the lung periphery (Stahlhofen et al., 1980; Newman and Clarke, 1983; Clay et al., 1986). The Pari-LC is also a breath -assisted, open vent nebuliser, and emitted droplets with a mean size of approximately $2 \mu \mathrm{m}$. However, the polydispersity of the droplets was greater than each of the alternative nebulisers (table 3.1). This is significant, as the mass of each droplet is related to the cube of the droplet size. Thus, in broadly heterodisperse aerosols such as this, a large proportion of the aerosolised liposomes will be contained within the larger droplets, which may be "non-respirable". It is therefore important that the "polydispersity" of droplet sizes within nebulised aerosols is considered, in addition to the mean size. The Sidestream, and Ventstream nebulisers, operated at higher flow rates yield aerosols with a mean size of approximately $2 \mu \mathrm{m}$, and a relatively reduced span (table 3.1). Therefore, the Sidestream and Ventstream nebulisers would be predicted to give maximal alveolar deposition, and minimal extra-pulmonary deposition, of the nebulisers tested, based upon droplet size considerations alone. However, it is important to note that other nebuliser variables, such as the aerosol output efficiency, will determine the mass of liposomes deposited within the lung.

As the driving gas flow rate was increased from 5 to $81 / \mathrm{min}$ the emitted droplet size produced by each nebuliser was reduced (fig. 3.6). This is consistent with many other studies (eg. Clay et al., 1983a; Newman et al., 1986; McCallion et al., 1995). Primary aerosol droplets are created as a result of the shearing action of the driving gas flow on the 


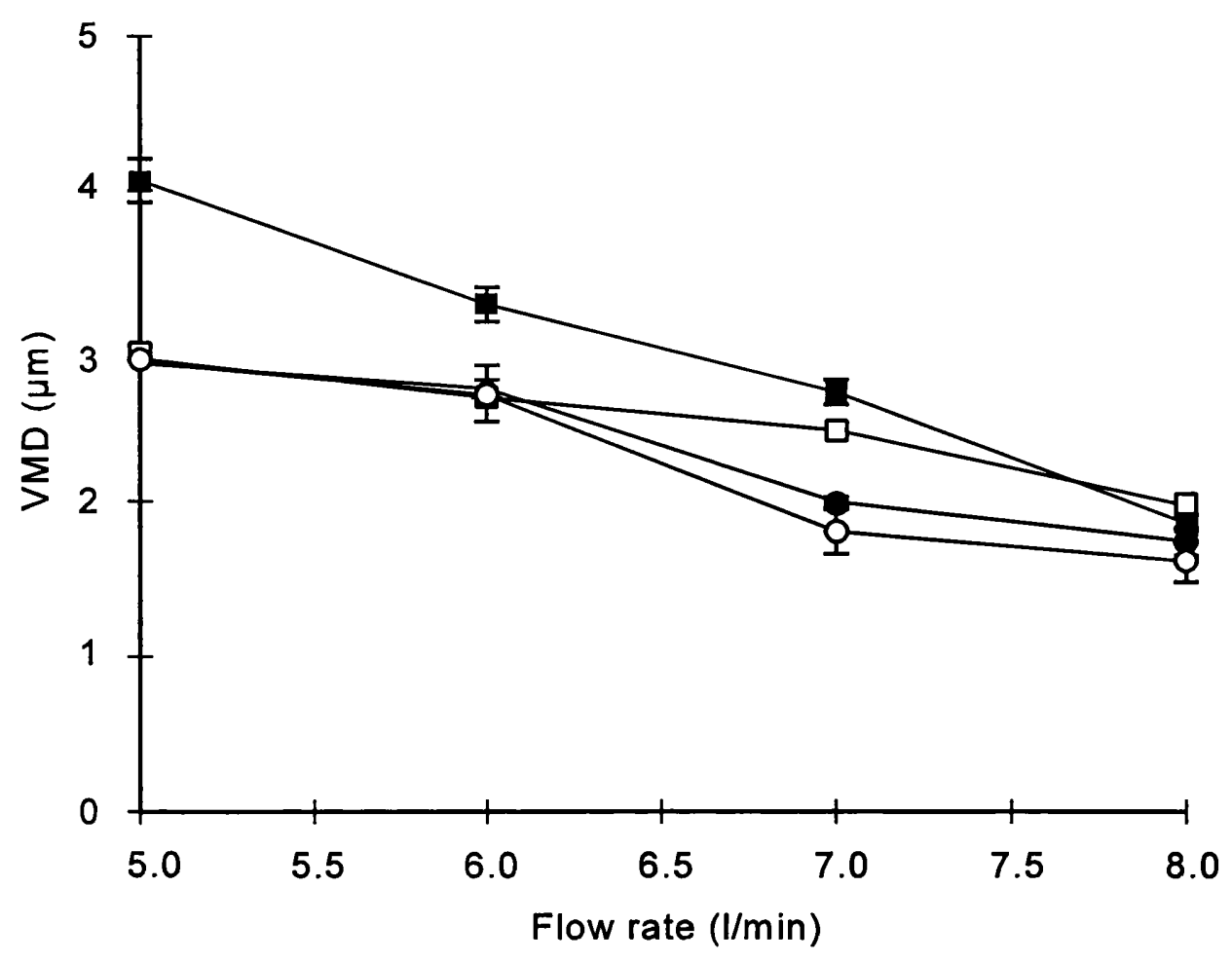

Figure 3.6 Effect of driving gas flow rate on the volume median diameter

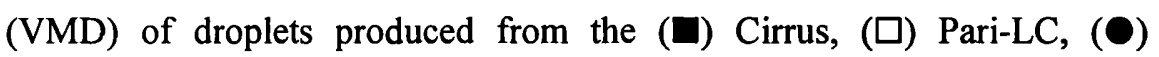
Sidestream and $(O)$ Ventstream nebulisers. $[n=6 ; \pm s d]$

liquid sheet formed from the nebuliser nozzle. At higher flow rates the energy available for droplet formation is increased, with the result that smaller droplets are formed. In addition, a higher flow rate increases the momentum of the droplets, increasing the probability of impaction on baffles, and other structures within the nebuliser. The Cirrus nebuliser yielded droplets that were most affected by a change in driving gas flow rate, with a $119 \%$ reduction in droplet size as the flow rate was increased from 5 to $8 \mathrm{l} / \mathrm{min}$. This was compared with $80 \%$ for the Sidestream, $66 \%$ for the Ventstream, and $48 \%$ for the PariLC. This is consistent with the results of a previous study of 11 nebulisers, in which the Cirrus was most influenced by a change in the gas flow rate (Newman et al., 1986). It is therefore apparent that the Cirrus nebuliser has a reduced efficiency baffling system compared to similar devices, which allows the emission of larger droplets produced by reduced driving gas flow rates. The larger droplets produced by the other nebulisers are more efficiently filtered out before emission, with the result that the emitted aerosol is less influenced by the driving gas flow rate. The Pari-LC apparently operates at lower flow rates more efficiently than the alternative nebulisers. This may be because it is principally 
designed to operate with the Pari-Master compressor, which gives a flow rate equivalent to gas flow at $4 \mathrm{l} / \mathrm{min}$. At the higher driving gas flow rates the observed difference between the droplet size produced by different models was less marked. This result is consistent with a previous study of four different jet nebuliser models (Clay et al., 1983a).

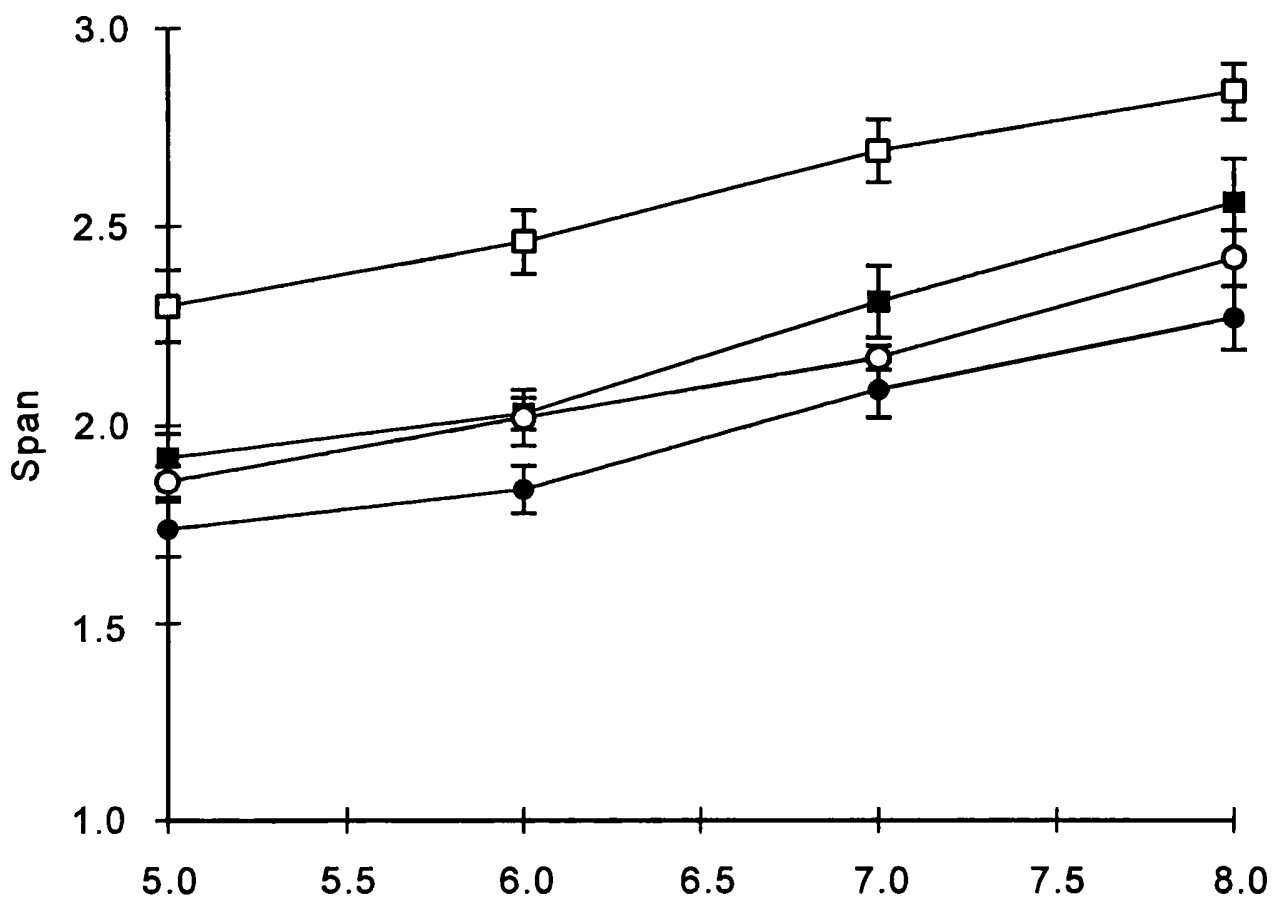

Figure 3.7 Effect of driving gas flow rate on the span of droplets produced from the ( $\square$ ) Cirrus, ( $\square$ ) Pari-LC, (๑) Sidestream and (O) Ventstream nebulisers. $[n=6 ; \pm s d]$

In contrast to the mean droplet size, the polydispersity of each of the nebulised aerosols was significantly increased by an increase in the driving gas flow rate from 5 to $81 / \mathrm{min}$ (fig. 3.7). The extent of the increase was similar for each nebuliser. This is in agreement with other studies of nebulised aerosols (Clay et al. 1983a; Newman et al., 1986; Niven and Brain, 1994). McCallion et al. (1995), failed to demonstrate consistent increases in span with an increase in driving gas flow rate from 6 to $8 \mathrm{l} / \mathrm{min}$. However, among the nebulisers tested were the Cirrus and Pari-LC, which indeed produced aerosols with a reduced span at the higher flow rates. 
The time taken for different nebulisers to reach the sputtering phase of nebulisation was highly dependent on the nebuliser model, and the driving gas flow rate. The time taken for the aerosol output to reach the sputtering stage was recorded as the time when the aerosol output first appeared intermittent, and yielded values of $820 \mathrm{~s}$ to $1210 \mathrm{~s}$ for the Cirrus (fig. 3.4a); approximately $310 \mathrm{~s}$ for the Pari-LC (fig. 3.4b); $340 \mathrm{~s}$ to $400 \mathrm{~s}$ for the Sidestream (fig. 3.5a); and 790 to $1090 \mathrm{~s}$ for the Ventstream (fig. 3.5b) at driving gas flow rates of 8 to $5 \mathrm{l} /$ minute. The reduction in nebulisation time with an increased gas flow rate is consistent with previous jet nebuliser studies (eg. Clay et al., 1983a; 1983b), and is the result of an increase in the rate at which fluid is entrained into the gas jet. Whilst the droplet size and efficiency of drug output are considered to be the principal determinants of the clinical efficacy of a nebulised formulation, the nebulisation time is important in terms of patient compliance, and for minimising nursing time (Clay et al., 1983b; Nebuliser Project Group, 1997). Furthermore, because nebulisers are often operated in clinical use for a fixed time period, regardless of the model used, it is important to determine the time taken for different nebuliser models to cease aerosol production. Just as differences in nebuliser design cause variations in the delivered aerosol size, so they influence the nebulisation time. Differences in baffle design between nebulisers affects the proportion of the primary aerosol that is emitted, and thus the mean size of the emitted aerosol. This influences the volume of fluid released, and hence the rate of aerosol output. Equally, differences in the dimensions, and orientation of the gas jets, will cause variations in the rate of aerosol entrainment. The Sidestream and Ventstream nebulisers gave very different nebulisation times, despite producing similar droplet sizes. This was due to the differences in the design of the vents in each device. The Sidestream nebuliser was the only device to incorporate an open vent. Supplementary air is fed into the chamber via the vent at all times during operation, which increases the output rate. Open vent nebulisers such as this are therefore associated with reduced nebulisation times. By contrast, the Ventstream incorporated a breath-assisted, open vent. This vent is designed to close as the patient exhales, preventing the passage of air through the nebuliser, and hence reducing output during expiration. The vent opens during inhalation, and in so doing promotes droplet entrainment. The drawback to such a design is that nebulisation times are prolonged, however aerosol output is synchronised with the patients breathing. During the present experiment the valve remained partially closed, as no inspiratory flow was provided, and hence there was a reduced output rate, compared with the Sidestream. 


\subsubsection{Effects of liposome concentration on droplet size}

Figures 3.8 to 3.10 show the mean droplet sizes of aerosols produced by the Cirrus, Pari-LC and Sidestream nebulisers, during nebulisation of eggPC and eggPC/chol (1:1) liposomes, of varying lipid concentrations. The Ventstream was omitted from the experiment as the nebuliser design was essentially the same as the Sidestream. Consequently, the aerosol would be expected to respond in a similar manner to changes in the nebulised fluid properties.

As determined in section 3.4.1, the aerosol output rate varied significantly between each of the different nebuliser models, as a function of nebuliser design. Because of this, the time taken to reach the sputtering phase of nebulisation was highly variable. Similarly, the droplet size varied to a significant extent between the different nebuliser models. The Cirrus nebuliser produced a mean aerosol VMD of 2.20 to $3.20 \mu \mathrm{m}$ (fig. 3.8), the Pari-LC 2.84 to $3.62 \mu \mathrm{m}$ (fig. 3.9), and the Sidestream 1.87 to $1.92 \mu \mathrm{m}$ (fig. 3.10), for lipid concentrations of 2.5 to $80 \mathrm{mg} / \mathrm{ml}$.

There was no apparent correlation between the physical properties of the dispersed liposomes and the emitted droplet size. The inclusion of cholesterol in eggPC liposomes makes the bilayers more rigid, and as a consequence causes a small increase in the viscosity of the fluid relative to liposomes constructed of eggPC alone (approximately $1 \%$; chapter 2). The droplet size produced by jet nebulisers may be influenced by such physicochemical changes (eg. Davis, 1978). However, the incorporation of cholesterol in the eggPC bilayers did not significantly influence the droplet size. It is likely that the small increase in viscosity produced by the inclusion of cholesterol was insufficient to produce demonstrable changes in the emitted aerosol cloud. In addition, there was no significant difference in the droplet size produced, by a change in liposome size from 1 to $5 \mu \mathrm{m}(\mathrm{p}<0.05)$. This result is consistent with several previous studies, which demonstrated that the size of nebulised aerosol droplets was independent of the liposome bilayer composition, and vesicle size (Farr et al., 1985; Taylor et al., 1990b; Niven et al., 1991a). However, the lipid concentration of the liposomes influenced the droplet size. The mean VMD of liposomal aerosols emitted from the Cirrus, Pari-LC and Sidestream nebulisers became significantly increased as the total lipid concentration of each liposome preparation was raised from 2.5 to $80 \mathrm{mg} / \mathrm{ml}(\mathrm{p}<0.05)$ (table 3.2 ; fig. 3.11). 

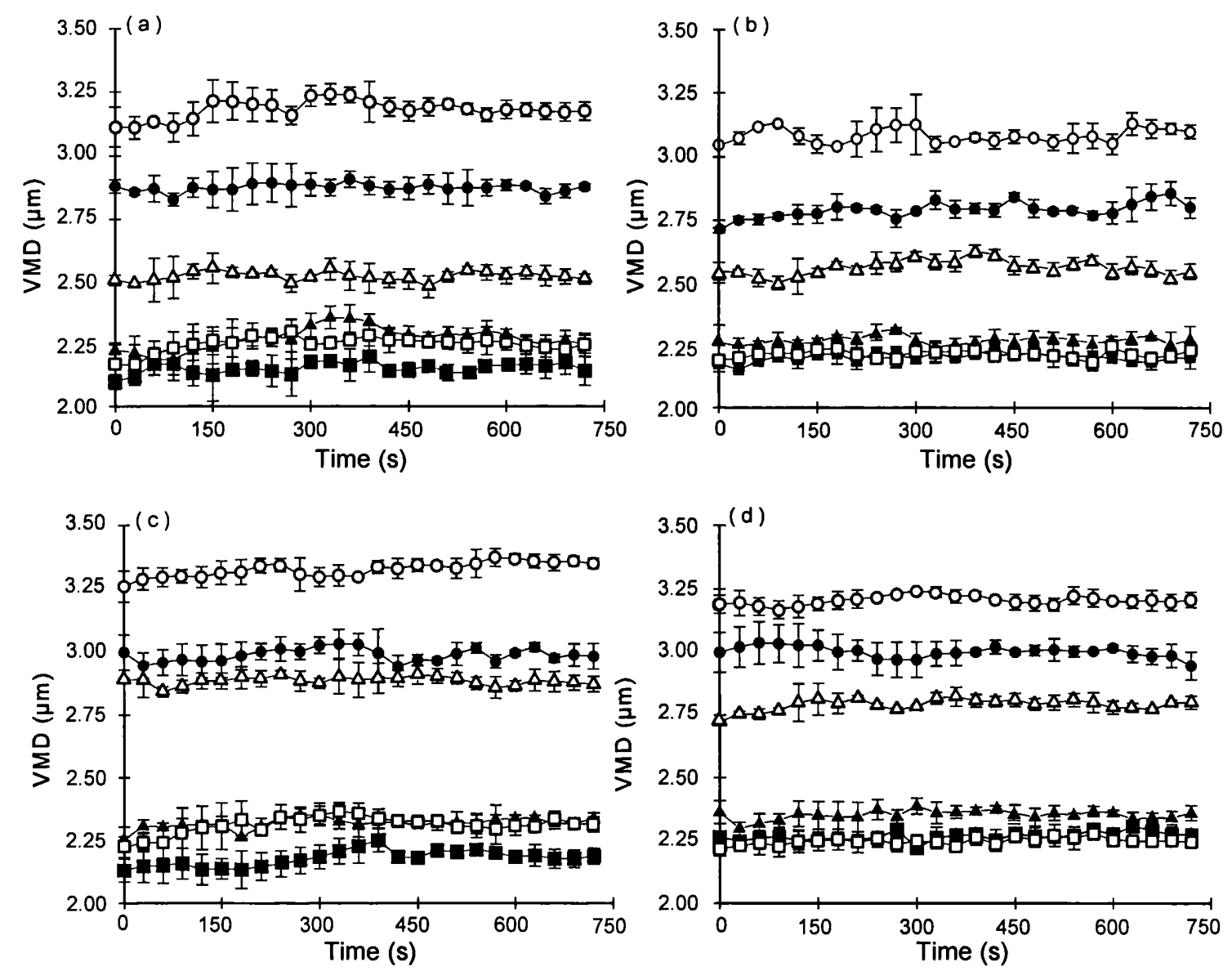

Figure 3.8 Mean VMD of droplets emitted from the Cirrus nebuliser with time, during nebulisation of (a) eggPC, $1 \mu \mathrm{m}$; (b) eggPC/chol, $1 \mu \mathrm{m}$; (c) eggPC, $5 \mu \mathrm{m}$; and (d) eggPC/chol, $5 \mu \mathrm{m}$ liposomes at lipid concentrations of $(\square) 2.5 \mathrm{mg} / \mathrm{ml},(\square) 5 \mathrm{mg} / \mathrm{ml},(\Delta) 10 \mathrm{mg} / \mathrm{ml},(\Delta) 20 \mathrm{mg} / \mathrm{ml},(\bullet) 40 \mathrm{mg} / \mathrm{ml}$, (०) $80 \mathrm{mg} / \mathrm{ml}$. [n $=3 ; \pm \mathrm{sd}]$ 

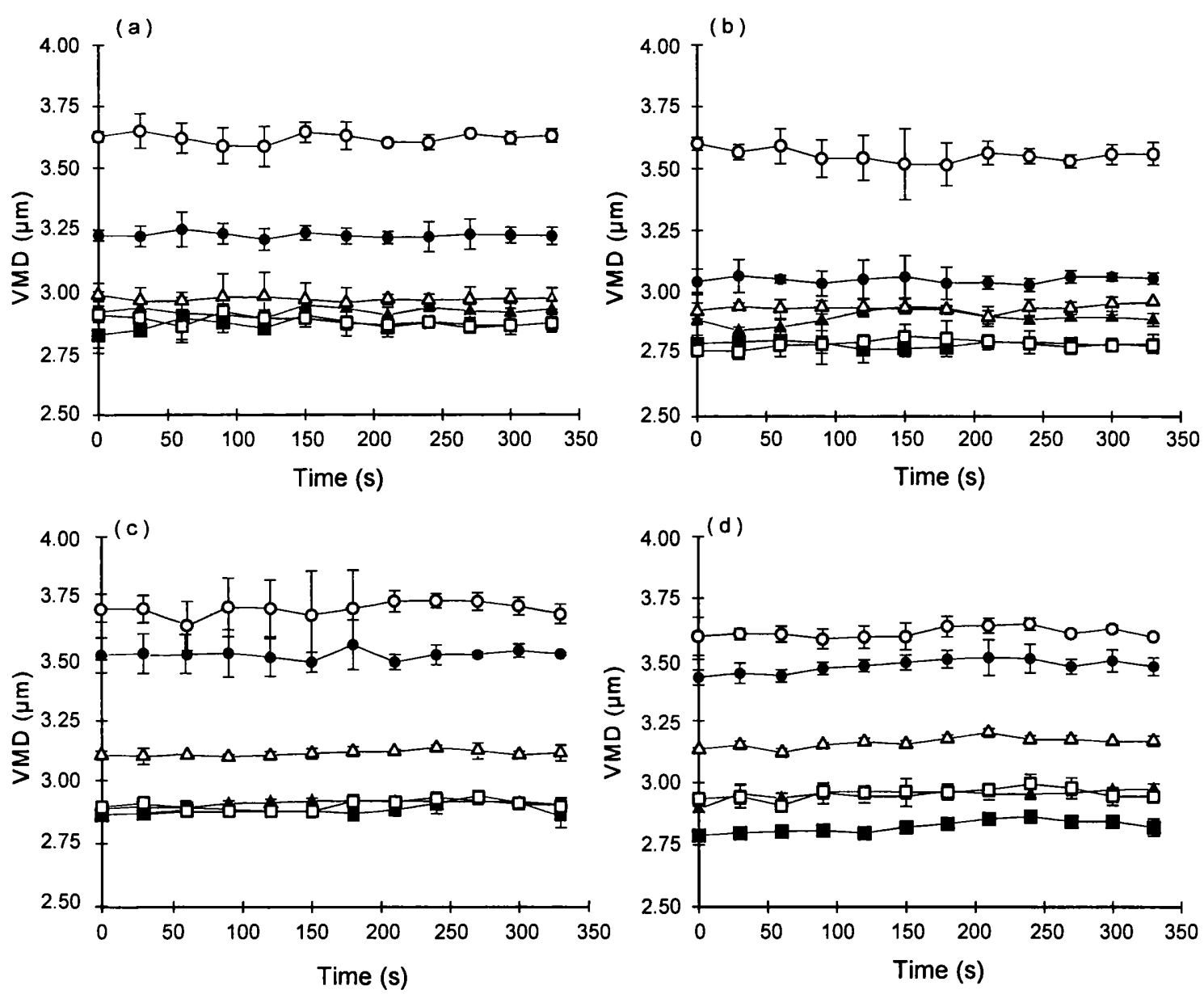

Figure 3.9 Mean VMD of droplets emitted from the Pari-LC nebuliser with time, during nebulisation of (a) eggPC, $1 \mu \mathrm{m}$; (b) eggPC/chol, $1 \mu \mathrm{m}$; (c) eggPC, $5 \mu \mathrm{m}$; and (d) eggPC/chol, 5 $\mu \mathrm{m}$ liposomes with lipid concentrations of (口) $2.5 \mathrm{mg} / \mathrm{ml}$, (口) $5 \mathrm{mg} / \mathrm{ml},(\boldsymbol{\Delta}) 10 \mathrm{mg} / \mathrm{ml},(\Delta) 20$ $\mathrm{mg} / \mathrm{ml},(\bullet) 40 \mathrm{mg} / \mathrm{ml}$, and (०) $80 \mathrm{mg} / \mathrm{ml}$. [n = 3; $\pm \mathrm{sd}]$ 

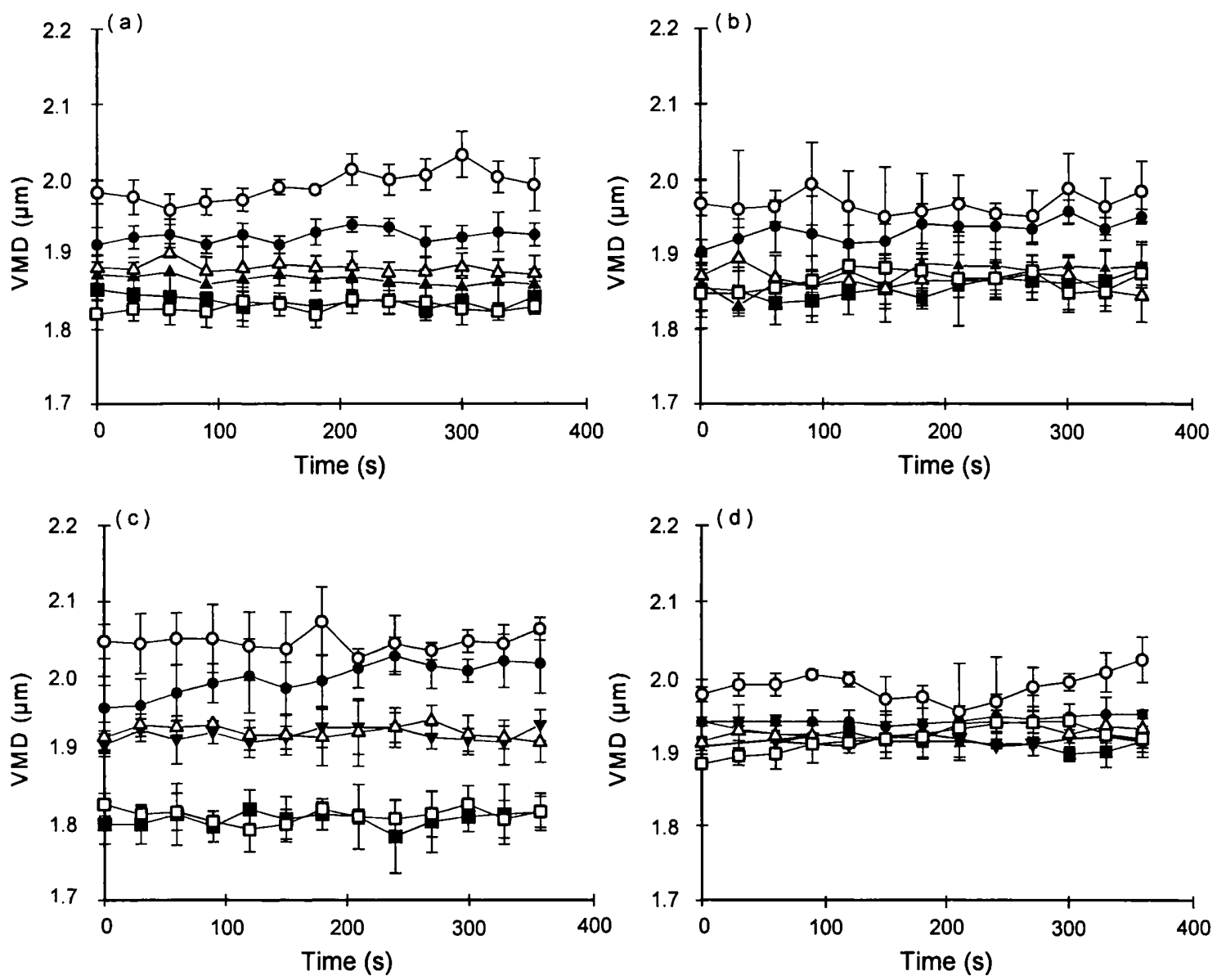

Figure 3.10 Mean VMD of droplets emitted from the Sidestream nebuliser with time, during nebulisation of (a) eggPC, $1 \mu \mathrm{m}$; (b) eggPC/chol, $1 \mu \mathrm{m}$; (c) eggPC, $5 \mu \mathrm{m}$; and (d) eggPC/chol, 5 $\mu \mathrm{m}$ liposomes with lipid concentrations of $(\square) 2.5 \mathrm{mg} / \mathrm{ml},(\square) 5 \mathrm{mg} / \mathrm{ml},(\Delta) 10 \mathrm{mg} / \mathrm{ml},(\Delta) 20$ $\mathrm{mg} / \mathrm{ml},(\bullet) 40 \mathrm{mg} / \mathrm{ml}$, and (०) $80 \mathrm{mg} / \mathrm{ml}$. [n = 3; $\pm \mathrm{sd}]$ 


\begin{tabular}{|c|c|c|c|}
\hline \multicolumn{4}{|c|}{$\begin{array}{l}\text { Table 3.2 Influence of the lipid concentration of nebulised liposomes on the mean } \\
\text { droplet VMD produced by various jet nebulisers. }[\mathrm{n}=3 ; \pm \mathrm{sd}]\end{array}$} \\
\hline \multirow[t]{2}{*}{ Nebuliser } & \multirow[t]{2}{*}{ Liposomes } & \multicolumn{2}{|c|}{$\begin{array}{l}\text { Mean increase in VMD with increase in } \\
\text { concentration from } 2.5 \text { to } 80 \mathrm{mg} / \mathrm{ml} \text {. }\end{array}$} \\
\hline & & $1 \mu \mathrm{m}$ & $5 \mu \mathrm{m}$ \\
\hline \multirow{2}{*}{ Cirrus } & eggPC/chol (1:1) & $40.0 \%( \pm 1.0)$ & $41.4 \%( \pm 3.0)$ \\
\hline & eggPC & $47.7 \%( \pm 0.7)$ & $52.8 \%( \pm 1.4)$ \\
\hline \multirow{2}{*}{ Pari-LC } & eggPC/chol (1:1) & $27.3 \%( \pm 0.5)$ & $27.9 \%( \pm 0.9)$ \\
\hline & eggPC & $26.4 \%( \pm 1.1)$ & $28.1 \%( \pm 1.7)$ \\
\hline \multirow{2}{*}{ Sidestream } & eggPC/chol (1:1) & $6.0 \%( \pm 0.8)$ & $3.8 \%( \pm 0.2)$ \\
\hline & eggPC & $8.4 \%( \pm 0.8)$ & $13.2 \%( \pm 1.2)$ \\
\hline
\end{tabular}

The extent of the concentration-induced droplet size change varied between the different nebulisers (table 3.2). The Sidestream nebuliser was least affected by a change in lipid concentration, producing a mean droplet size increase $( \pm \mathrm{sd})$ of $7.89( \pm 4.03) \%$ with an increase in lipid concentration from 2.5 to $80 \mathrm{mg} / \mathrm{ml}$ (fig. $3.11 \mathrm{e}, \mathrm{f}$ )(mean of each formulation). This compared with 26.91 ( \pm 1.25$) \%$ for the Pari-LC (fig. $3.11 \mathrm{c}, \mathrm{d}$ ), and $45.39( \pm 6.01) \%$ for the Cirrus (fig. $3.11 \mathrm{a}, \mathrm{b}$ ), over a similar concentration range. The response of the span values to the increased lipid concentration was less well defined than the droplet size changes (fig. 3.12). There was shown to be a marginal increase in the polydispersity of the aerosol droplets in response to an increase in the lipid concentration from 2.5 to $80 \mathrm{mg} / \mathrm{ml}$. However, this was only significant for the Cirrus and Pari-LC nebulisers $(\mathrm{p}<0.05)$. An increase in the polydispersity of nebulised aerosol droplets was previously demonstrated as the aerosolised droplet size reduced in response to an increased driving gas flow rate (section 3.4.1). However, the results of the lipid concentration experiments demonstrated a reverse trend, with an increase in the droplet size polydispersity evident as the droplet size rose in response to the increased lipid concentration (fig. 3.12). This indicates that the mechanisms producing the change in droplet size experienced as the flow rate, and the concentration were varied are different. 

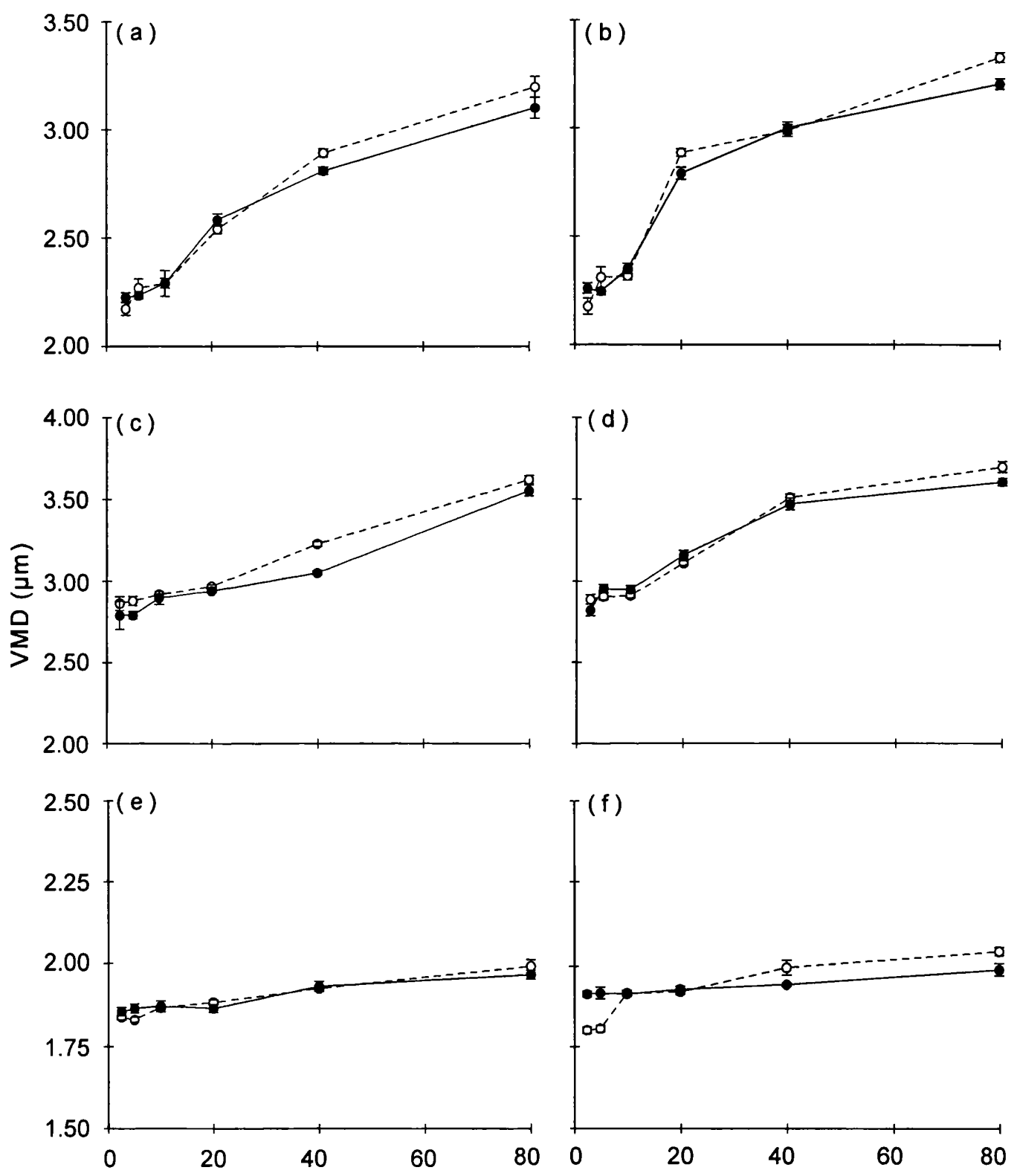

Lipid concentration $(\mathrm{mg} / \mathrm{ml})$

Figure 3.11 Change in the mean VMD of aerosol droplets produced from the nebulisation of $(0)$ eggPC and $(\bullet)$ eggPC/chol liposomes as the total lipid concentration was increased. (a) Cirrus, $5 \mu \mathrm{m}$ liposomes; (b) Cirrus, $1 \mu \mathrm{m}$ liposomes; (c) Pari-LC, $5 \mu \mathrm{m}$ liposomes; (d) Pari-LC, $1 \mu \mathrm{m}$ liposomes; (e) Sidestream, $5 \mu \mathrm{m}$ liposomes; (f) Sidestream, $1 \mu \mathrm{m}$ liposomes. $[\mathrm{n}=3 ; \pm$ sd] 

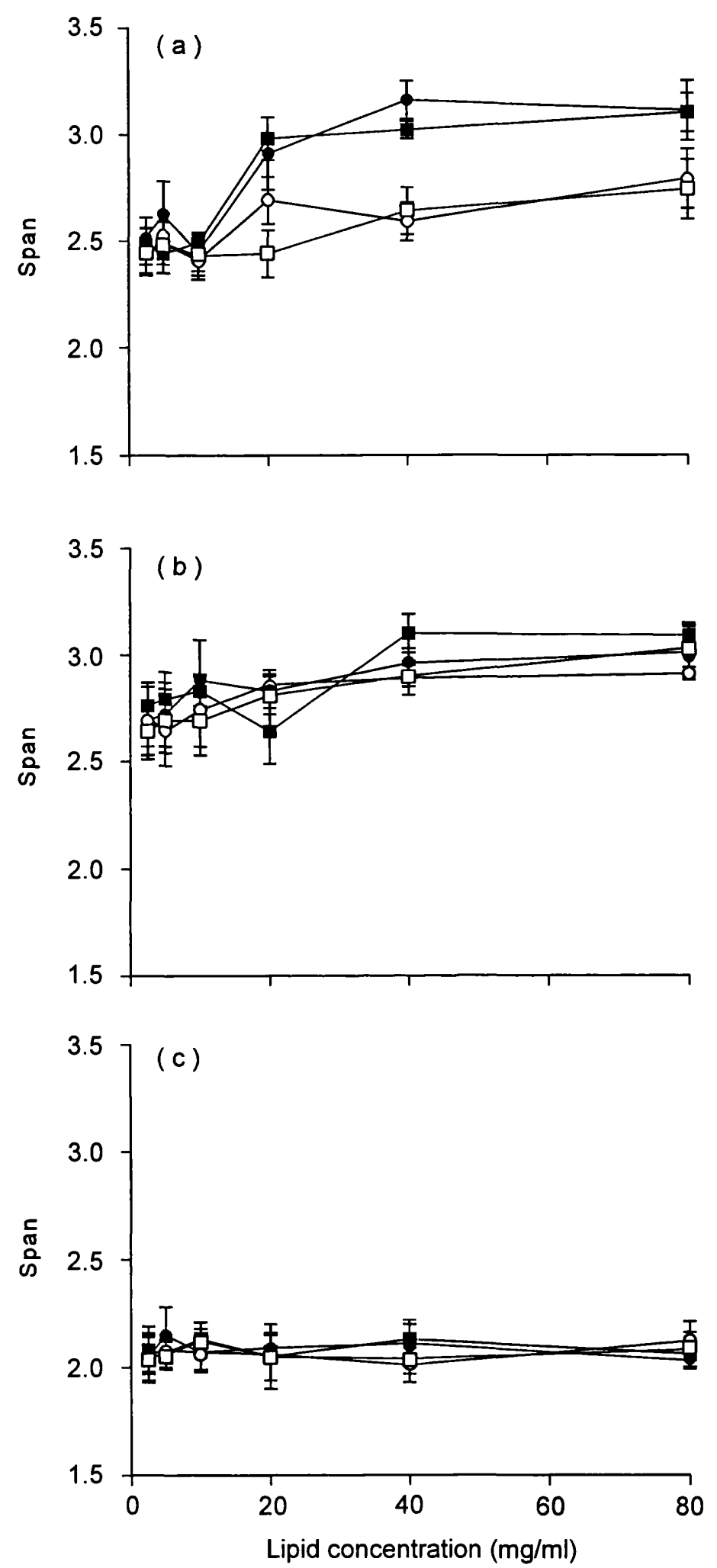

Figure 3.12 Influence of lipid concentration on the polydispersity of aerosol droplets

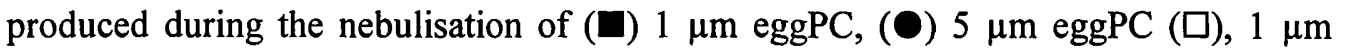
eggPC/chol (1:1) and (O) $5 \mu \mathrm{m}$ eggPC/chol (1:1) liposomes, using the (a) Cirrus (b) PariLC and ( c ) Sidestream nebulisers. [n=3; \pm sd] 
It would be useful to consider a model of the mechanism of droplet formation, so that the dependence of the nebulised droplet size on the lipid concentration of the liposome formulations may be explained. However, no general theory exists to explain the formation and break-up of droplets in nebulisers. Niven (1996) has stated that it would take a personal computer 28 years to quantitatively describe a spray produced over a millisecond period from a plain-jet orifice. Many of the droplet theories developed thus far are based on simple one-fluid atomizers. The situation in jet nebulisers is complicated by the fact that they are two-fluid devices, as high velocity gas is mixed with a moving liquid. Despite this, a model for droplet formation from simple nozzles was outlined by Byron (1987). The model proposed that liquid emerges from a nozzle as a hollow cone, which subsequently thins into sheets (fig. 3.13). The thickness of the sheet is determined by the design of the capillary that the fluid emerges from, the operating temperature, the rate of gas flow, and characteristics of the fluid, including viscosity, density, and surface tension. Turbulence in the fluid due to chaotic flow through the feed capillary, and disturbance by the driving gas, causes the sheet to ripple, leading to wave propagation, and stressing. This stress leads to holes being formed along the leading edge, which expand until adjacent holes coalesce.

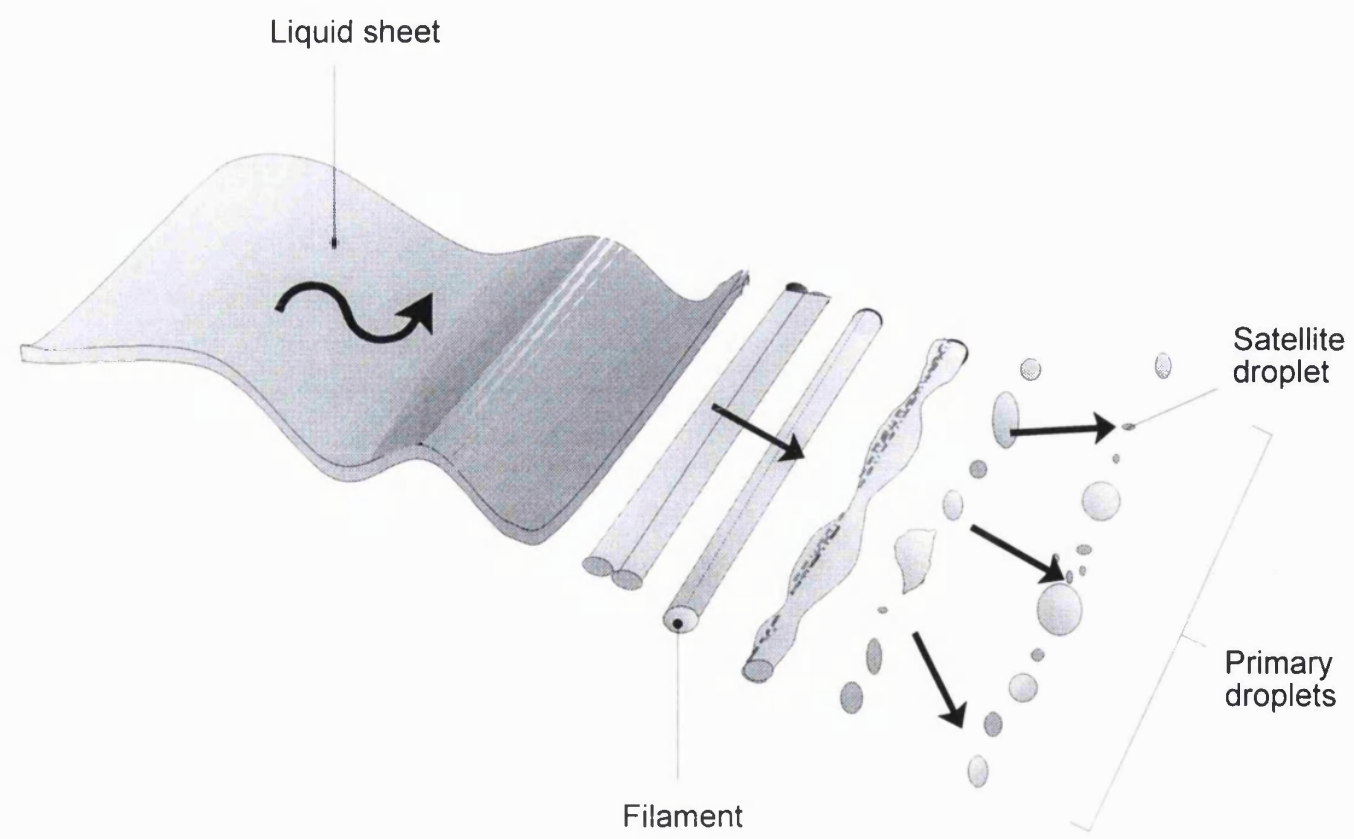

Figure 3.13 Schematic diagram of droplet formation from a nebuliser nozzle. 
Ultimately, a filament of fluid, approximately $10 \mu \mathrm{m}$ in diameter, is formed at each of the free edge surfaces. Thinning along each edge leads to the formation of weak spots, which cause the filament to break into droplets. These are termed "primary" droplets, and may constitute greater than $99 \%$ of the aerosol mass (Mercer, 1973). The primary droplets have a mean diameter that is greater than that of the filament from which they are formed, and as a consequence even the most carefully designed nozzle produces primary droplets greater than $20 \mu \mathrm{m}$ in diameter (Dombrowski and Munday, 1968). Only the satellite droplets, formed as adjacent large droplets draw away from the filament, fall within the respirable size range (Dombrowski and Munday, 1968).

Droplet formation in a jet nebuliser is aided by the shear energy of gas flow, employed to overcome the viscous and surface tension forces of the fluid. However, elevated surface tensions and viscosities impede distortion of the liquid surface, and as a consequence the onset of filaments, and resultant droplet formation is delayed. It is for this reason that fluids with elevated surface tensions and viscosities yield larger droplet sizes (Swift, 1980). However, the relationship between the fluid properties and the droplet size emitted from medical nebulisers remains unproven. Davis (1978) determined that droplet size increased as the concentration of propylene glycol solution was increased to $20 \% \mathrm{v} / \mathrm{v}$. However, the droplet size fell with further increases in concentration from $20 \%$ to $70 \% \mathrm{v} / \mathrm{v}$. Mercer (1981), stated that the spectrum of viscosities likely to be encountered in pharmaceutical solutions will have little impact on the primary droplet size emitted from jet nebulisers. However, the nebulisation time, and droplet size, of nebulised gentamicin has been shown to be greater than that of $0.9 \% \mathrm{w} / \mathrm{v}$ saline. This finding was attributed to the higher viscosity of the antibiotic solution (Newman et al. 1985). Such a result was subsequently confirmed in one nebuliser, but not in a second (Mallol, 1993). In contrast, Newman et al. (1987) could detect no correlation between the VMD of nebulised droplets, and the fluid characteristics. The relationship between nebulised droplet size and viscosity, already shown to be highly complex in simple solutions, is further complicated by the nonconventional properties of liposome dispersions. There have been no previous studies of the relationship between the concentration of liposomes and the nebulised droplet size they produce. The concentration of liposomes employed in pharmaceutical formulations may principally depend on the efficiency of drug entrapment, and the required dose. For this reason, a wide variety of liposome concentrations have been employed in aerosol studies. 
Chapter 2 demonstrated that there is a significant increase in viscosity as the lipid concentration of liposome formulations is increased over the range 2.5 to $80 \mathrm{mg} / \mathrm{ml}$. The rise in droplet size in the present experiment, with an increase in lipid concentration, may therefore be attributed to an increase in the viscosity of the liposomes. This is supported by the fact that there was a small increase in the emitted droplet size, between the initial and final three droplet size measurements, for each nebuliser studied (fig. 3.14). Though this was not statistically significant, there was clearly a trend towards an increased droplet size as nebulisation proceeded. Once again, the extent of the rise in VMD was found to be dependent on the nebuliser studied, with the Cirrus nebuliser yielding the largest increase $(2.1 \%)$, and the Sidestream the smallest $(0.71 \%)$. Such a phenomenon may be attributed to an increase in liposome viscosity as nebulisation proceeded. Solvent vapour saturates the driving gas as it leaves the nebuliser (Ferron et al., 1976), causing a marked fall in temperature (Clay et al., 1983b; Taylor et al., 1992), and a rise in the concentration of solute in the residual fluid (Ferron et al., 1976). This leads to an increase in the viscosity of the liposomes which may, as discussed above, cause an associated rise in the emitted droplet size. The Cirrus nebuliser has been shown to be more susceptible to changes in viscosity (table 3.2), and hence yielded the largest rise in droplet size during nebulisation. Conversely, the Sidestream nebuliser is capable of minimising such viscosity effects.

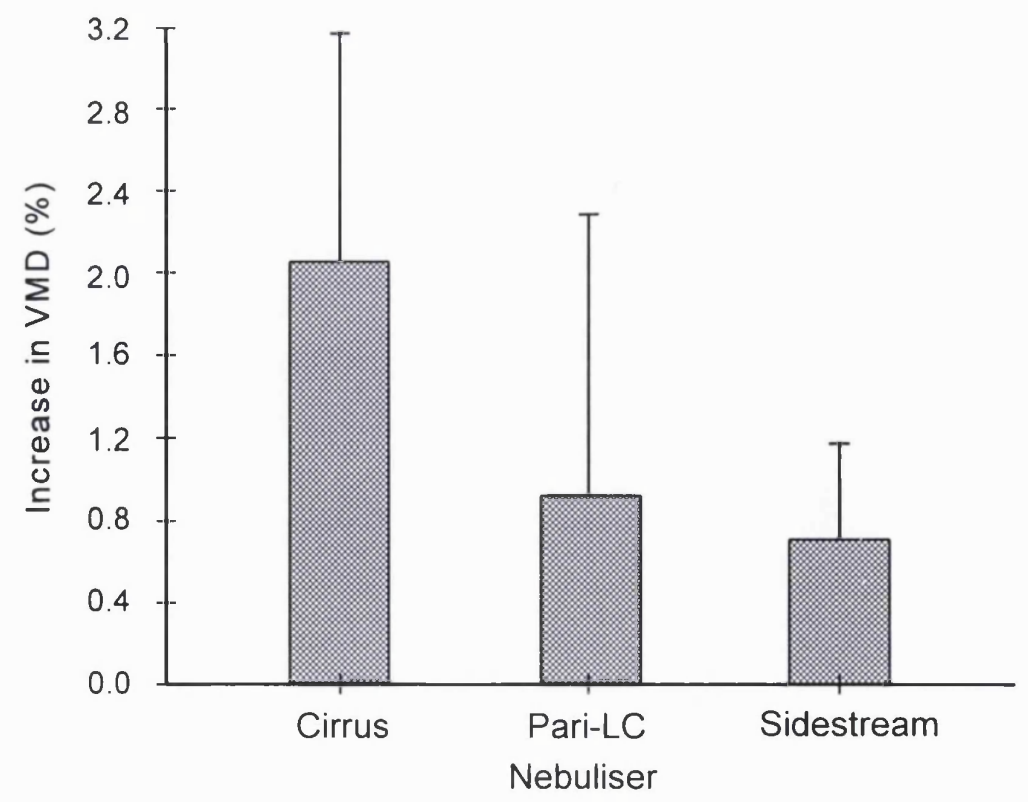

Figure 3.14 Increase in the mean droplet size (VMD) between the initial 90 seconds, and final 90 seconds of nebulisation, for the Cirrus, Pari-LC and Sidestream nebulisers. $[\mathrm{n}=3 ; \pm \mathrm{sd}]$. 
Further to the viscosity effects on droplet size, there may be effects mediated by a change in surface tension (Davis et al., 1978). However, Chapter 2 demonstrated that there was no significant change in the surface tension of the liposomes evident over the range of lipid concentrations studied in the present experiment. Furthermore, the effects of viscosity on nebulisation are more pronounced than the effects of surface tension (McCallion et al., 1995). As such, the change in nebulised droplet size, induced by an increase in lipid concentration, may be attributable to changes in viscosity, and not surface tension. However, it should be noted that the present results conflict with a recent report which demonstrated an inverse relationship between solution viscosity and droplet size, in two jet nebulisers, including the Pari-LC, over a similar viscosity range (McCallion and Patel, 1996). This suggests that properties of the liposomes themselves may be responsible for the observed increase in droplet size with elevated concentration. Interpretation of the results of the lipid concentration study is problematic, as the liposome systems are not simple onephase fluids. Each of the previous studies of the effects of the physicochemical properties of nebuliser fluids have involved simple solutions. However, liposomal dispersions possess properties that approximate to semi-solid suspensions. They have been shown to be delivered from nebulisers with sufficient stability to provide local sustained release of entrapped hydrophilic materials (Barker et al., 1994), yet, they are deformable to the extent that they may become significantly reduced in size during aerosolisation (Taylor et al., 1990b; Niven et al., 1991a).

Several studies have demonstrated that suspensions yield significantly different droplet sizes, when atomized, to those given by simple solutions. A study of an MDI system determined that a change in formulation from solution to suspension yielded a $95 \%$ increase in droplet size (Dalby and Byron, 1988). This was attributed to aggregation of the suspended particles, present as multiple inclusions within the aerosolised droplets. A similar process may be envisaged for nebulised liposome formulations. Stöber (1972) previously demonstrated the aggregation of particles during aerosolisation of latex sphere suspensions. These results were supported by McCallion et al. (1996), in a study of the nebulisation of latex spheres ranging in size from 0.605 to $11.9 \mu \mathrm{m}$. However, unlike the present case, the latter study detected no correlation between the concentration of the spheres and the emitted droplet size. However, this may be explained by their more dilute nature $(0.1 \%$ and $1 \% \mathrm{w} / \mathrm{v})$. 
Although used less commonly than solutions, a number of suspension formulations are available for nebulisation (eg. Pulmicort Respules ${ }^{\odot}$ suspension, Astra Pharmaceuticals). A suspension-containing aerosol, such as that produced from nebulised liposomes, may have a mean droplet size that is no smaller than that of the suspended particles, unless there is retention, or damage, of the dispersed particles within the residual fluid. In fact, the mean VMD of the nebulised droplets is likely to be significantly larger than that of the suspended particles, as a result of particle aggregation, and incomplete evaporation of the continuous phase. This is supported by a MDI study which observed a $57 \%$ reduction in respirable fraction, as the concentration of disodium fluoroscein suspension was increased from 0.1 to $2 \% \mathrm{w} / \mathrm{v}$ (Byron et al. 1989). This was attributed to an increase in the number of aggregates included within the aerosolised droplets, as the suspended particle concentration was increased. It must be borne in mind, that the MDI provides a poor comparison to nebulised systems, as the volatile solvent used in an MDI evaporates completely upon emission. Consequently, particle aggregation may have more pronounced effects. Nevertheless, aggregation-induced increases in droplet size may also occur during the nebulisation of liposomes. Liposome aggregation is a well documented phenomenon (Wong and Thompson, 1982), and its occurrence in the more concentrated samples may have led to the observed rise in droplet size. It may be hypothesised that the minimum size of satellite droplets, which are formed from filament break-up, will be increased by the presence of liposome aggregates within the fluid.

When a suspension is nebulised, the number of particles per droplet, and therefore the droplet size, will depend on the size of the aerosol droplet, and that of the particles (Raabe, 1968). However, the present study noted no difference in the droplet size produced from the 5 or $1 \mu \mathrm{m}$ mean size liposome formulations. This may have been due to liposomes larger than the mean droplet VMD becoming rapidly reduced in size during nebulisation, due to the shearing of bilayers by the high velocity gas jet (Taylor et al., 1990b; Niven et al., 1991a). As a consequence, the size of liposomes that were included in the aerosolised droplets may have been approximately equivalent for both the 5 and $1 \mu \mathrm{m}$ liposomes. This would cause the increase in droplet size, due to vesicle aggregation, to be equivalent for each liposome formulation. Alternatively, the reduced number of $5 \mu \mathrm{m}$ liposomes included within each droplet may have been compensated for by their greater vesicle size. Consequently, the $1 \mu \mathrm{m}$ liposomes may give an increased droplet size due to the formation 
of multiple vesicle aggregates. Whereas, the larger size of the $5 \mu \mathrm{m}$ liposomes limits their ability to form aggregates, but means the minimum droplet size that can be formed is larger. Thus, an equivalent rise in VMD, with increasing concentration, could be expected for each liposome size.

An increased droplet size may also have resulted from a phospholipid-mediated inhibition of evaporation from higher concentration droplets. Such a process would limit the shrinkage of droplets that occurs following generation into the dry gas jet, and a larger droplet size would be the result. Marks et al. (1983), suggested that the surface active nature of phospholipids may reduce water transport from a droplet, due to the formation of a condensed film at the air-water interface. However, such a barrier effect was likely to be very small, as even the most condensed phospholipid films exhibited minimal resistance to water transport (Marks et al., 1983). More significantly, the authors believed, were the likely bulk phase effects of liposomes. It was determined that phospholipid-saline dispersions yielded nebulised droplets that were less influenced by changes in humidity than the equivalent saline solution. This was attributed to an enhancement of droplet stability, brought about by the presence of liposomes in the liquid bulk. Liposomes form in an aqueous environment due to hydrogen bonding of the polar groupings of phospholipids to water molecules. Such an interaction may cause a barrier to the transport of water within the bulk phase. This would limit the evaporation occurring from the droplets, aerosolised into the relatively dry environment within the jet nebuliser chamber. More concentrated liposomes would therefore be predicted to provide a greater barrier to water transport, and thus maintain a larger droplet size, when dispersed in higher concentrations. The validity of this theory was tested by the study of an ultrasonic nebuliser (chapter 6). The air within an ultrasonic nebuliser is of high humidity due to the absence of gas flow. Therefore, an increase in droplet size tends to occur following atomisation. Hence the relationship between droplet size and lipid concentration may be very different between the two types of nebuliser.

The increase in droplet size with increasing lipid concentration may also have been due to the aforementioned shearing of liposome bilayers. Any process that reduces the amount of energy available to droplet formation may cause an increase in the primary droplet size. The greater number of bilayers present in the higher concentration samples, means a greater 
amount of atomisation energy may be expended in bilayer disruption, and consequently larger droplets would be formed. However, this hypothesis is not supported by the fact that the emitted droplet size produced by each nebuliser was independent of both the liposome size, and the bilayer phase state. It may be predicted that more energy would be expended disrupting the bilayers of liposomes which have a mean size greater than that of the aerosol droplets. Larger droplet sizes would therefore result from such a formulation. However, this was not the case. This disparity may be explained by consideration of the number of phospholipid bilayers in each formulation. The $1 \mu \mathrm{m}$ liposome formulations contain a greater number of vesicles. As such, the actual bilayer surface area exposed to the comminuting liquid front may be similar to that offered by the larger size liposomes. Consequently, the energy spent disrupting bilayers is constant, and hence the droplet size is unchanged, between each liposome size. The incorporation of cholesterol into liquidcrystalline state liposome bilayers results in an increase in the bilayer rigidity (Senior, 1987). As a result of this, eggPC/chol (1:1) liposomes are found to be more resistant than eggPC liposomes, to the shear forces associated with membrane extrusion and sonication (chapter 2). Therefore, cholesterol would be expected to cause an increase in the energy needed to disrupt bilayers during nebulisation. The inclusion of cholesterol in eggPC liposome bilayers would therefore yield a rise in the droplet size, if the absorbence of atomisation energy was indeed having demonstrable effects. However, this was not observed in the present study. A possible explanation is that fewer bilayers are disrupted when they are in the more rigid gel state. Therefore, a similar energy is absorbed by bilayer disruption during the nebulisation of liposomes with differing bilayer states.

The discrepancy between the extent of the rise in droplet size between different nebulisers, with increasing lipid concentration, is a consequence of differences in the design of each model. The size selective properties of the nebuliser baffles mean that the secondary aerosol may not reflect changes in the primary droplet size (O'Callaghan and Barry, 1997). Different nebuliser models, possessing characteristic baffling efficiencies, therefore produce different responses to changes in lipid concentration. It may be hypothesised that the Sidestream nebuliser contains baffles which compensate for the increase in the primary droplet size by increasing the number of large droplets that are filtered from the primary aerosol. As a result, the Sidestream minimises the lipid concentration effects on the emitted aerosol. This was further investigated in the study of aerosol output (section 3.4.3). 
It is significant to note that the Cirrus nebuliser gave the largest increase in emitted droplet size upon a change in the driving gas flow rate (section 3.4.1). This correlates with the fact that the Cirrus is most affected by a change in lipid concentration. It is therefore likely that the Cirrus nebuliser has an inefficient baffling system. Consequently, changes in the primary droplet size, resulting from variations in the lipid concentration, and/or driving gas flow rate, are reflected in the secondary aerosol size. However, the preceding study of the effects of driving gas flow rate (section 3.4.1) indicates that an increased baffling efficiency does not fully explain the ability to minimise lipid concentration-induced droplet size changes. The Pari-LC nebuliser was found to produce a rise in the mean droplet size which was approximately half that produced by the Sidestream, in response to a reducing gas flow rate. Such a result suggests that the Pari-LC nebuliser has more efficient baffles than the Sidestream. However, in the present experiment the droplet size produced by the Pari-LC was more sensitive to changes in lipid concentration, than that produced by the Sidestream.

The role of the nebuliser baffles in minimising lipid concentration effects was further investigated in the study of aerosol output (section 3.4.3). However, it is likely that additional nebuliser design factors influence the nebuliser response to changing fluid properties. These may include differences in the constituent part geometries and dimensions, local internal gas flow rates, and the incorporation of an open vent. Indeed, Davis (1978) concluded that the relationship between the physicochemical properties of a nebulised fluid and the droplet size was a complex function of the nebuliser design.

\subsubsection{The output of liposomal aerosols from jet nebulisers}

The results of section 3.4.2 demonstrated that the droplet size produced by nebulisation of liposomes using a variety of jet nebulisers, was increased as the total lipid concentration of the formulation increased. However, the Sidestream nebuliser minimised such concentration effects, compared with the other devices studied. It was postulated that the Sidestream nebuliser possessed highly efficient baffles that compensate for an increase in the primary aerosol droplet size by filtering out a greater proportion of the larger droplets. A consequence of maintaining a relatively constant droplet size over a wide range of lipid concentrations, due to baffling action, would therefore be a reduction in the total aerosol output produced over a fixed period, as a result of enhanced recycling of fluid. The aerosol output experiments of the present section investigated this. 


\begin{tabular}{|c|c|c|c|c|}
\hline \multirow{3}{*}{$\begin{array}{c}\text { Lipid } \\
\text { Concentration } \\
\text { (mg/ml) }\end{array}$} & \multicolumn{4}{|c|}{$\begin{array}{c}\text { Table 3.3 Output (\% of total mass added) of } \\
\text { eggPC/chol }(1: 1) \text { liposomes nebulised by two jet nebulisers. } \\
5 \mu \mathrm{m}[\mathrm{n}=3, \pm \mathrm{sd}] \text {, and } 1 \mu \mathrm{m} \text { mean size liposomes }[\mathrm{n}=4, \pm \mathrm{sd}]\end{array}$} \\
\hline & \multicolumn{2}{|c|}{ Pari-LC } & \multicolumn{2}{|c|}{ Sidestream } \\
\hline & $1 \mu \mathrm{m}$ & $5 \mu \mathrm{m}$ & $1 \mu \mathrm{m}$ & $5 \mu \mathrm{m}$ \\
\hline 5 & $\begin{array}{c}74.9 \\
( \pm 4.5)\end{array}$ & $\begin{array}{c}74.9 \\
( \pm 7.0)\end{array}$ & $\begin{array}{c}84.8 \\
( \pm 1.8)\end{array}$ & $\begin{array}{c}81.6 \\
( \pm 1.1)\end{array}$ \\
\hline 10 & $\begin{array}{c}72.6 \\
( \pm 9.5)\end{array}$ & $\begin{array}{c}72.4 \\
( \pm 6.4)\end{array}$ & $\begin{array}{c}82.4 \\
( \pm 3.3)\end{array}$ & $\begin{array}{c}83.8 \\
( \pm 5.1)\end{array}$ \\
\hline 20 & $\begin{array}{c}77.1 \\
( \pm 12.7)\end{array}$ & $\begin{array}{c}64.0 \\
( \pm 14.3)\end{array}$ & $\begin{array}{c}83.0 \\
( \pm 4.8)\end{array}$ & $\begin{array}{c}77.1 \\
( \pm 5.4)\end{array}$ \\
\hline 40 & $\begin{array}{c}71.3 \\
( \pm 12.0)\end{array}$ & $\begin{array}{c}65.6 \\
( \pm 12.6)\end{array}$ & $\begin{array}{c}83.4 \\
( \pm 4.4)\end{array}$ & $\begin{array}{c}79.6 \\
( \pm 3.0)\end{array}$ \\
\hline 60 & $\begin{array}{c}71.7 \\
( \pm 15.3)\end{array}$ & $\begin{array}{c}70.0 \\
( \pm 9.8)\end{array}$ & $\begin{array}{c}79.7 \\
( \pm 8.7)\end{array}$ & $\begin{array}{c}52.4 \\
( \pm 3.9)\end{array}$ \\
\hline 80 & $\begin{array}{c}67.4 \\
( \pm 15.0)\end{array}$ & $\begin{array}{c}47.2 \\
( \pm 16.0)\end{array}$ & $\begin{array}{c}73.3 \\
( \pm 7.7)\end{array}$ & $\begin{array}{c}51.2 \\
( \pm 9.5)\end{array}$ \\
\hline
\end{tabular}

The output of fluid from the Pari-LC nebuliser ranged from 47.2 to $77.1 \%$ of the total liposome mass added (table 3.3). Similarly, output from the Sidestream ranged from 51.2 to $84.8 \%$. The results indicate that each nebuliser produced a similar output of fluid during nebulisation of liposomes with a range of lipid concentrations $(p<0.05)$. However, the results also demonstrate that the Sidestream produced a more consistent output than the Pari-LC nebuliser, as the mean standard deviation between repeated measurements was approximately $50 \%$ less for the Sidestream. This supports the manufacturer's claim that the Sidestream device gives an increased reproducibility of output, compared with other models. This is apparently achieved by reducing the number of tooling parts used during manufacture. It must be borne in mind that the results may not reflect the true efficiency of the output of liposomes, as the output was determined by mass measurements, and took no account of the concentration of liposomes that remained within the nebuliser chamber. 


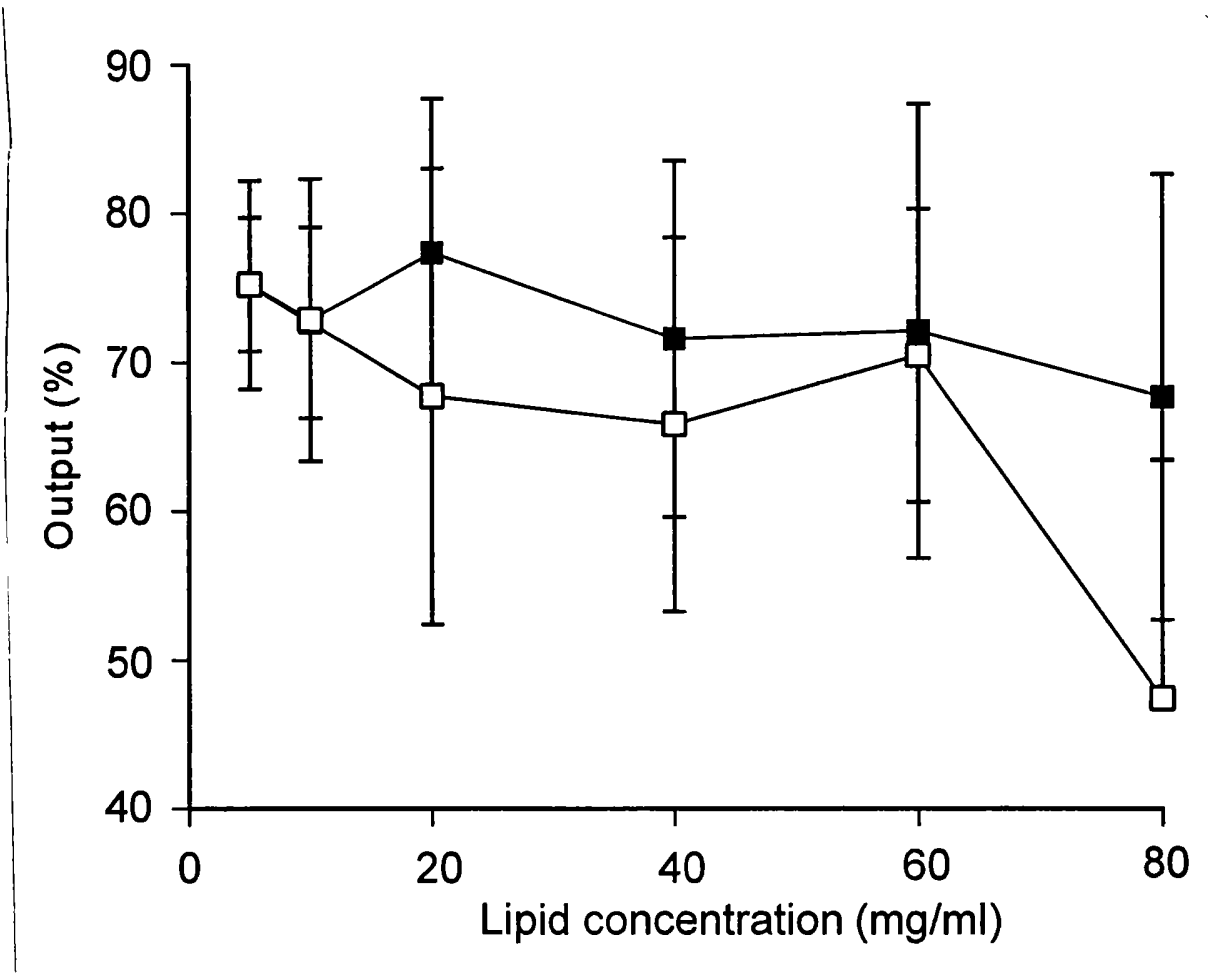

Figure 3.15 The output of nebulised (घ) $1 \mu \mathrm{m}$ and ( $\square$ ) $5 \mu \mathrm{m}$ mean size eggPC/chol (1:1) liposomes from the Pari-LC nebuliser as a function of the lipid concentration. $[ \pm$ sd; $n=4(\square), n=3(\square)]$

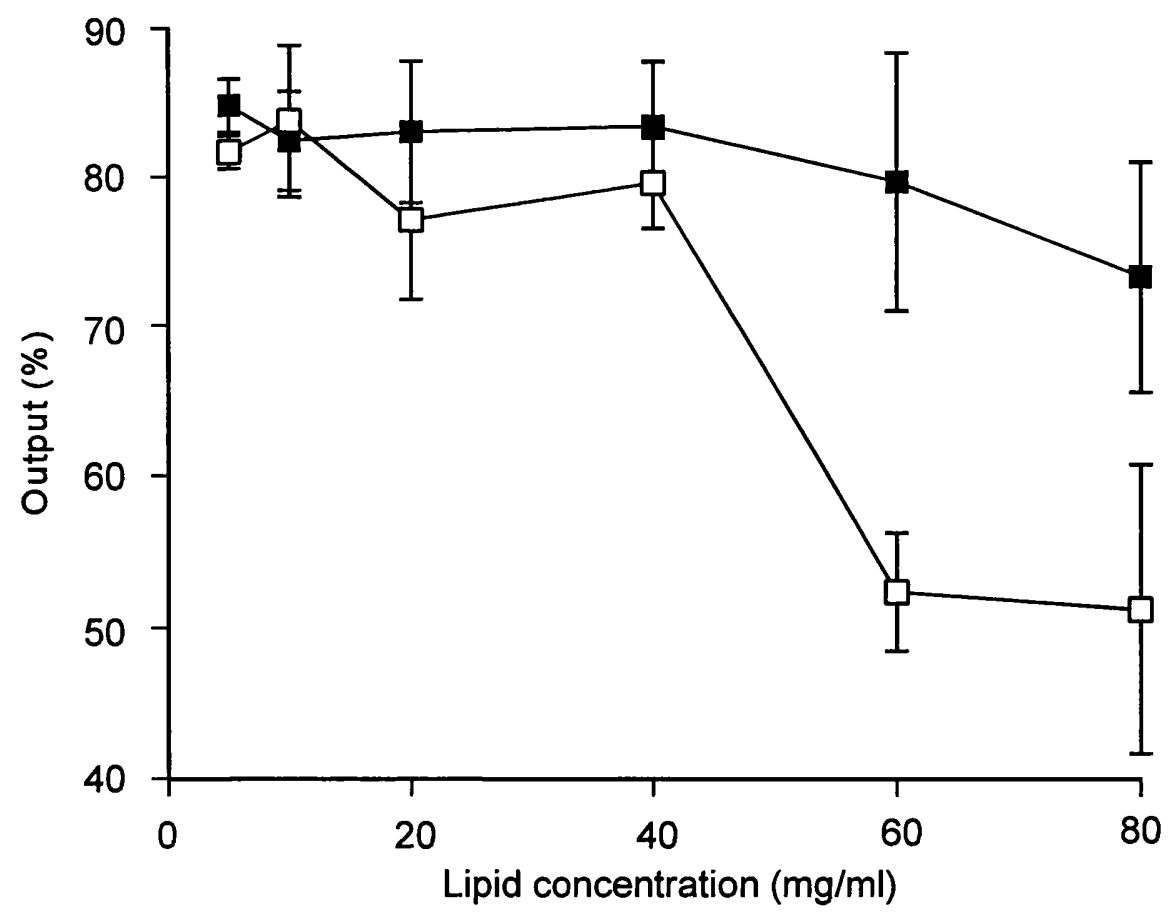

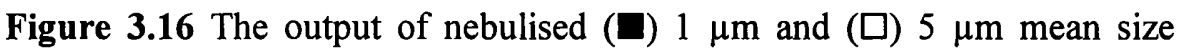
eggPC/chol (1:1) liposomes from the Sidestream nebuliser as a function of the lipid concentration. $[ \pm \mathrm{sd} ; \mathrm{n}=4(\square), \mathrm{n}=3$ (प)] 
An increase in the lipid concentration of the nebulised fluids from 5 to $80 \mathrm{mg} / \mathrm{ml}$ produced approximate falls in output of $10 \%$, and $37 \%$ for the 1 and $5 \mu \mathrm{m}$ liposomes nebulised with the Pari-LC (fig. 3.15). A similar change in concentration yielded a $14 \%$, and a $37 \%$ reduction in output for each liposome size nebulised with the Sidestream (fig. 3.16). The fall in output proved significant for both of liposome formulations nebulised with the Sidestream, but only for the $5 \mu \mathrm{m}$ mean size liposomes nebulised with the Pari-LC $(p<0.05)$. The results therefore go some way in supporting the hypothesis that the Sidestream nebuliser minimises the droplet size effects of an increased lipid concentration by filtering out a larger proportion of droplets from the primary aerosol. Therefore, despite an increase in the primary aerosol size, the size of the secondary aerosol emitted from the Sidestream is less affected by an increase in the lipid concentration. This also supports the finding that the droplet size produced by the Pari-LC nebuliser is influenced by a rise in lipid concentration to a greater extent. Although the baffles of the Pari-LC significantly reduce the output of the more concentrated $5 \mu \mathrm{m}$ liposomes, the filtering does not prevent a rise in the emitted droplet size. Indeed, the Pari-LC baffles had no significant influence on the output of aerosol when the concentration of smaller mean size liposomes was increased, and this co-coincides with a significantly greater increase in the emitted droplet size, compared with the Sidestream.

The Sidestream nebuliser may not resist the lipid concentration-induced droplet size changes through the filtering action of its baffles alone. Other factors, such as the diameter and geometry of capillaries, jets, and other internal structures, may additionally be involved. For instance, it may be due to the open vent design of the nebuliser. Multiple aggregates within the droplets may form at higher concentrations, which inhibit evaporation from the droplets. This is significant, as the open vent design of the Sidestream nebuliser has been implicated in ensuring that the nebuliser produces a small droplet size (O'Callaghan and Barry, 1997). Supplementary gas drawn through the vent results in droplet shrinkage due to evaporation. If such evaporation is inhibited by the presence of liposomes, the droplets will fail to reduce in size sufficiently, and hence become retained within the device. However, the baffles will still be responsible for filtering out the larger droplets that are so produced. Alternatively, the reduced aerosol output at higher lipid concentrations may have been due to a reduced tendency for the Sidestream nebuliser to damage liposome bilayers. A consequence of this would be the fact that the liposomes with a mean size larger than the 
aerosol size become retained within the nebuliser. There is likely to be a greater number of liposomes that are larger than the mean aerosol size in the higher concentration samples. This would lead to an increased retention of liposomes at higher concentrations, as the larger liposomes become aerosolised in larger droplets (Raabe, 1968). Therefore, a reduced aerosol output is produced at elevated lipid concentrations. This theory is further supported by the observation that the $5 \mu \mathrm{m}$ liposomes yielded a greater reduction in output as the liposome concentration became raised, compared with the $1 \mu \mathrm{m}$ formulation (fig. 3.16). However, this is also a reflection of the efficient nature of the Sidestream baffles. The nebuliser is able to filter out a significantly large proportion of the droplets which contain larger liposomes, thus restricting them to the nebuliser chamber. The concentrating effect of nebulisation upon differently sized liposomes was investigated in the experiments detailed in section 3.4.4.

It is clear that the Sidestream maintains relatively unchanged aerosol size characteristics over a range of lipid concentrations, at least in part through a reduction in aerosol output. This may be achieved through an efficient baffling action, or alternatively through a reduced tendency for droplet evaporation. Yet the mean aerosol output from the Sidestream nebuliser was similar to that produced by the Pari-LC. This was in addition to the fact that the Sidestream produced a smaller mean droplet size, a reduced spread of sizes, and a reduced nebulisation time. The Sidestream is thus likely to prove the more versatile of the two nebulisers when used to aerosolise liposomes to the alveolar region of the lung. However, the present results do not take into account the impact that the breath -assisted, open vent design of the Pari-LC may have on the aerosol output. Such a design prolongs nebulisation times, but reduces aerosol losses during expiration. The results also fail to consider the retention of liposomes within the nebuliser, or indeed their stability, during nebulisation.

The aerosol output rate from both the Pari-LC and Sidestream nebulisers was reduced in the latter half of nebulisation, prior to sputtering for each of the formulations studied (fig. 3.17 to 3.20). This was significant for each of the formulations studied in the Sidestream nebuliser (excepting the 80 and $40 \mathrm{mg} / \mathrm{ml}, 5 \mu \mathrm{m}$ liposomes), and for the 20,10 , and 5 $\mathrm{mg} / \mathrm{ml} 1 \mu \mathrm{m}$ liposomes, and the 10 , and $5 \mathrm{mg} / \mathrm{ml} 5 \mu \mathrm{m}$ liposomes, when nebulised with the Pari-LC $(\mathrm{p}<0.05)$. 


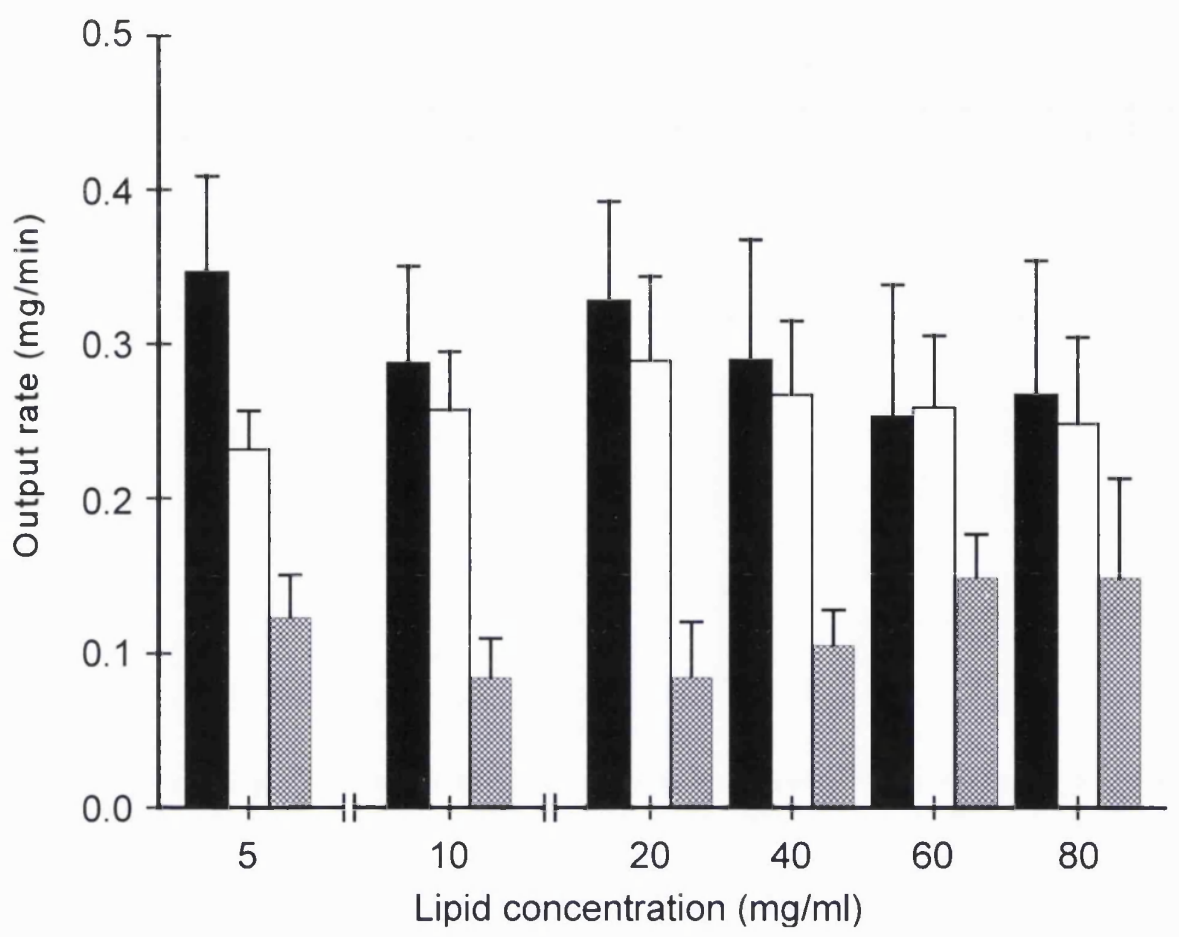

Figure 3.17 Rate of fluid output from the Pari-LC nebuliser during nebulisation of $1 \mu \mathrm{m}$ mean size eggPC/chol (1:1) liposomes. Output rate displayed as a function of time - ( $\square) 0$ to $50 \%$ of sputtering time, () 50 to $100 \%$ of sputtering time, $(\otimes)$ initial two minutes of sputtering. $[ \pm s d ; n=4]$

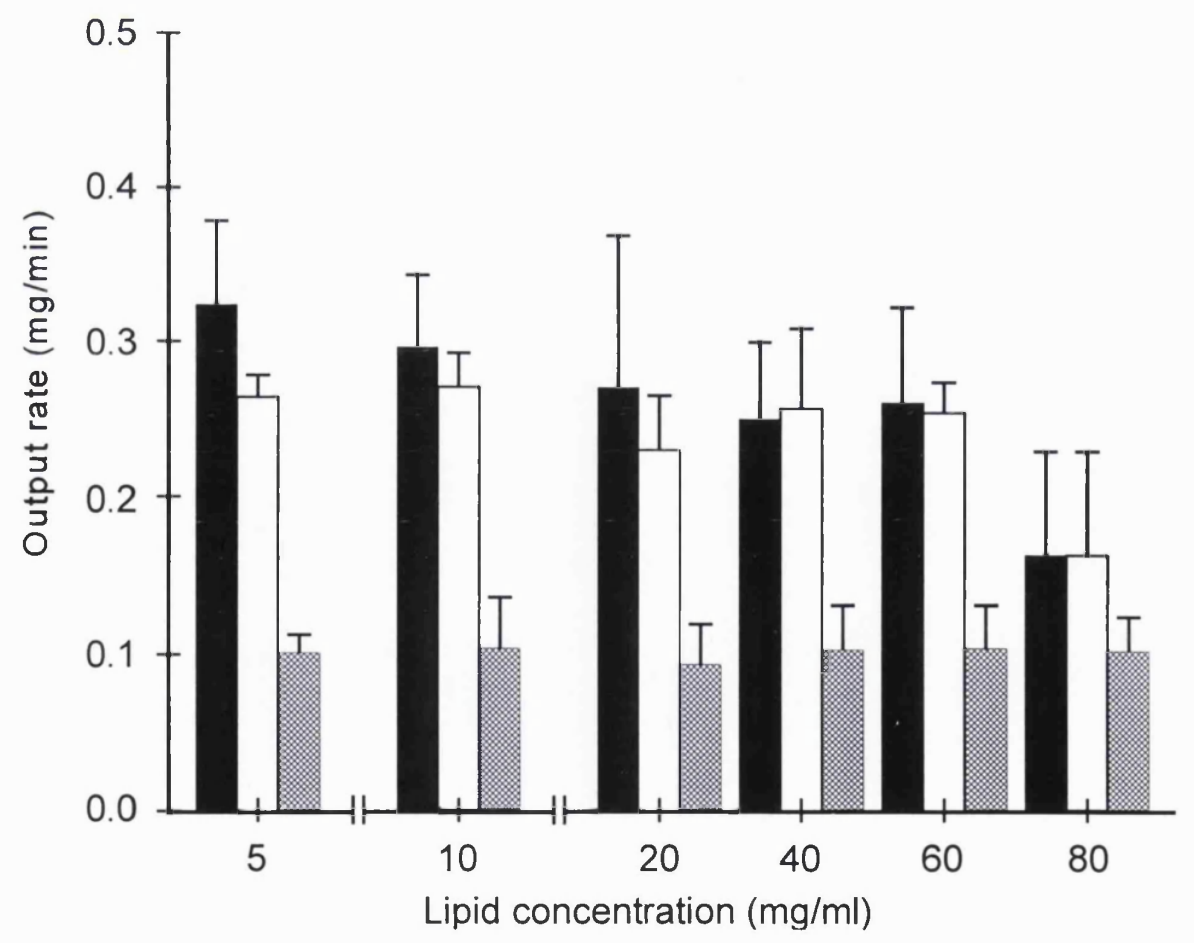

Figure 3.18 Rate of fluid output from the Pari-LC nebuliser during nebulisation of $5 \mu \mathrm{m}$ mean size

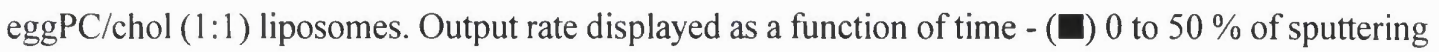
time, ( $\square$ ) 50 to $100 \%$ of sputtering time, $(\otimes)$ initial two minutes of sputtering. [ $\pm s d ; n=3$ ] 


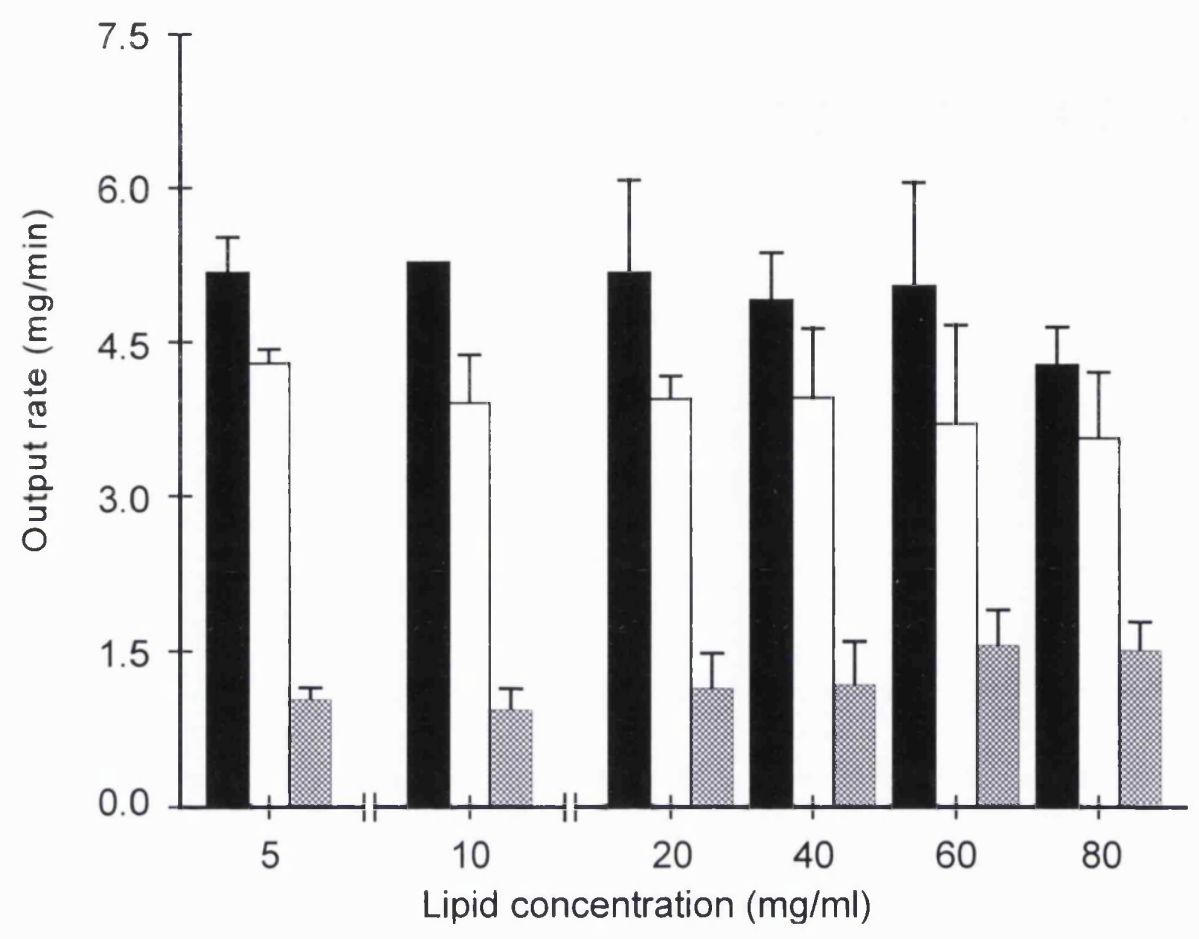

Figure 3.19 Rate of fluid output from the Sidestream nebuliser during nebulisation of $1 \mu \mathrm{m}$ mean size eggPC/chol (1:1) liposomes. Output rate displayed as a function of time - (-) 0 to $50 \%$ of sputtering time, $(\square) 50$ to $100 \%$ of sputtering time, $(\nabla)$ initial two minutes of sputtering. $[ \pm s d ; n=4]$

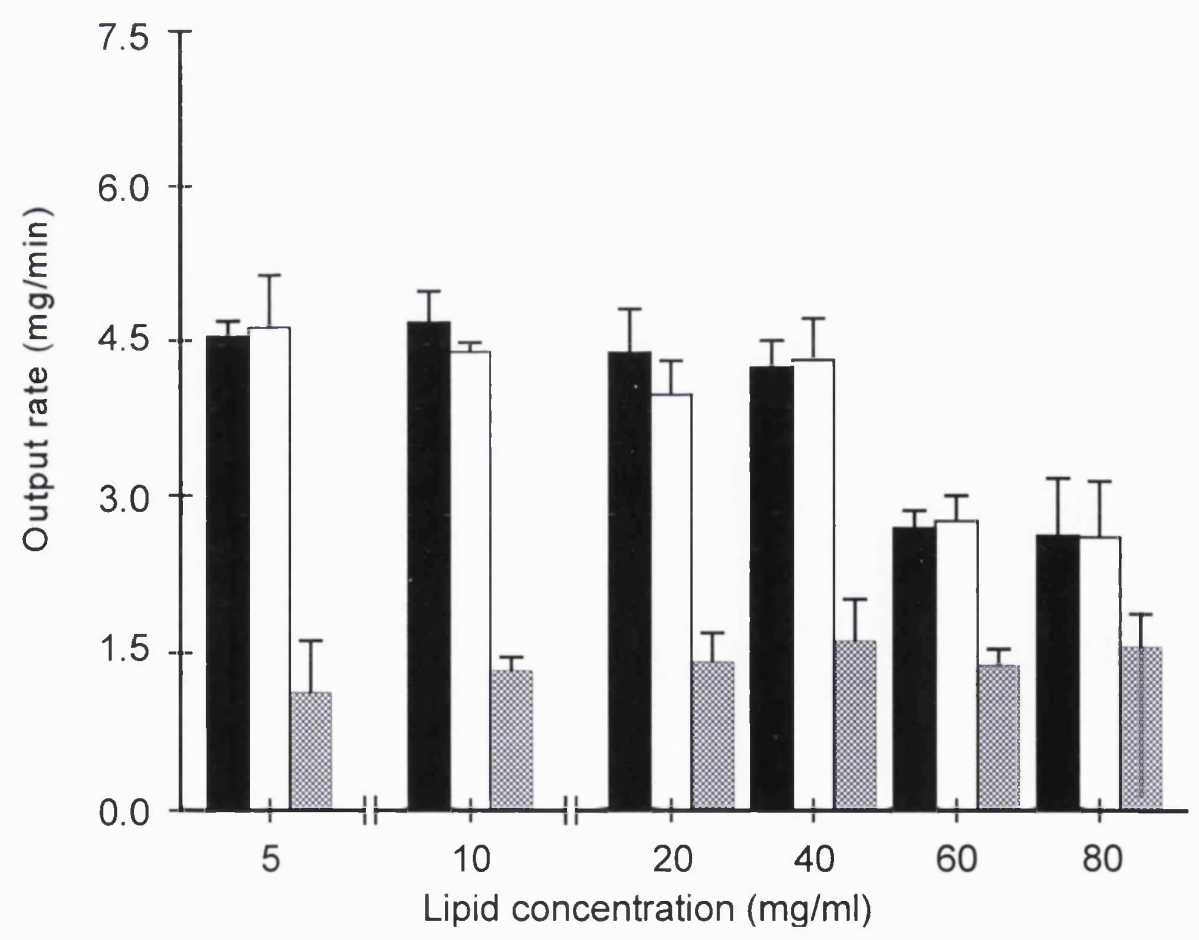

Figure 3.20 Rate of fluid output from the Sidestream nebuliser during nebulisation of $5 \mu \mathrm{m}$ mean size eggPC/chol (1:1) liposomes. Output rate displayed as a function of time - ( 0 ) to $50 \%$ of

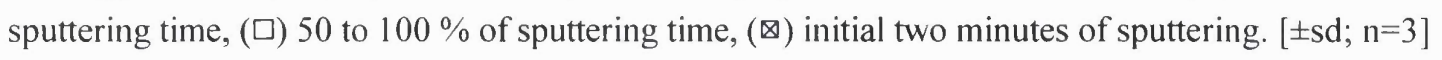


A fall in the residual fluid temperature, and a rise in the residual liposome concentration occurs during nebulisation, due to the evaporation of solvent (Ferron et al., 1976; Taylor et al., 1992). The fluid viscosity rises, and hence a reduction in the aerosol output occurs (eg. Clay et al., 1982). A liposome-containing droplet that becomes deposited on an internal structure within a nebuliser must return to the base of the fluid feed capillaries before it can be reatomised. The surface tension and viscosity of the nebulised fluid will, to a certain extent, determine the ease at which this occurs. Higher viscosity samples resist flow, and thus have a greater tendency to become retained on the nebuliser walls. In so doing, they become unavailable for reatomisation, and hence the aerosol output rate becomes reduced. The design of the plastic walls, and the geometries and construction of internal structures, may also determine the extent of the retention of impacted droplets, and hence the efficiency of output, from a particular device. Constituent parts which discourage gravitational flow of droplets to the nebuliser chamber will reduce the aerosol output. Air becomes drawn into the liquid feed capillaries at an earlier stage of nebulisation with higher viscosity fluids, with the result that the aerosol output may become less efficient. This may also cause the mean droplet size, and the spread of sizes (span) to fluctuate, and the aerosol output to be reduced, in a similar fashion to that observed during the sputtering phase of nebulisation. Each of these factors may explain the fact that there was a similar reduction in output from the Pari-LC and Sidestream devices when nebulising increased lipid concentration samples, despite the fact that larger droplets were emitted from the Pari-LC nebuliser.

The difference in aerosol output between the initial and latter stages of liposome nebulisation was reduced for the formulations which had an increased lipid concentration (that is 80,60 , and $40 \mathrm{mg} / \mathrm{ml}$ )(fig. 3.17 to 3.20 ). Concentration and temperature changes during nebulisation are likely to have a smaller relative effect for such high viscosity fluids. In addition, the increased viscosity of these formulations would have reduced the liposomal aerosol output rate in the earlier stage of nebulisation. This results in a prolonged nebulisation time, which may contribute to the reduced aerosol output experienced by the high concentration formulations. An increase in viscosity has been shown to reduce the aerosol output from nebulisers, due to an increased resistance of the nebulised fluid to disruption (Davis, 1978; Clay et al., 1982; McCallion et al., 1995). As a consequence fewer aerosol droplets, with a larger VMD, become aerosolised, and hence the total aerosol output 
is reduced. McCallion et al. (1995) noted that higher viscosity nebuliser fluids required longer to nebulise to dryness, and gave a greater volume of residual fluid at the end of nebulisation. In the present study the liposome dispersions were nebulised for a fixed period of time, calculated as the time taken for the aerosol output to cease from the $10 \mathrm{mg} / \mathrm{ml}$ lipid concentration formulation. Therefore, because the higher concentration samples were more viscous (chapter 2), produced larger droplets, and consequently took longer to nebulise, the volume remaining once nebulisation was halted was greater. As a result the total aerosol output was reduced, over the fixed time period, for the more concentrated liposome formulations. This fact was also reflected by a less marked fall in the aerosol output, between the initial and latter stages of nebulisation, for the more concentrated liposomes (fig. 3.17 to 3.20 ).

The concentration of dispersed, or dissolved materials, that occurs within residual nebuliser fluids during nebulisation, means that it is not necessarily the case that the reduced aerosol output observed in the latter stages of nebulisation would lead to a reduction in the number of respirable droplets that contain liposomes. It is instead possible, that as the residual liposome concentration became increased, in response to the evaporative losses and liposome retention, that the aerosol droplets contained a greater number of liposomes. Therefore, despite the fact that the aerosol output becomes reduced in the latter stages of nebulisation, the output of aerosolised liposomes from each of the jet nebulisers may in reality become increased.

The results indicated that the jet nebulisers produced reduced aerosol outputs from liposomes with a larger mean size. For each of the lipid concentrations tested (except for the $10 \mathrm{mg} / \mathrm{ml}$ liposomes nebulised with the Sidestream) the $5 \mu \mathrm{m}$ liposomes produced a reduced aerosol output, compared with the equivalent $1 \mu \mathrm{m}$ liposomes. However, the difference between the two formulations was only statistically significant for the 80 and 40 $\mathrm{mg} / \mathrm{ml}$ formulations nebulised with the Sidestream $(\mathrm{p}<0.05)$. Despite this, the results suggested that a size selective process may have been occurring towards the release of liposomes from each nebuliser. It may be proposed that larger liposomes are excluded from droplets that are emitted from the device, and thus a greater proportion of the $5 \mu \mathrm{m}$ liposome formulation became retained. This may be attributed to the fact that the mean droplet size produced from each nebuliser is about $2 \mu \mathrm{m}$. Consequently, a greater number 
relatively rigid eggPC/chol (1:1) liposomes within the $5 \mu \mathrm{m}$ mean size formulation were excluded from the secondary aerosol droplets that leave each device. The polydisperse nature of the liposomal aerosols produced from jet nebulisers means that a significant fraction of the large liposomes in the $5 \mu \mathrm{m}$ mean size formulations become aerosolised. In addition, a significant amount of liposome size reduction may occur. However, the greater majority of the large liposomes were included in the larger droplets of the primary aerosol that became recycled within the device. As a consequence, the larger liposomes tended to become retained within the nebuliser chamber, causing the total aerosol output to be reduced. Reducing the mean liposome size to $1 \mu \mathrm{m}$ ensured that majority of the liposomes became aerosolised, and consequently the aerosol output was relatively increased. The PariLC nebuliser has been shown to generate liposomal aerosols containing a greater spread of droplet sizes (as determined by the span value), compared with the Sidestream device (chapter 3). As a result, a greater proportion of the $5 \mu \mathrm{m}$ liposomes were emitted, and therefore the difference in output between the two liposome sizes was reduced compared with the Sidestream device (fig. 3.15 and 3.16). Retention of the larger liposomes within each nebuliser would increase the duration of their exposure to shear forces within the device, and creates the possibility of significant bilayer instability. This is further explored in the study of the residual liposomes that remain within the fluid in the nebuliser chamber following operation (chapter 4).

\subsubsection{Concentration of liposomes during nebulisation}

A method for the determination of the concentration of materials in residual nebuliser fluids was employed which allowed changes in the concentration of dissolved or dispersed materials induced by jet nebulisation to be determined. Using this method, the process of jet nebulisation was shown to significantly increase the concentration of a simple sodium chloride solution, which remained within the fluid reservoir of each nebuliser during operation (table 3.4 ). The increase in concentration ranged from approximately $14 \%$ in the Pari-LC nebuliser, to approximately $43 \%$ in the Respirgard II, and was attributable to the flow of high velocity, unsaturated gas through the nebuliser, which caused a significant loss of water vapour due to evaporation. Such evaporative losses are exacerbated by the large surface area presented by the recycling primary droplets. Consequently, nebulised fluids become increasingly more concentrated as nebulisation proceeds (Ferron et al., 1976). Similar findings were noted by Stapleton and Finlay (1995), with an $11 \%$ increase in the 
concentration of salbutamol sulphate within the chamber of a DeVilbiss Pulmo-Neb jet nebuliser following a 7 minute period of nebulisation. In another study, Cockroft et al. (1989) recorded a $62 \%$ rise in the concentration of residual saline solutions, following a 10 minute period of nebulisation with a Wright jet nebuliser.

\begin{tabular}{|c|c|c|}
\hline \multicolumn{2}{|c|}{$\begin{array}{c}\text { Table 3.4 Increase in the residual sodium chloride }(\mathrm{NaCl}) \text { concentration } \\
\text { following nebulisation with various jet nebulisers. [ } \pm \text { sd; } \mathrm{n}=3 \text { ] }\end{array}$} \\
\hline Nebuliser & $\begin{array}{c}\text { Concentration of residual } \\
\text { NaCl at sputtering } \\
(\mathrm{mg} / \mathrm{ml})\end{array}$ & $\begin{array}{c}\text { Increase in residual } \mathbf{~ N a C l} \\
\text { concentration following } \\
\text { nebulisation (\%) }\end{array}$ \\
\hline Cirrus & $\begin{array}{c}38.9 \\
( \pm 1.5)\end{array}$ & $\begin{array}{c}26.11 \\
( \pm 6.71)\end{array}$ \\
\hline Pari-LC & $\begin{array}{c}35.4 \\
( \pm 0.9)\end{array}$ & $\begin{array}{c}13.48 \\
( \pm 6.03)\end{array}$ \\
\hline Respirgard II & $\begin{array}{c}42.6 \\
( \pm 1.0)\end{array}$ & $\begin{array}{c}42.87 \\
( \pm 11.89)\end{array}$ \\
\hline Sidestream & 37.2 & 23.36 \\
& $( \pm 1.2)$ & $( \pm 3.88)$ \\
\hline
\end{tabular}

The concentration of sodium chloride remaining within each nebuliser at the sputtering time was related to the time taken for the nebuliser to reach sputtering (fig 3.21). Clearly, the longer that the residual nebuliser fluid is subjected to the flow of unsaturated gas, the greater the amount of solvent evaporation that will occur. However, the Sidestream deviated from the relationship, producing an increase in saline concentration that was statistically equivalent to that produced by the Cirrus, despite the fact that the Sidestream took significantly less time to complete nebulisation (479 s compared with $800 \mathrm{~s}$ ). Indeed, removing the Sidestream data from the plot of increase in saline concentration versus nebulisation time increases the correlation coefficient for the plot from 0.702 to 0.994 .

Evaporation occurring within the nebuliser chamber was therefore largely dependent on the duration of nebulisation. This was controlled by the rate of aerosol output, and hence the 
nebuliser design. A point to note is that the Sidestream nebuliser incorporates an open vent to increase the aerosol output rate. The expansion of gas above the Venturi jet draws air through the vent, which supplements gas flow through the device, yielding a more rapid output rate, thus reducing the nebulisation time. It has recently been suggested that the extra air flow through the device may lead to increased evaporation from the nebulised fluid (O'Callaghan and Barry, 1997). This may also lead to a more pronounced increase in the concentration of the dissolved drug. However, no evidence was presented to support this. The present results indicate that the open vent of the Sidestream nebuliser may indeed have demonstrable effects on the concentration of solute within the residual fluid following nebulisation. The additional gas flow through the device caused the evaporation occurring from nebulised saline to be significantly greater than that occurring within devices which lacked open vents, based on the nebulisation times they produce.

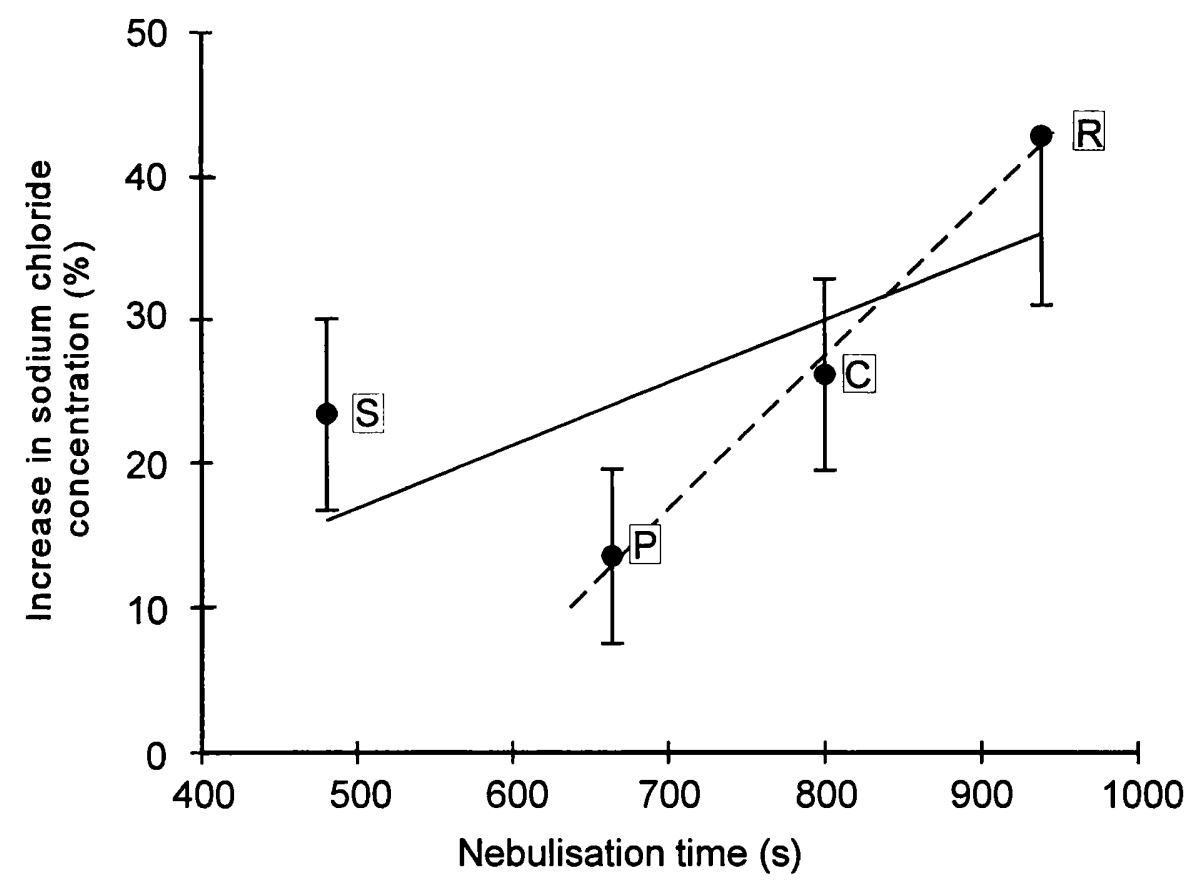

Figure 3.21 Increase in the residual sodium chloride concentration following nebulisation, as a function of the nebulisation time of each nebuliser studied $(\mathrm{C}=$ Cirrus, $\mathrm{P}=$ Pari-LC, $\mathrm{R}=$ Respirgard II, and $\mathrm{S}=$ Sidestream $)(-)$; and also for each nebuliser excluding the Sidestream (---). Equations for the lines of best fit: Sidestream included $(-), \mathrm{y}=0.0437 x$ $-5.00 ; \mathrm{r}=0.702$; and omitted $(--)$ from the plot: $\mathrm{y}=0.107 x-57.80, \mathrm{r}=0.994$. $[\mathrm{n}=3$; $\pm \mathrm{sd}]$ 


\begin{tabular}{|c|c|c|c|c|c|}
\hline \multicolumn{6}{|c|}{$\begin{array}{l}\text { Table } 3.5 \text { Increase in the residual lipid concentration following } \\
\text { nebulisation of variously sized eggPC/chol }(1: 1) \text { liposomes. [n=3; } \pm \text { sd] }\end{array}$} \\
\hline \multicolumn{6}{|c|}{$\begin{array}{c}\text { Experiment: (a) Concentration of lipid ( } \mathrm{mg} / \mathrm{ml}),(\mathrm{b}) \text { Increase in concentration } \\
(\%)(\mathrm{c}) \text { Increase in concentration adjusted for evaporation calculated from } \\
\text { nebulisation of saline (\%). }\end{array}$} \\
\hline \multicolumn{2}{|c|}{ Nebuliser/exp't } & $5 \mu \mathrm{m}$ lips & $2.5 \mu \mathrm{m}$ lips & $1 \mu \mathrm{m}$ lips & $0.6 \mu \mathrm{m}$ lips \\
\hline \multirow{3}{*}{ Cirrus } & $\mathbf{a}$ & $18.1(0.1)$ & $11.9(0.1)$ & $10.3(0.6)$ & $9.73(0.2)$ \\
\hline & $\mathbf{b}$ & $63.6(10.6) \%$ & $38.6(6.2) \%$ & $27.3(6.4) \%$ & $34.0(2.7) \%$ \\
\hline & c & $37.5(10.6) \%$ & $12.5(6.2) \%$ & $1.1(6.4) \%$ & $7.9(2.7) \%$ \\
\hline \multirow{3}{*}{ Pari-LC } & $\mathbf{a}$ & $15.6(0.6)$ & $13.1(3.2)$ & $10.2(0.3)$ & $8.97(0.7)$ \\
\hline & $\mathbf{b}$ & $38.0(3.75) \%$ & $42.9(12.1) \%$ & $23.4(2.7) \%$ & $25.8(2.7) \%$ \\
\hline & c & $24.5(3.75) \%$ & $29.4(12.1) \%$ & $9.91(2.7) \%$ & $12.4(2.7) \%$ \\
\hline \multirow{3}{*}{$\begin{array}{l}\text { Respirgard } \\
\text { II }\end{array}$} & $\mathbf{a}$ & $13.6(2.1)$ & $16.0(2.9)$ & $11.7(1.2)$ & $11.8(2.1)$ \\
\hline & $\mathbf{b}$ & $69.0(11.5) \%$ & $67.21(8.6) \%$ & $44.6(7.3) \%$ & $38.1(9.4) \%$ \\
\hline & c & $26.1(11.5) \%$ & $24.3(8.6) \%$ & $1.7(7.3) \%$ & $-4.7(9.4) \%$ \\
\hline \multirow{3}{*}{ Sidestream } & $\mathbf{a}$ & $22.5(0.7)$ & $14.3(1.8)$ & $11.6(0.6)$ & $9.6(0.5)$ \\
\hline & $\mathbf{b}$ & $94.3(6.2) \%$ & $96.5(14.3) \%$ & $45.0(3.9) \%$ & $43.6(3.6) \%$ \\
\hline & c & $70.9(6.2) \%$ & $73.1(14.3) \%$ & $21.6(3.9) \%$ & $20.2(3.6) \%$ \\
\hline
\end{tabular}

The results of the increase in residual lipid mass, following the nebulisation of liposomes, are presented in table 3.5. Subtracting the increase in concentration that was attributable to evaporation of solvent, as established from the study of saline, reveals that the nebulisation of liposomes yielded a significantly greater percentage rise in the residual dry mass, than was produced following the nebulisation of the simple saline solution $(p<0.05)$. The increase in lipid concentration ranged between 23 and $97 \%$ (table 3.5), compared with between 16 and $39 \%$ for the saline (table 3.4). It may be proposed that intercalation of phospholipid molecules between water molecules at the air-liquid interface, causes a reduction in the net forces of attraction between adjacent water molecules. This causes a reduction in the surface tension of the liposome dispersions, compared to that of the saline. 
Conversely, the addition of electrolytes, including $\mathrm{Na}^{+}$and $\mathrm{Cl}^{-}$, causes an increase in the surface tension of water (Myers, 1990). As a consequence, there was a greater evaporation of water during the nebulisation of liposomes, compared to the simple sodium chloride solution. Hence, the mass of liposomal lipid remaining within the fluid reservoir following nebulisation was significantly increased.

In contrast to the results obtained from the nebulisation of saline, there was no apparent correlation between the nebulisation time and the increase in the residual lipid mass of the liposomes (fig. 3.22 and 3.23). However, removing the contribution of the Sidestream nebuliser to the plots reveals a strong correlation between the nebulisation time and the increase in residual lipid mass. The Sidestream nebuliser is an open vent nebuliser, which, as proposed in section 3.4.2, significantly influences the manner in which the nebuliser handles liposomes. In contrast to the Sidestream, the Pari-LC nebuliser incorporates a breathe-assisted, open vent. Therefore, the Pari-LC vent only becomes relevant to the performance of the nebuliser when inspiratory breath flow is provided. As determined for the nebulisation of saline, the open vent design of the Sidestream nebuliser yielded a greater rise in the lipid concentration than would be predicted on the basis of nebulisation time. Thus, the open vent design of the Sidestream nebuliser influenced the residual masses obtained upon nebulisation of both the simple saline solution, and the liposomes.

The nebulisation of differently sized liposomes revealed that the retention of lipid within the nebuliser chamber during nebulisation was not solely dependent on the design of the nebuliser. There existed a direct correlation between the residual lipid concentration and the mean liposome size of the formulation (fig. 3.24 and 3.25). The residual mass was between 13 and $54 \%$ greater for the $5 \mu \mathrm{m}$ mean size liposomes than the $0.6 \mu \mathrm{m}$ liposomes (fig. 3.24). This indicates the existence of a size selective process, as postulated in the experiment investigating aerosol output from the Pari-LC and Sidestream (section 3.4.3). Although the results presented in the previous section failed to show conclusively a significantly reduced aerosol output for larger liposomes, thic present results support the observed trend towards such a relationship. It is thus clear, that the greater residual volumes produced by liposomes following nebulisation, compared with the saline solution, may be additionally due to a failure of the liposomes to become aerosolised, and not just to their influence on the surface tension. 
( a )

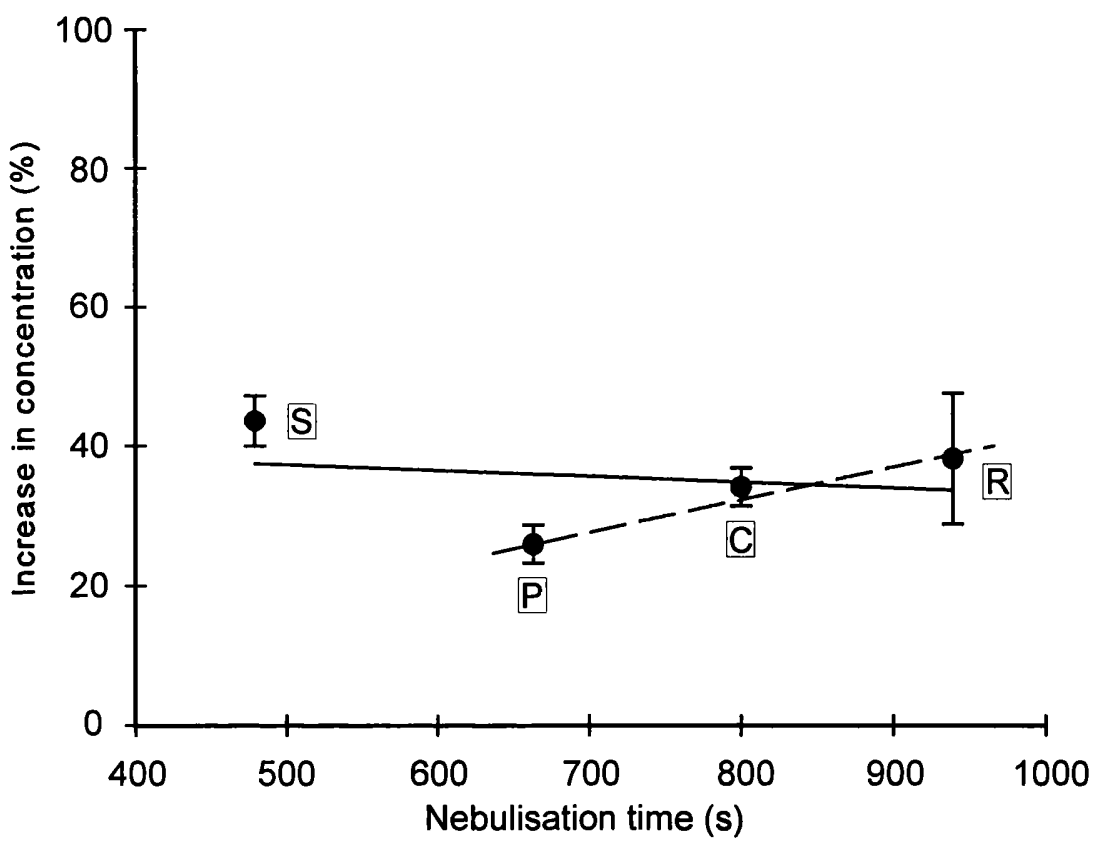

(b)

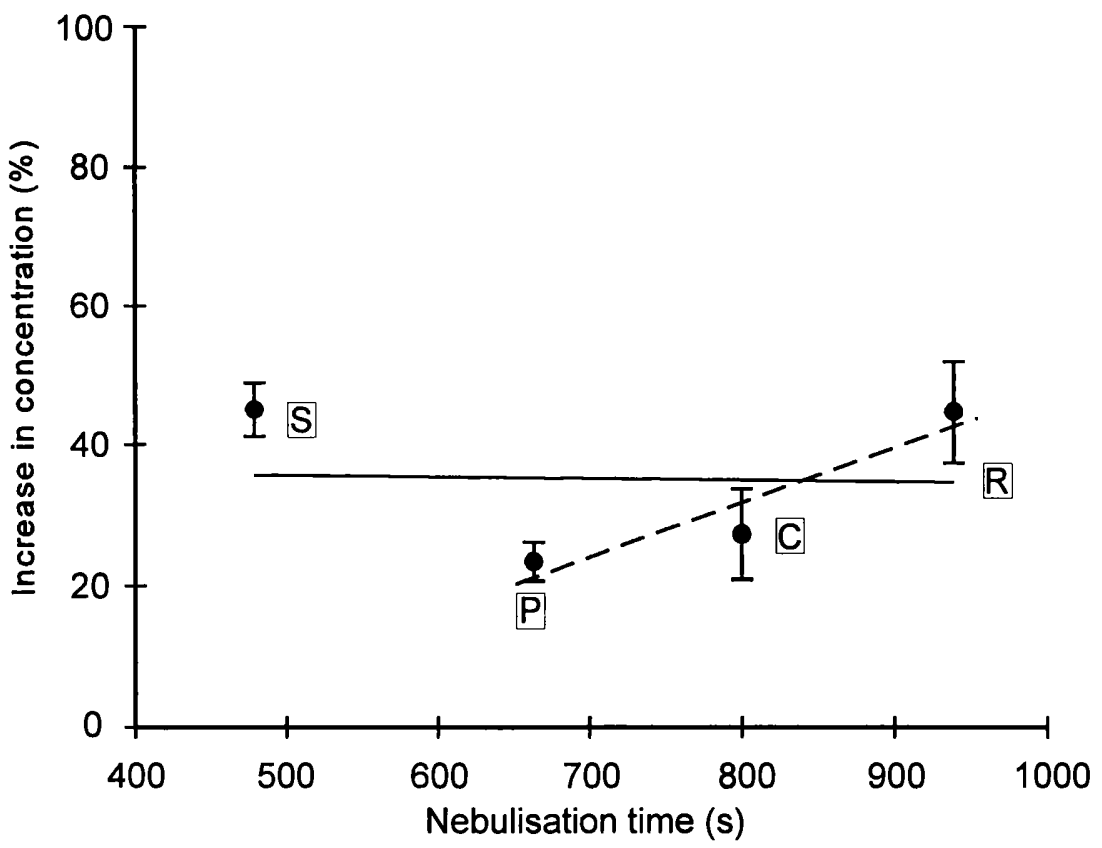

Figure 3.22 Increase in the residual lipid concentration of (a) approximately $0.6 \mu$ m mean size liposomes, and (b) $1 \mu \mathrm{m}$ mean size liposomes, as a function of the nebulisation time of different nebulisers. ( $C=$ Cirrus, $P=$ Pari-LC, $R=$ Respirgard II, and $S=$ Sidestream). Equations for the lines of best fit are shown with Sidestream included (-), and omitted (--) from the plot: (a) $0.6 \mu \mathrm{m}$ liposomes, Sidestream included: $\mathrm{y}=-0.00812 x+41.25, \mathrm{r}=$ 0.214; Sidestream omitted: $\mathrm{y}=-0.0442 x+2.738, \mathrm{r}=0.982$ (b) $1 \mu \mathrm{m}$ liposomes, Sidestream included: $\mathrm{y}=-0.00230 x+39.71, \mathrm{r}=-0.0397$; Sidestream omitted: $\mathrm{y}=$ $0.0769 x-29.85, \mathrm{r}=0.940 .[\mathrm{n}=3 ; \pm \mathrm{sd}]$ 
(a)

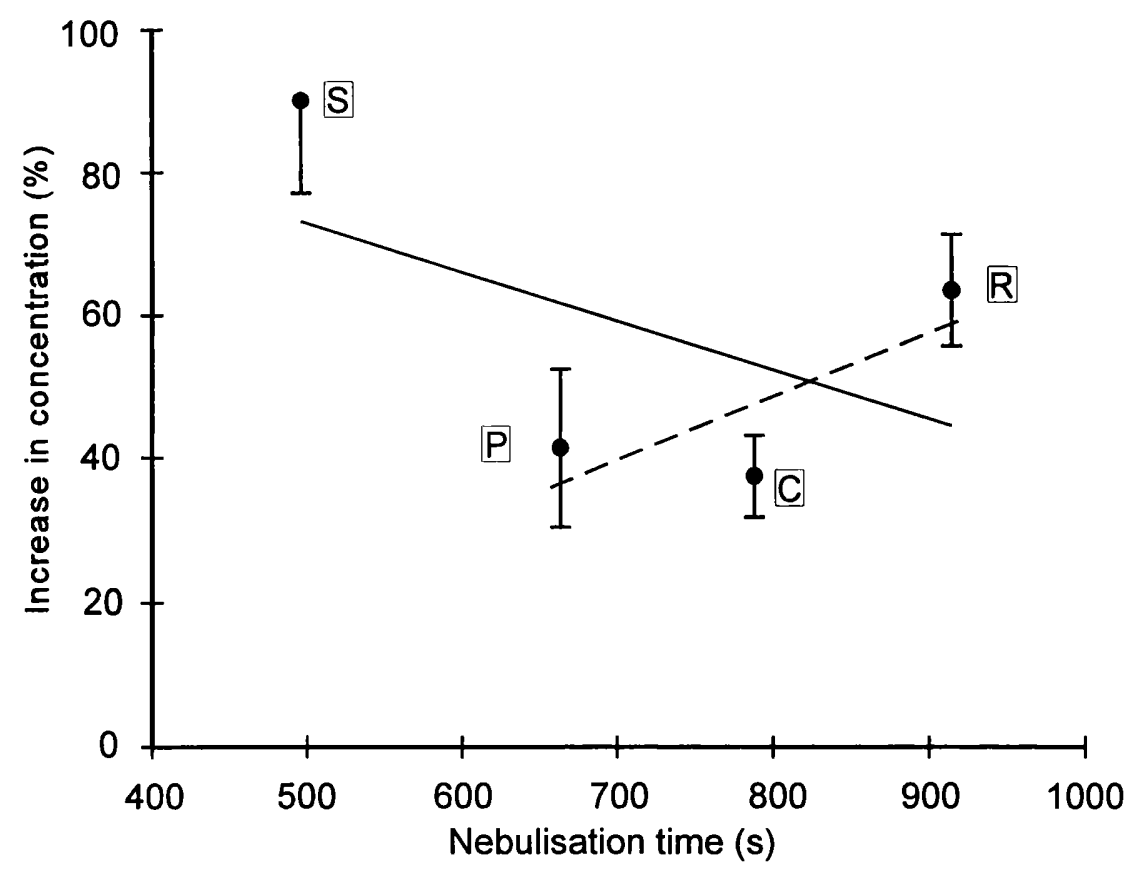

(b)

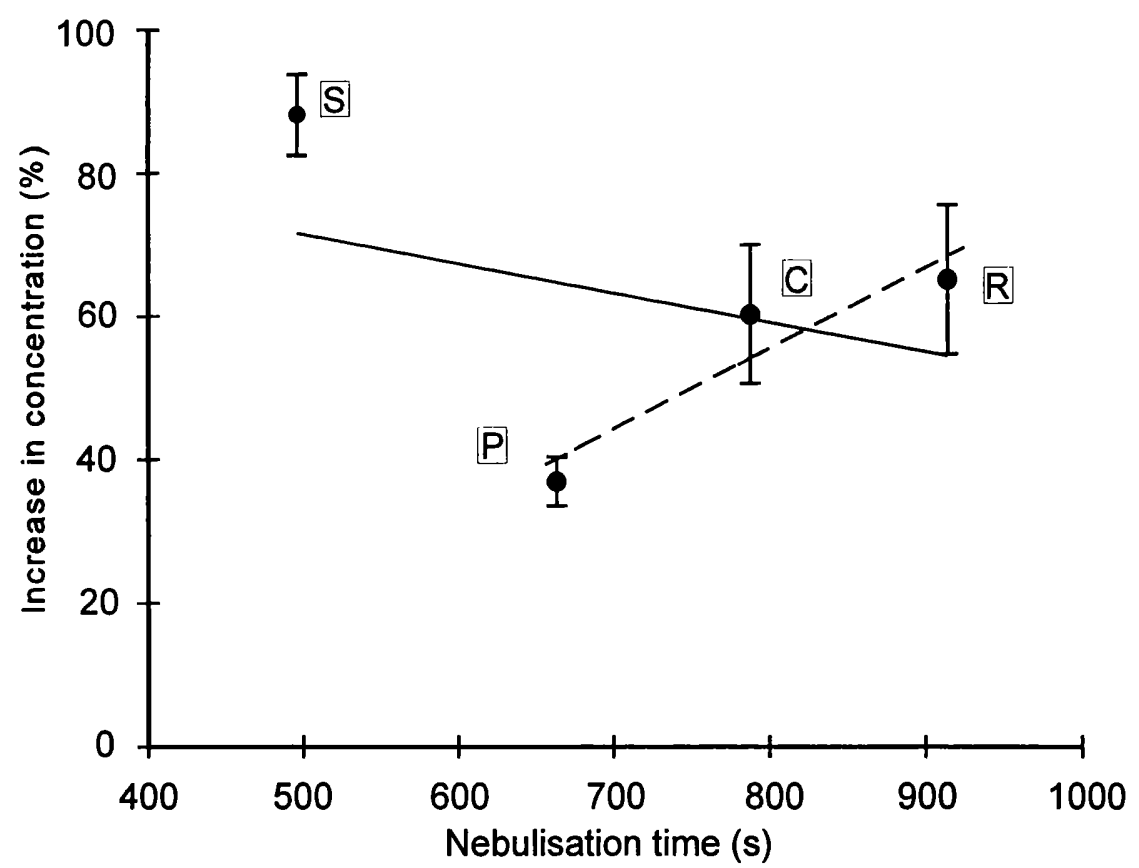

Figure 3.23 Increase in the residual lipid concentration of (a) $2.5 \mu \mathrm{m}$ mean size liposomes, and (b) $5 \mu \mathrm{m}$ mean size liposomes, as a function of the nebulisation time of different nebulisers. $(\mathrm{C}=$ Cirrus, $\mathrm{P}=$ Pari-LC, $\mathrm{R}=$ Respirgard II, and $\mathrm{S}=$ Sidestream). Equations for the lines of best fit are shown with Sidestream included (-), and omitted (--) from the plot: (a) $2.5 \mu \mathrm{m}$ liposomes, Sidestream included: $\mathrm{y}=-0.0688 x+110.83, \mathrm{r}=-0.507$; Sidestream omitted: $\mathrm{y}=-0.0883 x+21.10, \mathrm{r}=0.789$ (b) $5 \mu \mathrm{m}$ liposomes, Sidestream included: $\mathrm{y}=-0.0411 x+95.84, \mathrm{r}=-0.350$; Sidestream omitted: $\mathrm{y}=-0.112 x-32.85, \mathrm{r}=$ 0.934. $[\mathrm{n}=3 ; \pm \mathrm{sd}]$ 


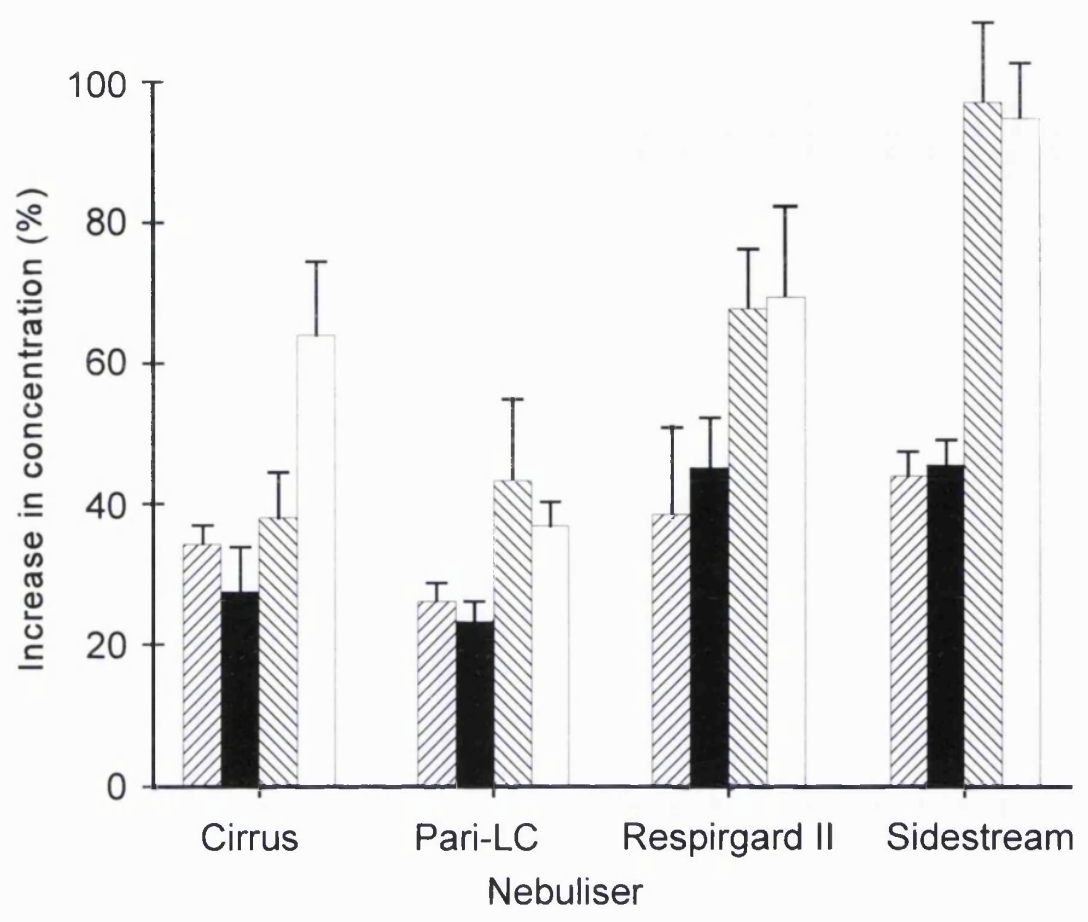

Figure 3.24 Increase in the residual lipid concentration following nebulisation of variously sized liposomes. Mean liposome size: (//I) $0.6 \mu \mathrm{m},(-) 1 \mu \mathrm{m}$, (III) $2.5 \mu \mathrm{m}$, and () $5 \mu \mathrm{m}$. [n=3; \pm sd]

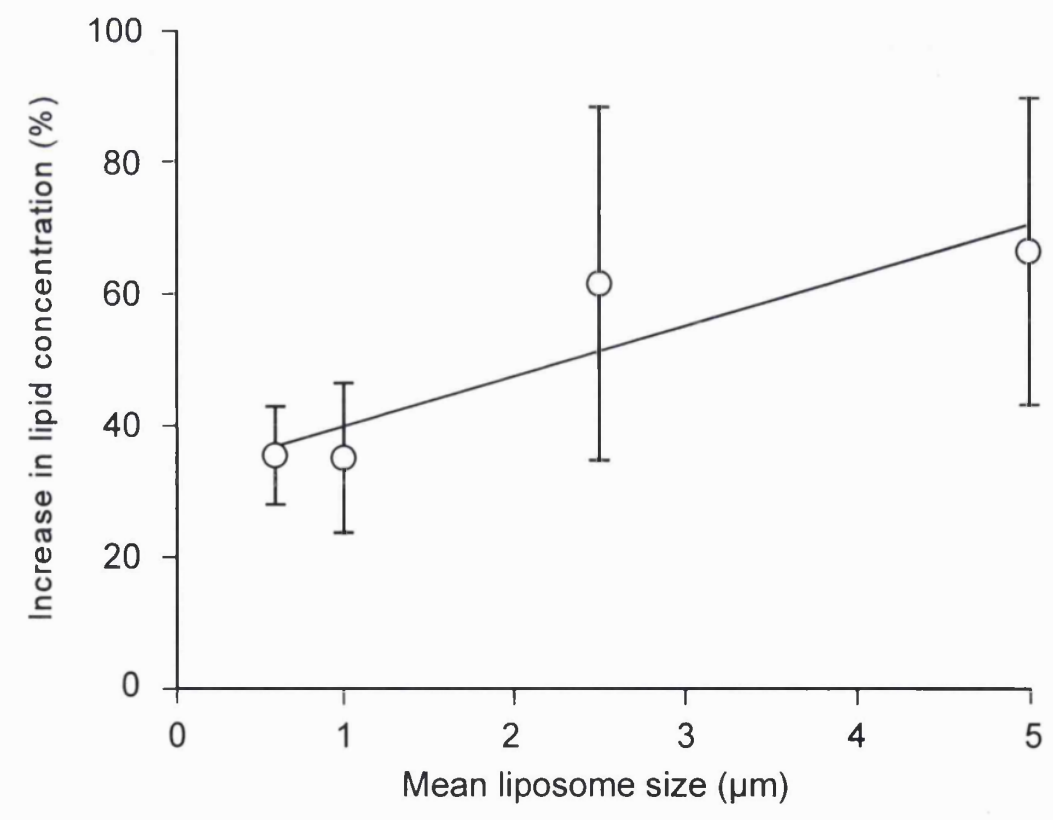

Figure 3.25 Increase in the residual lipid concentration of liposomes following nebulisation as a function of the mean liposome size (mean of all nebulisers studied). Equation of line of best fit shown: $y=7.576 x+32.54, r=0.910 .[n=3$; \pm sd] 
A number of authors have noted that the size reduction of liposomes during jet nebulisation may be minimised by reducing the initial mean vesicle size (Taylor et al., 1990b; Niven et al., 1991a). From this, it was hypothesised that larger liposomes are deformed and fractured by nebulisation, until reduced to a size that may be included within the emitted droplets. This was based on the finding that larger liposomes tended to yield a reduced total aerosol output (section 3.4.3). The present results add further weight to this hypothesis, as larger liposomes appear to be retained within the nebuliser to a greater extent than the equivalent small vesicles. For each nebuliser studied, there was no significant difference between the increase in lipid concentration occurring in the $0.6 \mu \mathrm{m}$ and $1 \mu \mathrm{m}$ mean liposome size samples $(\mathrm{p}<0.05)$. Therefore, reducing the liposome size below $1 \mu \mathrm{m}$ did not improve the output of the liposomes significantly. It may not be appropriate to consider the size of 0.6 $\mu \mathrm{m}$ attributed to the smallest liposomes as an accurate figure, as it was determined from the Malvern 2600c laser diffraction sizer, which has a lower limit of sensitivity of between 0.5 and $1 \mu \mathrm{m}$. However the $0.6 \mu \mathrm{m}$ liposome samples do represent liposomes with a mean size below $1 \mu \mathrm{m}$ as they were prepared by the continued sonication of $1 \mu \mathrm{m}$ liposomes. In each of the nebulisers studied there was an increase in the residual lipid concentration evident with an increase in the mean liposome size from 1 to $2.5 \mu \mathrm{m}(\mathrm{p}<0.05)$. Increasing the liposome size, as previously hypothesised, limits the number of liposomes that may be included in aerosolised droplets with a mean size less than that of the liposomes themselves. The heterodisperse nature of the aerosols, and the shearing of the liposomes within the nebuliser, allows a certain proportion of the larger liposomes to be aerosolised. However, because the mean droplet size produced by the Pari-LC, Respirgard II and Sidestream nebulisers was less than $2.5 \mu \mathrm{m}(2.15,1.70$, and $1.99 \mu \mathrm{m}$ respectively) a greater proportion of liposomes with a mean size of $2.5 \mu \mathrm{m}$ or greater became excluded from a greater number of the aerosol droplets. Reducing the liposome size to below $2.5 \mu \mathrm{m}$ increases the proportion of liposomes which may become aerosolised, and hence liposomes with a mean diameter of $1 \mu \mathrm{m}$ produce significantly reduced residual masses (fig. 3.26 and 3.27). The Cirrus nebuliser produced droplets which have a high degree of polydispersity, and a significantly larger size $(2.70 \mu \mathrm{m})$ (section 3.4 .1$)$. As a consequence, the Cirrus nebuliser aerosolised large liposomes more readily (fig. 3.26a). In contrast to the other nebulisers, an increase in the liposome size from 2.5 to $5 \mu \mathrm{m}$ produced a significantly increased residual mass in the Cirrus $(\mathrm{p}<0.05)$. This was because the $5 \mu \mathrm{m}$ liposome formulation was the only one to have a mean size greater than the aerosolised droplet size. 


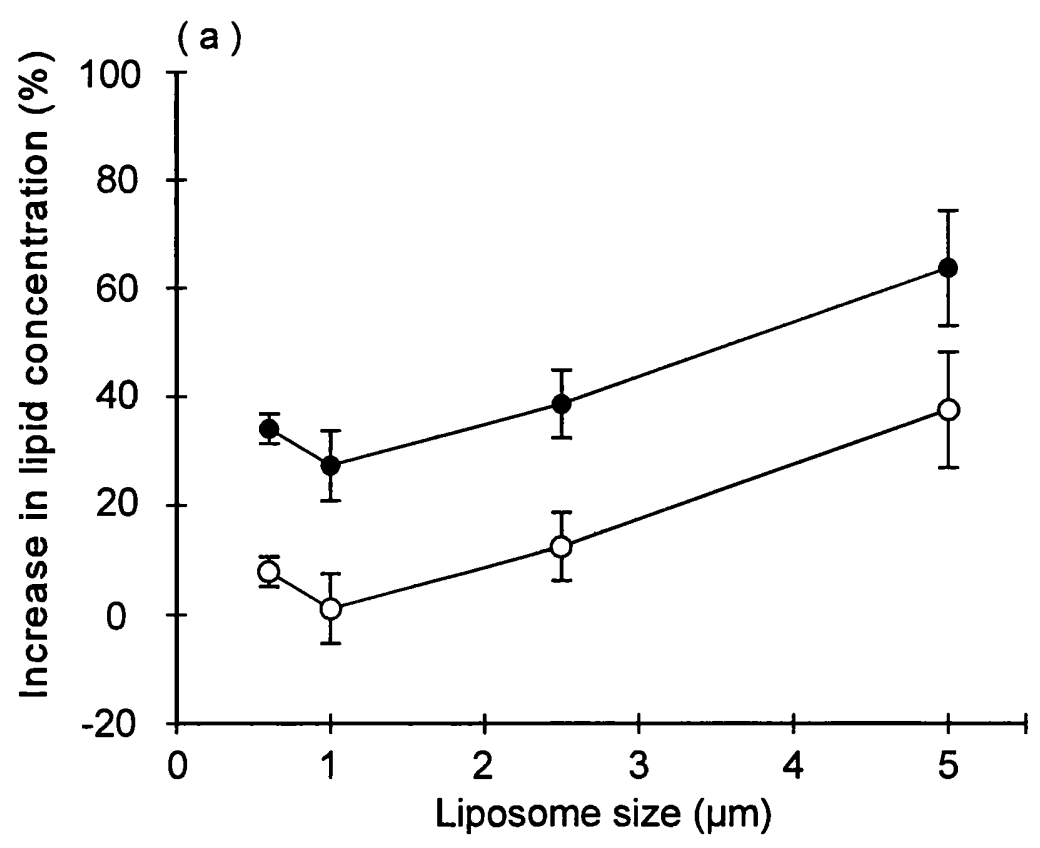

(b)

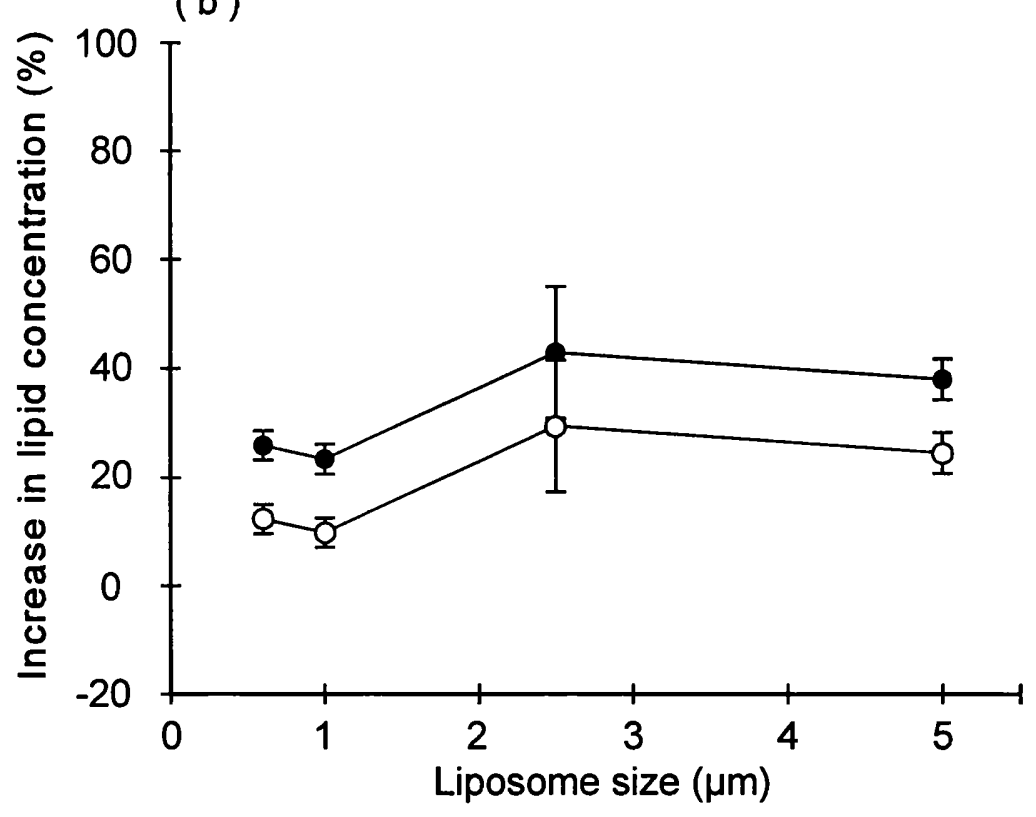

Figure 3.26 Increase in the residual lipid concentration of liposomes following nebulisation with the Cirrus (a) and Pari-LC (b) nebulisers. Data is plotted unadjusted ( $\bullet$ ) and adjusted $(0)$ for evaporative losses during nebulisation by subtracting the increase in residual sodium chloride concentration produced upon nebulisation of saline with each nebuliser (as detailed in table 3.5 ). [n=3; \pm sd] 

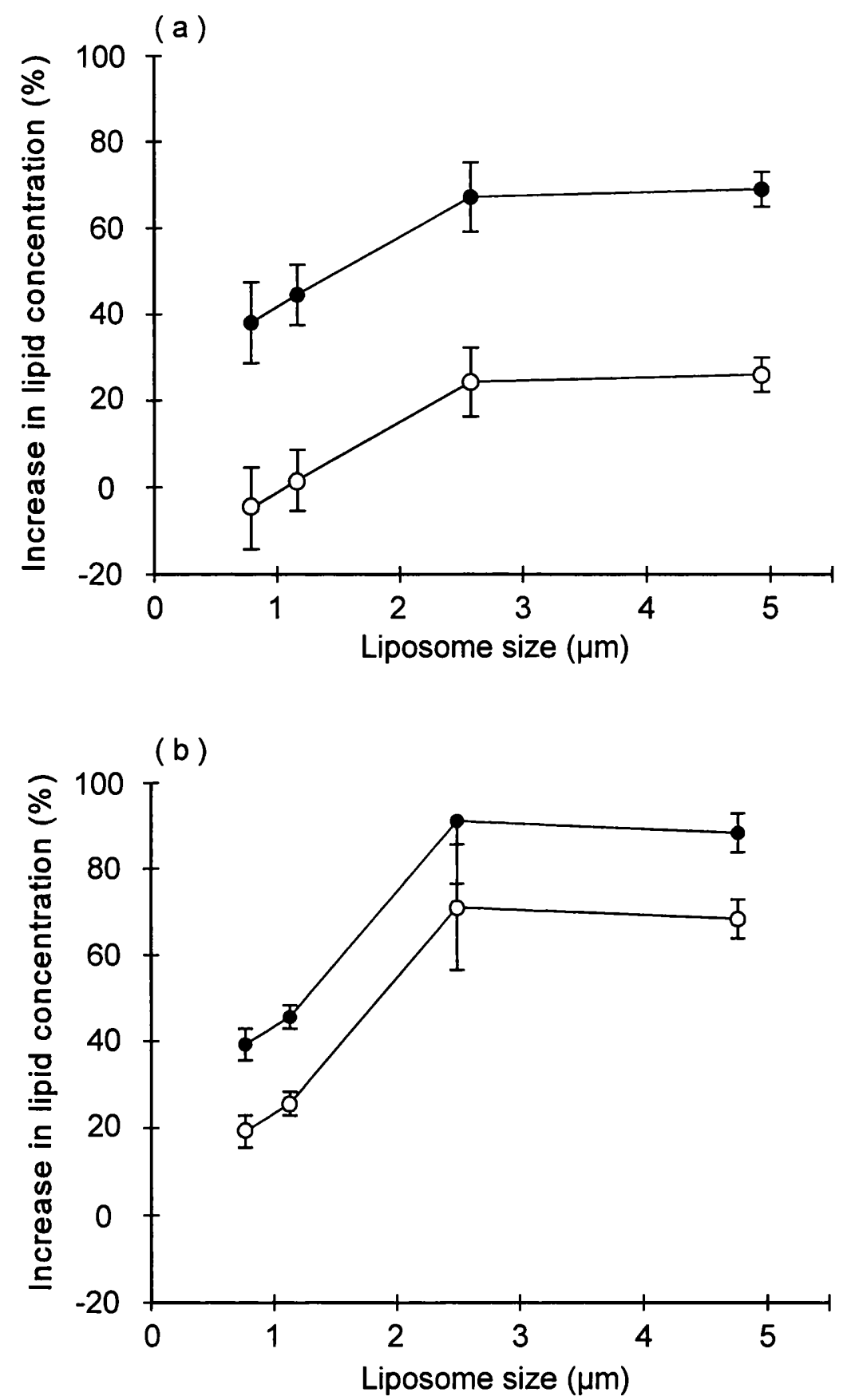

Figure 3.27 Increase in the residual lipid concentration of liposomes following nebulisation with Respirgard II (a) and Sidestream (b) nebulisers. Data is plotted unadjusted $(\bullet)$ and adjusted $(0)$ for evaporative losses during nebulisation by subtracting the increase in residual sodium chloride concentration produced upon nebulisation of saline with each nebuliser as detailed in (as detailed in table 3.5$)$. [n=3; $\pm \mathrm{sd}]$ 
From consideration of these findings, it is unsurprising to note that the results also demonstrate an apparent inverse relationship between the nebulised droplet size and the increase in the residual lipid concentration. As the droplet size is increased so the retention of liposomes is reduced (fig. 3.28). This supports the theory that exclusion of liposomes from smaller droplets was responsible for liposome retention. The liposomes were prepared with cholesterol in the bilayers, and as a consequence are resistant to size reduction during nebulisation (see chapter 4). This means that the liposomes with a mean size greater than that of the droplet size produced by the nebuliser were less able to be processed to a size that may be aerosolised. However, the Cirrus yields a droplet size of approximately 2.70 $\mu \mathrm{m}$, and therefore the greater proportion of the $2.5 \mu \mathrm{m}$ liposomes became aerosolised (fig. 3.28 to 3.30). Thus the difference in the concentration of $2.5 \mu \mathrm{m}$ and $5 \mu \mathrm{m}$ liposomes within the residual nebuliser fluid was more marked than for the alternative nebulisers (fig. $3.26 \mathrm{~b}$ and 3.27). The present findings for liposomes therefore agree with the hypothesis proposed by Dahlbäck (1994), that suspended drugs must be micronised to less than $2 \mu \mathrm{m}$ if they are to be efficiently delivered from nebulisers producing $3 \mu \mathrm{m}$ droplets.

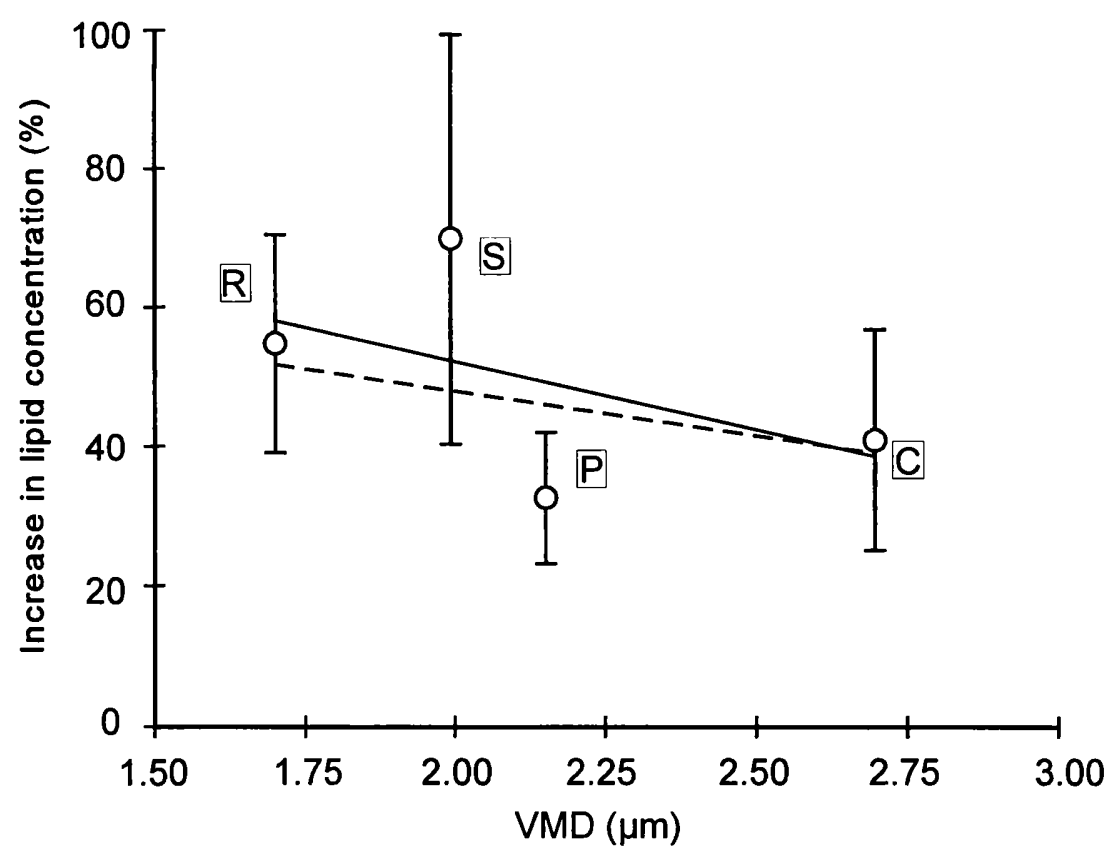

Figure 3.28 Increase in the residual lipid concentration of liposomes during nebulisation (mean of all liposome sizes) as a function of the volume median diameter (VMD) of droplets produced by various nebuliser models $(\mathrm{C}=$ Cirrus, $\mathrm{P}=$ Pari-LC, $\mathrm{R}=$ Respirgard II, and $\mathrm{S}=$ Sidestream). Equation for the line of best fit shown [-]: $\mathrm{y}=-19.594 x+91.313, \mathrm{r}=-0.501$. With Sidestream omitted [--]: $\mathrm{y}=-12.902 x+70.864, \mathrm{r}=-0.575 .[\mathrm{n}=3 ; \pm \mathrm{sd}]$ 


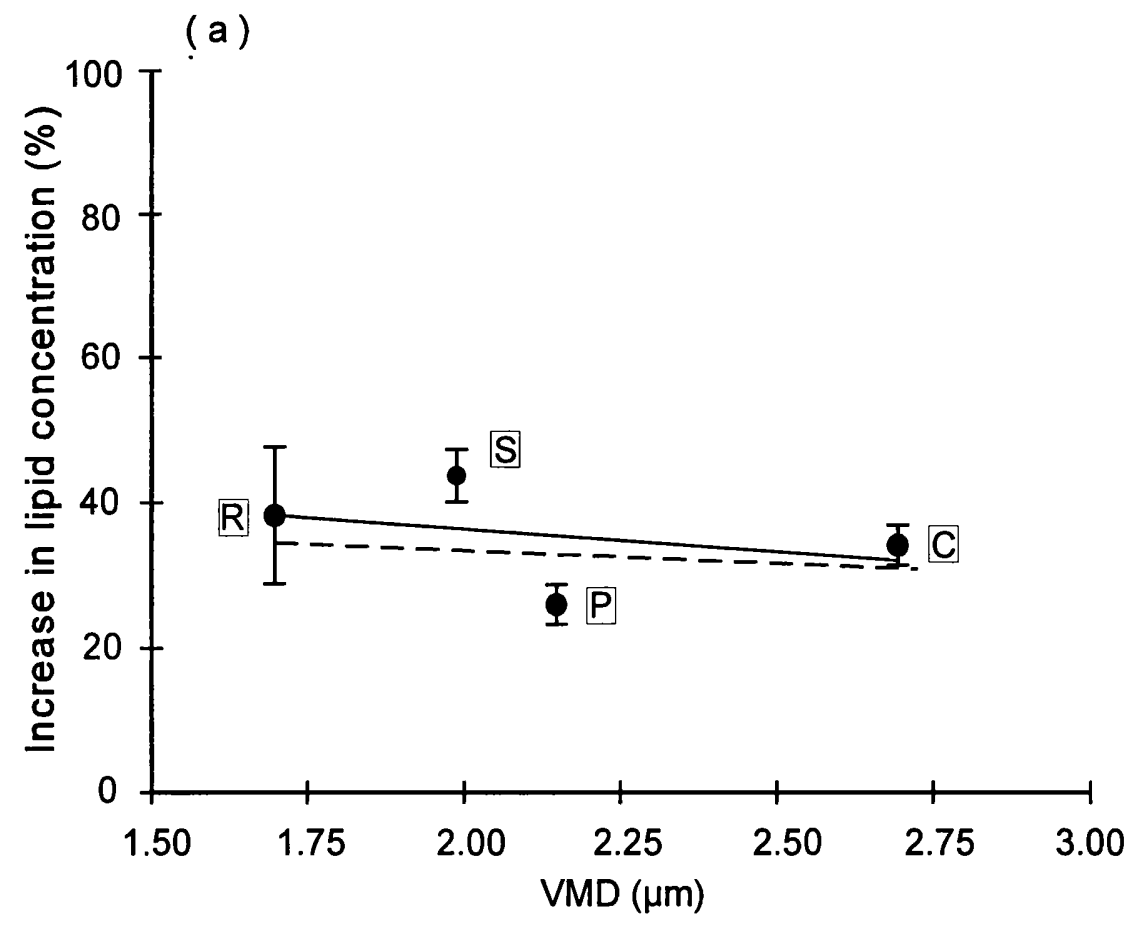

(b)

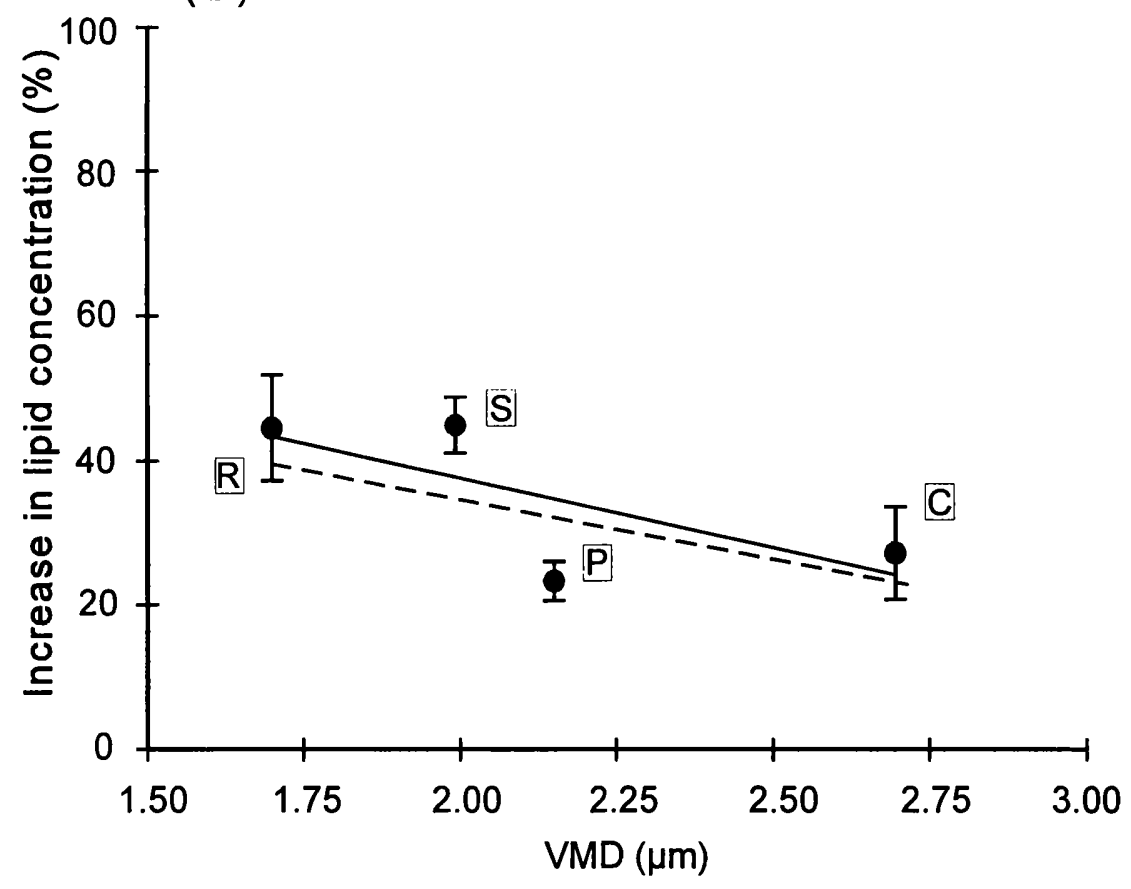

Figure 3.29 Increase in the lipid concentration of residual liposomes during nebulisation of approximately $0.6 \mu \mathrm{m}$ (a), and $1 \mu \mathrm{m}$ mean size liposomes (b), as a function of the mean droplet size (VMD) produced by the Cirrus (C), Pari-LC (P), Respirgard II (R), and Sidestream (S) nebulisers. Equations for the lines of best fit are shown with the Sidestream included (-), and omitted (---) from the plot: (a) $0.6 \mu \mathrm{m}$ liposomes, Sidestream included: $\mathrm{y}=-6.243 x+41.25, \mathrm{r}=-0.351$; Sidestream omitted: $\mathrm{y}=-3.441 x+40.19, \mathrm{r}=-0.236$ (b) $1 \mu \mathrm{m}$ liposomes, Sidestream included: $\mathrm{y}=-19.296$ $x+76.24, \mathrm{r}=-0.711 ;$ Sidestream omitted: $\mathrm{y}=-16.56 x-67.87, \mathrm{r}=0.731 .[\mathrm{n}=3 ; \pm \mathrm{sd}]$ 
(a)

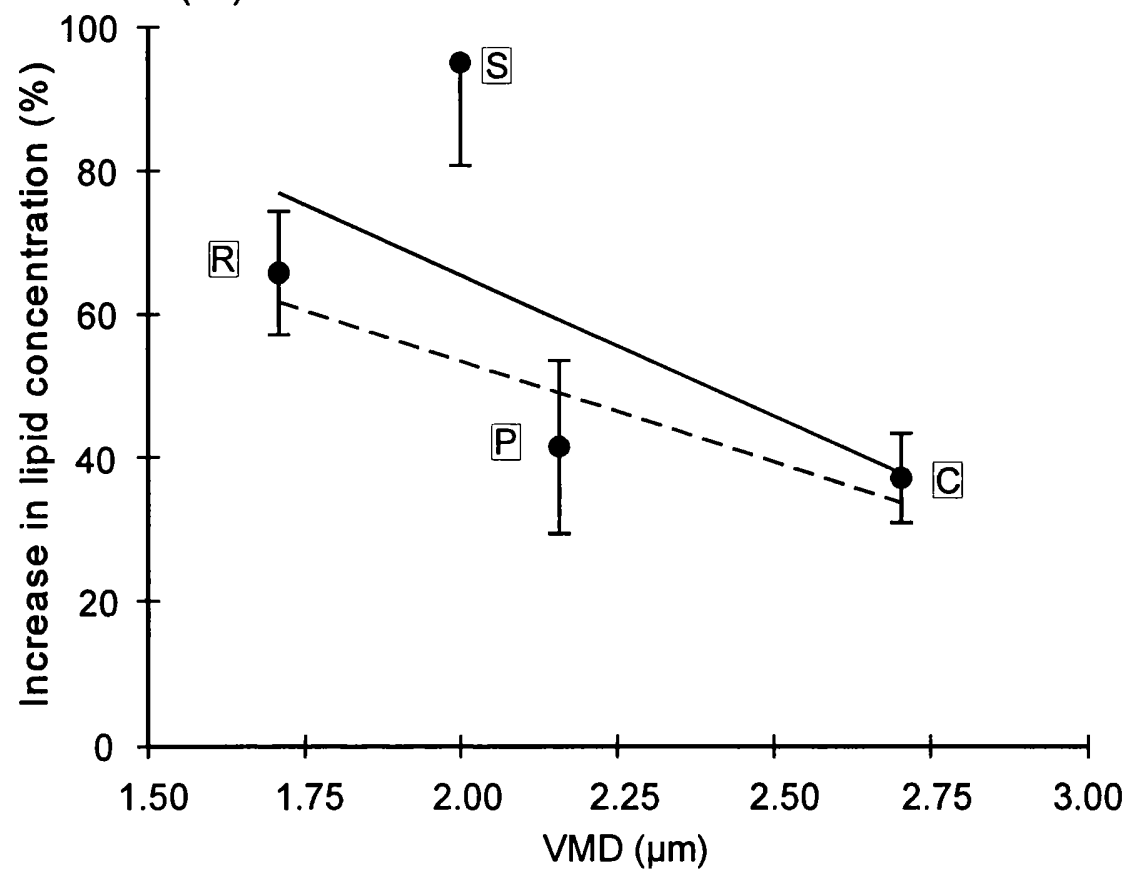

(b)

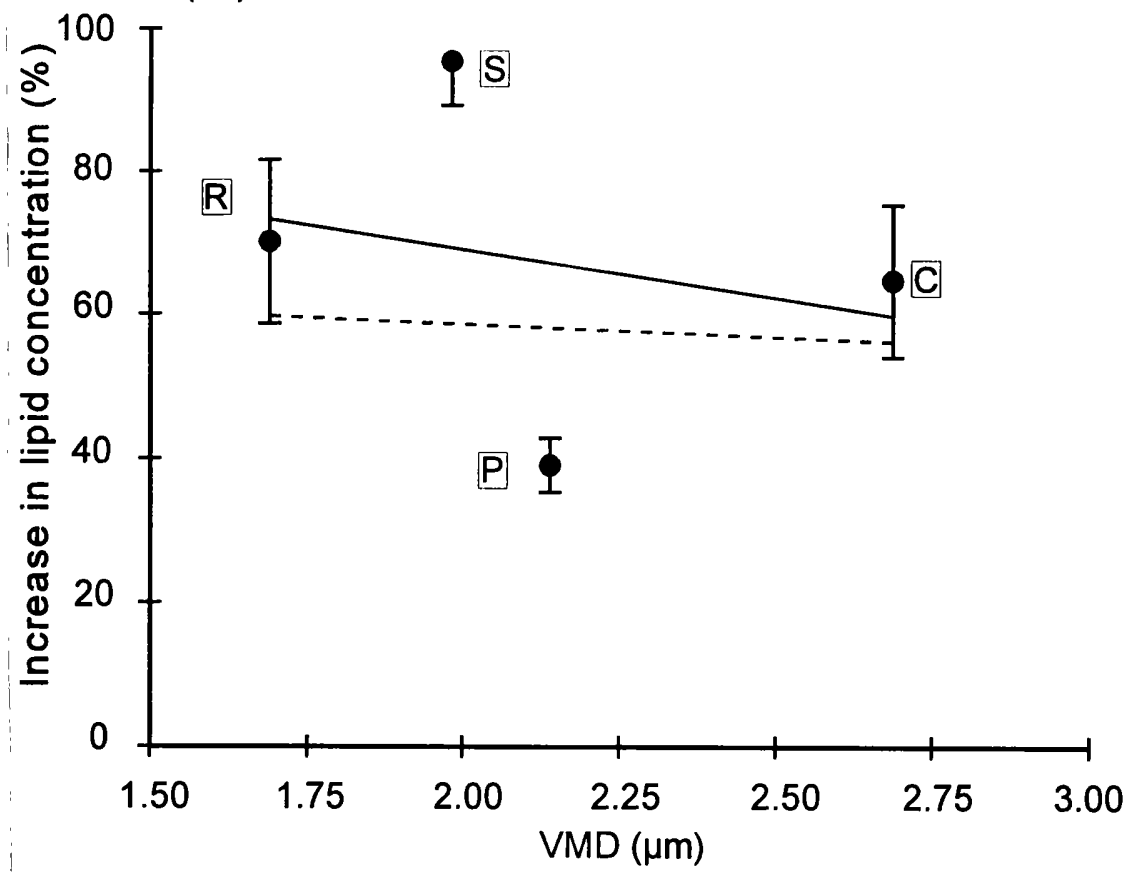

Figure 3.30 Increase in the lipid concentration of residual liposomes during nebulisation of approximately $2.5 \mu \mathrm{m}$ (a) and $5 \mu \mathrm{m}$ mean size liposomes (b), as a function of the mean droplet size (VMD) produced by the Cirrus (C), Pari-LC (P), Respirgard II (R), and Sidestream (S) nebulisers. Equations for the lines of best fit are shown with the Sidestream included (-), and omitted (---) from the plot: (a) $2.5 \mu \mathrm{m}$ liposomes, Sidestream included: $\mathrm{y}=-39.326 x+145.24, \mathrm{r}=-0.617$; Sidestream omitted: $\mathrm{y}=-28.043 x+110.76, \mathrm{r}=-0.906$ (b) $5 \mu \mathrm{m}$ liposomes, Sidestream included: $\mathrm{y}=-13.536$ $x+95.10, \mathrm{r}=-0.245$; Sidestream omitted: $\mathrm{y}=-3.57 x-+64.64, \mathrm{r}=0.108 .[\mathrm{n}=3 ; \pm \mathrm{sd}]$ 
An alternative explanation for the size selectivity exhibited by the nebulisers is that structures within the nebuliser impede the transport of larger liposomes to the site(s) of droplet formation. McCallion et al. (1996) noted a similar increase in the concentration of latex spheres to that recorded in the present experiment, when aerosolised with a variety of nebulisers. The increase in residual concentration was dependent on the latex sphere size in a similar manner to that produced in the present liposome study. For example 50 to 60 $\%$ of the $0.605 \mu \mathrm{m}$ latex spheres were nebulised. This fell to 7 to $40 \%$ of the $6.4 \mu \mathrm{m}$ spheres. These results were attributed to a loss of vehicle due to evaporation, and to physical blockage of the nebuliser orifice, and/or refluxing action of the suspension. In addition, greater settling of the larger latex spheres due to their increased density was suggested. Furthermore, suspensions of dexamethasone have been nebulised with limited success, due to variable output, potential clogging of jets, and poor nebulisation efficiency (Niven et al., 1991b). Over a 10 minute period there was a $14 \%$ increase in the concentration of dexamethasone solution during jet nebulisation. By contrast, there was a $38 \%$ increase in the concentration of dexamethasone suspension, indicating a preferential concentration of the suspended drug (Niven et al., 1991b). Such mechanisms may equally have applied to the liposome dispersions of the present study. The various orifices within each nebuliser were therefore examined to investigate the potential for blockage by larger liposomes.

\subsubsection{Examination of nebuliser component orifices}

The schematic diagrams and electron micrographs of figures 3.31 to 3.34 illustrate the diverse nature of the design of the different jet nebuliser models. This is responsible for the significant differences in the droplet sizes produced by each, the variability in nebulisation time, liposomal aerosol output, and response to varying lipid concentration.

\section{Cirrus (fig. 3.1a)}

The Cirrus nebuliser operates by forcing high velocity gas through a central jet with a diameter of $770 \mu \mathrm{m}$ (fig. 3.34a/b). The Cirrus differs from each of the other devices in that it lacks true fluid feed capillaries. Instead, the nebulised fluid is sandwiched between the wall of the central structure in which the gas jet orifice (fig 3.34a) is contained, and an outer cap which contains the fluid/gas orifice (fig. 3.34b). The fluid becomes drawn up the walls of these structures, and is blown out of the $510 \mu \mathrm{m}$ fluid/gas orifice (fig. 3.34b). 
Pari-LC (fig. 3.1b)

The Pari-LC nebuliser operates in a similar manner to the Cirrus, except the device has two capillary feed channels (fig. 3.32b), approximately $960 \mu \mathrm{m}$ in diameter, etched into the wall of the outer "cap". Fluid is drawn up these channels, response to the flow of gas through the $420 \mu \mathrm{m}$ diameter gas jet orifice (fig 3.32c), drilled into a cone-like structure over which the outer cap fits. The fluid and gas are mixed internally, and exit through an fluid/gas orifice with a diameter of approximately $1.3 \mathrm{~mm}$ by $3.0 \mathrm{~mm}$ (fig. $3.32 \mathrm{a}$ ).

\section{Respirgard II (fig. 3.1c)}

The Respirgard II nebuliser has four $1.40 \mathrm{~mm}$ fluid feed capillaries in the "cap" (fig. 3.33a) which fits over a central structure bearing a $450 \mu \mathrm{m}$ gas jet orifice (fig. 3.33c). Fluid and gas are mixed internally before leaving a $1 \mathrm{~mm}$ fluid/gas orifice (fig. $3.33 \mathrm{~b}$ ).

\section{Sidestream (fig. 3.1d)}

Fluid in the Sidestream is drawn up the gap formed between the central structure in the centre of the reservoir, and the baffle cap. The fluid is drawn through twin $840 \mu \mathrm{m}$ diameter fluid feed capillaries, adjacent to a central $530 \mu \mathrm{m}$ gas jet orifice (fig. 3.34c). The Sidestream nebuliser is the only device to mix the fluid and gas externally from the nozzle.

The differences in construction and design, evident from the micrographs, explain the unique characters of aerosols produced by different nebuliser models. There are wide variations in the size and orientation of the different functional structures within each device, which lead to the observed variations in the droplet size distribution, nebulisation time, liposomal aerosol output, and response to changing fluid properties. However, the dimensions of each of the various structures within each nebuliser appear to rule out liposome blockage as was proposed for latex sphere suspensions (McCallion et al., 1996)(fig. 3.32 to 3.34). The smallest orifice through which the liposomes must flow in each device varies between $510 \mu \mathrm{m}$ and $960 \mu \mathrm{m}$ in the nebuliser models studied. It is therefore unlikely that blockage of the capillaries was occurring, even if there was extensive aggregation of the liposomes. However, in each of the nebulisers studied, channels through which the liposomes must flow are formed only once the nebuliser is assembled. These channels may be implicated in the retention of liposomes, and their dimensions proved impossible to determine. 

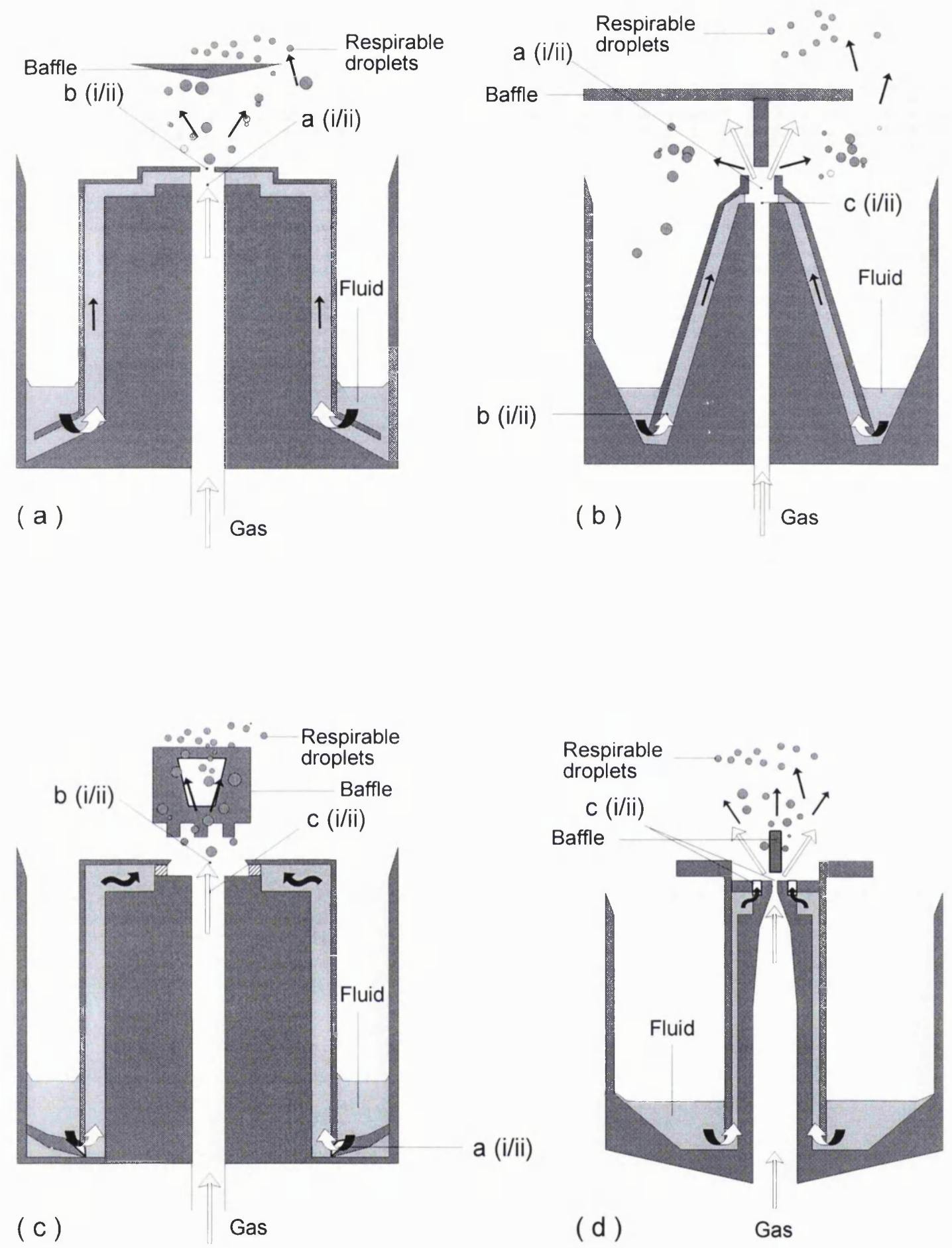

Figure 3.31 Schematic diagrams of the various orifices within each jet nebuliser, with baffles and unit top/mouthpiece removed (not to scale). (a) Cirrus, (b) Pari-LC, (c) Respirgard II, and (d) Sidestream. Letters/numerals in each diagram refer to the various scanning electron micrographs illustrated in figures 3.32 to 3.34 . 


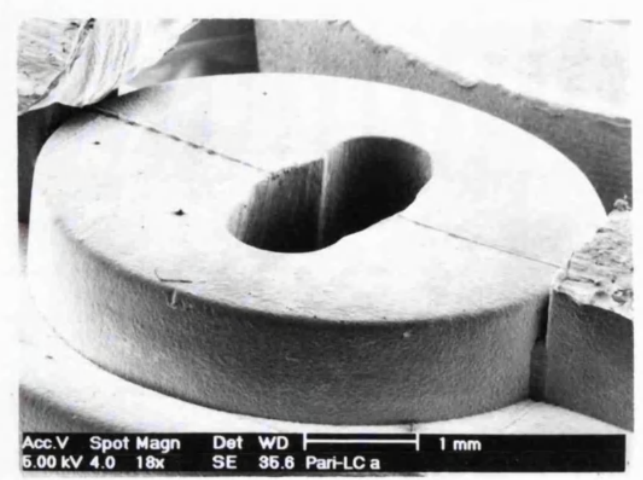

a(i) Pari-LC - gas and fluid orifice [1.30 mm]

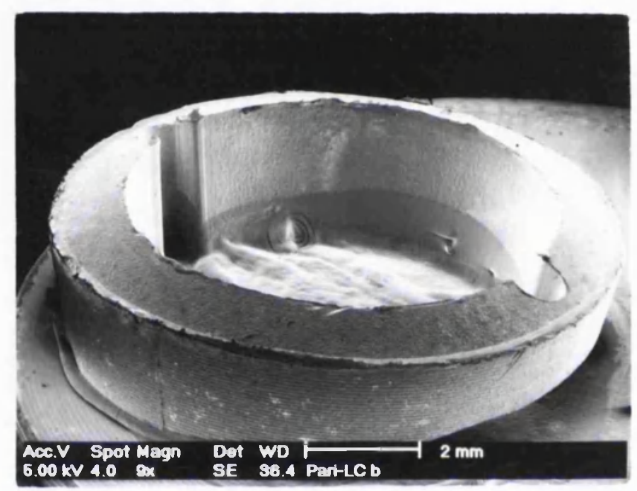

b(i) Pari-LC - gas and fluid orifice [960 $\mu \mathrm{m}]$

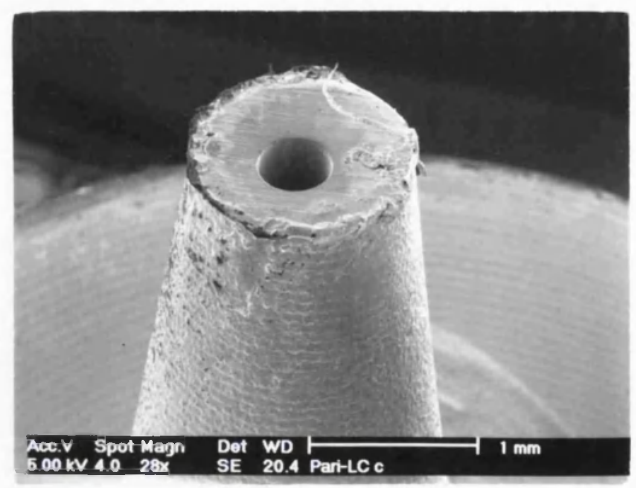

c(i) Pari-LC - gas jet orifice

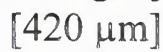

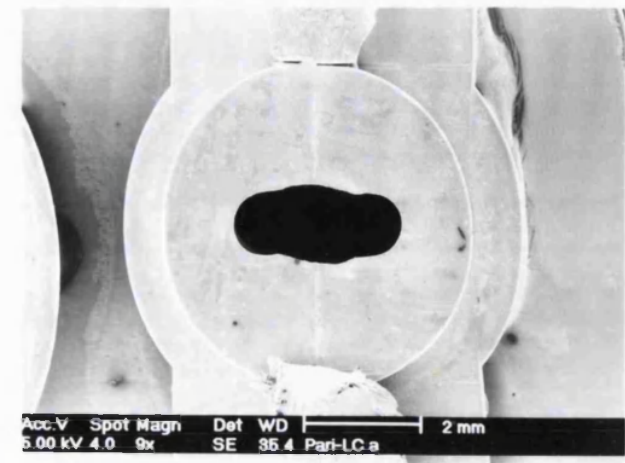

a(ii) Pari-LC - gas and fluid orifice

[1.30 mm]

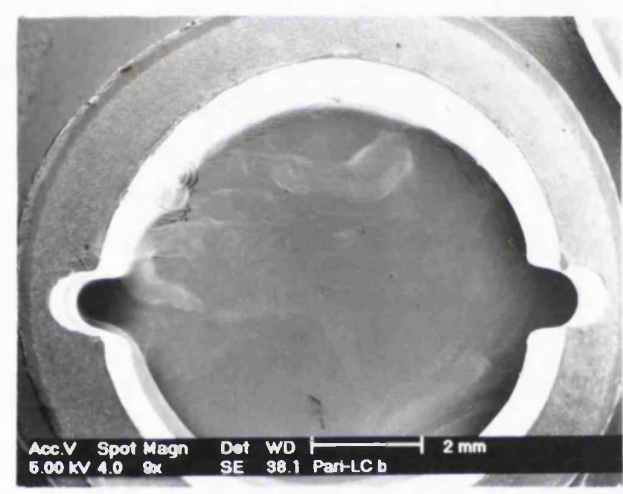

b(ii) Pari-LC - gas and fluid orifice [960 $\mu \mathrm{m}]$

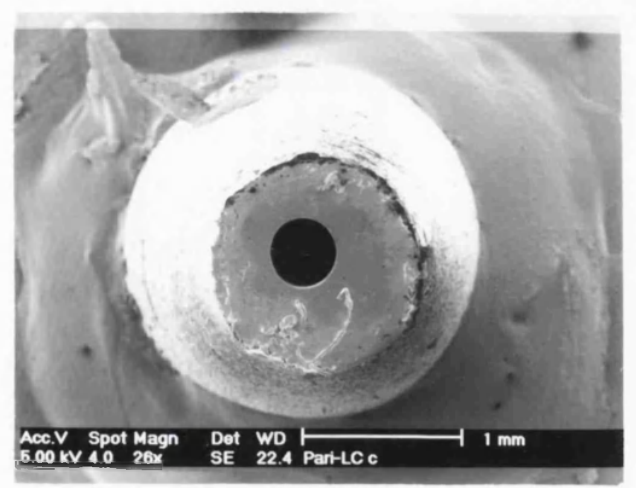

c(ii) Pari-LC - gas jet orifice [420 $\mu \mathrm{m}$ ]

Figure 3.32 Scanning electron micrographs of the various orifices within the Pari-LC jet nebuliser 


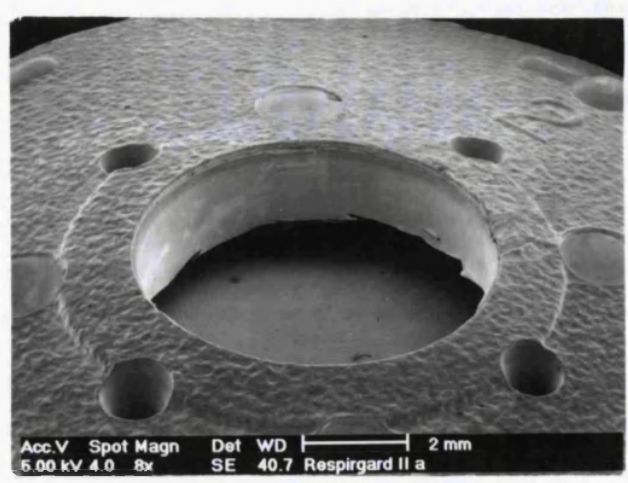

a(i) Respirgard II - fluid feed capillaries [1.40 mm]

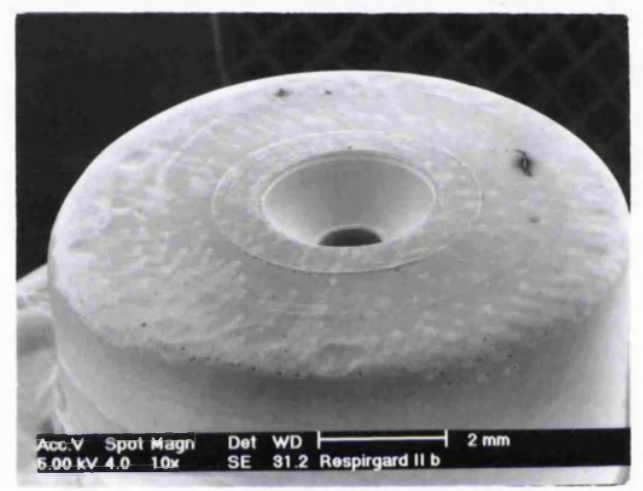

b(i) Respirgard II - gas and fluid orifice [1 mm]

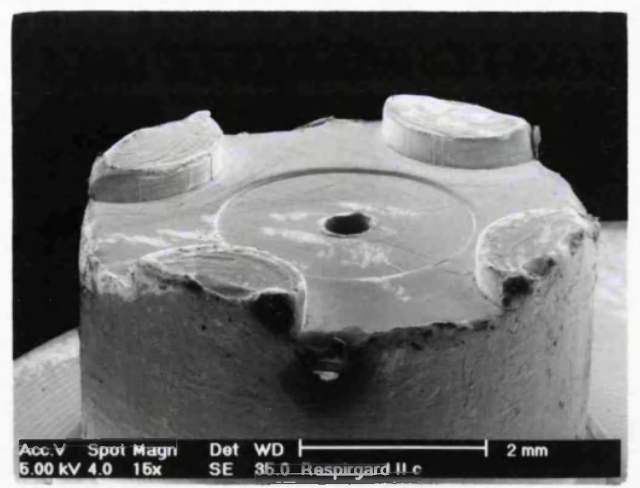

c(i) Respirgard II - gas jet orifice [450 $\mu \mathrm{m}]$

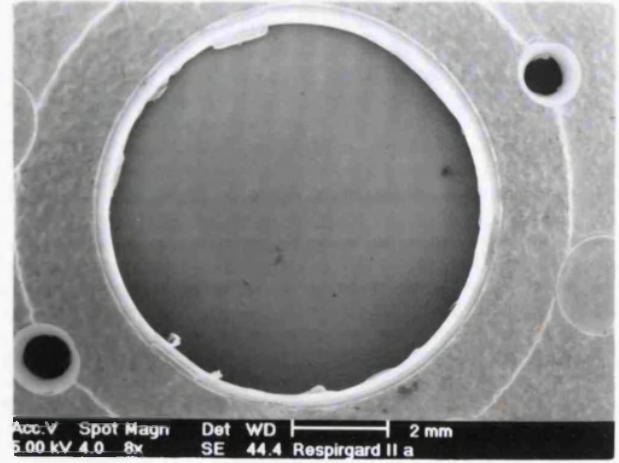

a(ii) Respirgard II - fluid feed capillaries $[1.40 \mathrm{~mm}]$

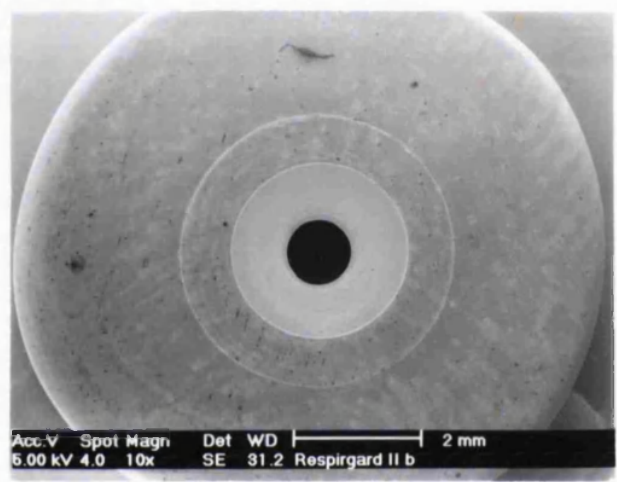

b(ii) Respirgard II - gas and fluid orifice [1 mm]

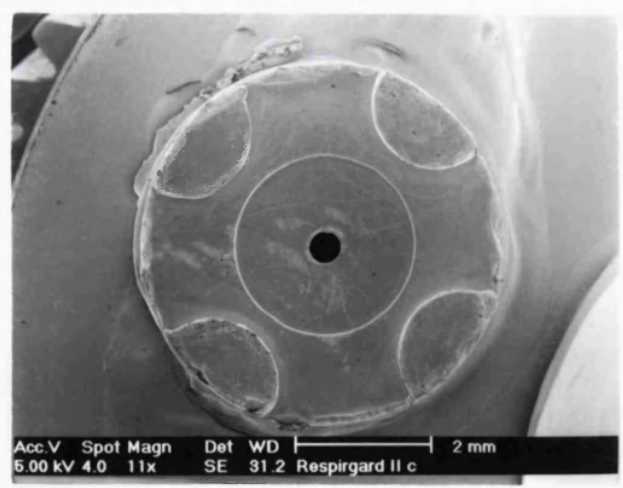

c(ii) Respirgard II - gas jet orifice $[450 \mu \mathrm{m}]$

Figure 3.33 Scanning electron micrographs of the various orifices within the Respirgard II jet nebuliser 


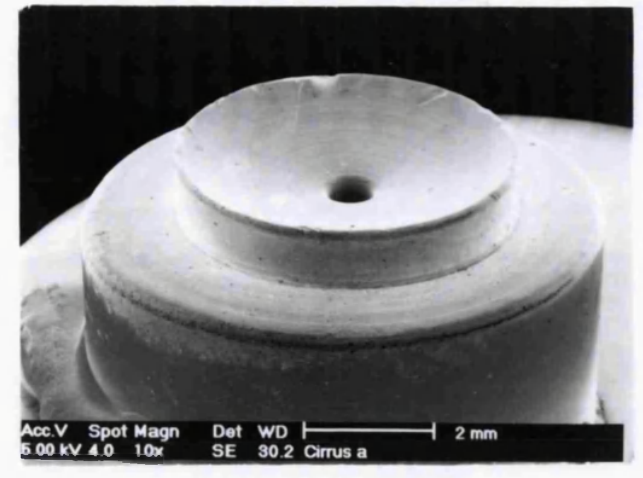

a(i) Cirrus - gas jet orifice

$[770 \mu \mathrm{m}]$

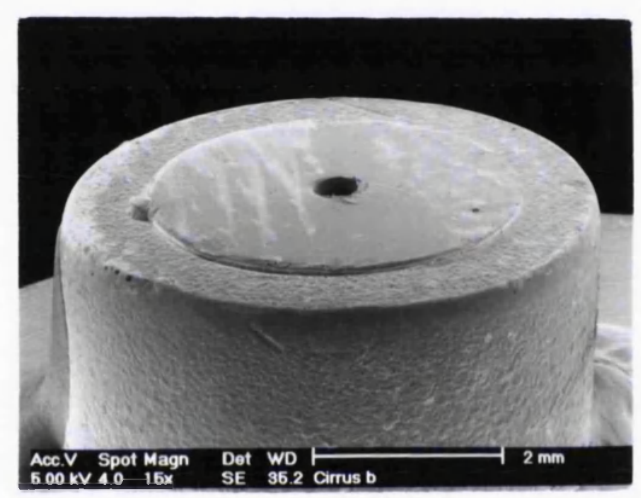

b(i) Cirrus - gas and fluid orifice [510 $\mu \mathrm{m}]$

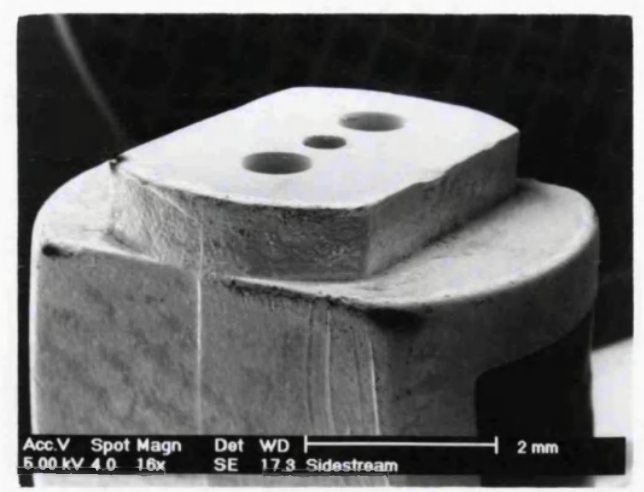

c(i) Sidestream - central gas jet and twin fluid feed capillaries

[Gas jet $=530 \mu \mathrm{m}$ ]

[Feed capillaries $=840 \mu \mathrm{m}]$

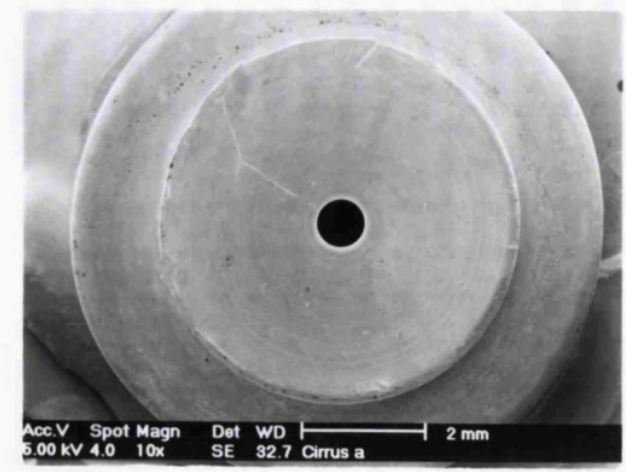

a(ii) Cirrus - gas jet orifice

$[770 \mu \mathrm{m}]$

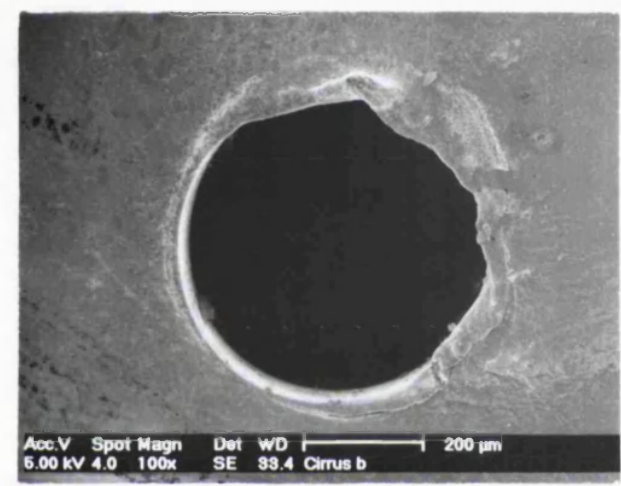

b(ii) Cirrus - gas and fluid orifice $[510 \mu \mathrm{m}]$

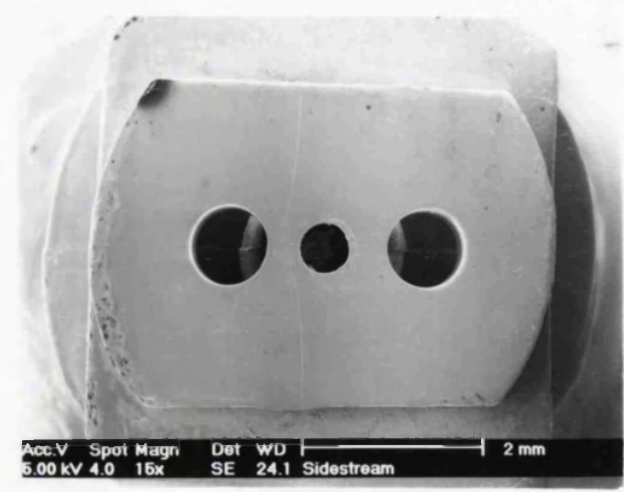

c(ii) Sidestream - central gas jet and twin fluid feed capillaries

[Gas jet $=530 \mu \mathrm{m}]$

[Feed capillaries $=840 \mu \mathrm{m}$ ]

Figure 3.34 Scanning electron micrographs of the various orifices within the Cirrus and Sidestream jet nebuliser 
The findings of the residual liposome study have important consequences for the output experiment results of section 3.4.3. As is the case with the majority of published studies of nebuliser output, the mass of aerosol released from the nebulisers was determined by measuring the mass of fluid lost during operation (eg. Clay et al., 1983b). The disadvantage to such a method is that it overestimates the output of liposomes during nebulisation, due to the significant evaporative loss of water vapour, and also the retention of liposomes occurring within the nebuliser. The results demonstrated that mass difference measurements, taken from liposome formulations with increased mean vesicle diameters, would significantly overestimate the liposome output. However, the results do confirm the various conclusions drawn from aerosol output study. Most notably, that the Sidestream nebuliser was found to give a significantly reduced output of aerosol during the nebulisation of $5 \mu \mathrm{m}$ liposomes. This has now been shown to be attributable to the failure of larger liposomes to become included within the smaller aerosol droplets which leave the device. However, the retention of liposomal lipid cannot be solely be attributed to the droplet size produced by the nebuliser, as the Sidestream yielded a significantly increased retention of liposomes to that produced by the Respirgard II, despite producing a larger droplet size. Therefore, other features of the nebuliser design must also contribute to the device's relative inability to nebulise liposomes with a mean size greater than the aerosol size. As previously stated, a number of researchers have noted that large liposomes become processed to a smaller size during nebulisation (Taylor et al., 1990b; Niven et al., 1991a). It is therefore likely that the retention of liposomes within the nebuliser may be in part controlled by the reduction in liposome size that a particular nebuliser causes during operation. A fall in the residual liposome size, in response to the shearing action of gas flow, would cause an increase in the number of liposomes that may become included within the droplets constituting the secondary aerosol mass. This would result in a reduced residual lipid concentration following nebulisation. Such a reduction in residual liposome size may be expected to be controlled, to an extent, by the design of the particular nebuliser model studied. Therefore, a feature of the Sidestream nebuliser design may produce a relatively low output of larger liposomes, attributable to the fact that the vesicles have a greater stability within the device. The relative stability of various liposome formulations to the nebulisation process was therefore investigated in chapter 4 , using a variety of nebuliser models. 


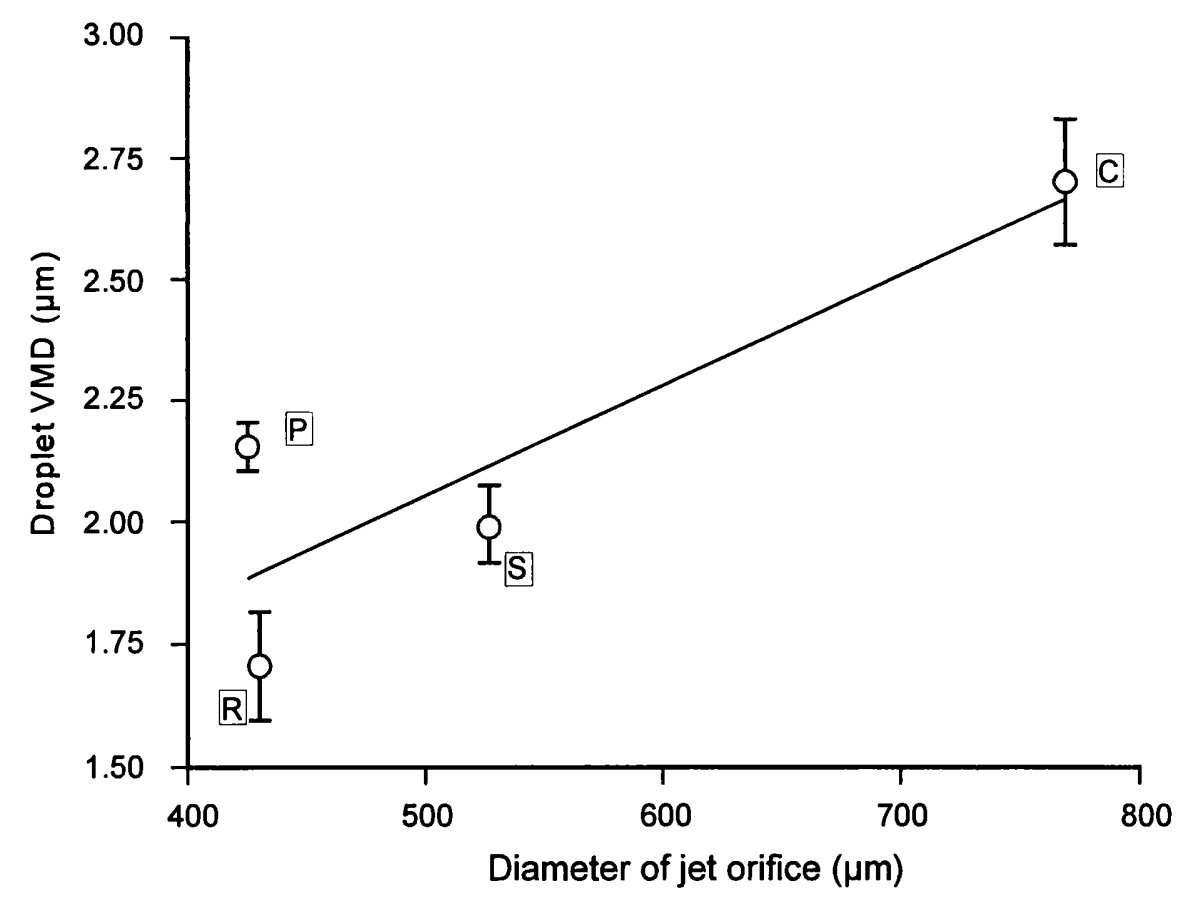

Figure 3.35 Relationship between the diameter of the gas jet orifice of various jet nebulisers $(C=$ Cirrus, $P=$ Pari-LC, $R=$ Respirgard II, and $S=$ Sidestream $)$ and the size of aerosol droplets produced from each device. [Equation of the line: $\mathrm{y}=0.0023 x+0.92 ; \mathrm{r}=0.873][ \pm \mathrm{sd} ; \mathrm{n}=3]$

It has been suggested that the mean size of droplets produced by a jet nebuliser is critically controlled by the size of the gas jet orifice (Nerbrink and Dahlbäck, 1994). The results from chapter 3 provides the first evidence to support this, as there is a direct correlation between the size of the gas jet orifice, and the droplet VMD (fig. 3.35). The Cirrus has the largest gas jet orifice, and accordingly produces the largest droplet size. A larger jet orifice means that driving gas is forced through the Venturi at a reduced velocity. This provides less shear energy for droplet formation, and larger droplets are therefore generated. The droplets are also aspirated from the nozzle at a reduced velocity, and hence a reduced fraction become impacted on the baffles. It is significant to note the similarity between the droplet size produced by the Pari-LC and the Sidestream, despite the Sidestream's larger jet. This confirms the Sidestream's efficient baffling system, and the impact of the open vent design. Additional gas drawn into the Sidestream device through the open vent increases droplet evaporation. As a result, a smaller droplet size is produced at a relatively low shear energy. Deviations from the relationship (the correlation coefficient, $r$, is 0.873 ) demonstrate that additional factors, such as the baffle efficiency, and rate of fluid delivery through the various channels, also influence the emitted droplet size. 


\subsection{Conclusions}

The mean droplet size within liposomal aerosols produced by different jet nebuliser models is found to be highly variable. This is due to significant variations in the nebuliser design and construction. Different nebuliser models, despite operating under the same basic principle, contain functional orifices which differ markedly in size, shape and orientation. For example, the diameter of the gas jet orifice differs by more than $50 \%$ between the Cirrus and Respirgard II nebulisers. This has demonstrable effects on the emitted aerosol size. A smaller gas jet orifice causes a more rapid flow of gas through the device, resulting in elevated shear forces, and an increased rate of aerosol entrainment, at a constant gas flow rate. Each of these will result in the generation of an aerosol with a smaller droplet size. Other components, such as the nebuliser baffles, may also be critical in determining the size of the emitted aerosol. In particular, an open vent nebuliser design appears to ensure that smaller droplets are produced, despite a relatively large gas jet orifice. This is likely to be due to an increase in droplet evaporation.

Despite such variations in design, each of the jet nebulisers investigated presently produced a mean aerosol droplet size of less than $5 \mu \mathrm{m}$. Each may therefore be predicted to give efficient deposition of liposomes within the lung. However, to target the alveolar region, a finer control of the droplet size produced by the aerosol delivery device is necessary. With this in mind, the Respirgard II and the Sidestream nebulisers may be most suitable, as they produced droplets with a mean VMD less than $2 \mu \mathrm{m}$. Such a droplet size is likely to produce maximal deposition of droplets in the alveolar region of the airways (Stahlhofen et al., 1980).

The operating conditions of the nebuliser may also influence the deposition of liposomes within the lung. The secondary aerosol droplet size is inversely proportional to the flow rate of gas through the device, due to its dependence on the shear energy and droplet inertia. The change in droplet size in response to changing flow rate is dependent on the nebuliser under study. The time taken to reach the sputtering phase is also inversely related to the driving gas flow rate, and varies markedly between different jet nebuliser models. The Sidestream nebuliser, operated at $8 \mathrm{l} / \mathrm{min}$ produces a small droplet size, and has a markedly reduced nebulisation time. This is attributable to the open vent design, which maximises the output rate, and the evaporation of aerosolised droplets. It may also be due to the high 
efficiency baffles of the device. As such, the Sidestream device may be the ideal nebuliser for achieving an increased pulmonary deposition of aerosol droplets, increased patient compliance, and reduced nursing times. However, the Ventstream and Pari-LC nebulisers are breathe-assisted, open vent nebulisers, which in clinical use may produce superior aerosol output and lung deposition, compared to the other less sophisticated devices (Knoch and Wunderlich, 1994).

The results demonstrate that the selection of nebuliser model, and operating conditions, in a clinical situation may be critical. In addition the nebulisation time must be selected with due regard to the nebuliser used. As the present study demonstrates, nebulising for a fixed time period, as is common in clinical situations, would lead to discrepancies in the proportion of dose that is delivered to the patient, should the same time period be used for different nebuliser models. The study of aerosol output by each nebuliser was necessary for a more complete understanding of the differences in nebuliser models with regard to nebulisation time.

The formulation of the liposomes may influence the liposomal aerosol performance. Whilst the droplet size is independent of the liposome size and bilayer composition, it is critically influenced by the lipid concentration. An increase in the lipid concentration causes a rise in the viscosity of the fluid, and an increase in the number of liposome bilayers within the system (chapter 2). This produces an associated rise in the secondary aerosol size, which may be attributed to various mechanisms, including an increased fluid viscosity, bilayer fracture, vesicle aggregation, or an inhibition of droplet evaporation. The extent of the droplet size increase is dependant upon the efficiency of the nebuliser baffles, and other design factors. As a result, a nebuliser such as the Sidestream, is capable of minimising the lipid concentration effects. However, this may be at the expense of a reduced output rate. The concentration of liposomes therefore has important consequences for the nebulisation time. Higher concentration formulations also produce protracted nebulisation times, due to a reduction in the aerosol output rate. This is attributable to the increased viscosity of high concentration liposome samples, and to the greater filtration of droplets, in response to the rise in the primary droplet size.

It is thus clear, that an increase in liposome concentration yields an associated increase in 
the droplet size of the primary aerosol, consistent with atomization theories, and that the resulting larger droplet size is compensated for by an increased retention of droplets within the nebuliser. This is evidenced by a reduction in output rate, and hence an increased nebulisation time, for the more concentrated samples. Such changes may have important consequences for the efficiency of liposome delivery to the respiratory tract. The droplet size produced by medical nebulisers is one of the most important determinants of the clinical efficacy of pulmonary delivered drugs (Ferron et al., 1981). Any shift in the secondary aerosol to a larger mean size, may potentially reduce the lung deposition of a delivered agent.

Whether the rise in droplet size caused by increasing lipid concentration would be significant enough to produce a demonstrable change in the deposition pattern in vivo is unclear, as there have been few clinical studies relating droplet size to deposition pattern. Studies by Hadfield et al. (1986) and Douglas et al. (1986), failed to demonstrate a significant change in clinical response upon changing droplet size. However, in each of these studies only the upper respiratory tract response was investigated. A subsequent study of carbenicillin, nebulised to cystic fibrosis patients, recorded a $50 \%$ reduction in lung deposition with an increase in the mean droplet size from 3.2 to $7.3 \mu \mathrm{m}$ (Newman et al., 1988). Furthermore, Patel et al. (1990) demonstrated an increased bronchodilation in asthmatic patients with a reduction in nebulised salbutamol droplet size from $5 \mu \mathrm{m}$ to 2.5 $\mu \mathrm{m}$. In each of these studies, the differences in the size of the droplets evaluated were greater than that resulting from an increase in the lipid concentration of liposomes from 2.5 to $80 \mathrm{mg} / \mathrm{ml}$. The maximal change in droplet size experienced upon changing lipid concentration in the present experiment was $40 \%$. It is therefore unclear whether such a droplet size increase will be important for inhaled liposomes. However, the nebulised droplet size must be controlled carefully for alveolar targeting, as the droplets depositing within this area lie within a narrowly defined size range. The deposition of droplets within the alveolar region may therefore be affected to a greater extent by the more subtle changes in droplet size brought about by an increase in lipid concentration. For instance, Vidgren et al. (1995) demonstrated that liposomal beclomethasone was retained in the lung for extended periods, when delivered by droplets with a mean size of $1.5 \mu \mathrm{m}$, compared with those having a mean size of $3.6 \mu \mathrm{m}$. Presumably, this was due to greater deposition of the liposomes in the non-ciliated alveolar regions, following delivery in the smaller droplets. 
Further in vivo studies are therefore required to confidently predict the clinical significance of varying the lipid concentration of nebulised liposomes. Clearly, increasing the lipid concentration of liposome formulations to compensate for poor drug entrapment would be counter productive if this resulted in a reduction in the respirable, or alveolar region depositing dose.

The output of aerosol from a nebuliser may be as critical in determining the clinical response as the droplet size (Nebuliser Project Group, 1997). The results of chapter 3 demonstrate that the Pari-LC and Sidestream nebulisers produce similar outputs of aerosol, despite significant differences in their design. The effect of an increased lipid concentration in both nebulisers is to reduce the aerosol output rate. This is due to the increase in the mean droplet size that a rise in the lipid concentration produces. Larger droplets result in an increase in the fraction of the primary aerosol that becomes retained on the jet nebuliser baffles.

There is evidence to suggest that the relatively small influence of lipid concentration on the secondary aerosol size produced by the Sidestream nebuliser is due to efficient baffling. In the Sidestream, differently sized liposomes consistently produce a fall in the aerosol output with an increasing lipid concentration. This was indicative of an increase in the fraction of droplets that became trapped on baffles. However, the Pari-LC gave a significantly reduced aerosol output with increasing concentration in the larger size liposomes studied, but not in the smaller. Therefore, it is likely that the reason that the mean size of droplets emitted from the Pari-LC nebuliser is greatly influenced by the lipid concentration, is because the baffles of the device fail to fully compensate for an increase in the primary aerosol size. There is also a suggestion that larger liposomes are associated with reduced aerosol outputs. This was apparent in both nebulisers studied. The larger liposomes apparently become included within a greater proportion of the large primary droplets that fail to escape from the nebuliser, and hence the aerosol output is reduced.

The present study determined the liposomal aerosol output using a simple method of measuring changes in the mass of the nebuliser fluid during operation. Despite the fact that this is commonly employed in published nebuliser studies, it is a flawed technique in so much as it fails to take into account the liposome concentration within the aerosol droplets, 
relative to the concentration within the nebuliser chamber. An increase in the concentration of the nebulised fluid occurs during operation, due to evaporative losses from the recycling primary droplets. In addition, there is the possibility of an exclusion of larger liposomes from the emitted secondary aerosol droplets. Therefore, in order to determine the output of liposomes accurately, a knowledge of the initial and final liposome concentration within the nebuliser fluid is required.

Section 3.4.4 determined that the Sidestream nebuliser retained a greater quantity of liposomes within the residual fluid than the Pari-LC. Thus, despite releasing a similar quantity of aerosol, the Sidestream is unlikely to yield a significantly increased delivery of liposomes. This serves to illustrate the limitations of the mass difference method for the determination of liposomal aerosol output. The Sidestream nebuliser manages to produce a similar output of aerosol at elevated lipid concentrations compared with the Pari-LC device, despite a relatively unchanged aerosol size. However, the study of the residual lipid concentration within each device indicates that nebulisation of liposomes with the Sidestream nebuliser is associated with an increased residual lipid concentration, and as such the Sidestream nebuliser fails to produce an increased output of liposomes compared with the Pari-LC.

The evaporation of solvent from a nebulised solution leads to an increase in the residual solute concentration. This was evidenced by an increase in the concentration of a sodium chloride solution, which remained within each nebuliser following operation. The amount of evaporation occurring is dependant on the nebuliser under study, and is related to the rate of aerosol output. Generally, longer nebulisation times allow greater evaporative losses to take place, and are thus associated with increased residual solution/dispersion concentrations. However, the Sidestream deviates from this pattern, producing significantly increased residual masses, despite producing the shortest nebulisation time. This is due to the influence of the open vent design of the Sidestream. Greater evaporation occurs from droplets in the Sidestream, due to the enhanced gas flow through the device during nebulisation. This design feature ensures a reduced droplet size, and rapid output rates are produced, but it also increases the residual fluid concentration, due to the recycling of primary droplets. 
The evaporation occurring from liposome dispersions during nebulisation has been shown to be greater than that occurring for a simple saline solution. This may be attributed to differences in surface tension. However, as the mean liposome size is increased so the residual masses become more elevated. This supports the proposed mechanism of liposome aerosolisation. Smaller liposomes are able to leave the nebuliser more readily as they enter a greater number of the smaller secondary aerosol droplets. As a result, smaller liposomes are associated with reduced residual lipid masses. It can therefore be concluded that the nebulisation of large liposomes is associated with a reduced total liposomal aerosol output. This is in addition to the recognised fact that larger liposomes are more unstable to the nebulisation process (Taylor et al., 1990b; Niven et al., 1991a).

The Sidestream nebuliser increased the retention of liposomes compared with similar jet nebulisers, due again to the open vent design. The nebuliser design is therefore an important factor in controlling the output of liposomes. In particular, the emitted droplet size may be important, as smaller secondary droplet sizes, despite providing greater respirable doses, are associated with an increased retention of liposomes. Large liposomes are excluded from the smaller droplets of the secondary aerosol cloud, and are thus retained within the nebuliser.

The proposed mechanism of liposome aerosolisation may have important clinical consequences. Previously, the only requisite for minimising the mean size of nebulised liposome formulations was when maintenance of the liposome bilayer integrity was important. The present study suggests that the mean liposome size should also be minimised, in order to maximise the liposomal aerosol output. However, the nature of the liposomes remaining within the nebuliser chamber requires investigation. The present experiment determined the concentration of lipid, and not liposomes, remaining within the nebuliser chamber following nebulisation. The residue remaining from the nebulisation of large liposomes may equally have consisted of phospholipid bilayer fragments. If this had been the case, then the output of liposomes from each of the jet nebulisers may have been constant, regardless of the mean vesicle size, with the generation of smaller liposomes from formulations with a larger mean vesicle size. This hypothesis was further investigated in the experiments detailed in chapter 4 . 


\section{THE STABILITY OF LIPOSOMES TO NEBULISATION}

\subsection{Introduction}

The aim of the experiments detailed in chapter 4 was to identify physical changes that occur in liposomes which remain within the chamber of jet nebulisers during operation. This was accomplished by studying the size characteristics of liposomes in residual nebuliser fluids, in conjunction with a study of the release of an entrapped aqueous marker from such liposomes. The mechanism by which liposomes of differing size, and bilayer composition, become aerosolised was also investigated, using a variety of nebuliser models, and driving gas flow rates.

Less than $1 \%$ of the droplets which are aspirated from a nebuliser nozzle(s) at any one time become emitted from the device (Mercer, 1973; Newman et al., 1987). The greater majority, representing the non-respirable fraction, become deposited on internal structures, and fail to leave the device. In this way, the emitted aerosol is conditioned to contain a greater proportion of smaller, more respirable droplets. This increases the proportion which are of an appropriate size for delivery to the lung, thus providing the potential for an increased efficacy. However, a drawback to droplet filtering, is that it causes protracted treatment times, and exposes the nebulised material to processes which may reduce its activity/drug carrier function (Niven, 1996). Sensitive materials have been shown to include protein and peptide drugs, which may be delivered via the lung as a convenient route for systemic delivery (eg. O'Hagan and Illum, 1990; Patton and Platz, 1992). Most protein and peptide drugs are developed as aqueous formulations, and so they are particularly suitable for delivery via nebulisers. Investigations into the nebulisation of proteins have been stimulated by recent studies demonstrating that protein and peptide formulations, including insulin, may produce significant systemic effects following pulmonary delivery (Elliott et al., 1987; Byron and Patton, 1994). A drawback to the delivery of such materials via a nebuliser is that they may become destabilised during aerosolisation. For instance, $75 \%$ of the initial activity of lactate dehydrogenase $(\mathrm{LDH})$ was lost during aerosolisation with a Collison nebuliser (Niven et al., 1994). A similar instability was exhibited by $\alpha$-interferon (Ip et al., 1995). The nebulisation of liposomes is associated with similar stability concerns, generating vesicles of a reduced size, and liberating entrapped materials (eg. Taylor et al., 1990b; Niven et al., 1991a). 
A number of factors have been identified as being responsible for such instabilities. The shear energy provided by gas flow through the jet nebuliser acts to disrupt the nebulised fluid into fine droplets, but also exposes dissolved or dispersed materials to significant levels of disruptive energy. Minimising the energy that is applied to a jet nebulised system, by reducing the gas flow rate, has been shown to significantly reduce the damage caused to proteins (Niven et al., 1994), and to liposomes (Niven et al., 1992a). However this may be detrimental to the aerosol characteristics (see chapter 3).

The vast air-fluid interface that is formed within a jet nebuliser during operation may also cause damage to sensitive materials. Hydrophobic regions which are normally located within the interior of a protein molecule, may establish contact with the hydrophobic air interface, resulting in the rearrangement of macromolecular groupings, and the consequent destruction of active sites (Andrews, 1991). Similar interactions may occur when proteins deposit on the plastic structures of the nebuliser chamber (Thurow and Geisen, 1984). It may be postulated that a similar mechanism destabilises liposomes. The lamellar nature of liposomes is dependent on the self-association, and subsequent internalisation, of the constituent phospholipid fatty acid groupings during hydration. Such structural arrangements may become altered if the liposome is exposed to the relatively hydrophobic air-liquid interface, or to plastic surfaces. In such an event, rearrangement of the acyl groupings, in order to associate with the more compatible hydrophobic environment, may be favoured, thus stressing the bilayer organisation, and ultimately leading to liposome disruption. Protein damage during nebulisation has also been ascribed to the rise in concentration that occurs as a result of evaporative losses from the nebulised fluid (Niven et al., 1994; chapter 3). This may cause the interaction of charged sites, establishment of van de Waal's forces, hydrogen bonding, and steric hindrance/interaction between adjacent macromolecules. Similar processes may also be envisaged for liposomes, which are known to exhibit aggregation, and fusion (Wong and Thompson, 1982).

The modern jet nebuliser represents a balance between achieving an acceptably short nebulisation time, and producing a suitably small droplet size. However, it is clear that the nebulisation of materials such as liposomes introduces additional stability considerations. A compromise may be required between the attainment of an aerosol with suitable characteristics for rapid and efficient delivery to the lung, whilst maintaining liposome 
stability. Consideration of the likely causes of liposome damage suggests that their stability may depend on the characteristics of the nebuliser, and also on the operating conditions. An ideal nebuliser would be one capable of performing at a $100 \%$ efficiency of respirable aerosol output, as a liposome would be exposed to the damaging process of aerosolisation on only a single occasion. The efficiency of aerosol output produced by a nebulised system may therefore be as critical as the droplet size characteristics, when determining its suitability for delivering liposomes. However, an output efficiency of $100 \%$ may only be achieved by removing the baffles from a jet nebuliser, such that all of the primary droplets become emitted from the device. With the baffle removed, the aerosol droplets would be unacceptably large (between 15 and >500 $\mu \mathrm{m}$ - Nerbrink and Dahlbäck, 1994), and the volume flow of fluid to the patient too rapid (between 1 and $3 \mathrm{ml} . \mathrm{s}^{-1}$ - Niven., 1996). An alternative strategy for reducing droplet recycling must therefore be sought, in order to improve the liposome stability. If the droplets that leave the nebuliser jet(s) are of a suitably small size such that each avoids impaction on baffles, prolonged exposure of liposomes to damaging processes may be avoided. However, this may only be achieved by an increase in the shear forces operating within the nebuliser, which may in turn enhance the damage done to the liposomes. The use of jet nebulisers designed to operate at higher flow rates, and possessing highly efficient baffles may give an increased delivery of liposomes to the peripheral regions of the lung, due to a reduction in droplet size. However, this may be at the expense of a poor liposome stability.

Different nebulisers have been shown to differ markedly with regard to the aerosol droplet size and output that they produce when nebulising liposome formulations. An important factor determining the ability of a particular nebuliser to aerosolise liposomes was the droplet size that the device produced (chapter 3). The droplet size in turn is controlled by the gas flow rate, the performance of the nebuliser baffles, the characteristic design of the constituent parts, and the physicochemical properties of the nebulised fluid. As a consequence, different nebuliser systems may be predicted to differ in the damage they cause to liposomes during operation. The experiments of chapter 4 were designed to investigate this. The droplet size produced by nebulisers is generally unaffected by changes in the liposome size and bilayer composition (Niven and Schreier, 1990; Niven et al., 1991a; Chapter 3). However, the liposomal aerosol output is reduced when large liposomes, or liposomes with rigid, gel state bilayers are nebulised (chapter 3). From this, it was 
postulated that large liposomes may only become included within a significant fraction of the smaller secondary aerosol droplets, following shear-induced bilayer rupture and subsequent reduction in vesicle size. Liposomes composed of eggPC/chol (1:1) have stable bilayers which resist bilayer rupture (chapter 2). This causes larger vesicles to be excluded from secondary droplets, and therefore results in a reduced liposomal aerosol output (chapter 3). This may result in the size of retained vesicles increasing during operation, as the smaller vesicle population becomes preferentially released. Alternatively, the size of the retained vesicles may decrease as the larger liposomes become fragmented. The experiments detailed in chapter 4 investigated the nature of the retained liposomes. In so doing, they may provide information regarding the mechanism of liposome aerosolisation, and that of the nebulisation process in general. It was also the intention to identify the formulation variables of the liposomes, and also the characteristics of the nebulisers, that improved liposome stability during nebulisation.

\subsection{Materials}

\section{Reagents}

Cholesterol, Anala-R (99-99.4\%)

$\mathrm{BDH}, \mathrm{UK}$

Dipalmitoyl phosphatidylcholine Sigma Chemicals, USA

Deionised water WR50 RO/Deioniser, Whatman, UK

Lecithin from eggs $(90 \%)$, purified Sigma Chemicals, USA

as detailed in section 2.3.1

Polycarbonate membrane filters, - $\quad$ Nucleopore, UK

$2.5 \mathrm{~mm}$ diameter

(1 3 , and $5 \mu \mathrm{m}$ pore size)

Triton $\mathrm{X}-100^{\circ}$ (iso-octylphenoxy

polyethoxyethanol), (98-102\%) - $\quad$ BDH, UK

\section{Nebulisers}

Cirrus nebuliser

- $\quad$ DHD Medical Products, USA

Pari-LC nebuliser

- Pari-Werk, Germany

Respirgard II nebuliser

- $\quad$ Marquest, USA

Sidestream nebuliser

- $\quad$ Medic Aid, UK 


\subsection{Methods}

\subsubsection{The effects of nebulisation on the size of residual liposomes}

EggPC and DPPC/chol (1:1) liposomes were prepared by the thin-film technique, as detailed in section 2.3.2. Deionised water was used as the aqueous phase, to yield a total lipid concentration of $10 \mathrm{mg} / \mathrm{ml}$. The mean liposome size was reduced to $5 \mu \mathrm{m}$ by membrane extrusion (see section 2.3.4.1), and $5 \mathrm{ml}$ of each formulation nebulised with the Cirrus, Pari-LC, Respirgard II, or Sidestream nebulisers, at a nitrogen gas flow rate of 8 $1 / \mathrm{min}$. The aerosol emitted from the eggPC liposomes was directed through the laser of the Malvern 2600c laser diffraction sizer, and 6 measurements made at regular intervals during nebulisation to sputtering, to determine the mean droplet VMD (as detailed in section 3.3.2). A sample of the liposomes was withdrawn from the nebuliser every 30 seconds, and analysed using the $63 \mathrm{~mm}$ lens of the laser diffraction sizer (as detailed in section 2.3.3).

\subsubsection{The influence of liposome size and nebulisation time on the stability of liposomes} to nebulisation

EggPC and eggPC/chol (1:1) liposomes were prepared by the thin-film technique, using deionised water as the aqueous phase, giving a lipid concentration of $20 \mathrm{mg} / \mathrm{ml}$. The mean liposome size was reduced to 5 or $2.5 \mu \mathrm{m}$ by membrane extrusion (section 2.3.4.1). Five $\mathrm{ml}$ of the eggPC liposomes was then nebulised using the Cirrus, Pari-LC, Respirgard II, or Sidestream nebulisers, operated at a gas flow rate of $7 \mathrm{l} / \mathrm{min}$, until dryness. The aerosol produced was directed through the laser of the Malvern 2600c laser diffraction sizer, and 6 measurements made at regular intervals during nebulisation to sputtering, in order to determine the mean droplet VMD. The time taken for the output of aerosol to reach the sputtering phase was recorded for each nebuliser by visual inspection. $5 \mathrm{ml}$ of each liposome formulation was then nebulised to $50 \%$ and $100 \%$ of this nebulisation time. Residual samples were drawn out using a Pasteur pipette at the end of each time interval, and analysed using the laser diffraction sizer.

\subsubsection{The release of SCG from SCG-containing liposomes during nebulisation}

EggPC and eggPC/chol (1:1) SCG-containing liposomes were prepared by the thin-film technique, as detailed in section 2.3.5, using $30 \mathrm{mg} / \mathrm{ml} \mathrm{SCG}$ solution as the aqueous phase, to a total lipid concentration of $80 \mathrm{mg} / \mathrm{ml}$. Liposomes were prepared in $25 \mathrm{ml}$ batches, and combined to produce a total volume of $500 \mathrm{ml}$. The liposomes were then diluted to a total 
lipid concentration of $40 \mathrm{mg} / \mathrm{ml}$ with deionised water. The liposomes were reduced in size by probe sonication ( $150 \mathrm{~W}, \mathrm{MSE}, \mathrm{UK})$ to $5,2.5$ or $1 \mu \mathrm{m}$, as detailed in section 2.3.4. The unentrapped SCG was removed prior to nebulisation by ultracentrifugation (MSE Superspeed 75 , pre-equilibrated to $10^{\circ} \mathrm{C}$ ) of six $10 \mathrm{ml}$ liposome samples at $200000 \mathrm{~g}$ for 30 minutes, as detailed in section 2.3.5. The supernatant was removed, and $10 \mathrm{ml}$ of deionised water was added to the resulting pellet. Contaminating titanium was separated from the liposomes by vortex mixing. The liposomes were then "washed" by adding $10 \mathrm{ml}$ of water, and respinning at $200000 \mathrm{~g}$ for a further 20 minutes. The supernatant was removed, and the resulting pellets were redispersed by the addition of $5 \mathrm{ml}$ of deionised water, followed by subjection to a 2 minute period of vortex mixing. The volume was then made up to $10 \mathrm{ml}$ with deionised water, and the liposome size established by laser diffraction. $5 \mathrm{ml}$ of the SCG-containing liposomes was then aerosolised with the Pari-LC and Sidestream nebulisers, operated at 4 or $7 \mathrm{l} / \mathrm{min}$ until sputtering. The liposomes within each nebuliser were sampled at 0 and $100 \%$ of the nebulisation time by withdrawl of 0.5 $\mathrm{ml}$ of the liposomes. Each sample was added to a centrifuge tube, and made up to $10 \mathrm{ml}$ with water. The tube was then respun in the centrifuge for a single time at $200000 \mathrm{~g}$ for 15 minutes. The supernatant was then analysed at a wavelength of $326 \mathrm{~nm}$ using a UV spectrophotometer (as detailed in section 2.3.5), and the unentrapped SCG following nebulisation determined. The initial concentration of unentrapped SCG prior to nebulisation was determined for each liposome formulation by redispersal of a spun liposome pellet to $10 \mathrm{ml}$ with deionised water, using a vortex mixer for 2 minutes. $1 \mathrm{ml}$ of the liposomes was then made up to $10 \mathrm{ml}$ with deionised water, and this was then spun for 15 minutes at $200000 \mathrm{~g}$, and the supernatant analysed at $326 \mathrm{~nm}$ using a UV spectrophotometer. Triton $\mathrm{X}-100$ was used to disrupt the liposome bilayers in order to assess the entrapment of SCG by the liposomes (as detailed in section 2.3.5). The fraction of entrapped SCG that became liberated from the liposomes during nebulisation was then calculated by reference to the solute entrapment at time zero. This was determined by redispersing a spun liposome pellet of each formulation to $10 \mathrm{ml}$ with deionised water. $0.5 \mathrm{ml}$ of the liposomes was then taken, $0.5 \mathrm{ml}$ of Triton X-100 (10\% v/v) added, followed by dilution to $10 \mathrm{ml}$ with deionised water. The liposomes were then incubated in sealed test tubes, at $56{ }^{\circ} \mathrm{C}$ in a water bath for 6 hours, cooled, and spun at $200000 \mathrm{~g}$ for 30 minutes. The resulting supernatant was analysed using a UV spectrophotometer, allowing the amount of SCG released during nebulisation to be calculated. 


\subsubsection{The collection of aerosolised liposomes}

EggPC and eggPC/chol $(1: 1)$ liposomes $(10 \mathrm{mg} / \mathrm{ml})$ were prepared by the thin-film technique with deionised water as the aqueous phase. The mean vesicle size of each formulation was reduced by membrane extrusion to 5 and $2.5 \mu \mathrm{m}$, as detailed in section 2.3.4. Five $\mathrm{ml}$ of each liposome formulation was then added to a Pari-LC, or Sidestream nebuliser. The nebuliser under study was operated at a driving gas flow rate of $7 \mathrm{l} / \mathrm{min}$, until the sputtering phase. The aerosol was directed into a glass twin impinger, (Copley Instruments, UK), operated at a gas flow throughput of $60 \mathrm{l} / \mathrm{min}$, and containing $7 \mathrm{ml}$ of deionised water in the upper stage, and $30 \mathrm{ml}$ in the lower stage. Once the aerosol output had reached $50 \%$ of the nebulisation time, a small sample of the residual liposomes remaining within each nebuliser chamber was withdrawn using a Pasteur pipette. The mean residual liposome size, and size distribution, were determined for a drop of each sample using the Malvern laser diffraction sizer, as detailed in section 2.3.3. The liposomes which had deposited within the upper and lower stages of the TI were then sampled and analysed. The TI was then cleaned thoroughly, and reassembled. The remaining residual liposomes were then nebulised to the sputtering phase (100\% of nebulisation time), and the residual liposomes within the nebuliser, and liposomes within the upper and lower stages of the TI analysed as previously detailed.

\subsubsection{The intra-nebuliser variability in the generation of liposomal aerosols}

Five different Pari-LC and Sidestream nebulisers were selected for investigation. EggPC and eggPC/chol (1:1) liposomes (10 $\mathrm{mg} / \mathrm{ml}$ lipid concentration) were prepared by the thinfilm method, and the mean liposome size reduced to $5 \mu \mathrm{m}$ by membrane extrusion. $5 \mathrm{ml}$ of each liposome formulation was then nebulised using each of the Pari-LC and Sidestream nebuliser devices, with a driving gas flow rate of 4 and $71 / \mathrm{min}$. The mean time taken for nebulisation to reach the sputtering phase was recorded for each model by visible inspection of the emitted aerosol. The mean droplet size was then determined for each model, following the protocol outlined in section 4.3.2. Nebulisation was then repeated to the nebulisation time particular to each model. The residual liposomes were sampled at $50 \%$ and $100 \%$ of the nebulisation time, by withdrawl of a small sample from the nebuliser chamber using a Pasteur pipette. The liposome size distribution characteristics of the residual liposomes remaining within each device were then analysed using the Malvern 2600c laser diffraction sizer (as detailed in section 4.3.2). 


\subsection{Results and discussion}

\subsubsection{The effects of nebulisation on the size of residual liposomes}

The mean droplet size produced by each nebuliser when operated to sputtering under the conditions of the experiment were recorded (table 4.1), in order that the stability of the different liposome formulations could be related to the mean droplet size produced by each device.

Table 4.1 The mean droplet VMD produced by various jet nebulisers during nebulisation of eggPC liposomes $(5 \mu \mathrm{m}$ mean size, $10 \mathrm{mg} / \mathrm{ml}$ lipid

\begin{tabular}{|c|c|}
\hline \multicolumn{2}{|c|}{ concentration) at $8 \mathrm{l} / \mathrm{min} .[\mathrm{n}=3 ; \pm \mathrm{sd}]$} \\
\hline Nebuliser & VMD $(\boldsymbol{\mu m})$ \\
\hline Cirrus & $2.70( \pm 0.12)$ \\
\hline Pari-LC & $2.15( \pm 0.05)$ \\
\hline Respirgard II & $1.70( \pm 0.11)$ \\
\hline Sidestream & $1.99( \pm 0.08)$ \\
\hline
\end{tabular}

Figure 4.1 shows the change in the size of liposomes remaining within the nebuliser chamber of the Cirrus, Pari-LC, Respirgard II and Sidestream jet nebulisers, during nebulisation. Each formulation underwent a progressive reduction in residual liposome size during nebulisation, indicating that vesicle damage was occurring. The results are consistent with a number of authors who have noted the processing of liposomes during studies of liposomal aerosols. Gilbert et al. (1988) demonstrated that the proportion of eggPC liposomes within the 100 to $750 \mathrm{~nm}$ size range was increased from $37 \%$ to $91 \%$ during nebulisation with a Collison nebuliser. The size reduction of large MLVs during jet nebulisation was further demonstrated by Vidgren et al. (1995). The damage done to liposomes in the present study was clearly dependent on the bilayer composition of the liposomes, and to an extent on the particular nebuliser used for aerosolisation. There was a significant difference $(\mathrm{p}<0.05)$ between the damage done to the eggPC and DPPC/chol $(1: 1)$ liposomes. Each nebuliser produced similar levels of damage to the residual liposomes, except for the Respirgard II, which produced significantly greater damage than each of the other nebulisers $(p<0.05)($ table 4.2$)$. 

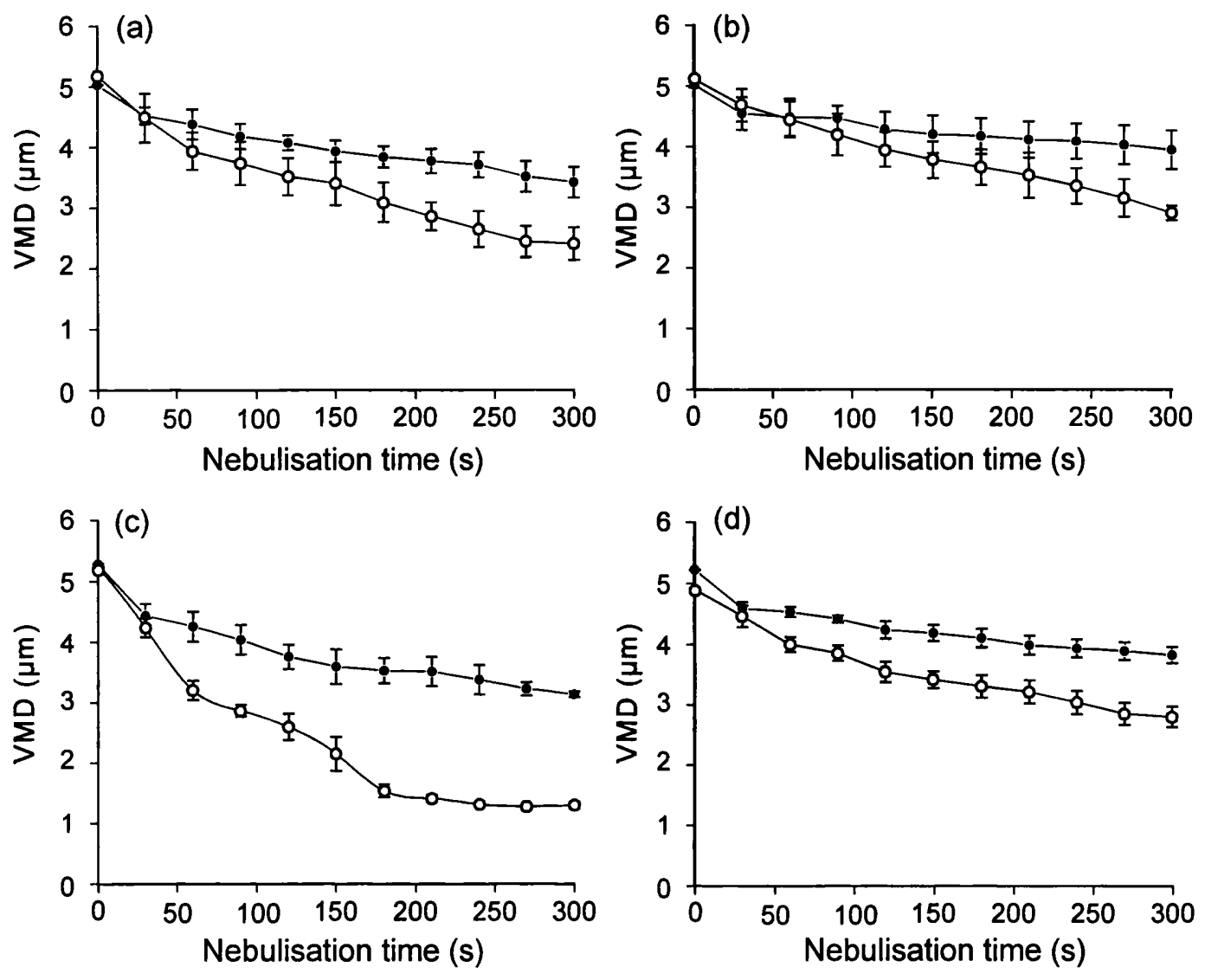

Figure 4.1 The mean size of residual eggPC $(O)$ and DPPC/chol $(1: 1)(\bullet)$ liposomes during nebulisation with the (a) Cirrus (b) Pari-LC (c) Respirgard II and (d) Sidestream nebulisers. $[ \pm$ sd; $n=3]$

As discussed in section 4.1 there are a number of factors contributing to the liposome instability observed during nebulisation. Principal amongst these is thought to be the shear energy that the liposomes are exposed to within the nebuliser (Taylor et al., 1990b; Niven et al., 1992a). The passage of high velocity gas through the device causes direct physical damage to the liposome bilayers, leading to bilayer rupture, and the generation of smaller liposomes. The following mechanism for liposome aerosolisation from jet nebulisers is proposed, based on the results of chapter 3 and chapter 4. As a droplet is produced from the liquid capillaries within the nebuliser it may take with it one or more liposome(s). A liposome with a diameter that is larger than the mean primary aerosol size will tend to be included within the droplets which have a VMD which is unsuitable for inhalation. Such 


\begin{tabular}{|c|c|c|c|c||}
\hline \multicolumn{5}{|c|}{ Table 4.2 Reduction in the mean size of $5 \mu \mathrm{m}$ liposomes following nebulisation, as a } \\
function of the bilayer composition. \\
[ \pm sd; $\mathrm{n}=3]$
\end{tabular}

droplets have an increased momentum, and thus tend towards deposition on baffle(s), before being returned to the residual fluid within the nebuliser reservoir. The larger liposomes of a nebulised formulation therefore become restricted to the $99 \%$ of the aerosol mass that is refluxed back to the residual fluid (Mercer et al., 1973). As a result, they are made available for reatomisation, and are repeatedly exposed to the shearing action of gas flow. Larger liposomes are therefore caught in a cycle of atomisation, shearing, and impaction, until processed to a size that is small enough for inclusion in the smaller satellite droplets which constitute the secondary aerosol. As a consequence, the mean size of larger liposomes falls, and the residual liposome concentration increases, during nebulisation (chapter 3). Such a mechanism explains the observation that the fall in mean liposome size is dependent on the bilayer composition of the liposomes, and to an extent on the nebuliser used (table 4.2). The fall in residual liposome size reflects the relative stability of the liposome bilayers to disruption. The DPPC/chol (1:1) liposome bilayers are more stable 
under the conditions of nebulisation, and as a result prove resistant to the size reduction induced by nebulisation. By contrast, the eggPC liposomes are composed of a phospholipid that is in the liquid-crystalline state under the conditions of the experiment. The bilayers are therefore relatively fluid, and hence more easily disrupted. The relative stability of DPPC/chol (1:1) liposomes to shear induced disruption was previously demonstrated during membrane extrusion, and probe sonication experiments (chapter 2). Liposomes with relatively stable bilayers have also been shown to yield reduced aerosol outputs, and significant residual volumes following nebulisation (chapter 3). Such results provide further evidence for the proposed mechanism for liposome nebulisation. Liposomes with rigid bilayers resist shearing to the extent that they yield concentrated residual fluids following nebulisation. This fluid contains liposomes which are likely to possess an increased mean size, relative to liquid-crystalline bilayer state liposomes.

The damage done to liposomes during nebulisation was also determined, to a limited extent, by the nebuliser used, as there was a significant difference in the reduction of residual liposome size caused by the Respirgard II to that caused by the other nebulisers $(p<0.05)$ (Appendix, table 1). Niven et al. (1991a) proposed that the mean size of liposomes for nebulisation should be reduced to below that of the aerosol droplets, to ensure maximum liposome stability. This suggests that the damage done to liposomes is dependent on the droplet size produced by the nebuliser, and indeed each of the nebulisers exhibited significantly less residual liposome processing than the Respirgard II, which produces the smallest droplet size (fig. 4.2).

As the mean aerosol size produced by the nebuliser is reduced, so the proportion of liposomes that are likely to be excluded from the secondary aerosol is increased. As a result, a greater number of liposomes are retained within the Respirgard II to undergo continued processing. This exposes the liposome bilayers to repeated shearing, and results in a more pronounced reduction in residual size. The liposomes, it is proposed, become released only once they are small enough to become included within the smaller droplets that constitute the secondary aerosol, which in the case of the Respirgard is significantly smaller than the alternative nebulisers. The smaller droplet size produced by the Respirgard II nebuliser also indicates that the shear forces operating within the nebuliser chamber are greater, and indeed the Respirgard II has one of the smallest gas jet orifices of the nebulisers 


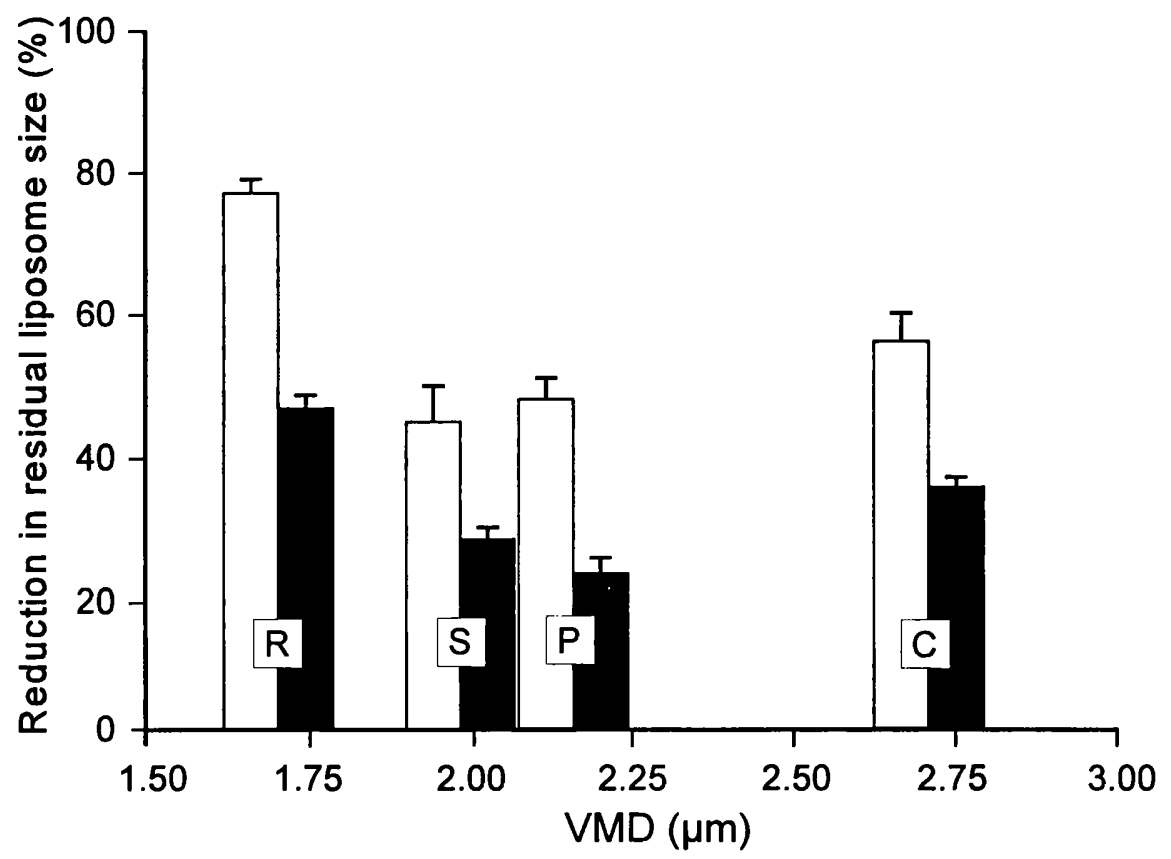

Figure 4.2 The relationship between the droplet size produced by a jet nebuliser ( $C=$ Cirrus, $\mathrm{P}=$ Pari-LC, $\mathrm{R}=$ Respirgard II, $\mathrm{S}=$ Sidestream) and the reduction in the mean residual liposome size. ( $\square$ ) eggPC and ( $\square$ ) DPPC/chol (1:1) liposomes. [ \pm sd; n=3]

studied. A smaller gas jet orifice leads to a more rapid entrainment of fluid from the jets, and an increased energy for fluid and bilayer disruption. The Respirgard II nebuliser also incorporates a sophisticated baffling system, which achieves the retention of primary droplets of a smaller minimum size. This would increase the recycling of liposomes, and therefore increase their damage. However, it is clear that the vesicle size reduction is not solely determined by the secondary aerosol size (fig. 4.2). The Sidestream nebuliser produces a similar decrease in residual liposome size to that produced by the Pari-LC and the Cirrus, despite the fact that the Sidestream produces droplets that are smaller than either device (VMD: Sidestream $=1.99 \mu \mathrm{m}$, Pari- $-\mathrm{LC}=2.15 \mu \mathrm{m}$, Cirrus $=2.70 \mu \mathrm{m})$. This may be because the Sidestream achieves a small aerosol size due to increased droplet evaporation, occurring in response to enhanced gas flow through the open vent. It is therefore likely, that the damage caused to liposomes during nebulisation is to an extent a function of the nebuliser design, with the efficiency of aerosol output, and the degree of shear within the 
nebuliser being principal factors determining the liposome stability. These in turn are controlled by the driving gas flow rate, and the design, dimensions and geometry of the nebulisers constituent parts (eg. baffles, capillaries, jets and vents). It is therefore the widely differing designs of each nebuliser that causes the observed variations in liposome processing between devices at similar gas flow rates. For example in the Respirgard II nebuliser gas is forced through an orifice with a diameter of $450 \mu \mathrm{m}$ (fig. 3.33c). The pressure drop, generated by the driving gas flow, draws fluid from the nebuliser reservoir surrounding the nozzle, which is then mixed with the gas stream. The resulting aerosol is then forced through an orifice with a diameter of $1 \mathrm{~mm}$ (fig. 3.3b), set within the nebuliser baffle that is adjacent to the jet orifice. In contrast, the Sidestream has a larger gas jet orifice through which the gas is forced $(530 \mu \mathrm{m}$, fig. $3.4 \mathrm{c})$, making the pressure drop that results from the passage of high velocity gas through the orifice reduced at the fixed flow rate. However, a small droplet size results from enhanced droplet evaporation. The primary aerosol droplets are also forced to leave the Sidestream nebuliser through a complex series of channels set in a baffle. This causes a proportion of the larger droplets to become deposited. By contrast, the Respirgard II uses a length of tubing attached to the nebuliser exit to filter out larger aerosol droplets. Such marked variations in nebuliser design may explain the significant differences in the damage each nebuliser causes to liposomes, in spite of the fact that the Respirgard II and Sidestream nebulisers produce similarly sized aerosols. The aerosol character, and liposome stability to nebulisation are each controlled by the complex interplay between the various components constituting a particular nebuliser model. The fact that these components differ so markedly between different nebuliser models, means that it is not possible to determine the particular features which are relevant to the relative liposome stability.

Figure 4.3 and table 4.3 demonstrate the effect that nebulisation has upon the spread of vesicle sizes within the residual liposome population. There was a marked reduction in the span value for the residual liposomes as nebulisation proceeded, for each nebuliser studied. This indicates that they were transformed into a more homogeneous population of vesicle sizes. 

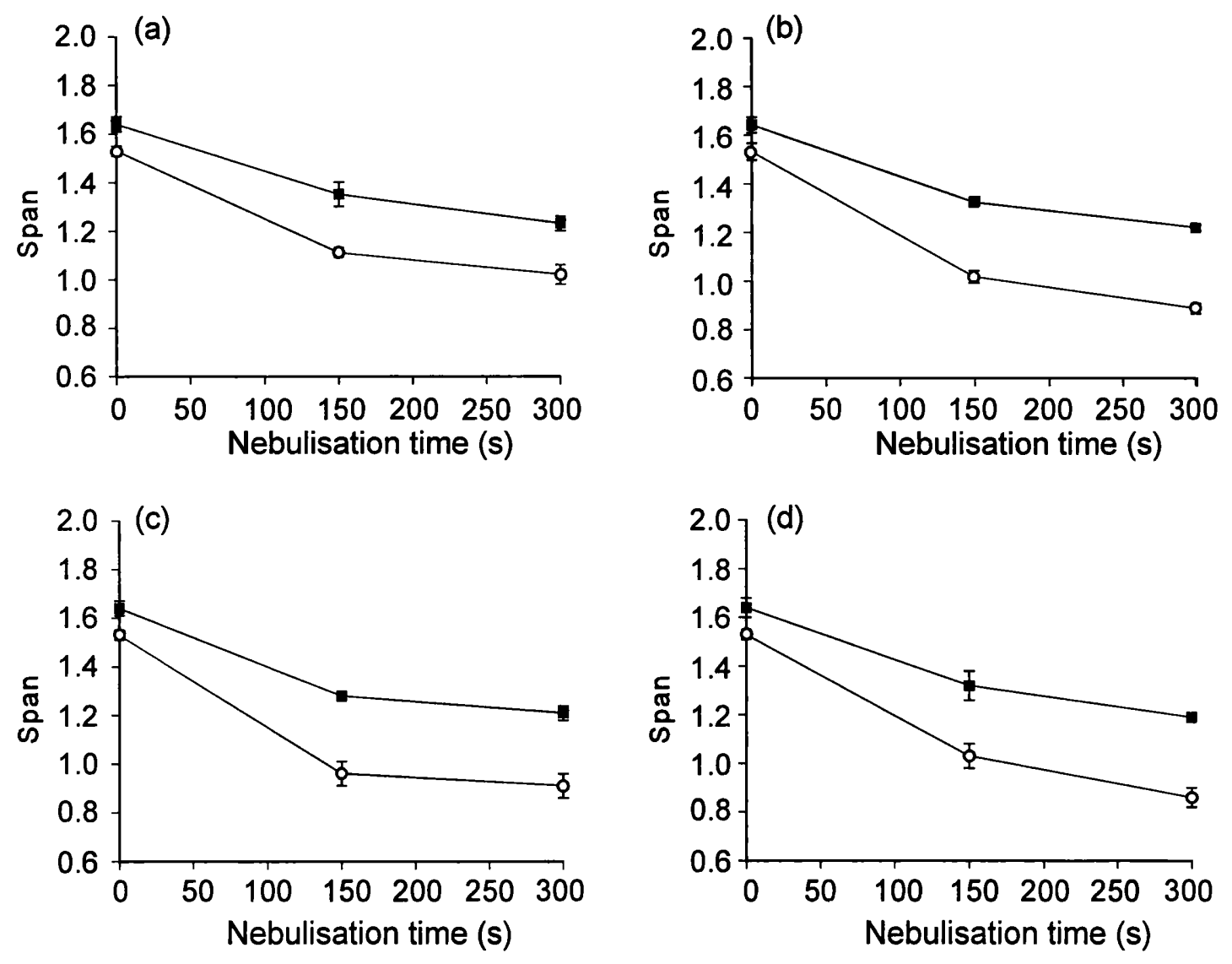

Figure 4.3 The effect of nebulisation with various jet nebulisers on the polydispersity (span) of vesicle sizes within eggPC (O) and DPPC/chol (1:1) (ש) residual liposomes. (a) Cirrus (b) Pari-LC ( c) Respirgard II (d) Sidestream nebulisers. [ \pm sd; $n=3]$

Table 4.3 Reduction in the span (\%) of residual $5 \mu \mathrm{m}$ mean size eggPC and DPPC/chol (1:1) liposomes during nebulisation with jet nebulisers.

\begin{tabular}{|c|c|c|}
\hline \multicolumn{3}{|c|}{$[ \pm \mathrm{sd} ; \mathrm{n}=3]$} \\
\hline Nebuliser & eggPC & eggPC/chol (1:1) \\
\hline Cirrus & $33.25( \pm 1.09)$ & $25.11( \pm 2.99)$ \\
\hline Pari-LC & $42.15( \pm 2.15)$ & $25.74( \pm 1.66)$ \\
\hline Respirgard II & $40.63( \pm 1.76)$ & $26.53( \pm 3.15)$ \\
\hline Sidestream & $43.89( \pm 3.20)$ & $27.34( \pm 1.49)$ \\
\hline
\end{tabular}


There was approximately a $40 \%$ and $25 \%$ reduction in the span of eggPC and DPPC/chol (1:1) liposomes respectively, during nebulisation to sputtering. Therefore the eggPC liposomes underwent a significantly greater change in span compared with the DPPC/chol $(1: 1)$ liposomes $(p<0.05)$, reflecting their increased sensitivity to nebulisation. The $\mathrm{DPPC} / \mathrm{chol}(1: 1)$ liposomes resist changes in span, to the extent that there was no significant difference between the change in span between different nebulisers $(p<0.05)$. Each nebuliser also produced a similar pattern of span change, with a less marked reduction in the latter half of nebulisation. This supports the theory that the liposome damage is caused by the preferential release of smaller liposomes. As the mean liposome size is reduced, so the residual liposome processing is lessened, and hence less impact is caused to the vesicle polydispersity.

\subsubsection{The influence of liposome size and nebulisation time on residual liposomes}

The previous experiment determined the stability of liposomes to nebulisation with different devices, operated for a fixed nebulisation time. Whilst this simulates many in clinical practice, each nebuliser gives widely varying aerosol output rates (chapter 3 ). The time taken for a nebuliser to reach sputtering is a reflection of the output efficiency, as nebulisers which recycle a greater number of aerosolised primary droplets are likely to produce extended nebulisation times. This may expose the liposomes to prolonged periods of shearing, and also to an increased number of reflux cycles. It may therefore prove more useful to study nebulisation over a period of time that reflects the efficiency of output of a particular nebuliser, such as operation until the aerosol output becomes intermittent (Kradjan and Lakshminarayan, 1985). In this way, the rate of liposome processing over the course of nebulisation to sputtering may be established, and this related to the rate of aerosol output. This may reveal more useful information concerning the processes causing liposome damage for a particular nebuliser. A nebuliser with an increased rate of liposome damage, yet a low rate of aerosol output, is likely to principally cause damage to the liposomes through prolonged exposure to shearing, resulting from an increased baffling efficiency. An increased rate of liposome damage, in conjunction with an efficient aerosol output, is likely to produce liposome damage through elevated shear forces within the nebuliser, rather than to prolonged vesicle recycling, as the liposome leave the nebuliser rapidly, yet are significantly damaged in the process. The present study therefore gives some indication of the baffling efficiencies, and shear forces within different devices. 
The mean droplet size, and time taken to reach the sputtering phase of nebulisation, for each jet nebuliser studied, is shown in table 4.4. Table 4.5, table 4.6, and fig. 4.4, show the progressive reduction in the residual liposome size within each nebuliser, that occurs during operation to sputtering.

Table 4.4 The mean droplet VMD, and time taken for the aerosol output to become intermittent for each jet nebuliser (calculated from the nebulisation of $20 \mathrm{mg} / \mathrm{ml} 5$ $\mu \mathrm{m}$ eggPC liposomes with a flow rate of $71 / \mathrm{min}) .[ \pm \mathrm{sd} ; \mathrm{n}=3]$

\begin{tabular}{|c|c|c|}
\hline Nebuliser & Droplet VMD $(\boldsymbol{\mu m})$ & $\begin{array}{c}\text { Nebulisation } \\
\text { Time (s) }\end{array}$ \\
\hline Cirrus & 2.83 & 600 \\
& $(0.01)$ & $(46)$ \\
\hline Pari-LC & 2.27 & 330 \\
& $(0.06)$ & $(29)$ \\
\hline Respirgard II & 1.81 & 740 \\
& $(0.04)$ & $(8)$ \\
\hline Sidestream & 2.05 & 286 \\
& $(0.02)$ & $(13)$ \\
\hline
\end{tabular}

The size reduction of residual liposomes induced in experiment 4.4 .2 was less than that demonstrated in experiment 4.4.1. This is likely to be a consequence of the fact that each of the nebulisers were operated at a reduced driving gas flow rate compared to that in the previous experiment. This was deemed necessary as the Sidestream nebuliser gave an extremely rapid aerosol output at $8 \mathrm{l} / \mathrm{min}$. Researchers suggest that open vent nebulisers need not be operated at elevated gas flow rates, as the gas flow is supplemented by air flow through the vent (O'Callaghan and Barry, 1997). A reduced flow rate of $7 \mathrm{l} / \mathrm{min}$ was therefore employed, in order to make a more realistic comparison with the other nebulisers studied. The results indicated that the liposomes were more stable to nebulisers operated at a reduced flow rate. Each of the nebulisers studied produced less liposome size reduction when operated at $71 / \mathrm{min}$, compared to that observed at $81 / \mathrm{min}$. This is consistent with the findings of Niven et al (1992a). The influence of gas flow rate on stability was further investigated in experiments detailed in sections 4.4.3 and 4.4.4. 
Table 4.5 Size of residual liposomes, following the nebulisation of liposome formulations with an initial mean size of 2.5 and $5 \mu \mathrm{m}$, to $50 \%$ and $100 \%$ of the nebulisation time. $[ \pm \mathrm{sd} ; \mathrm{n}=3]$

\begin{tabular}{|c|c|c|c|c|c|}
\hline Nebuliser & Liposome & Size & $0 \%$ & $50 \%$ & $100 \%$ \\
\hline \multirow{4}{*}{ Cirrus } & \multirow[t]{2}{*}{ eggPC } & $5 \mu \mathrm{m}$ & $\begin{array}{c}5.01 \\
(0.03)\end{array}$ & $\begin{array}{c}4.00 \\
(0.05)\end{array}$ & $\begin{array}{c}3.24 \\
(0.04)\end{array}$ \\
\hline & & $2.5 \mu \mathrm{m}$ & $\begin{array}{c}2.55 \\
(0.04) \\
\end{array}$ & $\begin{array}{c}1.99 \\
(0.06) \\
\end{array}$ & $\begin{array}{r}1.63 \\
(0.06) \\
\end{array}$ \\
\hline & \multirow{2}{*}{$\begin{array}{c}\text { eggPC/chol } \\
(1: 1)\end{array}$} & $5 \mu \mathrm{m}$ & $\begin{array}{c}5.04 \\
(0.03)\end{array}$ & $\begin{array}{c}4.10 \\
(0.36)\end{array}$ & $\begin{array}{c}3.85 \\
(0.20)\end{array}$ \\
\hline & & $2.5 \mu \mathrm{m}$ & $\begin{array}{c}2.48 \\
(0.02)\end{array}$ & $\begin{array}{c}2.11 \\
(0.03)\end{array}$ & $\begin{array}{c}2.05 \\
(0.06)\end{array}$ \\
\hline \multirow{4}{*}{ Pari-LC } & \multirow[t]{2}{*}{ eggPC } & $5 \mu \mathrm{m}$ & $\begin{array}{c}4.97 \\
(0.05)\end{array}$ & $\begin{array}{c}3.93 \\
(0.10)\end{array}$ & $\begin{array}{c}2.74 \\
(0.07)\end{array}$ \\
\hline & & $2.5 \mu \mathrm{m}$ & $\begin{array}{c}2.52 \\
(0.04)\end{array}$ & $\begin{array}{c}2.27 \\
(0.17)\end{array}$ & $\begin{array}{c}2.25 \\
(0.14)\end{array}$ \\
\hline & \multirow{2}{*}{$\begin{array}{c}\text { eggPC/chol } \\
(1: 1)\end{array}$} & $5 \mu \mathrm{m}$ & $\begin{array}{c}5.04 \\
(0.05)\end{array}$ & $\begin{array}{c}4.40 \\
(0.08)\end{array}$ & $\begin{array}{c}4.45 \\
(0.07)\end{array}$ \\
\hline & & $2.5 \mu \mathrm{m}$ & $\begin{array}{c}2.52 \\
(0.04)\end{array}$ & $\begin{array}{c}2.27 \\
(0.17)\end{array}$ & $\begin{array}{c}2.25 \\
(0.14)\end{array}$ \\
\hline \multirow{4}{*}{$\begin{array}{c}\text { Respirgard } \\
\text { II }\end{array}$} & \multirow[t]{2}{*}{ eggPC } & $5 \mu \mathrm{m}$ & $\begin{array}{c}5.06 \\
(0.05) \\
\end{array}$ & $\begin{array}{r}3.34 \\
(0.09) \\
\end{array}$ & $\begin{array}{c}2.81 \\
(0.08) \\
\end{array}$ \\
\hline & & $2.5 \mu \mathrm{m}$ & $\begin{array}{c}2.50 \\
(0.01) \\
\end{array}$ & $\begin{array}{c}2.00 \\
(0.01) \\
\end{array}$ & $\begin{array}{r}1.64 \\
(0.13) \\
\end{array}$ \\
\hline & \multirow{2}{*}{$\begin{array}{c}\text { eggPC/chol } \\
(1: 1)\end{array}$} & $5 \mu \mathrm{m}$ & $\begin{array}{r}5.02 \\
(0.02) \\
\end{array}$ & $\begin{array}{r}4.52 \\
(0.01) \\
\end{array}$ & $\begin{array}{r}4.16 \\
(0.01) \\
\end{array}$ \\
\hline & & $2.5 \mu \mathrm{m}$ & $\begin{array}{c}2.52 \\
(0.01) \\
\end{array}$ & $\begin{array}{r}2.22 \\
(0.04) \\
\end{array}$ & $\begin{array}{c}2.24 \\
(0.04) \\
\end{array}$ \\
\hline \multirow{4}{*}{ Sidestream } & \multirow[t]{2}{*}{ eggPC } & $5 \mu \mathrm{m}$ & $\begin{array}{c}5.02 \\
(0.02) \\
\end{array}$ & $\begin{array}{r}4.22 \\
(0.06) \\
\end{array}$ & $\begin{array}{c}4.01 \\
(0.03) \\
\end{array}$ \\
\hline & & $2.5 \mu \mathrm{m}$ & $\begin{array}{c}2.53 \\
(0.02) \\
\end{array}$ & $\begin{array}{c}2.00 \\
(0.10) \\
\end{array}$ & $\begin{array}{r}1.70 \\
(0.08) \\
\end{array}$ \\
\hline & \multirow{2}{*}{$\begin{array}{c}\text { eggPC/chol } \\
(1: 1)\end{array}$} & $5 \mu \mathrm{m}$ & $\begin{array}{c}5.02 \\
(0.01) \\
\end{array}$ & $\begin{array}{c}4.72 \\
(0.18) \\
\end{array}$ & $\begin{array}{c}4.53 \\
(0.10) \\
\end{array}$ \\
\hline & & $2.5 \mu \mathrm{m}$ & $\begin{array}{c}2.53 \\
(0.01)\end{array}$ & $\begin{array}{c}2.51 \\
(0.03)\end{array}$ & $\begin{array}{c}2.51 \\
(0.09)\end{array}$ \\
\hline
\end{tabular}


Table 4.6 Decrease (\%) in the mean size of residual liposomes, following the nebulisation of liposome formulations with an initial mean size of 2.5 and $5 \mu \mathrm{m}$, to $50 \%$ and $100 \%$ of the nebulisation time. [ $\pm \mathrm{sd} ; \mathrm{n}=3$ ]

\begin{tabular}{|c|c|c|c|c|c|}
\hline Nebuliser & Liposome & Size & $0-50 \%$ & $50-100 \%$ & $0-100 \%$ \\
\hline \multirow{4}{*}{ Cirrus } & \multirow[t]{2}{*}{ eggPC } & $5 \mu \mathrm{m}$ & $\begin{array}{l}20.11 \\
(0.76)\end{array}$ & $\begin{array}{l}18.99 \\
(1.77)\end{array}$ & $\begin{array}{l}35.29 \\
(0.80)\end{array}$ \\
\hline & & $2.5 \mu \mathrm{m}$ & $\begin{array}{l}21.84 \\
(2.88)\end{array}$ & $\begin{array}{l}18.18 \\
(4.34)\end{array}$ & $\begin{array}{l}35.97 \\
(5.76)\end{array}$ \\
\hline & \multirow{2}{*}{$\begin{array}{c}\text { eggPC/chol } \\
(1: 1)\end{array}$} & $5 \mu \mathrm{m}$ & $\begin{array}{l}18.62 \\
(6.77)\end{array}$ & $\begin{array}{c}5.41 \\
(12.07)\end{array}$ & $\begin{array}{l}23.55 \\
(3.91)\end{array}$ \\
\hline & & $2.5 \mu \mathrm{m}$ & $\begin{array}{l}15.03 \\
(1.32)\end{array}$ & $\begin{array}{l}2.82 \\
(3.86)\end{array}$ & $\begin{array}{l}17.43 \\
(3.14)\end{array}$ \\
\hline \multirow{4}{*}{ Pari-LC } & \multirow[t]{2}{*}{ eggPC } & $5 \mu \mathrm{m}$ & $\begin{array}{l}20.97 \\
(2.19)\end{array}$ & $\begin{array}{l}30.24 \\
(2.29)\end{array}$ & $\begin{array}{l}44.90 \\
(0.59)\end{array}$ \\
\hline & & $2.5 \mu \mathrm{m}$ & $\begin{array}{l}19.90 \\
(3.63)\end{array}$ & $\begin{array}{l}17.52 \\
(4.12) \\
\end{array}$ & $\begin{array}{l}34.03 \\
(1.26)\end{array}$ \\
\hline & \multirow{2}{*}{$\begin{array}{c}\text { eggPC/chol } \\
(1: 1)\end{array}$} & $5 \mu \mathrm{m}$ & $\begin{array}{l}12.70 \\
(1.61)\end{array}$ & $\begin{array}{l}+1.15 \\
(2.04)\end{array}$ & $\begin{array}{l}11.70 \\
(1.77)\end{array}$ \\
\hline & & $2.5 \mu \mathrm{m}$ & $\begin{array}{c}9.98 \\
(7.95) \\
\end{array}$ & $\begin{array}{c}0.70 \\
(5.85)\end{array}$ & $\begin{array}{l}10.77 \\
(6.66)\end{array}$ \\
\hline \multirow{4}{*}{$\begin{array}{c}\text { Respirgard } \\
\text { II }\end{array}$} & \multirow[t]{2}{*}{ eggPC } & $5 \mu \mathrm{m}$ & $\begin{array}{l}33.94 \\
(2.13) \\
\end{array}$ & $\begin{array}{l}15.84 \\
(2.88) \\
\end{array}$ & $\begin{array}{l}44.43 \\
(1.24)\end{array}$ \\
\hline & & $2.5 \mu \mathrm{m}$ & $\begin{array}{l}20.11 \\
(0.57)\end{array}$ & $\begin{array}{l}19.32 \\
(6.69)\end{array}$ & $\begin{array}{l}35.55 \\
(5.24)\end{array}$ \\
\hline & \multirow{2}{*}{$\begin{array}{c}\text { eggPC/chol } \\
(1: 1)\end{array}$} & $5 \mu \mathrm{m}$ & $\begin{array}{l}10.02 \\
(2.30) \\
\end{array}$ & $\begin{array}{l}7.93 \\
(2.47) \\
\end{array}$ & $\begin{array}{l}17.19 \\
(1.51) \\
\end{array}$ \\
\hline & & $2.5 \mu \mathrm{m}$ & $\begin{array}{l}11.90 \\
(1.12)\end{array}$ & $\begin{array}{l}+0.92 \\
(2.70)\end{array}$ & $\begin{array}{l}11.08 \\
(3.00)\end{array}$ \\
\hline \multirow{4}{*}{ Sidestream } & \multirow[t]{2}{*}{ eggPC } & $5 \mu \mathrm{m}$ & $\begin{array}{l}15.99 \\
(1.26)\end{array}$ & $\begin{array}{l}4.81 \\
(1.31)\end{array}$ & $\begin{array}{l}20.04 \\
(0.28)\end{array}$ \\
\hline & & $2.5 \mu \mathrm{m}$ & $\begin{array}{l}20.96 \\
(3.29)\end{array}$ & $\begin{array}{l}11.94 \\
(3.68)\end{array}$ & $\begin{array}{l}30.45 \\
(2.77)\end{array}$ \\
\hline & \multirow{2}{*}{$\begin{array}{c}\text { eggPC/chol } \\
(1: 1)\end{array}$} & $5 \mu \mathrm{m}$ & $\begin{array}{c}6.03 \\
(3.87) \\
\end{array}$ & $\begin{array}{l}3.96 \\
(3.02) \\
\end{array}$ & $\begin{array}{l}9.82 \\
(2.00) \\
\end{array}$ \\
\hline & & $2.5 \mu \mathrm{m}$ & $\begin{array}{c}0.66 \\
(0.61) \\
\end{array}$ & $\begin{array}{c}1.59 \\
(2.00) \\
\end{array}$ & $\begin{array}{c}2.25 \\
(1.88) \\
\end{array}$ \\
\hline
\end{tabular}



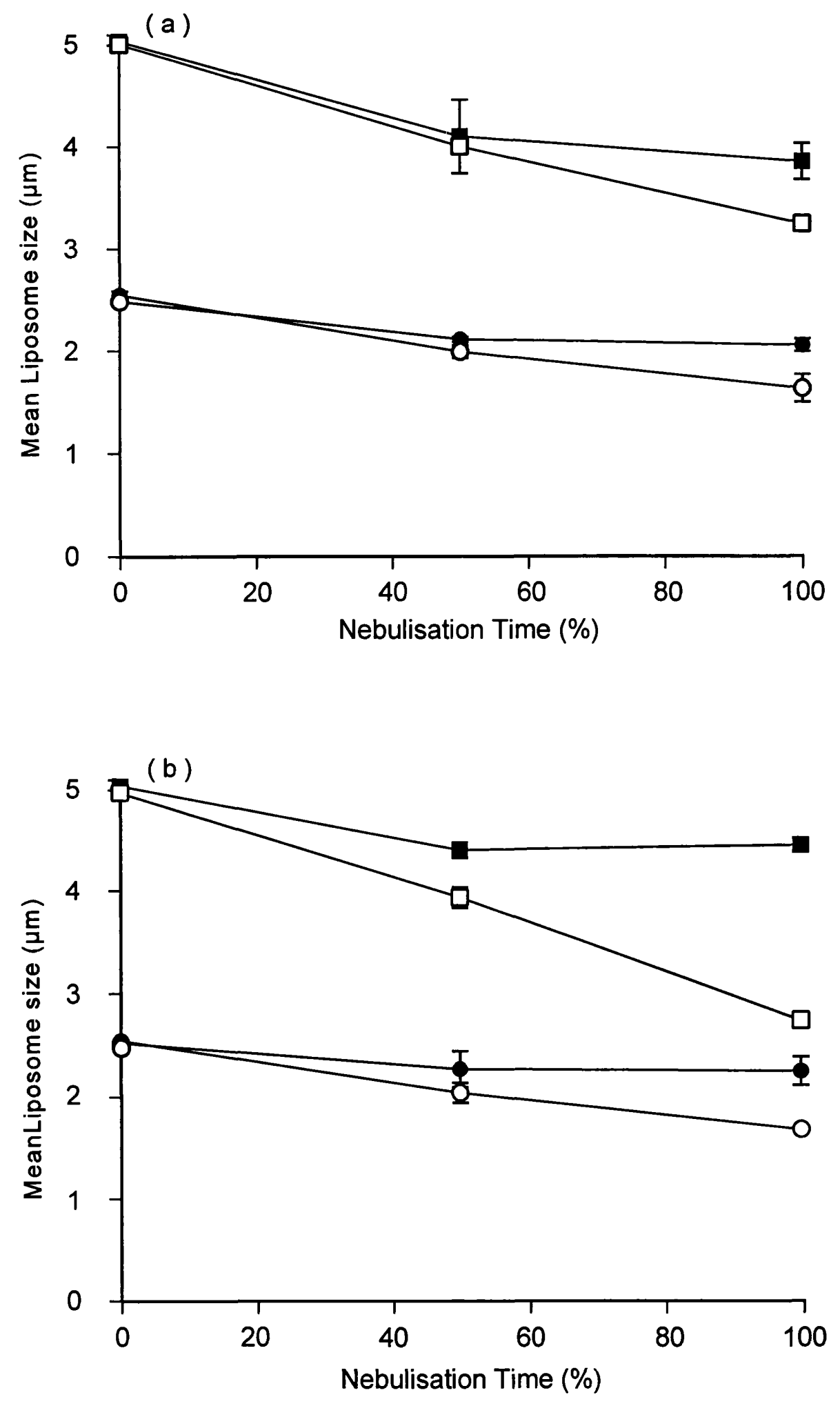

Figure 4.4 The effect of nebulisation with the (a) Cirrus and (b) Pari-LC nebuliser, on the residual liposome size of eggPC ( $\square$ O), and eggPC/chol (1:1) (ロO) liposomes, with an initial mean size of $5 \mu \mathrm{m}(\square \square)$ and $2.5 \mu \mathrm{m}(\mathrm{O})$. [ $\left.{ }^{\mathrm{s} d} \mathrm{~s} ; \mathrm{n}=3\right]$ 


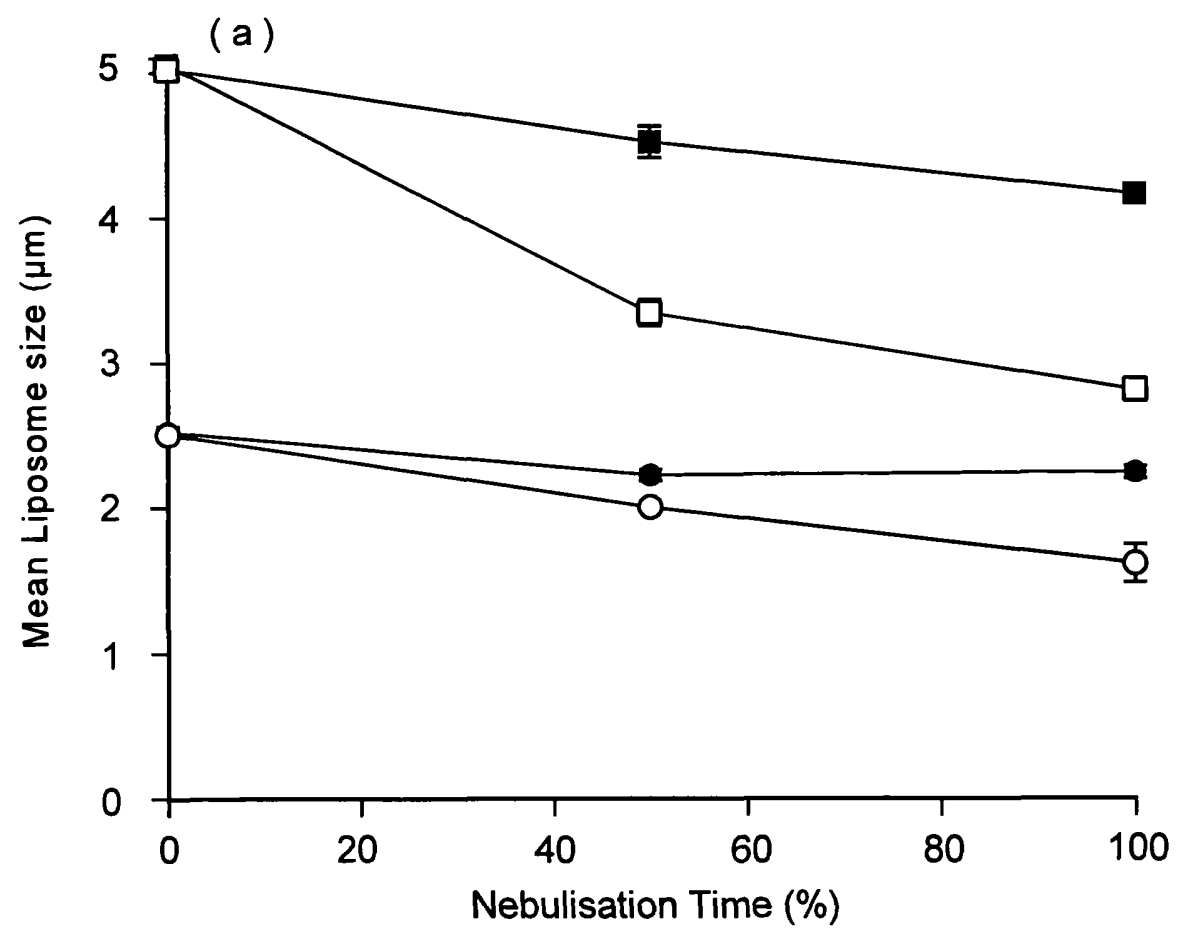

(b)

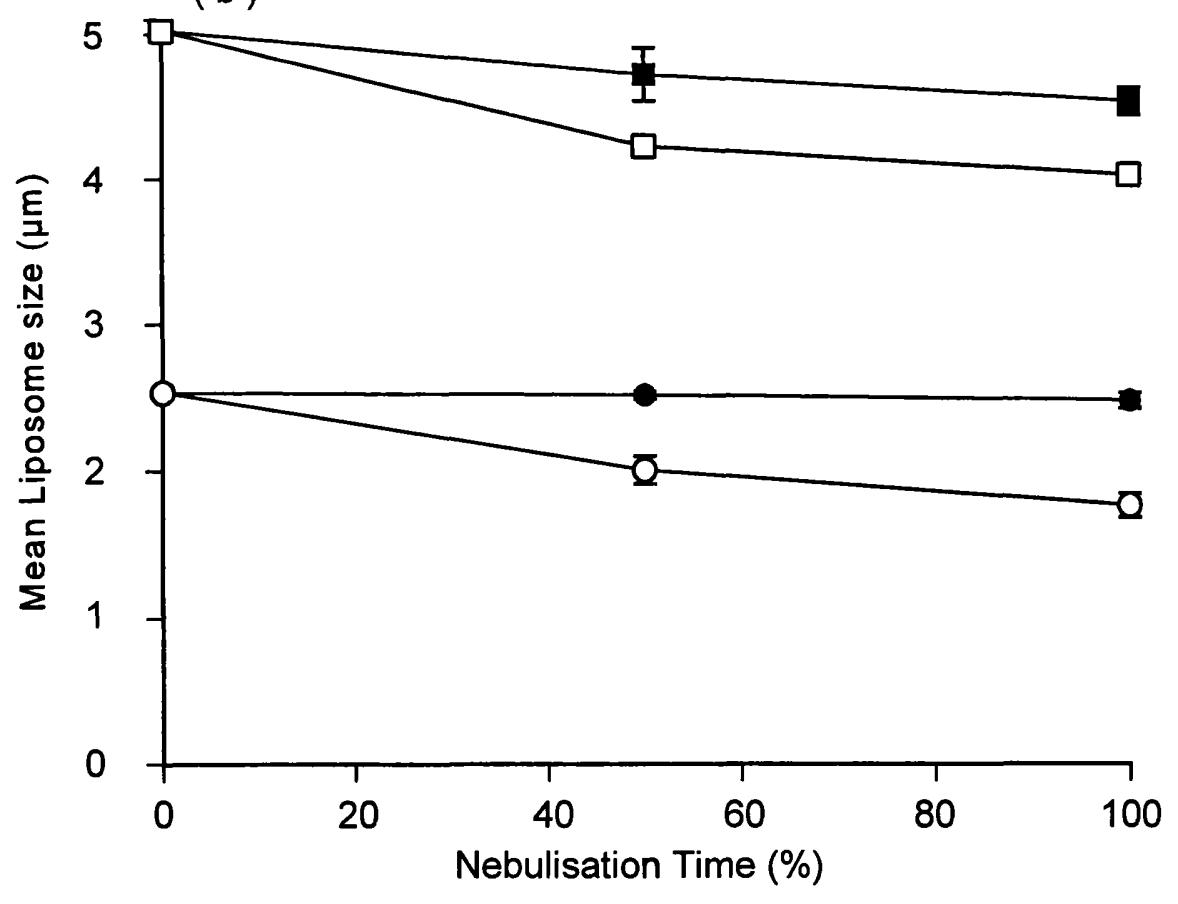

Figure 4.5 The effect of nebulisation with the (a) Respirgard II, and (b) Sidestream

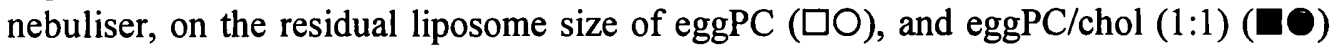
liposomes, with an initial mean size of $5 \mu \mathrm{m}(\square \square)$ and $2.5 \mu \mathrm{m}\left(\mathrm{O}^{\circ}\right)$. [ $\pm \mathrm{sd} ; \mathrm{n}=3$ ] 
The fluid eggPC liposomes underwent a reduction in size that was 2 and 4 times greater than that undergone by the eggPC/chol (1:1) liposomes, for the 5 and $2.5 \mu \mathrm{m}$ initial mean size liposomes respectively. Inclusion of equimolar cholesterol into eggPC bilayers eliminates the sharp phospholipid gel to liquid crystalline transition, and thus imparts a rigidity to the bilayer at temperatures above the $T_{m}$ (Ladbroke et al., 1968). As a result, the eggPC/chol (1:1) liposomes exhibited a stability similar to that observed for the DPPC/chol (1:1) liposomes (fig. 4.1). Each nebuliser significantly differed in the damage caused to residual liposomes generated from the $5 \mu \mathrm{m}$ liposome formulation $(\mathrm{p}<0.05)$ (Appendix, table 2). However, there was less of a difference evident for the $2.5 \mu \mathrm{m}$ liposome formulations, with a significant difference only evident between the Cirrus and the Respirgard II or Sidestream (for the eggPC/chol (1:1) liposomes); and between the Respirgard and Sidestream nebulisers (for the eggPC liposome liposomes)(Appendix, table 3). The differences in the handling of $5 \mu \mathrm{m}$ liposomes by each nebuliser is due to variations in the baffling efficiencies, and shear forces operating within each nebuliser as detailed in section 4.4.1. However, unlike the previous experiment the differences in liposome damage between the nebulisers for the eggPC/chol (1:1) liposomes, were largely significant for the $5 \mu \mathrm{m}$ formulations, with only the Sidestream and Pari-LC nebulisers yielding similar liposome damage. The rate of liposome size reduction produced by each nebuliser was significantly reduced in the latter stage of nebulisation (ie. 50 to $100 \%$ of nebulisation time), when compared to the initial stage (ie 0 to $50 \%)($ table 4.7$)(\mathrm{p}<0.05)$. This is a consequence of the fall in the residual liposome size undergone as the nebulisation proceeded. As the mean residual liposome size fell, so the liposome damage became selflimiting. In addition, the aerosol output rate from jet nebulisers has been shown to be significantly reduced in the latter stages of nebulisation (chapter 3). This causes the liposomes to become aerosolised less frequently, with the result that they are exposed to shearing forces to a reduced extent. As a consequence, liposome processing becomes reduced. It may equally be argued, that as the residual volume falls, the probability of a liposome becoming aerosolised from the residual fluid, and thus exposed to the shearing action of gas flow, is increased. However, the supply of fluid for aerosolisation is limited when the residual volume dwindles, by the return of deposited fluid to the feed capillaries. This will take a fixed time, regardless of the volume remaining in the chamber. Therefore, as sputtering is approached the fluid volume in the reservoir is insufficient to maintain the aerosol output rate, and hence the liposomes undergo less recycling. 


\begin{tabular}{|c|c|c|c|c|c|}
\hline Nebuliser & Liposome & $\begin{array}{c}\text { Initial } \\
\text { liposome } \\
\text { Size }\end{array}$ & $0-50 \%$ & $50-100 \%$ & $0-100 \%$ \\
\hline \multirow{4}{*}{ Cirrus } & \multirow[t]{2}{*}{$\begin{array}{c}\text { eggPC/chol } \\
(1: 1)\end{array}$} & $5 \mu \mathrm{m}$ & $\begin{array}{l}187 \\
(67)\end{array}$ & $\begin{array}{c}50 \\
(104)\end{array}$ & $\begin{array}{l}119 \\
(21)\end{array}$ \\
\hline & & $2.5 \mu \mathrm{m}$ & $\begin{array}{l}75 \\
(7) \\
\end{array}$ & $\begin{array}{c}12 \\
(16) \\
\end{array}$ & $\begin{array}{l}38 \\
(8) \\
\end{array}$ \\
\hline & \multirow[t]{2}{*}{ eggPC } & $5 \mu \mathrm{m}$ & $\begin{array}{r}201 \\
(7)\end{array}$ & $\begin{array}{l}152 \\
(16)\end{array}$ & $\begin{array}{l}177 \\
(45)\end{array}$ \\
\hline & & $2.5 \mu \mathrm{m}$ & $\begin{array}{l}111 \\
(16) \\
\end{array}$ & $\begin{array}{c}72 \\
(15) \\
\end{array}$ & $\begin{array}{c}92 \\
(15)\end{array}$ \\
\hline \multirow{4}{*}{ Pari-LC } & \multirow[t]{2}{*}{$\begin{array}{c}\text { eggPC/chol } \\
(1: 1)\end{array}$} & $5 \mu \mathrm{m}$ & $\begin{array}{l}232 \\
(30)\end{array}$ & $\begin{array}{l}-17 \\
(33)\end{array}$ & $\begin{array}{l}107 \\
(17)\end{array}$ \\
\hline & & $2.5 \mu \mathrm{m}$ & $\begin{array}{l}92 \\
(73)\end{array}$ & $\begin{array}{c}7 \\
(47)\end{array}$ & $\begin{array}{c}50 \\
(31)\end{array}$ \\
\hline & \multirow[t]{2}{*}{ eggPC } & $5 \mu \mathrm{m}$ & $\begin{array}{l}380 \\
(41) \\
\end{array}$ & $\begin{array}{l}433 \\
(14) \\
\end{array}$ & $\begin{array}{l}405 \\
(32) \\
\end{array}$ \\
\hline & & $2.5 \mu \mathrm{m}$ & $\begin{array}{l}184 \\
(33)\end{array}$ & $\begin{array}{l}131 \\
(36)\end{array}$ & $\begin{array}{r}158 \\
(7)\end{array}$ \\
\hline \multirow{4}{*}{$\begin{array}{c}\text { Respirgard } \\
\text { II }\end{array}$} & \multirow[t]{2}{*}{$\begin{array}{c}\text { eggPC/chol } \\
(1: 1)\end{array}$} & $5 \mu \mathrm{m}$ & $\begin{array}{l}82 \\
(19)\end{array}$ & $\begin{array}{l}58 \\
(20)\end{array}$ & $\begin{array}{l}70 \\
(1)\end{array}$ \\
\hline & & $2.5 \mu \mathrm{m}$ & $\begin{array}{l}49 \\
(5)\end{array}$ & $\begin{array}{l}-3 \\
(10)\end{array}$ & $\begin{array}{l}23 \\
(7)\end{array}$ \\
\hline & \multirow[t]{2}{*}{ eggPC } & $5 \mu \mathrm{m}$ & $\begin{array}{l}278 \\
(19)\end{array}$ & $\begin{array}{c}86 \\
(17)\end{array}$ & $\begin{array}{r}182 \\
(5)\end{array}$ \\
\hline & & $2.5 \mu \mathrm{m}$ & $\begin{array}{l}82 \\
(2)\end{array}$ & $\begin{array}{l}63 \\
(22)\end{array}$ & $\begin{array}{c}72 \\
(11)\end{array}$ \\
\hline \multirow{4}{*}{ Sidestream } & \multirow[t]{2}{*}{$\begin{array}{c}\text { eggPC/chol } \\
(1: 1)\end{array}$} & $5 \mu \mathrm{m}$ & $\begin{array}{l}128 \\
(82)\end{array}$ & $\begin{array}{l}80 \\
(64)\end{array}$ & $\begin{array}{l}103 \\
(21)\end{array}$ \\
\hline & & $2.5 \mu \mathrm{m}$ & $\begin{array}{c}7 \\
(7)\end{array}$ & $\begin{array}{c}17 \\
(21)\end{array}$ & $\begin{array}{l}12 \\
(10)\end{array}$ \\
\hline & \multirow[t]{2}{*}{ eggPC } & $5 \mu \mathrm{m}$ & $\begin{array}{l}337 \\
(27)\end{array}$ & $\begin{array}{l}85 \\
(24)\end{array}$ & $\begin{array}{l}211 \\
(13)\end{array}$ \\
\hline & & $2.5 \mu \mathrm{m}$ & $\begin{array}{l}222 \\
(34)\end{array}$ & $\begin{array}{l}101 \\
(35)\end{array}$ & $\begin{array}{l}162 \\
(14)\end{array}$ \\
\hline
\end{tabular}


The present results have implications for the selection of nebulisers for generating liposomal aerosols. A small aerosol size generally requires increased shear forces within the nebuliser. However, the evaporation of droplets in response to gas flow through the open vent of the Sidestream may additionally contribute, causing an increased rate of aerosol output, and reduced damage to the liposomes, relative to the other nebulisers. The damage to residual liposomes is, like the output rate, droplet size, and aerosol response to changing fluid properties, a complex function of the nebuliser design. This may explain the poor correlation between the droplet size and the damage caused to residual liposomes (fig. 4.6). Nebulisers producing small aerosol droplets are likely to cause the greatest damage to residual liposomes, as they are associated with increased shear forces, and/or more efficient baffles. However, the Sidestream produces significantly smaller droplets than the Cirrus, yet causes significantly less bilayer damage $(p<0.05)$. Therefore, the damage caused by nebulisers to retained liposomes may be independent of the droplet size characteristics of the nebuliser, particularly if the nebuliser is less reliant upon shear energy to control the droplet size (eg. Sidestream). It is instead, a complex function of the relative performance of the baffles, of the disruptive energy provided by shear forces, and other design features peculiar to a nebuliser. These control the efficiency of aerosol production and output, and also the fate of the aerosolised liposomes. The Sidestream nebuliser may be considered, of those tested, to be an ideal nebuliser for delivering liposomes to the peripheral lung. It produces a short nebulisation time $(286 \mathrm{~s})$, relatively small droplets $(1.99 \mu \mathrm{m})$, and the least damage to the retained liposomes. This is because the smaller droplet size produced by the Sidestream is in part achieved through the evaporation of droplets, due to supplemental gas flow through the open vent, rather than solely due to elevated shear forces. However, a consequence of the increased liposome stability is that the Sidestream retains a significantly greater proportion of large liposomes within the chamber, compared with the other nebulisers (chapter 3). The present results indicate that this is because the Sidestream causes less damage to liposomes, due to the fact that a small secondary aerosol size is achieved by evaporation. The larger liposomes are less likely to become reduced in size, causing them to become restricted to larger droplets, and hence the output of liposomes in the secondary aerosol is reduced. In contrast, the Respirgard II creates increased shear forces, and so causes greater liposome damage. Therefore the Respirgard II nebuliser includes liposomes in the aerosol more readily, compared with the Sidestream. Hence, the residual lipid concentration is reduced, despite having a more prolonged nebulisation time. 

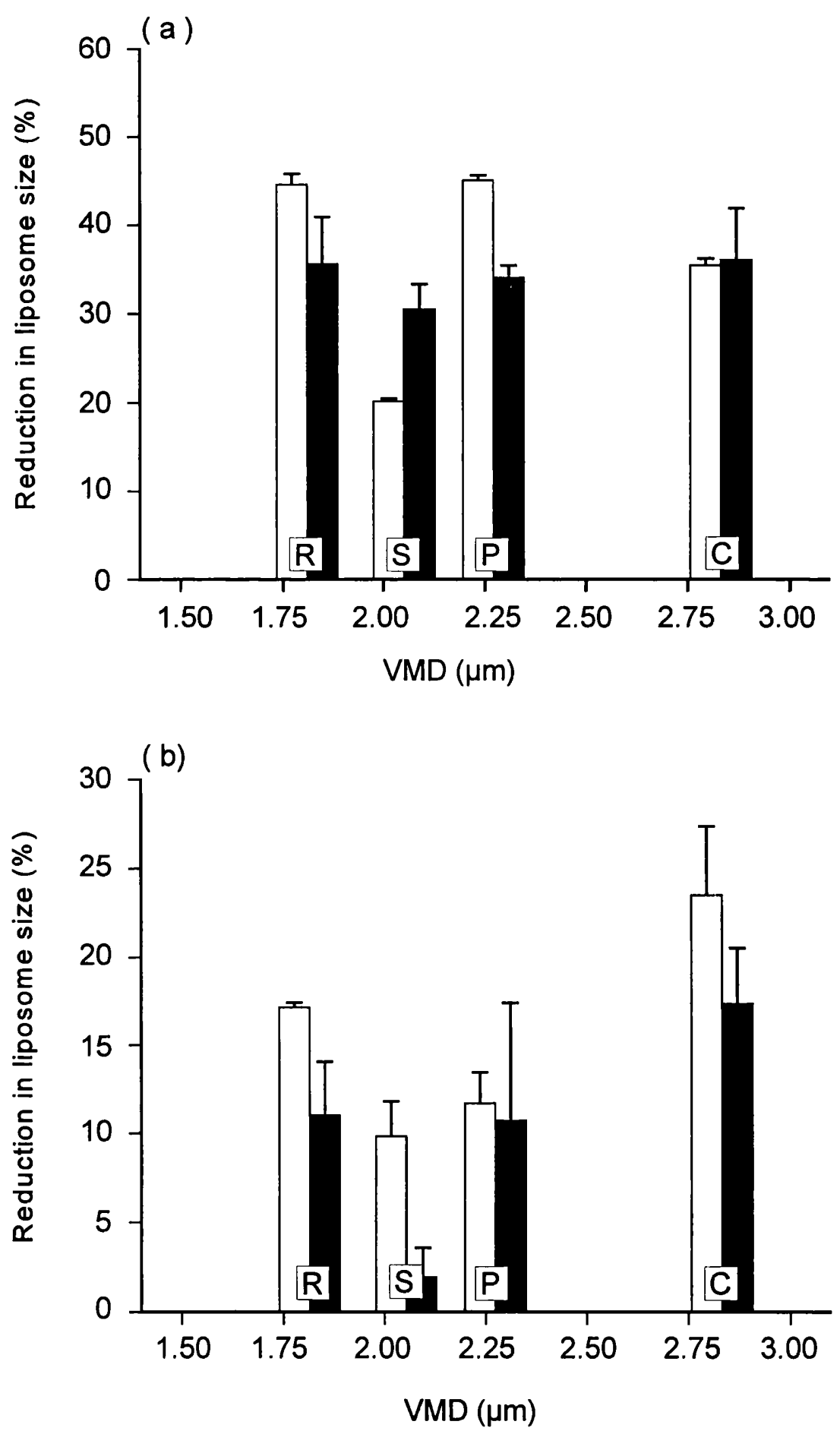

Figure 4.6 The relationship between the droplet size produced by a jet nebuliser and the reduction in the mean vesicle size caused to residual (a) eggPC and (b) eggPC/chol (1:1)

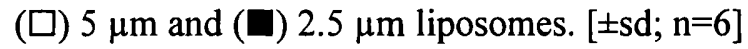


The postulated theory that larger liposomes are excluded from aerosolised droplets, until they are processed to a size that is small enough to be included in the droplets comprising the secondary aerosol mass, is supported by the results illustrated in table 4.8 .

\begin{tabular}{|c|c|c|}
\hline \multicolumn{3}{|c|}{$\begin{array}{l}\text { Table 4.8 Rate of residual liposome size reduction during } \\
\text { nebulisation as a function of the initial liposome size, and } \\
\text { bilayer composition (mean of all nebulisers })(\mathrm{nm} / \mathrm{min}) \text {. } \\
\qquad[ \pm \mathrm{sd} ; \mathrm{n}=3]\end{array}$} \\
\hline Liposome & $5 \mu \mathrm{m}$ & $2.5 \mu \mathrm{m}$ \\
\hline eggPC/chol (1:1) & $\begin{array}{r}100 \\
( \pm 21)\end{array}$ & $\begin{array}{c}31 \\
( \pm 17)\end{array}$ \\
\hline eggPC & $\begin{array}{c}244 \\
( \pm 109)\end{array}$ & $\begin{array}{c}121 \\
( \pm 46)\end{array}$ \\
\hline
\end{tabular}

The $2.5 \mu \mathrm{m}$ liposomes exhibited a damage rate that was three and five times less than that produced by the equivalent $5 \mu \mathrm{m}$ liposomes, for eggPC/chol (1:1) and eggPC liposomes respectively (table 4.8) (mean of all of nebulisers studied). The smaller liposomes became included in an increased fraction of the secondary aerosol droplets produced by each jet nebuliser, compared with the large $5 \mu \mathrm{m}$ liposomes. Consequently, the smaller liposomes were able to leave each device promptly, avoiding prolonged exposure to the shear energy that causes bilayer disruption. In so doing, they also yielded a reduced residual volume (chapter 3). Smaller liposomes are therefore exposed to the damaging shear forces of nebulisation for shorter periods, and are, as a result, inherently more stable. They also yield improved aerosol outputs, as demonstrated in chapter 3 . A consequence of these facts is that in most instances there was no significant difference between the handling of the smaller liposomes by each of the different nebulisers.

\subsubsection{The release of SCG from SCG-containing liposomes during nebulisation}

Bilayer instability during nebulisation is likely to be accompanied by the release of liposomally entrapped hydrophilic material. This was investigated using SCG-containing liposomes. The entrapment of SCG by each of the liposome formulations observed in the present experiment was in close agreement with the figures recorded in chapter 2 (table 
4.9). Entrapment efficiencies ranged from approximately $4 \%$ for the eggPC liposomes to approximately $5 \%$ for the eggPC/chol (1:1) liposomes. As determined in chapter 2 the inclusion of equimolar cholesterol within the liposome bilayers produced an increase in the recorded entrapment efficiency. However, this increase was only statistically significant for the $1 \mu \mathrm{m}$ formulations. An increased entrapped volume often results from the inclusion of cholesterol in liposomes, due to expansion of the lipid bilayers (McIntosh, 1978). There was also a trend, although not statistically significant $(\mathrm{p}<0.05)$, towards an increase in the liposomal entrapment of SCG, as the mean vesicle size of the liposome formulations increased.

\begin{tabular}{|c|c|c|c|}
\hline \multicolumn{4}{|c|}{$\begin{array}{c}\text { Table 4.9 Entrapment of SCG by variously sized eggPC } \\
\text { and eggPC/chol (1:1) liposomes (\%/100 mg lipid). } \\
{[ \pm \mathrm{sd} ; \mathrm{n}=3]}\end{array}$} \\
\hline $\begin{array}{c}\text { Size/ } \\
\text { Composition }\end{array}$ & $\mathbf{1} \boldsymbol{\mu m}$ & $\mathbf{2 . 5} \boldsymbol{\mu m}$ & $\mathbf{5} \boldsymbol{m}$ \\
\hline eggPC & 4.00 & 4.18 & 4.25 \\
& $(0.33)$ & $(0.34)$ & $(0.31)$ \\
\hline eggPC/chol & 4.88 & 4.81 & 5.09 \\
$(\mathbf{1 : 1 )}$ & $(0.44)$ & $(0.38)$ & $(0.59)$ \\
\hline
\end{tabular}

The Pari-LC and Sidestream nebulisers were studied, as they represent modern open vent nebulisers, commonly cited in aerosol research publications. They produce comparable aerosol characteristics, yet are based on markedly different designs (see section 3.4.5). The purpose of the vent is to provide supplementary gas flow to the aerosol during the patient's inspiratory phase of breathing. The Sidestream nebuliser has a relatively simple open vent, through which air is drawn at all times during nebulisation. Such a design may cause enhanced evaporation of solvent from aerosolised droplets during operation, thus providing smaller aerosol sizes at reduced driving gas flow rates (see chapter 3). In contrast, the PariLC nebuliser represents a higher technology device, incorporating as it does a breatheassisted, open vent. Such a vent opens fully only when inspiratory airflow is provided by the patient. 
Each nebuliser was studied at relative extremes of driving gas flow, with flow rates of 4 and $7 \mathrm{l} / \mathrm{min}$ used. This allowed the relationship between the driving gas flow rate and the liposome stability during nebulisation to be investigated. A loss of entrapped SCG from the liposomes occurred during nebulisation, as evidenced by the increase in the unentrapped SCG concentration within the residual fluid (table 4.10, fig. 4.7 and 4.8). The fraction of liposomally entrapped SCG that became released during nebulisation, ranged from 32.78 to $82.37 \%$ for the Pari-LC nebuliser (fig. 4.7), and from 24.94 to $68.91 \%$ for the Sidestream (fig. 4.8). The release of SCG from the SCG-containing liposomes appeared to be dependent on the initial liposome size and bilayer composition, and to an extent on the nebuliser model and driving gas flow rate used (fig 4.7 and 4.8).

The results of SCG release during nebulisation confirmed the relatively unstable nature of eggPC liposomes (fig. 4.7 and 4.8). The eggPC liposomes yielded a $65.06 \%$ greater release of entrapped SCG during nebulisation, compared with liposomes that incorporated equimolar cholesterol. This is due to the stabilising effect that cholesterol has upon liposome bilayers. Just as cholesterol increases the resistance of liposomes to ultrasonic and extrusion mediated size-reduction, to entrapped solute efflux, and to liposome size reduction during nebulisation, so it increases the retention of a soluble marker during nebulisation. The eggPC liposomes have relatively fluid bilayers, which makes them sensitive to the shear forces created by gas flow within the nebuliser. As a result, the liposome bilayers readily become disrupted, rapidly reduce in size, and release the entrapped aqueous phase, and associated SCG, to the continuous phase.

The relative ease of SCG release from the eggPC liposomes also made the influence of the mean liposome size less critical in determining liposome stability. There was only a significant difference in SCG release evident between the 5 and $1 \mu \mathrm{m}$ liposome formulations, when nebulised with the Pari-LC $(\mathrm{p}<0.05)$. However, the effects of eggPC liposome size were more critical for the Sidestream nebuliser, with only the 5 and $2.5 \mu \mathrm{m}$ mean size formulations giving insignificant differences $(p<0.05)$. 


\begin{tabular}{|c|c|c|c|c|c|c|}
\hline \multicolumn{7}{|c|}{$\begin{array}{l}\text { Table 4.10 Loss of SCG }(\mu \mathrm{g} / \mathrm{ml} \text { of residual liposomes, and } \%) \text {, from eggPC and } \\
\text { eggPC/chol (1:1) }(2.5 \text { and } 5 \mu \mathrm{m} \text { initial mean size) liposome formulations, during } \\
\text { nebulisation at gas flow rates of } 4 \text { and } 71 / \mathrm{min} .[ \pm \mathrm{sd} ; \mathrm{n}=3]\end{array}$} \\
\hline \multirow{2}{*}{$\begin{array}{l}\text { Flow } \\
\text { rate }\end{array}$} & \multirow{2}{*}{$\begin{array}{r}\text { Size } \\
(\mu \mathrm{m})\end{array}$} & \multirow{2}{*}{$\begin{array}{c}\text { SCG } \\
\text { loss }\end{array}$} & \multicolumn{2}{|c|}{ eggPC } & \multicolumn{2}{|c|}{ eggPC/chol $(1: 1)$} \\
\hline & & & Pari-LC & Sidestream & Pari-LC & Sidestream \\
\hline \multirow{6}{*}{$\begin{array}{c}7 \\
1 / \min \end{array}$} & \multirow[t]{2}{*}{5} & $\mu \mathrm{g} / \mathrm{ml}$ & $\begin{array}{r}456.91 \\
(33.25) \\
\end{array}$ & $\begin{array}{r}336.20 \\
(7.24) \\
\end{array}$ & $\begin{array}{r}380.45 \\
(21.91) \\
\end{array}$ & $\begin{array}{l}214.04 \\
(11.97) \\
\end{array}$ \\
\hline & & $\%$ & $\begin{array}{l}82.37 \\
(7.42)\end{array}$ & $\begin{array}{l}68.91 \\
(4.02)\end{array}$ & $\begin{array}{c}56.71 \\
(0.539)\end{array}$ & $\begin{array}{l}42.74 \\
(2.39)\end{array}$ \\
\hline & \multirow[t]{2}{*}{2.5} & $\mu \mathrm{g} / \mathrm{ml}$ & $\begin{array}{r}331.67 \\
(28.78) \\
\end{array}$ & $\begin{array}{r}341.23 \\
(2.85) \\
\end{array}$ & $\begin{array}{r}270.74 \\
(16.56) \\
\end{array}$ & $\begin{array}{r}231.75 \\
(11.40) \\
\end{array}$ \\
\hline & & $\%$ & $\begin{array}{l}74.45 \\
(6.46)\end{array}$ & $\begin{array}{l}64.80 \\
(2.85)\end{array}$ & $\begin{array}{l}43.83 \\
(2.54)\end{array}$ & $\begin{array}{l}44.86 \\
(2.20)\end{array}$ \\
\hline & \multirow[t]{2}{*}{1} & $\mu \mathrm{g} / \mathrm{ml}$ & $\begin{array}{l}378.64 \\
(26.83)\end{array}$ & $\begin{array}{c}219.33 \\
(8.80)\end{array}$ & $\begin{array}{c}232.37 \\
(9.62)\end{array}$ & $\begin{array}{l}137.22 \\
(5.35)\end{array}$ \\
\hline & & $\%$ & $\begin{array}{l}70.82 \\
(5.01)\end{array}$ & $\begin{array}{l}45.92 \\
(1.71)\end{array}$ & $\begin{array}{l}37.98 \\
(1.19)\end{array}$ & $\begin{array}{l}26.65 \\
(1.03)\end{array}$ \\
\hline \multirow{6}{*}{$\begin{array}{c}4 \\
1 / \min \end{array}$} & \multirow[t]{2}{*}{5} & $\mu \mathrm{g} / \mathrm{ml}$ & $\begin{array}{l}443.91 \\
(38.48) \\
\end{array}$ & $\begin{array}{l}309.09 \\
(37.15) \\
\end{array}$ & $\begin{array}{r}252.18 \\
(19.27) \\
\end{array}$ & $\begin{array}{c}268.15 \\
(9.7) \\
\end{array}$ \\
\hline & & $\%$ & $\begin{array}{l}77.30 \\
(6.86) \\
\end{array}$ & $\begin{array}{l}64.66 \\
(7.77) \\
\end{array}$ & $\begin{array}{l}43.95 \\
(2.64) \\
\end{array}$ & $\begin{array}{l}43.36 \\
(1.57) \\
\end{array}$ \\
\hline & \multirow[t]{2}{*}{2.5} & $\mu \mathrm{g} / \mathrm{ml}$ & $\begin{array}{l}326.93 \\
(20.99) \\
\end{array}$ & $\begin{array}{r}339.86 \\
(24.53) \\
\end{array}$ & $\begin{array}{l}301.72 \\
(10.26) \\
\end{array}$ & $\begin{array}{r}223.69 \\
(11.72) \\
\end{array}$ \\
\hline & & $\%$ & $\begin{array}{l}65.90 \\
(4.20) \\
\end{array}$ & $\begin{array}{l}63.32 \\
(4.57) \\
\end{array}$ & $\begin{array}{l}50.54 \\
(1.72) \\
\end{array}$ & $\begin{array}{l}38.89 \\
(2.04) \\
\end{array}$ \\
\hline & \multirow[t]{2}{*}{1} & $\mu \mathrm{g} / \mathrm{ml}$ & $\begin{array}{l}271.27 \\
(34.15) \\
\end{array}$ & $\begin{array}{l}211.06 \\
(10.05) \\
\end{array}$ & $\begin{array}{r}187.44 \\
(12.67) \\
\end{array}$ & $\begin{array}{l}157.46 \\
(6.58) \\
\end{array}$ \\
\hline & & $\%$ & $\begin{array}{l}58.88 \\
(7.41) \\
\end{array}$ & $\begin{array}{l}47.25 \\
(2.25) \\
\end{array}$ & $\begin{array}{l}32.78 \\
(2.22) \\
\end{array}$ & $\begin{array}{l}24.94 \\
(1.05)\end{array}$ \\
\hline
\end{tabular}


(a)

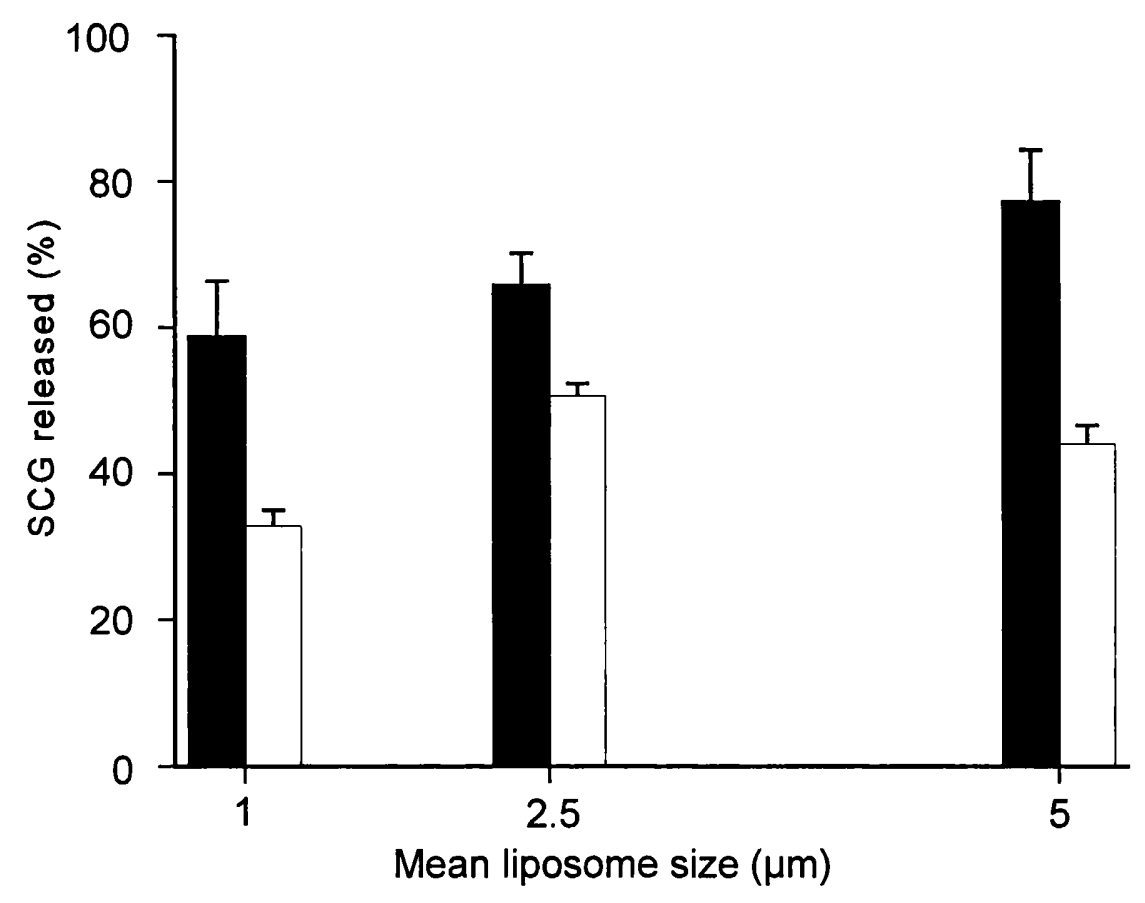

(b)

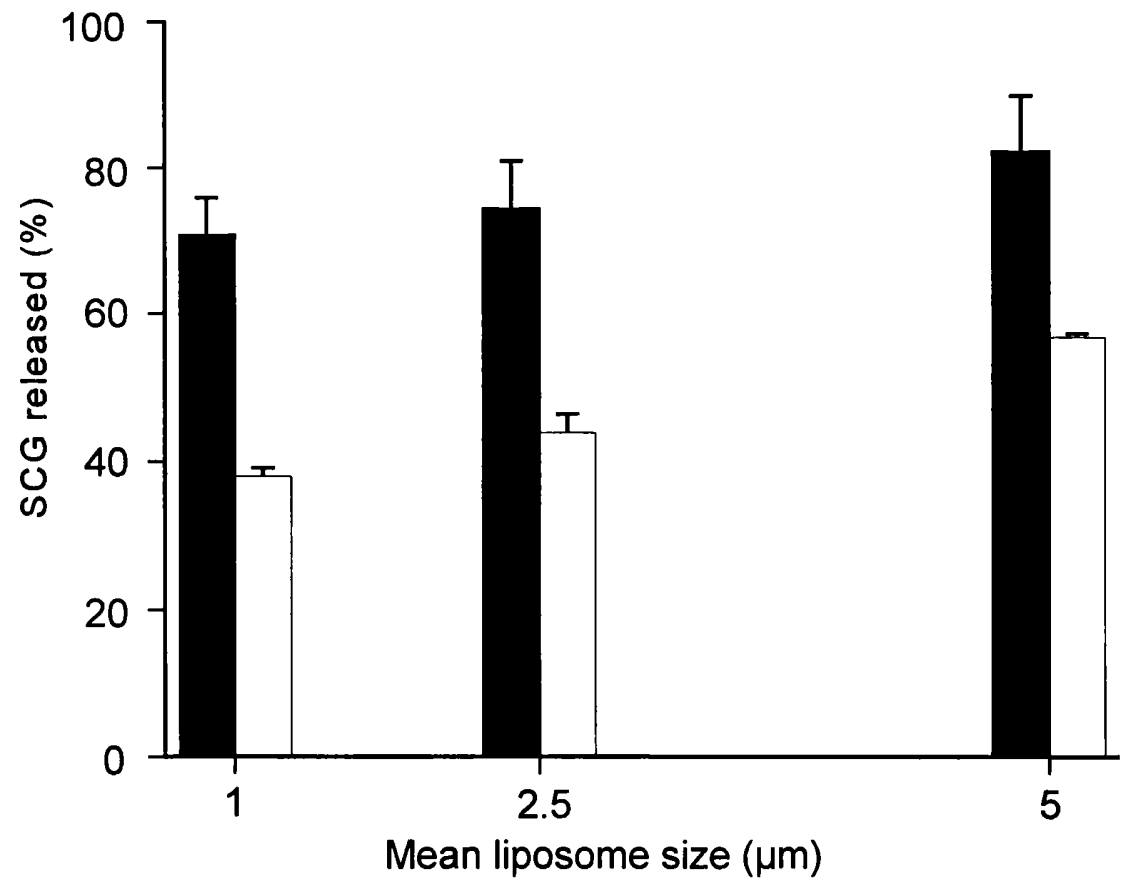

Figure 4.7 The proportion of liposomally entrapped SCG released from 5, 2.5 and $1 \mu \mathrm{m}$ mean size ( $\square$ ) eggPC and ( $\square$ ) eggPC/chol (1:1) liposomes, following nebulisation with the Pari-LC nebuliser, operated at a driving gas flow rate of (a) $4 \mathrm{l} / \mathrm{min}$ and (b) $7 \mathrm{l} / \mathrm{min}$. [n=3; \pm sd] 
(a)

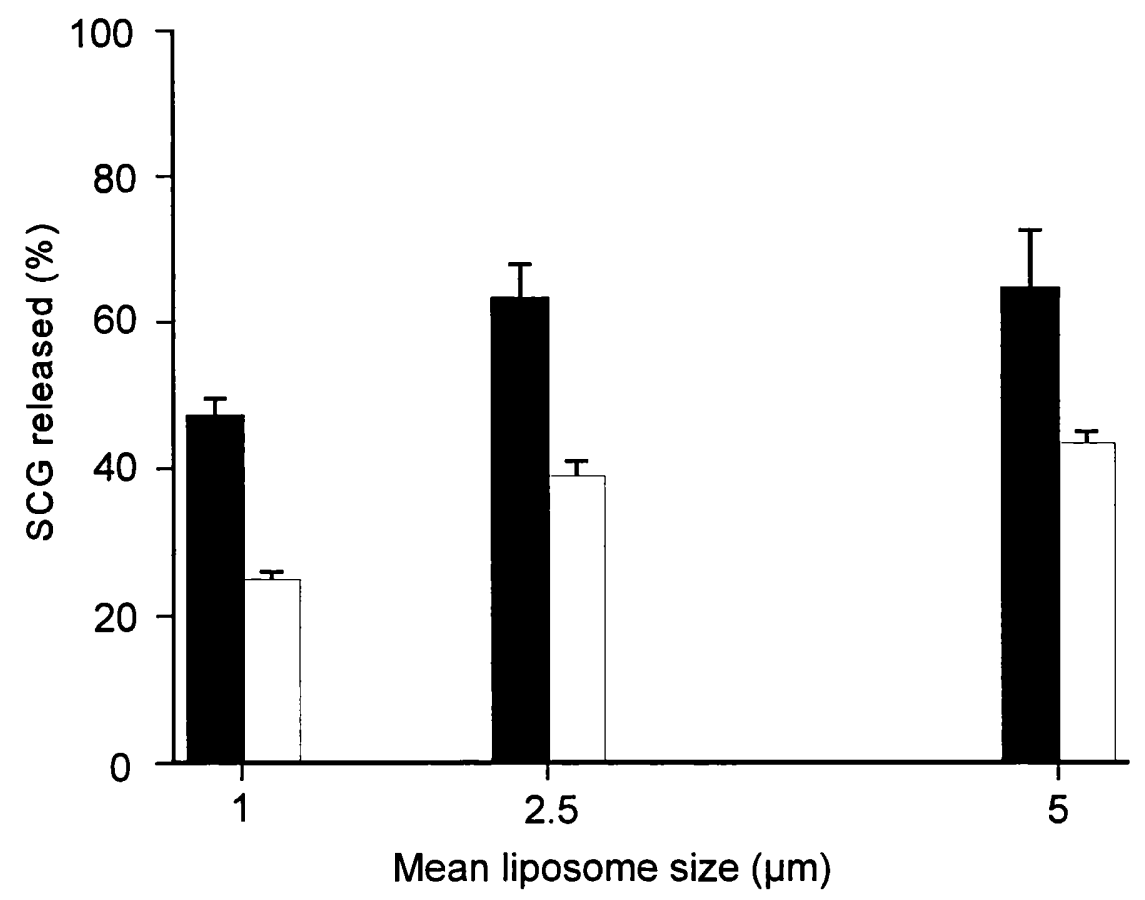

(b)

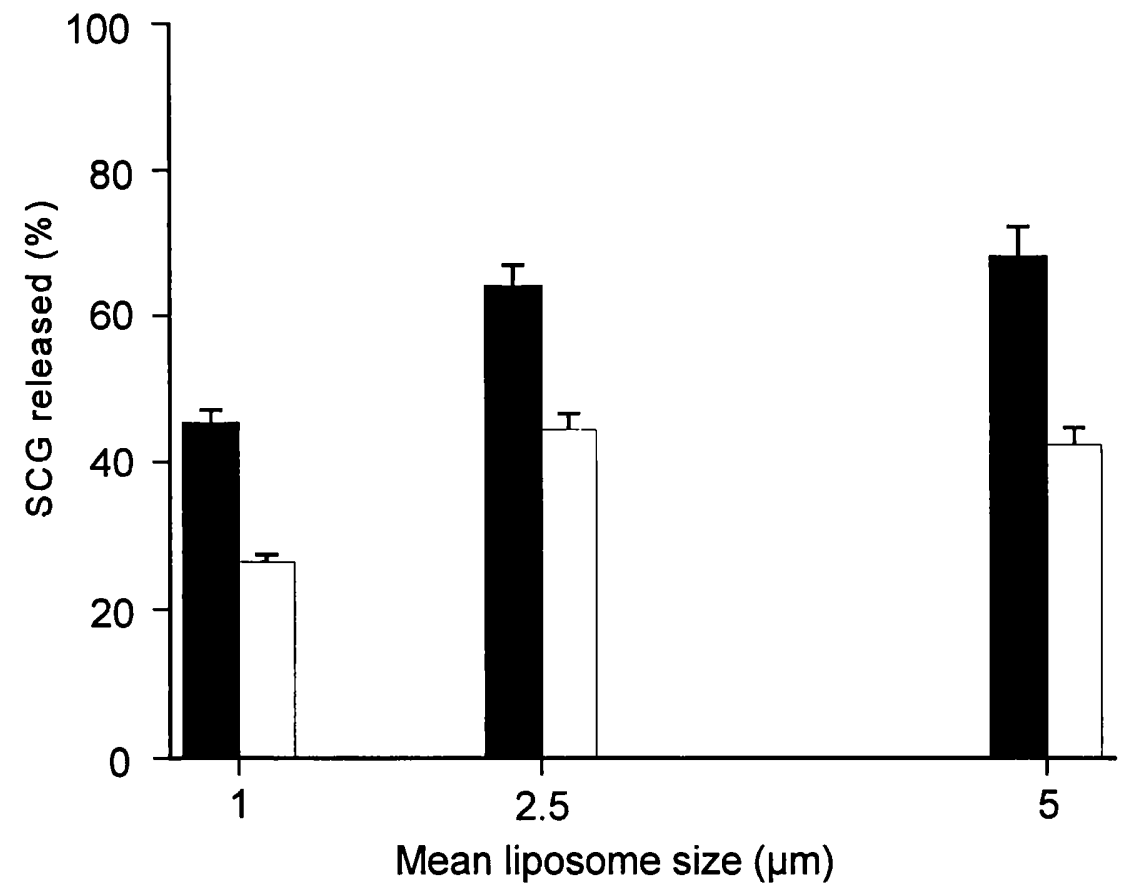

Figure 4.8 The proportion of liposomally entrapped SCG, released from 5, 2.5 and $1 \mu \mathrm{m}$ mean size ( $\square$ ) eggPC and $(\square)$ eggPC/chol (1:1) liposomes, following nebulisation with the Sidestream nebuliser, operated at a driving gas flow rate of (a) $4 \mathrm{l} / \mathrm{min}$ and (b) $7 \mathrm{l} / \mathrm{min}$. $[n=3 ; \pm s d]$ 
The retention of entrapped solutes during nebulisation may to a certain extent depend on the characteristics of the particular solute/drug studied, as well as the liposome bilayer composition. For instance, Wichert et al. (1992) demonstrated that amikacin was more readily released from cholesterol-containing SPC liposomes, than those composed of SPC alone. However, this is in contrast to the effects of cholesterol demonstrated in the present study, and also those presented by Taylor et al. (1990b), and Niven and Schreier (1990), using SCG and CF respectively. This may be due to the fact that amikacin is a polycationic molecule at physiological $\mathrm{pH}$, and is likely to behave differently to both $\mathrm{CF}$ and SCG, which possess negatively charged groupings.

Niven et al. (1992a) suggested that the operating characteristics of the nebuliser system would be a factor in determining the stability of liposomes to nebulisation. Despite this, no studies have compared the stability of liposomes to nebulisation with different nebulisers. The present study selected two nebulisers, that gave similar nebulisation times (the Pari-LC and the Sidestream) for study. Nebulisation of SCG-containing liposomes with the Pari-LC nebuliser was associated with a greater release of SCG compared with the Sidestream, for each of the formulations, and flow rates studied. This indicates that the Pari-LC nebuliser damages liposome bilayers to a greater extent during operation. This supports the results of the liposome size reduction experiment which demonstrated that the Pari-LC caused a greater reduction in the residual liposome size than the Sidestream during nebulisation. Despite this, it is unclear whether the stability of liposomes to nebulisation can be simply measured by assessing residual liposome size changes. The picture may be complicated by the fact that disruption of bilayers may result in larger liposomes being produced, as a result of bilayer fusion. This has previously been demonstrated with large DPPC liposomes, which were shown to increase in size during ultrasonic nebulisation (Barber and Shek, 1989). The results of the residual liposome size study seem to discount this, although the reannealling of smaller liposomes and bilayer fragments is also likely to have occurred.

The present results therefore support the previous hypothesis that the processing of liposomes during nebulisation is highly dependent on the design and build characteristics of the particular nebuliser under study. A consequence of this, is that despite producing larger droplets, with a greater span of sizes (chapter 3), the Pari-LC causes the release of significantly more entrapped SCG during operation. As previously postulated, this may be 
due to the open vent design of the Sidestream, which ensures a small droplet size, due through evaporation, without necessitating damagingly elevated shear forces. The release of SCG from the liposomes may also be influenced by the internal surface area of the nebuliser, as bilayer packing changes may result from contact with hydrophobic surfaces.

The release of SCG to the continuous phase during nebulisation was apparently dependent on the gas flow rate used to drive the nebulisers (fig. 4.8). Operation of the Pari-LC and the Sidestream nebulisers at $7 \mathrm{l} / \mathrm{min}$ yielded an increase in SCG release of 19 and $2.52 \%$ for the Pari-LC, and 8.84 and $6.58 \%$ for the Sidestream (mean of each liposome size, for eggPC and eggPC/chol (1:1) liposomes respectively), compared with $4 \mathrm{l} / \mathrm{min}$. This is in agreement with the previous observation that the residual liposome damage was increased by changing the flow rate from 7 to $81 / \mathrm{min}$ (section 4.4 .1 and 4.4.2). However, the differences prove insignificant when tested $(p<0.05)$. An increased release of SCG at the higher flow rate may result from the greater shear energy generated, and the increased rate of fluid aspiration, which causes more rapid fluid recycling within the nebuliser. The primary droplets that contain liposomes have an increased momentum, and thus become impacted on baffles more readily. This causes an increased droplet recycling, and thus exposes the liposomes to an increased duration of bilayer damaging processes. However, solute evaporation during nebulisation must also be considered. The concentration of both liposomes and dissolved solutes has been shown to markedly increase during nebulisation, as a significant percentage of the nebuliser output is composed of water vapour (chapter 3 ). Higher driving gas flow rates may be expected to cause an increased level of solvent evaporation, and thus produce a more concentrated SCG solution following nebulisation. It is therefore difficult to separate the contributions of evaporative losses, and bilayer damage, to the observed increase in SCG concentration. Although the present results were not statistically conclusive, the trend supports the findings of Niven et al. (1992a), which described an approximately linear relationship between the air pressure and CF release from soyPC/DPPG (9:1) liposomes, during nebulisation. The authors concluded that of all the variables involved in the nebulisation of liposomes, it was the driving gas flow rate that had the greatest influence on the retention of entrapped solutes. The failure of the present experiment to show a significant relationship between flow rate and SCG release, may be a consequence of the nebulisers selected for study, or the fact that the experiment was only performed in triplicate. Alternatively, the flow rates may not have differed sufficiently. 


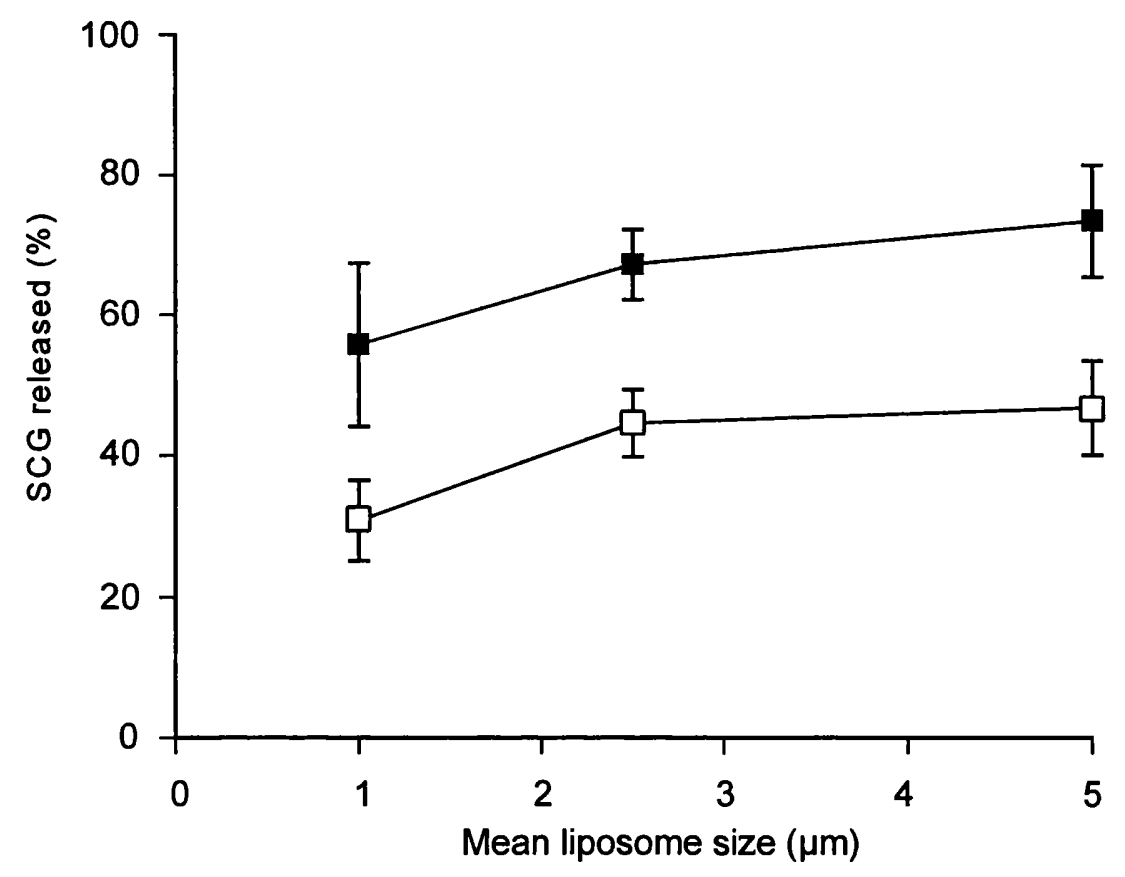

Figure 4.9 The relationship between the initial size of $(\square)$ eggPC and $(\square)$ eggPC/chol (1:1) liposomes, and the fraction of entrapped SCG released during nebulisation (mean of all nebulisers and flow rates). $[ \pm s d ; n=3]$

The release of entrapped SCG from eggPC liposomes was increased by $34.1 \%$ (mean of Pari-LC and Sidestream), with a change in the initial size of the liposomes from 1 to $5 \mu \mathrm{m}$ (fig. 4.9). This is in close agreement with the results published by Niven et al. (1991a), which demonstrated an increase in CF release from SPC/DPPG liposomes of $39.1 \%$, as the mean vesicle size was increased from 1 to $5 \mu \mathrm{m}$, following nebulisation with a Collison nebuliser operated at $4 \mathrm{l} / \mathrm{min}$. Taylor et al (1990b) had previously demonstrated that 50.8 $\%$ of the SCG originally entrapped within $5.4 \mu \mathrm{m}$ eggPC/chol (1:1) MLVs was lost following nebulisation into an MLI. This figure was reduced to $31.9 \%$ for $3.4 \mu \mathrm{m}$ eggPC/chol (1:1) REVs, and to $16.6 \%$ and $14.2 \%$ for $1.2 \mu \mathrm{m} \mathrm{eggPC/chol} \mathrm{(1:1)} \mathrm{and} \mathrm{DPPC}$ REVs, respectively. The greater stability of small liposomes to nebulisation was further illustrated by Wichert et al. (1992). The increased solute losses experienced by larger liposomes results from the fact that they are more exposed to the continually forming airfluid interface of the primary droplets, and therefore to the shearing action of gas flow, than smaller liposomes. Secondly, as was demonstrated in chapter 3, unless they are processed 
to a smaller size the larger liposomes remain excluded from the secondary aerosol droplets, and therefore become retained within the nebuliser chamber. In so doing, they undergo repeated and prolonged atomisation. With a reduction in the mean vesicle size to $1 \mu \mathrm{m}$ the liposome becomes small enough to be included in a greater proportion of the secondary aerosol droplets without requiring extensive size reduction. As a result, the liposome output is increased (chapter 3), and consequently the observed bilayer damage, vesicle size reduction, and entrapped solute losses are all significantly reduced.

It is significant that the increase in SCG release is more marked with an increase in the mean liposome size from $1 \mu \mathrm{m}$ to $2.5 \mu \mathrm{m}$, than with an increase in size from 2.5 to $5 \mu \mathrm{m}$ (fig. 4.9). This is consistent with the results obtained for the increase in residual liposome concentration during nebulisation (fig. 3.19). It is thus apparent, that $2.5 \mu \mathrm{m}$ represents a significant vesicle size with regard to being aerosolised. Liposome sizes below $2.5 \mu \mathrm{m}$ yield a markedly improved liposome output (chapter 3), and also possess an increased bilayer stability (fig. 4.9). This is because liposomes with a size greater than $2.5 \mu \mathrm{m}$ are retained within the nebuliser to a greater extent, with the result that they are significantly more unstable. A similar plateau in solute release at a mean size of above $2 \mu \mathrm{m}$ was observed by Niven et al. (1991b). This was attributed to the fact that the majority of solute was contained within liposomes that were larger than the droplet size produced by the nebuliser. Such results support the previously proposed mechanism for liposome aerosolisation. Liposomes with a size greater than the mean aerosol size are excluded from a greater proportion of the secondary aerosol droplets. As such, it is principally the smaller vesicles of the initial liposome population that become aerosolised, along with suitably small "daughter" liposomes, yielded from the disruption of larger "parent" liposomes. Nebulisers producing larger droplets generally demand less liposome processing prior to liposomeaerosol formation, and hence tend to cause less disruption to the residual liposomes. This is supported by the finding that the Cirrus nebuliser yielded residual liposome volumes that were of a reduced concentration. A fall in the residual liposome size occurs during nebulisation, despite the fact that smaller liposomes have a greater propensity for emission in the secondary aerosol. The products of the disruption of larger liposomes must therefore be significantly small, and in large enough quantities, to compensate for the preferential emission of the smaller vesicle fraction. If this had not been the case, a rise in the residual liposome size would have been observed, as the smaller liposomes of the residual liposome 
dispersion became released.

The importance of using liposomes that have mean sizes substantially below that of the mean VMD of the aerosol droplets was proposed by Niven et al (1991b). The current results support this. However, the situation is complex as the Pari-LC nebuliser produces greater solute release and size reduction than the Sidestream, despite producing larger droplet sizes with a broader distribution (chapter 3). Therefore, the design and construction of the particular nebuliser used is critical in determining the liposome stability. This, as stated previously, is likely to be a consequence of the open vent design of the Sidestream, which ensures smaller droplets are formed at a lower shear energy.

\subsubsection{The collection of aerosolised liposomes}

As previously determined (section 4.4.3), there was a marked decrease in the residual liposome size as nebulisation proceeded, for both the eggPC and eggPC/chol (1:1) liposome formulations (table 4.10 and 4.11). The inclusion of cholesterol in the liposomes stabilised the liposome bilayers to nebulisation as previously observed, such that the fall in residual liposome size was less marked for the eggPC/chol (1:1) liposomes. Similarly, the change in residual liposome size was controlled by their initial size, with the $5 \mu \mathrm{m}$ liposomes experiencing a more pronounced fall in size, compared with the equivalent $2.5 \mu \mathrm{m}$ liposomes.

The use of the TI allowed the size characteristics of the aerosolised liposomes to be investigated (fig. 4.11 and 4.12). The results of the present experiment demonstrate that the size of liposomes deposited within the TI was primarily determined by the size of the secondary aerosol droplets within which the liposome becomes emitted. The upper stage of the TI collected aerosol droplets that had a nominal aerodynamic particle size cut-off of approximately $6.4 \mu \mathrm{m}$ (Hallworth and Westmoreland, 1987). The "respirable" aerosol dose that penetrated the lower impingement stage was therefore composed of droplets possessing a mean aerodynamic diameter that was less than this size. Because by definition $50 \%$ of the aerosol volume delivered to each stage of the TI is greater than the declared cut-off size, and because of the polydisperse nature of nebulised aerosol clouds, there was a wide spread of liposome sizes within each stage of the TI (fig. 4.12 to 4.13 ). 
Table 4.11 The size of residual liposomes, and liposomes deposited within the upper and lower stages of the TI, following nebulisation of $5 \mu \mathrm{m}$ liposomes with the PariLC and Sidestream nebulisers, for $50 \%$ and $100 \%$ of the nebulisation time $(\mu \mathrm{m})$.

\begin{tabular}{|c|c|c|c|c|c|}
\hline \multicolumn{6}{|c|}{$[ \pm \mathrm{sd} ; \mathrm{n}=3]$} \\
\hline & & \multicolumn{2}{|c|}{ eggPC } & \multicolumn{2}{|c|}{ eggPC/chol (1:1) } \\
\hline Nebuliser & & $50 \%$ & $100 \%$ & $50 \%$ & $100 \%$ \\
\hline \multirow{3}{*}{ Pari-LC } & Residual & $3.74(0.08)$ & $3.33(0.13)$ & $4.58(0.04)$ & $4.00(0.09)$ \\
\hline & Upper TI & $4.37(0.11)$ & $4.16(0.62)$ & $4.80(0.16)$ & $4.61(0.07)$ \\
\hline & Lower TI & $3.25(0.15)$ & $3.16(0.41)$ & $3.15(0.03)$ & $3.01(0.08)$ \\
\hline \multirow{3}{*}{ Sidestream } & Residual & $3.97(0.27)$ & $3.67(0.03)$ & $4.62(0.09)$ & $4.22(0.09)$ \\
\hline & Upper TI & $4.21(0.13)$ & $3.97(0.69)$ & $4.20(0.42)$ & $4.53(0.23)$ \\
\hline & Lower TI & $3.06(0.42)$ & $2.97(0.17)$ & $2.59(0.12)$ & $2.81(0.09)$ \\
\hline
\end{tabular}

Table 4.12 The size of residual liposomes, and liposomes deposited within the upper and lower stages of the TI, following nebulisation of $2.5 \mu \mathrm{m}$ liposomes with the PariLC and Sidestream nebulisers, for $50 \%$ and $100 \%$ of the nebulisation time $(\mu \mathrm{m})$.

\begin{tabular}{|c|c|c|c|c|c|}
\hline \multicolumn{2}{|c|}{} & \multicolumn{2}{c|}{ eggPC } & \multicolumn{1}{c|}{ eggPC/chol (1:1) } \\
\hline Nebuliser & & $\mathbf{5 0 \%}$ & $\mathbf{1 0 0} \%$ & $\mathbf{5 0} \%$ & $\mathbf{1 0 0} \%$ \\
\hline \multirow{4}{*}{ Pari-LC } & Residual & $2.13(0.04)$ & $1.70(0.01)$ & $2.75(0.02)$ & $2.86(0.02)$ \\
\cline { 2 - 7 } & Upper TI & $3.29(0.08)$ & $4.6(0.27)$ & $2.92(0.02)$ & $2.96(0.01)$ \\
\cline { 2 - 7 } & Lower TI & $2.13(0.04)$ & $3.49(0.01)$ & $2.75(0.02)$ & $2.86(0.02)$ \\
\hline \multirow{4}{*}{ Sidestream } & Residual & $2.56(0.03)$ & $2.20(0.03)$ & $2.56(0.02)$ & $2.83(0.03)$ \\
\cline { 2 - 7 } & Upper TI & $2.68(0.10)$ & $3.25(0.13)$ & $3.37(0.23)$ & $3.62(0.02)$ \\
\cline { 2 - 7 } & Lower TI & $2.36(0.04)$ & $2.20(0.03)$ & $1.68(0.05)$ & $2.13(0.03)$ \\
\hline
\end{tabular}



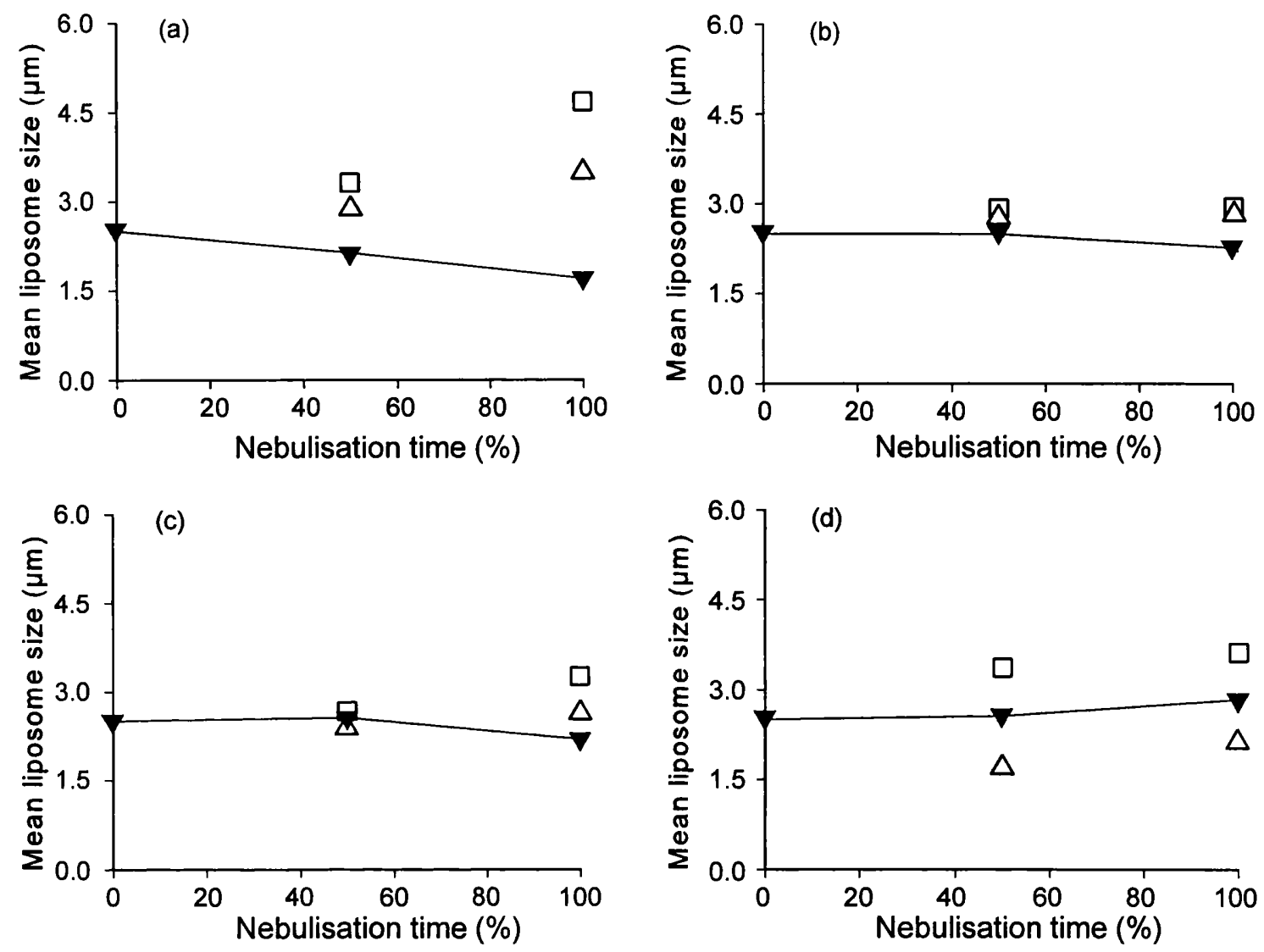

Figure 4.10 The mean size of residual liposomes $(\nabla)$, and liposomes deposited in the upper $(\square)$, and lower $(\Delta)$ stages of the twin impinger, during nebulisation of liposomes with an initial mean size of $2.5 \mu \mathrm{m}$. [ $\pm \mathrm{sd} ; \mathrm{n}=3$ ]

(a) Pari-LC nebuliser, eggPC liposomes, (b) Pari-LC nebuliser, eggPC/chol (1:1) liposomes,

(c) Sidestream nebuliser, eggPC liposomes, (d) Sidestream nebuliser, eggPC/chol (1:1) liposomes. 

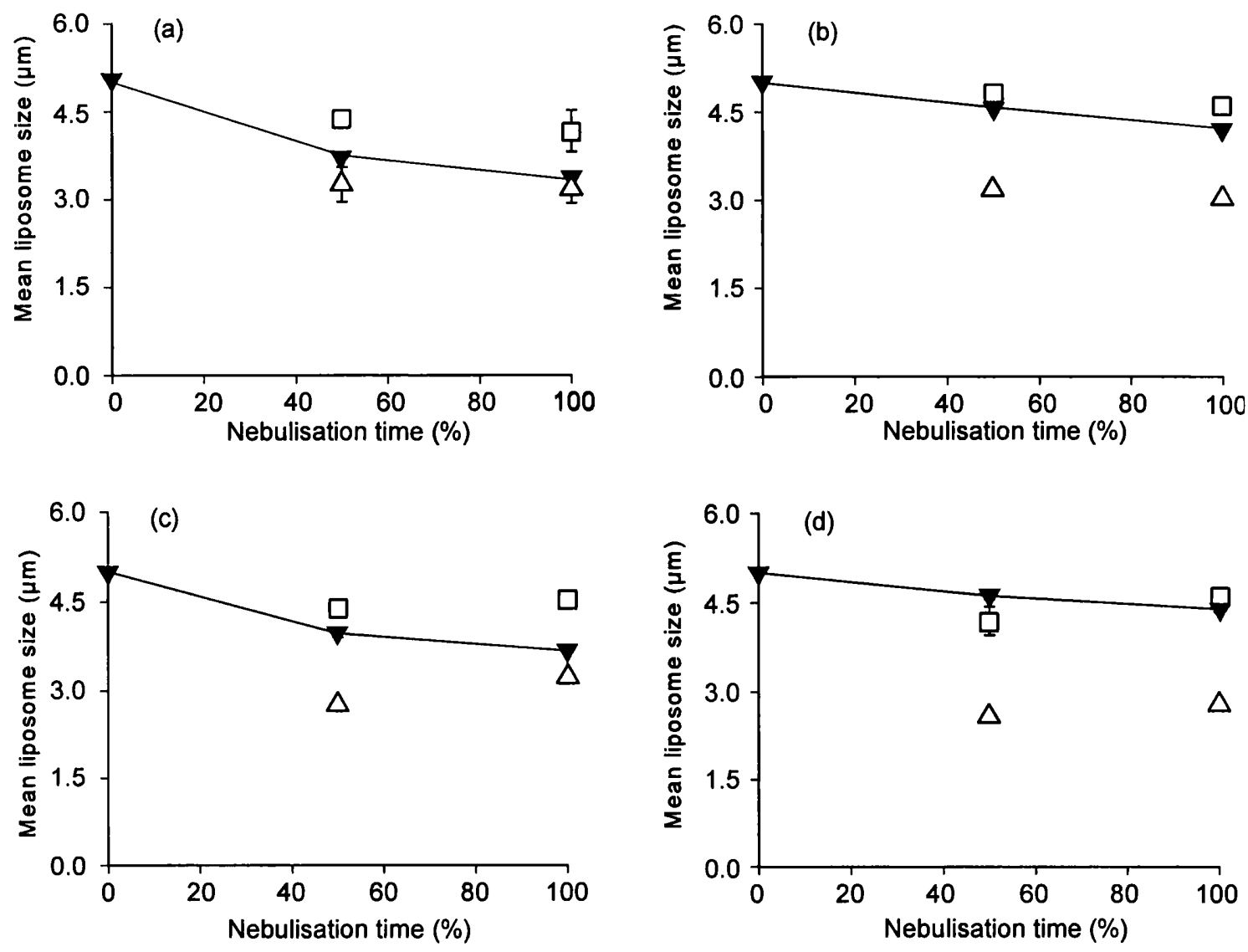

Figure 4.11 The mean size of residual liposomes $(\nabla)$, and liposomes deposited in the upper $(\square)$, and lower $(\Delta)$ stages of the twin impinger during nebulisation of liposomes with an initial mean size of $5 \mu \mathrm{m}$. [ $\pm \mathrm{sd}$; $\mathrm{n}=3]$

(a) Pari-LC nebuliser, eggPC liposomes, (b) Pari-LC nebuliser, eggPC/chol (1:1) liposomes,

(c) Sidestream nebuliser, eggPC liposomes, (d) Sidestream nebuliser, eggPC/chol (1:1) liposomes. 

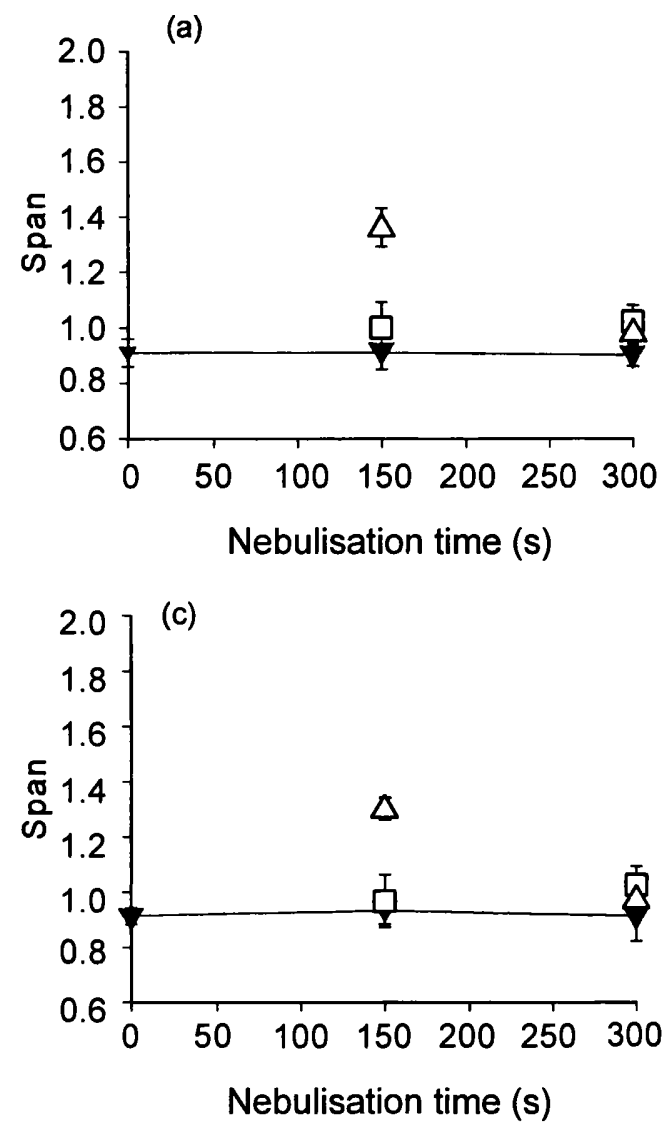

(b)
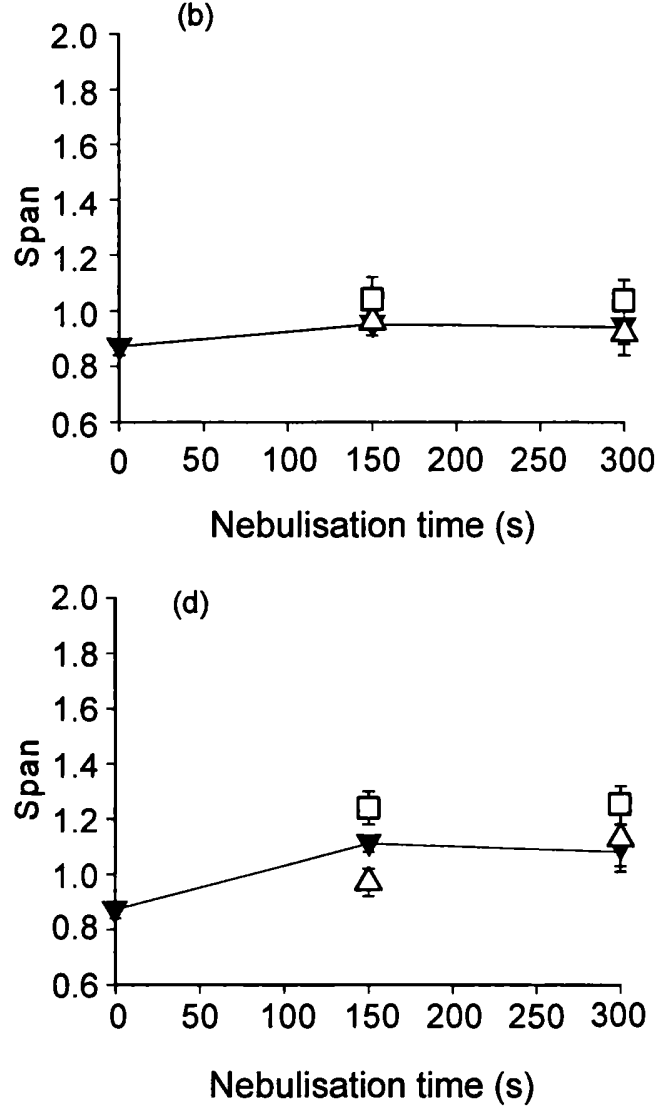

Figure 4.12 The distribution of vesicle sizes in the population of residual liposomes $(\nabla)$ and liposomes deposited in the upper $(\square)$, and lower $(\Delta)$ stages of the twin impinger, during nebulisation of liposomes with an initial mean size of $2.5 \mu \mathrm{m}$. [ $\pm \mathrm{sd} ; \mathrm{n}=3$ ]

(a) Pari-LC nebuliser, eggPC liposomes, (b) Pari-LC nebuliser, eggPC/chol (1:1) liposomes, (c) Sidestream nebuliser, eggPC liposomes, (d) Sidestream nebuliser, eggPC/chol (1:1) liposomes. 

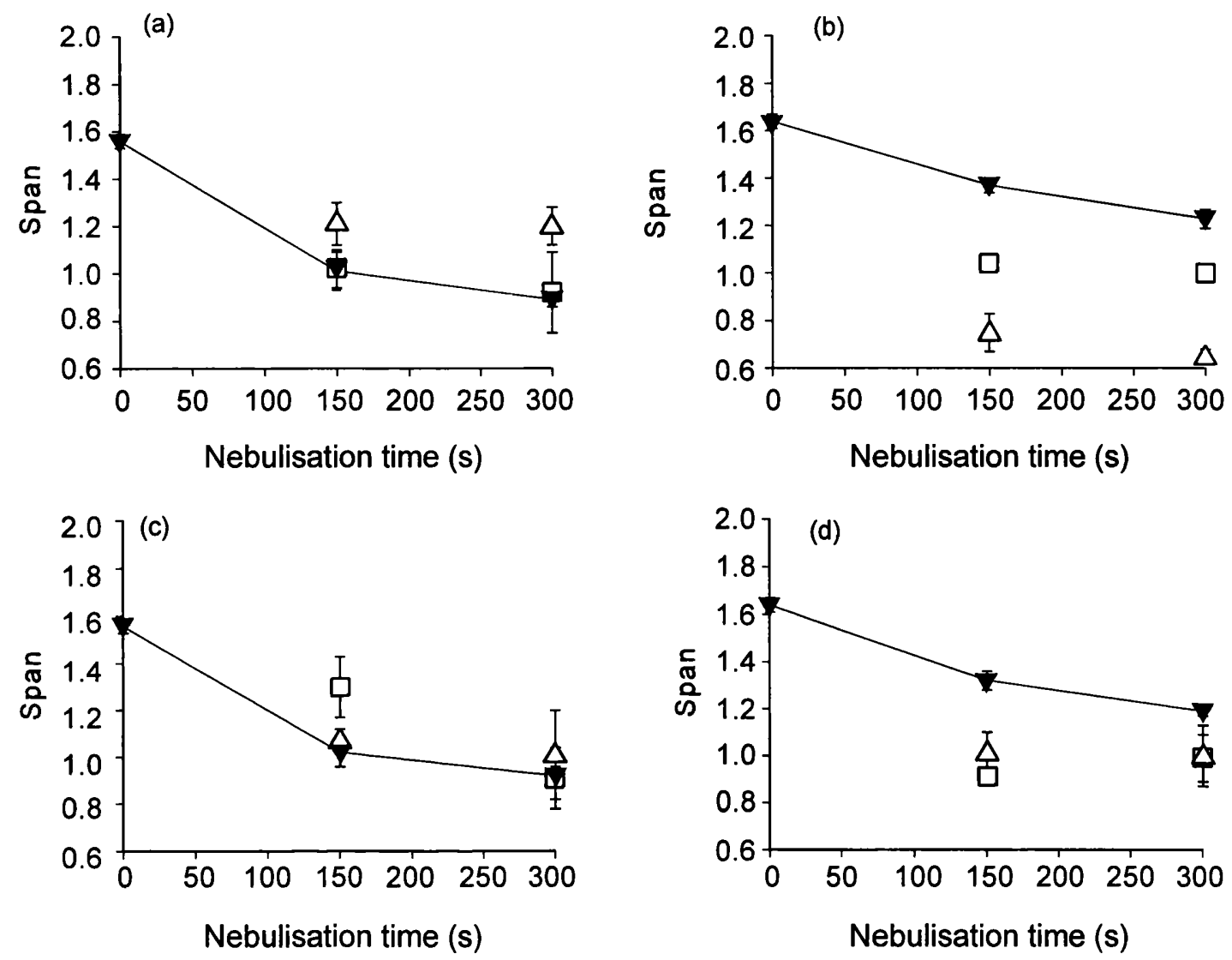

Figure 4.13 The distribution of vesicle sizes in the population of residual liposomes $(\nabla)$, and liposomes deposited in the upper $(\square)$, and lower $(\Delta)$ stages of the twin impinger, during nebulisation of liposomes with an initial mean size of $5 \mu \mathrm{m}$. [ $\pm \mathrm{sd} ; \mathrm{n}=3$ ]

(a) Pari-LC nebuliser, eggPC liposomes, (b) Pari-LC nebuliser, eggPC/chol (1:1) liposomes,

(c) Sidestream nebuliser, eggPC liposomes, (d) Sidestream nebuliser, eggPC/chol (1:1) liposomes. 
The results clearly demonstrate that the larger droplets collecting within the upper stage of the TI contained liposomes that had a larger diameter than those depositing in the lower stage. Such a result support the hypothesis that liposomes with a large mean diameter become excluded from the smaller of the aerosolised droplets. This is manifested as an increase in the residual liposome concentration as nebulisation proceeds (chapter 3), and greater entrapped solute losses (section 4.4.4). The larger mean diameter of droplets depositing in the upper stage of the TI means an increased proportion of the larger liposomes from the liposome population are aerosolised in these secondary aerosol droplets. The mean vesicle size of liposomes sampled from the upper stage was therefore relatively large. By contrast, the smaller diameter of droplets depositing within the lower stage limits the size of the liposomes that may be deposited. As a result, the lower stage contains liposomes of a reduced diameter compared with the upper stage.

The size of eggPC liposomes which deposited within the TI, following nebulisation of 2.5 $\mu \mathrm{m}$ mean size liposomes with the Pari-LC nebuliser, was found to be very much larger than the residual liposome size (table 4.11). Of the two nebulisers studied, the Pari-LC produces the most significant reduction in residual size for these liposomes. This result may therefore indicate that the liposomes become fused and/or aggregated during, or following, aerosolisation, in response to the increased bilayer fracture.

The mean size of liposomes deposited within the TI was generally larger when delivered by the Pari-LC nebuliser compared with delivery from the Sidestream (table 4.11 and 4.12). However, this was only significant $(p<0.05)$ for 6 out of the 16 results. Despite this, it does seem to support the theory that the Pari-LC, due to its larger secondary aerosol size and larger span of droplet sizes, releases a greater proportion of liposomes with a larger diameter from the residual liposome dispersion. This also results in the Pari-LC producing reduced residual masses compared with the Sidestream (chapter 3). The Sidestream concentrates large liposomes within the residual fluid, and thus the aerosol output is comprised of smaller vesicle sizes. This may support the theory that liposomes included within the droplets inhibit evaporation, due to an inhibition of water transport in the fluid bulk. Such an effect would be increased by an elevated lipid concentration, and would have more of an impact on the Sidestream nebuliser which, due to its open vent design, is reliant on droplet evaporation to achieve a smaller secondary aerosol droplet size. 
The eggPC liposomes which deposited within the lower stage of the TI did so with an increased mean diameter compared with the equivalent eggPC/chol (1:1) liposomes, for each nebuliser and liposome formulation studied. This may have been due to fusion of liposomes during, or after, aerosolisation. The lower stage of the TI contains liposomes deposited from aerosol droplets with a mean size below $6.4 \mu \mathrm{m}$, and as such, the included liposomes may have been subjected to greater stresses during droplet formation, than liposomes deposited in larger droplets within the upper stage. The liposomes in the smaller droplets may therefore have undergone greater bilayer fracture, in order to become included in such small droplets. Consequently, the propensity for an increase in liposome size, as a result of the fractured bilayers refusing into larger liposomes, may be greater, particularly for the liquid-crystalline eggPC liposomes, at the ambient temperature. The fusion of eggPC/chol $(1: 1)$ bilayers is hindered at ambient temperature due to the stability of the gelstate bilayers, and hence the deposited liposomes retained a smaller mean size.

For liposomes depositing within the upper stage the picture is less clear. The $5 \mu \mathrm{m}$ initial size eggPC/chol (1:1) liposomes, which deposited within the upper stage of the TI, were considerably larger than the equivalent eggPC liposomes. A possible explanation for this is that the eggPC/chol $(1: 1)$ liposomes resist deformation and fracture upon nebulisation, due to the increased bilayer stability imparted by the inclusion of cholesterol. As a result, they are largely restricted to deposition within the upper stage of the TI. Consequently, the liposomes deposit in the upper stage with a larger mean size than the eggPC liposomes, which have relatively fluid bilayers that fracture readily during nebulisation. This has been previously evidenced by the smaller residual liposome size exhibited by eggPC liposomes during nebulisation. The lower stage of the TI gives the reverse trend, that is eggPC/chol (1:1) liposomes deposited with a reduced mean size, relative to the equivalent eggPC liposomes. The relative ease of shearing of the eggPC liposome bilayers may again be responsible for this. Larger liposomes within the eggPC population may be sheared relatively easily, to a size that approximates to the mean size of the aerosol droplets (ie. approximately $6.4 \mu \mathrm{m}$ ). The smaller droplets depositing in the lower stage therefore contain a high proportion of liposomes generated from larger vesicles within the initial population, having a size approximating to that of the droplets. By contrast, the rigid bilayers of the eggPC/chol (1:1) liposomes render them more resistant to shearing, and it is only the smaller vesicles of the original liposomes that are carried over to the lower stage. 
The mean size of the liposomes that deposited within the TI generally failed to reflect the changes that occurred in the residual liposomes during nebulisation. Only the $5 \mu \mathrm{m}$ initial size liposomes, aerosolised by the Pari-LC, deposited with a size that was smaller at the end of nebulisation compared with that at the $50 \%$ time. This is perhaps not surprising, as the Pari-LC has been shown to cause significantly greater residual liposome size reduction than the Sidestream during nebulisation, and this reduction is significantly greater with the larger liposomes (section 4.4.3). However, despite the fact that the $2.5 \mu \mathrm{m}$ residual liposomes become progressively smaller during nebulisation, the mean size of the liposomes that deposit on each stage of the TI did not become reduced. Indeed, each of the liposome formulations deposited in the TI, following nebulisation with the Sidestream nebuliser, deposited with a significantly increased mean diameter at the end of nebulisation, compared with the $50 \%$ stage $(\mathrm{p}<0.05)$. This may be attributed to the significantly increased residual liposome concentration that has been shown to be produced by the Sidestream during nebulisation (chapter 3). The Sidestream nebuliser causes less damage to residual liposomes (section 4.4.2), and as a result the liposomal aerosol output is reduced (chapter 3). This causes the concentration of residual liposomes to be increased more than three times greater than that occurring within the Pari-LC nebuliser during operation (chapter 3). This pronounced rise in liposome concentration may encourage the fusion of liposomes during aerosolisation, as they come into increasingly intimate contact with each other as nebulisation proceeds. The result of this, is that the deposited liposome size increases with time. This is supported by the fact that the size of liposomes depositing in the TI with time was dependent on the presence or absence of cholesterol in the liposome bilayers. The size of eggPC liposomes depositing within the TI with time was found to vary according to the nebuliser under study. EggPC liposomes emitted from the Pari-LC deposited with a markedly increased diameter at the $100 \%$ time stage. By contrast, the size of the deposited eggPC/chol (1:1) liposomes remained relatively unchanged with time. This suggests that the failure of the deposited vesicle size to reflect changes in the residual liposome size was indeed a result of fusion of the fluid eggPC bilayers following nebulisation. The eggPC/chol $(1: 1)$ bilayers are relatively stable, and hence the depositing liposomes remain relatively unchanged in size. There is no obvious pattern in the nebulisation of liposomes with differing bilayer compositions using the Sidestream nebuliser. This, and the fact that the size of liposomes depositing in the TI varies between each device, suggests that the liposomes delivered by each nebuliser are processed differently. 
The eggPC liposomes, depositing within the lower stage of the TI at the $50 \%$ nebulisation time, generally had significantly larger span values than the equivalent eggPC/chol $(1: 1)$ liposomes (with the exception of $5 \mu \mathrm{m}$ liposomes nebulised with the Sidestream)(fig. 4.12 and 4.13) $(\mathrm{p}<0.05)$. This may be further evidence for the aggregation, and fusion, of the aerosolised and deposited eggPC liposomes. The situation at the end of nebulisation is less clear however, with similar span values being exhibited for the liposomes depositing within each stage. The failure of the deposited liposome size to decrease with time, in a similar manner to the residual size, may also have been due to the rise in the residual liposome concentration that is experienced within the nebuliser with time. This causes an increase in the viscosity of the residual fluid, producing an associated rise in the emitted droplet size (chapter 3). Such a rise in the emitted droplet size with time would cause the larger liposomes in the residual fluid to be aerosolised without damage, and hence the size of liposomes depositing in the TI remained relatively constant (Pari-LC), or increased (Sidestream), despite a fall in the residual liposome size. Further to this, the secondary aerosol may not reflect the full effect that a rise in the residual concentration has upon the primary aerosol. It is conceivable, that a rise in the residual liposome concentration may have produced a more significant increase in the primary aerosol size within the Sidestream nebuliser, than the changes in the secondary aerosol size would suggest, due to the efficient baffling exhibited by the device. Therefore, despite the fact that the emitted aerosol size remained relatively unchanged, the secondary droplets were produced from a population of primary droplets containing an increased fraction of large liposomes. As a result, the liposomes within the TI failed to reflect the reducing size of the residual liposomes.

The span values of liposomes depositing within the TI following nebulisation provided interesting results. The span of the residual liposomes generated from the $2.5 \mu \mathrm{m}$ liposome formulation was relatively unchanged during nebulisation (fig. 4.12), reflecting their greater stability to nebulisation, and is consistent with the study of the residual liposome size. By contrast, the instability of larger liposomes to nebulisation caused the span of the residual liposomes generated from the $5 \mu \mathrm{m}$ liposome formulation to undergo a marked fall during nebulisation (fig. 4.13). The liposomes that deposited within the TI therefore had span values that generally reflected changes occurring in the mean vesicle size of the residual liposomes. 


\subsubsection{The intra-nebuliser variability in the generation of liposomal aerosols}

Different nebulisers have been shown to vary in the quantity, and quality, of aerosol produced during liposome nebulisation (chapter 3). Furthermore, the liposomes are processed differently depending on the particular device used. The production of aerosol droplets is dependent on the performance of baffles, jets and other structures within a nebuliser. With the design and build of nebulisers varying considerably between models, it is not surprising that liposomes are processed differently by different nebulisers. Further to this, it may be predicted that individual nebulisers of the same model, that share a common design and build, would give a highly reproducible liposome handling. To test this hypothesis, nebulisation using five different Pari-LC and Sidestream devices was examined. The mean aerosol characteristics produced by the Pari-LC and Sidestream nebulisers are presented in table 4.13. The droplets produced by the Pari-LC have a broader size distribution than those produced by the Sidestream, with a mean span value of 2.33 and 2.72 , at $4 \mathrm{l} / \mathrm{min}$ and $7 \mathrm{l} / \mathrm{min}$ respectively, compared with 1.61 and 2.06 produced by the Sidestream. This is consistent with the results of chapter 3. For both nebulisers an increase in the driving gas flow rate produced a rise in the recorded span value. This increase in the polydispersity of the aerosols is in agreement with previous jet nebuliser evaluations (Clay et al. 1983a; Newman et al., 1986; Niven and Brain 1994), and with the experiments of chapter 3. The size of the aerosol droplets produced from the Pari-LC nebuliser, operated at the reduced gas flow rate of $41 / \mathrm{min}$, was particularly variable in nature. This is surprising, in view of the fact that the Pari-LC nebuliser is designed for use in conjunction with the Pari-Master ${ }^{\circledR}$ (Pari-Werk) compressor, which operates at a flow rate equivalent to $4 \mathrm{l} / \mathrm{min}$.

Table 4.13 The mean VMD and span of aerosols emitted from the Pari-LC and Sidestream nebulisers at different driving gas flows. [ $\pm \mathrm{sd} ; \mathrm{n}=3]$

\begin{tabular}{|c|c|c|c|}
\hline & & $4 \mathrm{l} / \mathrm{min}$ & $7 \mathrm{l} / \mathrm{min}$ \\
\hline \multirow[t]{2}{*}{ Pari-LC } & VMD $(\mu \mathrm{m})$ & $3.37( \pm 0.31)$ & $2.10( \pm 0.14)$ \\
\hline & Span & $2.33( \pm 0.10)$ & $2.87( \pm 0.11)$ \\
\hline \multirow[t]{2}{*}{ Sidestream } & $\operatorname{VMD}(\boldsymbol{\mu m})$ & $3.23( \pm 0.17)$ & $1.91( \pm 0.16)$ \\
\hline & Span & $1.61( \pm 0.04)$ & $2.06( \pm 0.11)$ \\
\hline
\end{tabular}


(a)

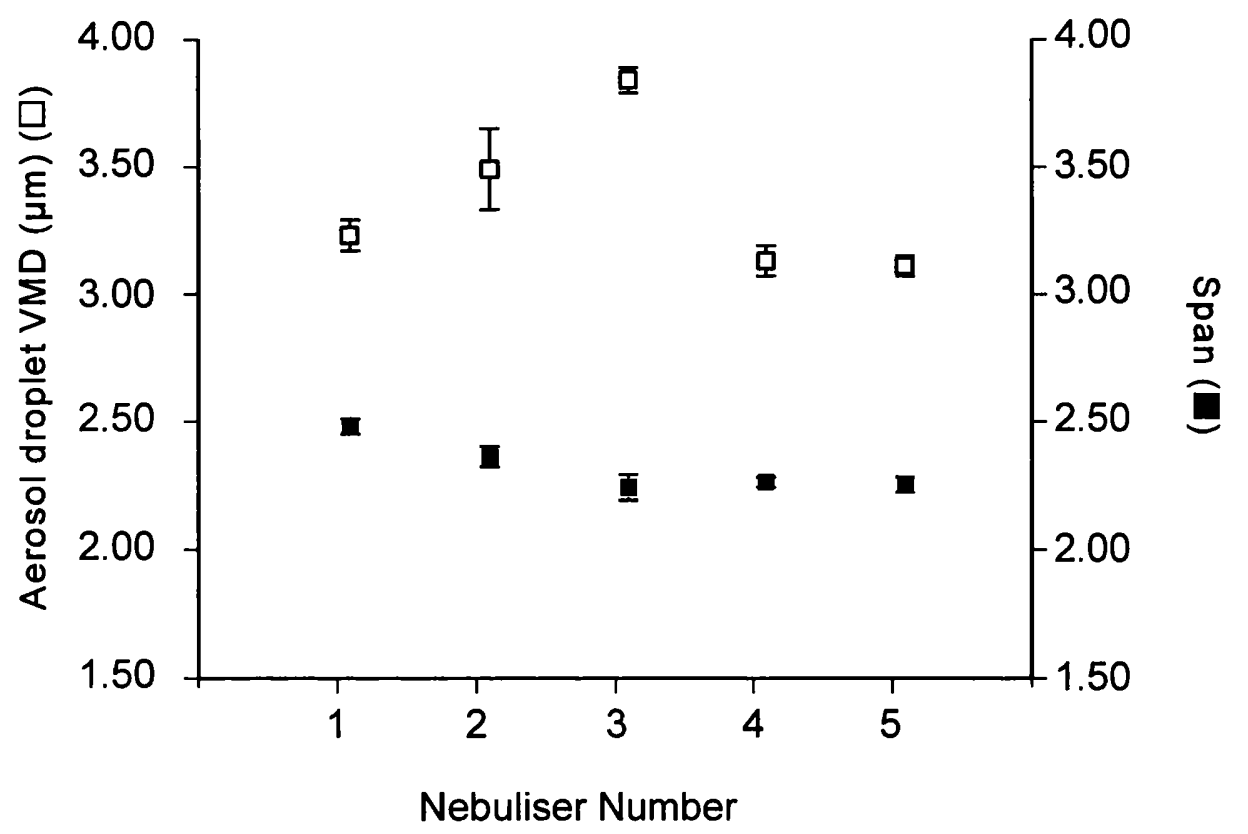

(b)

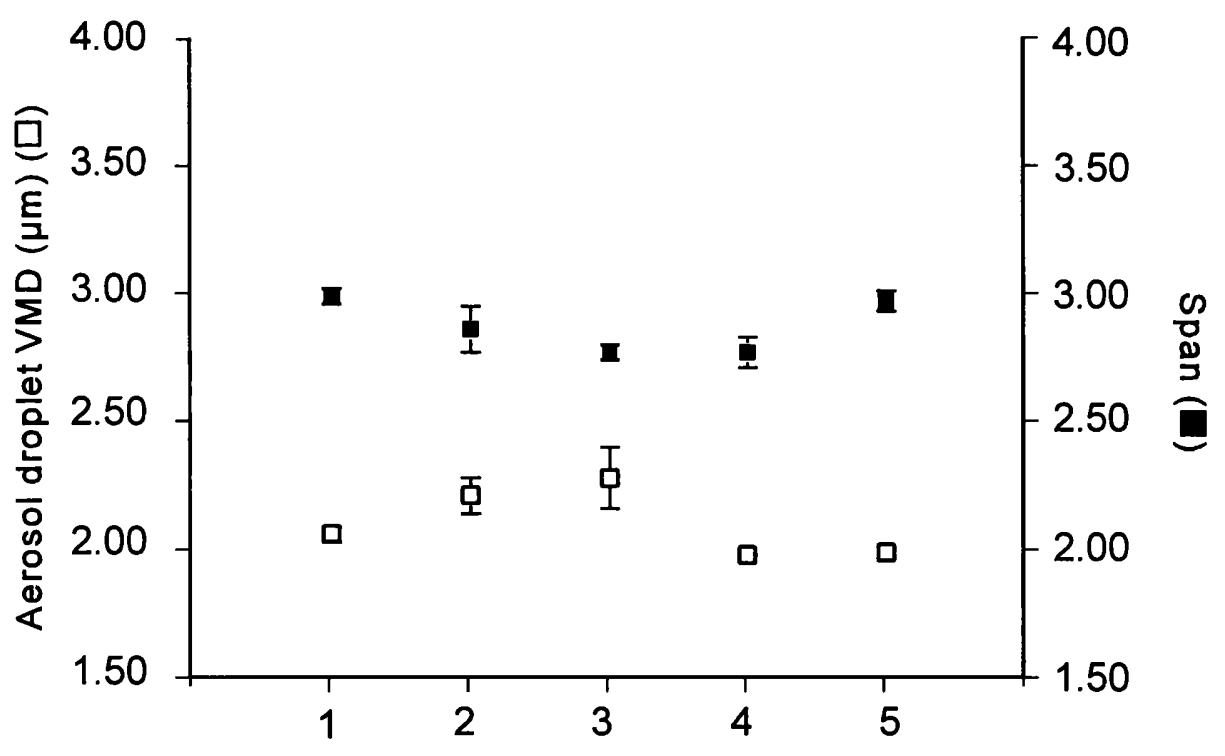

Nebuliser Number

Figure 4.14 The mean volume median diameter (VMD) and span of aerosols produced from eggPC/chol (1:1) liposomes, using five different jet nebulisers of the same model (numbered 1 to 5), driven at driving gas flow rates of 4 and $7 \mathrm{l} / \mathrm{min}$. (a) Pari-LC nebuliser, $4 \mathrm{l} / \mathrm{min}$ gas flow rate and (b) Pari-LC nebuliser, $7 \mathrm{l} / \mathrm{min}$ gas flow rate. [ \pm sd; $n=3$ ] 
( a )

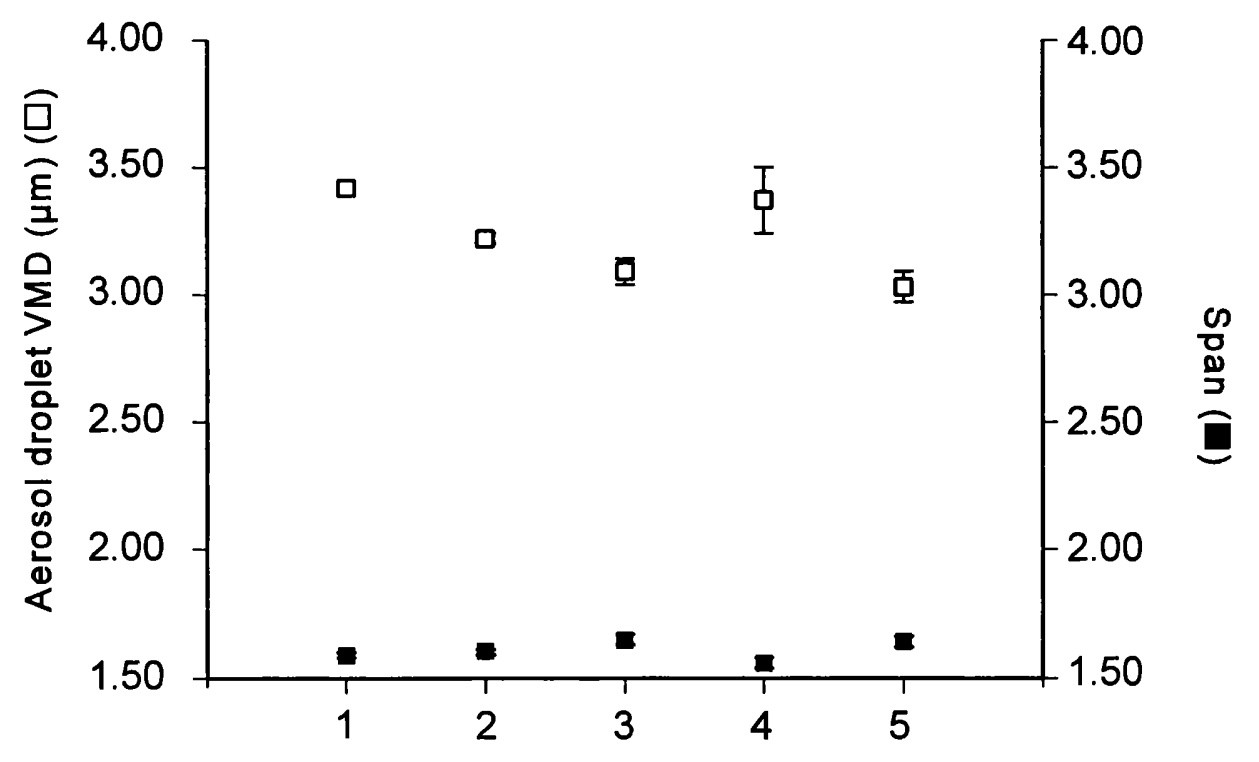

Nebuliser Number

(b)

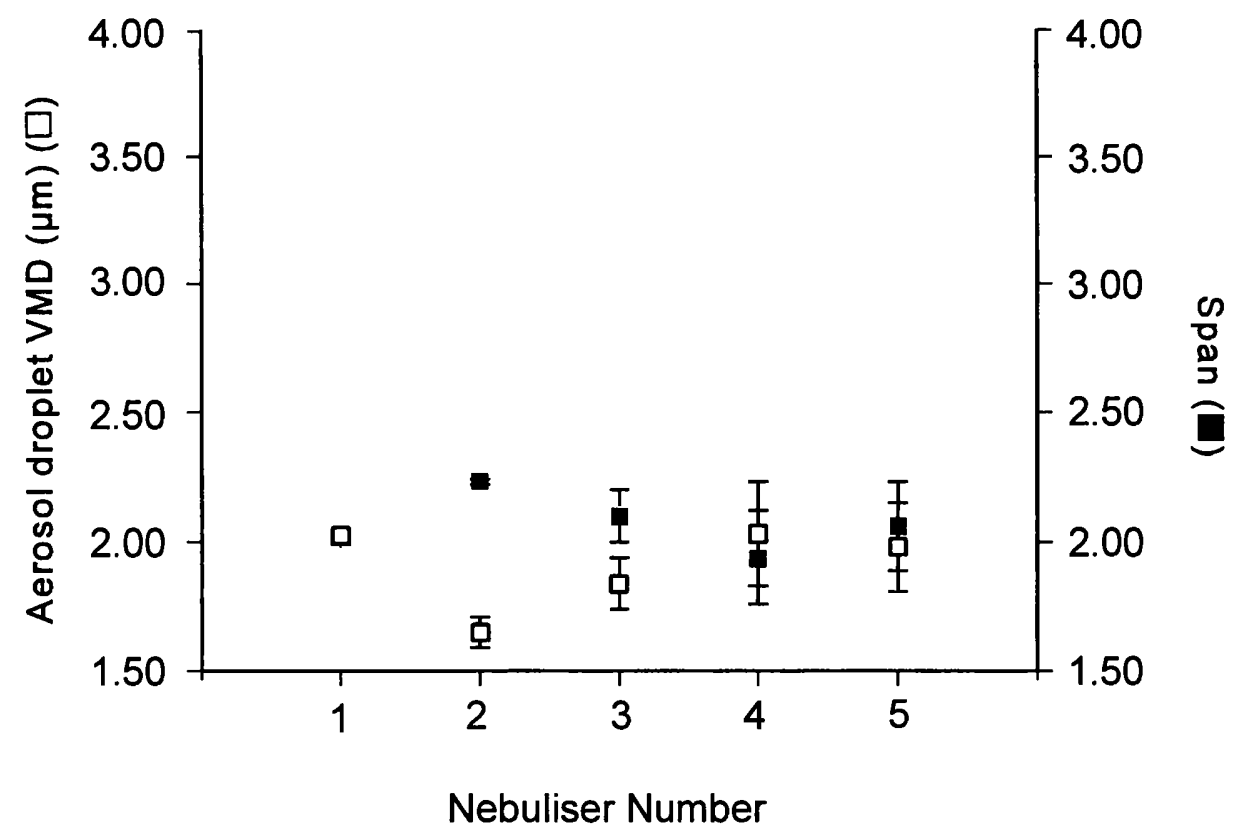

Figure 4.15 The mean volume median diameter (VMD) and span of aerosols produced eggPC/chol (1:1) liposomes, using five different jet nebulisers of the same model (numbered 1 to 5), driven at driving gas flow rates of 4 and $7 \mathrm{l} / \mathrm{min}$. (a) Sidestream nebuliser, $4 \mathrm{l} / \mathrm{min}$ gas flow rate and (b) Sidestream nebuliser, $7 \mathrm{l} / \mathrm{min}$ gas flow rate. $[ \pm \mathrm{sd}$; $n=3$ ] 
The droplet size produced by different nebulisers of the same model varied between devices (fig. 4.14 and 4.15). This was most evident with the Pari-LC nebuliser operated at $4 \mathrm{l} / \mathrm{min}$, which produced droplets that varied by as much as $18 \%(0.71 \mu \mathrm{m})$ between devices. This was reduced to $13 \%(0.3 \mu \mathrm{m})$ at the higher flow rate. The Sidestream produced a similar variability in the emitted aerosol size to that given by the Pari-LC. At $41 / \mathrm{min}$ the droplet VMD varied by $11 \%(0.33 \mu \mathrm{m})$ between individual nebulisers, and by $23 \%(0.38 \mu \mathrm{m})$ at $7 \mathrm{l} / \mathrm{min}$.

Alvine et al. (1992) postulated that the gas jet orifice within the jet nebuliser was the most important determinant of the droplet size produced by a particular nebuliser model. It had been previously proposed that smaller gas jet orifices resulted in the generation of reduced aerosol sizes (Lourenço and Cotromanes, 1982; Nerbrink and Dahlbäck, 1994), and the results of chapter 3 confirmed this. The modern jet nebulisers, typified by those in the present study, are devices constructed from relatively inexpensive injection moulded plastics (Muers, 1997). This may make the manufacture of consistently sized functional orifices difficult (Alvine et al., 1992), leading to variations in the emitted droplet size, as demonstrated in the present study. For instance, the gas jet orifice may not be centred correctly in the nebuliser reservoir. Such a fault is shown in the SEM image taken of a PariLC device (fig. 3.32c). There is also a highly irregular orifice evident in the Cirrus nebuliser (fig. 3.34b).

Previous authors have noted that aerosol output variations occur between individual nebulisers of a certain brand, as a result of inconsistent manufacture (Newman et al., 1987; Nerbrink and Dahlbäck, 1994). Massey et al. (1982) specifically traced the significant variability in output, that occurred between different DeVilbiss 646 nebulisers, to variation in the wear of baffles, and to variation in assembly. Supporting this, Hollie et al. (1991) identified irregularities in the manufacture of the nebuliser chamber and capillary tube, as being responsible for the observed variations in mass output from the DeVilbiss 646 nebuliser. These irregularities were compounded by the random nature of nebuliser assembly prior to use. This was in addition to the effects of damage and wear to nebuliser structures, during continued operation. 


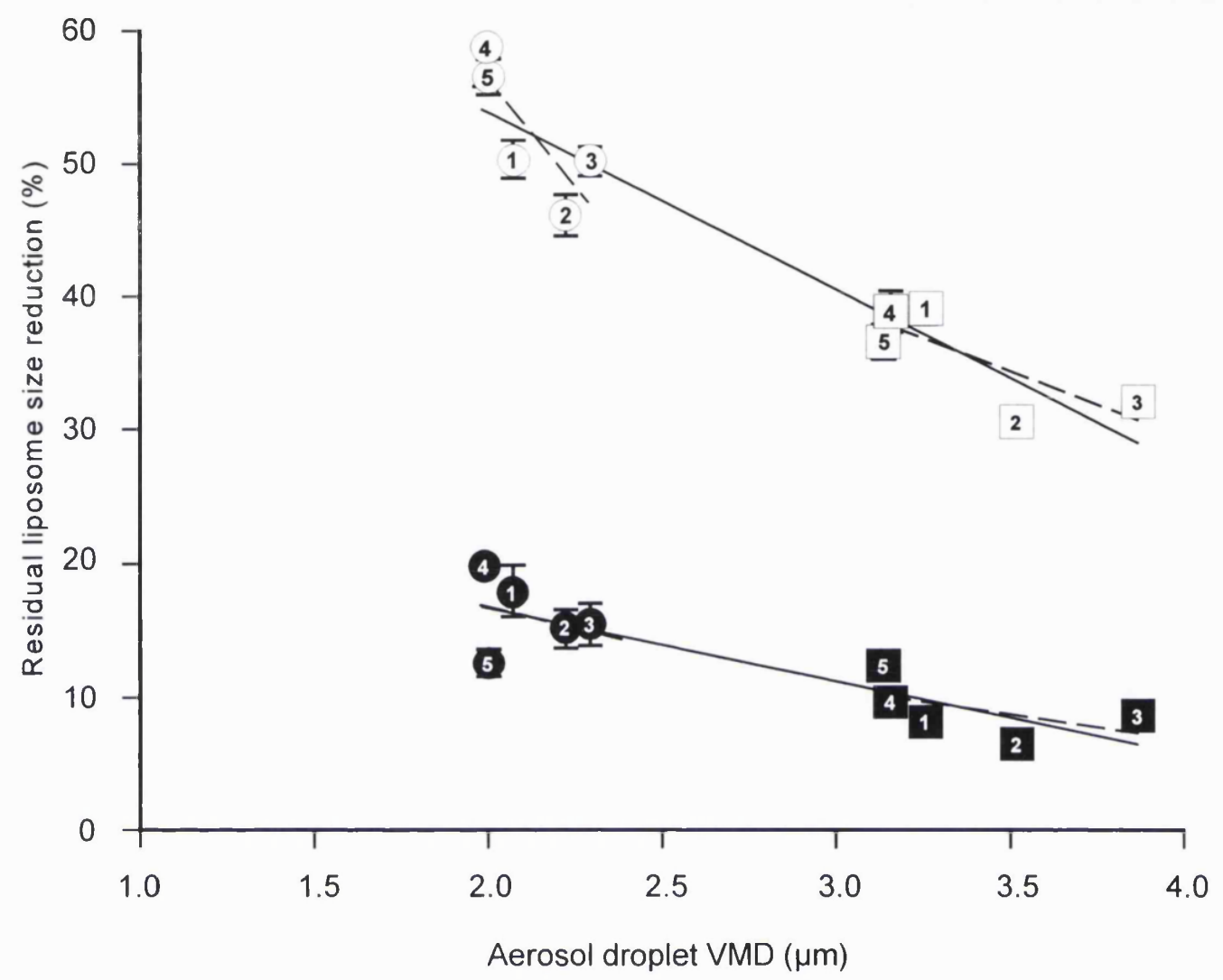

Figure 4.16 The relationship between the mean aerosol droplet size produced by five different Pari-LC nebulisers (numbered $\mathbf{0}$ to $\mathbf{6}$ ) (operated at driving gas flow rates of 4 $\mathrm{l} / \mathrm{min}(\square \square)$ and $7 \mathrm{l} / \mathrm{min}(\bullet \bigcirc)$ ) and the reduction in the residual eggPC $(\square \bigcirc)$ and eggPC/chol (1:1) (घ) liposome size. Equations of lines of best fit shown, eggPC/chol (1:1) liposomes (7 l/min)(--O--): y - $4.87 x+26.37(\mathrm{r}=-0.681)$; eggPC/chol $(1: 1)$ liposomes $(4 \mathrm{l} / \mathrm{min})(--\square--): \mathrm{y}=-3.61 x+21.2(\mathrm{r}=-0.517)$; eggPC liposomes $(7 \mathrm{l} / \mathrm{min})(--$ ---): $\mathrm{y}=-30.47 x+116.00(\mathrm{r}=-0.802)$; eggPC liposomes $(4 \mathrm{l} / \mathrm{min})(--\mathbf{- -}): \mathrm{y}=-9.86$ $x+68.50(\mathrm{r}=-0.607) .[ \pm \mathrm{sd} ; \mathrm{n}=6]$

Figures 4.16 and 4.17 attempt to correlate the reduction in the mean size of residual liposomes in each of five models of Pari-LC and Sidestream nebuliser, with the mean droplet size that each device produced. The figures clearly illustrate the stabilising effect that cholesterol has upon liposome bilayers, rendering them more resistant to the damaging processes that occurred during nebulisation. The results also demonstrate that the reduction 


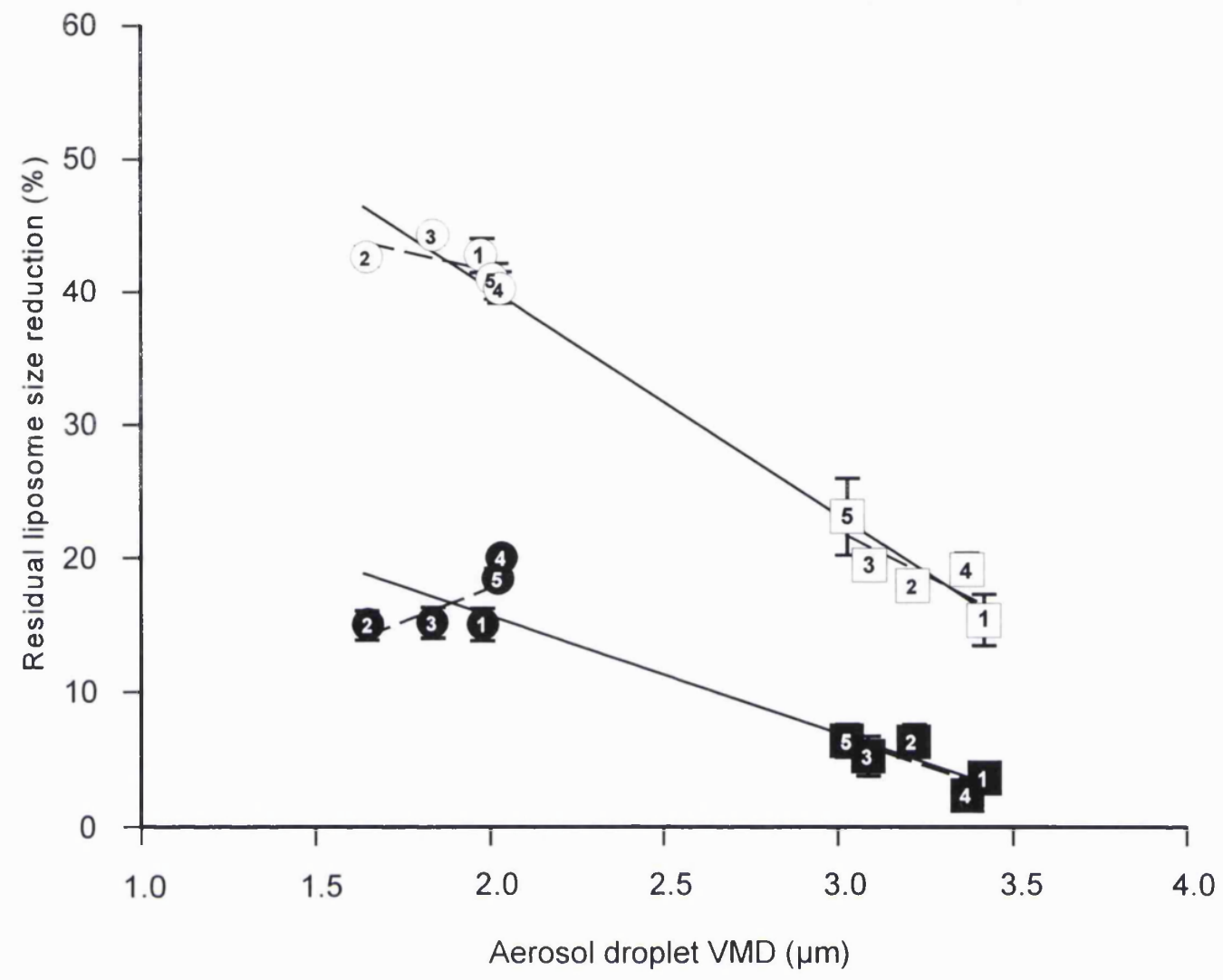

Figure 4.17 The relationship between the mean aerosol droplet size produced by five different Sidestream nebulisers (numbered $\mathbf{0}$ to 5 ) (operated at driving gas flow rates of $4 \mathrm{l} / \mathrm{min}(\square \square)$ and $7 \mathrm{l} / \mathrm{min})(\bigcirc)$ and the reduction in the residual eggPC $(\square \bigcirc)$ and eggPC/chol (1:1) ( $\mathbf{0})$ liposome size. Equations of lines of best fit shown, eggPC/chol (1:1) liposomes (7 1/min)(--O--): y - $9.94 x-2.30(\mathrm{r}=-0.681)$; eggPC/chol $(1: 1)$ liposomes $(4 \mathrm{l} / \mathrm{min})(--\square--)$ : $\mathrm{y}=-8.44 x+3.19(\mathrm{r}=-0.792)$; eggPC liposomes $(7 \mathrm{l} / \mathrm{min})(--$ $--): \mathrm{y}=-5.82 x+53.15(\mathrm{r}=0.595)$; eggPC liposomes $(4 \mathrm{l} / \mathrm{min})(--\mathbf{a}--): \mathrm{y}=-13.24 x+$ $61.67(\mathrm{r}=-0.804) .[ \pm \mathrm{sd} ; \mathrm{n}=6]$

in residual liposome size was dependent on the driving gas flow rate, as the higher flow rates were associated with increased residual liposome size reduction. The results are therefore consistent with the trend for increasing SCG release at higher flow rates, revealed in section 4.4.4. More significantly, the results show an apparent relationship between the droplet size produced by an individual device, and the damage done to residual liposomes. 
The relationship is most striking for the eggPC liposomes nebulised with the Pari-LC at each driving gas flow rate, and for each liposome formulation nebulised at $4 \mathrm{l} / \mathrm{min}$ with the Sidestream. The correlation between the droplet size, and the liposome damage in the Sidestream is less clear at the higher flow rate studied, with no relationship evident for the eggPC/chol $(1: 1)$ liposomes. This is a further demonstration of the difference in the operation of the two devices.

The data therefore reveal a correlation between the liposome stability, and the size of the secondary aerosol droplets that the individual nebuliser emits. This contrasts with the results of the investigation of different nebuliser models which revealed little correlation between the residual liposome size reduction, and the droplet size produced by a particular nebuliser model (section 4.4.2). It is therefore clear, that when different models of nebuliser are compared, the differences in their design and construction masks the relationship between the secondary aerosol size and the processing of the residual liposomes. Between individual nebulisers of the same model, such design and build variations are absent, and consequently a correlation between the liposome processing and the secondary aerosol size is evident. Thus, nebulisers that produce relatively small droplets for a particular model are associated with a significantly increased damage to residual liposomes $(p<0.05)$. This may be attributed to a local increase in the shear forces within the nebuliser jets, or to an increased baffle efficiency, or both. Such differences may have resulted from fluctuations in the build, or orientation of constituent parts, particularly the jet orifice (Alvine et al., 1992). It is clear that variations in the build, integrity and assembly of structures within nebuliser devices of the same model, cause demonstrable changes to both the aerosol character, and to the damage caused to nebulised liposomes.

The present observations support the proposed mechanism for liposome aerosolisation. Nebulisers producing a smaller mean droplet size exclude a greater number of liposomes of a given size from the secondary aerosol than nebulisers producing larger droplets. As a consequence, nebulisers producing a smaller aerosol size cause greatest damage to liposomes, due to continued exposure of the liposomes to shear forces within the nebuliser. The situation in the Sidestream is less clear, as this particular nebuliser is not solely reliant upon the shearing action of gas flow to provide a small droplet size. 


\subsection{Conclusions}

A drawback to the use of jet nebulisers for the generation of liposomal aerosols is that they may cause significant damage to liposomes during operation. Such damage is attributable to bilayer shearing by the high velocity gas flow, and possibly to conformational changes which occur as the phospholipids interact with hydrophobic surfaces within the nebuliser. Each of these mechanisms is controlled to an extent by the design features which are peculiar to a particular nebuliser model. This is evidenced by the marked differences in the liposome damage, and entrapped solute release, that different nebuliser models produce during operation. The damage to residual liposomes occurring during nebulisation may therefore be characteristic of the nebuliser used. The relationship between operating conditions, nebuliser design, and the damage done to liposomes is complex, and masks its relationship with the droplet size produced by the nebuliser. This is illustrated by the fact that there is a strong correlation between the aerosol size produced by different devices of the same model and the observed liposome stability. Pari-LC devices which produce relatively small droplets, are generally associated with an increased liposome damage. This is due to variations in the nebuliser construction, assembly, and perhaps also wear resulting from repeated use. As a result, certain devices operate with increased shear forces, and/or higher baffling efficiencies. This results in significant differences in the emitted droplet size, and hence a variation in the liposome stability. The situation in the Sidestream nebuliser is less clear, as the device incorporates an open vent. This makes the droplet size less reliant on the gas flow. The open vent promotes droplet evaporation, allowing the generation of smaller droplets, without necessitating elevated shear forces. Consequently, liposomes are more stable to nebulisation with the Sidestream. Liposome damage is also controlled by the operating conditions, as higher driving gas flow rates cause an increased shearing action, and a larger air-liquid interface within the nebuliser. The aerosolisation processes involved in jet nebulisation may therefore limit the effectiveness of liposomal aerosols for the delivery of hydrophilic drugs.

The stability of liposomes to nebulisation may be improved by control of the formulation characteristics. Reducing the mean liposome size increases the fraction of vesicles that are emitted promptly with the respirable aerosol, so reducing their recycling within the nebuliser, and lessening their exposure to damaging processes. This leads to a markedly reduced liposome processing, and hence maximises the retention of entrapped solutes. 
The liposome stability may also be improved by the use of liposomes with bilayers that are not in the liquid crystalline state at room temperature. Liposomes with relatively fluid bilayers, such as those composed of eggPC, are particularly unstable to nebulisation. They are rapidly processed to a smaller vesicle size, and release a greater proportion of entrapped hydrophilic solute. Bilayer stabilising agents, such as cholesterol, increase the rigidity of eggPC bilayers. The liposomes in this state resist nebuliser-induced destabilisation, and thus retain entrapped contents more readily. However, the improvement in liposome stability must be balanced with the reduced liposome output that it may yield (chapter 3 ). The liposome characteristics also determine the nature of the vesicles depositing following aerosolisation. The size of liquid-crystalline state liposomes is generally larger than that produced by the equivalent gel state liposomes. This suggests that a degree of vesicle fusion, and/or aggregation, may occur following atomisation.

The present results highlight the importance of characterising all aspects of a nebuliser's performance when selecting a device for the generation of liposomal aerosols. This extends to the likely variation in aerosol character between different nebulisers of the same model. 


\section{FREEZE DRYING OF LIPOSOMES FOR NEBULISATION}

\subsection{Introduction}

The application of liposome technology to drug delivery has thus far failed to fulfil its early potential (Talsma and Crommelin, 1992). Among the reasons for this, is that liposomes are inherently unstable. Much of laboratory based liposome research neglects issues of stability, as liposomes are prepared "as and when" required. Only once their research advances to the product development stage, does their relative instability usually become highlighted. A prerequisite of pharmaceuticals is that they exhibit relative stability upon storage, with a shelf-life in the order of years generally required (Crommelin and Schreier, 1994). Products with short shelf-lives are unlikely to be granted a product licence. It is therefore important that the physical and chemical stability of a product is considered during development.

\subsubsection{Physical stability of liposomes}

MLVs and LUVs are relatively unstable colloidal systems, which may precipitate and flocculate within a matter of hours, forming MLV formulations with a larger mean size (Lasic, 1993). SUVs generally form optically clear dispersions, with vesicles small enough to remain dispersed indefinitely (Szoka and Papahadjopoulos, 1980). However, the mean size of SUVs will also tend to increase during storage. This is because it is energetically favourable for SUVs to aggregate and fuse, in order to relieve the high curvature stresses inherent in their structures (Hauser and Irons, 1972; Suurkuusk et al., 1976; New, 1990). In fact, both SUVs and LUVs will, given time, become transformed into MLVs, and ultimately into a two phase system of giant flat lipid bilayers in excess water (Lasic, 1988). As the drug carrier characteristics of liposomes are intrinsically dependent on their size and bilayer characteristics, such formulation changes may directly influence the in vivo fate of entrapped drugs (Senior, 1987).

The disruption of bilayers that occurs during liposome fusion will be associated with a loss of entrapped hydrophilic materials. MLVs, due to the increased number of bilayers that they possess, exhibit a superior solute retention, compared with LUVs or SUVs (New, 1990; Szoka and Papahadjopoulos, 1980). By careful control of the temperature of storage, the physical stability of the liposomes may be maximised. Liposomes stored at a temperature below the $T_{m}$ of the bilayers, retain entrapped materials for significantly extended periods, 
due to their more rigid nature. However, drug losses will increase as the excitation of phospholipid molecules rises in response to an elevation of the temperature above the $T_{m}$ (De Gier et al., 1968; Jacobson et al., 1975; Papahadjopoulos et al., 1973b). Therefore, liposomes composed of phospholipid bilayers with an elevated $\mathrm{T}_{\mathrm{m}}$, or those incorporating cholesterol as a bilayer stabilising agent, are associated with an improved drug retention (De Gier et al., 1968; Szoka and Papahadjopoulos, 1980; Crommelin and van Bommel, 1984; Crommelin and Schreier, 1994). An increased rate of vesicle fusion may also occur as the storage temperature increases, due to the increased interaction of phospholipid molecules at higher temperatures. The incorporation of a charged lipid into liposome bilayers may limit aggregation, but this may be at the expense of other liposome properties, such as size, number of lamellae, and the entrapped aqueous volume (Crommelin and Schreier, 1994).

\subsubsection{Chemical stability of liposomes}

Liposomal phospholipids are prone to chemical instability, which modifies the liposome properties, accelerating their break-down, and altering drug release. The most common constituents of liposomes, the PCs, are particularly sensitive to ester bond hydrolysis, and peroxidation of unsaturated acyl bonds, in an aqueous environment. The two acyl ester bonds of PC are sensitive to hydrolysis, yielding lysoPC. LysoPC increases the permeability of the bilayers, promoting fusion and associated drug losses (Riaz et al., 1988). Free fatty acids introduce negative charges to the lipid/water interface, which may dramatically change the bilayer organization and dynamics, altering drug release, and also interactions with biological structures (Inoue and Kitagawa, 1974; Grit et al., 1989; Grit and Crommelin, 1992; Grit et al., 1993). Lipid peroxidation is a particular problem for phospholipids derived from natural sources, as they contain a significant level of polyunsaturation. A synthetic phospholipid, such as DPPC, possesses a greater degree of saturation, and is thus a more stable molecule (Grit et al., 1993). The oxidation process occurs via free radical reactions, yielding a wide variety of peroxide decomposition products, which significantly increase the permeability of the liposome bilayers (Hunt and Tsang, 1981).

A number of strategies may be employed to reduce the chemical degradation of lipids. The rate of hydrolysis may be adequately described by a biphasic Arrhenius curve, with a break 
observed at the $T_{m}$ of the bilayer phospholipid(s) (Grit et al., 1993). The rate of hydrolysis therefore increases with a rise in temperature. It is also catalysed by metal ions, certain organic molecules, and extremes of $\mathrm{pH}$ (Grit et al., 1993). Storage of the liposomes in the absence of light and oxygen, will slow the reactive processes. For example, after 6 months there was no detectable signs of degradation, and less than $5 \%$ leakage from glucose containing liposomes, following their maintenance at $22{ }^{\circ} \mathrm{C}, \mathrm{pH} \mathrm{7.4}$, and in the absence of oxygen and light (Hunt and Tsang, 1981). By contrast, the presence of oxygen and light yielded complete loss of glucose within 48 hours. Protective measures against peroxidation may also be employed. For instance, the shelf-life of liposomes may be doubled by the inclusion of $10 \mathrm{~mol} \%$ of the antioxidant, $\alpha$-T (Hung and Tsang, 1981). Other approaches include the use of chelating agents such as EDTA (Crommelin and Schreier, 1994). Such strategies increase the stability of liposomal pharmaceuticals, but are limited by the need to design specific formulations for specific applications. Ideally, the method selected for the long-term stabilisation of liposomes should be applicable to a wide variety of liposomal formulations. As a consequence, first generation liposome products have been commonly developed as dried solids, for reconstitution prior to administration (eg. AmBisome ${ }^{\circledR}$ ).

\subsubsection{Freeze drying of liposomes}

Stable, dried liposomal powders may be produced by freeze drying (eg. Crowe et al., 1986), or spray drying (eg. Goldbach et al, 1993). Spray drying is disadvantaged by the fact that heat is applied up to approximately $110^{\circ} \mathrm{C}$, thus creating the potential for significant liposome instability. In contrast, freeze drying has yielded more promising results (eg. Crowe et al., 1986).

Although non-ideal, the freeze dried product affords protection against many of the processes causing liposome instability in aqueous dispersions, and thus yields a product with a greatly extended shelf-life. Freeze drying of aqueous products exploits the fact that under certain conditions of temperature and pressure, water will exist in equilibrium between the solid and vapour phase. At a vapour pressure of $610 \mathrm{Nm}^{-2}$, and a temperature of $0.0075^{\circ} \mathrm{C}$, water will co-exist in equilibrium between the liquid, gas and solid phase. This point is known as the eutectic point. If the pressure is reduced below $610 \mathrm{Nm}^{-2}$ then absorption of heat from the environment by the solid water will cause sublimation of the ice directly to the vapour phase (fig. 5.1). 


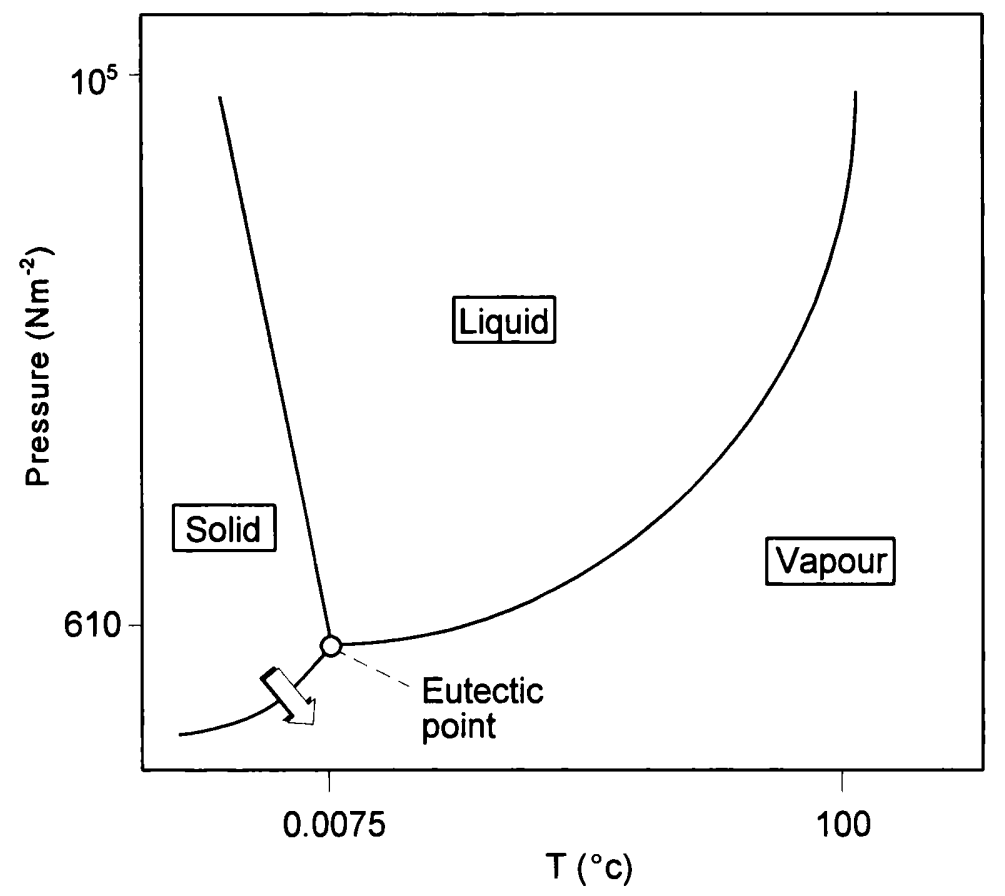

Figure 5.1 The phase diagram and eutectic point for water (not to scale). Freeze drying exploits the equilibrium established between ice and vapour (sublimation, $\Rightarrow$ ) ) below the eutectic point.

Freeze-driers use a vacuum in order to lower the pressure to below the eutectic point, and also to remove the large volumes of vapour produced by drying. Heat of sublimation is commonly applied by the ambient environment. However, to speed the process, heat may also be supplied to the frozen solid. Freeze drying has a number of advantages compared with alternative methods of drying. Most importantly, the drying occurs at very low temperatures, thus minimising decomposition processes such as hydrolysis. This is also a distinct advantage for liposomes, in which temperature dependent phase transitions may induce fusion and entrapped material losses. The product is also dried to form a network of dehydrated solid, occupying the same volume as the original solution. It thus forms a light and porous product, which is easy to disperse (Travers, 1988). There are, however, certain disadvantages of freeze drying. Firstly the addition of a drying and rehydration step may considerably increase costs (Boylan and Fites, 1990), which in the case of liposomal products, is likely to already be expensive. This is exacerbated by the hygroscopic nature of the product, which necessitates special packaging requirements. Secondly, whilst avoiding instabilities induced at high temperatures, the freezing and dehydration processes exert a high degree of stress on the liposomes. Freezing may cause phase transitions changes, osmotic stress, and the expansion of bilayers, due to ice formation (Talsma et al, 
1990b; Hauser, 1993). Liposome bilayers are reliant upon hydrogen bonding between water molecules and the polar head groups of the constituent phospholipids for stability. Loss of these water molecules during drying therefore causes a significant change in bilayer behaviour, and a loss of integrity. Lateral stresses are exerted on the membrane, leading to membrane deformation, lateral phase separations, and gel to liquid-crystalline, or lamellar to hexagonal phase state transitions (Mobley and Schreier, 1994). Such changes lead to bilayer fractures, fusion, and vesicle aggregation. This is evidenced by a significant loss of entrapped material (up to $100 \%$, Crowe et al., 1985), and changes in the liposome size distribution (Crowe et al., 1986). Such deleterious changes during freeze drying may be minimised by the inclusion of various excipients, called cryoprotectants, within the formulation. Among the most successful cryoprotectants are disaccharide sugars. The use of these sugars is based on studies demonstrating that anhydrobiotic organisms, such as fungal spores and yeasts, survive prolonged desiccation due to the high concentration of disaccharides (up to about $60 \%$ ) which they contain. The presence of trehalose, a nonreducing disaccharide of $\alpha$-D-glucose, has been shown to preserve these organisms in a fully viable condition, such that full functional viability resumes upon rehydration many years later (Crowe and Crowe, 1988). A variety of sugars, including lactose, dextrose, sucrose, maltose and trehalose, have been investigated for their ability to afford similar protection to liposomal membranes during dehydration. Of these, trehalose has proved most successful, protecting liposomes during freeze drying, such that up to $100 \%$ of the entrapped contents may be retained upon rehydration (Crowe et al, 1985).

\section{Mechanism of cryoprotection}

The precise mechanism of protection afforded by disaccharides, such as trehalose, to bilayer membranes during freeze drying remains contentious, and literature concerning the subject is often contradictory. Trehalose, and similar sugars, are thought to protect liposomes through their action on two destabilising mechanisms, which occur during freeze drying. The first involves the inhibition of vesicle fusion, and aggregation, during dehydration (Rudolph, 1988). The second, influences the phase transition state changes which occur in phospholipid bilayers during dehydration (the "water replacement hypothesis"; Rudolph, 1988, Crowe and Crowe, 1992). Aggregation, and fusion of liposomes during freeze drying, may be inhibited by sugar concentrations as low as $10 \% \mathrm{w} / \mathrm{w}$. In contrast, protection against the phase transition changes is only demonstrable at concentrations of 
approximately $75 \% \mathrm{w} / \mathrm{w}$ (Crowe et al, 1986). The two stabilisation processes are therefore thought to operate through discrete mechanisms (Engel et al., 1994). The inhibition of liposome fusion and aggregation is largely attributed to the glass-like sugar matrix which covers the liposome surface upon dehydration. The vitrification of trehalose inhibits the long range motion of phospholipid molecules, and as such prevents diffusion-dependent degradation processes from occurring (Mobley and Schreier, 1994). The sugar molecules provide a physical barrier between adjacent liposomes, thus preventing the apposition of bilayers, and their resultant fusion (Harrigan et al., 1990; Engel et al., 1994). By contrast, the maintenance of a stable bilayer transition state during freeze drying is thought to be due to the direct interaction of sugar and phospholipid molecules within the liposome bilayer. The water replacement hypothesis, proposes that sugar molecules replace the water that is lost between hydrated phospholipid molecules during dehydration (Crowe et al., 1985). The sugars act to maintain the hydrogen bonded structure of polar groups, such as phosphates, within the bilayers, thus ensuring that a stable bilayer is retained in the absence of water. This improves the retention of entrapped solutes, and reduces fusogenic bilayer interactions. The incorporation of sugars between the polar head regions also increases the phospholipid spacing within the bilayer, reducing the van der Waals interactions of adjacent acyl chains, and thus fluidises the bilayer. The effect of this is to greatly reduce the $T_{m}$ of the bilayers in the dry state. For example, a trehalose-mediated reduction in $\mathrm{T}_{\mathrm{m}}$ of $80^{\circ} \mathrm{C}$ has been recorded for dry DPPC (Crowe et al., 1986). The phase transition that takes place when liposome bilayers constructed from liquid-crystalline state phospholipids are hydrated, is thus avoided. The occurrence of such phase transitions is associated with a marked increase in the release of entrapped solutes, and hence entrapped solutes are retained to a greater extent upon rehydration in the presence of a cryoprotectant (Crowe et al., 1985).

The experiments detailed in this chapter were designed to assess the potential application of freeze drying for the preservation of liposomal formulations designed for nebulisation. The ability to produce a dehydrated liposomal formulation, suitable for rehydration prior to nebulisation, would be an obvious advantage from a stability point of view. However, the size of the rehydrated liposomes may be of concern, as the mean vesicle size has been shown to determine the stability of the liposomes to nebulisation (chapter 4), and also the liposomal aerosol output (chapter 3). 


\subsection{Materials}

\section{Reagents}

Cholesterol, Anala-R (99-99.4\%)

$\mathrm{BDH}, \mathrm{UK}$

Deionised water

WR50 RO/ Deioniser, Whatman UK

Lecithin from eggs (90\%), purified

Sigma Chemicals, USA

as detailed in section 2.3.1

Polycarbonate membrane filters, - $\quad$ Nucleopore, UK

$2.5 \mathrm{~mm}$ diameter

$(1,3$, and $5 \mu \mathrm{m}$ pore size $)$

Trehalose (99\%)

BDH, UK

\section{Nebuliser}

Pari-LC nebuliser

Pari-Werk, Germany

\subsection{Methods}

\subsubsection{Freeze drying of liposomes}

EggPC and eggPC/chol (1:1) liposomes were prepared by the thin-film method, to a lipid concentration of $10 \mathrm{mg} / \mathrm{ml}$, using deionised water as the aqueous phase (section 2.3.2) Liposomes were additionally prepared in the presence of trehalose (eggPC/tr and eggPC/chol/tr), dissolved in the aqueous phase to a concentration of $1 \mathrm{~g}$ of trehalose per 1 $\mathrm{g}$ of total liposomal lipid. In this way the trehalose was entrapped within the hydrated bilayers of the resulting liposomes. The mean size of the liposomes was reduced by extrusion through polycarbonate membrane filters (as detailed in section 2.3.4.1) to a mean size of 5, 2.5 or $1 \mu \mathrm{m}$. Each liposome sample was freeze dried using the Edwards Micro Modulyo freeze-drier (Edwards Ltd., UK). Fifty $\mathrm{ml}$ of the liposome dispersion was added to drying vessels, and frozen in a freezer at $-20^{\circ} \mathrm{C}$. The vessels were then attached to the freeze-drier, and dried for 12 hours. The freeze dried samples were stored in vacuum sealed vessels, at 1 to $5^{\circ} \mathrm{C}$ and in darkness, until required for analysis. Prior to use, the freeze dried liposome samples were rehydrated with $50 \mathrm{ml}$ of deionised water at ambient temperature followed by a 2 minute period of vortex mixing in order to thoroughly redisperse the liposomes. The mean liposome size, and size distribution characteristics, of the liposome samples, before and after freeze drying and rehydration, was then determined using the Malvern 2600C laser diffraction sizer (as detailed in section 2.3.3). 


\subsubsection{Nebulisation of rehydrated freeze dried liposomes}

Twenty-five $\mathrm{ml}$ of eggPC/chol (1:1) liposomes were prepared with, or without trehalose (1 $\mathrm{g}$ trehalose to $1 \mathrm{~g}$ lipid), by the thin-film method to a total lipid concentration of $10 \mathrm{mg} / \mathrm{ml}$. The mean liposome size was reduced to approximately 2.5 or $1 \mu \mathrm{m}$ by extrusion. The liposomes were then freeze dried by the method detailed in section 5.3.1. The liposomes were rehydrated prior to use with deionised water in $25 \mathrm{ml}$ batches, and redispersed using a vortex mixer for 2 minutes.

\section{Examination of the freeze dried liposomes}

Four $50 \mathrm{ml}$ samples of $1 \mu \mathrm{m}$, or $2.5 \mu \mathrm{m}$ mean diameter eggPC/chol (1:1) liposomes, prepared with or without trehalose ( $1 \mathrm{~g}$ trehalose per $1 \mathrm{~g}$ lipid), were freeze dried as detailed in section 5.3.1. Following drying, approximately $50 \mathrm{mg}$ of the freeze dried liposomes was removed from the dry mass, and mounted on a scanning electron microscopy (SEM) sample stage. The solid was then vacuum coated with a $30 \mathrm{~nm}$ thick layer of gold. Finally, the liposomes were examined under a scanning electron microscope (Phillips XL20, Phillips, Cambridge, UK), and electron micrographs taken at an accelerating voltage of 10 to $15 \mathrm{kV}$.

\section{Nebulisation}

The remaining freeze dried samples of each formulation were reconstituted with $50 \mathrm{ml}$ of deionised water, and redispersed thoroughly at room temperature, using vortex mixing for 2 minutes. The initial size of the liposomes was then determined using laser diffraction sizing (as detailed in section 2.3.3). Five $\mathrm{ml}$ of the liposomes under study was then added to each of three Pari-LC nebulisers. The nebulisers were operated consecutively, at a driving gas flow rate of $7 \mathrm{l} / \mathrm{min}$, until $50 \%$ of the sputtering time. The aerosol released from each nebuliser was entrained into the TI with a gas throughput rate of $60 \mathrm{l} / \mathrm{min}$. The liposomes remaining within the nebuliser chamber, and those deposited in the upper and lower stages of the TI, were then analysed by laser diffraction, following the method previously described in section 4.3.4. The TI was then cleaned and reassembled. Each nebuliser was then operated once more from the $50 \%$ to $100 \%$ of nebulisation time into the TI. The residual liposomes, and the liposomes deposited within each stage of the TI during this time were then analysed as before. 


\subsection{Results}

\subsubsection{Freeze drying of liposomes}

The results demonstrated that freeze drying of liposomes caused the mean vesicle size to be significantly increased upon subsequent reconstitution (table 5.1). Each liposome formulation produced a mean reconstituted liposome size of between 1.61 and $7.67 \mu \mathrm{m}$ following freeze drying. This represented an increase of between $11.67 \%$ to $585.78 \%$ (table 5.1) (fig. 5.2), following freeze drying and rehydration. Such a size increase represents the aggregation and fusion of liposomes in response to the freeze drying process (Engel et al., 1994; Mobley and Schreier, 1994). The drying of the liposomes removes the hydration barrier to vesicle fusion. Aggregates form, which encourage the interaction, and resultant fusion, of the bilayers. As dehydration proceeds the liposomes concentrate, increasing the opportunities for bilayer interactions.

The inclusion of cholesterol in liquid-crystalline state bilayers has been shown to stabilise the liposomes to the shear stresses induced by a variety of processes, including membrane extrusion, probe sonication (chapter 2), and nebulisation (chapter 4). Cholesterol also reduces the release of entrapped hydrophilic solutes through liposome bilayers that would normally be in the fluid state at room temperature (chapter 4). It may therefore be predicted that cholesterol would impart a similar resistance to the fusogenic processes induced by freeze drying. However, the present results demonstrate that the inclusion of cholesterol had no significant effect on the maintenance of the original size characteristics of liposomes during their freeze drying $(\mathrm{p}<0.05)$. This is consistent with similar studies involving the freezing and dehydration of cholesterol-containing liposomes (Crommelin and Van Bommel, 1984; Strauss, 1992), and also their spray drying (Goldbach et al., 1993).

Each of the liposome formulations freeze dried in the absence of trehalose, yielded a reconstituted size of approximately $7 \mu \mathrm{m}$, and therefore the reconstituted liposome size was independent of the initial liposome size. This was the case, regardless of whether cholesterol was included in the liposome bilayers. The $1 \mu \mathrm{m}$ mean size liposomes therefore underwent a more pronounced change in the reconstituted size, of approximately $600 \%$, following freeze drying. This was compared to an increase of approximately $200 \%$ for the $2.5 \mu \mathrm{m}$ liposomes, and $50 \%$ for the $5 \mu \mathrm{m}$ initial mean size liposome formulations. 


\begin{tabular}{|c|c|c|c|c|c|c|}
\hline \multicolumn{7}{|c|}{$\begin{array}{l}\text { Table 5.1 The size characteristics of eggPC and eggPC/chol (1:1) liposome } \\
\text { formulations, freeze dried in the presence or absence of trehalose (tr). }[ \pm \text { sd; } n=6]\end{array}$} \\
\hline \multirow[b]{2}{*}{ Liposome } & \multicolumn{2}{|c|}{$\begin{array}{l}\text { Before freeze } \\
\text { drying }\end{array}$} & \multicolumn{2}{|c|}{$\begin{array}{l}\text { After freeze drying } \\
\text { and rehydration }\end{array}$} & \multicolumn{2}{|c|}{$\begin{array}{l}\text { Mean change } \\
(\%) \\
\end{array}$} \\
\hline & $\begin{array}{l}\text { Size } \\
(\mu \mathrm{m})\end{array}$ & Span & $\begin{array}{l}\text { Size } \\
(\mu \mathrm{m})\end{array}$ & Span & $\begin{array}{l}\text { Size } \\
(\mu \mathrm{m})\end{array}$ & Span \\
\hline eggPC & 5.04 & 1.47 & 7.67 & 2.98 & 52.31 & 102.95 \\
\hline$(5 \mu \mathrm{m})$ & $(0.04)$ & $(0.04)$ & $(0.54)$ & $(0.20)$ & $(10.67)$ & (13.37) \\
\hline eggPC & 2.53 & 1.30 & 6.92 & 2.29 & 179.19 & 76.03 \\
\hline$(2.5 \mu \mathrm{m})$ & $(0.05)$ & $(0.02)$ & $(0.53)$ & $(0.33)$ & (11.47) & $(25.66)$ \\
\hline eggPC & 1.02 & 0.98 & 7.00 & 2.13 & 585.78 & 117.10 \\
\hline$(1 \mu \mathrm{m})$ & $(0.06)$ & $(0.09)$ & $(0.64)$ & $(0.09)$ & $(62.31)$ & $(9.47)$ \\
\hline eggPC/chol & 5.00 & 1.64 & 7.21 & 2.35 & 44.23 & 43.29 \\
\hline$(5 \mu \mathrm{m})$ & $(0.02)$ & $(0.09)$ & $(0.68)$ & $(0.32)$ & $(13.54)$ & $(19.54)$ \\
\hline eggPC/chol & 2.51 & 1.03 & 7.23 & 2.30 & 187.85 & 119.74 \\
\hline$(2.5 \mu \mathrm{m})$ & $(0.07)$ & $(0.07)$ & $(0.61)$ & $(0.16)$ & $(24.25)$ & (19.89) \\
\hline eggPC/chol & 1.06 & 0.94 & 6.96 & 2.12 & 556.76 & 125.36 \\
\hline$(1 \mu \mathrm{m})$ & $(0.11)$ & $(0.03)$ & $(0.66)$ & $(0.23)$ & $(62.15)$ & $(24.24)$ \\
\hline eggPC/tr & 5.04 & 1.94 & 6.06 & 1.36 & 20.34 & 36.65 \\
\hline$(5 \mu \mathrm{m})$ & $(0.09)$ & $(0.09)$ & $(0.20)$ & $(0.07)$ & $(3.90)$ & $(1.77)$ \\
\hline eggPC/tr & 2.50 & 1.21 & 2.79 & 1.29 & 11.67 & 6.20 \\
\hline$(2.5 \mu \mathrm{m})$ & $(0.04)$ & $(0.06)$ & $(0.26)$ & $(0.04)$ & $(10.24)$ & $(2.99)$ \\
\hline eggPC/tr & 1.01 & 1.01 & 1.71 & 1.19 & 69.14 & 17.49 \\
\hline$(1 \mu \mathrm{m})$ & $(0.05)$ & $(0.04)$ & $(0.07)$ & $(0.05)$ & $(6.73)$ & $(5.30)$ \\
\hline eggPC/chol/tr & 5.05 & 1.09 & 6.09 & 1.31 & 20.99 & 19.88 \\
\hline$(5 \mu \mathrm{m})$ & $(0.02)$ & $(0.09)$ & $(0.11)$ & $(0.10)$ & & $(9.06)$ \\
\hline eggPC/chol/tr & 2.53 & 1.04 & 2.94 & 1.09 & 16.21 & 5.45 \\
\hline$(2.5 \mu \mathrm{m})$ & $(0.07)$ & $(0.11)$ & $(0.23)$ & $(0.05)$ & $(9.08)$ & $(4.44)$ \\
\hline eggPC/chol/tr & 1.00 & 0.82 & 1.61 & 1.24 & 60.50 & 52.24 \\
\hline$(1 \mu \mathrm{m})$ & $(0.05)$ & $(0.08)$ & $(0.09)$ & $(0.03)$ & $(8.87)$ & $(4.04)$ \\
\hline
\end{tabular}


(a)

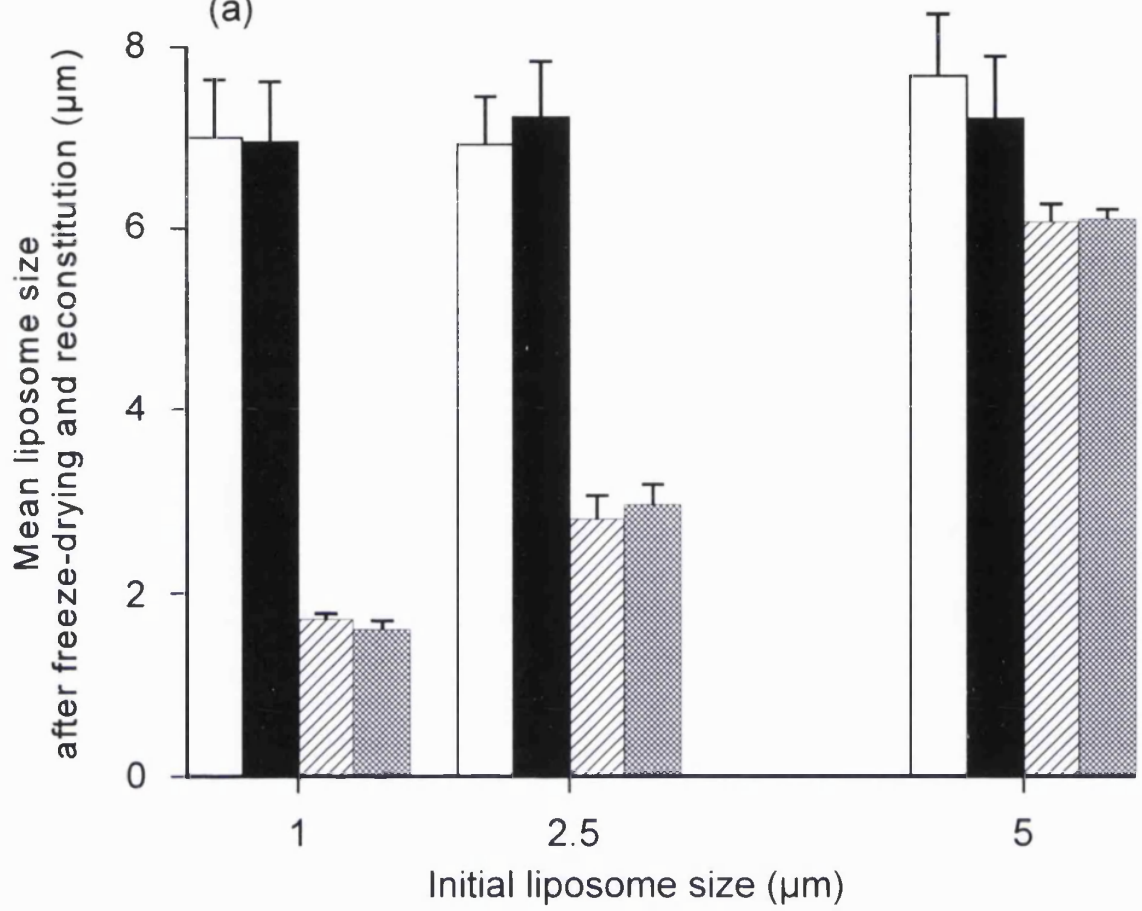

(b)

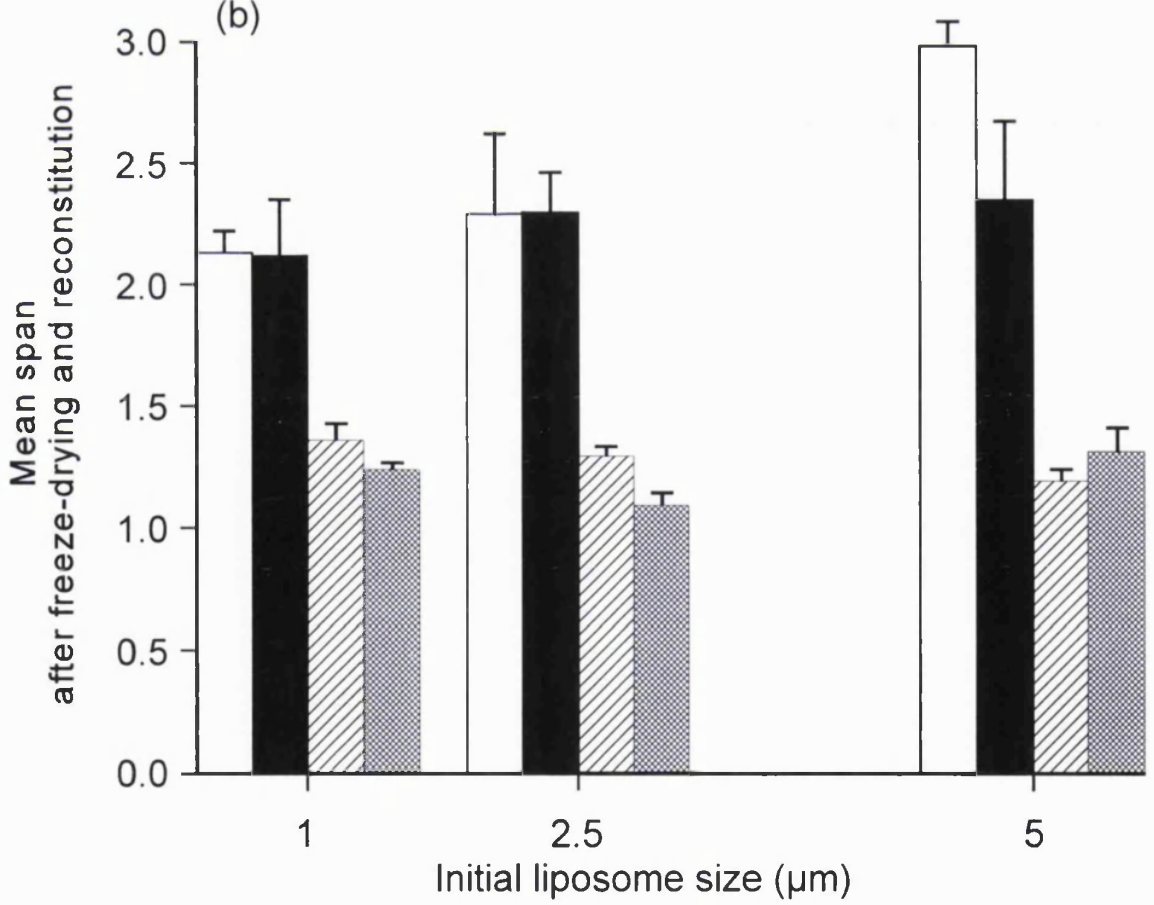

Figure 5.2 (a) The mean size and (b) polydispersity (as determined by the span) of reconstituted eggPC ( $\square / /)$ and eggPC/chol (1:1) (⿴囗) liposome formulations previously freeze dried in the presence $(/ /, \otimes)$ and absence $(\square \square)$ of trehalose. $[ \pm s d ; n=6]$ 
The polydispersity of liposome sizes was also increased by freeze drying and subsequent rehydration (figure 5.2b). This was demonstrated by an increase in the span of the rehydrated liposomes from an initial 1 to 1.5 , to approximately 2 to 3 . This represented an increase in the span of the liposomes of between 70 and $125 \%$, in liposomes freeze dried without trehalose. No pattern could be identified with regard to the increase in the span values experienced by the differently sized liposomes following freeze drying.

The inclusion of trehalose within each formulation significantly improved the maintenance of the original size characteristics of the liposomes, upon rehydration following freeze drying. The formulations freeze dried in the presence of the disaccharide yielded a significantly smaller liposome size, following freeze drying and rehydration, compared with the formulations freeze dried in the absence of trehalose $(p<0.05)$. In contrast to the formulations in which trehalose was absent, those containing trehalose yielded a reconstituted liposome size that was dependent on the mean size of the formulation prior to freeze drying. The final liposome size when freeze dried in the presence of trehalose was approximately $1.5,3$, and $6 \mu \mathrm{m}$ for the $1,2.5$ and $5 \mu \mathrm{m}$ initial liposome size preparations, respectively (table 5.1). These sizes represent an increase in the rehydrated liposome size following freeze drying of approximately $50 \%$ for the initial $1 \mu \mathrm{m}$ mean size liposome formulation, $15 \%$ for the $2.5 \mu \mathrm{m}$, and $20 \%$ for the $5 \mu \mathrm{m}$ liposomes. The size distribution of liposomes, rehydrated following freeze drying, was therefore less influenced by freezedrying when trehalose was included in the formulation. The liposomes exhibited an increase in polydispersity upon freeze drying of between 5 and $50 \%$, compared to between 70 and $125 \%$ when trehalose was absent. It was therefore apparent, that trehalose aided the maintenance of the original liposome size distribution and, by inference, stabilised the liposomes during freeze drying. A number of theories have been proposed to explain the protection afforded by trehalose to liposomes during freeze drying.|Principal amongst them is the formation of a glass-network by the trehalose molecules, and the replacement by the disaccharide, of the water of hydration between adjacent bilayer phospholipid molecules. The motion of phospholipid molecules in the bilayer becomes restricted. such that adjacent liposome bilayers have a reduced tendency to interact when dehydrated. As a result, fusogenic events are minimised (Rudolph, 1988). There may also be stabilisation of the liposomes, through the effects that trehalose has on the transition state of the phospholipid bilayers (Crowe, 1988). 
(a)

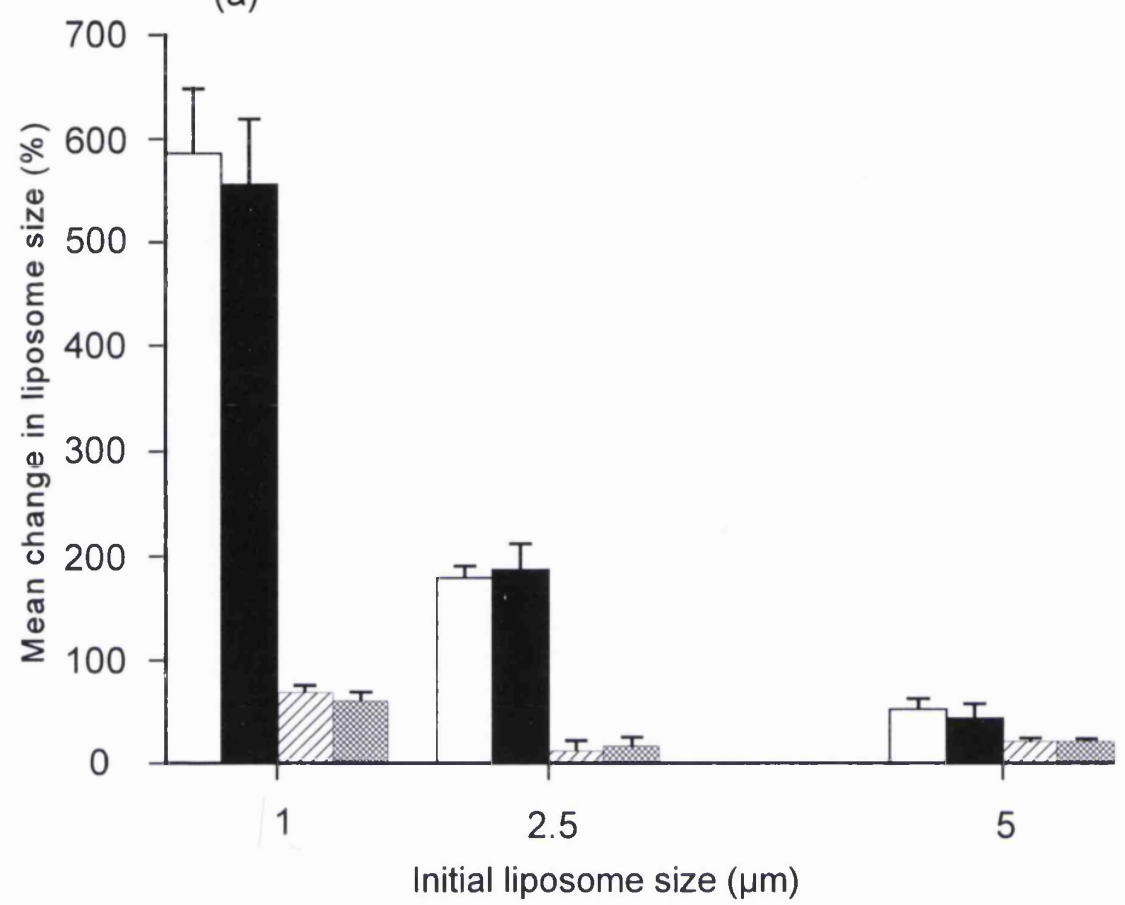

(b)

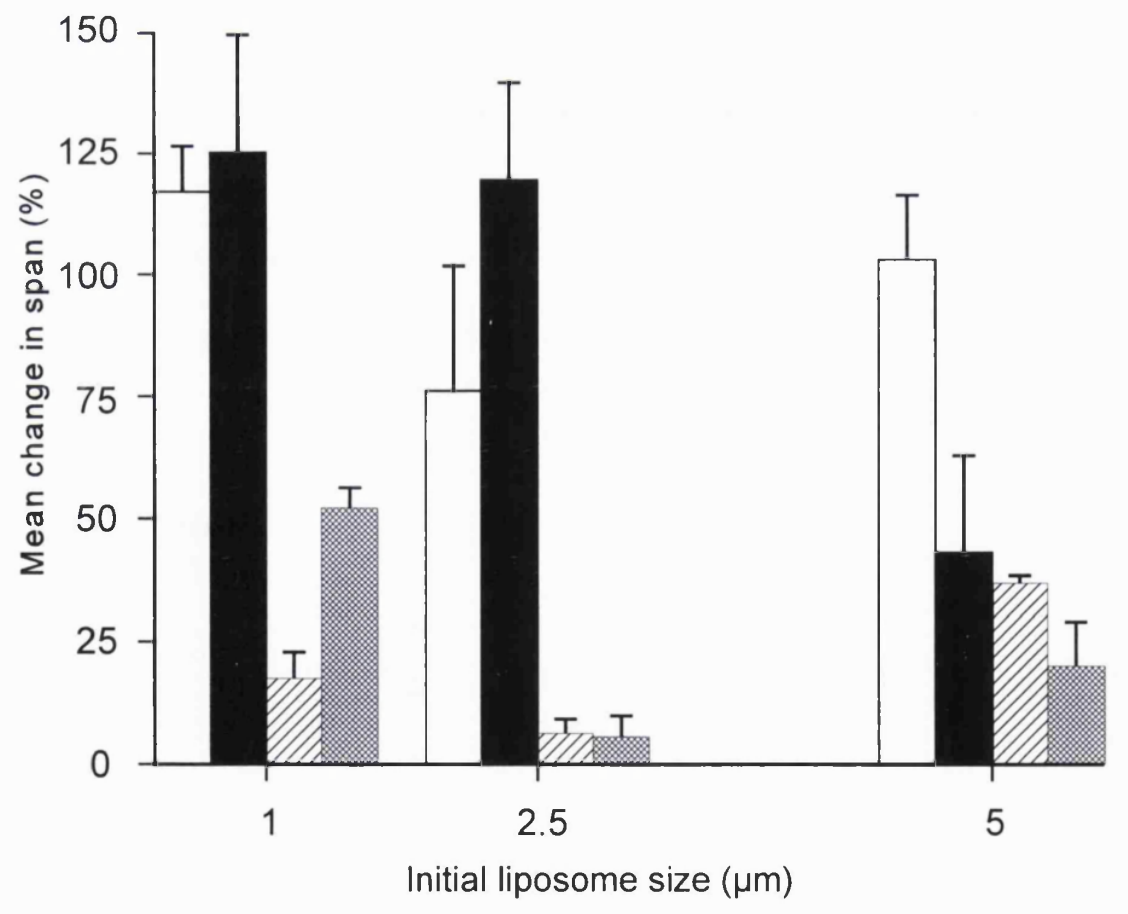

Figure 5.3 The change in (a) the mean size and (b) the polydispersity (span) of reconstituted eggPC ( $\square / /)$ and eggPC/chol (1:1) (⿴囗) liposome formulations following freeze drying in the presence $(/ /, \nabla)$ and absence $(\square \square)$ of trehalose. [ \pm sd; n=6] 
The mean vesicle size of the initial liposome formulations has a significant influence on the change in size distribution characteristics, undergone by formulations containing trehalose during freeze drying (fig. 5.3). The change in mean vesicle size undergone by the 5 and 2.5 $\mu \mathrm{m}$ liposome formulations, during freeze drying, does not significantly differ $(\mathrm{p}<0.05)$. However, the increase in the mean size of the $1 \mu \mathrm{m}$ liposome formulations is significantly greater than that of the 5 , or the $2.5 \mu \mathrm{m}$ formulations. It is not clear why this is the case, however it may be related to the increased bilayer surface area within the smaller liposome formulations.

It is clear that the use of trehalose allows the preparation of a dried liposome product which, upon rehydration, possesses vesicle size characteristics that approximate to those of the initial liposome formulation. However, the initial $5 \mu \mathrm{m}$ mean size liposomes generated a dried product which, upon rehydration, yielded a mean liposome size that was significantly greater than the size accepted as being representative of a respirable particle/droplet (Nebuliser Project Group, 1997), and that of the aerosols produced by common jet nebulisers (chapter 2). As such, the formulation may be relatively unsuitable for development as a reconstitutable liposome product for nebulisation, as larger liposome sizes are associated with an increased bilayer instability during nebulisation (chapter 4). However, they may be more suitable for the delivery of hydrophobic agents, in which the maintenance of bilayer integrity is less critical. Indeed, the majority of the studies involving the nebulisation of liposomal formulations for the carriage of hydrophobic materials, have used relatively large multilamellar liposomes (eg. Debs et al, 1987; Meisner et al., 1989a; Waldrep et al., 1993; Vidgren et al., 1995; Waldrep et al., 1997). That said, the results of chapter 3 demonstrate that aerosol output from nebulisers becomes less efficient as the mean size of the liposomes is increased above that of the aerosol droplets (chapter 3 ). Freeze dried formulations with an initial liposome size of $5 \mu \mathrm{m}$ may therefore be generally unsuitable for nebulisation. However, the 1 and $2.5 \mu \mathrm{m}$ initial mean size liposomes yielded liposome sizes that may be more suitable. The reconstituted liposomes were of a size that has proven to be more stable to nebulisation (chapter 4), and which yields a more efficient aerosol output (chapter 3).

\section{Microscopic analysis of freeze dried liposomes}

The freeze drying of 1 and $2.5 \mu \mathrm{m}$ mean diameter liposome formulations yielded a disc of 
solid on the floor of the drying vessel. The liposome mass that was formed was found to differ significantly in appearance, depending on whether trehalose was included within the formulation. A cream coloured, waxy mass was formed when liposomes were freeze dried in the absence of trehalose. This contrasted to the formulations containing trehalose, which yielded a pure white granular mass. The trehalose-containing formulations also appeared to have dried more thoroughly as the dry solid had a large number of cracks and fissures. The waxy nature of the dried formulations lacking trehalose appeared to hinder redispersion, and consequently they appeared to redisperse less readily than those containing trehalose.

SEM examination of the dried liposomes revealed that the liposome morphology within the freeze dried mass differed significantly, depending on the presence of trehalose within the formulation (fig. 5.4 to 5.7). The SEM images demonstrate that in the absence of trehalose the freeze dried liposomes clumped together in tightly packed aggregates, and/or show signs of a loss of liposome integrity (fig. 5.4 and 5.5). As a result, the mean size of the liposomes was increased significantly following rehydration. Such a size increase is exacerbated by fusion of the liposomes, brought about by the intimate contact between adjacent bilayers. This is supported by the observation that where discrete vesicles are visible, they possess diameters that are significantly greater than the initial mean size of the formulation. The aggregation of the liposomes during freeze drying also means that water would have difficulty in penetrating the aggregated liposome particles, when added for reconstitution. Incomplete aggregate disruption was thus likely to have occurred, and this may have contributed to the large size of the rehydrated trehalose-free liposomes, following freeze drying. However, continued vortex mixing for as long as 10 minutes failed to produce any additional reduction in the mean liposome size.

The influence of the cryoprotectant, trehalose, on the freeze drying behaviour of liposomes is clear from the electron micrographs. The dehydrated liposomes appear as more discrete entities, with little aggregation evident. As a result the observed size characteristics following rehydration were relatively similar to those of the original liposome formulation prior to drying. The existence of the dehydrated liposomes as discrete liposomes would also aid their redispersal upon rehydration. 

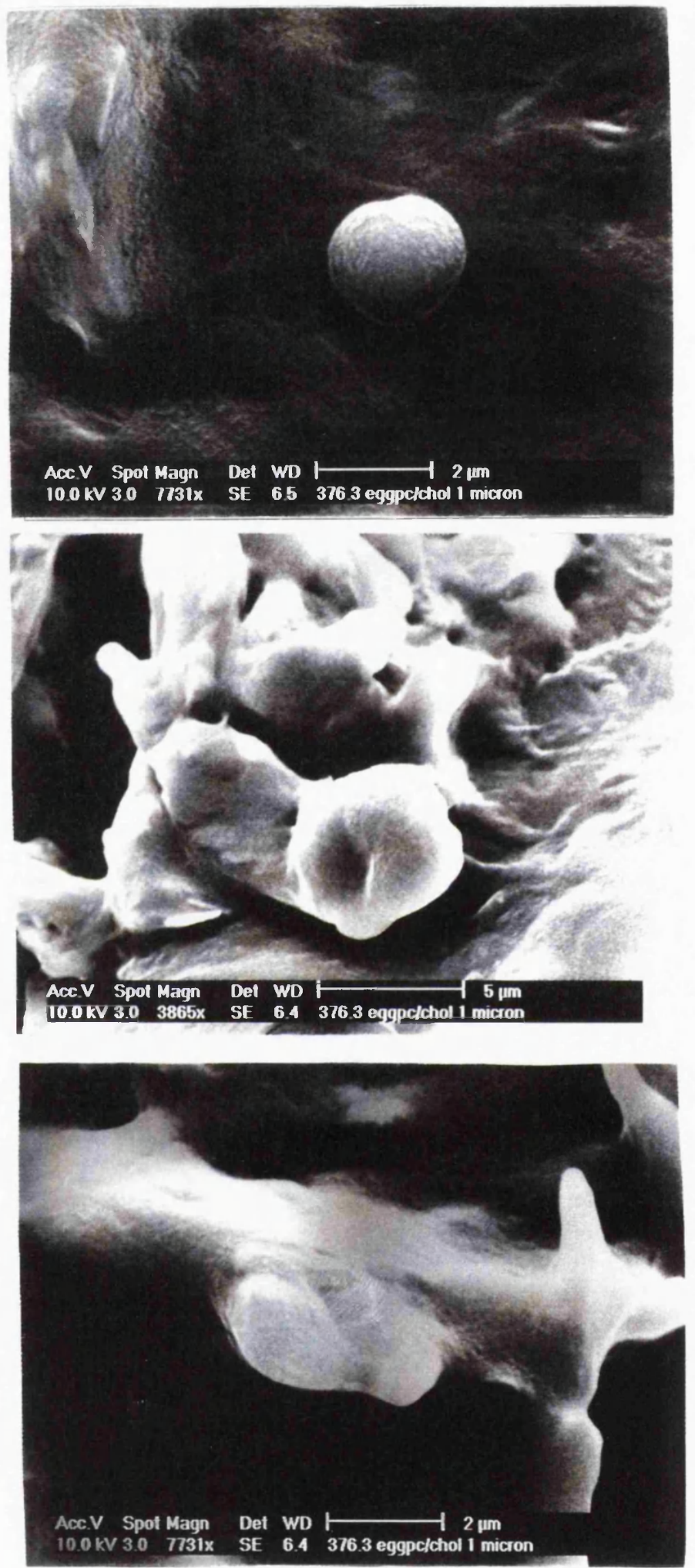

Figure 5.4 Scanning electron micrographs of freeze dried eggPC/chol (1:1) liposomes (initial mean size $1 \mu \mathrm{m}$ ). 

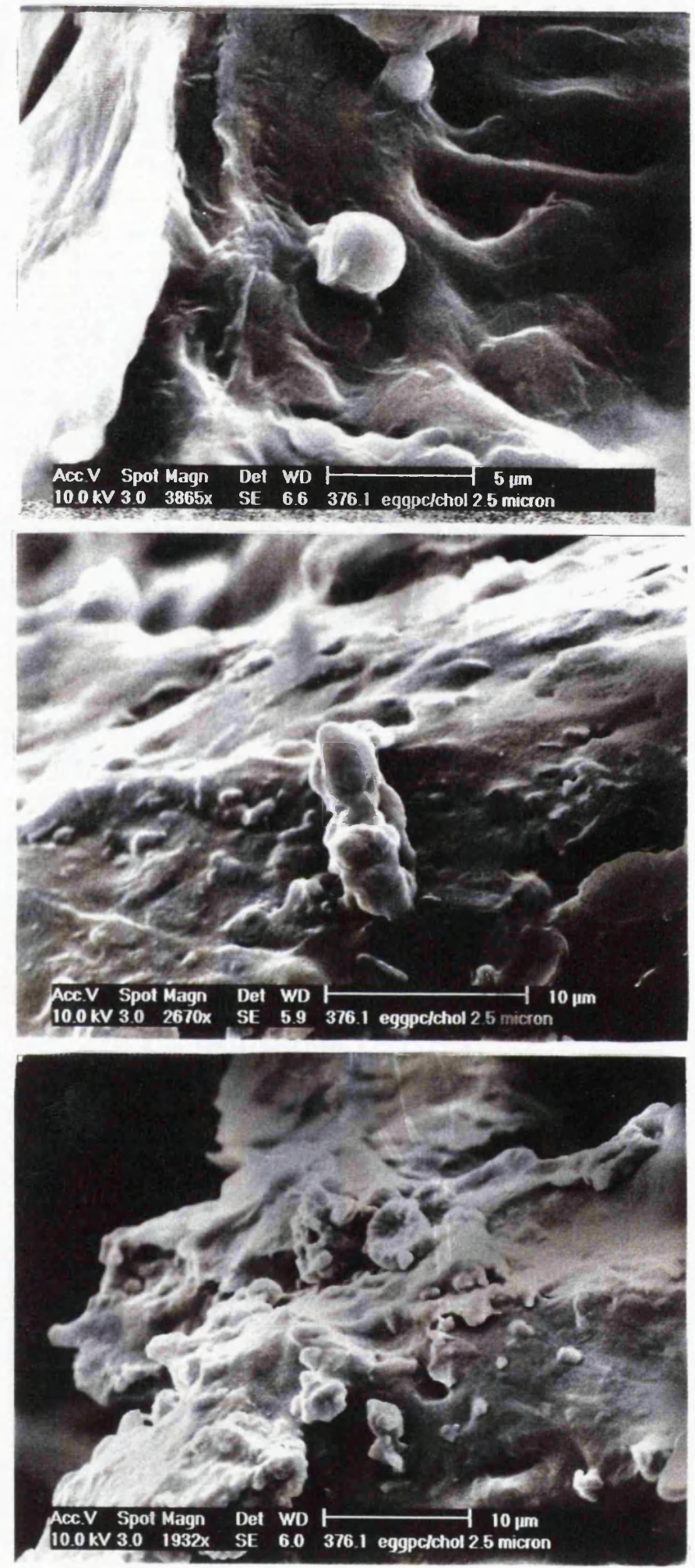

Figure 5.5 Scanning electron micrographs of freeze dried eggPC/chol (1:1) liposomes (initial mean size $2.5 \mu \mathrm{m}$ ). 

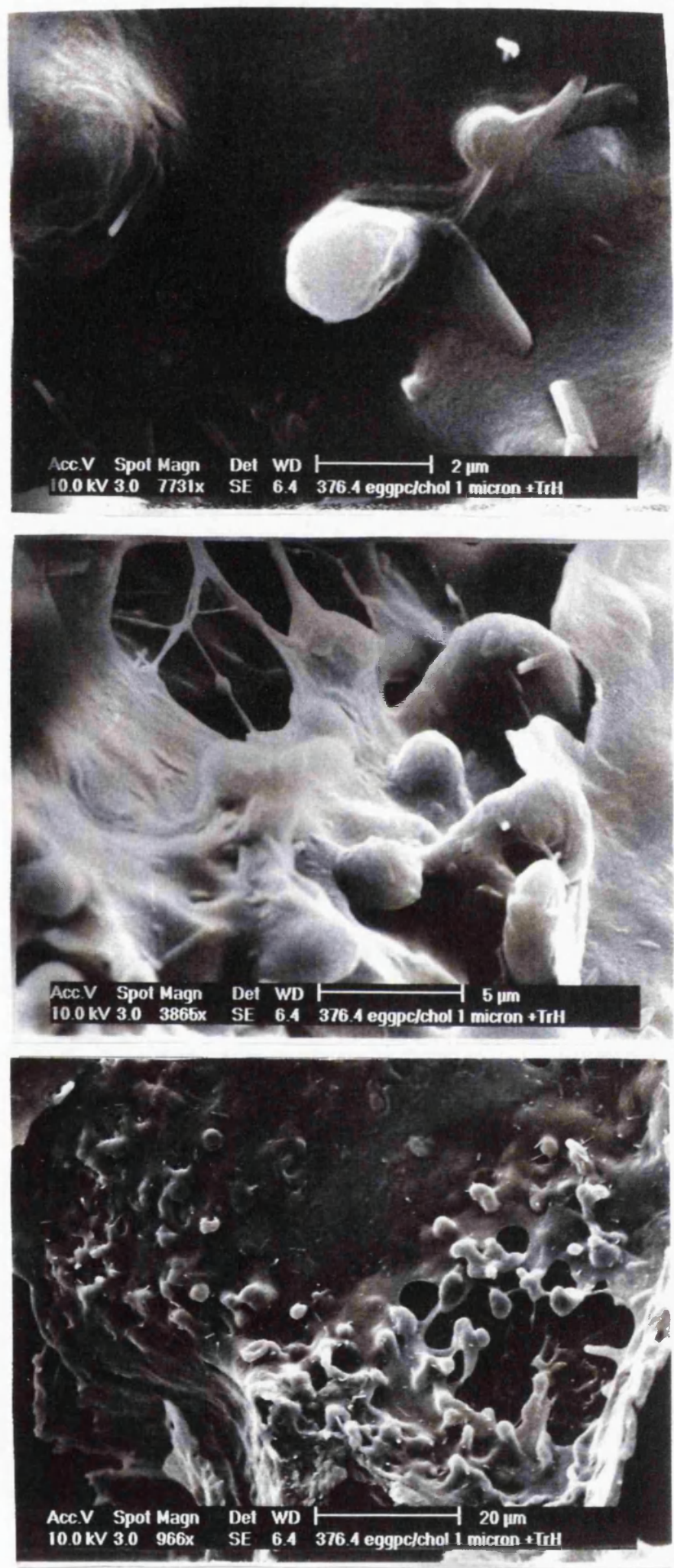

Figure 5.6 Scanning electron micrographs of freeze dried eggPC/chol (1:1) liposomes (initial mean size $1 \mu \mathrm{m}$ ), prepared with the cryoprotectant trehalose. 

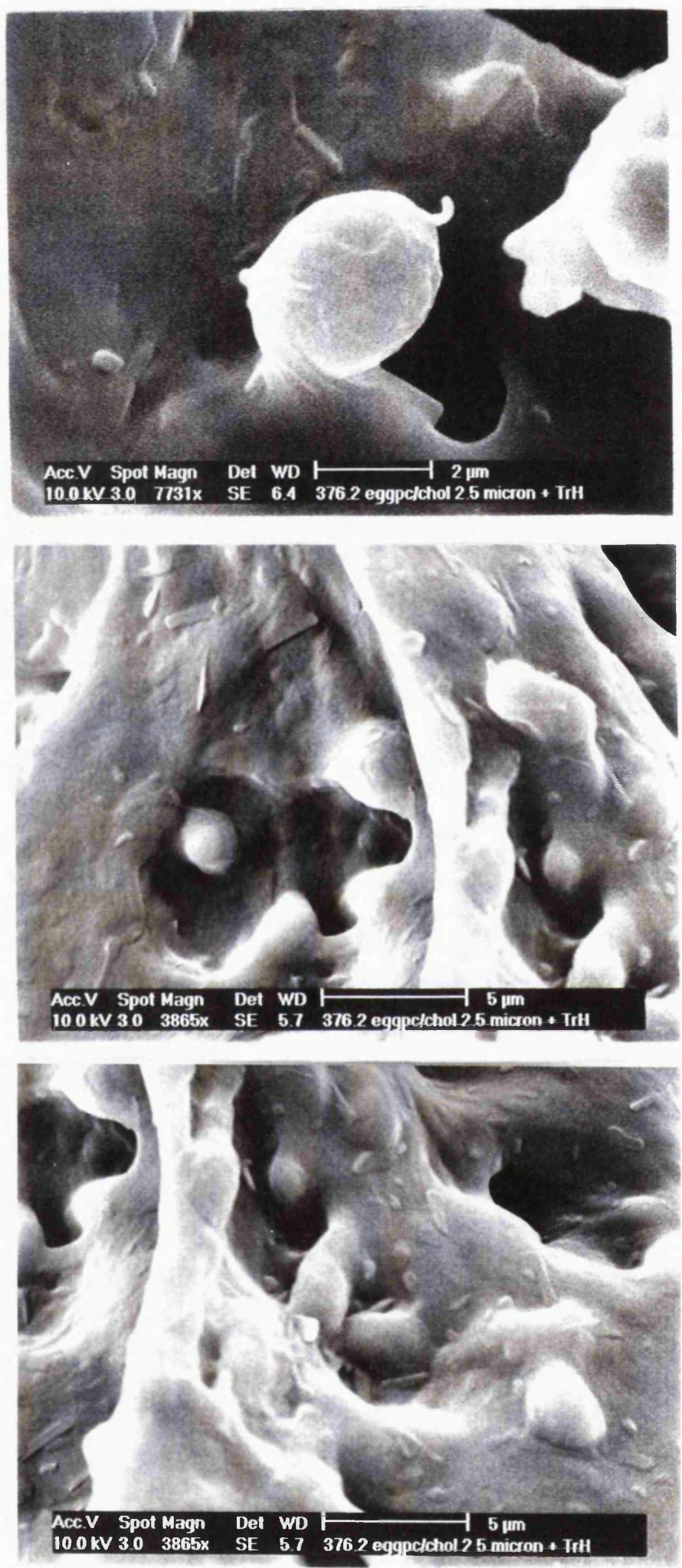

Figure 5.7 Scanning electron micrographs of freeze dried eggPC/chol $(1: 1)$ liposomes (initial mean size $2.5 \mu \mathrm{m}$ ) prepared with the cryoprotectant trehalose. 
A feature of the micrographs taken of the trehalose-containing formulations is the presence of rod-like structures linking adjacent dehydrated liposomes (fig. 5.6 and 5.7). In a number of instances processing of the sample had fractured these connections, causing them to appear as small "tails" on the liposome surface. It is hypothesised that these structures are the disaccharide spacer structures, proposed by certain researchers (Harrigan et al., 1990; Engel et al., 1994), which form as the trehalose establishes a glass-matrix. These structures distance adjacent liposomes, and thus prevent the dehydrated liposome bilayers from interacting. Consequently, the aggregation of liposomes, and their resultant fusion, is hindered. As a result of this, the initial liposome size characteristics of the trehalosecontaining formulations were maintained. The glass matrix also aided liposome redispersal, as water readily penetrated the hydrophilic trehalose mass, liberating the liposomes with relatively unchanged size characteristics to those of the formulation prior to freeze drying. The proposed mechanism for the cryoprotective effect of trehalose is illustrated diagrammatically in figure 5.8 .

(a) Liposomes with trehalose

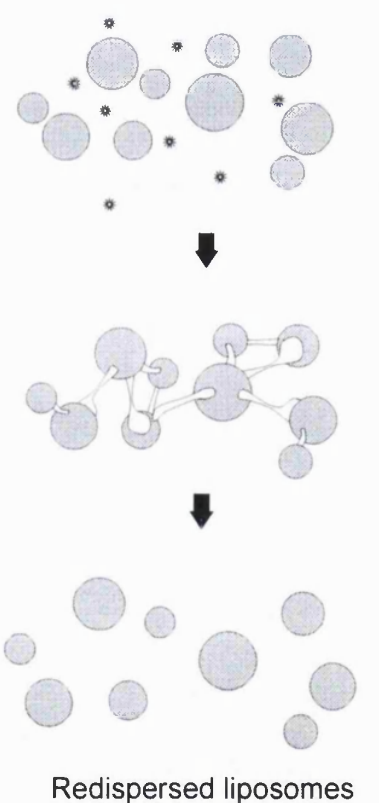

(b) Liposomes without trehalose

Freeze-drying
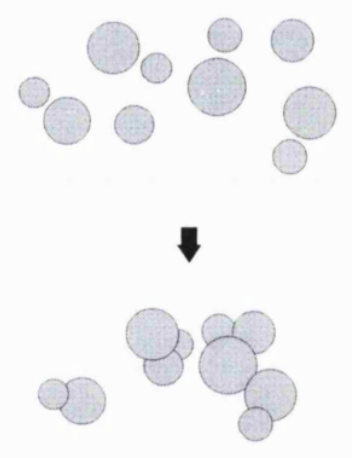

Rehydration

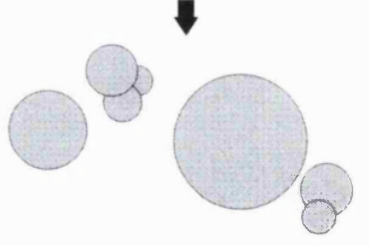

Redispersed liposomes

Figure 5.8 Proposed mechanism of the trehalose-mediated stabilisation of liposomes during freeze drying and subsequent rehydration. (a) Trehalose forms a glass matrix upon dehydration, which coats the liposomes, and forms 'spacers' which prevent adjacent vesicles from interacting. Trehalose also aids the redispersal of the liposomes upon subsequent rehydration. (b) In the absence of trehalose, the liposomes concentrate, and form aggregates. The dehydrated bilayers readily interact, leading to bilayer fusion. Water added during rehydration has difficulty penetrating the tightly packed aggregates. 


\subsubsection{Nebulisation of rehydrated freeze dried liposomes}

The nebulisation of previously freeze dried liposomes was studied using the Pari-LC nebuliser. The Pari-LC is a commonly cited device in nebuliser research, and gave aerosol characteristics that were approximately representative of the jet nebulisers studied previously in the project.

Table 5.2 The VMD $(\mu \mathrm{m})$ and span of aerosol droplets produced during the nebulisation of freeze dried and rehydrated liposomes (fd), and non-freeze dried liposomes, with the Pari-LC nebuliser. The liposomes were prepared with (+tr) or without (-tr) trehalose.

$[ \pm \mathrm{sd} ; \mathrm{n}=3]$

\begin{tabular}{|c|c|c|c|}
\hline \multicolumn{4}{|c|}{ (a) Initial liposomes reduced in size to a mean of $1 \mu \mathrm{m}$} \\
\hline & $\operatorname{eggPC} /$ chol $(1: 1)$ & $\begin{array}{c}\operatorname{eggPC} / \text { chol }(1: 1) \\
(\text { fd)(-tr) }\end{array}$ & $\begin{array}{c}\operatorname{eggPC} / \operatorname{chol}(1: 1) \\
(\mathrm{fd})(+\mathrm{tr})\end{array}$ \\
\hline VMD ( $\mu \mathrm{m})$ & $2.19(0.05)$ & $2.31(0.03)$ & $2.18(0.07)$ \\
\hline span & $2.57(0.05)$ & $2.67(0.05)$ & $2.61(0.04)$ \\
\hline \multicolumn{4}{|c|}{ (b) Initial liposomes reduced in size to a mean of $2.5 \mu \mathrm{m}$} \\
\hline & $\operatorname{eggPC} /$ chol $(1: 1)$ & $\begin{array}{c}\text { eggPC/chol (1:1) } \\
\text { (fd)(-tr) }\end{array}$ & $\begin{array}{c}\text { eggPC/chol (1:1) } \\
(\text { fd })(+ \text { tr })\end{array}$ \\
\hline VMD $(\mu \mathrm{m})$ & $2.31(0.08)$ & $2.37(0.03)$ & $2.21(0.03)$ \\
\hline span & $2.63(0.03)$ & $2.74(0.04)$ & $2.62(0.05)$ \\
\hline
\end{tabular}

Upon nebulisation, the initial $1 \mu \mathrm{m}$ and $2.5 \mu \mathrm{m}$ mean size eggPC/chol (1:1) (non-freeze dried) liposome formulations yielded aerosols which did not differ significantly in size $(p<0.05)$ (table 5.2). Therefore, the droplet size produced by the nebuliser, as determined in chapter 2, was independent of the mean size of the liposome formulation, and also the presence of trehalose. However, the droplets produced upon the nebulisation of liposomes previously freeze dried in the absence of trehalose were slightly larger than those produced from the other formulations. Although this was only statistically significant $(p<0.05)$ for the $1 \mu \mathrm{m}$ mean size formulation, it correlates with a previous study involving the nebulisation of latex suspensions using the Pari-LC nebuliser, which demonstrated a similar trend in the emitted droplet size changes that occurred as the sphere size was increased (fig. 
5.9) (McCallion et al, 1996). The $6.40 \mu \mathrm{m}$ diameter latex spheres used by McCallion et al. (1996) may be considered equivalent to the 1 and $2.5 \mu \mathrm{m}$ eggPC/chol (1:1) liposome formulations of the present study which had undergone freeze drying in the absence of trehalose (having a mean size of 6.96 and $7.41 \mu \mathrm{m}$ respectively). Equally, the 1.16 and 2.97 $\mu \mathrm{m}$ latex spheres approximate to the 1 and $2.5 \mu \mathrm{m}$ liposomes redispersed after freeze drying in the presence of trehalose (yielding mean sizes of 1.61 and $2.94 \mu \mathrm{m}$ respectively). Of the latex sphere formulations studied by McCallion et al. (1996), the $1.16 \mu \mathrm{m}$ spheres yielded the smallest aerosol size (droplet VMD $=2.35 \mu \mathrm{m}$ ). Similarly, in the present study the liposomes with the smallest mean diameter following redispersal produced the smallest aerosol droplet size (droplet VMD $=2.18 \mu \mathrm{m}$ ). An increase in latex sphere size to $2.97 \mu \mathrm{m}$ yielded a comparable droplet size (droplet $\mathrm{VMD}=2.41 \mu \mathrm{m}$ ), to that obtained in the present study when the mean redispersed liposome size was increased to $2.94 \mu \mathrm{m}$ (droplet VMD $=2.21 \mu \mathrm{m}$ ). With a further increase in the latex sphere size to $6.40 \mu \mathrm{m}$, the emitted droplet size was reported as being $2.58 \mu \mathrm{m}$. Again, this is a similar increase in the emitted droplet size with increasing suspended particle size, as that recorded when eggPC/chol $(1: 1)$ liposomes which had previously been freeze dried in the absence of trehalose (mean size $=7.23 \mu \mathrm{m})$ were nebulised (droplet VMD $=2.37 \mu \mathrm{m})$.

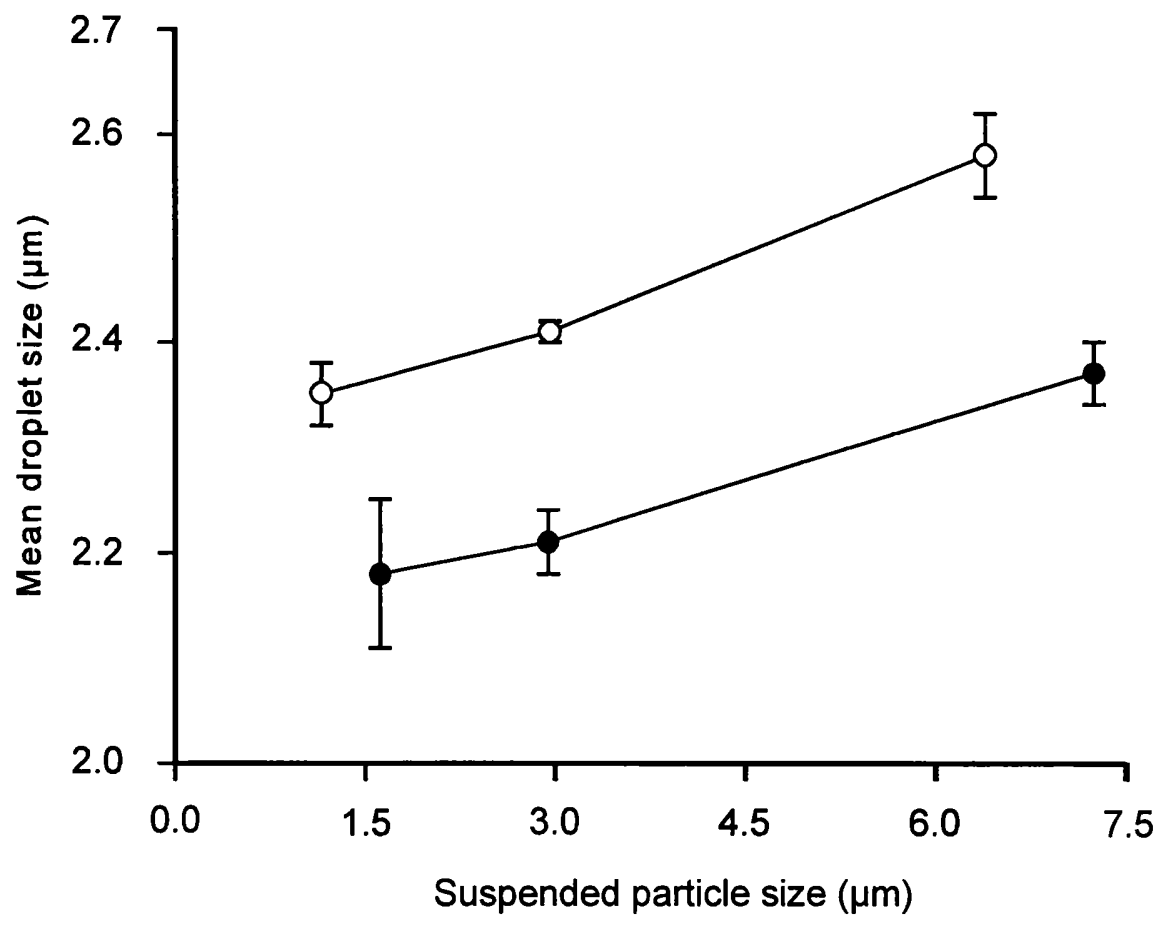

Figure 5.9 A comparison between the change in droplet size recorded by McCallion et al., (1996) as the size of suspended latex spheres is increased (0) to that produced as the redispersed liposome size (following freeze drying) was increased in the present study $(\bullet)$. [(०): \pm se; $n=5][(\bullet): \pm s e ; n=3]$ 
Therefore, in each study there was a trend towards an increased droplet size, with an increase in the size of the dispersed phase, be they true suspensions or liposomes. Although only statistically significant for the $1 \mu \mathrm{m}$ initial mean size formulations, it appeared that the large liposome aggregates produced upon freeze drying without trehalose, caused the droplet size emitted upon their nebulisation to be relatively large. Indeed, an increase in the emitted droplet size has been demonstrated in a previous study of the aerosolisation of suspended aggregates delivered from a MDI (Dalby and Byron, 1988). Furthermore, the previously noted increase in droplet size, produced by an increase in lipid concentration of liposomes, may have been due to the presence of liposome aggregates at higher concentrations. The mechanisms for the increase in droplet size arising from such aggregation were speculated upon in chapter 3 . However, the freeze drying of liposomes only significantly influenced the droplet size for the $1 \mu \mathrm{m}$ liposomes freeze dried without trehalose. This is despite the fact that the mean size of the liposomes being nebulised may be as much as seven times larger in the liposomes that had been freeze dried without trehalose. A possible explanation for this is that the liposome aggregates were disrupted during nebulisation, and thus had a similar size for each liposome formulation for the greater part of the nebulisation time. The residual liposome size, and that of the aerosolised liposomes, was therefore investigated (fig. 5.10).

Table 5.3 The rate of liposome size reduction during nebulisation of liposomes freeze dried (fd) in the presence/absence of trehalose (+tr/-tr), and that of non-freeze dried (nfd) eggPC/chol (1:1) liposome formulations ( $\mathrm{nm} / \mathrm{min})$.

\begin{tabular}{|c|c|c|}
\hline \multicolumn{2}{|c|}{$[ \pm \mathrm{sd} ; \mathrm{n}=3]$} & $\begin{array}{c}\mathbf{5 0} \text { to } \mathbf{1 0 0} \\
\text { nebulisation of }\end{array}$ \\
\hline $\mathbf{2 . 5} \boldsymbol{\mu m}$ initial size (nfd) & $\begin{array}{c}\mathbf{0} \text { to } \mathbf{5 0} \% \text { of } \\
\text { nebulisation }\end{array}$ & $127(25)$ \\
\hline $\mathbf{5} \boldsymbol{\mu m}$ initial size (nfd) & $233(30)$ & $46(50)$ \\
\hline $\mathbf{1} \boldsymbol{\mu m}$ initial size (fd)(-tr) & $622(36)$ & $156(78)$ \\
\hline $\mathbf{2 . 5} \boldsymbol{\mu m}$ initial size (fd)(-tr) & $767(11)$ & $64(70)$ \\
\hline $\mathbf{1} \boldsymbol{\mu m}$ initial size (fd)(+tr) & $58(25)$ & $-1.3(12)$ \\
\hline $\mathbf{2 . 5} \boldsymbol{\mu m}$ initial size (fd)(+tr) & $65.5(29)$ & $62(25)$ \\
\hline
\end{tabular}


The response of the residual liposomes to nebulisation was found to depend on whether the formulation had been previously freeze dried, and also whether the freeze drying had taken place in the presence of trehalose (fig. 5.10).
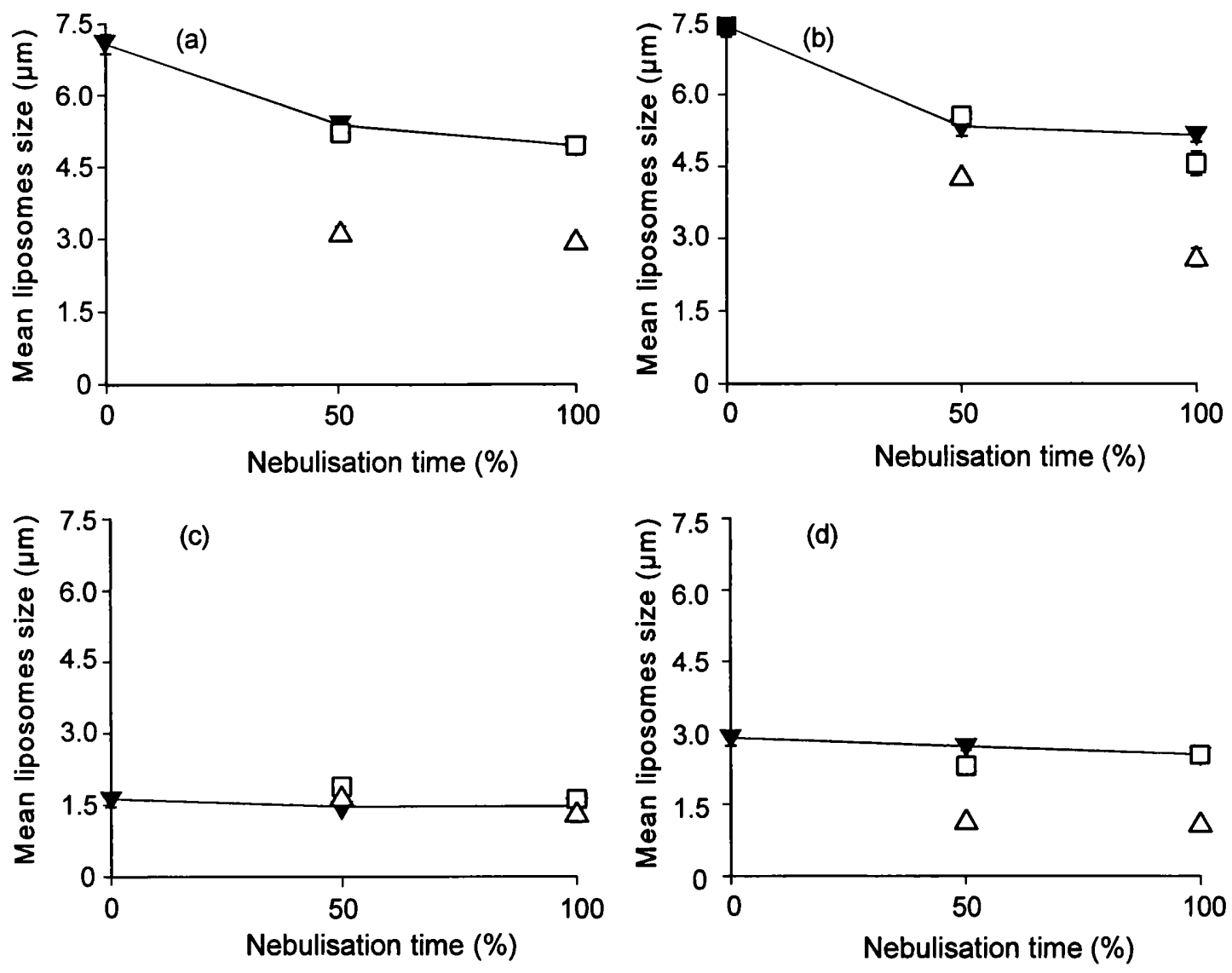

Figure 5.10 The mean size of freeze dried and reconstituted eggPC/chol (1:1) liposome formulations, during nebulisation into a twin-impinger. (a) $1 \mu \mathrm{m}$ initial mean liposome size. (b) $2.5 \mu \mathrm{m}$ initial mean liposome size. (c) $1 \mu \mathrm{m}$ initial mean liposome size, containing trehalose. (d) $2.5 \mu \mathrm{m}$ initial mean liposome size, containing trehalose. The liposomes were sampled from $(\boldsymbol{\nabla})$ the nebuliser chamber (residual liposomes), ( $\square$ ) the upper stage and $(\Delta)$ the lower stage of the twin impinger. [ \pm sd; $n=3]$ 
Liposomes freeze dried in the absence of trehalose experienced a rapid reduction in residual size during the first half of nebulisation ( 0 to $50 \%$ of the nebulisation time). Indeed, the level of liposome processing seen in the earlier stages of nebulisation was greater than that observed for $5 \mu \mathrm{m}$ mean size non-freeze dried eggPC/chol (1:1) liposomes (fig. 5.11). This may to a certain extent reflect the increased mean size of the liposomes within the previously freeze dried formulation (approximately $7 \mu \mathrm{m}$ compared with $5 \mu \mathrm{m}$ ). The larger size causes an increased resistance to aerosolisation, making the liposomes less able to be included within secondary aerosol droplets. They thus became retained for extended periods within the nebuliser chamber, and as a result were transformed in size to a greater extent (fig. 5.11).

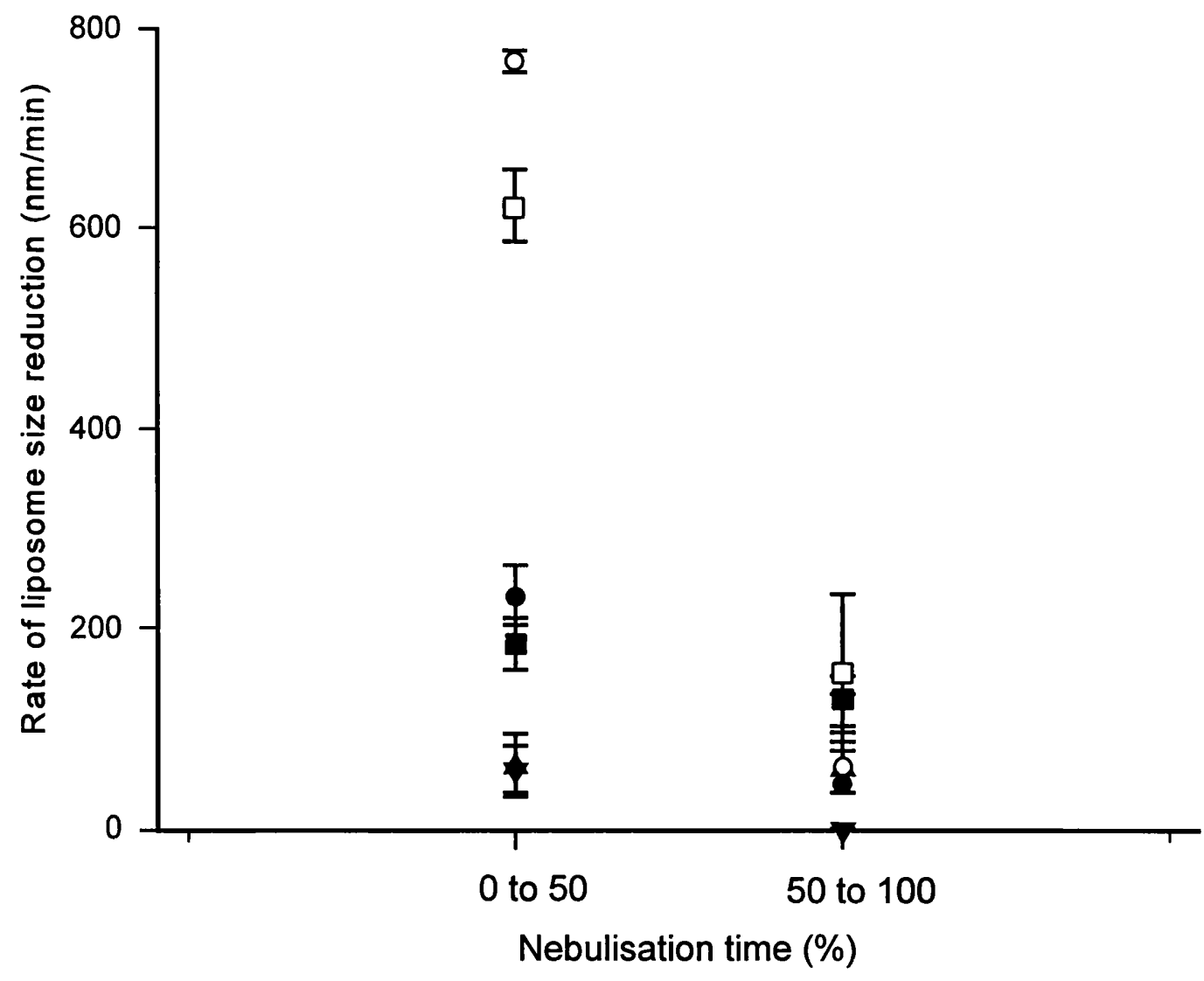

Figure 5.11 A comparison between the rate of residual liposome size reduction during the initial and latter stages of the Pari-LC nebulisation of conventional eggPC/chol (1:1)

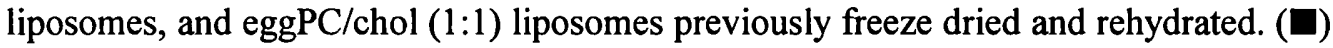
$2.5 \mu \mathrm{m}$ non-freeze dried liposomes (from chapter 4), (0) $5 \mu \mathrm{m}$ non-freeze dried liposomes (from chapter 4), (口) $1 \mu \mathrm{m}$ liposomes freeze dried without trehalose, (O) $2.5 \mu \mathrm{m}$ liposomes freeze dried without trehalose, $(\nabla) 1 \mu \mathrm{m}$ liposomes freeze dried with trehalose, (A) $2.5 \mu \mathrm{m}$ liposomes freeze dried with trehalose. [ \pm sd; $n=3$ ] 
The pronounced size reduction exhibited by freeze dried formulations lacking trehalose, may also indicate that the shearing action of droplet formation caused the disruption of liposomes aggregates that remain within the dispersions. These aggregates may be disrupted more easily than the relatively stable gel-state phospholipid bilayers of the liposomes themselves, and thus the mean residual liposome size fell sharply in the early stages. The rate of residual liposome size reduction for the freeze dried eggPC/chol (1:1) liposomes was therefore significantly greater than that produced by the non-freeze dried $5 \mu \mathrm{m} \mathrm{eggPC} / \mathrm{chol}$ (1:1) liposomes (table 5.3; fig. 5.11), despite having a similar size. This may also account for the fact that there is little significant difference between the droplet size produced from freeze dried and non-freeze dried liposomes formulations, despite the significant difference in the initial liposome size. Once $50 \%$ of the nebulisation time was reached, the majority of liposome aggregates had become disrupted, and the liposomes that remained were predominantly discrete vesicles, formed from the fusion of smaller liposomes during freeze drying. As a result, the trehalose-lacking, freeze dried formulations (fig. $5.10 \mathrm{a} / \mathrm{b}$ ), experienced a fall in the residual liposome size in the latter stages of nebulisation, which was of a magnitude similar to that observed for the non-freeze dried eggPC/chol (1:1) liposome formulation studied in chapter 4 (table 5.3). In addition, it was previously determined that the damage caused to liposomes by nebulisation was greatest in the initial stages, when the liposomes have an increased mean size (chapter 4). As the liposomes become reduced in size, so the processing of the liposomes becomes size-limiting. This therefore contributes to the reduced liposome processing that is attributable to the completion of aggregate disruption. Finally, as the residual fluid volume falls so the output of aerosol from the Pari-LC becomes reduced (chapter 3). As a result, the rate of liposome damage falls towards sputtering.

The change in the distribution of liposome sizes (span) within the residual fluid was similar for each formulation, and correlates with the results previously obtained for non-freeze dried liposomes (chapter 4)(fig. 5.12). That is, there was a small reduction in the polydispersity of vesicle sizes, associated with the reduction in the mean residual liposome size during nebulisation. Freeze drying therefore appeared to have had little impact on the changes in the polydispersity of residual liposomes that occur during nebulisation, regardless of whether trehalose was present. 

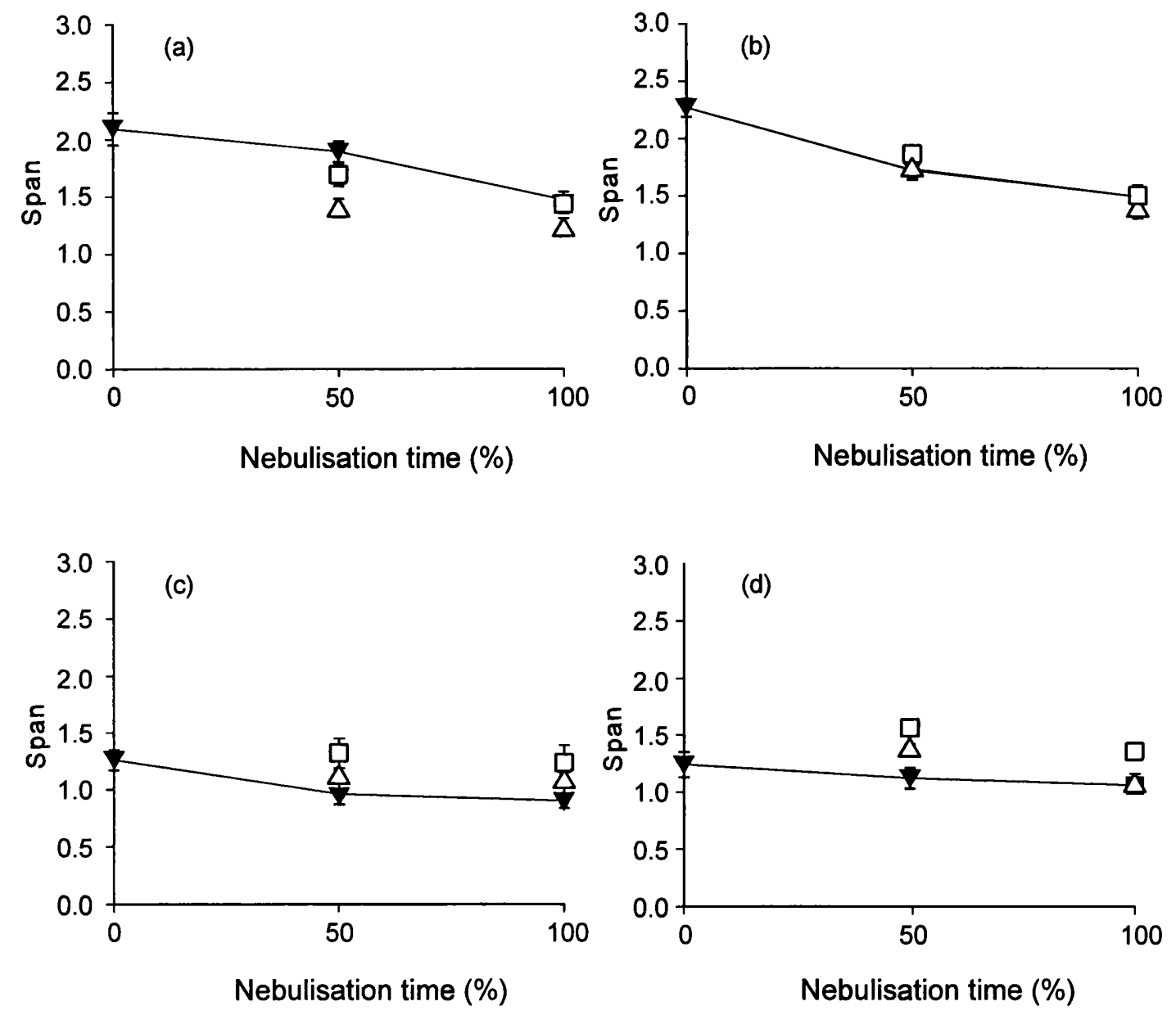

Figure 5.12 The polydispersity (span) of freeze dried and reconstituted eggPC/chol (1:1) liposomes during nebulisation into a twin-impinger. (a) $1 \mu \mathrm{m}$ initial mean liposome size. (b) $2.5 \mu \mathrm{m}$ initial mean liposome size. (c) $1 \mu \mathrm{m}$ initial mean liposome size, containing trehalose. (d) $2.5 \mu \mathrm{m}$ initial mean liposome size, containing trehalose. The liposomes were sampled from $(\boldsymbol{\nabla})$ the nebuliser chamber (residual liposomes) ( $\square$ ) the upper stage, and $(\Delta)$ the lower stage of the twin impinger. [ \pm sd; $n=3$ ]

The pattern of liposome deposition within each stage of the TI, was similar to that obtained with non-freeze dried liposomes, and was largely unaffected by the presence of trehalose in the formulation (fig. 5.10, 5.12). As previously determined (chapter 4), liposomes that deposited within the upper stage of the TI had the largest mean size, due to the fact that the upper stage collects the largest droplets from the aerosol. The larger liposomes of the formulation tend to be restricted to the non-respirable droplets, and thus deposit predominantly within the upper stage of the TI. The size of the liposomes depositing in 
each stage of the TI was markedly smaller for the liposomes that had been previously freeze dried in the presence of trehalose, and reflected the smaller rehydrated liposome size (chapter 4). Similarly, the liposomes which had been freeze dried without trehalose, had a large reconstituted size, due to aggregation and fusion, which caused them to produce aerosolised liposomes with a significantly larger mean size. The larger size of the liposomes freeze dried in the absence of trehalose also rendered them unstable to nebulisation, and the deposited size was therefore significantly reduced in the latter stage of nebulisation. This may in part be due to the processing of aggregates within the nebuliser chamber. In contrast, the liposomes previously freeze dried in the presence of trehalose yielded small liposomes upon rehydration. They were thus stable to nebulisation, and produced deposited liposomes with a consistent size at both times of nebulisation. The $1 \mu \mathrm{m}$ initial mean liposome size formulation, that was freeze dried with trehalose, yielded liposomes which deposited within the TI with a mean size that did not significantly differ between the upper and lower stages. This was because the liposomal aerosol was produced from a population of liposomes with a relatively small mean size $(1.61 \mu \mathrm{m})$. Consequently, the aerosolised liposomes had an approximately equal distribution between the larger droplets deposited within the upper stage, and the smaller ones deposited within the lower stage. Similar findings have been published in a study of nebulised polymer sphere suspensions (Lashmar et al., 1994). "Polybeads" with a diameter of $1 \mu \mathrm{m}$ were evenly distributed between four stages of a MLI. However, as the polybead size was increased to $3 \mu \mathrm{m}$ a greater percentage of the beads deposited in the upper stages of the MLI. The $2.5 \mu \mathrm{m}$ initial size liposomes freeze dried with trehalose support this. The reconstituted size for these liposomes was approximately $3 \mu \mathrm{m}$ and, as a result, the larger liposomes were restricted to the non-respirable droplets. The size of liposomes deposited in the TI reflected this, as the upper stage contained liposomes with an increased mean size.

\subsection{Conclusions}

Freeze drying may address certain of the long-term stability concerns which significantly limit the applicability of liposomes to drug delivery. However, the freezing and dehydration processes involved expose liposome bilayers to stresses that cause demonstrable changes in liposome structure. Extensive aggregation and fusion of vesicle results, regardless of the initial liposome size, or the bilayer phase state. This generates liposomes with a greatly increased mean size and polydispersity, and the loss of any entrapped hydrophilic materials 
is likely to occur. Due to their large rehydrated size, the redispersed liposomes have a reduced stability to nebulisation, and, based on the results of chapter 3 , may give a relatively inefficient aerosol output.

The problems inherent with liposomal freeze drying may be overcome with the use of a cryoprotectant, such as trehalose. The disaccharide stabilises liposomes during freeze drying, yielding a dried product which, upon rehydration, retains the approximate vesicle size characteristics of the original formulation. Microscopic analysis indicates that the cryoprotection afforded by trehalose is at least in part due to the formation of a glassnetwork of dehydrated sugar molecules. The dehydrated liposomes are maintained as discrete entities, and hence the interaction of adjacent liposome bilayers is minimised. Liposome aggregation and fusion is therefore reduced, and hence the liposome size following subsequent rehydration is relatively unchanged. Trehalose also aids the redispersion of the formulation, as there are fewer hydrophobic aggregates for the aqueous phase to penetrate. Water molecules readily penetrate between the liposomes, which carry a hydrophilic coat of sugar molecules, and the liposomes are thus liberated from the sugar matrix relatively unchanged with regard to size.

The nebulisation of freeze dried and redispersed liposomes is to an extent dependent on their vesicle size characteristics. The large liposomes resulting from freeze drying in the absence of trehalose are more prone to bilayer damage, and as a result they become rapidly transformed to a reduced mean size. This is primarily due to the disruption of vesicle aggregates. However, significant bilayer disruption may also result. Freeze drying therefore produces liposomes that may be unsuitable for nebulisation. However, the addition of a cryoprotectant, such as trehalose, during preparation largely overcomes these problems. The redispersed liposomes have size characteristics that are similar to the formulation prior to freeze drying, and consequently they are nebulised in a similar manner to non-freeze dried liposomes. Liposomes with a small mean size may be freeze dried and redispersed in the presence of trehalose with relatively unchanged size characteristics. As a result, the liposomes are relatively stable to nebulisation, and become evenly distributed within the aerosol. Trehalose therefore allows a stable, dried liposomal product to be formed, which may be reconstituted prior to use without influencing the size characteristics of the aerosol, or the liposomes that it contains. 


\section{ULTRASONIC NEBULISATION OF LIPOSOMES}

\subsection{Introduction}

Nebulisers have thus far been the devices of choice for the generation of liposomal aerosols. This is largely because they are capable of delivering large aerosol volumes, and the liposomes may be prepared by conventional techniques, without any additional processing, except the removal of unentrapped drug where appropriate (Taylor and Farr, 1993). All reported studies of liposome delivery to the human lung have specifically employed jet nebulisers (Farr et al., 1985; Taylor et al., 1989; Barker et al., 1994; Vidgren et al., 1995; Purcell and Corris, 1995; Waldrep et al., 1997). However, jet nebulisers may damage liposomes due to the repeated exposure of bilayers to the shearing action of the gas jet (Taylor et al., 1990b; Niven et al., 1992a; chapter 4). The major determinants of liposome stability to jet nebulisation have been shown to be the size of the liposomes relative to the size of the aerosolised droplets (Taylor et al., 1990b; Niven et al., 1991a; chapter 4), the air pressure employed to generate the aerosol (Niven et al., 1992a), the phase state of the bilayer constituents (chapter 4), and the design characteristics of the nebuliser (chapter 4). The alternative device for the nebulisation of liposomes is the ultrasonic nebuliser. First introduced in the early 1960s, the ultrasonic nebuliser was initially used for air humidification, avoiding the need for steam production, and thus allowing the maintenance of a normal ambient temperature in respiratory care units (Greenspan, 1996). They have since been used to deliver a wide array of drugs to the lung. However, the jet nebuliser is more commonly used for drug delivery in the UK (O'Callaghan and Barry, 1997). This is largely because of their simpler operation, and lower cost (Waldman et al., 1987). Ultrasonic nebulisers are too expensive to be produced as disposable units, and thus run the risk of bacterial contamination during repeated use (Nebuliser Project Group, 1997). However, they prove popular with patients for bronchodilator therapy, and are more widely used elsewhere in the world (Clarke, 1988; O'Callaghan and Barry, 1997). Ultrasonic nebulisers also yield more efficient outputs of aerosol, which is independent of the airflow, and have a significantly increased droplet concentration (Phipps and Gonda, 1990). Consequently, the nebulisation time is reduced. They also do not rely on a continuous flow of compressed air, and are therefore far quieter in operation. Ultrasonic nebulisers operate very differently to jet nebulisers, and as the stability of liposomes to jet nebulisation is controlled by the design of the device, they may handle liposomes very differently. 


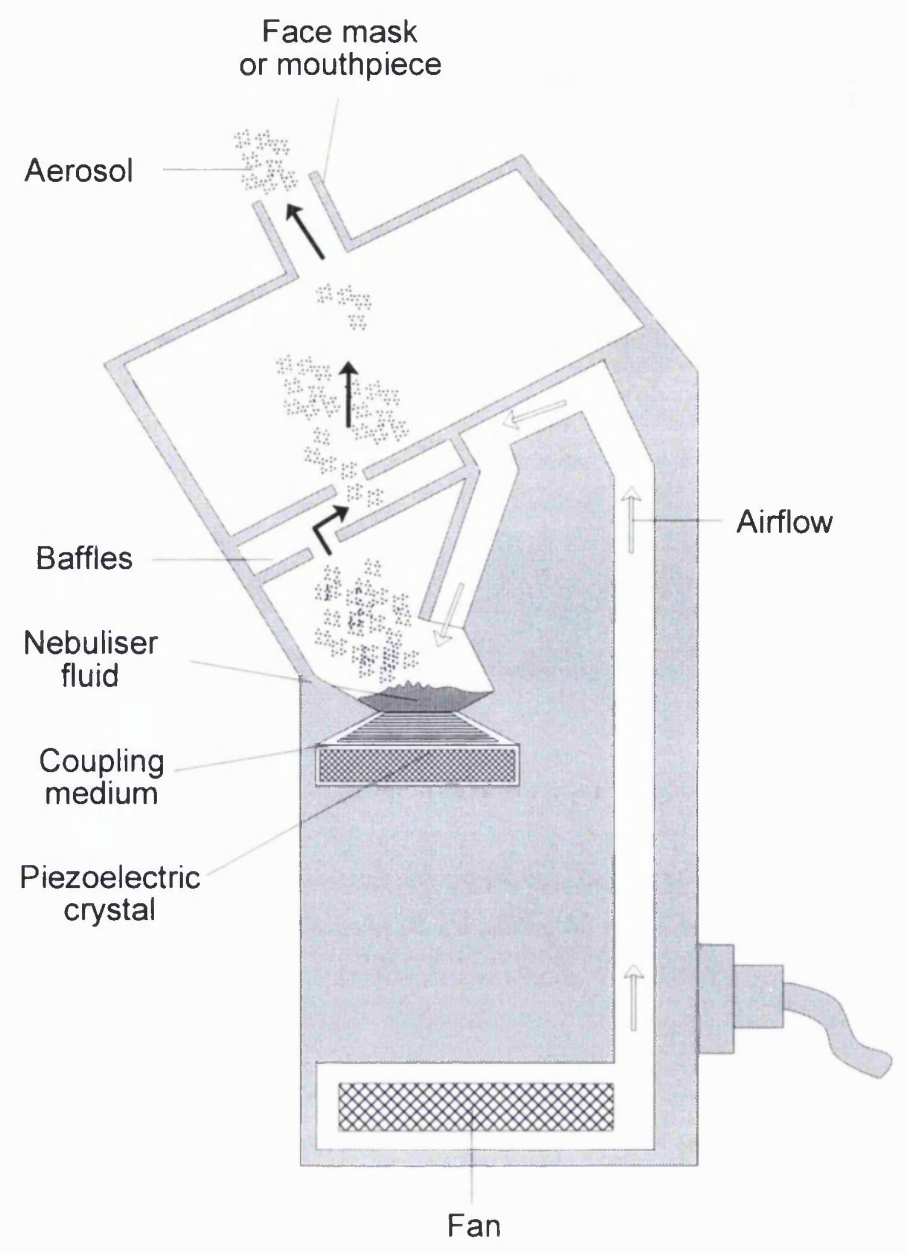

Figure 6.1 Schematic diagram of a typical ultrasonic nebulizer.

\section{Droplet formation in ultrasonic nebulisers}

In an ultrasonic nebuliser (fig. 6.1), the energy necessary for liquid atomisation is generated by high frequency vibrations (1-2 MHZ) in a piezoelectric crystal (transducer), driven by an alternating electrical field (Clarke, 1988). As the oscillating electrical energy is supplied to the crystal, it undergoes small physical displacements at the same frequency as the applied energy. The vibrations are transmitted through a coupling fluid into the nebuliser chamber, resulting in periodic hydraulic shocks within the fluid. The precise mechanism of droplet formation from the body of liquid remains unclear. The capillary wave theory describes droplet formation due to the generation and propagation of waves at the fluid surface (Peskin and Raco, 1963). Vertical capillaries are formed on the fluid surface which, when of a sufficiently high amplitude, form a highly concentrated (up to $200 \mu \mathrm{g}$ of water per litre of air) fountain of droplets (Boucher and Kreuter, 1968; Lourenço and Cotromanes, 
1982). A number of researchers, including Lang (1962), have provided evidence to show that the size of these droplets (count median diameter, CMD) is a function of the excitation frequency $(F)$, the surface tension $(\sigma)$, and the liquid density $(\rho)$ :

$$
C M D=0.34\left(8 \pi \sigma / \rho F^{2}\right)^{1 / 3}
$$

[equation 6.1]

A second theory describes the formation of droplets by ultrasonic nebulisers (Sölner, 1936; Gerhenzon and Eknadiosyants, 1964). This cavitation theory proposes that the vibration of the bulk liquid causes the formation of cavitation bubbles. As the bubbles rise, the internal pressure equilibrates to that of the external atmosphere, causing it to implode. Turbulence at the liquid surface results from bubble bursting, and fluid fragments are thrown into the air. In fact, a more popular theory is that droplets are formed through the interaction of these two mechanisms. That is, the capillary waves generated at the fluid surface are formed by cavitation disturbances (Boguslavskii and Eknadiosyants, 1969). The crests of these waves release droplets, the smaller of which are inhaled. Aerosol droplet release then occurs, either through entrainment into the inspiratory air-flow, or through the aid of an internal fan.

The droplet size produced from ultrasonic nebulisers is generally larger than that produced by jet nebulisers. Indeed, the majority of ultrasonically generated aerosols have a mean size that is unsuitable for the efficient targeting of the alveolar region (Mercer, 1981; Sterk et al., 1984; Matthys and Köhler, 1985; Phipps and Gonda, 1990; Hardy et al., 1993). However, the spread of droplet sizes within an aerosol produced by ultrasonic nebulisation is generally smaller than that produced from jet nebulisers (Mercer, 1981). Despite this, the aerosol is broadly heterodisperse in nature, and therefore a significant fraction of the primary aerosol droplets have a diameter that is too large to be efficiently inhaled. Therefore, in common with the gas jet driven devices, ultrasonic nebulisers employ baffles in order to filter out the larger droplets. The absence of high velocity gas flow through the nebuliser means that evaporative losses occur to a far lesser extent, compared with jet nebulisers. As a consequence, there is less of an increase in solute concentration during operation. 
The greatest proportion of the energy generated by the piezoelectric crystal in ultrasonic nebulisers is transformed into heat (Mercer et al., 1968). As a result, the temperature of fluid within the liquid reservoir may rise by as much as $20^{\circ} \mathrm{C}$ during operation (Taylor and Hoare, 1993). This heating does not affect most conventional drugs for inhalation (Goddard et al., 1992). Indeed, it may be beneficial for poorly soluble drugs, which precipitate in response to the cooling induced by jet nebulisation (Taylor and Hoare, 1993). However, it may lead to the chemical breakdown of certain sensitive materials. These include certain antibiotics (Gale, 1985), proteins (eg. Wigley et al., 1971), and possibly the imaging agent ${ }^{99 m}$ Tc-DTPA (Waldman et al., 1987). Indeed, heating is a significant enough problem that certain ultrasonic nebuliser models include a thermal cut-off switch, which halts nebulisation once the residual fluid reaches a critical temperature. However, this cut-off may not operate until the temperature of the reservoir fluid has become significantly elevated (Niven et al., 1995). Ip et al. (1995) determined that the Microstat nebuliser increased the temperature of nebulised solutions to $80^{\circ} \mathrm{C}$ during operation. The heating effects of other nebulisers were moderated by the cooling air provided by internal fans. Ip et al. (1995) went on to demonstrate that $\alpha$-interferon was stable to nebulisation with an ultrasonic nebuliser, provided that the fluid temperature was maintained at a constant $25^{\circ} \mathrm{C}$ throughout nebulisation.

The heating problems associated with ultrasonic nebulisers, and the fact that they are less commonly used in clinical situations in the UK, has meant that ultrasonic nebulisers have generally been avoided for the delivery of liposomes, which are known to be sensitive to fluctuations in temperature. Despite this, eggPC liposomes, with a mean size of $281 \mathrm{~nm}$ or smaller, were reported to be stable to nebulisation with a DeVilbiss ultrasonic device (Barber and Shek, 1989). However, DPPC liposomes with a mean diameter of $499 \mathrm{~nm}$ significantly increased in size within the nebuliser reservoir during operation, suggesting the fusion of vesicles. Such behaviour would lead to a loss of entrapped hydrophilic materials. The aim of the experiments in this chapter was to advance the knowledge regarding the stability of liposomes to ultrasonic nebulisation. The effects of ultrasonic nebulisation on the size of liposomes delivered from, and those remaining within an ultrasonic nebuliser, were therefore investigated. This was then compared with the results obtained from the studies of jet nebulisers. 


\subsection{Materials}

Reagents

Cholesterol, Anala-R (99-99.4\%) - $\quad$ BDH, UK

Deionised water $\quad$ - WR50 RO/ Deioniser, Whatman UK

Lecithin from eggs (90\%), purified - Sigma Chemicals, USA

as detailed in section 2.3.1

\section{Nebuliser}

Medix Electronic nebuliser $\quad$ - $\quad$ Medic-Aid Ltd, UK

\subsection{Methods}

\subsubsection{Preparation of liposomes}

EggPC and eggPC/chol (1:1) liposomes were prepared by the thin-film method (as detailed in section 2.3.2), with lipid concentrations of 2.5, 10, 20 and $40 \mathrm{mg}$ lipid component per $\mathrm{ml}$ of aqueous phase. The mean liposome size was reduced to approximately $5 \mu \mathrm{m}$ by repeated extrusion through polycarbonate membrane filters (see section 2.3.4). The size distribution of liposomes in this, and subsequent stages of the study, was determined by laser diffraction analysis, using a $63 \mathrm{~mm}$ lens, as detailed in section 2.3.3 (Malvern 2600c, Malvern Instruments, UK).

\subsubsection{Characterization of liposomal aerosols produced by the ultrasonic nebuliser} Aerosols were produced from eggPC, or eggPC/chol (1:1) liposomes (lipid concentration $10 \mathrm{mg} / \mathrm{ml}$ ), using the Medix Electronic ultrasonic nebuliser, operated at its highest power setting, with a $5 \mathrm{ml}$ fill volume. The emitted aerosol droplet size was measured with a laser diffraction particle size analyser (63 mm lens; Malvern 2600c, Malvern instruments, UK), as detailed in section 3.3.2. The liposomes were nebulised, and the aerosol sized for a total of $10 \mathrm{~min}$. At 0,5 and $10 \mathrm{~min}, 100 \mu \mathrm{l}$ samples were removed from the reservoir, and the liposome VMD, and span values, determined by laser diffraction as detailed in section 4.3.4.

\subsubsection{Characterization of liposomes delivered in aerosols produced by the ultrasonic} nebuliser

The aerosol delivered from the Medix Electronic nebuliser was collected by directing it into 
the $\mathrm{TI}$, as detailed in the study of jet nebulisers (section 4.3.4). Aerosols were generated from $5 \mathrm{ml}$ of eggPC, or eggPC/chol (1:1) liposomes, for $5 \mathrm{~min}(\mathrm{t}=0$ to $5 \mathrm{~min})$, and the aerosol drawn through the TI at $60 \mathrm{l} / \mathrm{min}$, by means of a vacuum pump. Alternatively, the nebuliser was run for $5 \mathrm{~min}$ (without aerosol being directed into the TI), before the aerosol was directed into the TI for a further $5 \mathrm{~min}$ period (i.e. $\mathrm{t}=5$ to $10 \mathrm{~min}$ ). The size distribution of the liposomes deposited in each stage of the TI during nebulisation, from $t$ $=0$ to $5 \mathrm{~min}$, and $\mathrm{t}=5$ to $10 \mathrm{~min}$, was determined by laser diffraction analysis (as detailed in section 2.3.3).

\subsection{Results and discussion}

The mean size of the aerosols produced over 10 minutes from all liposome preparations was calculated to be between 4.3 and $5.1 \mu \mathrm{m}$ (fig. 6.2 and 6.3). The liposomal aerosols generated from the ultrasonic nebuliser were therefore comprised of larger droplets than those produced by each of the jet nebulisers studied in chapter 3 . Indeed, the mean aerosol sizes were close to the limit accepted as being suitable for efficient pulmonary deposition (Nebuliser Project Group, 1997). This larger size may in part be due to coagulation of droplets, resulting from the high concentration of the ultrasonically nebulised aerosol (Brain and Valberg, 1979). Liposomes delivered by the present ultrasonic device would therefore tend towards deposition higher in the respiratory tract than those delivered by jet nebulisation. This makes the Medix Electronic nebuliser less suitable for the targeting of liposomes to the non-ciliated alveolar region of the lung, which demands droplet sizes of approximately $2 \mu \mathrm{m}$ (Stahlhofen, 1980). Therefore, based on aerosol droplet size predictions alone, the use of such an ultrasonic nebuliser may be contraindicated for liposome formulations designed to produce a sustained release of drugs deposited within the lung.

For all lipid concentrations of the eggPC liposome formulations, and each of the eggPC/chol (1:1) liposomes, except the $40 \mathrm{mg} / \mathrm{ml}$ lipid concentration, the droplet size increased significantly during nebulisation $(p<0.05)$ (fig. 6.2a and 6.2b respectively). This contrasted with a study of dilute suspensions of monodisperse latex spheres (mean sizes ranging from 0.605 to $11.90 \mu \mathrm{m})$. The latex spheres, which were of comparable concentrations, exhibited no time-dependent behaviour during nebulisation with the same Medix Electronic ultrasonic nebuliser (McCallion et al., 1996). 

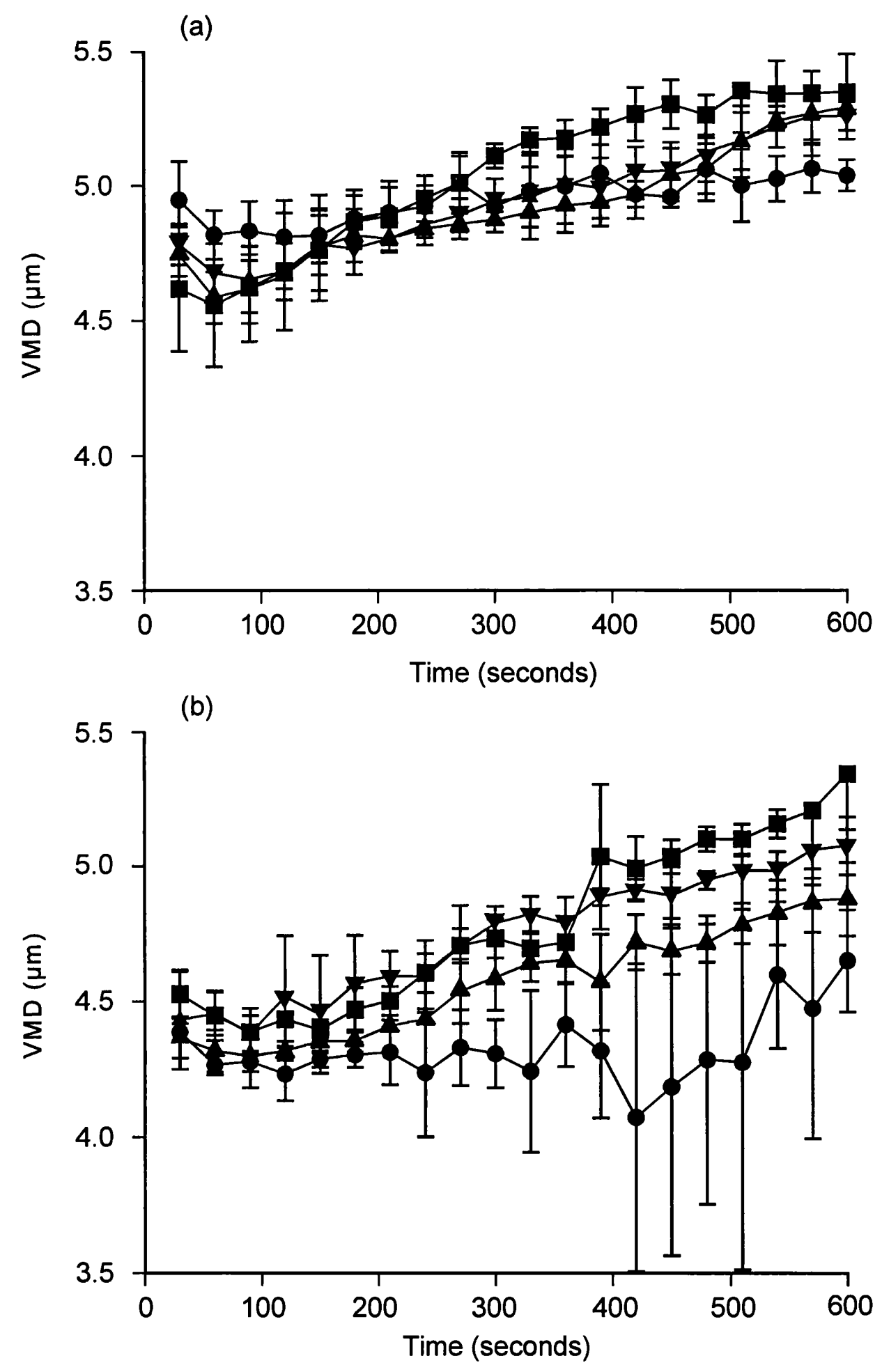

Figure 6.2 The mean droplet size (VMD) of liposome aerosols produced from the Medix Electronic nebuliser, during the nebulisation of (a) eggPC and (b) eggPC/chol (1:1) liposomes of varying lipid concentration. (ロ) $2.5 \mathrm{mg} / \mathrm{ml}(\boldsymbol{\nabla}) 10 \mathrm{mg} / \mathrm{ml}(\Delta) 20 \mathrm{mg} / \mathrm{ml}(\bullet) 40 \mathrm{mg} / \mathrm{ml}$. [ $\pm \mathrm{sd} ; \mathrm{n}=4$ ] 

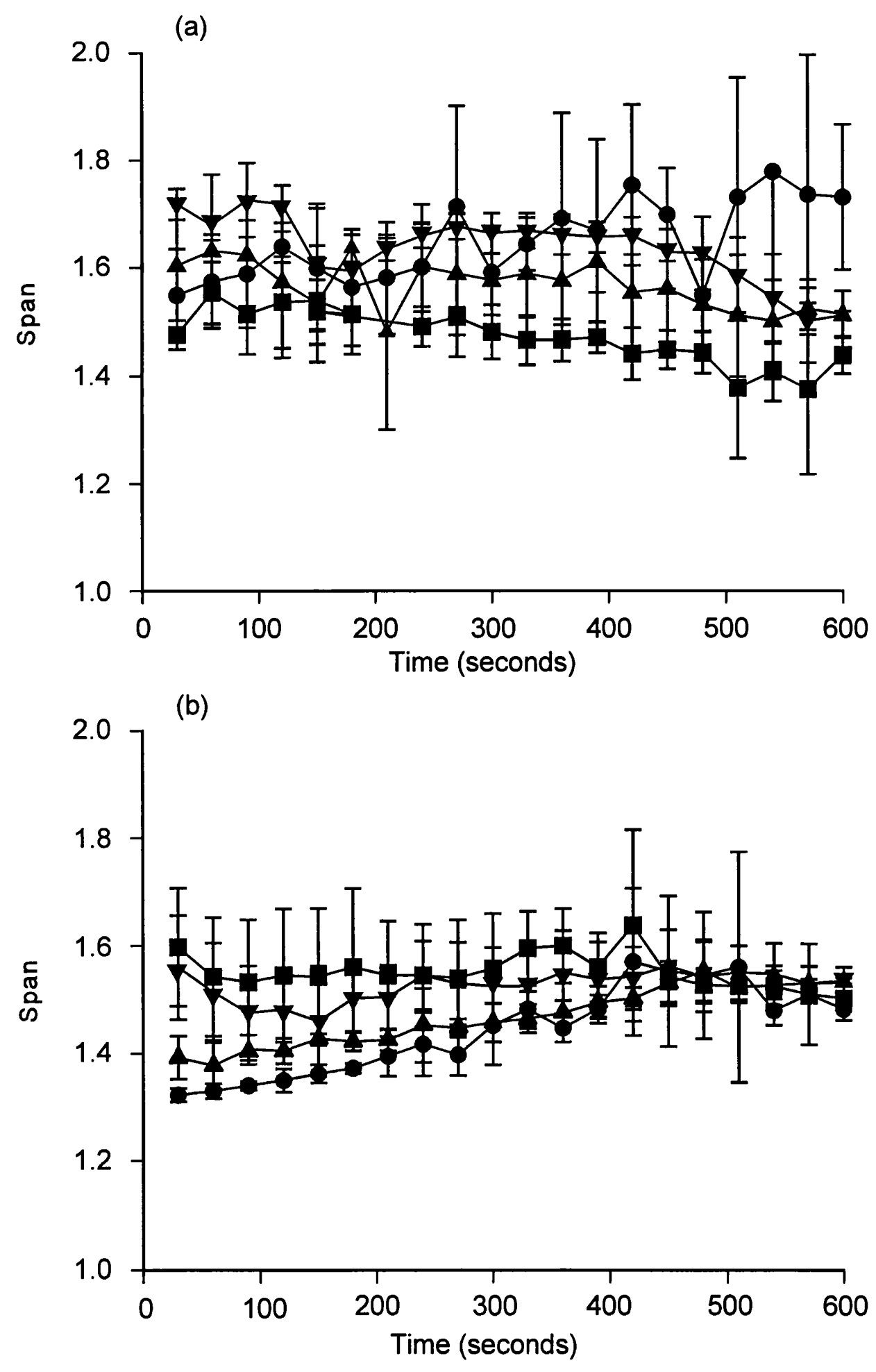

Figure 6.3 The polydispersity of liposome aerosols produced from the Medix Electronic nebuliser, during the nebulisation of (a) eggPC and (b) eggPC/chol (1:1) liposomes of varying lipid concentration. (অ) $2.5 \mathrm{mg} / \mathrm{ml} \mathrm{( \nabla )} 10 \mathrm{mg} / \mathrm{ml}(\Delta) 20 \mathrm{mg} / \mathrm{ml}(\bullet) 40 \mathrm{mg} / \mathrm{ml}$. [ \pm sd; $n=4]$ 
The reason for the time-dependent increase in aerosol size is unclear, but mirrors a trend evident in the jet nebulisers (chapter 3 ). In the case of the jet nebulisers this was attributed, in part, to a rise in the viscosity of the residual fluid as the liposomes become concentrated due to evaporative losses. However, ultrasonic nebulisation is not normally associated with significant concentration effects, and indeed, the increase in fluid temperature that occurs during ultrasonic nebulisation is likely to cause a fall in viscosity. It is evident from the literature that different ultrasonic nebuliser and medication systems yield different timedependent aerosol behaviour. Newman et al. (1987) noted a fall in the droplet size from the DeVilbiss Pulmosonic nebuliser, during a study of hypertonic saline. Similarly, Taylor and Hoare (1993) noted a marked decrease in the size of pentamidine aerosols produced from the Medix Electronic nebuliser with time. This behaviour was attributed to the rise in the residual fluid temperature during operation. However, studies with the Mist- $\mathrm{O}_{2}-\mathrm{Gen}$ ultrasonic nebuliser, with saline solution, failed to produce any demonstrable change in aerosol size during nebulisation (Phipps and Gonda, 1990).

The presently observed time-dependent response of the aerosol, as for jet nebulisation, may be attributable to the physical presence of the dispersed liposomes. It is likely that a significant number of the liposomes within the nebuliser chamber failed to become aerosolised, as they had a mean size that was approximately equal to the mean size of the aerosol. Ultrasonic nebulisers are recognised as being poor devices for the delivery of suspensions (O'Callaghan and Barry, 1997). Therefore, concentration of the liposomes may have occurred, as a consequence of exclusion from droplets. Such a retention of large liposomes was previously recorded for jet nebulisers (chapter 3). The viscosity of the nebuliser fluid, and thus the droplet size, may have risen in response to this increase in concentration. A previous study demonstrated that the aerosol size produced by ultrasonic nebulisers is proportional to the fluid viscosity (McCallion et al., 1995). In addition, Dahlbäck (1994), proposed that large particles have difficulty rising up the crests of capillary waves at the fluid surface, resulting in a poor output. This theory is further supported by the fact that the eggPC/chol $(1: 1)$ liposomes produced a larger rise in the emitted droplet size with time, than the equivalent eggPC liposomes. EggPC/chol (1:1) liposomes have stable bilayers, and are thus more resistant to degradation. As a result, they are likely to have been excluded from a greater proportion of the aerosolised droplets, with the result that the concentration effect during nebulisation was more pronounced. The 
consequent rise in viscosity would therefore be greater, and hence a larger rise in the aerosol size was recorded. Indeed, the eggPC/chol (1:1) liposomes produced highly variable mean droplet sizes (fig. 6.2b), and spans of droplet size (fig. 6.3b), particularly at elevated lipid concentrations. The viscosity of a fluid influences the threshold amplitude of oscillation $\left(\mathrm{A}_{\mathrm{t}}\right)$ required to generate capillary waves at the fluid surface thus (Niven et al., 1995):

$$
A_{t}=4 \frac{v}{F \lambda_{k}}
$$

Where:

[equation 6.1]

$v=$ kinematic viscosity $(\eta / \rho), F=$ frequency, $\lambda_{k}$ capillary wavelength

Therefore, a rise in the viscosity of the nebulised fluid, as the liposomes became more concentrated, would have led to aerosol production becoming more inefficient. Hence, the size of droplets that break away from capillary waves at the fluid surface will be limited by the size of liposomes that remain within the fluid (and thus the concentration). This residual size is likely to be larger for the more stable eggPC/chol (1:1) liposomes. Whether, such a rise in the liposome concentration is reflected in the size of the secondary aerosol will be determined by the efficiency of the nebuliser baffles. In support of the present finding was a study demonstrating that a rise in fluid viscosity significantly reduced the output of ultrasonic nebulisers (Niven et al., 1995). In fact, attempts to nebulise concentrations of 80 $\mathrm{mg} / \mathrm{ml}$ were abandoned in the present study, due to the considerably variable and sporadic aerosol output they yielded. This may be due to the viscosity effects of the liposomes influencing aerosol generation through equation 6.1 , since the viscosity of eggPC and eggPC/chol (1:1) liposomes increases linearly with concentration (chapter 2), and aerosol output is inversely proportional to viscosity (McCallion et al., 1995). A previous study of the Medix Electronic ultrasonic nebuliser also determined that high viscosity liquids failed to become nebulised efficiently (McCallion et al., 1995). It is difficult to speculate on the factors that cause the observed rise in VMD with time, whilst the precise mechanism of droplet formation within ultrasonic nebulisers remains to be conclusively proven.

The results failed to demonstrate an increase in droplet size as the lipid concentration was raised (fig. 6.2a and 6.2b), and the size of the liposomal aerosols generated from the eggPC 
liposome formulations was independent of the lipid concentration. Furthermore, for eggPC/chol $(1: 1)$ liposomes, there was a significant decrease $(p<0.05)$ in aerosol size as the concentration was increased to $80 \mathrm{mg} / \mathrm{ml}$ (figure 6.4). A failure to attribute the increase in aerosol size with time, to the residual liposome concentration, may instead indicate that it is due to changes in the nature of the liposomes themselves.

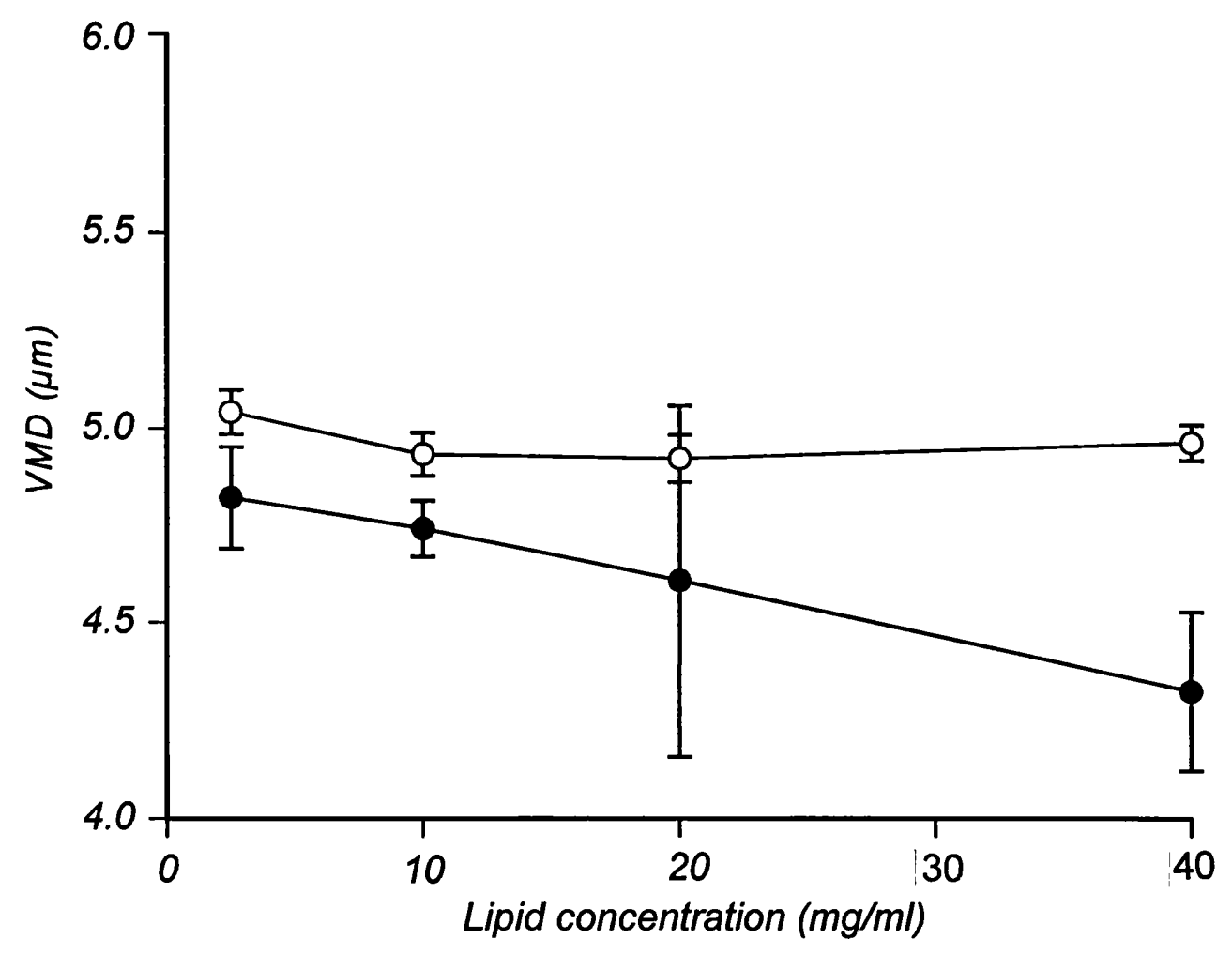

Figure 6.4 The influence of lipid concentration on the volume median diameter (VMD) of droplets formed during the nebulisation of eggPC (0) and eggPC/chol $(1: 1)(\bullet)$ liposomes with the Medix Electronic ultrasonic nebuliser. [ $\pm \mathrm{sd}: \mathrm{n}=4]$

These results contrast with those found when liposomes of the same size and lipid composition where nebulised with the Cirrus, Pari-LC and Sidestream jet nebulisers (chapter 3). These jet nebuliser devices exhibited an increase in droplet size as the concentration was increased. The mechanism of droplet formation, and the structure of the component parts differs significantly between jet and ultrasonically driven nebulisers. It is therefore unsurprising that the relationship between liposome concentration, and aerosol size differs between each. For instance, aerosol droplets are emitted into a high humidity 
environment in the ultrasonic device, reducing the tendency for droplet evaporation. By contrast, gas flow through the jet nebuliser causes significant droplet evaporation. An inhibition of droplet evaporation was suggested as a possible mechanism responsible for the observed rise in droplet size produced from the jet nebuliser with increasing lipid concentration. The results of the ultrasonic nebuliser study do not discount this. Significant evaporation of droplets is unlikely to occur in ultrasonically generated aerosols, and hence concentration effects will not be mediated through evaporative effects. Consequently, the linear relationship between lipid concentration and droplet size recorded in jet nebulisers is not observed in the ultrasonic nebuliser.

The present results suggest that the liposomal bilayer composition influences the aerosol response to changing lipid concentration in the ultrasonic nebuliser. This contrasts with the results obtained from the jet nebulisers, which demonstrated no difference in the droplet size response to bilayer composition (chapter 3). Large eggPC/chol (1:1) liposomes have previously been shown to be resistant to disruption during jet nebulisation, and accumulate significantly in the fluid (chapter 4). Large eggPC/chol (1:1) liposomes in the region of capillary wave formation are also likely to be excluded from the droplets forming the emitted aerosol, as they resist deformation and fracture. As a result only smaller droplets are released from the higher concentration liposome formulations, as a greater proportion of the residual nebuliser fluid contains liposomes larger than the mean emitted droplet diameter. The fluid viscosity also rises, making aerosol production inefficient. The eggPC liposomes are relatively fluid, and hence aerosolise more readily. Therefore, little impact is made on the aerosol production as the lipid concentration is increased.

Changes in the mean size of the residual liposomes during nebulisation indicated that there was a significant instability to ultrasonic nebulisation. The results support the hypothesis that the different nebuliser response to increases in the concentration of the different liposome formulations, was attributable to differences in the ability of the liposomes to become aerosolised. EggPC liposomes underwent a marked reduction in size during nebulisation (table 6.1). However, the liposomes incorporating cholesterol underwent significantly less size reduction $(\mathrm{p}<0.05)$. The eggPC liposome bilayers were in the liquidcrystalline state during the experiment, were therefore inherently unstable, and hence rapidly became reduced in size. 
Table 6.1 Mean size and span of liposomes remaining in the nebuliser during nebulisation in a Medix Electronic nebuliser.

\begin{tabular}{|c|c|c|c|c|}
\hline \multicolumn{2}{|c|}{$[ \pm \mathrm{sd} ; \mathrm{n}=3]$} \\
\cline { 2 - 5 } & \multicolumn{2}{|c|}{ eggPC } & \multicolumn{2}{c|}{ eggPC/chol (1:1) } \\
\cline { 2 - 5 } & Size $(\boldsymbol{\mu m})$ & Span & Size $(\boldsymbol{\mu m})$ & Span \\
\hline $\mathbf{t}=\mathbf{0 ~ s}$ & $5.17(0.02)$ & $1.81(0.02)$ & $4.71(0.10)$ & $1.53(0.18)$ \\
\hline $\mathbf{t}=\mathbf{3 0 0} \mathbf{~ s}$ & $1.20(0.04)$ & $2.88(1.62)$ & $3.75(0.06)$ & $1.37(0.02)$ \\
\hline $\mathbf{t}=\mathbf{6 0 0} \mathbf{~ s}$ & $0.97(0.05)$ & $3.74(5.16)$ & $4.11(0.13)$ & $1.75(0.08)$ \\
\hline
\end{tabular}

EggPC liposomes have been shown to be particularly susceptible to damage during jet nebulisation (chapter 4). The present results indicate that, although ultrasonic and jet nebulisers operate on very different principles, and produce aerosol droplets of different sizes (approximately $5 \mu \mathrm{m}$ for the ultrasonic nebuliser, and 2 to $3.5 \mu \mathrm{m}$ for the previous study of jet nebulisers), they each produce similar effects on eggPC liposomes. The ultrasonically nebulised eggPC liposomes apparently become disrupted in a similar manner to that experienced during ultrasonic probe sonication (chapter 2)(fig. 6.5). During ultrasonic nebulisation, droplets are formed through capillary wave generation at the surface of the liquid (Lang, 1962), through hydraulic shocks produced by imploding cavitation bubbles waves near the liquid surface (Gerhenzon and Eknadiosyants, 1964), and/or by a combination of these processes (Boguslavskii and Eknadiosyants, 1969). Each of these processes, in conjunction with the continued recycling of liposomes within the nebuliser, would physically disrupt the relatively fluid eggPC vesicles.

The present results contrast with those presented by Barber and Shek (1989), which showed that small eggPC and DPPC liposomes, sized in the reservoir of an ultrasonic device, were unchanged during nebulisation, but that larger DPPC liposomes were increased in size. However, the span of the residual liposomes became increased during nebulisation in the present study (table 6.1). This may indeed indicate aggregation and fusion of the liposomes, which are recycled within a heated environment. Supporting this, the span values of the eggPC liposomes exhibit a particularly wide variability once $300 \mathrm{~s}$ of nebulisation was reached. However, this may have been because at that time most of the liposomes were about $1 \mu \mathrm{m}$ in size, which represents the lower limit of sensitivity of the Malvern sizer. 

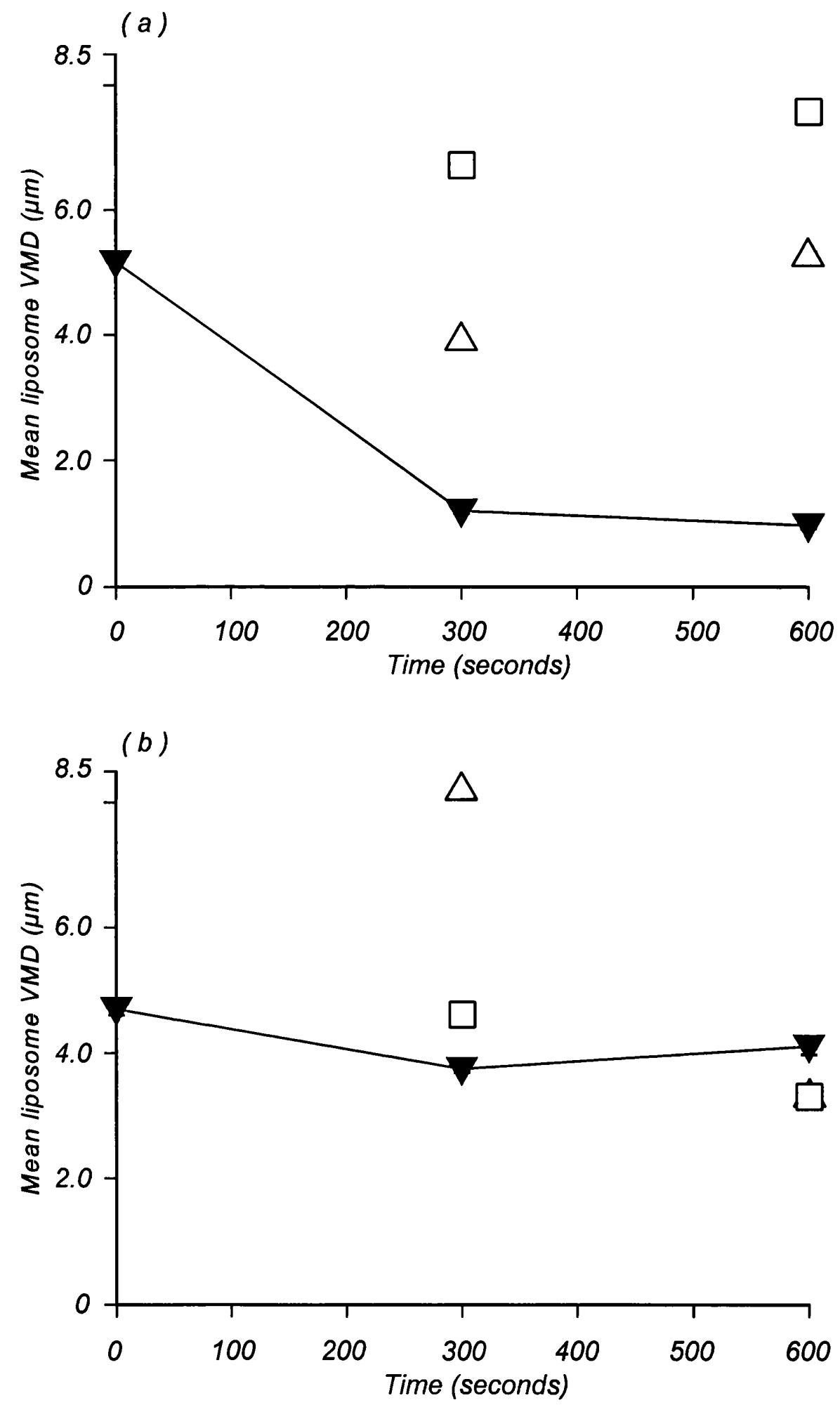

Figure 6.5 The mean size of residual liposomes $(\nabla)$ and liposomes deposited in the upper stage ( $\square$ ) and lower stage $(\Delta)$ of the twin impinger following nebulisation with the Medix Electronic ultrasonic nebuliser. (a) eggPC liposomes, (b) eggPC/chol (1:1) liposomes. [ \pm sd; $n=4$ ] 
Compared to eggPC liposomes, those composed of eggPC/chol (1:1) showed a less marked change in size during nebulisation, although a small shift to a reduced mean size was still evident (table 6.1 and fig. 6.5). Again, this is consistent with the effects of jet nebulisation (chapter 4). Inclusion of equimolar cholesterol into the liposome bilayers modulates the fluidity of the phospholipid bilayer, imparting to it a rigidity that restricts the movement of the phospholipid hydrocarbon chains above their transition temperature (Ladbroke et al., 1968). As a consequence, eggPC/chol (1:1) liposomes were more resistant than eggPC liposomes to the disruptive forces during ultrasonic nebulisation. This is reflected by a consistent residual liposome size, and a relatively unchanged span of the eggPC/chol (1:1) liposomes during ultrasonic nebulisation (table 6.1).

The degrading of materials contained within the reservoir of ultrasonic nebulisers during operation is a recognised phenomenon. Niven et al. (1995) speculated that the damage done to sensitive materials would be greater during ultrasonic nebulisation, than during jet nebulisation. Not only is a significant amount of energy dissipated as heat within the ultrasonic nebuliser, but also the energy for droplet formation, which produces enormous g-forces, is directed through the entire volume of the nebulised fluid, and indeed, is sufficient to cause disruption of covalent bonds (Boucher and Kreuter, 1968). This contrasts with jet nebulisers, which produce a fall in the fluid temperature during operation, and present only a small fraction of the fluid volume to the shearing forces at any one time. The present results support this, in so much as the eggPC liposomes were reduced in size to a greater extent during ultrasonic nebulisation, than during jet nebulisation (chapter 4). A similar finding prompted Wigley et al. (1971), to deliver insulin to rabbits using a jet nebuliser, having determined that ultrasonic nebulisation led to a loss of activity. Furthermore, Sato et al. (1992) determined that alpha-interferon was degraded at a low ultrasonic frequency of $80 \mathrm{KHz}$. This was attributed to cavitation within the fluid. Of more relevance to liposomes, were studies performed by Notter et al. (1982), which demonstrated the loss of surface activity in DPPC dispersions, following ultrasonic nebulisation. Köhler and Matthys (1984), further reported the loss of lung surfactant activity during ultrasonic nebulisation, a finding that was attributed to degradation of the phospholipids by the high energy ultrasonic waves. In fact, a number of mechanisms have been proposed for the loss of protein integrity, experienced during ultrasonic nebulisation, each of which may equally apply to liposomes (Niven et al., 1996). Firstly thermal inactivation occurs, due to the 
temperature of the fluid becoming elevated above the $\mathrm{T}_{\mathrm{m}}$ of the protein (eg. $\mathrm{LDH}, 56^{\circ} \mathrm{C}$ ) during nebulisation (Niven et al., 1995). Indeed, the temperature within the droplet fountain at the fluid surface may far exceed that in the body of the residual fluid, where it is most common to monitor temperature changes during nebulisation. A "hot-spot" is thus present within the nebuliser, through which droplets are continually recycled. This exposes all of the retained protein macromolecules to melting transitions, and rapidly leads to denaturation. Further to this, Cipolla et al. (1994) determined that aggregation of recombinant human deoxyribonuclease only occurred in ultrasonic type nebulisers, and was likely to be due to the elevated temperature of the nebuliser fluid exceeding the $T_{m}$ of the protein. Liposomes experience melting-transitions at a similar temperature range to $\mathrm{LDH}$, and therefore may undergo similar temperature-dependent transformations. Such degradation may result in the loss of liposomal phospholipid bilayer integrity, and hence the observed reduction in the liposome size. Such instability effects, would be in addition to the direct disrupting action of sonic waves on the liposome bilayers. Thus, despite the similarity between the instability effects of jet and ultrasonic nebulisation, the mechanism causing instability is likely to be very different in each. Supporting this, Niven et al. (1995) determined that the degrading of LDH by each type of nebuliser followed very different kinetics. Whilst loss of activity in the jet nebuliser followed first order kinetics, ultrasonic degradation was non-linear, indicating the presence of more than one process. In addition to thermal inactivation, the loss of LDH activity during ultrasonic nebulisation was attributed to protein interaction within the droplets, which led to the production of insoluble aggregates (Niven et al., 1994). This may explain the finding that the mean vesicle size of the eggPC/chol (1:1) liposomes increased significantly during nebulisation from $300 \mathrm{~s}$ to $600 \mathrm{~s}$.

In addition to these destabilising processes may be the proposed degradation of liposomes through interaction of the phospholipid bilayers with hydrophobic surfaces (see chapter 4), which may equally apply to both jet and ultrasonic nebulisers. The molecules at the surface of protein solutions have been shown to aggregate at hydrophobic contact areas (Henson et al., 1970), causing them to precipitate and form a thixotropic gel. Such instability reactions may lead to bilayer disruption in liposomes, which depend on an aqueous environment for stability. 
The TI allowed the liposomes aerosolised from the ultrasonic nebuliser to be investigated, and thus compared with the results produced during jet nebulisation (chapter 4). As previously described, the cut-off diameter for the lower stage of the TI is $6.4 \mu \mathrm{m}$. The median size of aerosols produced by the Medix Electronic nebuliser in this study varied between 4.1 and $5.5 \mu \mathrm{m}$ (as determined by laser diffraction). By definition $50 \%$ of the aerosol volume is greater than this size, and the widely distributed nature of the aerosol clouds (mean span values were 1.57 and 1.53 for eggPC and eggPC/chol (1:1) aerosols respectively) meant that a proportion of the droplets were greater than $6.4 \mu \mathrm{m}$ in size.

\begin{tabular}{|c|c|c|c|c|c|}
\hline \multicolumn{6}{|c|}{$\begin{array}{l}\text { Table } 6.2 \text { The size of residual liposomes, and liposomes } \\
\text { deposited within each stage of the TI, following nebulisation to } 300 \mathrm{~s} \text { and } 600 \mathrm{~s} \text { with } \\
\text { the Medix Electronic ultrasonic nebuliser. }[ \pm \mathrm{sd} ; \mathrm{n}=4]\end{array}$} \\
\hline \multirow{2}{*}{ Nebuliser } & \multirow{2}{*}{$\begin{array}{l}\text { Liposomes } \\
\text { sampled } \\
\text { from: }\end{array}$} & \multicolumn{2}{|c|}{ eggPC } & \multicolumn{2}{|c|}{$\operatorname{eggPC/chol~}(1: 1)$} \\
\hline & & $300 \mathrm{~s}$ & $600 \mathrm{~s}$ & $300 \mathrm{~s}$ & $600 \mathrm{~s}$ \\
\hline \multirow{3}{*}{ Medix } & Residual & $1.20(0.04)$ & $0.97(0.05)$ & $3.75(0.06)$ & $4.11(0.13)$ \\
\hline & Upper $T I$ & $6.70(0.01)$ & $7.56(0.18)$ & $4.60(0.02)$ & $3.35(0.01)$ \\
\hline & Lower $T I$ & $3.91(0.03)$ & $5.26(0.07)$ & $8.20(0.04)$ & $3.36(0.01)$ \\
\hline
\end{tabular}

\begin{tabular}{|c|c|c|c|c|c|}
\hline \multicolumn{6}{|c|}{ nebulisers. $[ \pm$ sd; $n=3]$} \\
\hline \multirow{2}{*}{ Nebuliser } & \multirow{2}{*}{$\begin{array}{c}\text { Liposomes } \\
\text { sampled } \\
\text { from: }\end{array}$} & \multicolumn{2}{|c|}{$\operatorname{eggPC}$} & \multicolumn{2}{|c|}{ eggPC/chol (1:1) } \\
\hline & & $50 \%$ & $100 \%$ & $50 \%$ & $100 \%$ \\
\hline \multirow{3}{*}{ Pari-LC } & Residual & $3.74(0.08)$ & $3.33(0.13)$ & $4.58(0.04)$ & $4.22(0.09)$ \\
\hline & Upper $T I$ & $4.37(0.11)$ & $4.16(0.62)$ & $4.80(0.16)$ & $4.61(0.07)$ \\
\hline & Lower $T I$ & $3.25(0.15)$ & $3.16(0.41)$ & $3.15(0.03)$ & $3.01(0.08)$ \\
\hline \multirow{3}{*}{ Sidestream } & Residual & $3.97(0.27)$ & $3.67(0.03)$ & $4.62(0.09)$ & $4.00(0.09)$ \\
\hline & Upper TI & $4.21(0.13)$ & $3.97(0.69)$ & $4.20(0.42)$ & $4.53(0.23)$ \\
\hline & Lower $T I$ & $3.06(0.42)$ & $2.97(0.17)$ & $2.59(0.12)$ & $2.81(0.09)$ \\
\hline
\end{tabular}


During the first 5 minutes of ultrasonic nebulisation the mean size of eggPC liposomes, collected in the upper and lower stage of the impinger, was 6.70 and $3.91 \mu \mathrm{m}$ respectively (table 6.2), compared with their initial mean size of $5.14 \mu \mathrm{m}$ (fig. 6.5). As determined for the jet nebulisers (chapter 4), the fractionation of the aerosol within the TI was reflected in the size of liposomes deposited on the respective stages, with the smallest liposomes being delivered in the smaller droplets. The same fractionating effect was seen with the eggPC liposomes collected between 5 and $10 \mathrm{~min}$. However, the size of eggPC liposomes on each stage was increased in the latter half of nebulisation, with mean sizes of 7.56 and $5.26 \mu \mathrm{m}$ respectively (fig. 6.5; table 6.2). This contrasts with the overall decrease in size to $1.2 \mu \mathrm{m}$ that occurs in the liposomes remaining within the nebuliser chamber (table 6.2), but reflects the time dependent increase in aerosol droplet size that occurred during nebulisation (fig. 6.1). A precise explanation of these findings cannot be given, especially since laser diffraction sizing gives a size distribution based on volume, and hence large liposomes, or aggregates, will have a much greater influence on the distribution than smaller liposomes. However, the data suggest that larger liposomes are conveyed in the largest droplets of the aerosol output from a nebuliser, whilst smaller liposomes are restricted to the smaller droplets, in a similar manner to that observed for the jet nebulisers. Over 10 minutes the mean size of the aerosol increases, permitting delivery of the larger liposomes which remain in the nebuliser chamber. In addition, fusion of the relatively fluid eggPC bilayers during and after aerosolisation is likely, particularly as the elevated temperature of ultrasonically nebulised droplets makes adjacent phospholipid bilayers more susceptible to interactions.

The time-dependent increase in deposited liposome size follows a similar trend recorded for the Sidestream jet nebuliser (chapter 4). However, the size of eggPC liposomes depositing in each stage of the TI was very much larger than the size recorded for the jet nebulisers studied in chapter 4 (summarised in table 6.2 and 6.3). This may be due to the large differences between the droplet size between the jet nebulisers, and that of the ultrasonic nebuliser $($ Pari-LC $=2.15 \mu \mathrm{m}$, Sidestream $=1.99 \mu \mathrm{m}$, Medix Electronic $=5.00$ $\mu \mathrm{m}$, approximately). The larger droplets produced by the Medix allow a greater number of large liposomes to become aerosolised. In addition, the elevated temperature of fluid within the Medix may encourage the fusion of liposomes present as multiple inclusions within an aerosolised droplet. Conversely, the temperature of fluid within the jet nebulisers falls. 
In the first 5 minutes of nebulisation, the mean size of eggPC/chol (1:1) liposomes collected in the upper and lower stages of the impinger was 4.60 and $8.20 \mu \mathrm{m}$ respectively (Table 6.2), compared with a residual size of $4.71 \mu \mathrm{m}$. This result demonstrates an opposite trend in aerosol fractionation to that found for eggPC liposomes, as the lower stage apparently collected larger vesicles than the upper stage, despite collecting smaller droplets. This finding also contrasts with the results determined for the jet nebulisers. An explanation for this may be the formation of liposomal aggregates within the lower chamber. Between 5 and $10 \mathrm{~min}$ the mean size of eggPC/chol (1:1) liposomes collected in the upper and lower stage of the impinger was 3.35 and $3.36 \mu \mathrm{m}$ respectively (Table 6.2), which compared to the size of the residual liposomes of 3.75 and $4.11 \mu \mathrm{m}$. It is therefore apparent that, for the stable eggPC/chol (1:1) liposomes, changes in the residual liposome size were more critical in determining the deposited liposome size, rather than the changes occurring in the aerosol size. Indeed, the liposomes deposited within the TI were smaller than those delivered from the jet nebulisers (table 6.2), despite the increased droplet size produced by the ultrasonic device. This may be a reflection of the relative stability of the cholesterol containing liposomes to ultrasonic nebulisation. It is likely that the droplets deposited in the TI contained discrete multiple eggPC/chol (1:1) liposomes, whereas eggPC aerosols tended to contain large aggregates, and fused liposomes.

\section{Conclusions}

The Medix Electronic nebuliser produced liposomal aerosols with a mean size of approximately $5 \mu \mathrm{m}$, which is typical for the ultrasonic type nebuliser. This aerosol size was relatively insensitive to changes in the liposome formulation, as determined for jet nebulisers (chapter 3). Such a droplet size may be predicted to give efficient deposition within the lungs of patients (Nebuliser Project Group, 1997). However, the principle site of deposition is likely to be within the conducting airways, as a mean droplet size of below about $2 \mu \mathrm{m}$ is required for penetration of the alveolar region (Stahlhofen et al., 1980; Newman and Clarke, 1983). Indeed, nebulisers producing droplet sizes of about $5 \mu \mathrm{m}$ are usually intended for the delivery of bronchodilator drugs, which act within the bronchioles. In a study of four jet nebulisers, Ilowite et al. (1991) found that the Fisoneb nebuliser, a relabelled Medix Electronic device, gave a significantly greater incidence of extrapulmonary side-effects, compared with the Respirgard II. This was attributed solely to the larger aerosol size produced by the Fisoneb, which led to an increased deposition 
within extrapulmonary regions. The increased droplet size produced by the Medix Electronic may therefore be predicted to produce an increased incidence of extrapulmonary adverse effects, compared with the jet nebulisers previously investigated. Of course, such adverse effects may be minimised by the encapsulation of aerosolised drug within liposomes, and will also depend on the total output of the devices. However, the site of deposition produced by the ultrasonically generated aerosol may also limit the duration of drug leakage from deposited liposomes, as the droplets will tend to deposit in sites where mucociliary clearance operate most efficiently.

The size of aerosols produced during ultrasonic nebulisation of eggPC and eggPC/chol (1:1) liposomes increased in size during nebulisation in a similar fashion, but a greater degree, to that determined for jet nebulisers. The reasons for this remain unclear, but may be due to an increase in the fluid viscosity as the concentration of liposomes remaining within the nebuliser increased. The size of liposomes remaining in the nebuliser reservoir during ultrasonic nebulisation decreased for both formulations, but this was more marked for the eggPC liposomes. This result is similar to that determined for jet nebulisers, and reflects the instability of liquid-crystalline phase bilayers, typified by eggPC. Reduction of vesicle size is indicative of liposome break-up during nebulisation, and has previously been correlated with the loss of entrapped hydrophilic materials from liposomes in jet nebulisers (Taylor et al., 1990b; Niven et al., 1991a; chapter 4). Inclusion of cholesterol in liposomes above the bilayer $T_{m}$ is thus necessary to promote vesicle stability during ultrasonic nebulisation. However, the extra stability imparted to the liposomes by cholesterol may have a detrimental effect on the aerosol output. The results also indicate that the ultrasonic device has difficulty in aerosolising the more rigid cholesterol-containing liposomes. The emitted droplet size was highly variable from such formulations, particularly for higher lipid concentrations, and in the later stages of nebulisation. This is likely to be due to exclusion of larger liposomes from the emitted aerosol, causing a rise in the concentration, and thus the viscosity, of the residual fluid.

As determined for jet nebulisers there was a good correlation between the size of eggPC liposomes deposited in an impinger, and the size of aerosols collected on each stage. However, the depositing vesicle size was significantly greater for the ultrasonic nebuliser, due to the increased droplet sizes it produces. It may also have been a result of the 
aggregation, or fusion processes, occurring as the temperature within the ultrasonic nebuliser chamber became increased. Aerosolised eggPC/chol (1:1) liposomes, deposited in the impinger with a size that was independent of the size of aerosol droplets, and instead reflected that of the liposomes remaining within the nebuliser chamber.

This study indicates that large liposomes may be delivered from ultrasonic nebulisers and that, although liposome damage occurs regardless of the bilayer composition, cholesterol containing formulations seem most appropriate for the delivery of hydrophilic agents. The delivery of hydrophilic materials by ultrasonic nebulisation is not likely to be achieved using liquid crystalline phase liposomes such as eggPC, due to their relative instability. However, they may remain suitable for the delivery of hydrophobic materials, for which the maintenance of bilayer integrity is less crucial. Indeed, the more stable aerosol produced from eggPC liposomes may make them more appropriate to such an application. There has been recent interest in the formulation of the hydrophobic glucocorticoid, beclomethasone dipropionate, in liposomes in order to reduce the incidence of extrapulmonary adverse effects, associated with its inhalation from MDIs (Vidgren et al., 1995). The present study suggests that liposomal drugs, such as beclomethasone dipropionate, may be delivered to appropriate regions within the lung (ie. the conducting airways) using an ultrasonic nebuliser. However, the applicability of ultrasonic nebulisation may be limited, as the output of liposomal aerosol may be poor, due to liposome retention within the device. In particular, the device appears to have difficulty aerosolising more concentrated liposome formulations.

The results support the conclusions drawn from the nebulisation of proteins (Niven et al. 1995), in so much as there are different formulation requirements for the delivery of liposomes, depending on whether aerosolisation is achieved by ultrasonically or gas jet driven devices. It also appears that ultrasonic nebulisers may be relatively poor delivery devices for suspended particulates, due to particle retention. This is the case whether the particulates are conventional non-deformable suspensions (Niven et al., 1991b; O'Callaghan and Barry, 1997), or liposomes designed to be stable to aerosolisation. However, aerosol output may be improved by reducing the size of the liposomes, as was found to be the case in jet nebulised suspensions (Dahlbäck, 1994), and liposomes (chapter 4). 


\section{CONCLUSION}

There are many different nebulisers available to the clinician for the purpose of generating therapeutic aerosols, with new and updated models being continuously introduced. As a result, there is often no standard nebuliser for a particular therapy. The selection of a nebuliser is frequently made subjectively, with the result that a wide variety of nebulisers may be used for the same drug therapy regime (Kendrick et al., 1997). Jet nebulisers have been most commonly used for the nebulisation of novel aerosol formulations, such as liposomes and proteins, as they are aqueous based systems, and are widely used in clinical practice. However, each nebuliser model may have significantly differing designs, and this causes variability in the emitted aerosol output characteristics. This problem is compounded by the fact that the driving gas flow, which controls the aerosol size characteristics and nebulisation time, is rarely specified. This may be acceptable if the performance of the nebulised system is systematically assessed prior to use, however this is rarely the case. Instead, the choice of nebuliser is often made without reference to the precise droplet size, or the output that the device may generate, when used in conjunction with a particular formulation. Similarly, nebulised fluids are almost exclusively marketed without reference to a suitable nebuliser system.

Recently, guidelines for clinicians have been published regarding nebuliser use. This was an attempt to address the fact that "...their [nebulisers] widespread use has preceded much needed, more fundamental work in optimising drug delivery" (Nebuliser Project Group, 1997). The guidelines provide useful advice to clinicians regarding the principles, and aims of nebulised drug therapy. However, they provide scant information regarding the selection of appropriate nebulisers, and operating conditions for particular applications. Indeed, the guidelines largely serve to demonstrate the bewildering array of nebulisers that may be used for therapy, and the wide ranging operating conditions that may also be employed. For instance, a list of 41 commercially available jet nebulisers has been compiled (Kendrick et al., 1997). For $42 \%$ of these devices no details of the likely droplet size could be found, and for $64 \%$ there was no information regarding the nebulisation time. The problems of nebuliser variation are increasingly being compounded by the fact that designs are becoming more and more sophisticated, and thus diversifying. Nebuliser research now embraces designs including "open vents" (eg. Sidestream ${ }^{\circledR}$, Medic-Aid), "breath -assisted, 
open (single) vents" (eg Pari-LC ${ }^{\circledR}$, Pari-Werk), and "breath assisted, open (double) vents" (eg. Pari-LC Plus ${ }^{\circledR}$, Pari-Werk). At the same time, more conventional jet nebulisers remain widely used. This seems a remarkable situation, considering that the dose delivered between these nebulisers may vary by $100 \%$ or more (O'Callaghan and Barry, 1997).

Ambiguity towards the selection of nebuliser and conditions is a recognised problem in nebulised drug therapy. However, with the development of more complex inhalation systems, such as proteins, suspensions, and liposomes, problems arising from this may be more significant. Liposomes are highly complex systems, and small changes in their formulation characteristics may lead to significant variations in their clinical performance. Consequently, liposomal aerosol research has largely investigated the liposome component, during the carriage and release of agents in the lung. However, without optimisation of the delivery system (ie. the nebuliser), the performance of the delivered carrier may be irrelevant. Certainly, it complicates the interpretation of in vivo results. The present study has redressed this situation.

A representative sample of nebulisers, although operating under very similar principles, generate liposomal aerosols with widely varying characteristics. The most significant internebuliser variability is seen in the aerosol output rate. This has important implications for patient compliance, and nursing time, and is due to variations in the design of jets, baffles and vents within the device. For instance, the open vent design of the Sidestream nebuliser successfully achieves a reduction in nebulisation time. This may, to a significant degree, be due to heightened evaporative losses. The liposomal aerosol output rate is also controlled by the driving gas flow rate, with increased flow rates providing a more rapid aerosol entrainment, and thus reduced nebulisation times. It is therefore important that a particular nebuliser system is characterised prior to use with a particular liposomal formulation, in order to determine the nebulisation time. Operation of different nebulisers for the same fixed time, would result in significant variations in liposome dose. The droplet size produced by different jet nebulisers may also be controlled by the driving gas flow rate. However, little control may be made of the aerosol emitted from ultrasonic devices, as they generally operate with a limited number (usually one or two) of power settings.

Despite significant differences in size, and orientation, of functional components, such as 
gas jets, fluid feed capillaries, and the presence or absence of vents, each of the nebulisers tested presently produces "respirable" liposomal aerosols (that is with a mean VMD less than about $5 \mu \mathrm{m}$ ). Therefore, in terms of droplet size, each device is suitable for depositing liposomal aerosols in the lung. However, only certain devices, in this study the Respirgard II and Sidestream jet nebulisers, generate liposomal aerosols with a mean droplet size less than $2 \mu \mathrm{m}$ at all flow rates. Such nebulisers would be more suitable for liposome deposition within the alveolar region of the lung, where sustained liposome residence, and therefore prolonged drug release, may be predicted. The Cirrus and Pari-LC nebulisers were capable of producing aerosols with a mean size of $2 \mu \mathrm{m}$, but only at elevated gas flow rates. This may be a significant disadvantage when nebulising liposomes, as the phospholipid bilayers exhibit reduced stability to increased gas flow rates.

The generation of small droplet sizes correlates with a reduced diameter of the gas jet within the jet nebuliser. A smaller diameter causes a higher velocity gas flow through the device. The shearing of the fluid, and also the momentum of the droplets, is therefore greater, resulting in the generation of an aerosol with a reduced mean droplet size. However, the baffles within the device also contribute to the control of the emitted droplet size, by trapping larger droplets before emission. There is also evidence to suggest that extra gas flow through the open vent of the Sidestream nebuliser promotes the evaporation of aerosol droplets, such that a smaller droplet size is emitted.

Such variations in the liposomal aerosol size between different devices may result in marked changes in the distribution, and fate of the liposomes within the lung, and therefore the formulation's efficacy. In addition, the choice of an appropriate nebuliser and gas flow rate may permit the targeting of specific regions of the respiratory tract. However, even if a particular nebuliser is characterised for use with a specific liposome formulation, there remains significant variability in the aerosol characteristics between jet nebulisers of the same model (that is intra-nebuliser variation). This may be a result of the inconsistent manufacture of the nebuliser reservoir, liquid-feed capillaries, jet orifices and other constituent structures. The most widely used jet nebulisers are recommended for disposal after three months of use, although certain "durable" devices (eg. Sidestream) may be used for up to 12 months (Nebuliser Project Group, 1997). However, to be economic jet nebulisers are mass produced from cheap plastics. Such plastics may prove difficult to 
machine accurately, and imperfections in the geometry, and dimensions of functional orifices have been identified. In addition, the plastics forming these components may become stressed, deform, and fracture during use. The structure, orientation, and integrity of the component parts of a nebuliser are all critical in determining the emitted aerosol character. As a consequence, the emitted droplet size is found to vary to an extent between nebulisers of the same model. These problems are compounded by the fact that the functional components of many nebulisers are formed upon assembly of the device prior to use, which may vary randomly. Although the intra-nebuliser variation in droplet size is less than the inter-nebuliser variation, it is still significant between certain devices. This may not be significant enough to yield significant differences in clinical response. However, nebuliser manufacturers should strive to make devices more reproducible, as any optimisation of aerosol output would benefit therapy. This may be particularly important for formulations which are expensive (eg. rhDNase), or drugs which have significant adverse effects (eg. steroids). It is also particularly relevant for sophisticated systems, such as liposomes, which produce a modulated drug release, that may be highly dependant on the liposome stability, and the site of deposition within the lung. For instance, the intranebuliser variation in droplet size inherent in the Pari-LC nebuliser, was correlated with fluctuations in the stability of liposomes to nebulisation. This is because the droplet size and liposome stability are each dependent on the shear energy of gas flow through the device. This in turn depends on such factors as the size, and orientation of the gas jet orifice. The Sidestream nebuliser is more reliant on evaporation of droplets for the achievement of a small aerosol size, and the relationship between the droplet size and liposome damage may be less distinct for such a device.

The need to evaluate the nebuliser component of liposomal aerosols, rather than solely the liposomal component, is highlighted by the fact that the droplet size produced by jet nebulisers is largely insensitive to changes in liposome size, or bilayer composition. Liposomes that have bilayers in either the gel, or liquid-crystalline state may therefore be jet nebulised without influencing the emitted aerosol size. Further to this, the mean liposome size may be increased to at least $5 \mu \mathrm{m}$ without influencing the droplet size. Therefore, liposomes may be designed to provide suitable drug release characteristics in the lung, without fear of influencing their deposition profile. However, this may not be the case with ultrasonic nebulisers, as large cholesterol-containing liposomes yielded a highly 
variable aerosol output, especially at elevated concentrations. This indicates that the ultrasonic device has difficulty in aerosolising "stable" liposomes with a relatively large mean size, and supports the current view that ultrasonic devices are largely unsuitable for generating suspension aerosols (O'Callaghan and Barry, 1997). This serves to illustrate the different principles of operation involved in jet and ultrasonic devices, and indicates that jet nebulisers may be more suitable for aerosolising such formulations.

Despite the relative insensitivity of liposomal aerosols to formulation changes, the liposome size and bilayer composition each prove critical in determining the liposome stability to nebulisation, and also the rate and extent of liposomal aerosol output. These factors may be as important in determining the therapeutic performance of the formulation as the emitted droplet size (Nebuliser Project Group, 1997). Liposomes with a mean diameter greater than the nebulised droplet size become excluded from a significant proportion of the smaller droplets constituting the primary aerosol (fig. 8.1). Aerosolised liposomes therefore become fractionated within the aerosol cloud as a function of their size. Consequently larger liposomes would be predicted to deposit higher in the respiratory tract, as they are included in larger droplets. The sustained release of entrapped hydrophilic materials provided by larger multilamellar liposomes, would therefore tend to occur in the ciliated regions of the lung. In such regions mucociliary clearance is most rapid, and may limit the duration of drug release. In addition, larger liposomes are restricted to larger droplets which have an elevated inertia, and therefore tend to impact on baffles, and other internal structures, before aerosol emission. A further consequence of the aerosolisation mechanism, is that liposomes with a mean size greater than the mean aerosol droplet size yield a reduced output. The liposomes become preferentially retained within the nebuliser, and therefore yield elevated residual masses. Larger liposomes are therefore associated with reduced liposomal aerosol doses. Additionally, the retention of larger liposomes within a jet nebuliser chamber means that such liposomes experience sustained recycling within the device. The bilayers are exposed to prolonged shearing by the gas jet, and possibly to other destabilising processes, such that bilayers become fractured, and the liposomes reduce in size. Larger liposomes are therefore associated with a significant loss of entrapped aqueous materials, particularly if the bilayers are in the more fluid liquid-crystalline phase state, under the conditions of aerosolisation. 


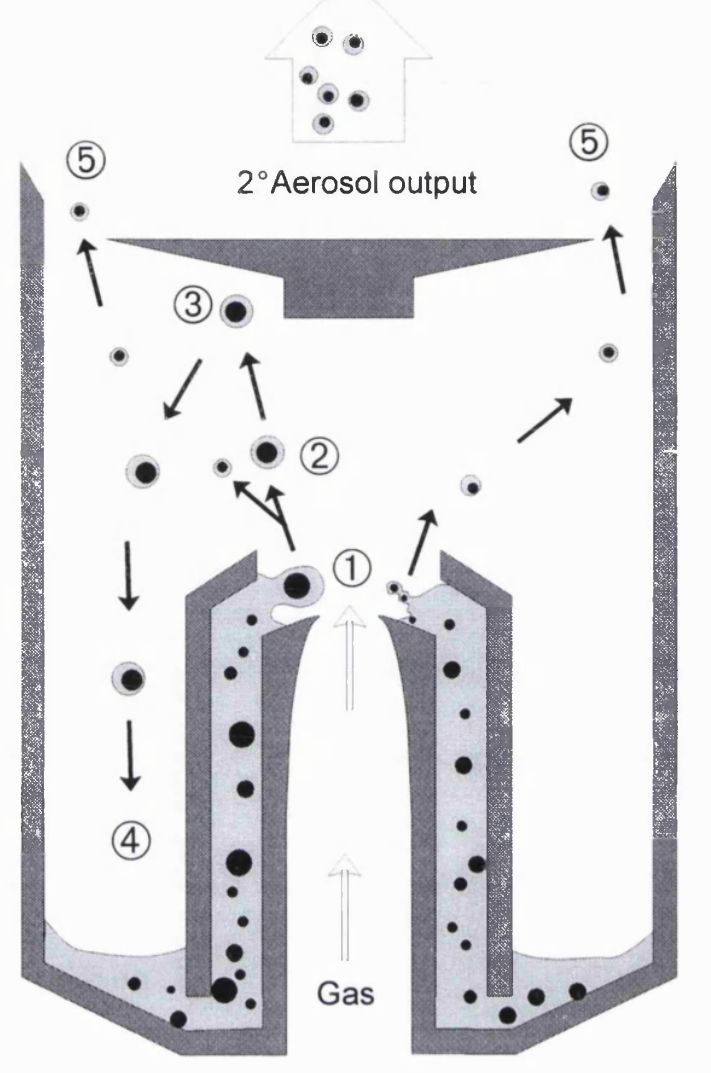

Figure 8.1 Proposed mechanism of liposome aerosolisation from a jet nebuliser. Shear energy provided by gas flow (1) provides energy for fluid atomisation. However, this disruptive energy also causes fracture of the bilayers of larger liposomes (2). Liposomes with a diameter larger than the mean secondary $\left(2^{\circ}\right)$ aerosol size $(\bullet)$ are restricted to larger droplets within the primary aerosol. Such droplets impact on baffle(s) (3), and are thus retained within the nebuliser (4). Continued recycling of the liposomes causes repeated exposure to destabilising forces, until the liposomes are small enough to be included within the secondary aerosol that leaves the device $(\bullet)(5)$. Size reduction of liposomes is facilitated by bilayers in the liquidcrystalline phase state (eg. eggPC). Conversely less fluid liposomes (eg. eggPC/chol) resist bilayer disruption.

The liposomal aerosol output is to an extent dependent on the droplet size generated by the device. Jet nebulisers that produce smaller droplet sizes, are generally associated with an increased retention of liposomes. The liposomes become excluded from a greater proportion of the smaller aerosol droplets emitted from nebulisers such as the Respirgard II, and as such, the aerosol output becomes reduced. However, other nebuliser design factors may complicate this relationship. For instance, the open vent in the Sidestream causes an increase in evaporative losses from the aerosolised droplets. Whilst this allows 
a small droplet size to be produced, it also results in a greater concentration of liposomes in the residual fluid during nebulisation. The nebuliser design is thus important in determining the stability, and thus the retention, of liposomally entrapped materials. The relationship between the operating conditions, the nebuliser design, and the damage done to liposomes is complex, and masks the predicted relationship between the emitted droplet size (and thus the shear energy), and the bilayer damage. For example, the Sidestream nebuliser, despite producing small droplets, causes relatively little damage to liposomes. This is due to the fact that the device achieves a small droplet size through additional mechanisms, such as solvent evaporation, rather than solely through elevated shear forces. However, an inevitable consequence of an increased liposome stability, is that a greater proportion of the liposomes fail to become reduced to a size that may be aerosolised. As a result, the Sidestream nebuliser retains a greater volume of liposomes during nebulisation. There is, therefore, a compromise to be made between obtaining an efficient liposome output, yet a favourable liposome stability.

Liposomal bilayer destabilisation also results from the ultrasound waves produced by ultrasonic nebulisers. As with jet nebulisers, a particularly significant reduction in the size of liquid crystalline phase state liposomes is observed during ultrasonic nebulisation. Cholesterol-containing bilayers prove more resistant to destabilising processes in both the ultrasonic and jet driven devices, enabling the liposomes to better retain their initial size, and also any entrapped materials. However, the resistance of the gel state liposomes to size reduction indicates that they will be retained within the nebuliser to a greater extent, and this may have a detrimental effect on the aerosol output.

It can be concluded that the energy required to disperse fluids into an aerosol will inevitably lead to damage of liposomes which have a mean size greater than a significant proportion of the aerosolised droplets. This is the case whether the energy is supplied by high velocity gas flow, or ultrasound. The obvious answer to the problems of liposome instability, and retention, during nebulisation, would be to use liposomes processed to a mean size less than that of the aerosol droplets. If the mean size of the liposomes is reduced to below that of the aerosol droplets then an increased liposome stability, and liposome output, is observed. The smaller liposomes become included within a greater number of the droplets that escape the device, without requiring extensive processing. The milling of dispersed drug particles 
to a size significantly less than the nebulised aerosol size, was previously shown to be necessary in order to maximise suspension aerosol output (Dahlbäck, 1994). It is thus clear that rigid liposomes, such as those composed of eggPC/chol (1:1), behave in a similar manner during nebulisation, to non-deformable suspensions. Whilst size reduction obviates problems such as bilayer instability, and liposome retention, it is not without its disadvantages. Clearly, the extra processing involved would increase the product cost. Furthermore, the multilamellarity of larger liposomes may provide a more prolonged drug release, and also a reduced clearance by biological factors (Fielding and Abra, 1992). For instance, alveolar macrophages achieve a more rapid, and efficient clearance of particles which are smaller than $3 \mu \mathrm{m}$ in size (Bowden, 1987). Prior to the present study, the only established requirement for reducing the size of liposomes for nebulisation, was in order to maximise the retention of entrapped aqueous phase materials during aerosolisation. However, the present finding that the liposome size is critical in determining output during nebulisation, is as relevant when using liposomes to solubilise hydrophobic drugs.

Despite being largely insensitive to changes in the liposome characteristics, the liposomal aerosol output, and droplet size, are significantly influenced by the lipid concentration of the formulation. The situation in ultrasonic nebulisers is less clear than in jet nebulisers, but it appears that the ultrasonic device is unable to efficiently aerosolise more concentrated liposome formulations. This may be attributed to the elevated viscosity of high concentration samples, which resists the disruption of the fluid into droplet-forming capillary waves. However, whilst the process of droplet formation in ultrasonic nebulisers remains undefined, it is difficult to speculate on the precise mechanism responsible for this. Ultrasonic nebulisers have previously been shown to be relatively inefficient devices for the delivery of conventional suspensions (Niven et al., 1991b; McCallion et al., 1996; O'Callaghan and Barry, 1997). With this in mind, jet nebulisers may prove more appropriate for the nebulisation of liposomes, particularly formulations with a large mean vesicle size, stable bilayers or with an elevated concentration. However, in jet nebulisers there is a direct correlation between the concentration of the liposomes, and the emitted droplet size, and rate of liposomal aerosol output. This is consistent with atomisation theories, and may be due to the resistance of higher viscosity fluids to the disruptive action of gas flow. Other contributory factors may include the absorption of disruptive energy by bilayers, a resistance of droplet evaporation through bulk phase interactions with liposomes, 
or aggregation of the liposomes during aerosolisation. The response of nebulisers to varying fluid properties, such as viscosity, has previously been shown to be a complex function of the nebuliser constituent parts (Davis, 1978). In particular, the filtering action of the nebuliser baffles may mask the concentration effects. This explains the variable response of different nebulisers to a changing liposome concentration. The Cirrus and Pari-LC nebulisers produce significantly increased droplet sizes when nebulising higher concentration liposome formulations. By contrast, the Sidestream nebuliser is able to minimise the effects of concentration. This illustrates the significant differences in the design of different nebulisers. In particular, it may reflect the impact that the open vent design of the Sidestream nebuliser has upon the mechanism of aerosol formation.

The results of the present study highlight the variable nature of aerosols produced from different nebulisers, which is attributable to wide variations in their design, and build. Medical nebulisers have been widely used in hospitals, and homes for many decades with little attempt having been made to standardise their use. This may in part be due to the fact that many inhaled drugs, such as salbutamol, have a very wide therapeutic range. Despite this, the clinical response, and the adverse effects, resulting from nebuliser therapy may be significantly influenced by the choice of nebuliser, and operating conditions for a particular formulation. Periodically, research is published which evaluates the clinical effectiveness of different nebulisers for use with a specific formulation (eg. pentamidine, Thomas et al., 1991a). Whilst this provides useful information, it is only applicable to the particular nebuliser and drug combination involved, as the formulation characteristics may significantly alter the aerosol quality. In addition, the commercial nebuliser market is constantly changing, and often by the time research has been published the nebulisers have been superseded. This is now of particular relevance, as nebulisers are becomingly increasingly sophisticated and diverse in nature, due to the desire to improve their output efficiency. The introduction of novel designs, such as breath-enhanced systems, and the fact that they are increasingly being used to deliver more potent, and sophisticated formulations, means that standardisation of nebulisers is now overdue. The efficacy of complex formulations, such as such as proteins, peptides and liposomes, may be particularly sensitive to the site of deposition within the lung. Furthermore, such products are too expensive to accept a poor delivery efficiency. In particular, liposomes and proteins are sensitive to destabilisation by nebulisation, which has now been shown to be controlled by 
the nebuliser device, and the operating conditions used.

The need to produce reproducible aerosols from nebuliser devices is arguably as important as ensuring a reproducible dissolution rate between solid oral dosage forms. Despite this, there remain no hard and fast rules concerning nebuliser use. Nebulised drugs continue to be granted product licences without the need to specify the precise nebuliser type or model, or the required operating conditions. The present study provides useful information regarding the mechanism of liposome aerosolisation with nebulisers. It demonstrates that more stable liposomes may be prepared by careful control of the bilayer composition, the vesicle size characteristics, and the nebuliser system. Such variables will also influence the nebulised liposome output. It highlights the variability of the performance of different nebuliser models when used to aerosolise liposomes, and indicates that developments in nebuliser technology may have important implications for the design, and the performance of liposomal aerosols.

The present research also explored likely future developments of liposomal aerosol products. The question of liposome instability on storage requires addressing, before nebulised liposomal products are marketed. Freeze drying may be a suitable approach for achieving a shelf-life that is acceptable to regulatory authorities. Freeze dried liposomes may be prepared relatively easily. The dehydrated product proves simple to redisperse, and generates an aerosol that is unchanged from that produced by the conventional liposomes, provided a cryoprotectant such as trehalose is used in the formulation. Without a cryoprotectant, extensive liposome aggregation and fusion occurs, causing a significant increase in the rehydrated liposome size. This is significant, in view of the fact that the nebulisation of larger liposomes is associated with bilayer breakdown, and a reduced liposomal aerosol output. Trehalose has also been shown to maximise the retention of entrapped hydrophilic agents during freeze-drying, and subsequent rehydration (Crowe et al., 1985).

Nebulisers were at one time perceived as being old-fashioned, and inefficient. However, nebuliser designs are becoming increasingly more sophisticated, and nebulised therapy has been significantly improved by such advances. Whilst MDI devices remain the mainstay of inhalation therapy, their reliance on ozone-depleting, and "greenhouse gas" propellants 
limits their projected use (Tansey, 1995). However, the alternative DPI devices are unsuitable for the elderly, young, and debilitated patients, and are less efficient than nebulisers in providing a respirable dose (Zainudin et al., 1990). In addition, both MDI and DPI devices are unable to deliver large dose volumes, or aqueous based formulations. Consequently, nebulisers are likely to be increasingly used in a clinical setting. The recent introduction of comprehensive guidelines regarding nebuliser therapy serves to illustrate the secure future of nebulised inhalation therapy (Nebuliser Project Group, 1997). It also highlights the lack of comprehensive data regarding nebuliser use. The development of novel inhaled formulations for delivery by nebuliser, such as liposomes and protein drugs, demands that the process of nebulisation in different devices is better understood. This $\mathrm{PhD}$ project illustrates the importance of standardising nebuliser operation, and highlights the many variables that influence the generation of liposomal aerosols.

\section{Future work}

Nebulisation is a complex, and relatively unexplored process. It is made even more complex when the aerosolised formulation is a two phase systems such as liposomes. The present $\mathrm{PhD}$ project investigated a number of factors which may influence the nebulisation of liposomes. These included the driving gas flow rate, nebuliser model, liposome size and liposome bilayer composition. However, there are additional variables, such as fill volume, uniformity of performance on repeated use, and temperature or humidity effects, which also require consideration. A complete investigation of all the factors involved in the generation of respirable liposomal aerosols was beyond the time-scale allowable for the project, however such work may clearly form the basis of future research.

In keeping with most scientific research, the present study raised as many questions as it provided answers. For instance, the Sidestream nebuliser appeared to handle liposomes in a significantly different manner to the other nebuliser models tested. This was interpreted as being in part due to the influence of the open vent incorporated in the device. The results suggested that the Sidestream was less reliant on the shear energy of gas flow for the attainment of a small droplet size. It was proposed that this made the liposomes more stable within the device during operation. This hypothesis clearly requires further testing. This may be accomplished by the operation of the device using humidified gas flow, thus revealing whether the evaporation of the droplets was indeed having demonstrable effects 
on the aerosol size, and liposome stability.

The present study used SCG as a marker for drug release during nebulisation. It would be useful to study a range of drugs with differing physicochemical properties, as the incorporated drug may itself influence the aerosol characteristics, and the liposome response to nebulisation. For instance, the incorporation of hydrophobic drugs into the liposome may influence the bilayer packing, and therefore the liposome stability during aerosolisation. If this is the case, the advantages of the inclusion of bilayer cholesterol may not be relevant in situations where hydrophobic agents were being entrapped. In addition, certain hydrophilic drugs may influence the viscosity and surface tension of the continuous phase, which may alter the emitted aerosol character.

A limited number of liposome bilayer compositions, principally eggPC in the presence or absence of cholesterol, were used in the present experiments. The conclusion was drawn that gel state, or cholesterol-containing liposomes, were more appropriate for the carriage of hydrophilic drugs to the lung. However, eggPC is unlikely to be used in liposomal products, due to its relative impurity. Many other bilayer compositions may therefore be more appropriate to the design of liposomes for delivery to the lung, and these will require similar investigation.

The present study chose to use large multilamellar liposomes in the aerosolised formulations. This was because their processing during nebulisation yielded useful information regarding the mechanism of aerosolisation. However, smaller liposomes than those used presently may be more appropriate for the delivery of drugs by liposomal aerosol, due to the increased stability and aerosol output which they may provide. Future studies should therefore investigate whether factors such as the choice of bilayer composition, nebuliser, or gas flow rate are as important to the nebulisation of small submicron liposomes, as they are to the larger liposomes studied presently.

Having shown that liposomes are damaged by nebulisation, a number of strategies for minimising this were proposed. Reducing the vesicle size and driving gas flow rate, selecting a particular nebuliser model, or including cholesterol in the bilayers may all be appropriate. A more novel approach may be to employ stabilising agents, designed to 
protect the liposomes during nebulisation. Perhaps this may be achieved by adjusting the viscosity of the continuous phase, such that the damaging shear forces, which are responsible for bilayer instability, are absorbed. However, such an approach may cause detrimental effects to the aerosol quality.

The results of liposome stability during nebulisation may be extrapolated to the likely stability of other sensitive materials, such as proteins, to nebulisation. It may be useful to investigate the validitity of such a comparison. For instance, the Sidestream nebuliser was found to damage liposomes to a lesser extent than the other nebulisers studied. This may make the device suitable for the delivery of other sensitive formulations, such as enzymes, for the therapy of cystic fibrosis. However, such a conclusion may only be made following studies with the relevant proteins.

The present project studied many of the variables which influence the generation of nebulised liposomal aerosols in vitro. However, conclusions regarding the suitability of different nebulisers for the delivery of liposomal aerosols to the lung, cannot be made without supporting in vivo data. In vitro data provides only a limited reflection of a nebulised aerosol's potential efficacy, as patient factors, such as breathing pattern, presence of disease, and age, will influence the aerosol performance. Whilst the present project highlighted areas where liposomal aerosol generation may be optimised, it is the clinical end point of therapy that is the primary concern of medicinal product development. True standardisation of nebulisers for the generation of liposomal aerosols therefore requires additional human studies. For instance, different jet nebulisers generate liposomal aerosols with significantly different droplet sizes. In vivo studies are required to determine whether such variability will significantly influence the deposition and clearance of nebulised liposomal drugs within the lung. Likewise, the droplet size effects, caused by an increase in the lipid concentration may, or may not, be significant in determining the in vivo performance of a liposomal aerosol.

The testing of liposomal aerosols in patients with relevant disease states is required. To date, only a very limited study in asthmatics has been performed (Waldrep et al., 1997). Much has been made of the potential of liposomes to achieve the sustained release of entrapped agents within the lung. However, the success of such a strategy depends on the 
efficient targeting of the lung, in particular the non-ciliated alveolar airways, where clearance of particulates is slow. The penetration of liposome-containing droplets to these regions may be restricted in conditions which are characterised by airway narrowing, excessive mucus secretion and/or limited tidal volumes. Diseases such as asthma, chronic obstructive airways disease and bronchitis may therefore limit the residence time of an inhaled liposome within the lung. The delivery of liposomal aerosols to diseased lung therefore requires further evaluation. In addition other patient variables, such as breathing pattern, age etc require investigation.

The need for in vivo data is particularly important, as the nebulisers may operate in a significantly different manner in the clinical situation to that revealed in vitro. The results of the $\mathrm{PhD}$ project indicate that certain features of the nebuliser design, such as the incorporation of an open vent in the Sidestream, play an especially important role in determining the liposomal aerosol character. Such features also determine the aerosol response to changes in the physicochemical nature of the liposomes. Nebulisers are becoming increasingly reliant on breath-enhancement systems. These systems improve aerosol output, but prove difficult to investigate accurately in vitro, as the inspiratory flow patterns of the patient are not modelled. This makes the acquirement of in vivo data all the more necessary. 


\section{APPENDIX}

Table 4.3 The significance of the difference in liposome stability to nebulisation with different jet nebulisers. [ $\mathrm{p}<0.05]$.

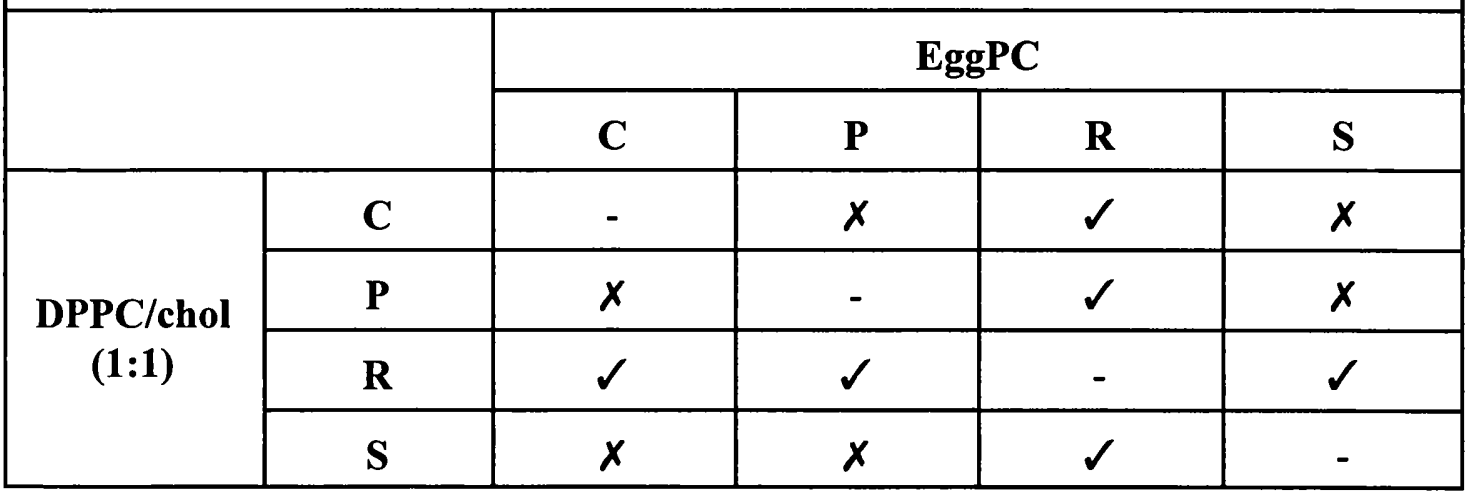

Table 4.8 The significance of the difference in the stability of eggPC/chol (1:1) liposomes (mean size $2.5 \mu \mathrm{m}$ or $5 \mu \mathrm{m}$ ) to nebulisation with jet nebulisers. [p<0.05]

\begin{tabular}{|c|c|c|c|c|c|}
\hline \multicolumn{3}{|c|}{$\begin{array}{c}\text { Initial liposome size/ } \\
\text { nebuliser used. }\end{array}$} & \multicolumn{5}{|c|}{$2.5 \mu \mathrm{m}$} \\
\cline { 2 - 6 } & $\mathrm{C}$ & $\mathrm{P}$ & $\mathrm{R}$ & $\mathrm{S}$ \\
\hline \multirow{3}{*}{$5 \mu \mathrm{m}$} & $\mathrm{C}$ & - & $x$ & $\checkmark$ & $\checkmark$ \\
\cline { 2 - 6 } & $\mathrm{P}$ & $\checkmark$ & - & $x$ & $x$ \\
\cline { 2 - 6 } & $\mathrm{R}$ & $\checkmark$ & $\checkmark$ & - & $\checkmark$ \\
\cline { 2 - 6 } & $\mathrm{S}$ & $\checkmark$ & $x$ & $\checkmark$ & - \\
\hline
\end{tabular}

Table 4.9 The significance of the difference in the stability of eggPC liposomes (mean size of $2.5 \mu \mathrm{m}$ or $5 \mu \mathrm{m}$ ) to nebulisation with jet nebulisers. [ $\mathrm{p}<0.05]$

\begin{tabular}{|c|c|c|c|c|c|}
\hline \multicolumn{2}{|c|}{$\begin{array}{c}\text { Initial liposome size/ } \\
\text { nebuliser used }\end{array}$} & \multicolumn{5}{|c|}{$2.5 \mu \mathrm{m}$} \\
\cline { 2 - 6 } & $\mathrm{C}$ & $\mathrm{P}$ & $\mathrm{R}$ & $\mathrm{S}$ \\
\hline \multirow{3}{*}{$5 \mu \mathrm{m}$} & $\mathrm{C}$ & - & $x$ & $x$ & $x$ \\
\cline { 2 - 6 } & $\mathrm{P}$ & $\checkmark$ & - & $x$ & $x$ \\
\cline { 2 - 6 } & $\mathrm{R}$ & $\checkmark$ & $x$ & - & $\checkmark$ \\
\cline { 2 - 6 } & $\mathrm{S}$ & $\checkmark$ & $\checkmark$ & $\checkmark$ & - \\
\hline
\end{tabular}

\section{Key:}

$C=$ Cirrus, $P=$ Pari-LC, $R=$ Respirgard $I I, S=$ Sidestream

$\checkmark$ significant, $X$ insignificant 


\section{REFERENCES}

Abra, R.M., Hunt, C.A. and Lau, D.T., Liposome disposition in vivo. VI: Delivery to the lung. J. Pharm. Sci., 73 (1984) 203-206

Adamson, I.Y.R. and Bowden, D.H., Dose response of the pulmonary macrophage system to various particulates and its relationship to transepithelial passage of free particulates. Exp. Lung Res., 2 (1981) 165-175

Adjei, A. and Garren, J., Pulmonary delivery of peptide drugs: Effect of particle size on bioavailability of leuprolide acetate in healthy male volunteers. Pharm. Res., 7 (1990) $565-569$

Agostini, C., Trentin, L., Zambello, R. and Semenzato, G., HIV-1 and the lung. Am. Rev. Resp. Dis., 147 (1993) 1038-1049

Allen, T.M. and Chonn, A., Large unilamellar liposomes with low uptake into the reticuloendothelial system. FEBS Lett., (1987) 42-64

Allen, S.D., Sorenson, K.N., Nejdl, M.J., Durant, C. and Proffitt, R.T., Prophylactic efficacy of aerosolised liposomal (AmBisome) and non-liposomal (Fungizone) amphotericin B in murine pulmonary aspergillosis. J. Antimicrob. Chemother., 34 (1994a) 1001-1013

Allen, S.D., Sorenson, K.N., Nejdl, M.J. and Proffitt, R.T., Efficacy of aerosolised liposomal amphotericin B $\left(\mathrm{AmBisome}^{\circledR}\right)$ as a prophylactic treatment in immune compromised murine model of pulmonary aspergillosis. J. Control. Rel., 8 (1994b) 348349

Alton, E.W.F.W., Middleton, P.G., Caplen, N.J., Smith, S.N., Steel, D.M., Munkonge, F.M., Jeffrey, P.K., Geddes, D.M., Hart, S.L., Williamson, R., Fasold, K.I., Miller, A.D., Dickinson, P., Stevenson, B.J., McLachlan, G., Dorin, J.R. and Porteus, D.J., Noninvasive liposome mediated gene delivery can correct the ion transport defect in cystic fibrosis mutant mice. Nature Genet., 5 (1993) 135-142

Alvine, G.F., Rodgers, P., Fitzsimmons, K.M. and Ahrens, R.C., Disposable jet nebulizers, how reliable are they? Chest, 101 (1992) 316-319

Andrews, A.T., Partial denaturation and renaturation of $\beta$-lactoglobulin at air-water interfaces. Biochem. Soc. Trans., 19 (1991) 272S

Arakawa, M., Kondo, T. and Tamamushi, B., Flow properties of microcapsule suspensions as a model of blood. Biorheol., 12 (1975) 57-66 
Atkins, P.J., Barker, N.P. and Mathieson, D., The design and development of inhalation drug delivery systems. In Hickey, A.J. (Ed) Pharmaceutical Inhalation Aerosol Technology, Marcell Dekker, New York. (1992) pp61-82

Bally, M.B., Nayar, R., Masin, D., Hope, M.J., Cullis, P.R. and Mayer, L.D., Liposomes with entrapped doxorubicin exhibit extended blood residence times. Biochim. Biophys. Acta, 1023 (1990) 133-139

Bangham, A.D., Comments after presentations. Ann. NY Acad. Sci., 308 (1979) 411-423

Bangham, A.D., Standish, M.M. and Watkins, J.C., Diffusion of univalent ions across the lamellae of swollen phospholipids. J. Mol. Biol., 13 (1965) 238-252

Bangham, A.D., Hill, M.W. and Miller, N.G., Preparation and use of liposomes as models of biological membranes. In Korn, E.D. (Ed.) Methods Membr. Biol., Plenum Press, New York. 1 (1974) 1-68

Barber, R.F. and Shek, P.N., Liposome stability during ultrasonic nebulization. Proc. The Thirty-Third Harden Conference, The Biochemical Society, Ashford. (1989) p53

Barenholz, Y., Amselem, S. and Lichtenberg, D., A new method for preparation of phospholipid vesicles (liposomes) - French press. FEBS Lett., 99 (1979) 210-214

Barker, S.A., Taylor, K.M.G. and Short, M.D., The deposition and clearance of liposome entrapped ${ }^{99 \mathrm{~m}}$ Tc-DTPA in the human respiratory tract. Int. J. Pharm., 102 (1994) 159-165

Batzri, S. and Korn, E.D., Single bilayer liposomes prepared without sonication. Biochim. Biophys. Acta, 298 (1973) 1015-1019

Bennett, B., Tyson, E., Mah, S., de Groot, J.S., Hegde, S.G., Terao, S. and Teitelbaum, Z., Sustained delivery of detirelix after pulmonary administration of liposomal formulations. J. Contr. Rel., 32 (1994) 27-35

Bergers, J., Den Otter, W., Dullens, H.F.J., Kerkvliet, C.T.M. and Crommelin, D.J.A., Interleukin-2-containing liposomes: Interaction of interleukin-2 with liposomal bilayers and preliminary studies on application in cancer vaccines. Pharm. Res., 10 (1993) 17151721

Bingham, K.L., Meyrick, B., Christman, B., Magnuson, M., King, G. and Berry, L.C., In vivo transfection of murine lungs with a functioning prokaryotic gene using a liposome vehicle. Am. J. Med. Sci., 298 (1989) 278-281

Boguslavskii, Y.Y. and Eknadiosyants, O.K., Physical mechanism of the acoustic atomization of a liquid. Sov. Phys. Accoust., 15 (1969) 14-21 
Boucher, R.M.G. and Kreuter, J., The fundamentals of the ultrasonic atomization of medicated solutions. Ann. Allergy, 26 (1968) 591-600

Bowden, D.H., Macrophages, dusts, and pulmonary diseases. Environ. Lung Res., 12 (1987) 89-107

Boylan, J.C. and Fites, A.L., Parenteral products. In Banker, G.S. and Rhodes, C.T. (Ed.) Modern Pharmaceutics, Marcel-Dekker, New York. (1990) pp491-538

Brain, J.D. and Valberg, P.A., Deposition of aerosols in the respiratory tract. Am. Rev. Resp. Dis., 120 (1979) pp1325-1373

Brain, J.D. and Blanchard, J.D., Mechanisms of particle deposition and clearance. In Morén, F., Dolovich, M.B., Newhouse, M.T. and Newman, S.P. (Eds.) Aerosols in Medicine. Principles, Diagnosis and Therapy, Elsevier, London (1993) pp27-60

Brinkman, H.C., The viscosity of concentrated suspensions and solutions. J. Chem. Phys., 10 (1952) p571

Briscoe, P., Caniggia, I., Graves, A., Benson, B., Huang, L., Tanswell, K. and Freeman, B.A., Delivery of superoxide dismutase to pulmonary epithelium via $\mathrm{pH}$-sensitive liposomes. Am. J. Phys., 268 (1995) 374-380

British National Formulary, Number 32, Mehta, D.K. and Prassad, A.B. (Eds.) BMA and RPSGB, England. (1996) 135-137

Brockerhoff, H., Model of interaction of polar lipids, cholesterol and proteins in biological membranes. Lipids, 9 (1974) 645-650

Broderick, R. and Tulenko, T., Mechanism of cholesterol induced arterial supersensitivity. Fed. Proc., 43 (1984) 350 (a383)

Buckley, B.J., Transwell, A.K. and Freeman, B.A., Liposome-mediated augmentation of catalase in alveolar Type-II cells protects against $\mathrm{H}_{2} \mathrm{O}_{2}$ injury. J. Appl. Physiol., 63 (1987) 359-367

Burton, J.A. and Schanker, L.S., Absorption of sulphonamides and antitubercular drugs from the rat lung. Xenobiotica, 23 (1974) 291-296

Byron, P.R., Pulmonary Targetting With Aerosols. Pharm. Tech., 11 (1987) 42-56

Byron, P.R., Dalby, R.N. and Hickey, A.J., Optimised inhalation aerosols. I. The effects of spherical baffle size and position upon the output of several pressurized nonaqueous suspension formulations. Pharm. Res., 6 (1989) 225-229

Byron, P.R. and Patton, J.S., Drug delivery via the respiratory tract. J. Aerosol Med., 7 (1994) 49-75 
Cabral Marques, H.M., Hadgraft, J., Kellaway, I.W. and Taylor, G., Studies of cyclodextrin inclusion complexes. III. The pulmonary absorption of $\beta$-, DM- $\beta$ - and HP- $\beta$-cyclodestrins in rabbits. Int. J. Pharm., 77 (1991) 297-302

Camner, P., Philipson, K., Friberg, L., Holma, B., Larsson, B. and Svedberg, J., Human tracheobronchial clearance studies. Arch. Environ. Health, 22 (1971) 444-449

Canny, G.J. and Levison, H., Aerosols - therapeutic use and delivery in childhood asthma. Ann. Allergy, 60 (1988) 11-20

Canonico, A.E., Conary, J.T., Meyrick, B.O. and Brigham, K.L., In vivo expression of a CMV promoter driven human $\alpha-1$ antitrypsin $(\alpha-1 \mathrm{AT})$ gene after intravenous or airway administration of DNA/liposome complex. Am. Rev. Respir. Dis., 145 (1992) A200

Caplen, N.J., Alton, E.W.F.W.K., Middleton, P.G., Dorin, J.R., Stevenson, B.J., Gao, X., Durham, S., Jeffrey, P.K., Hodson, M.E., Coutelle, C., Huang, L., Porteus, D.J., Williamson, R., Alton, E.W.F.W. and Geddes, D.M., Liposome mediated CFTR gene transfer to the nasal epithelia of patients with cystic fibrosis. Nature Med., 1 (1995) 39-46

Cevc, G., Lipid properties as a base for membrane modelling and rational liposome design. In Gregoriadis, G. (Ed.) Liposome Technology, Vol 1. (2nd Edn.) CRC Press, New York (1992) pp1-36

Cevc, G. and Marsh, D., Self-assembly. In: Phospholipid Bilayers: Physical Principles and Models. Wiley-Interscience, New York (1987) pp30-56

Chapman, D., Peel, W.E., Kingston, B. and Lilley, T.H., Lipid phase transitions in model membranes. The effect of ions on phosphatidylcholine bilayers., Biochim. Biophys. Acta, 464 (1977) 260-275

Cipolla, D.C., Clark, A.R., Chan, H.-K., Gonda, I. and Shire, S.J., Assessment of aerosol delivery systems for recombinant human deoxyribonuclease. S.T.P. Pharma. Sciences, 4 (1994) 50-62

Clarke, A.R., The use of laser diffraction for the evaluation of the aerosol clouds generated by medical nebulizers. Int. J. Pharm., 115 (1995) 69-78

Clarke, S.W., Inhaler therapy. Quart. J. Med., 67 (1988) 355-368

Clarke, S.W. and Pavia, D., Lung mucus production and mucociliary clearance: Methods of assessment. In Tattersfield, A.E. and Howell, J.B.L. (Eds.) Respiratory System, Macmillan, London (1981) pp85-94

Clay, M., Pavia, D., Newman, S.P. and Clarke, S.W., Efficiency of jet nebulizers in the production of therapeutic aerosols. Thorax, 37 (1982) 788 
Clay, M., Pavia, D., Clarke, S.W. and Clarke, S.W., Factors influencing the size distribution of aerosols from jet nebulisers. Thorax, 38 (1983a) 755-759

Clay, M., Pavia, D., Newman, S., Lennard-Jones, T. and Clarke, S.W., Assessment of jet nebulisers for lung aerosol therapy. Lancet, ii (1983b) 592-594

Clay, M., Pavia, D. and Clarke, S.W., Effect of aerosol particle size on bronchodilatation with nebulised terbutaline in asthmatic patients. Thorax, 41 (1986) 364-368

Claypool, W.D., Wand, D.L., Chander, A. and Fisher, A.B., An ethanol/ether soluble apoprotein from rat lung surfactant augments liposome uptake by isolated granular pneumocytes. J. Clin. Invest., 74 (1984) 677-684

Clejan, S., Bittman, R., Deroo, P.W., Isaacson, Y.A. and Rosenthal, A.F., Permeability properties of sterol-containing liposomes from analogues of phosphatidylcholine-lacking acyl groups. Biochem., 18 (1979) 2118-2125

Cockroft, D.W., Thomas, S., Hurst, M. and Gore, B.P., Importance of evaporative water losses during standardized nebulized inhalation provocation tests. Chest, 96 (1989) 506509

Colthorpe, P., Farr, S.J., Taylor, G., Smith, I.J. and Wyatt, D., The pharmacokinetics of pulmonary-delivered insulin: A comparison of intratracheal and aerosol administration to the rabbit. Pharm. Res., 9 (1992) 764-768

Coutelle, C. and Williamson, R., Liposomes and viruses for gene therapy of cystic fibrosis. J. Aerosol Med., 9 (1996) 79-88

Craig, I.R., Riley, M.R. and Cooke, N.J., Prescribing of nebulized bronchodilators - can we change bad habits? Respir. Med., 83 (1989) 333-334

Crapo, J.D., Barry, B.E., Gehr, P., Bachofen, M. and Wiebel, E.R., Cell number and cell characteristics of the normal human lung. Am. Rev. Respir. Dis., 126 (1982) 332-337

Crommelin, D.J.A. and Van Bommel, L.M.G., Stability of liposomes on storage: Freezedried, frozen or as an aqueous dispersion. Pharm. Res., 1 (1984) 159-163

Crommelin, D.J.A. and Schreier, H., Liposomes. In Kreuter, J. (Ed.) Colloidal Drug Delivery Systems, Marcel Dekker, New York (1994) 73-190

Crowe, L.M., Crowe, J.H., Rudolph, A., Womersley, C. and Appel, L., Preservation of freeze-dried liposomes by trehalose. Arch. Bioch. Biophys., 242 (1985) 240-247

Crowe, L.M., Crowe, J.H., Womersley, C., Reid, D., Appel, L. and Rudolph, A., Prevention of fusion and leakage in freeze-dried liposomes by carbohydrates. Biochim. Biophys. Acta, 861 (1986) 131-140 
Crowe, L.M. and Crowe, J.H., Trehalose and dry dipalmitoylphophatidylcholine revisited. Biochim. Biophys. Acta, 946 (1988) 193-201

Crowe, L.M. and Crowe, J.H., Preservation of liposomes by freeze-drying. In Gregoriadis, G. (Ed.) Liposome Technology, Vol 1 (2nd Edn.) CRC Press, New York (1992) 229-253

Cullis, P.R., Hope, M.J., Bally, M.B., Madden, T.D., Mayer, L.D. and Janoff, A.S., Liposomes as pharmaceuticals. In Ostro, M.J. (Ed.) Liposomes: From Biophysics to Therapeutics. Marcel Dekker, New York. (1987) pp39-72

Cullis, P.R., Mayer, L.D., Bally, T.D., Madden, T.D. and Hope, M.J., Generating and loading of liposomal systems for drug-delivery applications. Adv. Drug Del. Rev., 3 (1989) 267-282

Dahlbäck, M., Behaviour of nebulizing suspensions and solutions. J. Aer. Sci. 7(suppl. 1) (1994) S13-S15

Dalby, R.N. and Byron, P.R., Comparison of output particle size distributions from pressurised aerosols formulated as solutions or suspensions. Pharm. Res., 5 (1988) 36-39

Dalby, R.N., Byron, P.R., Shepard, H.R. and Papahadjopoulos, E., CFC propellant substitution: P-134a as a potential replacement for P-12 in MDIs. Pharm. Tech., 14 (1990) 26-33

Damani, L.A., Metabolism of drugs in the respiratory tract. In Ganderton, D. and Jones, T. (Eds.) Drug Delivery To The Respiratory Tract, Ellis Horwood, Chichester (1987) pp4757

Davies, D.S., Pharmacokinetics of inhaled substances. Postgrad. Med. J., 51(suppl. 7) (1975) 69-75

Davis, S.S., Physico-chemical studies on aerosol solutions for drug delivery. I: Waterpropylene glycol systems. Int. J. Pharm., 1 (1978) 71-83

Davis, S.S., Elson, G. and Whitmore, J., Physico-chemical studies on aerosol solutions for drug delivery II. Water-propylene glycol-ethanol systems. Int. J. Pharm., 1 (1978) 85-93

De Gier, J., Mandersloot, J.G. and van Deenen, L.L.M., Lipid composition and permeability of liposomes. Biochim. Biophys. Acta, 150 (1968) 666-675

Deamer, D.W. and Bangham, A.D., Large volume liposomes by an ether vaporization technique. Biochim. Biophys. Acta, 443 (1976) 629-634 
Debs, R.J., Straubinger, R.M., Brunette, E.N., Lin, J.M., Lin, E.J., Montgomery, A.B., Frend, D.S. and Papahadjopoulos, D.P., Selective enhancement of pentamidine uptake in the lung by aerosolisation and delivery in liposomes. Am. Rev. Respir. Dis., 135 (1987) 731-737

Debs, R., Liu, Y., Zhong, W. and Tu, Q., Aerosolized liposome-mediated gene delivery. J. Aerosol Med., 7 (1994) 238

Dennis, J.H., Drug nebuliser design and performance: Breath enhanced jet vs constant output jet vs ultrasonic. J. Aerosol Med., 8 (1995) 277-280

Dennis, J.H. and Hendrick, D.J., Design characteristics for drug nebulizers. J. Med. Eng. Tech., 16 (1992) 63-68

Dickenson, G. and Smalldone, G.C., Particle deposition and failure of prophylaxis using aerosolised pentamidine. Am. Rev. Respir. Dis., 131 (1990) A150

Djordjevich, L., Kashani, A., Miller, I.F. and Ivankovich, A.D., Measurements of viscosity of synthetic erythrocyte suspensions. Biorheol., 24 (1987) 207-213

Dombrowski, N. and Munday, G., Spray Drying. In Blakeborough, N. (Ed.) Biochemical and Biological Engineering Sciences, Academic Press, London (1968) 209-320

Dorman, R.G., The atomization of liquids in a flat spray. Br. J. Appl. Physiol., 3 (1952) 189-192

Douglas, R.B., The physiology of the lung., In Ganderton, D. and Jones, T.M., (Eds.) Drug Delivery to the Respiratory Tract, V.C.H. and Ellis Horwood, Chichester (1987) 11-26

Douglas, J.G., Leslie, M.J., Crompton, G.K. and Grant, I.W.B., A comparative study of two doses of salbutamol nebulised at 4 and 8 litres per minute in patients with chronic asthma. Br. J. Dis. Chest, 80 (1986) 55-58

Effros, R.M., Epithelial permeability. In Morén, F., Dolovich, M.B., Newhouse, M.T. and Newman, S.P. (Eds.) Aerosols in Medicine. Principles, Diagnosis and Therapy, Elsevier Science, Amsterdam (1993) pp27-60

Egbaria, K. and Weiner, N., Liposomes as a topical drug delivery system. Adv. Drug Deliv. Rev., 5 (1990) 287-300

Ellens, H., Rustum, Y., Mayhew, E. and Ledesma, E., Distribution and metabolism of liposome-encapsulated and free 1-B-D-Arabinofuranosylcytosine (Ara-C) in dog and mouse tissues. J. Pharmacol. Exp. Ther., 222 (1982) 324-330 
Elliott, R.B., Edgar, B.W., Pilcher, C.C., Quested, C. and McMaster, J., Parenteral absorption of insulin from the lung in diabetic children. Aust. Paediatr. J., 23 (1987) 293297

Engel, A., Bendas, G., Wilhelm, F., Mannova, M., Ausborn, M. and Nuhn, P., Freeze drying of liposomes with free and membrane-bound cryoprotectants - the background of protection and damaging processes. Int. J. Pharm., 107 (1994) 99-110

Enna, S.J. and Schanker, L.S., Absorption of saccharides and urea from the rat lung. Am. J. Physiol., 222 (1972a) 409-414

Enna, S.J. and Schanker, L.S., Absorption of drugs from the rat lung. Am. J. Physiol., 223 (1972b) 1227-1231

Everard, M.L., Hardy, J.G. and Miller, A.D., Comparison of aerosol deposition in the lungs of healthy adults following oral and nasal inhalation. Thorax, 48 (1993) 586-591

Everard, M.L., Evans, M. and Milner, A.D., Is tapping jet nebulisers worthwhile ? Arch. Dis. Child., 70 (1994) 538-539

Farr, S.J., Kellaway, I.W., Parry-Jones, D.R. and Woolfrey, S.G., ${ }^{99 \mathrm{~m}-T e c h n e t i u m ~ a s ~ a ~}$ marker of liposomal deposition and clearance in the human lung. Int. J. Pharm., 26 (1985) 303-316

Farr, S.J., Kellaway, I.W. and Carman-Meakin, B., Assessing the potential of aerosolgenerated liposomes from pressurised pack formulations. J. Contr. Rel., 5 (1987) 119-127

Farr, S.J., Kellaway, I.W. and Carman-Meakin, B., Comparison of solute partitioning and efflux in liposomes formed by a conventional and an aerosolised method. Int. J. Pharm., 51 (1989) 39-46

Fell, J.T., Surface and interfacial phenomena. In Aulton M.E. (Ed.) Pharmaceutics: The Science of Dosage Form Design, Churchill Livingstone , Edinburgh (1988) pp50-61

Ferron, G.A., Kerrebijn, K.F. and Weber, J., Properties of aerosols produced with three nebulizers. Am. Rev. Respir. Dis., 114 (1976) 899-908

Ferron, G.A., Hornick, S., Kreling, W.G. and Haider, B., Comparison of experimental and calculated data for the total and regional deposition in the human lung. J. Aerosol Sci., $16(1981)$ 133-143

Fick, R.B., Jr. and Stillwell, P.C., Controversies in the management of pulmonary disease due to cystic fibrosis. Chest, 95 (1989) 1319-1327 
Fidler I.J., Raz, A., Fogler, W.E., Kirsh, R., Bugelski, P. and Poste, G., Design of liposomes to improve delivery of macrophage augmenting agents to alveolar macrophages. Cancer Res. , 40 (1980) 4460-4466

Fielding, R.M. and Abra, R.M., Factors affecting the release rate of terbutaline from liposome formulations after intratracheal instillation in the guinea pig. Pharm. Res., 9 (1992) 220-223

Fisher, A.B., Chander, A. and Richerter, J., Uptake and degradation of natural surfactant by isolated rat granular pneumocytes. Am. J. Physiol., 253 (1987) c792-c796

Florence, A.T. and Attwood, D., Physicochemical Principles of Pharmacy, Macmillan Press, London (1988) pp173-227

Forsgren, P., Moody, J., Gerdin, B., Axelson, B. and Dahlbäck, M., Intrapulmonary deposition of aerosolized corticosteroid liposome in a porcine model of early ARDS induced by endotoxaemia. Acta. Chir. Scand., 156 (1990) 423-431

Forrest, J.B., Lower airway: Structure and function. In Morén, F., Dolovich, M.B., Newhouse, M.T. and Newman, S.P. (Eds.), Aerosols in Medicine. Principles, Diagnosis and Therapy., Elsevier, London (1993) pp27-60

Freeman, B.A., Young, S.L. and Crapo, J.D., Liposome-mediated augmentation of superoxide dismutase in endothelial cells prevents oxygen injury. J. Biol. Chem., 258 (1983) 12534-12542

Gabizon, A., Goren, D., Ramu, A. and Barenholz, Y., Design, characterization and antitumour activity of adriamycin-containing phospholipid vesicles. In Gregoriadis, G., Senior, G. and Poste, G. (Eds.) Targeting of Drugs With Synthetic Systems, Plenum Press, New York (1986) pp229-238

Gale, A.E., Drug degeneration during ultrasonic nebulization. J. Aerosol Sci.. 16 (1985) 265

Gehr, P., Bachofen, M. and Weibel, E.R., The normal human lung: Ultrastructure and morphometric estimation of diffusion capacity. Respir. Physiol., 32 (1978) 121-140

Geiger, K., Gallagher, M.L. and Hedley-White, J., Cellular distribution and clearance of aerosolized dipalmitoyl lecithin. J. Appl. Physiol., 39(5) (1975) 759-766

Gerhenzon, E.L. and Eknadiosyants, O.K., The nature of liquid atomization in an ultrasonic fountain. Sov. Phys. Accoust., 12 (1964) 156-162

Gilbert, B.E., Liposomal aerosols in the management of pulmonary infections. J. Aerosol Med., 9 (1996) 111-122 
Gilbert, B.E., Six, S.Z., Wilson, P.R., Wyde, P.R. and Knight, V., Small particle aerosols of enviroxime-containing liposomes. Antiviral Res., 9 (1988) 355-365

Gilbert, B.E., Wyde, P.R. and Wilson, S.Z., Aerosolized liposomal amphotericin B for treatment of pulmonary and systemic Cryptococcus neoformans infections in mice. Antimicrob. Agents Chemother., 36 (1992) 1466-1471

Gilbert, B.E., Wyde, P.R., Lopez-Berestein, G. and Wilson, S.Z., Aerosolized amphotericin B-liposomes for treatment of systemic Candida infections in mice. Antimicrob. Agents Chemother., 38 (1994) 356-359

Gilbert, B.E. and Proffitt, R.T., Aerosolised amBisome treatment of pulmonary Cryptococcus neoformans infection in mice. J. Aerosol. Med., 9 (1996) 263-276

Glukhov, S.A., The theory and calculation of an ejection type sprayer. Med. Technol., 6 (1969) 20-26

Goddard, R.F., Mercer, T.T., O’Neill, R.L., Flores, R.L. and Sanchez, R., Output characteristics and clinical efficacy of ultrasonic nebulizers. J. Asthma Res., 5 (1992) $355-368$

Goldbach, P., Brochart, H. and Stamm, A., Spray-drying of liposomes for a pulmonary administration. II. Retention of encapsulated materials. Drug Dev. Ind. Pharmacy, 19 (1993) 2624-2636

Gonda, I., Therapeutic aerosols. In Aulton, M.E., (Ed.) Pharmaceutics: The Science of Dosage Form Design, Churchill Livingstone, Edinburgh (1988) pp341-358

Gonda, I., Aerosols for delivery of therapeutic and diagnostic agents to the respiratory tract. Crit. Rev. Ther. Drug Carr. Syst., 6 (1990) 273-313

Gonda, I., Targeting by deposition. In Hickey, A.J. (Ed.) Pharmaceutical Inhalation Aerosol Technology, Marcell Dekker, New York. (1992) pp61-82

Gonda, I. and Byron, P.R., Perspectives on the biopharmacy of inhalation aerosols., Drug Dev. Ind. Pharm., 4 (1978) 243-259

Gonzalez-Rothi, R.J., Cacace, J., Straub, L. and Schreier, H., Liposomes and pulmonary alveolar macrophages: Functional and morphological interactions. Exp.Lung Res., 17 (1991) 687-705

Greenspan, B.J., Ultrasonic and electrohydrodynamic methods for aerosol generation. In Hickey, A.J. (Ed.) Inhalation Aerosols: Physical and Biological Basis for Therapy, Marcel Dekker, New York (1996) pp273-307 
Gregoriadis, G., Immunological adjuvants: A role for liposomes. Immunol. Today, 11 (1990) 89-97

Gregoriadis, G. and Florence, A.T., Liposomes in drug delivery: Clinical, diagnostic and ophthalmic potential. Drugs, 45 (1993) 15-28

Grit, M., de Smidt, J.H., Struijke, A. and Crommelin, D.J.A., Hydrolysis of phosphatidylcholine in aqueous liposome dispersions. Int. J. Pharm., 50 (1989) 1-6

Grit, M. and Crommelin, D.J.A., The effect of ageing on the physical stability of liposome dispersions. Chem. Phys. Lipids, 62 (1992) 113-122

Grit, M., Underberg, W.J.M. and Crommelin, D.J.A., Hydrolysis of saturated soybean phosphatidylcholine in aqueous liposome dispersions. J. Pharm. Sci., 82 (1993) 362-366

Grossman, J., The evolution of inhaler technology. J. Asthma, 31 (1994) 55-64

Gruner, S.M., Materials properties of liposomal bilayers. In Ostro, M.J. (Ed.) Liposomes: From Biophysics to Therapeutics. Marcel Dekker, New York. (1987) pp1-38

Gruner, S.M., Lenk, R.P., Janoff, S. and Ostro, M.J., Novel multilayered lipid vesicles: Comparison of physical characteristics of multilamellar liposomes and stable plurilamellar vesicles. Biochem., 24 (1985) 2822-2842

Gunstone, F.D., Fatty acid structure. In Gunstone, F.D., Harwood, J.L. and Padley, F.B. (Eds.) The Lipid Handbook, Chapman and Hall, London (1994) pp1-19

Hadfield, J.W., Windebank, W.J. and Bateman, J.R.M., Is driving gas flow rate clinically important for nebuliser therapy? Br. J. Dis. Chest, 80 (1986) 50-54

Hallman, M., Epstein, B.L. and Gluck, L., Analysis of labelling and clearance of lung surfactant phospholipids in rabbit. J. Clin. Invest., 68 (1981) 742-751

Hallworth, G.W., The formulation and evaluation of pressurised metered-dose inhalers. In Ganderton, D. and Jones, T.M. (Eds.) Drug Delivery to the Respiratory Tract. Ellis Horwood Ltd., Chichester (1987) pp87-118

Hallworth, G.W. Particle size analysis of therapeutic aerosols. In Morén, F., Dolovich, M.B., Newhouse, M.T. and Newman, S.P. (Eds.) Aerosols in Medicine. Principles, Diagnosis and Therapy. (1993) pp351-374

Hallworth, G.W. and Andrews, U.G., Size analysis of suspension inhalation aerosols by inertial separation methods. J. Pharm. Pharmacol., 28 (1976) 50-54

Hallworth, G.W. and Westmoreland, D.G., The twin impinger: A simple device for assessing the delivery of drugs from metered dose pressurised aerosol inhalers. J. Pharm. Pharmacol., 39 (1987) 966-972 
Hansen, O.R. and Pederson, S., Optimal inhalation technique with terbutaline Turbuhaler. Eur. Respir. J., 2 (1989) 637-639

Hardy, J.G., Newman, S.P. and Knoch, M., Lung deposition from four nebulizers. Respir. Med., 87 (1993) 461-465

Harrigan, P.R., Madden, T.D. and Cullis, P.R., Protection of liposomes during dehydration or freezing. Chem. Phys. Lipids, 52 (1990) 139-149

Harris, R.L., Jr. and Fraser, D.A., A model for deposition of fibers in the human respiratory system. Am. Ind. Hyg. Assoc. J., 37 (1976) 37-44

Harwood, J.L., Lipid structure., In Gunstone, F.D., Harwood, J.L. and Padley, F.B. (Eds.) The Lipid Handbook, Chapman and Hall, London (1994) pp21-46

Hasson, D. and Mizrhai, J., The drop size of fan spray nozzles. Trans. Instn. Chem. Engrs., 39 (1961) 415-422

Hauser, H., Stabilising of liposomes during spray drying. In Gregoriadis, G. (Ed.) Liposome Technology.Vol 1. (2nd Edn.) CRC Press, New York (1993) 197-251

Hauser, H. and Irons, L., The effect of ultracentrifugation on the chemical and physical properties of aqueous egg yolk lecithin dispersions. Hope-Seyler's Z. Physiol. Chem., 353 (1972) 1579-1590

Haynes, R.H. and Burton, A.C., Role of the non-Newtonian behaviour of blood in hemodynamics., Am. J. Physiol., 197 (1959) 943-950

Haynes, R.H., The rheology of blood. Trans. Soc. Rheol., 5 (1961) 85-101

Hazinski, T.A., Liposome-mediated transfer of fusion genes to the intact lung. Sem. Perinatol., 16 (1992) 200-204

Hazinski, T.A., Ladd, P.A. and DeMatteo, C.A., Localisation and induced expression of fusion genes in the rat lung. Am. J. Respir. Cell Mol. Biol., 4 (1991) 206-209

Henson, A.F., Mitchell, J.R. and Mussellwhite, P.R., The surface coagulation of proteins during shaking. J. Colloid Interface Sci., 32 (1970) 162-165

Hess, D., Horney, D. and Sneyder, T., Medication performance of eight small volume, hand-held nebulizers: Effect of diluent volume, gas flow rate, and nebulizer model. Respir. Care., 54 (1989) 717-723

Hilman, B., Aerosol deposition and delivery of therapeutic aerosols. J. Asthma, 28 (1991) 239-242 
Hinds, W.C., Macher, J.M. and First, M.W., Size distribution of aerosols produced by the Laskin aerosol generator using substitute materials for DOP. Am. Ind. Hyg. Assoc., 44 (1983) 495-500

Ho, J., Generation and analysis of liposome aerosols. In Shek, P.N. (Ed.) Liposomes in Biomedical Applications, Harwood Academic, Amsterdam (1995) pp199-208

Ho, K.K.L., Kellaway, I.W. and Tredree, R.L., The determination of aerosol output during size analysis by laser diffraction. J. Pharm. Pharmacol., 39 (suppl.) (1987) 77P

Ho, K.K.L., Farr, S.J., Kellaway, I.W. and Tredree, R.L., In vitro - in vivo correlation of nebuliser performance. J. Pharm. Pharmacol., 40 (suppl.) (1988) 12P

Hollie, M.C., Malone, R.A., Skifca, R.M. and Nelson, H.S., Extreme variability in aerosol output of the DeVilbiss jet nebulizer. Chest, 100 (1991) 1339-1344

Holzner, P.M. and Müller, B.W., Particle size determination of metered dose inhalers with inertial separation methods: Apparatus A and B (BP), Four Stage Impinger and Andersen Mark II Cascade Impactor. Int. J. Pharm., 116 (1995) 11-18

Huang, C., Studies on phosphatidylcholine vesicles. Formation and physical characteristics. Biochem., 8 (1969) 344-352

Hughes, B.J., Kennel, S., Lee, R. and Huang, L., Monoclonal antibody targeting of liposomes to mouse lung in vivo. Cancer Res., 49 (1989) 6214-6220

Hung, O.R., Whynot, S.C., Varvel, J.R., Shafer, S.L. and Mezei, M., Pharmacokinetics of inhaled liposome-encapsulated fentanyl. Anaesth., 83 (1995) 277-284

Hunt, C.A., Rustum, Y.M., Mayhew, E. and Papahadjopoulos, D., Retention of cytosine arabinoside in mouse lung following intravenous administration in liposomes of different size. Drug Metab. Disp., 7 (1979) 124-128

Hunt, C.A. and Tsang, S., $\alpha$-tocopherol retards autoxidation and prolongs the shelf-life of liposomes. Int. J. Pharm., 8 (1981) 101-110

Hurley, P.K., Smye, S.W. and Cunliffe, H., Assessment of antibiotic aerosol generation using commercial jet nebulizers. J. Aer. Med., 7 (1994) 217-228

Hwang, K.J., Padki, M.M., Chow, D.D., Essien, H.E., Lai, J.Y. and Beaumier, P.L., Uptake of small liposomes by non-reticuloendothelial tissues. Biochim. Biophys. Acta, 901 (1987) 88-96

Ilowite, J.S., Gorvoy, J.D. and Smalldone, G.C., Quantitative deposition of aerosolized gentamicin in cystic fibrosis. Am. Rev. Resp. Dis., 136 (1987) 1445-1449 
Ilowite, J.S., Baskin, M.I., Sheetz, M.S. and Abd, A.G., Delivery dose and regional distribution of aerosolized pentamidine using different delivery systems. Chest, 99 (1991) $1139-1144$

Inoue, K. and Kitagawa, T., Effect of exogenous lysolecithin on liposomal membranes: Its relation to membrane fluidity. Biochim. Biophys. Acta, 363 (1974) 361-372

Ip, A.Y., Arakawa, T., Silvers, H., Ransone, M. and Niven, R.W., Stability of recombinant consensus interferon to air-jet and ultrasonic nebulization. J. Pharm. Sci., 84 (1995) $1210-1214$

Israelachvili, J.N., Mitchell, D.J. and Ninham, B.W., Theory of self-assembly of lipid bilayers and vesicles. Biochim. Biophys. Acta, 470 (1977) 185-201

Israelachvili, J.N., Marcelja, S. and Horn, R., Physical principles of membrane organization. Quart. Rev. Biophys., 13 (1980) 121-200

Ivey, H., Roth, S. and Kattwinkel, J., Nebulization of sonicated phospholipids (PL) for treatment of respiratory distress syndrome (RDS) of infancy. Paediatr. Res., 11 (1977) 573 (A1207)

Jacobson, K. and Papahadjopoulos, D., Phase transitions and phase separations in phospholipid membranes induced by changes in temperature, $\mathrm{pH}$, and concentration of bivalent ions. Biochem., 14(1) (1975) 152-161

Johnson, S.M., The effect of charge and cholesterol on the size and thickness of sonicated phospholipid vesicles. Biochim. Biophys. Acta, 397 (1973) 27-41

Johnson, A.R. and Davenport, J.B., Physical chemistry of lipids. In Johnson, A.R. and Davenport, J.B. (Eds.) Biochemistry and methodology of lipids., Wiley-Interscience. New York (1971) pp64-83

Johnson, M.A., Newman, S.P., Bloom, R., Talaee, N. and Clarke, S.W., Delivery of albuterol and ipratropium bromide from two nebulizer systems in chronic stable asthma. Chest, 96 (1989) 6-10

Jopski, B., Pirkl, V. and Schmidt, K.-H., Viscosity measurements of suspensions of haemoglobin-containing liposomes of varying lipid composition. Biomat., Art. Cells, Art. Org., 18 (1990) 344-358

Juliano, R.L., Stamp, D. and McCullough, H.N., Pharmacokinetics of liposomeencapsulated antitumour drugs and implications for therapy. Ann. NY Acad. Sci., 308 (1979) 411-423 
Juliano, R.L. and McCullough, H.N., Controlled delivery of an antitumour drug: Localised action of liposome encapsulated cytosine arabinoside administered via the respiratory tract. J. Pharmacol. Exp. Ther., 214 (1980) 381-387

Jurima-Romet, M., Barber, R.F., Demeester, J. and Shek, P.N., Distribution studies of liposome-encapsulated glutathione administered to the lung. Int. J. Pharm., 63 (1990) 227-235

Jurima-Romet, M. and Shek, P.N., Lung uptake of liposome entrapped glutathione after intratracheal administration. J. Pharm. Pharmacol., 43 (1991) 6-10

Kamarei, A., Wong, A. and McCalden, T., Methodology for efficacy study of liposome bronchodilators in long duration experiments. Proc. West. Pharmacol. Soc., 32 (1989) $33-35$

Kato, A., Arakawa, M. and Kondo, T., Flow properties of hemolysate-loaded liposomes. Biorheol., 20 (1983) 593-601

Kendrick, A.H., Smith, E.C. and Wilson, R.S.E., Selecting and using nebuliser equipment. Thorax, 52 (Suppl. 2) (1997) S92-S101

Keough, K.M., Oldfield, E., Chapman, D. and Benyon, P., Carbon-13 and proton nucleated magnetic resonance of unsonicated model and mitochondrial membranes. Chem. Phys. Lip., 10 (1973) 37-50

Khanna, C., Hasz, D.E., Klausner, J.S. and Anderson, P.M., Aerosol delivery of Interleukin-2 liposomes is non-toxic and biologically effective: Canine studies. Clin. Cancer Res., 2 (1996) 721-734

King, R.J. and Clements, J.A., Lipid synthesis and surfactant turnover in the lungs. In Fishman, A.P., Fisher, A.B. and Geiger, S.R. (Eds.) Handbook of Physiology, Section 3, Volume 1, Respiration., American Physiological Society, Bethesda (1985) pp9309-336

Kirby, L., Clarke, J. and Gregoriadis, G., Effect of the cholesterol content of small unilamellar liposomes on their stability in vivo and in vitro. Biochem. J., 186 (1980) 591598

Knoch, M. and Wunderlich, E., In vitro assessment of a new efficient nebulizer system for continuous operation. Proc. Respir. Drug Del. IV, Aerosol Society, Portishead (1994) 265-271

Kochetov, V.I., Klepikov, E.S. and Garaishin, R.M., Effect of surface active agents on spraying of a liquid. Colloid J. USSR, 27 (1965) 164-170 
Köhler, D. and Matthys, H., Correspondence. J. Aerosol Sci., 15 (1984) 519-520

Kradjan, W.A. and Lakshminarayan, S., Efficiency of air-compressor-driven nebulizers. Chest, 87 (1985) 512-516

Ladbroke, B.D., Williams, R.M. and Chapman, D., Studies on lecithin-cholesterol-water interactions by differential scanning calorimetry and X-ray diffraction. Biochem. Biophys. Acta, 150 (1968) 333-340

Ladbroke, B.D. and Chapman, D., Thermal analysis of lipids, proteins and biological membranes. A review and summary of some recent studies. Chem. Phys. Lipids, 3 (1969) 304-319

Lang, R.J., Ultrasonic atomization of liquid. J. Accoust. Soc. Am., 34 (1962) 6-8

Lashmar, U.T., Provasi, D. and Poole, S.W., Evaluation of the delivery of suspensions from an ultrasonic nebuliser with and without the lung surfactant "Exosurf". Int. J. Pharm., 103 (1994) 119-123

Lasic, D.D., The mechanism of vesicle formation. Biochim. J., 256 (1988) 1-11

Lasic, D.D., Letter to the editor on the thermodynamic stability of liposomes. J. Colloid Interf. Sci., 140(1) (1990) 302-304

Lasic, D.D. Preparation of liposomes. In Lasic, D.D. (Ed.) Liposomes: From Physics to Applications. Elsevier, Amsterdam (1993) pp63-171

Lauweryns, J.M. and Baert, J.H., Alveolar clearance and the role of the pulmonary lymphatics. Am. Rev. Resp. Dis., 115 (1977) 625-683

Leong, K.H., Theoretical principles and devices used to generate aerosols for research. In Hickey, A.J. (Ed.) Pharmaceutical Inhalation Aerosol Technology, Marcel Dekker, New York (1992) pp129-159

Less, M.J. and Schanker, L.S., Lung $\mathrm{pH}$ and pulmonary absorption of non-volatile drugs in the rat. Drug Metab. Disp., 5 (1977) 174-178

Lewis, R.A., Nebulisers for lung aerosol therapy. Lancet, 2 (1983) 849

Lidgate, D.M., Felgner, P.L., Fleitman, J.S., Whatlry, J. and Fu, R.C-C., In vitro and in vivo studies evaluating a liposome system for drug solubilisation. Pharm. Res., 5 (1988) 759764

Lim, J.G.P., Farr, S.J. and Kellaway, I.W., Studies of hydrophobic microspheres for controlled drug pulmonary delivery. J. Pharm. Pharmacol., 41 (suppl.) (1989) 8P

Liu, F., Shao, Z., Kildig, D.O and Mitra, A.K., Pulmonary delivery of free and liposomal insulin. Pharm. Res., 10 (1993) 228-232 
Lopez-Berestein, G.R., Liposomes as carriers of anti-fungal drugs. Ann. N. Y. Acad. Sci., 544 (1988) 590-597

Lorenzetto, G.E. and Lefebvre, A.H., Measurements of drop size on a plain-jet airblast atomizer. AIAA J., 15 (1977) 1006-1010

Lourenço, R.V. and Cotromanes, E., Clinical aerosols: I. Characterization of aerosols and their diagnostic uses. Arch. Intern. Med., 142 (1982) 2163-2171

Lundberg, B., Properties of mixed vesicles of lecithin:cholesterol up to a 2:1 molar ratio. Chem. Phys. Lipids, 18 (1977) 212-220

Mabrey, S. and Sturtevant, J.M., High-sensitivity differential scanning calorimetry in the study of biomembranes and related model systems. In Korn, E.W. (Ed.) Methods in Membrane Biology, 9 (1978) 237-274

Mallol, J., Particle size distribution for jet nebulizers commonly employed in the pediatric clinical setting. J. Aerosol Med., 6 (1993) 213-219

Marks, L.B., Oberdörster, G. and Notter, R.H., Generation and characterization of aerosols of dispersed surface active phospholipids by ultrasonic and jet nebulization. J. Aer. Sci., 14 (1983) 683-694

Marriott, C., Rheology and the flow of fluids. In Aulton, M.E. (Ed.) Pharmaceutics: The Science of Dosage Form Design, Churchill Livingstone, Edinburgh (1988) pp17-37

Massey, D.G., Miyauchi, D. and Fournier-Massey, G., Nebulizer function. Bull. Europ. Physiopath. Resp., 18 (1982) 665-671

Matthys, H. and Köhler, D., Pulmonary deposition of aerosols by different mechanical devices. Respiration, 48 (1985) 269-276

May, K.R., The Collison nebulizer: Description, performance and application. Aerosol Sci., 4 (1973) 235-243

Mayer, L.D., Hope, M.J. and Cullis, P.R., Solute distributions and trapping efficiencies observed in freeze-thawed multilamellar vesicles. Biochim. Biophys. Acta., 858 (1986) $161-168$

Mayhew, E. and Rustum, Y., Effect of liposome entrapped chemotherapeutic agents in mouse primary and metastatic tumours. Biol. Cell, 47 (1983) 81-85

Mayhew, E., Lazo, R., Vail, W.J., King, J. and Green, A.M., Characterisation of liposomes prepared using a microemulsifier. Biochim. Biophys. Acta, 775 (1984) 169-174 
McCalden, T.A., Fielding, R.M., Mihalko, P.J. and Kaplan, S.A., Sustained bronchodilator therapy using inhaled liposomal formulations of beta- 2 adrenergic agonists. In Prescott, L.F. and Nimmo, W.S. (Eds.) Novel Drug Delivery and Its Therapeutic Applications Proceedings of the third international conference on drug absorption, Edinburgh (1989a) 297-303

McCalden, T.A., Particulate systems for drug delivery to the lung. Adv. Drug Del. Rev., 5 (1990) 253-263

McCalden, T.A., Abra, R.A. and Mihalko, P.J., Efficacy of a liposome formulation of the bronchodilator metaproterenol in the guinea pig. J. Lip. Res., 1 (1989b) 211-222

McCallion, O.N.M., Taylor, K.M.G., Thomas, M. and Taylor, A.J., Nebulization of fluids of different physicochemical properties with air-jet and ultrasonic nebulizers. Pharm. Res., 12 (1995) 1682-1688

McCallion, O.N.M., Taylor, K.M.G., Thomas, M. and Taylor, A.J., Nebulisation of monodisperse latex sphere suspensions in air-jet and ultrasonic nebulisers. Int. J. Pharm., 133 (1996) 203-214

McCallion, O.N.M. and Patel, M.J., Viscosity effects on nebulization of aqueous-solutions. Int. J. Pharm., 130 (1996) 245-249

McCullough, H.N. and Juliano, R.L., Organ-selective action of an antitumour drug: Pharmacologic studies of liposome-encapsulated $\beta$-cytosine arabinoside administered via the respiratory system of the rat. J. Natl. Cancer Inst., 63 (1979) 727-731

McGurk, J.G., Ross, A.R. and Gilroy, G.C., A novel method for the in situ preparation of liposomes using an aerosol delivery system. J. Pharm. Pharmacol., 38 (suppl.) (1986) 20P

McIntosh, T.J., The effect of cholesterol on the structure of phosphatidylcholine bilayers. Biochim. Biophys. Acta 513 (1978) 43-58

McPherson, S.P., Humidifiers and nebulizers. In McPherson, S.P. (Ed.) Respiratory Therapy Equipment, (3rd edition), Mosby, St. Louis (1985) pp119-162

Meisner, D., Pringle, J. and Mezei, M., Liposomal pulmonary drug delivery I. In vivo disposition of atropine base in solution and liposomal form following endotracheal instillation to the rabbit lung. J. Microencaps., 6 (1989a) 379-387

Meisner, D., Pringle, J. and Mezei, M., Liposomal ophthalmic drug delivery: III. Pharmacodynamic and biodisposition studies of atropine. Int. J. Pharm., 55 (1989b) 105113 
Mercer, T.T., Production and characterisation of aerosols. Arch. Intern. Med., 131 (1973) $39-50$

Mercer, T.T., Production of therapeutic aerosols: Principles and techniques. Chest, 80 (suppl.) (1981) 813-818

Mercer, T.T., Goddard, B.F. and Flores, R.L., Output characteristics of three ultrasonic nebulizers. Ann. Allergy, 26 (1968) 18-27

Mihalko, P.J., Schreier, H. and Abra, R.M., Liposomes: A pulmonary perspective. In Gregoriadis, G. (Ed) Liposomes as Drug Carriers, Recent Trends and Progress, WileyInterscience, Chichester (1988) pp679-694

Miles, P.R., Castronova, V. and Bowman, L., Catabolism of rat surfactant disaturated phosphatidylcholines during incubation of alveolar lavage materials in vitro at $37^{\circ} \mathrm{C}$. Biochem. Biophys. Acta, 836 (1985) 39-44

Minty, B., Jordam, C. and Jones, J.G., Rapid improvement in abnormal pulmonary epithelial permeability after stopping cigarettes. Br. Med. J., 282 (1981) 1183-1186

Mobley, W.C. and Schreier, H., Phase transition temperature reduction and glass formation in dehydroprotected lyophilised liposomes. J. Control. Rel., 31 (1994) 73-87

Morén, F., Aerosol dosage forms and formulations. In Morén, F., Dolovich, M.B., Newhouse, M.T. and Newman, S.P. (Eds.) Aerosols in Medicine. Principles, Diagnosis and Therapy. Elsevier, London (1993) pp27-60

Morrow, P.E., Evaluation of inhalation hazards based upon the respirable dust concept and the philosophy and application of selective sampling. Am. Ind. Hyg. Assoc. J., 25 (1964) 213-236

Morrow, P.E., Aerosol characterization and deposition. Am. Rev. Resp. Dis., 110 (1974) 8899

Morrow, P.E., Factors determining hygroscopic aerosol deposition in airways. Physiol. Rev., 66 (1986) 330-376

Muers, M.F., Overview of nebuliser treatment. Thorax, 52 (suppl. 2) (1997) S25-S30

Myers, D., Liquid-fluid interfaces. In Myers, D. (Ed) Surfaces, Interfaces, and Colloids. Principles and Applications. (1990) 139-174

Myers, M.A., Niven, R.W., Straub, L.E., Wichert, H., Schreier, H., Hood, C.I. and Gonzalez-Rothi, R.J., Pulmonary effects of chronic exposure to liposome aerosols in mice. Exp. Lung Res., 19 (1993) 1-19 
Navari, R.M., Gainer, J.L. and Updike, O.L., Blood flow modelling with microcapsular suspensions. Ind. Engng Chem Fund., 8 (1969) 615-620

Nebuliser Project Group, of the British Thoracic Society Standards of Care Committee, Current best practice for nebuliser treatment. Thorax, 52 (suppl. 2) (1997) S1-S106

Nerbrink, O. and Dahlbäck, M., Basic nebulizer function. J. Aerosol Med., 7 (suppl. 1) (1994) 7-11

New, R.R.C., Characterization of liposomes. In New, R.R.C. (Ed.) Liposomes: A Practical Approach., Oxford University Press, Oxford (1990) pp105-162

New, R.R.C., Chance, M.L., Thomas, S.C. and Peters, W., Antileishmanial activity of antimonials entrapped in liposomes. Nature, 272 (1978) 55-56

Newman, S.P., Aerosol physiology, deposition, and metered dose inhalers. Allergy Proc., 12 (1991) $42-45$

Newman, G.C. and Huang, C., Structural studies on phosphatidylcholine-cholesterol mixed vesicles. Biochem., 14 (1975) 3363-3369

Newman, S.P., Pavia, D. Morén, F., Sheahan, N.F. and Clark, S.W., Deposition of pressurized aerosols in the human respiratory tract. Thorax, 36 (1981) 52-55

Newman, S.P. and Clarke, S.W., Therapeutic aerosols. I. Physical and practical considerations. Thorax, 38 (1983) 881-886

Newman, S.P., Pellow, P.G.D., Clay, M.M. and Clarke, S.W., Evaluation of jet nebulisers for use with gentamicin solution. Thorax, 40 (1985) 670-676

Newman, S.P., Pellow, P.G.D. and Clarke, S.W., Droplet size distributions of nebulised aerosols for inhalation therapy. Clin. Phys. Physiol. Meas., 7 (1986) 139-146

Newman, S.P., Pellow, P.G.D. and Clarke, S.W., Dropsizes from medical atomizers (nebulisers) for drug solutions with different viscosities and surface tensions. Atom. Spray Tech., 3 (1987) 1-11

Newman, S.P., Woodman, G. and Clarke, S.W., Deposition of carbenicillin aerosols in cystic fibrosis: Effects of nebuliser system and breathing pattern. Thorax, 43 (1988) 318322

Nicalau, C. and Cudd, A., Liposomes as carriers of DNA. Crit. Rev. Therap. Drug Carrier Syst., 6 (1989) 239-271

Niven, R.W., Modulated drug therapy with inhalation aerosols. In Hickey, A.J. (Ed) Pharmaceutical Inhalation Technology, Marcel Dekker, New York. (1992) pp29-59 
Niven, R.W., Atomization and nebulizers. In Hickey, A.J. (Ed.) Inhalation Aerosols: Physical and Biological Basis for Therapy, Marcel Dekker, New York (1996) pp273-307

Niven, R.W. and Byron, P.R., Solute absorption from the airways of the isolated rat lung.

I. The use of absorption data to quantify drug dissolution or release in the respiratory tract. Pharm. Res., 5 (1988) 574-579

Niven, R.W. and Schreier, H., Nebulization of liposomes I. Effects of lipid composition. Pharm. Res., 7 (1990) 1127-1133

Niven, R.W., Speer, M. and Schreier, H., Nebulization of liposomes II. The effects of size and modelling of solute release profiles. Pharm. Res., 8 (1991a) 217-221

Niven, R.W., Haver, K. and Brain, J.D., Output characteristics of jet nebulizers containing solutions vs suspensions of steroids for inhalation therapy. Pharm. Res., 8 (1991b) S167

Niven, R.W., Carajal, T.M. and Schreier, H., Nebulization of liposomes III. The effects of operating conditions and local environment. Pharm. Res., 9 (1992) 515-520.

Niven, R.W., Steven, A.Y., Mittelman, S.D., Farrar, C., Arakawa, T. and Prestrelski, S.J., Protein nebulisation: I. Stability of lactate dehydrogenase and recombinant granulocytecolony stimulating factor to air-jet nebulization. Int. J. Pharm., 109 (1994) 17-26

Niven, R.W. and Brain, J.D., Some functional aspects of air-jet nebulizers. Int. J. Pharm., 104 (1994) 73-85

Niven, R.W., Ip, A.Y., Mittelman, S., Prestrelski, S.J. and Arakawa, T., Some factors associated with the ultrasonic nebulization of proteins. Pharm. Res., 12 (1995) 53-59

Notter, R.H. and Morrow, P.E., Pulmonary surfactant: A surface chemistry viewpoint. Ann. Biomed. Eng., 3 (1975) 119-159

Notter, R.H., Smith, S., Taubold, R.D. and Finkelson, J.N., Path dependence of adsorption behaviour of mixtures containing dipalmitoyl phosphatidylcholine. Ped. Res., 16 (1982) $515-519$

O'Callaghan, C. and Barry, P.W., The science of nebulised drug delivery. Thorax, 52 (Suppl. 2) (1997) S31-S44

O'Driscoll, B.R.C. and Cochrane, G.M., Emergency use of nebulised bronchodilator drugs in British hospitals. Thorax, 42 (1987) 491-493

O'Hagan, D.T. and Illum, L., Adsorption of peptides and proteins from the respiratory tract and the potential for development of a locally administered vaccine. Crit. Rev. Drug Car. Syst., 7 (1990) 35-97 
Olson, F., Hunt, C.A., Szoka, F.C., Vail, W.J. and Papahadjopoulos, D., Preparation of liposomes of defined size distribution by extrusion through polycarbonate membranes. Biochim. Biophys. Acta., 557 (1979) 9-23

Omri, A., Beaulac, C., Bouhajib, M., Montplasir, S., Sharkawi, M. and Lagacé, J., Pulmonary retention of free and liposome-encapsulated tobramycin after intratracheal administration in uninfected rats and rats infected with Pseudomonas aeruginosa. Antimicrob. Agent. Chemother., 38 (1994) 1090-1095

Padfield, J.M., Principles of drug administration to the respiratory tract. In Ganderton, D. and Jones, T.M. (Eds.) Drug Delivery to the Respiratory Tract, Ellis Horwood, Chichester (1987) pp76-86

Padley, F.B., Gunstone, F.D. and Harwood, J.L., Occurrence and characteristics of oils and fats. In Gunstone F.D., Harwood, J.L. and Padley, F.B. (Eds.) The Lipid Handbook, Chapman and Hall, London (1994) pp38-49

Padmanabhan, R.V., Gudapatay, R., Liener, I.E., Schwartz, B.A. and Hoidal, J.R., Protection against pulmonary oxygen toxicity in rats by the intratracheal administration of liposome-encapsulated superoxide dismutase or catalase. Am. Rev. Resp. Dis., 135 (1985) $731-737$

Papahadjopoulos, D., Liposomes and their uses in biology and medicine. Ann. NY Acad. Sci., 308 (1978) 1-462

Papahadjopoulos, D. and Watkins, J.C., Phospholipid model membranes II. Permeability properties of hydrated liquid crystals. Biochim. Biophys. Acta 135 (1967) 639-651

Papahadjopoulos, D., Poste, G. and Shaeffer, B.E., Fusion of mammalian cells by unilamellar lipid vesicles. Influence of lipid surface charge, fluidity and cholesterol. Biochim. Biophys. Acta, 323 (1973a) 23-42

Papahadjopoulos, D., Jacobson, K., Nir, S. and Isac, T., Phase transitions in phospholipid vesicles. Fluorescence and permeability measurements concerning the effect of temperature and cholesterol. Biochim. Biophys. Acta, 311 (1973b) 330-348

Patel, P., Mukai, D. and Wilson, A.F., Dose-response effects of two sizes of monodisperse isoproteronol in mild asthmatics. Am. Rev. Respir. Dis., 141 (1990) 357-360

Patton, J.S., McCabe, J.G., Hansen, S.E. and Daugherty, A.L., Absorption of human growth hormone from the rat lung. Biotech. Ther., 1 (1990) 213-228

Patton, J.S. and Platz, R.M., Routes of delivery: Case studies II. Pulmonary delivery of peptides and proteins for systemic action. Adv. Drug Del. Rev., 8 (1992) 176-196 
Pavia, D., Thompson, M.L., Clarke, S.W. and Shannon, H.S., Effect of lung function and mode of inhalation on penetration of aerosols into the lung. Thorax, 32 (1977) 194-197

Peskin, R.L. and Raco, R.J., Ultrasonic atomization of liquids. J. Acoust. Soc. Am., 35 (1963) 1378-1381

Petenazzo, A., Jobe, A., Machiko, I., Abra, R.M., Hogue, E. and Mihalko, P.J., Clearance of phosphatidylcholine and cholesterol from liposomes, liposomes loaded with metaproterenol, and rabbit surfactant from adult rabbit lungs. Am. Rev. Resp. Dis., 139 (1989) 752-758

Phipps, P.R. and Gonda, I., Droplets produced by medical nebulizers: Some factors affecting their size and solute concentration. Chest, 97 (1990) 1327-1333

Porstendörfer, J., Gerbhart, J. and Röbig, G., Effect of evaporation on the size distribution of nebulized aerosols. J. Aerosol Sci., 8 (1977) 371-380

Purcell, I.F. and Corris, P.A., Use of nebulised liposomal amphotericin B in the treatment of Aspergillus fumigatus empyema. Thorax, 50 (1995) 1321-1323

Raabe, O.G., The dilution of monodisperse suspensions for aerosolization. Am. Ind. Hyg. Assoc. J., 29 (1968) 439-443

Radhakrish, R., Mihalko, P.H. and Abra, R.M., Apparatus for the delivery of dehydrated liposomes by inhalation - useful in the treatment of bronchoconstriction and for systemicaction drug delivery. US Patent Application 4895719 (1990)

Reifenrath, R., Surfactant action in bronchial mucus transport. In Cosmi, E.V. and Scarpeli, E.M. (Eds.) Pulmonary Surfactant System, Elsevier, London (1983) 339-347

Rhoden, V. and Goldin, S.M., Formation of unilamellar lipid vesicles of controllable dimensions by detergent analysis. Biochem., 18 (1979) 4173-4176

Riaz, M., Weiner, N. and Martin, F., Liposomes. In Lieberman, L. (Ed.) Pharmaceutical Dosage Forms: Disperse Systems, Vol. 2., Marcel Dekker, New York (1988) pp567-703

Rizkalla, A.A. and Lefebvre, A.H., Influence of liquid properties on airblast atomizer spray characteristics. Trans. Am. Soc. Mech. Eng., (1974) 1-5

Robinson, N., A light scattering study of lecithin., Trans. Far. Soc., 56 (1960) 126-1264

Rudolph, A.S., The freeze-dried preservation of liposome encapsulated hemoglobin: A potential blood substitute. Cryobiol., 27 (1988) 277-284

Salzman, G.A. and Pyszczynski, D.R., Oropharangyeal candidiasis in patients treated with beclomethasone dipropionate delivered by metered-dose inhaler alone and with the aerochamber. J. Allergy Clin. Immunol., 81 (1988) 424-428 
Sato, Y., Sato, M., Kita, M. and Kishida, T., A comparison of $28 \mathrm{kHz}$ and $160 \mathrm{kHz}$ ultrasonic aerosolization of interferon-alpha. J. Aerosol Med., 5 (1992) 59-64

Schäfer, K.P., Molecular aspects of lung surfactant proteins and their use as pulmonal carriers. In Junginger, H.E. (Ed.) Drug Targetting and Delivery. Concepts in Dosage Form Design, Ellis Horwood, Chichester (1992) pp155-166

Schanker, L.S., Drug absorption from the lung. Biochem. Pharmacol., 27 (1978) 381-385

Schreier, H., McNicol, K.J., Ausborn, M., Soucy, D.W., Derendorf, H., Stecenko, A.A. and Gonzalez-Rothi, R.J., Pulmonary delivery of amikacin liposomes and acute liposome toxicity in the sheep. Int. J. Pharm., 87 (1992) 183-193

Schreier, H., Mobley, W.C., Concessio, N., Hickey, A.J. and Niven, R.W., Formulation and in vitro performance of liposome powder aerosols. S.T.P. Pharma Sciences, 4 (1994) 3844

Searls, E.M. and Snyder, F.M., Relation of viscosity to drop-sizes. J. Econ. Entomol., 29 (1936) $1167-1170$

Senior, J.H., Fate and behaviour of liposomes in vivo: A review of controlling factors. Crit. Rev. Ther. Drug Carr. Sys., 3 (1987) 123-193

Serafini, S.M., Wanner, A. and Michaelson, E.D., Mucociliary transport in central and intermediate size airways: Effects of aminophyllin. Bull. Europ. Physiopath. Resp., 12 (1976) 415-422

Shek, P.N., Jurima-Romet, M., Barber, R.F. and Demeester, J., Liposomes: Potential for inhalation prophylaxis and therapy. J. Aerosol Med., 1 (1988) 257-258

Shek, P.N., Suntres, Z.E. and Brooks, J.I., Liposomes in pulmonary applications: Physicochemical considerations, pulmonary distribution and antioxidant delivery. J. Drug Target., 2 (1994) 431-442

Shew, R.L. and Deamer, D., A novel method for encapsulation of macromolecules in liposomes. Biochim. Biophys. Acta, 816 (1985) 1-8

Simonds, A.K., Newman, S.P., Johnson, M.A., Talaee, N., Lee, C.A. and Clarke, S.W., Aerosolised pentamidine. Lancet, i (1989a) 221-222

Simonds, A.K., Newman, S.P., Johnson, M.A., Talaee, N., Lee, C.A. and Clarke, S.W., Simple nebuliser modification to enhance alveolar deposition of pentamidine. Lancet, ii (1989b) 953

Singleton, W.S., Gray, M.S., Brown, M.L. and White, J.L., Chromatographically homogenous lecithin from egg phospholipids. J. Am. Oil Chem. Soc., 42 (1965) 53-56 
Six, H.R., Gilbert, B.E., Wyde, P.R., Wilson, S.Z. and Knight, V., Liposomes as carriers of enviroxime for use in aerosol therapy of rhinovirus infections. In Lopez-Bernstein, G. and Fidler, I. (Eds.) Liposomes in the Therapy of Infectious Diseases and Cancer, Liss, New York (1989) 229-238

Smalldone, G.C., Perry, R.J., Bennett, W.D., Messina, M.S., Zwang, J. and Ilowite, J., Interpretation of 24 hour lung retention in studies of mucociliary clearance. J. Aerosol Med., 1 (1988) 11-20

Smith, R. and Tanford, C., The critical micelle concentration of 1- $\alpha$-dipalmitoyl phosphatidylcholine in water and water/methanol systems. J. Mol. Biol., 67 (1972) 75-83

Smith, S.J. and Bernstein, J.A., Therapeutic uses of lung aerosols. In Hickey, A.J. (Ed.) Inhalation Aerosols: Physical and Biological Basis for Therapy, Marcel Dekker, New York (1996) pp233-272

Smolensky, M.H., D’Alonao, G.E., Kunkel, G. and Barnes, P.J., Day-night patterns in bronchial patency and dyspnea - daily and unequally divided twice daily theophylline dosing schedules. Chronobiol. Int., 4 (1987) 303-317

Smye, S.W., Shaw, A., Norwood, H.M. and Littlewood, J.M., Some factors influencing the efficiency of a jet nebuliser system. Clin. Phys .Physiol. Meas., 11 (1990) 167-175

Sölner, K., The mechanism of the formation of fogs by ultrasonic waves., Trans. Faraday Soc., 32 (1936) 1532-1536

Stahlhofen, W., Gebhart, J. and Heyder, J., Experimental determination of the regional deposition of aerosol particles in the human respiratory tract. Am. Ind. Hyg. Assoc. J., 41 (1980) $385-398$

Stapleton, K.W. and Finlay, W.H., Determining solution concentration within aerosol droplets output by jet nebulizers. J. Aerosol Sci., 26 (1995) 137-145

Sterk, P.J., Plomp, A., Crobach, M.J.J.S., van de Vate, J.F. and Quanjer, P.H., The physical properties of a jet-nebuliser and their relevance to the histamine provocation test, Bull. Europ. Physiopath. Resp., 19 (1983) 27-36

Sterk, P.J., Plomp, A., Crobach, M.J.J.S., van de Vate, J.F. and Quanjer, P.H., Physical properties of aerosols produced by several jet- and ultrasonic nebulizers. Physiopath. Respir., 20 (1984) 65-72

Stern, N., Riklis, S., Kalina, M. and Tietz, A., The catabolism of lung surfactant by alveolar macrophages. Biochim. Biophys. Acta, 877 (1986) 323-333 
Stöber, W., Dynamic shape factors of nonspherical aerosol particles. In Mercer, T.T., Morrow, P.E. and Stöber, W. (Eds.) Assessment of Airborne Particles, C.C. Thomas, Springfield. (1972) pp249-289

Strauss, G., Freezing and thawing of liposome suspensions. In Gregoriadis, G. (Ed) Liposome Technology, Vol. 1 (2nd Edn.) CRC Press Inc. New York (1992) pp197-219

Stuart, B.O., Deposition of inhaled aerosols. Arch. Intern. Med., 131 (1973) 60-73

Suntres, Z.E., Hepworth, S.R. and Shek., P.N., Protective effect of liposome-associated $\alpha$ tocopherol against paraquat induced acute lung toxicity. Biochem. Pharmacol., 45 (1992) 1811-1818

Suntres, Z.E., Hepworth, S.R. and Shek, P.N., Pulmonary uptake of liposome-associated $\alpha$-tocopherol following intratracheal instillation in rats. J. Pharm. Pharmacol., 45 (1993) 514-520

Suntres, Z.E. and Shek, P.N., Incorporation of $\alpha$-tocopherol in liposomes promotes the retention of liposome-associated glutathione in the rat lung. Int. Care Med., 20 (1994) S91

Suntres, Z.E. and Shek, P.N., Liposome-associated antioxidants for pulmonary applications. In Shek, P.N. (Ed.) Liposomes in Biomedical Applications, Harwood Academic, Amsterdam (1995) pp199-208

Suurkuusk, J., Lentz, B.R., Barenholz, Y., Biltonen, R.L. and Thomson, T.E., A calorimetric and fluorescent probe study of the gel-liquid crystalline phase transition in small, single-lamellar dipalmitoylphosphatidylcholine vesicles. Biochem., 15 (1976) 1393-1401

Swift, D.L., Aerosols and humidity therapy. Am. Rev. Respir. Dis., 122 (1980) 71-77

Szoka, F.C., The future of liposomal drug delivery. Biotech. Appl. Biochem., 12 (1990) 496-500

Szoka, F. and Papahadjopoulos, D., Procedure for preparation of liposomes with large internal aqueous volumes and high capture by reverse-phase evaporation. Proc. Natl. Acad. Sci. USA, 75 (1978) 4194-4198

Szoka, F. and Papahadjopoulos, D., Comparative properties and methods of preparation of lipid vesicles (liposomes). Ann. Rev. Biophys. Bioeng., 9 (1980) 467-508

Taeusch, H.W., Clements, J. and Benson, M., Exogenous surfactant for human lung disease. Am. Rev. Resp. Dis., 128 (1983) 791-794 
Takada, M., Yuzuriha, T., Katayama, K., Iwamoto, K. and Sunamoto, J., Increased lung uptake of liposomes coated with polysaccharides. Biochim. Biophys. Acta, 802 (1984) 237-244

Talsma, H., Özer, A.Y., Van Bloois, L. and Crommelin, D.J.A., The size reduction of liposomes with a high pressure homogenizer (Microfluidizer ${ }^{\mathrm{TM}}$ ). Characterization of prepared dispersions and comparison with conventional methods. Drug. Dev. Ind. Pharm., 16 (1990a) 2167-2191

Talsma, H, van Steenbergen, M.J. and Crommelin, D.J., The cryopreservation of liposomes: 3. Almost complete retention of a water-soluble marker in small liposomes in a cryoprotectant containing dispersion after a freezing/thawing cycle. Int. J. Pharm., 77 (1990b) 119-126

Talsma, H. and Crommelin, D.J.A., Liposomes as drug delivery systems, part I: Preparation. Pharm. Tech. Intern., Nov. (1992) 24-29

Tanford, C., Introduction. In Tanford, C. (Ed) The Hydrophobic Effect. Formation of Micelles and Biological Membranes, Wiley Interscience, New York. (1980) pp1-20

Tansey, I.P., MDIs - past, present and future. Drug Delivery to the Lung VI, The Aerosol Society, London (1995) p5

Task Group on Lung Dynamics, Deposition and retention models for internal dosimetry of the human respiratory tract. Health Phys., 12 (1966) 173-207

Taylor, K.M.G., Taylor, G., Kellaway, I.W. and Stevens, J., The influence of liposomal encapsulation on sodium cromoglycate pharmacokinetics in man. Pharm. Res., 6 (1989) 633-636

Taylor, K.M.G., Taylor, G., Kellaway, I.W. and Stevens, J., Drug entrapment and release fom multilamellar and reverse-phase evaporation liposomes., Int. J. Pharm., 58 (1990a) $49-55$

Taylor, K.M.G., Taylor, G., Kellaway, I.W. and Stevens, J., The stability of liposomes to nebulisation. Int. J. Pharm., 58 (1990b) 57-61.

Taylor, K.M.G., Venthoye, G. and Chawla, A., Pentamidine isethionate delivery from jet nebulisers. Int. J. Pharm., 85 (1992) 203-208

Taylor, K.M.G. and Farr, S.J., Liposomes for drug delivery to the respiratory tract. Drug Dev. Ind. Pharm., 19 (1993) 123-142.

Taylor, K.M.G. and Hoare, C., Ultrasonic nebulisation of pentamidine isethionate. Int. J. Pharm., 98 (1993) 45-49 
Thomas, S.H.L., Langford, J.A., George, R.D.J. and Geddes, D.M., Improving the efficiency of drug administration with jet nebulisers. Lancet., i (1988) 126

Thomas, S.H.L., O’Doherty, M.J., Page, C.J., Nunan, T.O. and Bateman, N.T., Which apparatus for inhaled pentamidine? A comparison of deposition via eight nebulisers. Eur. Respir. J., (1991a) 616-622

Thomas, D.A., Myers, M.A., Wichert, B.M., Schreier, H. and Gonzalez-Rothi, R.J., Acute effects of liposome aerosol inhalation on pulmonary function in healthy human volunteers. Chest, 99 (1991b) 1268-1270

Thompson, D.C., Pharmacology of therapeutic aerosols. In Hickey, A.J. (Ed.) Pharmaceutical Inhalation Technology. Marcel Dekker, New York. (1992) 29-59

Thurow, H. and Geisen, K., Stabilisation of dissolved proteins against denaturation at hydrophobic interfaces. Diabetologia, 27 (1984) 212-218

Toogood, J.H., Jennings, B., Baskerville, J. and Johansson, S.-L., Clinical use of spacer systems for corticosteroid inhalation therapy: A preliminary analysis. Eur. J. Resp. Dis., 63 (suppl. 122) (1982) 100-107

Travers, D.N., Drying. In Aulton, M.E. (Ed.) Pharmaceutics: The Science of Dosage Form Design, Churchill Livingstone, Edinburgh (1988) pp629-646

Tsuchida, E., Nishide, H. and Ohno, H., Liposome/heme as a totally synthetic oxygen carrier. Biomat., Art, Cells, Art., Org., 16 (1988) 313-319

Vemuri, S. and Rhodes, C.T., Preparation and characterisation of liposomes as therapeutic systems: A review. Pharmaceutica Acta Helvetiae, 70 (1995) 95-111

Vidal-Naquet, A., Gossage, J.L., Sullivan, T.P., Haynes, J.W., Gilruth, B.H. and Beissenger, R.L., Liposome-encapsulated haemoglobin as an artificial red blood cell: Characterization and scale-up. Biomat., Art. Cells, Art. Org., 16 (1989) 531-552

Vidgren, M., Waldrep, J.C., Arppe, J., Black, K., Rodarte, J.A., Cole, W. and Knight, V.,

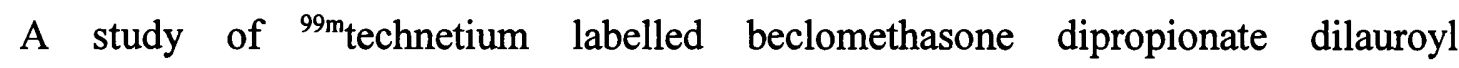
phosphatidylcholine liposome aerosol in normal volunteers. Int. J. Pharm., 115 (1995) $209-216$

Virchow, J.C., Kroegel, C. and Mätthys, H., Antiasthma therapy: What is on the horizon? Clin. Pharmacokinet., 27 (1994) 85-93

Waldman, D.L., Weber, D.A., Oberdorster, G., Drago, S.R., Utell, M.J., Hyde, R.W. and Morrow, P.E., Chemical breakdown of technetium-99m DTPA during nebulisation. $J$. Nucl. Med., 28 (1987) 378-382 
Waldrep, J.C., Pulmonary delivery of anti-inflammatory glucocorticoids and immunosuppressive cyclosporin-A by liposome aerosols. J. Aerosol Med., 8 (1995) 171P

Waldrep, J.C., Scherer, P.W., Keyhani, K. and Knight, V., Cyclosporin A liposome aerosol: Particle size and calculated respiratory deposition. Int. J. Pharm., 97 (1993) 205-212

Waldrep, J.C., Keyhani, K., Black, M. and Knight, V., Operating characteristics of 18 different continuous-flow jet nebulisers with beclomethasone dipropionate liposome aerosol. Int. J Pharm., 105(1) (1994) 106-110

Waldrep, J.C., Gilbert, B.E., Knight, C.M., Black, B.S., Scherer, P.W., Knight, V. and Eschenbacher, W., Pulmonary delivery of beclomethasone liposome aerosol in volunteers. Tolerance and safety. Chest, 111 (1997) 316-323

Walkenhurst, W. and Dautrebande, L., Experimental observations on various factors influencing weight, number, flow rate and size distribution of aerosol particles. Arch. Int. Pharmacodyn., 150 (1964) 264-294

Weg, J.G., Balk, R.A., Theralt, S., Jenkinson, S.G., Shah, J.B., Zaccardelli, D., Horton, J. and Pattishall, E.N., Safety and potential efficacy of an aerosolised surfactant in human sepsis-induced adult respiratory distress syndrome. J. Am. Med. Assoc., 272 (1994) 14331438

Weibel, E.R., Morphometry of the Human Lung, Springer-Verlag, Berlin (1963) pp1-151 Weibel, E.R., Gehr, P., Haies, D., Gil, J. and Bachofen, M., The cell population of the normal lung. In Bouhuys, M.D. (Ed.), Lung Cells in Disease, Elsevier, Amsterdam (1976) pp3-16

Weinstein, J.N., Yoshikami, S., Henkart, P., Blumenthal, R. and Hagins, W.A., Liposomecell interaction: Transfer and intracellular release of a trapped fluorescent marker. Science, 195 (1977) 489-492

Wells, R.E., Denton, R. and Merill, E.W., Measurement of viscosity of biologic fluids by a cone plate viscometer. J. Lab. Clin. Med., 57 (1961) 646-656

Wells, R.E., Merrill, E.W. and Gabelnick, H., Shear-rate dependence of viscosity of blood: Interaction of red cells and proteins. Trans. Soc. Rheol., VI (1962) 19-24

Whitham, M.E. and Eagle, A.M., Alternative propellants: proprietary rights, toxicological issues and projected licensing problems. Proc. Drug Del. Lung IV, (1994) 203-209 
Wichert, B.V, Gonzalez-Rothi, R.J., Straub, L.E., Wichert, B.M. and Schreier, H., Amikacin liposomes: Preparation, characterization, and in vitro activity against Mycobacterium avium-intracellulare infection in alveolar macrophages. Int. J. Pharm., 78 (1992) 227-235

Wigley, F.M., Londono, J.H., Scott, H.W., Shipp, J.C. and Waldmann, R.H., Insulin across respiratory mucosae by aerosol delivery. Diabetes, 20 (1971) 552-556

Williams, M.S., Conversion of lamellar body membranes into tubular myelin in alveoli of fetal rat lungs. J. Cell. Biol., 72 (1977) 260-277

Wong, M. and Thompson, T.E., Aggregation of dipalmitoyl phosphatidylcholine vesicles. Biochem., 21 (1982) 4133-4138

Woodle, M.C. and Lasic, D.D., Sterically stabilised liposomes. Biochim. Biophys. Acta, 1113 (1992) 171-199

Woolfrey, S.G., Taylor, G., Kellaway, I.W. and Smith, A., Pulmonary absorption of liposome encapsulated 6-carboxyfluorescein. J. Controlled Rel., 5 (1988) 203-209

Wyde, P.R., Six, H.R., Wilson, S.Z., Wyde, P.R. and Knight, V., Activity against rhinoviruses, toxicity, and delivery in aerosol of enviroxime in liposomes. Antimicrob. Agents Chemother., 32 (1988) 890-895

Yoshimura, K., Rosenfeld, M.A., Nakamura, H., Scherer, E.M., Pavirani, A., Lecocq, J.-P. and Crystal, R.G., Expression of the human cystic fibrosis transmembrane conductance receptor gene in the mouse lung after in vivo intratracheal plasmid-mediated gene transfer. Nucleic Acids Res., 20 (1992) 3233-3240

Zachman, R.D. and Tsao, F.H.C., Pulmonary uptake of liposomal phosphatidylcholine. Pediatr. Res., 14 (1980) 24-27

Zainudin, B.M.Z., Biddiscombe, M., Tolfree, S.E.J., Short, M. and Spiro, S.G., Comparison of bronchodilator responses and deposition patterns of salbutamol inhaled from a pressurized metered dose inhaler, as a dry powder, and as a nebulised solution. Thorax, 45 (1990) 469-473 